\title{
Abstracts from the 2020 International Congress of Meat Science and Technology and the AMSA Reciprocal Meat Conference
}

\section{Animal Welfare}

\section{1 - PERFORMANCE AND MEAT QUALITY OF WATER-RESTRICTED XHOSA GOATS SUPPLEMENTED WITH VITAMIN C}

O. F. Akinmoladun ${ }^{1,2 *}$, F. Fon ${ }^{3}$, C. Mpendulo ${ }^{1}$, and O. Okoh ${ }^{4},{ }^{1}$ Department of Livestock and Pasture Science, University of Fort Hare, Alice, South Africa, ${ }^{2}$ Animal and Environmental Biology, Adekunle Ajasin University, Akungba-Akoko, Nigeria, ${ }^{3}$ Agriculture, University of Zululand, Kwadlangezwa, ${ }^{4}$ Applied Chemistry, University of Fort Hare, Alice, South Africa, *201821379@ufh.ac.za

Objectives: The continuous shortfall in rainfall patterns especially in the dry zones of the world could result in preslaughter stress (due to limited water intake [WI]) as well as affect the meat quality of animals. This study evaluated the effect of water restriction periods with or without single and/or multiple vitamin C (VC) supplementations on performance and meat quality of Xhosa goats.

Materials and Methods: Goats (42) were distributed into 7 treatment groups in a completely randomized design: without water restriction (GI, control), water restrictions of $70 \%$ (GII) and 50\% (GIII) of ad libitum WI, water restrictions of $70 \%(\mathrm{GIV})$ and $50 \%(\mathrm{GV})$ of ad libitum WI plus $3 \mathrm{~g} \mathrm{VC}$ daily, and water restrictions of $70 \%$ (GVI) and $50 \%$ (GVII) of ad libitum WI plus $3 \mathrm{~g}$ VC and an extra $5 \mathrm{~g} \mathrm{VC}$ given every $8 \mathrm{~d}$. VC was administered per os. Animal adaptation was for $14 \mathrm{~d}$ and $75 \mathrm{~d}$ for data collection. Water restriction percentages were calculated based on daily ad libitum intake of the control group after rebating loss due to evaporation. Water restriction- $70 \%$ and water restriction- $50 \%$ groups did receive drinking water daily at a level of $70 \%$ and $50 \%$ of the total WI recorded in the $100 \%$-water group, respectively. The efficiency of water use was determined by finding the ratio of WI to dry matter intake (WI:DMI). At the end of the trial, slaughtering was done following standard procedures. $\mathrm{pH}$ was measured in the Longissimus lumborum) muscle between the 12th and 13th ribs, $45 \mathrm{~min}$ and $24 \mathrm{~h}$ post-slaughter. Four pieces ( $2 \mathrm{~cm}$ thick) were carefully cut from the Longissimus lumborum muscle of the carcass and randomly assigned for color, drip loss, cooking loss, and Warner-Bratzler Shear force determination. Meat color (lightness, $L^{*}$; redness, $a^{*}$; and yellowness, $b^{*}$ ) was measured after $24 \mathrm{~h}$ of slaughter from the longissimus muscle. Data obtained were analyzed using the generalized linear model of SAS (SAS Institute Inc., Cary, NC).

Results: The depression in final weight due to water restriction was lessened following VC supplementation. The DMI decreased $(P<0.05)$ in response to water restriction levels and rose in the VC-treated groups. The WI decreased $(P<0.05)$ as the percentage of ad libitum water given decreased. The WI:DMI decreased $(P<0.05)$ with an increase in levels of water restriction. Regardless of VC concentration and/or supplementation, all the waterrestricted groups had decreased $L^{*}$ (lightness) $(P=$ $0.0002)$ and $b^{*}$ (yellowness) $(P=0.0048)$. However, the water-restricted treated groups tended to increase $(P=$ $0.0058) a^{*}$ (redness) values compared to the water-restricted untreated and control groups. The drip loss, $\mathrm{C}^{*}, \mathrm{pH}_{45 \mathrm{~min}}$, and $\mathrm{pH}_{24 \mathrm{~h}}$ were not affected $(P>0.05)$ by levels of water restriction and $\mathrm{VC}$ supplementation. Regardless of VC supplementation, cooking loss and shear force significantly increased $(P<0.05)$ with water restriction levels.

Conclusion: This study demonstrates that the decreased body weight due to suboptimal WI could be lessened with daily VC supplementation. Supplementation of VC did improve meat redness $\left(a^{*}\right)$, which is important to consumers when making purchasing choices. Multiple VC failed to additively improve on the positive outcomes from a single VC dosage. Despite the limited WI, the increase in meat shear force did not exceed the medium tenderness range $\left(26.40-33.31 \mathrm{~N} / \mathrm{cm}^{2}\right)$.

Keywords: goat, meat quality, performance, vitamin C, water restriction 


\section{2 - MEAT QUALITY OF PIGS TRANSPORTED TO SLAUGHTER USING TWO NOVEL TRAILER DESIGNS VERSUS A STANDARD POT-BELLY TRAILER UNDER CANADIAN CONDITIONS}

K. Moak ${ }^{1,2^{*}}$, S. Conte ${ }^{2}$, R. Bergeron ${ }^{1}$, B. M. Bohrer ${ }^{1}$, G. A. Ferreira $^{3}$, J. Vero ${ }^{3}$, G. Aboagye ${ }^{4}$, S. Khalife ${ }^{1}$, A. Thompson $^{1}$, A. M. Bridi ${ }^{3}$, N. Devillers ${ }^{2}$, and L. Faucitano ${ }^{2}$, ${ }^{1}$ Animal Biosciences, University of Guelph, Guelph, Ontario, Canada, ${ }^{2}$ Swine Welfare and Behaviour, Agriculture and Agri-Food Canada, Sherbrooke, Canada, ${ }^{3}$ Departamento de Zootecnia, Universidade Estadual de Londrina, Londrina, Brazil, ${ }^{4}$ Animal Production, Università di Bologna, Bologna, Italy,*kmoak@uoguelph.ca

Objectives: This study evaluated and compared effect of 2 novel trailer designs against the current standard North American pot-belly (PB) trailer on meat quality of pigs during short transport under Canadian summer and winter conditions.

Materials and Methods: A total of 36 loads of pigs ( 3 trailers, 2 seasons, 6 replicates per trailer design per season) were transported from 9 similar commercial farms to a commercial processing facility located in Southern Ontario (1 h $44 \mathrm{~min}$ average trip) over both summer 2019 (July-August) and winter 2020 (January-February). Within each trailer, 9 sentinel crossbred barrows (27 sentinel pigs per replicate), weighing $130 \pm$ $0.29 \mathrm{~kg}$ on average, were randomly selected from the same finishing pen and either allocated to (1) a standard passively ventilated PB featuring internal ramps, (2) a modified PB equipped with fan-assisted ventilation and water drinkers, or (3) a triple flat-deck trailer (designed by Pezzaioli, Italy) equipped with fully hydraulic middle and top deck, fan-assisted ventilation, water misters, an adjustable rooftop compartment, and water drinkers. These sentinel pigs were allocated and mixed with nonsentinel pigs to 3 test compartments in each trailer ( 3 sentinel pigs/3 compartments/trailer) with an average trailer density of $5.6 \mathrm{ft}^{2} / \mathrm{pig}$ in summer and $5 \mathrm{ft}^{2} /$ pig in winter. Meat quality of sentinel pigs was assessed at $24 \mathrm{~h}$ postmortem by measuring ultimate $\mathrm{pH}(\mathrm{pHu})$ and drip loss in the longissimus muscle, and $\mathrm{pHu}$ in the semimembranosus muscle and adductor muscle. Data were analyzed using SAS mixed procedure (SAS Institute Inc., Cary, NC) with the trailer compartment as the experimental unit.

Results: The quality of the longissimus muscle was influenced by the trailer type $\times$ season and compartment $\times$ season interaction, with the $\mathrm{pHu}$ value tending to be lower in pigs transported in the modified PB compared to those transported in the Pezzaioli trailer during winter $(P=$ 0.06 ), and drip loss was lower (less exudative) in pigs shipped in the bottom front compartment compared to the middle compartment $(1.45 \%$ vs. $1.9 \%)$ during summer $(P=0.04)$, regardless of trailer type. The $\mathrm{pHu}$ of the semimembranosus and adductor muscles was lower in pigs transported in the middle deck compartment compared to those transported in the top rear compartment of the PB trailer during summer $(P=0.02$ and $P=0.04$, respectively). A lower $\mathrm{pHu}$ value was found in the adductor muscle of pigs transported in the PB bottom front compartment compared to the top rear compartment during winter $(P=0.01)$.

Conclusion: Overall, the results of this study show the negative effect of the compartment location within trailer on meat quality. The loading order and the low deck height may explain the reduced meat quality in pigs transported in the middle and bottom decks, respectively, within the PB trailer. The shared result of the bottom front compartment on pork exudation between all trailers, perhaps due to low deck height impeding sufficient airflow and handling ease, indicates that this compartment may need to be redesigned in all trailers. Both summer and winter had an additive effect on meat quality variation, implying that improving climate control in specific trailer compartments should be made to ensure improved meat quality.

Keywords: meat quality, pigs, season, transport, vehicle design

\section{3 - DOES CHARCOAL REDUCE SKATOLE AND INDOLE IN THE CHYME OF PIGLETS?}

F. Witte ${ }^{1}$, D. C. Schubert ${ }^{2}$, C. Visscher ${ }^{2}$, V. Heinz ${ }^{1}$, and N. Terjung ${ }^{1 *},{ }^{1}$ Product Innovation, DIL e. V., Quakenbrück, Germany, ${ }^{2}$ Institute of Animal Production, University of Veterinary Medicine Hannover, Hannover, Germany, *n.terjung@dil-ev.de

Objectives: Boar taint occurs by combining 3-methyl-indole (skatole) and 5 $\alpha$-androstenone (androstenone). The presence of skatole and indole in the large intestine is caused by fermentation of L-tryptophan in the colon (Deslandes et al., 2001; Lundström et al., 2009; Visscher et al., 2018). A clear correlation of concentration of these indoles in the intestine and their concentration in pigs' fat tissue has been demonstrated (Borg et al., 1993). Charcoal is known for its good adsorption capacity and already has been fed to pigs to reduce boar taint (Jen and Squires, 2011). Thus, application of a coated charcoal that remains available until the intestine to reduce boar-taint-causing substances might be possible. The hypothesis of this study was that coated charcoal adsorbs more skatole and indole than natural charcoal and control feed.

Materials and Methods: Intact male piglets $(n=12$; mother: German Landrace $\times$ German Edelschwein; father: Pietrain), born from 3 sows, were fattened for $21 \mathrm{~d}$, beginning at $25 \pm 1 \mathrm{~d}$ of life. Diet consisted of either $2 \% \mathrm{w} / \mathrm{w}$ natural charcoal or $4 \% \mathrm{w} / \mathrm{w}$ coated charcoal including $2 \% \mathrm{w} / \mathrm{w}$ charcoal (COATED CC) that were added to control feed. As control, 4 piglets were solely fattened with control feed. To prevent loading prior to colon, charcoal was coated with $50 \%$ rapeseed fat having a high melting point. Directly after 
euthanasia, chyme from caecum and colon was retrieved and kept frozen until analyses. Preparation of chyme samples was conducted by established method adapted from Gibis, Dehnhard, and Fischer (1991) and analyzed by ultraperformance liquid chromatography-tandem mass spectrometry (ACQUITY-UPLC-System, Waters/API4000, AB Sciex). An external reference of skatole and indole was used to calibrate. Results were given in $\mu \mathrm{g} / \mathrm{kg}$ initial substance. Therefore, in a second step, moisture of chyme was evaporated by a drying chamber at $103^{\circ} \mathrm{C}$ and then dry matter calculated. Significant differences were determined using one-way analysis of variance in Sigma Plot 14.0 (Systat Software Inc., San Jose, CA), followed by a Tukey test $(P<0.05)$.

Results: Besides significant differences between skatole or indole contents of animals in each feeding group, indole is significantly less abundant in the colon chyme of piglets fed with coated charcoal as well as skatole in the caecum chyme of piglets of NATURAL and COATED CC (Table 1). In the colon chyme, indole is reduced from control group $17 \mu \mathrm{g} / \mathrm{g}$ dry matter to $2 \mu \mathrm{g} / \mathrm{g}$ dry matter in the COATED CC, and in the caecum chyme, skatole is reduced from 28 to $3 \mu \mathrm{g} / \mathrm{g}$ dry matter, respectively. Moreover, tendencies towards reduced skatole contents in the colon chyme are obvious.

Conclusion: Using coated charcoal seems to contribute to reductions in skatole and indole content in the chyme. NATURAL CC also reduced indoles but not as significant as COATED CC. However, considering that chyme only gives possibility of boar taint reduction, more research is needed. A feeding approach with a larger number of boars is running currently. Initial results of skatole and indole in the chyme do not support our findings here. In this study, we could not prove whether there have been interactions of charcoal on piglets' diet. Thus, variations can be due to diet but also caused by animal-individual issues. Nevertheless, skatole and androstenone reduction in fat will determine success of a boar-taint reduction by charcoal in pigs' diet.

Acknowledgments: This study was conducted within $\mathrm{AiF} 20221 \mathrm{~N}$ of FEI, promoted by BMWi.

Keywords: boar taint, charcoal, coating, indole, skatole

\section{Consumer Topics}

\section{4 - A COMPARISON OF CONSUMER RESPONSE USING PAPER AND DIGITAL BALLOTS FOR EATING QUALITY ASSESSMENT OF BEEF STEAKS}

\author{
K. T. Mahagan ${ }^{1 *}$, A. J. Garmyn ${ }^{1}$, J. F. Legako ${ }^{1}$, and M. F. \\ Miller ${ }^{1},{ }^{1}$ Animal and Food Sciences, Texas Tech University, \\ Lubbock, TX, USA, *kyle.mahagan@ttu.edu
}

Objectives: The ultimate goal of Meat Standards Australia is the continual improvement to the predictive capability of the carcass grading system, where carcass inputs can be used to predict eating quality of a variety of cuts in the beef carcass. According to Meat Standards Australia protocol, consumer data, which have been collected from thousands of consumers globally, have historically been collected on paper ballots with 100-mm line scales for evaluation of tenderness, juiciness, liking of flavor, and overall liking. Ballots are then scored either digitally or by hand before compiling the consumer data. These methods can be quite timeconsuming and leave room for human error during the scoring and data entry processes. The use of electronic devices equipped with sensory software or digital ballots has

Table 1: Mean \pm standard error of the mean of indole and skatole $(\mu \mathrm{g} / \mathrm{g} \mathrm{DM})$ contents in colon and caecum chyme of piglets from groups fed with solely control feed or additionally $2 \%$ natural charcoal or $4 \%$ coated charcoal with $2 \%$ charcoal. Significant differences between indole and skatole contents in one group as well as between feeding groups are depicted by lower-and uppercase letters, resp., and were determined using One Way ANOVA and Tukey test $(p<0.05) .(n=3)$

\begin{tabular}{|c|c|c|c|c|c|c|c|c|c|c|c|}
\hline \multirow{3}{*}{$\begin{array}{c}\text { Colon } \\
1\end{array}$} & \multicolumn{5}{|c|}{ Indole ( $\mu \mathrm{g} / \mathrm{g} \mathrm{DM})$} & \multicolumn{6}{|c|}{ Skatole ( $\mu \mathrm{g} / \mathrm{g} D M)$} \\
\hline & \multicolumn{2}{|c|}{ Control } & \multicolumn{2}{|c|}{ Natural CC } & \multirow{2}{*}{$\frac{\text { Coated CC }}{1.3^{\circ} \pm 0.0}$} & \multicolumn{2}{|c|}{ Control } & \multicolumn{2}{|c|}{ Natural CC } & \multicolumn{2}{|c|}{ Coated CC } \\
\hline & $12.2^{\circ}$ & \pm 0.9 & $1.4^{\circ}$ & \pm 0.0 & & $169.0^{\circ}$ & \pm 11.6 & $84.1^{\circ}$ & \pm 9.4 & $1.3^{\circ}$ & \pm 0.0 \\
\hline 2 & $8.8^{\circ}$ & \pm 0.3 & $5.1^{\circ}$ & \pm 1.0 & $3.1^{b} \pm 0.4$ & $38.0^{b}$ & \pm 1.4 & $35.2^{b}$ & \pm 2.7 & $51.7^{b}$ & \pm 4.5 \\
\hline 3 & $23.5^{b}$ & \pm 3.3 & $27.6^{b}$ & \pm 2.1 & $1.7^{\circ} \pm 0.0$ & $73.3^{c}$ & \pm 5.1 & $69.0^{\circ}$ & \pm 5.1 & $38.4^{4}$ & \pm 0.5 \\
\hline 4 & $24.1^{b}$ & \pm 1.8 & $33.0^{\circ}$ & \pm 2.0 & $1.6^{\circ} \pm 0.0$ & $4.2^{d}$ & \pm 0.4 & $13.3^{b}$ & \pm 0.8 & $20.6^{d}$ & \pm 1.6 \\
\hline Mean & $17.1^{\mathrm{A}}$ & \pm 2.0 & $16.8^{A}$ & \pm 3.8 & $1.9^{8} \pm 0.2$ & 71.1 & \pm 17.3 & 50.4 & \pm 8.0 & 28.0 & \pm 5.3 \\
\hline \multicolumn{12}{|l|}{ Caecum } \\
\hline 1 & $9.3^{\circ}$ & \pm 1.2 & $2.5^{\circ}$ & \pm 0.0 & $27.9^{\circ} \pm 1.9$ & $26.9^{\circ}$ & \pm 6.3 & $3.7^{\circ}$ & \pm 0.6 & $2.9^{\circ}$ & \pm 0.0 \\
\hline 2 & $5.9^{\circ}$ & \pm 1.1 & $12.7^{\circ}$ & \pm 0.9 & $2.7^{b} \pm 0.5$ & $2.3^{b}$ & \pm 0.0 & $2.0^{\circ}$ & \pm 0.0 & $2.3^{b}$ & \pm 0.1 \\
\hline 3 & $8.2^{\circ}$ & \pm 0.5 & $45.8^{\mathrm{b}}$ & \pm 4.9 & $2.4^{b} \pm 0.0$ & $81.2^{\mathrm{a}}$ & \pm 5.8 & $2.9^{3}$ & \pm 0.0 & $2.6^{\circ b}$ & \pm 0.2 \\
\hline 4 & $23.9^{\circ}$ & \pm 4.6 & $11.2^{\circ}$ & \pm 1.2 & $2.4^{b} \pm 0.1$ & $2.4^{b}$ & \pm 0.0 & $2.8^{\circ}$ & \pm 0.4 & $2.2^{s b}$ & \pm 0.0 \\
\hline Mean & 11.8 & \pm 2.2 & 18.0 & \pm 4.7 & $8.8 \pm 3.1$ & $28.2^{A}$ & \pm 9.1 & $2.9^{8}$ & \pm 0.2 & $2.5^{8}$ & \pm 0.1 \\
\hline
\end{tabular}


emerged as an efficient means of collecting data from sensory trials. The purpose of this study was to determine whether consumers scored beef palatability traits differently on paper versus with digital ballots.

Materials and Methods: Beef subprimals were collected to represent 4 treatments with inherent variation in eating quality: USDA Select eye of round aged $7 \mathrm{~d}$ postmortem, USDA Select strip loin aged $7 \mathrm{~d}$ postmortem, USDA Choice tenderloin aged $21 \mathrm{~d}$ postmortem, and USDA Prime strip loin aged $21 \mathrm{~d}$ postmortem. Accessory muscles, external fat, and connective tissue were removed from subprimals. Muscles were fabricated into $2.5 \mathrm{~cm}$ steaks and further divided into 2 equal halves for consumer testing. Consumers $(n=360)$ were served 8 cooked samples divided into 2 blocks representing the 2 ballot types. Select strip loin samples were always served in the first and fifth position to start each ballot block, with the remaining 3 treatments served in a randomized order between the latter 3 positions within each ballot block. Consumers rated each steak sample for tenderness, juiciness, flavor liking, and overall liking on either a paper or digital ballot, and then rated the paired steak halves on the opposite ballot during the second block of sample testing. Both ballots utilized 100-mm line scales for the scoring of palatability traits, with reference hash marks at 20,40,60, and $80 \mathrm{~mm}$.

Results: No interactions between treatment and ballot type were observed $(P>0.05)$ for any palatability traits. Ballot type influenced $(P<0.02)$ all traits, as consumers scored tenderness, juiciness, flavor liking, and overall liking greater $(P<0.05)$ on paper ballots compared to digital ballots, regardless of treatment. The smallest margin between paper and digital ballots was observed for tenderness, where scores only differed by 1.8 units. However, juiciness, flavor liking, and overall liking all differed by 3.4 units in favor of paper ballots. Treatment influenced $(P<0.05)$ all palatability traits. Overall, Choice tenderloin were liked the most, followed by Prime strip loin, Select strip loin, and Select eye of round, with a significant decline $(P<0.05)$ between each treatment.

Conclusion: Consumers scored palatability traits greater on paper ballots compared to digital ballots, and these findings were consistent across treatments. The findings of this study need to be taken into consideration when comparing studies conducted with paper ballot versus a digital ballot or vice versa. Furthermore, an adjustment factor may be necessary to accurately compare data collected using the 2 ballot types.

Keywords: consumer, digital ballot, palatability, sensory panels

\section{5 - CONSUMER ACCEPTANCE OF GRASS- FED BEEF USING DIFFERENT FEEDING SYSTEMS}

T. L. Duarte ${ }^{1 *}$, S. Klopatek ${ }^{1}$, B. Bolkenov ${ }^{1}$, L. Yang ${ }^{1}$, J. Oltjen ${ }^{1}$, and X. Yang ${ }^{1},{ }^{1}$ Animal Science, University of California Davis, Davis, CA, USA, *tlduarte@ucdavis.edu
Objectives: Growing consumer concern over issues of sustainability, animal welfare, and health have led to an increased demand for products like grass-fed beef. As consumers' interest in grass-fed beef continues to rise, so too does the need for research that evaluates the impacts that feeding methods have on the palatability of the final meat product. Therefore, the objective of this study was to evaluate consumers' preference on the sensory attributes of beef steaks from animals raised on conventional grain-fed (CON; $n=12$ ), 20-mo grass-fed (GF20; $n=12$ ), 25-mo grass-fed (GF25; $n=12$ ), and 20-mo grass-fed 45-d grain-finished (GG; $n=9$ ) feeding systems.

Materials and Methods: Consumer sensory panel evaluations were conducted at the University of California, Davis. At each panel day, steaks were thawed at $4^{\circ} \mathrm{C}$ for $24 \mathrm{~h}$ prior to cooking. They were then cooked to an internal temperature of $71^{\circ} \mathrm{C}$ using a George Foreman clamshell. Internal temperature was measured at the geometric center of each steak using a $\mathrm{K}$ thermocouple thermometer. Following cooking, steaks were rested for $3 \mathrm{~min}$ and then cut into ten $1.5 \mathrm{~cm}$ by $1.5 \mathrm{~cm}$ cubes. Samples were then placed into glass bowls prelabeled with a unique 3-digit random number, covered with tin foil, and stored in an insulated food warmer. One hundred and twenty untrained participants evaluated samples over a period of 6 sessions. Each participant tasted and evaluated 4 samples per session meaning that each sample was evaluated by 10 consumers. At the beginning of each session, participants were asked to fill out a background survey. Each participant was given 2 pieces of steak cubes per sample and asked to cleanse their palate between samples using unsalted saltine crackers, apple juice, and water. They were then asked to evaluate the likeness of tenderness, flavor, juiciness, and overall acceptance using a 9-point hedonic scale $(1=$ dislike extremely and $9=$ like extremely). All the data were analyzed using the KruskalWallis test to determine the significance of the treatments in R. Dunn's test with $P$ value adjustment following Bonferroni methods was used for post hoc pair-wise comparisons. The alpha level was defined as 0.05 .

Results: Consumer scores for all sensory attributes ranged from 5.18 to 6.46 across all the treatment groups. However, the scores of likeness of all the attributes, namely tenderness, juiciness, and flavor, were rated higher $(P<$ $0.05)$ for beef steaks in the CON group when compared to both or at least one grass-fed group (GF20 and GF25). For instance, the mean score of steaks in the CON group was 6.45 compared to that of the steaks in GF20 (5.50) and GF25 (5.51). Additionally, consumers rated steaks in the CON group as having a significantly higher overall acceptance than either the GF20 or GF25 groups. No difference was detected by consumers between GG and the CON or either of the two grass-fed groups for any sensory attributes.

Conclusion: The results suggested that consumers recruited in the current study prefer the striploin steaks of cattle raised on grain-finished diet (either conventional 
feeding system or grass feeding system with additional $45 \mathrm{~d}$ on grain-finished diet) compared to those from grass-fed systems regardless of length of feeding period. This would also indicate that there may not be a significant benefit to switching to grass feeding systems as consumers still prefer the taste of conventional beef to that of grass-fed.

Keywords: beef, consumer preference, grass-fed, taste panel

\section{6 - CONSUMER PERCEPTION, WILLINGNESS TO PAY, TENDERNESS, AND RETAIL DISPLAY OF NONENHANCED, ENHANCED, AND HIGH-QUALITY PORK LOINS}

D. Cassens ${ }^{1 *}, \quad$ K. Hearn ${ }^{2}, \quad$ R. Ramanathan ${ }^{2}$, D. VanOverbeke $^{2}$, and G. Mafi ${ }^{2}$, ${ }^{1}$ Department of Animal Science, Tarleton State University, Stephenville, TX, USA, ${ }^{2}$ Department of Animal \& Food Sciences, Oklahoma State University, Stillwater, TX, USA, *cassens@tarleton.edu

Objectives: The objective of this study was to evaluate the effects of 3 different commercially available pork loins on retail display, trained and consumer sensory perceptions, and consumer intent to purchase.

Materials and Methods: Enhanced (E; $n=10)$, nonenhanced (NE; $n=10)$, and high-quality (HQ, $n=10)$ pork loins were selected from a commercial food distribution company. The $\mathrm{E}$ loins were labeled as containing up to a $12 \%$ solution of water and salt, and HQ loins contained a greater amount of intramuscular fat. Loins were wet aged for $21 \mathrm{~d}$ and cut into $2.54-\mathrm{cm}$-thick chops and randomly assigned to retail display, palatability analysis, or WarnerBratzler shear (WBS) force analysis. For retail display, chops $(n=27)$ were randomly assigned to 1 of 3 packaging treatments: polyvinyl chloride overwrap (PVC), carbon monoxide modified atmospheric packaging, and high-oxygen modified atmospheric packaging. Visual color for muscle color (MC) and surface discoloration were recorded on day 0,2 , and 4 of retail display. Additionally, instrumental color was taken using a Hunterlab Miniscan XE Plus spectrophotometer for $L^{*}$ and $a^{*}$ values on day $0-4$. For trained sensory panel and WBS force $(n=90)$, chops were evaluated at 3 different degrees of doneness $\left(63^{\circ} \mathrm{C}, 68^{\circ} \mathrm{C}\right.$, and $\left.74^{\circ} \mathrm{C}\right)$. Trained panelists evaluated overall tenderness, juiciness, and pork flavor. A consumer retail survey $(n=$ 129) was conducted to determine consumer perception of pork color, marbling, and degree of doneness. Consumer sensory panelists $(n=50)$ evaluated overall, flavor, juiciness, and tenderness like for chops cooked to $68^{\circ} \mathrm{C}$. A splitplot design with repeated measures was utilized. Pork loin treatment was the whole-plot and packaging type was the split-plot factor with retail display as the repeated measure. For WBS force and sensory evaluation, a completely randomized design was utilized. Data were analyzed using the PROC GLM procedure of SAS (version 9.4; SAS Institute Inc., Cary, NC).

Results: HQ chops packaged in PVC had among the highest $L^{*}$ and the lowest $a^{*}$ values indicative of a brighter, less red color. Chops packaged in carbon monoxide- and high-oxygen modified atmospheric packaging had similar $\mathrm{MC}$ and surface discoloration scores on day 2 and 4 of retail display along with a lower MC score compared to chops packaged in PVC. For the consumer retail survey, a higher percent (17.8) of consumers chose HQ pork chops first over $\mathrm{E}$ and NE chops based on the pictures provided in the survey. Of participants that consume beef at medium-rare, only $19.2 \%$ would consume pork at medium-rare, with the majority of participants $(59.6 \%)$ consuming pork at a medium degree of doneness. The $\mathrm{E}$ loins had lower $(P<0.05)$ shear values compared to NE loins. In addition, there was no difference $(P>0.05)$ between HQ and E, or HQ and NE, loins for WBS force values. Trained panelists found no difference $(P>0.05)$ in tenderness for $\mathrm{E}$ chops when cooked at 3 different degrees of doneness. Consumer panelists ranked the $\mathrm{E}$ chops the highest for overall like, tenderness like, and juiciness like, compared to HQ and NE loins.

Conclusion: In conclusion, HQ chops possessed a brighter, less red color with a higher consumer acceptability compared to E and NE chops. Lastly, E pork chops were more tender with less variation when cooking at various endpoint temperatures. The results suggest that $\mathrm{E}, \mathrm{NE}$, and $\mathrm{HQ}$ pork loins available in the market have different quality parameters at retail and as a cooked product on consumer acceptability.

Keywords: color, consumer perception, enhanced, marbling, pork loins

\section{7 - CONSUMER PERCEPTIONS ABOUT BENEFICIAL EFFECTS ON HUMAN HEALTH OF MEAT PRODUCTS PROCESSED WITH NATURAL INGREDIENTS}

C. A. Torres-Martínez ${ }^{1}$, G. R. Torrescano-Urrutia ${ }^{2}$, B. D. M. Torres-Martínez ${ }^{3}$, A. Pelayo-Ochoa ${ }^{1}$, N. O. Huerta-Leidenz ${ }^{4 *}$, A. Sánchez-Escalante ${ }^{3}$, and R. D. Vargas Sánchez ${ }^{2},{ }^{1}$ Department of Psychology, Universidad Autónoma de Occidente, Los Mochis, Mexico, ${ }^{2}$ Coordinación de Tecnología de Alimentos de Origen Animal, Centro de Investigación en Alimentación y Desarollo, A.C., Mexico, ${ }^{3}$ Coordinación de Tecnología de Alimentos de Origen Animal, Centro de Investigación en Alimentación y Desarrollo, A.C., Hermosillo, Mexico, ${ }^{4}$ Department of Animal and Food Sciences, Texas Tech University, Lubbock, TX, USA, *rey.vargas@ciad.mx 
Objectives: To study objective was to learn about consumer perceptions about alleged health benefits of meat products formulated with natural, functional ingredients.

Materials and Methods: An exploratory consumer survey was conducted in a sample of meat consumers $(n=$ 192) of the municipality of Hermosillo, Sonora State, Mexico. The questionnaire was previously approved by a small group of experts in the area of human psychology and food technology and was administered in person (face to face) using the paper-and-pen mode to randomly selected meat buyers in local supermarkets. Besides demographics (gender, age, and scholarship), consumers responded to a total of 9 questions - 3 about their knowledge and perceptions of functional foods and 6 regarding the acceptance of functional meat products and their alleged effects on mental health and well-being. Data were subjected to analysis of variance and a Tukey test $(P<0.05)$ for mean separation.

Results: More women than men, between 25 and $40 \mathrm{y}$ of age, participated; the majority of individuals aged $25 \mathrm{y}$ or younger had completed college education $(P<0.05)$. At the start of the interview, more than $60 \%$ of the consumers expressed that they did not know the meaning of functional foods, but once this concept was explained to them, more than half of the interviewees indicated that they had consumed these products, and $>80 \%$ believed that its consumption could help to improve human health $(P<0.05)$. In regard to consumer acceptance of functional meat products (Figure 1), all women younger than $25 \mathrm{y}$ of age and men between 25 and 40 y of age with a college background indicated that they would be willing to consume meat products processed with carrot peel and romerillo leaves to mitigate stress and improve mood $(P<0.05)$. Also, the results indicated (Figure 1) that they would be willing to consume chorizo processed with spinach because it would increase cognitive abilities $(P<0.05)$.

Conclusion: The local consumer's perception of functional foods, as well as their willingness to accept meat products processed with natural ingredients, are based on the positive belief that these products would improve physical
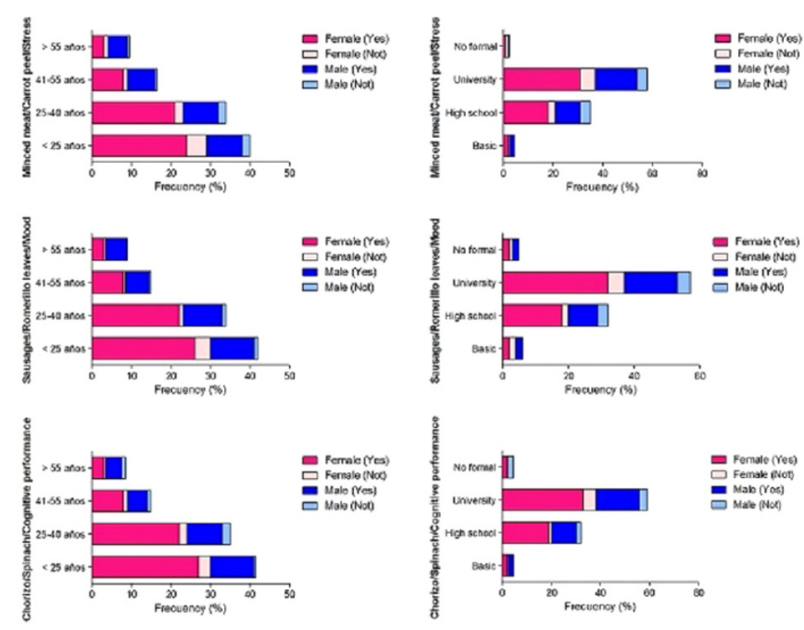

and mental health. These attitudinal responses represent valuable information for designing marketing strategies of meat-based functional, natural foods.

Keywords: consumer acceptance, functional foods, health, meat

\section{8 - SENSORY AND QUALITY DIFFERENCES OF PAIRED DRY-AGED LAMB LOINS}

\author{
K. Wall ${ }^{1 *}$, C. Kerth ${ }^{1}$, J. Boles ${ }^{2}$, and R. Miller ${ }^{1},{ }^{1}$ Animal \\ Science, Texas A\&M University, College Station, TX, \\ USA, ${ }^{2}$ Animal and Range Sciences, Montana State \\ University,Bozeman, MT, USA, *wall4165@tamu.edu
}

Objectives: The objective of this study was to determine the flavor and texture differences between fresh and dry-aged lamb loin chops.

Materials and Methods: Lambs $(n=10)$ of a contemporary group were fed a concentrate diet for $60 \mathrm{~d}$ prior to harvest. Carcasses (average $57.3 \mathrm{~kg}, 0.27 \mathrm{~cm}$ fat thickness) were halved, and loins were randomly assigned an aging treatment of no age (frozen day 0) or 14-d dry age (average temperature of $4^{\circ} \mathrm{C}, 55 \%$ relative humidity). Loins $(n=20)$ were frozen whole and shipped to College Station, Texas, for sensory analysis. Loins were cut into $1.9-\mathrm{cm}$ thick-chops, labeled, and individually packaged. Samples were thawed in $4^{\circ} \mathrm{C}$ overnight and cooked to $71^{\circ} \mathrm{C}$ internal temperature on a flat-top electric grill. A 5-member sensory panel was trained on flavor and texture attributes specific to lamb with a 16-point intensity scale. The panel rated each sample individually and reported a consensus intensity score for each attribute. Representative cubes from sensory analysis were quick frozen for gas chromatography-mass spectrometry analysis. Samples $(5.0 \mathrm{~g})$ were broken into a scintillation vial, heated to $60^{\circ} \mathrm{C}$ using a heating block, and collected for $30 \mathrm{~min}$ with a Solid Phase Micro-Extraction fiber. Samples were run on a GC/MS and tentatively identified using the Wiley library. Samples were run in a REML application with rep as a random sensory effect using JMP Pro 15 (SAS Institute Inc., Cary, NC). Multivariate tools were utilized to explore the relationships between sensory and $\mathrm{GC} /$ MS results.

Results: No differences $(P>0.05)$ were found between the aging treatments for lamb identity, brown, roasted, sweet, bitter, sour, umami, fat-like, bloody/serumy, metallic, liverlike, musty/earthy, lanolin, mutton, or juiciness. Dry-aged loin samples were more $(P<0.04)$ salty than no age $(1.9$ vs. 1.5 , respectively). Dry-aged loins tended to be more green $(P=0.078)$ than those without age ( $1.5 \mathrm{vs} .1 .1$, respectively). No age loins received lower $(P<0.001)$ muscle fiber and connective tissue ratings than dry-aged (10.1 vs. 12.7 and 11.7 vs. 12.8 , respectively). Of the volatile aromatic compounds identified $(n=62)$, a 1.6 -fold decrease $(P<$ $0.05)$ in $\log$ Total Ion Count of 2-pentyl furan and 
hentriacontane and a 0.5 -fold increase $(P<0.05)$ for thiobismethane and 2-methyl-butanal was found with 14-d dry aging time.

Conclusion: Dry aging lamb loins for $14 \mathrm{~d}$ enhanced tenderness of the samples with no disadvantage to flavor profiles.

Keywords: gas chromatography-mass spectrometry, lamb, sensory

\section{9 - PERCEPTION OF LAMB EXTRINSIC ATTRIBUTES BY BRAZILIAN CONSUMER AT PURCHASE TIME}

H. V. Battagin ${ }^{1}$, B. Panea ${ }^{2}$, and M. A. Trindade ${ }^{1 *},{ }^{1}$ Food Engineering Department, University of São Paulo, Pirassununga, Brazil, ${ }^{2}$ Animal Production and Health Unit, Centro de Investigación y Tecnología Agroalimentaria de Aragón, Zaragoza, Spain,*trindadema@usp.br

Objectives: In Brazil, the consumption of lamb is small (0.7 $\mathrm{kg} /$ inhabitant $/ \mathrm{y}$ ). Because of this, a great part of the population does not know what factors to take into account when choosing this meat at purchase time. A consumer can be considered frequent when he/she eats lamb at least once a month. Thus, this study aimed to investigate the degree of importance that consumers ascribe to certain lamb extrinsic attributes at the time of purchase and whether there is a difference between the behavior of frequent and nonfrequent consumers.

Materials and Methods: An online survey was carried out using the Google Forms tool during the months of May and June 2019 to obtain responses of a convenience sample of consumers from all over Brazil. The survey was made available via email and Facebook in groups of 20 universities (from the main Brazilian ones), and the criterion for selecting participants was only their interest in taking part in the study. Questions about lamb buying habits, in relation to the frequency of consumption and extrinsic attributes to the meat that are taken into account at the time of purchase, were asked. For this, a scale ranging from "very important," "important," "unimportant," and "very unimportant" was used. Consumers were grouped into clusters according to the frequency of consumption, and the data on the importance given to the researched attributes were studied according to the relative frequency. Chi-squared tests (at the 5\% significance level) were carried out to investigate whether there was a relationship between the degree of importance attributed to lamb extrinsic factors and the frequency of consumption and whether there were attributes considered more important.

Results: At the end of the survey, 1,457 valid responses had been collected. Three groups (clusters) of consumers were separated in terms of frequency of lamb consumption: 172 consumers $(11.8 \%)$ were considered frequent (consumers who consume lamb meat "once or more a week," "2 to 3 times a month," or "once a month"), 444 (30.5\%) were nonfrequent (consumers who responded "less than once a month"), and the rest (57.8\%) were considered non-lamb consumers (consumers who responded "never consume lamb"). Table 1 show the relative frequencies of lamb consumer clusters (frequent and nonfrequent) regarding the importance they give to "organic production," "quality label," "ease of cooking," "price," and "type of packaging." Organic production of animals was the least important item for both groups. The ease of cooking and type of packaging attributes were of intermediate importance, and the price and presence of quality label appeared as the most important ones in the purchasing decision-making process. Price was the only attribute that received different $(P<0.05)$ degrees of importance among clusters and was considered more important for nonfrequent consumers. Non-lamb consumers were not asked about this topic because, if they do not consume lamb, they generally do not buy it.

Conclusion: Only a small portion (11.8\%) of the convenience sample of Brazilian consumers that responded to the online survey eat lamb frequently (at least once a month). Among the evaluated extrinsic attributes that are taken into account at the time of purchase, the most important are the presence of quality label and price. The behavior of frequent

Table 1. Relative frequency (\%) of the importance given by frequent and non-frequent consumers to extrinsic attributes of lamb at purchasing time

\begin{tabular}{|c|c|c|c|c|c|c|}
\hline Cluster & Importance & $\begin{array}{c}\text { Organic production } \\
\left(\mathrm{p}^{*}=0,26\right)\end{array}$ & $\begin{array}{l}\text { Quality label } \\
\qquad(\mathrm{p}=0,18)\end{array}$ & $\begin{array}{l}\text { Ease of cooking } \\
\qquad(\mathrm{p}=0,28)\end{array}$ & $\begin{array}{c}\text { Price } \\
(p=0,02)\end{array}$ & $\begin{array}{c}\text { Type of packaging } \\
(\mathrm{p}=0,98)\end{array}$ \\
\hline \multirow[t]{4}{*}{ Frequent consumers $(n=172)$} & Very unimportant & 36.0 & 18.0 & 19.2 & 15.7 & 20.3 \\
\hline & Unimportant & 34.9 & 22.7 & 24.4 & 30.8 & 29.1 \\
\hline & Important & 20.4 & 30.2 & 41.9 & 36.6 & 34.9 \\
\hline & Very important & 8.7 & 29.1 & 14.5 & 16.9 & 15.7 \\
\hline \multirow[t]{4}{*}{ Non-frequent consumers $(n=444)$} & Very unimportant & 34.9 & 16.4 & 14.9 & 12.8 & 19.4 \\
\hline & Unimportant & 42.6 & 17.1 & 31.5 & 21.2 & 30.6 \\
\hline & Important & 15.8 & 39.0 & 38.7 & 41.7 & 34.2 \\
\hline & Very important & 6.7 & 27.5 & 14.9 & 24.3 & 15.8 \\
\hline
\end{tabular}

*Pearson's chi-square test. 
and nonfrequent consumers differs only in relation to price, which is considered more important for the second group.

Keywords: consumer behavior, frequency of consumption, price, quality label, sheep meat

\section{0 - EFFECT OF SIRE BREED AND AGE AT HARVEST ON SENSORY AND VOLATILE ATTRIBUTES OF LAMB LOIN CHOPS}

\author{
K. Wall ${ }^{1 *}$, C. Kerth ${ }^{1}$, Z. Hicks ${ }^{1}$, R. Miller ${ }^{1}$, T. Murphy ${ }^{2,3}$, W. \\ Stewart ${ }^{4}$, and J. Boles ${ }^{3},{ }^{1}$ Animal Science, Texas A\&M \\ University, College Station, TX, USA, ${ }^{2}$ Genetics, Breeding, \\ and Animal Health Research Unit, USDA-ARS, Clay Center, \\ NE, USA, ${ }^{3}$ Animal and Range Sciences, Montana State \\ University, Bozeman, MT, USA, ${ }^{4}$ Animal Science, University \\ of Wyoming, Laramie, WY, USA, *wall4165@tamu.edu
}

Objectives: The objective of this study was to identify flavor differences of lamb loin chops due to sire breed, preharvest age, and degree of doneness (DOD).

Materials and Methods: Rambouillet (RAM), South African Meat Merino, and Suffolk rams were bred to RAM ewes to produce lambs for this study in Bozeman, Montana. Wether lambs $(n=60 ; 20 /$ breed; 30/age) were randomly assigned to one of 2 finishing groups (11 or 18 mo of age at slaughter) and fed a concentrate diet for approximately $60 \mathrm{~d}$ before harvesting at a commercial facility. Frozen, boneless loin samples were shipped to Texas A\&M University and cut into 1.9 -cm-thick chops, randomly assigned a DOD $\left(63^{\circ} \mathrm{C}\right.$ or $\left.71^{\circ} \mathrm{C}\right)$, and packaged individually. Samples were thawed at $4^{\circ} \mathrm{C}$ overnight, cooked on an electric flat-top grill, and served as cubes for sensory evaluation. A 5-member panel of trained sensory panelists provided a consensus evaluation of each sample for flavor and texture attributes using a 16-point hedonic intensity scale. Representative cubes were quick frozen and broken apart for gas chromatography-mass spectrometry analysis. Pieces of each sample $(5.0 \mathrm{~g})$ were placed in a $20 \mathrm{~mL}$ scintillation vial and heated to $60^{\circ} \mathrm{C}$ for a 30-min collection period with a Solid Phase MicroExtraction fiber. Samples were run on a gas chromatography-mass spectrometry and tentatively identified using the Wiley Spectrum Library. Collagen amount and solubility were determined from the raw sample. Statistical analyses were performed using JMP Pro 15 software (SAS Institute Inc., Cary, NC) with an alpha value of 0.05 . Mean separations of the trial were determined using analysis of variance with sire breed, age at slaughter, and DOD and interactions as main effects with day included as a random effect for sensory analysis. Principal component analysis and correlations were used to explore trends between flavor and texture attributes.

Results: Younger lambs were more bitter $(P<0.05)$ and had lower muscle fiber tenderness and higher connective tissue amount scores $(P<0.001)$ than older lambs $(8.9$ vs. 11.7 and 10.8 vs. 12.3 , respectively). A lesser DOD resulted $(P<0.05)$ in juicier, more cardboardy, more bloody/serumy, more sour, more roasted, and less fat-like intensity of flavor. Although lamb identity did not differ $(P>0.05)$, mutton increased $(P<0.05)$ with lambs sired by RAM. Volatile aromatic compounds $(n=47)$ identified contributed sweet, dairy, green, citrus, roasted, sulfurous, waxy, and fermented aroma compounds. Suffolk-sired lambs had greater $(P<$ $0.01)$ total amount of collagen than either South African Meat Merino or RAM (5.85 vs. 3.62 and $4.44 \mathrm{mg}$ collagen $/ \mathrm{g}$, respectively). No differences $(P>0.05)$ in collagen solubility were detected.

Conclusion: Sire breed of lambs has an influence on flavor and tenderness of lamb chops. Preharvest age impacts the texture of the meat but did not induce off-flavors.

Keywords: gas chromatography-mass spectrometry, lamb, sensory analysis

\section{1 - BRISKET - BACK TO THE BASICS}

K. Wall ${ }^{1 *}$, C. Kerth ${ }^{1}$, S. Curry ${ }^{1}$, Z. Hicks ${ }^{1}$, J. W. Savell ${ }^{1}$, and A. Adams ${ }^{2},{ }^{1}$ Animal Science, Texas A\&M University, College Station, TX, USA, ${ }^{2}$ Technical Services, Sadler's Smokehouse,Tyler, TX, USA, *wall4165@tamu.edu

Objectives: The objective of this study was to identify an ending temperature for optimizing beef brisket yield and tenderness.

Materials and Methods: USDA Choice beef briskets $(n=96)$, with deckle fat removed and trimmed to $0.8 \mathrm{~cm}$ fat cover, were selected in 2 separate batches from representative commercial lots and randomly assigned a final temperature $\left(75^{\circ} \mathrm{C}, 80^{\circ} \mathrm{C}, 85^{\circ} \mathrm{C}\right.$, or $\left.90^{\circ} \mathrm{C}\right)$ and hold time $(0$ or $3 \mathrm{~h})$ combination. Briskets were cooked in duplicate batches $(n=$ 6/batch; 16 total batches) that were blocked by brisket raw weight. Commercial, single-truck smokehouses were utilized to cook the briskets. Yield information was recorded before doneness was examined using a unique brisket poker connected to a texture analyzer to measure the force that a 1-cm-diameter ball needs to penetrate the cooked brisket. Briskets were chilled, separated into point and flat pieces, and sliced for further analyses. Warner-Bratzler shear force (WBSF) was conducted from a 2.54-cm-thick slice immediately for each portion, where max peak force was recorded. Slice shear force (SSF) and trained sensory slices $(0.9 \mathrm{~cm}$ thick) were reheated via sous vide method to $63^{\circ} \mathrm{C}$. Slices from each flat and point were served to a 5 -member trained panel to evaluate and come to a consensus on the degree of cohesiveness, cohesiveness of mass, muscle fiber tenderness, moisture release, and connective tissue using a 16point hedonic intensity scale. Data were analyzed using JMP Pro 15 software (SAS Institute Inc., Cary, NC). Differences in yield, poke, WBSF, SSF, and collagen were 
found utilizing analysis of variance with assigned final temperature, hold, and their interaction as main effects with rep included as a random block effect. Linear contrasts were used to identify influential temperature and/or hold classifications. Linear regression models were generated for each analysis, and pairwise correlations were measured.

Results: Hot and chilled cook yield differed $(P<0.001)$ by temperature and hold time. Briskets cooked to $90^{\circ} \mathrm{C}$ with a 3-h hold time had the greatest loss in chilled yield, whereas briskets cooked to $75^{\circ} \mathrm{C}$ with 0 -h hold time had the greatest cooked product yield (55.2\% vs. $70.2 \%$, respectively). Poke force decreased $(P<0.001)$ with increased temperature and increased hold time. Poking briskets at the central location resulted in more reliable $\left(R_{\text {adj }}^{2}=0.60\right)$ tenderness outcome than poking at the flat or point. Overall, increased ending temperature and increased hold time resulted in less $(P<$ $0.01)$ cohesiveness, cohesiveness of mass, and moisture release as well as more $(P<0.01)$ muscle fiber tenderness and absence of connective tissue. WBSF and SSF values of the point decreased $(P<0.05)$ with increased temperature and hold time. Moderate correlations $(P<0.001)$ existed between yield evaluation, sensory attributes, and instrumental texture measurements with the exception of moisture release, which had no $(P>0.05)$ correlation.

Conclusion: Endpoint temperature and hold time affect brisket tenderness differently between the flat and point. Compared to $90^{\circ} \mathrm{C}$ with a 3-h hold, briskets cooked to $85^{\circ} \mathrm{C}$ with a 3 -h hold achieved enhanced yield $(56.0 \%$ vs. $59.3 \%$, respectively) without sacrifice to tenderness attributes.

Keywords: beef brisket, collagen, cooking endpoint temperature

\section{2 - CONSUMERS PREFER BEEF FROM FEEDLOT CROSSBRED ANGUS $\times$ NELORE STEERS AND HEIFERS OVER BULLS}

P. L. M. Garbossa ${ }^{1 *}$, T. S. Martins ${ }^{1}$, A. M. Ferrinho ${ }^{2}$, A. Pereira $^{3}$, A. G. V. do $\mathrm{Barco}^{4}$, C. F. de $\mathrm{Sousa}^{2}$, F. A. Cassimiro $^{1}$, G. V. de Moura $^{2}$, H. C. Rocha ${ }^{1}$, I. H. S. Fuzikawa $^{2}$, J. L. Gemelli ${ }^{2}$ J. A. de Sá $^{2}$, J. P. S. Silva ${ }^{5}$, J. A. Muñoz ${ }^{2}$, L. F. Mueller ${ }^{2}$, T. A. J. Sant'Ana ${ }^{1}$, T. R. Amorim $^{2}$, M. D. Poleti ${ }^{3}$, and A. S. C. Pereira ${ }^{1},{ }^{1}$ Department of Animal Nutrition and Production, School of Veterinary Medicine and Animal Science, University of São Paulo (FMVZ/USP), São Paulo, Brazil, ${ }^{2}$ Department of Animal Science, Faculty of Animal Science and Food Engineering, University of São Paulo (FZEA/USP), Pirassununga, Brazil, ${ }^{3}$ Department of Veterinary Medicine, Faculty of Animal Science and Food Engineering, University of São Paulo (FZEA/USP), Pirassununga, Brazil, ${ }^{4}$ Department of Biomedicine, Hermínio Ometto Fundation (Uniararas), Araras, Brazil, ${ }^{5}$ Department of Animal Science,
Federal Rural University of The Amazon (UFRA), Paragominas, Brazil, *pollyana_matioli@usp.br

Objectives: Beef tenderness is designated as a very important sensory trait by consumers, which has a great influence on purchase decision. Therefore, this work aimed to evaluate the influence of the sex class (SC; bull, heifer, and steer) of feedlot crossbred Angus $\times$ Nelore cattle on beef sensory traits.

Materials and Methods: F1 Angus $\times$ Nelore cattle (50 bulls, 50 steers, and 50 heifers) at $11 \mathrm{mo}$ of age were raised under diet composed of $86 \%$ concentrate (mainly corn) and 14\% roughage (Brachiaria hay) in dry matter for $150 \mathrm{~d}$ and harvested. The final mean weight was $488.9 \pm 30.7 \mathrm{~kg}, 452.5 \pm 24.9 \mathrm{~kg}$, and $431.3 \pm 26.3 \mathrm{~kg}$ for bulls, steers, and heifers, respectively. An acceptance test was performed with 126 untrained panelists who evaluated overall liking, flavor, tenderness, and juiciness of Longissimus muscle steaks. Three samples were offered to each panelist, referring to the $3 \mathrm{SC}$ in the aging time of $14 \mathrm{~d}$. For overall liking, a structured 9-point hedonic scale (where 1 = "dislike extremely," and 9= "like extremely") was used. For the other traits, a 5-point structured scale was used (where $1=$ "very bland, very tough or very dry," and $5=$ "very intense, very tender or very juicy" according to the flavor, tenderness, and juiciness; respectively). The data were analyzed using a randomized block design by a mixed linear model, including the fixed effect of SC, the random effects of the block (panelist), and residue. The procedures for the analysis of shear force (SF) were performed according to AMSA (2016). Significant effects for the SC were assessed using the PDIFF option, significance was declared when $P<0.05$, and means were compared by the Tukey test (5\% of significance).

Results: Steaks from steers were scored by panelists as more tender and juicier $(P<0.05)$ than bull counterparts;

Table 1. Effect of sex classes on sensory traits, and Warner-Bratzler Shear Force of beef aged for 14 days

\begin{tabular}{llllllr}
\hline \multirow{2}{*}{ Variable } & \multicolumn{3}{c}{ Sex Classes } & & \\
\cline { 2 - 5 } & Steer & Heifer & Bull & SEM & P-value \\
\hline Overall Liking & $7.24^{\mathrm{a}}$ & $6.97^{\mathrm{ab}}$ & $6.83^{\mathrm{b}}$ & 0.08 & 0.03 \\
Flavor & 7.08 & 6.83 & 6.84 & 0.08 & 0.17 \\
Flavor Intensity & 3.14 & 3.11 & 3.03 & 0.03 & 0.29 \\
Tenderness & $7.32^{\mathrm{a}}$ & $6.87^{\mathrm{ab}}$ & $6.56^{\mathrm{b}}$ & 0.09 & $<0.01$ \\
Tenderness Intensity & $4.02^{\mathrm{a}}$ & $3.80^{\mathrm{ab}}$ & $3.58^{\mathrm{b}}$ & 0.05 & $<0.01$ \\
Juiciness & $7.03^{\mathrm{a}}$ & $6.81^{\mathrm{ab}}$ & $6.63^{\mathrm{b}}$ & 0.08 & 0.02 \\
Juiciness Intensity & $3.87^{\mathrm{a}}$ & $3.60^{\mathrm{b}}$ & $3.55^{\mathrm{b}}$ & 0.04 & $<0.01$ \\
WBSF 14 days, kg & $6.20^{\mathrm{b}}$ & $6.55^{\mathrm{b}}$ & $7.67^{\mathrm{a}}$ & 0.20 & $<0.01$ \\
\hline
\end{tabular}

Distinguished superscript lower-case letters differ by Tukey test with $P<0.05$.

SEM: Standard Error of the Mean.

WBSF 14 days: Warner-Bratzler Shear Force at 14 days of aging. 
however, no differences in flavor and flavor intensity were detected $(P<0.05)$. On the other hand, steaks from heifers presented intermediate values with no differences compared with the other SC $(P<0.05)$, except for juiciness intensity that was equal to bull steaks. Steer and heifer steaks presented lower SF values than bull steaks. There were no difference for all sensory traits between steaks from heifers and bulls (Table 1). This can be explained by the fact that no difference was found between the SF of steers and heifers.

Conclusion: Steers' steaks produced more desirable palatability attributes, followed by heifer steaks, which indicates the possibility of improving consumer acceptance and marketing opportunities for the Brazilian beef industry in quality-oriented niche markets.

Keywords: beef tenderness, consumer preference, sensory analysis, shear force

\section{3 - CONSUMER SENSORY EVALUATION OF PLANT-BASED GROUND BEEF ALTERNATIVES IN COMPARISON TO GROUND BEEF OF VARIOUS FAT PERCENTAGES}

S. Davis ${ }^{1 *}$, K. M. Harr ${ }^{1}$, S. B. Bigger ${ }^{2}$, D. U. Thomson ${ }^{3}$, M. D. Chao ${ }^{1}$, J. L. Vipham ${ }^{1}$, M. D. Apley ${ }^{2}$, D. A. Blasi ${ }^{1}$, S. M. Ensley ${ }^{2}$, M. D. $\mathrm{Haub}^{4}$, M. D. Meisner ${ }^{2}$, A. J. Tarpoff $^{1}$, K. Olson ${ }^{1}$, and T. G. O'Quinn ${ }^{1},{ }^{1}$ Animal Science and Industry, Kansas State University, Manhattan, KS, USA, ${ }^{2}$ College of Veterinary Medicine, Kansas State University, Manhattan, KS, USA, ${ }^{3}$ Animal Science, Iowa State University, Ames, IA, USA, ${ }^{4}$ Nutrition, Dietetics, and Health, Kansas State University, Manhattan, KS, USA, *sgdavis@ ksu.edu

Objectives: The objective of this study was to determine whether current plant-based protein ground beef alternatives (GBA) offer similar palatability characteristics to ground beef (GB) patties of varying fat percentages.

Materials and Methods: Fifteen different production lots ( $n=15 /$ fat level) of $1.36 \mathrm{~kg} \mathrm{~GB}$ chubs of 3 different fat levels $(10 \%, 20 \%$, and $30 \%)$ were collected from retail markets in the Manhattan, Kansas, area. Additionally, GBA products including a soy-protein-based Foodservice GBA (FGBA), a pea-protein-based Retail GBA (RGBA), and a traditional soy-protein-based GBA (TGBA) $(n=15$ production lots $/$ product) currently available through commercial channels were collected from retail markets and a commercial foodservice chain. All GB and GBA treatments were formed into 151-g patties (approximately $13-\mathrm{cm}$ diameter; $1-\mathrm{cm}$ thick) and frozen at $-40^{\circ} \mathrm{C}$ until consumer sensory analysis. Patties were cooked to $71^{\circ} \mathrm{C}$ on a clamshell-style grill, cut into 6 equally sized wedges, and served within $5 \mathrm{~min}$ of cooking to consumers. Consumers $(n=120)$ were fed 6 samples ( 1 wedge/sample) in a random order and evaluated sample appearance, juiciness, tenderness, overall flavor liking, beef flavor liking, texture liking, and overall liking on continuous line scales verbally anchored at the ends and midpoints. Additionally, consumers rated each trait as either acceptable or unacceptable. All data were analyzed as a completely randomized design.

Results: All 3 GB samples rated higher $(P<0.05)$ than the 3 GBA samples for appearance, overall flavor, beef flavor, and overall liking. Retail GBA rated lowest $(P<0.05)$ for appearance, overall flavor, texture, and overall liking. Of the GBA samples, FGBA rated highest $(P<0.05)$ for juiciness, beef flavor, and texture liking, and TGBA rated lowest $(P<0.05)$ for juiciness. However, FGBA rated higher $(P<$ 0.05 ) for tenderness than the $20 \%$ fat GB samples. Moreover, of the GBA samples, FGBA and TGBA rated similar $(P>$ 0.05 ) for appearance, tenderness, overall flavor liking, and overall liking. Among the GB samples, no differences $(P>0.05)$ were found for appearance, juiciness, overall flavor liking, beef flavor liking, or overall liking. For the percentage of samples rated acceptable for each palatability trait, all $3 \mathrm{~GB}$ treatments had a higher $(P<0.05)$ percentage of samples rated acceptable for appearance, overall flavor, beef flavor, texture, and overall liking than the 3 GBA. Retail GBA had the lowest $(P<0.05)$ percentage of samples rated acceptable for appearance, overall flavor, texture, and overall liking. Traditional GBA had the lowest $(P<0.05)$ percentage of samples rated acceptable for juiciness. Among the GBA samples, FGBA had the highest $(P<$ 0.05 ) percentage of samples rated acceptable for juiciness and beef flavor liking. Furthermore, among the GBA treatments, FGBA and TGBA had a similar $(P>0.05)$ percentage of samples rated acceptable for appearance, flavor, texture, and overall liking.

Conclusion: These results indicate GB samples had higher ratings than GBA samples for most palatability traits evaluated. Moreover, a higher percentage of samples were rated as acceptable for GB than for GBA. This clearly indicates that the eating experience provided by GBA is different than that provided by traditional GB. Thus, consumers who purchase GBA should not expect the same eating quality as they would receive with GB.

Keywords: alternative proteins, consumer, ground beef, ground beef alternative, palatability

\section{4 - THE APPLICATION OF DESIGN THINKING IN FOODSERVICE: DRY AGED SHEEPMEAT CASE STUDY}

M. Hastie ${ }^{*}$, H. Ashman ${ }^{1}$, and R. Warner ${ }^{1},{ }^{1}$ Faculty of Veterinary and Agricultural Science, the University of Melbourne, Melbourne, Australia, *hastiem@student. unimelb.edu.au 
Objectives: Industry and government bodies are considering strategies to increase cull ewe meat consumption within Australia. Dry aging may improve cull ewe meat consumer acceptability. In order to understand the optimal dry-aged sheepmeat product formats from foodservice and consumer perspectives, design thinking principles were applied to a study combining qualitative and quantitative consumer methodologies.

Materials and Methods: This study's methodology is outlined against the design thinking process.

1. Understanding user needs: Chefs were provided with 2 entire 35-day dry-aged cull ewe carcasses. Dry-aged sheepmeat supply chain participants attended an interactive half-day workshop in which they observed butcher and chef preparation of the carcasses and tasted a range of dishes that were prepared from the carcasses. During the workshop, insights and challenges from the perspective of consumer, butcher, chef, and producer were captured in facilitated group discussions and 1:1 interviews.

2. Problem definition: Insights gathered from stage 1 were incorporated and prioritized in a briefing document.

3. Ideation: Twenty-five food service professionals were briefed on the outcomes of stages 1 and 2 and subsequently participated in a facilitated ideation session. After assessment by the group, 20 concept dishes were put forward for testing.

4. Concept development/testing: The 20 concepts generated from the ideation session were turned into image or product stimuli used in 2 perceptual mapping sessions. All participants were food service professionals.

5. Consumer testing: Eight concept dishes were selected for testing with consumers $(n=26)$ and rated for liking and premiumness.

Results: Key findings included the fact that many chefs were not experienced with cooking cull ewe meat and often showed a preference for loin cuts, mistakenly assuming that meat from cull ewes had similar properties to lamb and would be suitable for grilling. After experiencing cull ewe meat, their preference changed to slow-cooked leg and shoulder cuts. Concept testing indicated dry-aged cull ewe meat suited a range of dishes covering the full range of economy to premium dishes and were suited to menus ranging from classic to "foodie" (a menu focused on delivering new or novel eating experiences). Figure 1 is an example concept map. All 8 dry-aged mutton concept dishes achieved liking scores $>7$ and therefore were highly acceptable. Overall, there were differences in liking $(P<0.001)$; the 5 most liked dishes (statistically similar) scored $>8 / 9$. These dishes included pitas (marinated silverside roasted at $160^{\circ} \mathrm{C}$ to internal temperature of $65^{\circ} \mathrm{C}$ ), shoulder (braised at $180^{\circ} \mathrm{C}$ for $5 \mathrm{~h}$ ), pho (sous vide backstrap at $58^{\circ} \mathrm{C}, 10 \mathrm{~h}$ ), piccata (3-mm topside slice, crumbed and shallow fried), and smoked mutton salad (silverside; smoked $10 \mathrm{~min}$, roast at $120^{\circ} \mathrm{C}$ to internal temperature of $\left.68^{\circ} \mathrm{C}\right)$. Premiumness ratings for the 5 most liked dishes differed $(P<0.001)$; the most premium and statistically similar scored $>4.7 / 5$ (shoulder and smoked mutton salad), and the least premium (pitas and piccata) scored 3.71 and 3.69/5, respectively.

Conclusion: The study highlighted the need for cooking guidelines for cull ewe meat. Foodservice product preference was driven by eating quality, convenience, and versatility of cuts, with braising of large cuts or sous vide of small cuts providing consistent quality and easy preparation in the restaurant setting. Consumer testing confirmed the acceptability of these dishes.

Keywords: consumer acceptance, cull ewe, design thinking, foodservice

\section{5 - BEEF BRISKET SUBJECTIVE MEASURE OF DONENESS AND TENDERNESS}

S. Curry ${ }^{1 *}$, K. Wall ${ }^{1}$, C. Kerth ${ }^{1}$, Z. Hicks ${ }^{1}$, J. Savell ${ }^{1}$, and A. Adams ${ }^{2},{ }^{1}$ Animal Science, Texas A\&M University, College Station, TX, USA, ${ }^{2}$ Technical Services, Sadler's Smokehouse, Henderson, TX, USA, *wall4165@tamu.edu

Objectives: The objective of this study was to identify the final temperature of beef briskets without the use of instrumentation.

Materials and Methods: USDA Choice beef briskets $(n=96)$, with deckle fat removed and trimmed to $0.8 \mathrm{~cm}$ fat, were selected and randomized into separate groups and assigned a treatment combination for cooking. Final temperatures were $75^{\circ} \mathrm{C}, 80^{\circ} \mathrm{C}, 85^{\circ} \mathrm{C}$, or $90^{\circ} \mathrm{C}$, and hold time consisted of $0 \mathrm{~h}$ or $3 \mathrm{~h}$. Measurements, including weight, length, flop, $\mathrm{pH}$, and initial temperature, were taken from the raw briskets. Length of the brisket was measured with the fat side up and on the side closest to the forerib from the top of the point to the flat. For the flop measurement, the brisket was allowed to fall over the side of a flat surface, using the segment of the point and flat muscle overlay as the point of bending in the "flop." Horizontal and vertical length of the brisket, lean side up, when flopped were measured using a framing square. Flop distance was determined using the Pythagorean theorem. Briskets were cooked in commercial, single-truck smokehouses. Measurements to determine yield and degree of doneness were recorded. Pokes by a texture analyzer fitted with a custom 1-cm-diameter spherical head were done in triplicate over 3 locations: the flat, center (where the natural seam attaches the 2 muscles), and point. Briskets were chilled, separated into point and flat, and sliced. WarnerBratzler shear force (WBSF) was conducted immediately on a 2.54-cm-thick slice. Slice shear force and sensory were conducted on slices $(0.9 \mathrm{~cm}$ thick) proximate to the WBSF slice after being chilled and reheated to $63^{\circ} \mathrm{C}$ utilizing the sous vide method. A sensory panel consisting of 5 trained members evaluated the degree of cohesiveness, cohesiveness of mass, muscle fiber tenderness, moisture release, and connective tissue using a 16-point hedonic intensity scale of each slice. Differences in means were detected using analysis of variance 


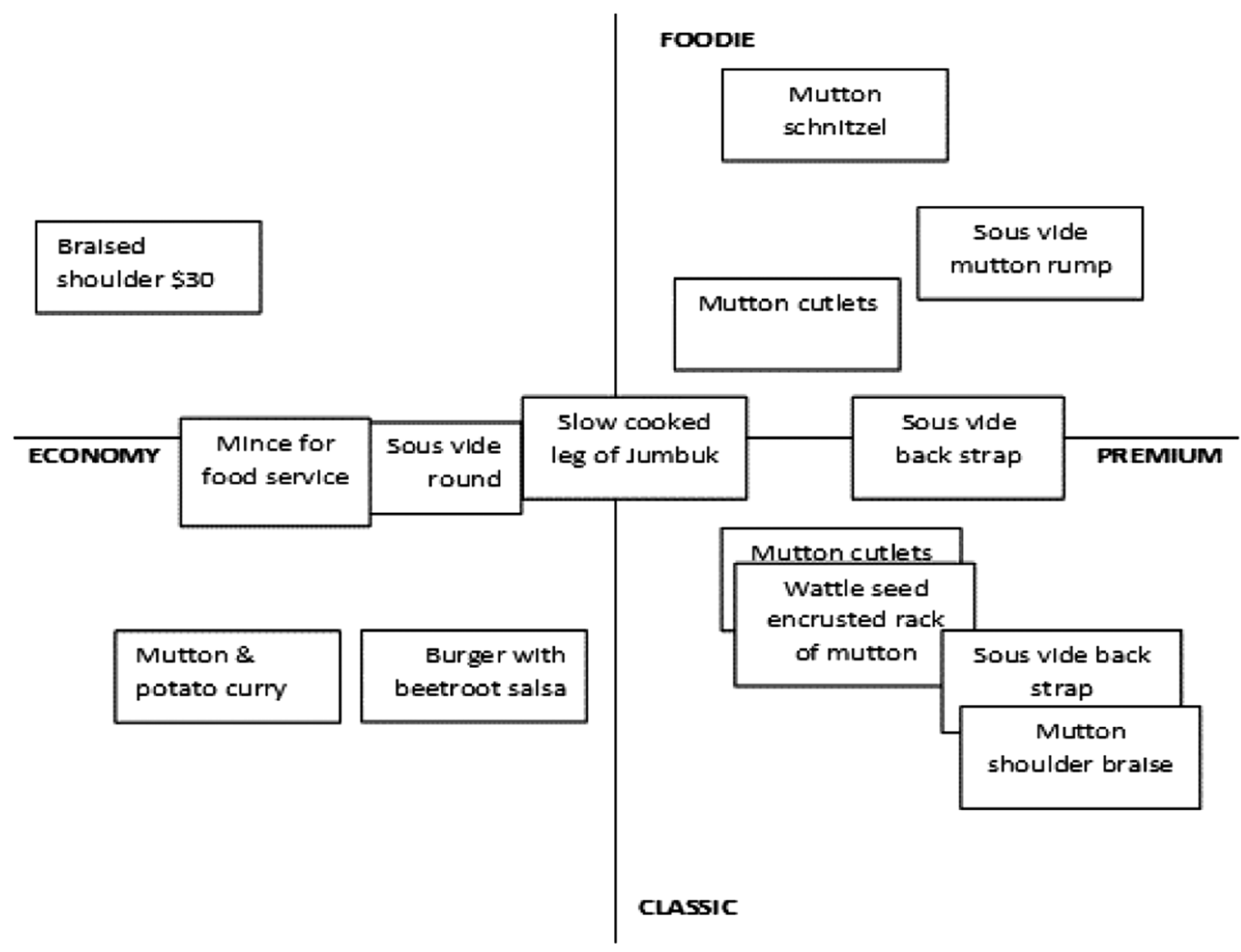

Figure 1. Concept map from focus group 2. illustrating positioning of concept dishes plotted against eating experience; economy to premium (x-axis) and menu type; classic to foodie (Y-axis). Foodie refers to a menu focussed on delivering new or novel eating experiences.

and Student $t$ mean separation with JMP Pro 15 (SAS Institute Inc., Cary, NC), using final temperature, hold time, and their interaction as fixed effects for each muscle. Pearson's pairwise correlations were used to show the relationships of flop data to data from instrumentation.

Results: Briskets cooked to $90^{\circ} \mathrm{C}$ with a 3-h hold time had the greatest loss in hot cooked yield, whereas briskets cooked to $75^{\circ} \mathrm{C}$ with 0 -h hold time had the greatest hot cooked product yield $(56.0 \%$ vs. $73.2 \%$, respectively). Flop angle of cooked briskets was dependent on final internal temperature as an increase in final temperature resulted in a narrower $(P<$ $0.05)$ angle. Increased angle of the cooked briskets resulted in greater $(P<0.05)$ cohesiveness, cohesiveness of mass, moisture release, tenderness, and amount of connective tissue scores. Angle of cooked briskets was weakly positively correlated $(P<0.05)$ to WBSF, point poke, center poke, and flat poke $(r=0.21,0.33,0.39$, and 0.31 , respectively). WBSF and slice shear force values decreased $(P<0.05)$ as the raw length of the briskets increased.

Conclusion: Final temperature influences yield, texture, and sensory attributes of brisket. The degree of flop with increased final temperature for cooked briskets was positively related to instrumental measurements of tenderness and may be used to detect when briskets are done.

Keywords: beef brisket, tenderness

\section{6 - CONSUMER ATTITUDES TO ARTIFICIAL MEAT IN CHINA}

M. Liu ${ }^{1}$, P. Dong ${ }^{1}$, Y. Zhang ${ }^{1}$, L. Zhu ${ }^{1}$, L. Niu ${ }^{1}$, X. Luo ${ }^{1}$, and Y. Mao ${ }^{1 *},{ }^{1}$ College of Food Science and Engineering, Shandong Agricultural University, Taian, China, *maoyanwei@163.com

Objectives: China is the largest producer and consumer of meat in the world. The meat consumption amount was predicted to increase from 158.3 to 330.4 million tons between 2010 and 2050, and meat production probably cannot meet this large demand of consumers. At the same time, animal farming places a severe burden on the environment, which raises people's concerns about the sustainable development of animal husbandry. In order to solve these challenges and meet the needs of consumers, artificial meat has received extensive attention in the meat industry, academia, and media. However, the attitude of Chinese consumers towards artificial meat is not explicit. Therefore, the objective of the study was to investigate consumers' attitudes towards cultured meat $(\mathrm{CM})$ and plant-based meat (PBM) and their purchase preference in artificial meat production schemes in China.

Materials and Methods: This research was based on an online survey on the website https://www.wjx.cn/. Participants answered questions including questions about gender, age, education, frequency of consuming meat, and 
familiarity with artificial meat. Then a brief introduction of artificial meat was provided: CM, a kind of animal protein produced by stem cells from an animal and cultured in a suitable medium. PBM is a plant protein product with meat texture and flavor produced through specific processing technology. Respondents also answered about their willingness to purchase CM or PBM, respectively, which was scored by a 5-point rating scale: will not purchase (1 point), would like to know more information and then to determine ( 2 points), would like to try artificial meat ( 3 points), would like to replace part of traditional meat with artificial meat (4 points), would like to replace all traditional meat with artificial meat (5 points). In addition, the participants answered what kinds of production schemes they would like to choose, including CM, PBM, a mixture of PBM and CM (PBM\&CM), and a mixture of artificial meat and traditional meat (AM\&TM). The survey lasted for $1 \mathrm{wk}$, and 3,691 valid responses were obtained. All statistical analysis was performed using SPSS software (IBM Corp., Armonk, NY).

Results: According to this survey, $68.2 \%$ of the 3,691 responders would like to try or replace traditional meat with PBM, with an average score of 3.63 of willingness to purchase, and $64.92 \%$ of responders would like to try or replace traditional meat with CM, giving an average score of 3.61. Moreover, the results also showed that responders preferred $\operatorname{PBM}($ mean $=2.96, \mathrm{SD}=1.13)$ to $\mathrm{CM}($ mean $=2.88, \mathrm{SD}=$ 1.12). In terms of production schemes, the results showed that $40.75 \%$ of consumers chose PBM, and $26.98 \%$, $14.03 \%$, and $18.23 \%$ of consumers chose PBM\&CM, $\mathrm{CM}$, and AM\&TM, respectively (Figure 1). Therefore, PBM will be a relatively popular product among artificial meat products for Chinese consumers.
Conclusion: Chinese consumers have a positive attitude towards purchasing both PBM and CM, and PBM was more preferred in the production schemes of artificial meat. Artificial meat is supposed to be a solution to meet the requirements of meat consumption, and PBM should be paid more attention in this moment.

Keywords: consumer preference, cultured meat, plantbased meat

\section{7 - CONSUMERS' ACCEPTANCE AND PURCHASE INTENT OF LAMB MEAT WITH EDIBLE COATING AT BLIND AND INFORMED CONDITIONS}

R. T. Nassu ${ }^{1 *}$, R. A. Mauricio ${ }^{2}$, C. S. Cordeiro ${ }^{2}$, and V. C. Francisco ${ }^{2},{ }^{1}$ Food Technology, Embrapa Pecuaria Sudeste, Sao Carlos, Brazil, ${ }^{2}$ Alimentos e Nutricao, FCFAr/UNESP, Araraquara, Brazil, *renata.nassu@embrapa.br

Objectives: Packaging affects physicochemical and sensory characteristics of meat. The use of edible coatings is an alternative to extend the shelf life of meat, but consumer acceptance of this type of product is not widely investigated. In this study, lamb meat with chitosan edible coating was evaluated regarding consumers' acceptance and purchase intent in blind and with-label-information conditions.

Materials and Methods: Longissimus muscles from male lambs obtained from a butcher shop were cut into 2-cm-thick steaks, randomized equally and distributed into

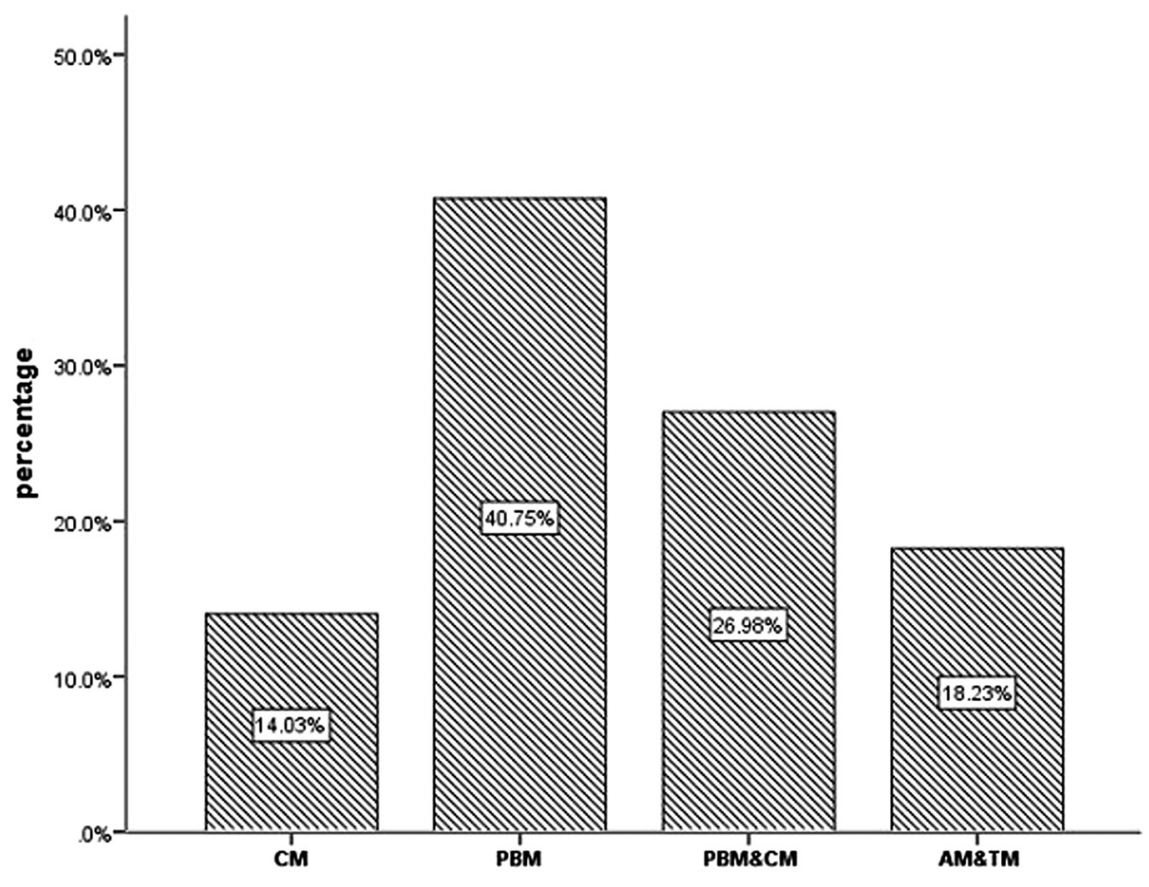

Figure 1. The consumer preference to different manufacturing scheme 
2 treatments: control (no coating) and coated with chitosan $(1 \% \mathrm{w} / \mathrm{v}) / 0.5 \%$ glycerol $(\mathrm{w} / \mathrm{v})$ solubilized in $1 \%$ lactic acid $(\mathrm{v} / \mathrm{v})$. The lamb meat was grilled (internal temperature, $\left.80^{\circ} \mathrm{C}\right)$, salted ( $1 \mathrm{~g}$ sodium chloride), cut in cubes $(1.5 \mathrm{~cm}$ of side), individually wrapped in aluminum foil, and kept warm at $60^{\circ} \mathrm{C}$, in a controlled temperature oven. One-hundred and fifty-three consumers evaluated the samples using a 9-point ( $1=$ disliked extremely; $9=$ liked extremely) scale for sensory acceptance and a 5-point $(1=$ certainly would not buy; 5 = certainly would buy) scale for purchase intent. The control (noncoated) and chitosan-coated samples were served in a randomized order, in 2 sessions: blind condition (no label) and informed condition (with a label indicating the use of the chitosan coating). The obtained data were analyzed by analysis of variance. Cluster analysis by Ward's method was also performed to verify whether there were different groups according to the responses.

Results: The control treatment (noncoated) and chitosancoated in the blind condition showed the same values (7.2) and were higher $(P<0.05)$ than those from informed condition ( 6.3 for both treatments), indicating that the information in the label affected the product's acceptance negatively. Purchase intent values were the same (3.5) for chitosancoated samples in the blind and informed conditions and lower $(P<0.05)$ than control samples, in both conditions, blind (4.0) or informed (3.8), indicating that label information did not affect this parameter, differently from sensory acceptance. After segmentation by cluster analysis, in group $1(n=40)$, the chitosan-coated with information sample showed the highest values $(P<0.05)$ for sensory acceptance (7.1) and purchase intent (3.9). In group $2(n=52)$, no difference $(P>0.05)$ was found for sensory acceptance between samples for both blind condition (noncoated $=7.9$; chitosan-coated $=8.2$ ) and informed condition (noncoated = 7.7; chitosan-coated $=7.8$ ), and purchase intent was not different $(P>0.05)$ among all the samples, ranging from 4.2 to 4.3. Finally, in group $3(n=55)$, a significant difference $(P$ $<0.05$ ) was found between control samples in the blind (6.9) and informed (4.9) conditions, and the same for the chitosancoated (blind $=7.1$; informed $=4.4$ ) samples for sensory acceptance, but purchase intent was not different $(P>$ $0.05)$ between control samples in the both conditions (3.9) and chitosan-coated samples (blind $=2.7$; informed $=2.8$ ).

Conclusion: The label information affected sensory acceptance and purchase intent of chitosan-coated lamb meat, and according to cluster analysis, different consumer responses were found.

Acknowledgments: We acknowledge FAPESP process number 2016/18232-3 and Higher Education Personnel Improvement Coordination-Brazil (CAPES) Financing Code 001.

Keywords: consumer behavior, lamb, sensory analysis

\section{8 - USE OF A MEAT SCIENCE COURSE FOR EVALUATION OF CONSUMER KNOWLEDGE AND APPLICATION OF MEAT THERMOMETERS}

\author{
H. R. Smith ${ }^{1 *}$, J. Gallado ${ }^{1}$, A.-G. Parnell ${ }^{1}$, D. Mulvaney ${ }^{1}$, \\ and J. T. Sawyer ${ }^{1},{ }^{1}$ Animal Sciences, Auburn University, \\ Auburn, AL,USA,*jts0109@auburn.edu
}

Objectives: The objectives of the current study were to equip course participants with knowledge of meat cookery and proper endpoint temperatures in an effort to reduce the incidence of food-borne illnesses. In addition, it was intended that the course participants in this survey would enhance their accuracy, frequency, and comfort level when using a thermometer for cooking meat products.

Materials and Methods: Undergraduates participating in a meat science course have limited knowledge of meat cookery and food safety. In an effort to enhance the undergraduate consumer education on use of food safety measures during meat cookery, a module on meat cookery and meat thermometers was incorporated into the Muscle Foods course within the Department of Animal Sciences. ANSC 3700 is a 4-credit course taught each spring semester with an annual enrollment of up to 60 participants. Muscle Foods is designed to introduce the theories and principles of meat animal production from conception to consumer. Students participate in 1-h lectures (3/wk) and a 2-h "hands-on" laboratory experience $(1 / \mathrm{wk})$ at the Lambert-Powell Meat Laboratory. During the first week of the course, a survey (PRETEST) on the use and application of meat thermometers was provided electronically to each student within the course using Qualtrics online survey software. In a 5-min survey, participants were asked level of intent on purchasing, using, and owning a meat thermometer. In addition, questions were asked about degree of doneness, methods to determine degree of doneness, and frequency of using a thermometer when cooking meat. Demographic questions were asked to aid in identifying previous potential influencers of meat thermometer use. During the course lecture and laboratory meetings, educational material was presented to course participants regarding the use of meat cookery methods and various thermometer models (dial vs. digital). The information on measuring meat and food internal cooking temperature occurred on 8 different occasions throughout the 16-wk course. With the interruption of courses due to coronavirus disease 2019, delivery of content transitioned from face to face to a virtual classroom. To summarize the use and application of a meat thermometer during cooking, a video produced by the North American Meat Institute was provided to course participants. Following the video, course participants completed a 5-min POSTTEST survey, which included the same PRETEST questions.

Results: Responses (PRETEST) from course participants indicate that $70 \%$ can measure meat cookery using a thermometer. However, participants (67.24\%) also deem 
that cutting into a hamburger is used for determining degree of doneness. Course participants $(65.52 \%)$ measuring internal temperature of hamburgers use $71.1^{\circ} \mathrm{C}$ as the safest internal temperature, whereas the use of a thermometer on multiple occasions (3.5\%) needs to be addressed.

At the conclusion of the course (POSTTEST), respondents indicated $(51.06 \%)$ that they were extremely confident in purchasing the correct thermometer. Additionally, at the conclusion of this course, respondents $(100 \%)$ were more confident in measuring internal meat temperature. Respondents $(72.34 \%)$ also deemed that $71.1^{\circ} \mathrm{C}$ was a safer internal temperature for hamburgers.

Conclusion: Results suggest that providing training on use and adoption of meat thermometers for cooking is beneficial to undergraduate consumer knowledge of meat cookery. In addition, training on meat cookery and internal meat doneness should possibly occur earlier than a collegiate meat science course.

Keywords: consumer survey, meat cookery

\section{9 - A COMPARISON OF WET AND DRY AGING ON THE SENSORY PROPERTIES OF BONELESS PORK LOINS}

D. J. Hanson ${ }^{1}$, T. A. Tennant ${ }^{2}$, and J. A. Ascencio ${ }^{1}$, ${ }^{1}$ Department of Food, Bioprocessing and Nutrition Sciences, North Carolina State University, Raleigh, NC, USA, ${ }^{2}$ Department of Agricultural Sciences, West Texas A\&M University, Canyon, TX, USA, *dana_hanson@, ncsu.edu

Objectives: Wet aging (WA) is the predominant form of postmortem aging for pork; however, there is growing consumer interest in dry-aged (DA) meat. The goal of this study was to compare differences between WA and DA boneless pork loins using consumer and trained panelist sensory analysis.

Materials and Methods: Boneless pork loins, Institutional Meat Purchase Specifications $413(n=45)$, were cut across the medial plane and randomly assigned to either WA or DA treatments. Both treatments consisted of 15 loin sections aged for 10,20 , and $30 \mathrm{~d}$, respectively. WA samples were stored at $3^{\circ} \mathrm{C}$ in vacuum-sealed packages. DA samples were stored (unpackaged) within an environmental chamber (Stagionello Maturmeat, Arredo Inox Srl., Crotone, Italy), at $3{ }^{\circ} \mathrm{C}$ and $75 \%$ relative humidity. At the end of each aging period, loin sections were weighed and trimmed prior to being cut into $12-\mathrm{mm}$-thick sensory chops. Sensory samples were stored at $-25^{\circ} \mathrm{C}$ for future sensory testing. Samples for consumer and trained sensory panels were prepared by thawing, for $24 \mathrm{~h}$ at $3^{\circ} \mathrm{C}$, prior to being cooked to an internal temperature of $66^{\circ} \mathrm{C}$, using a Taylor clamshell grill (Model \#L820-23). Cooked chops were allowed to rest $1 \mathrm{~min}$ before being cut into
$2.5 \times 2.5 \times 1.2 \mathrm{~cm}^{2}$ samples. Samples were evaluated using consumer acceptance tests $(n=126)$ and descriptive analysis with highly trained panelists $(n=6)$ following Spectrum $®$ methods. Consumers were asked overall liking, flavor, freshness, texture, moisture, meaty flavor liking, and purchase intent of samples. Trained panelists evaluated the samples for aromatics, basic tastes, and texture. Pork loins were randomly assigned to a $2 \times 3$ factorial; fixed effects were aging method (DA and WA) and aging length (10, 20, and $30 \mathrm{~d})$. Consumer and descriptive analysis data were analyzed using two-way analysis of variance, Kruskal-Wallis nonparametric, and chi-squared test of XL STAT (Addinsoft, New York, NY).

Results: Physical properties of pork loins were greatly affected due to dehydration and trimming loss in loins. Samples aged 10, 20, and $30 \mathrm{~d}$ lost 10.12\%, 21.92\%, and $31.6 \%$ of their initial raw weight, respectively. Overall eating experience was not improved due to either aging method. When evaluating the consumer acceptance test, WA and DA were not significant $(P>0.05)$ factors for overall liking, flavor, freshness, texture, moisture, meaty flavor liking, and purchase intent of pork. DA panel results did not reveal any meaningful differences in pork aroma, flavor, or texture across aging methods or duration.

Conclusion: WA and DA are both equally liked and accepted by pork consumers and trained panelists. Dry aging boneless pork loins does not appear to increase consumer acceptability.

Keywords: dry aging, pork, sensory analysis

\section{Education and Extension Tools}

\section{0 - NEEDS ASSESSMENT OF FUNDAMENTAL MEAT SCIENCE TRAINING/CONTINUED EDUCATION FOR EXTENSION EDUCATORS AND YOUTH AGRICULTURAL ADVISORS TO DETERMINE THE APPETITE FOR INNOVATIVE AND NONTRADITIONAL TRAINING PRACTICES}

\author{
B. M. C. Garcia ${ }^{1 *}$, J. N. Rumble ${ }^{2}$, and L. G. Garcia ${ }^{1},{ }^{1}$ Animal \\ Science/Meat Science, The Ohio State University, \\ Columbus, OH, USA, ${ }^{2}$ Agricultural Communication Educa- \\ tion Leadership, The Ohio State University, Columbus, $\mathrm{OH}$, \\ USA, *garcia.951@osu.edu
}

Objectives: The objective was to determine whether a need exists for extending traditional meat science content to extension educators and youth agricultural advisors, 
enhancing their understanding of the relationship between the livestock and meat industries in the state of Ohio.

Materials and Methods: A needs assessment and preference survey was sent to extension educators and youth agricultural advisors across the state. The survey included 19 questions and gave respondents the opportunity to provide additional comments if desired. Categories included questions regarding the need, importance, and role of meat science, the availability of meat science training, the facilitation of training, resource availability, and respondent's willingness to attend training if available. The survey included a Likert scale with choices ranging from "strongly disagree" to "strongly agree."

Results: A total of 112 surveys were completed and collated for assessment with 40 respondents providing additional comments. When asked questions regarding the importance/ awareness of meat science across students, exhibitors, and producers, $97.13 \%$ of respondents "agreed" or "strongly agreed." When asked questions prompted to measure their need and desire for training, 76.61\% "agreed" or "strongly agreed" to a need. When asked whether willing to attend a local 2-d workshop, $68.32 \%$ of respondents indicated that "yes" they would attend, whereas $27.72 \%$ indicated that they would "maybe" attend. Finally, respondent location information was collected to better understand training needs by region. Comments included such themes as overall support and need for additional training, willingness to host or facilitate training, need for exhibitors to understand relationship between raising animals and end product, and concerns about resource availability. One respondent commented, "Our advisory committee mentioned in our last meeting how butchering and knowledge of meat science was becoming a lost art in our area. They would fully support a program like this."

Conclusion: Meat science is a unique area of focus that not all understand, yet it makes up a large portion of the U.S. economy. The results of the survey suggest that extension educators and youth agricultural advisors have a need for, and understand the importance of, additional education in the area of meat science. The population surveyed expressed a strong will to attend additional training programs if provided. This supports a data-driven decision to move forward with the development of a training program focused in the field of meat science by means of a nontraditional practice in academia.

Keywords: education, extension, meat science, Ohio, training

\section{1 - ADAPTING FOOD PROCESSING TO DECONTAMINATE N95 RESPIRATORS DURING THE 2019 NOVEL CORONAVIRUS PANDEMIC}

J. P. Schweihofer ${ }^{1 *}$, T. Conklin ${ }^{2}$, J. Baker ${ }^{3}$, W. Degg ${ }^{3}$, J. Hofman $^{2}$, J. Klausner ${ }^{4}$, and J. W. Dwyer ${ }^{2},{ }^{1}$ Michigan
State University Extension, Michigan State University, Port Huron, MI, USA, ${ }^{2}$ Michigan State University Extension, Michigan State University, East Lansing, MI, USA, ${ }^{3}$ Sparrow, Sparrow Health System, Lansing, MI, USA, ${ }^{4}$ Mechanical Engineering, Michigan State University, East Lansing, MI, USA, *grobbelj@msu.edu

Objectives: The coronavirus disease 2019 pandemic caused an abrupt shift in university practices and a great need for personal protective equipment (PPE) by medical providers, first responders, and food plant personnel. The objective was to utilize food manufacturing equipment and food safety principles to decontaminate PPE for required personnel.

Materials and Methods: The Michigan State University Extension dry heat decontamination system for N95 respirators (masks) was developed. Logistics for labeling, collecting, and transporting used masks were established with a partnering health system provider. Personnel decontaminating masks wore appropriate PPE. A spiral oven (Unitherm/ Marlen XSSO-12-1.1-6AG-T, Riverside, MO) was set to $82.2^{\circ} \mathrm{C}$ with a $45-\mathrm{min}$ residence time and fan speed of $95 \%$ and masks placed on a coffee filter on the oven belt. A calibrated datalogger (HOBO, Bourne, ME) was included in each batch to verify that the critical control point of $75^{\circ} \mathrm{C}$ for 30 min was met. Validation was conducted for biological indicators, mask integrity, and fit testing. Vegetative bacteria (S. aureus ATCC 29213, K. pneumoniae ATCC 700603, E. coli ATCC 25922, P. aeruginosa ATCC 27853, K. pneumoniae ATCC 27736, K. pneumoniae ATCC 35657, K. oxytoca ATCC 49131, E. aerogenes ATCC 13048, E. cloacae ATCC 23355) were used to determine $8 \log$ reduction biological effectiveness of the dry heat decontamination system in killing bacteria and viruses such as the novel coronavirus. Mask integrity including filtration efficiency and pressure drop was conducted on masks $(n=3)$ at new and after 5 , 10,15 , and 20 heat cycles. Fit testing was completed on 6 masks after 10,15, and 20 heat cycles. Cross-contamination was minimized by developing a no-touch system for the masks, and the facility utilized was divided between used and decontaminated masks with separate foot traffic. Decontaminated masks were packaged in clear plastic bags, sealed, labeled, and boxed to return to the originating facility and original mask user.

Results: One hundred percent of masks passed fit testing after 10 cycles of dry heat decontamination. It remained at $100 \%$ passing after the masks were decontaminated for 15 and 20 cycles $(P=1.00)$. The fit test has a confidence interval of $54 \%-100 \%$ at $95 \%$ probability. Biological growth validation results indicated that there was no growth of the vegetative bacteria tested after going through the dry heat decontamination system $(P=0.1)$. The dry heat decontamination system was effective for no biological growth at $48 \mathrm{~h}$, mask integrity and fit testing passed through 20 cycles. An emergency use authorization application was submitted to the U.S. Food and Drug Administration with accompanying risk assessments and is currently being reviewed. 
Conclusion: The Michigan State University Extension dry heat decontamination system for N95 respirators is effective at extending the use of the critical PPE through 20 uses during times of shortages and the coronavirus disease 2019 pandemic. Dry heat treatment utilizing food safety principles and processing equipment has potential for global implementation during periods of extreme PPE shortages, including pandemics.

Keywords: decontaminate, food processing, pandemic, personal protective equipment

\section{2 - FEEDER CALF RETAINED OWNERSHIP EDUCATIONAL PROGRAM: ALABAMA PASTURE TO RAIL}

A. Tigue ${ }^{1}$, H. R. Smith ${ }^{1 *}$, C. L. Bratcher ${ }^{2}$, L. K. Anderson ${ }^{1}$, E. Blythe ${ }^{3}$, S. P. Rodning ${ }^{1}$, and J. T. Sawyer ${ }^{1},{ }^{1}$ Animal Sciences, Auburn University, Auburn, AL, USA, ${ }^{2}$ College of Agriculture, Texas Tech University, Lubbock, TX, USA, ${ }^{3}$ College of Agriculture, Auburn University, Auburn, AL, USA, *jts0109@auburn.edu

Objectives: The Alabama Pasture to Rail is a retained ownership program that provides a mechanism for Alabama beef producers to collect feeder calf performance, health, and carcass data, for enhanced management decisions. The majority of beef cattle throughout the southeastern U.S. are marketed as stocker or feeder calves. Traditional marketing platforms through regional stockyards result in cattle being transitioned to the Midwest region of the U.S. for the confined finishing phase.

Materials and Methods: Prior to shipping, all cattle arrive at a centralized location where body weights are recorded, feeder calf grades are assigned, and cattle are identified with both radio frequency and visual ear tags. Following processing phase in Alabama, cattle are transported to the Hy-Plains Feedyard in Montezuma, Kansas. Harvest dates are determined by feedyard management as the projected date of animal finishing approaches. Cattle are sold on a carcass merit grid, where premiums and discounts are applied using USDA Yield and Quality grades. After harvest and chilling, carcass characteristics were captured electronically. Live weight is estimated using the individual hot carcass weight and the carcass yield percentage of the harvest group. In addition, average daily gain is also a calculated estimate, using the estimated live weight. Data were analyzed with linear models using the GLIMMIX procedure of SAS version 9.4 (SAS Institute Inc., Cary, NC) with explanatory variables as gender (steer, heifer) and year $(2017,2018,2019)$ and response variables as initial weight, live weight, average daily gain, hot carcass weight, days on feed, ribeye area, backfat, and marbling, in addition to quality and yield grade.

Results: Results indicate that feeder cattle prices and total production costs were greatest in 2019, whereas carcass value and payable amount were greater $(P<0.05)$ during 2018, with profit being the greatest $(P<0.05)$ in 2017. Cattle participants in 2018 provided the greatest $(P<$ $0.05)$ initial weight, live weight, average daily gain, and hot carcass weight coupled with the least $(P<0.05)$ days on feed. For 2018 , carcass produced greater $(P<0.05)$ ribeye areas, less backfat, and higher marbling scores. In contrast, 2019 provided a different perspective of carcass traits as shown by the least $(P<0.05)$ amount of muscling in the ribeye area, subsequently resulting in the highest numerical carcass yield grade. The economic value by gender suggests that steer carcass value and payable amount were greater $(P<0.05)$ than heifers. Nonetheless, the data suggest that heifers can result in a greater $(P<0.05)$ price per hundredweight consuming less $(P<0.05)$ total cost, resulting in a greater $(P<0.05)$ profit margin. These results indicate that steers began the project with greater $(P<0.05)$ initial weight, ultimately resulting in greater $(P<0.05)$ live weight, average daily gain, and hot carcass weight at the conclusion of the finishing phase. Gender differences for steer and heifer carcass traits indicate that steers resulted in significantly larger $(P<0.05)$ ribeye area, less backfat, and lower numerical carcass yield grade. Conversely, it was intriguing that heifer carcasses displayed greater $(P<0.05)$ marbling in the ribeye, greater backfat, greater calculated yield grade, and less muscling in the ribeye area $(P<0.05)$.

Conclusion: Analysis of Alabama Pasture to Rail results suggests the importance of providing growth, carcass, and financial data for southeast beef producers. A greater knowledge of feedyard performance and carcass merit could aid beef producers of the southeast in production management decisions.

Keywords: beef cattle, carcass data, feedlot performance, retained ownership

\section{3 - EXPLORING THE MEAT INDUSTRY IN PUERTO RICO}

K. Perez ${ }^{1 *}$, I. M. Santana ${ }^{1}$, K. I. Domenech ${ }^{2}$, M. T. Rodriguez ${ }^{3}$, and L. G. Garcia ${ }^{1},{ }^{1}$ Animal Sciences, THE OHIO STATE UNIVERSITY, Columbus, OH, USA, ${ }^{2}$ Agricultural Sciences, University of Puerto Rico-Mayaguez, Mayaguez, Puerto Rico, ${ }^{3}$ Agricultural, Communication, Education, and Leadership, THE OHIO STATE UNIVERSITY, Columbus, OH, USA, *garcia.625@osu.edu

Objectives: The purpose of this qualitative research study was to explore the perceptions of the meat industry in Puerto Rico. The first of its kind, necessary questions served as the initial step in an attempt to understand the 
current situation of the meat industry, primarily focusing on beef.

Materials and Methods: Thirty semi-structured interviews were conducted using a validated group of experts involved in the beef industry over a 3-month time frame. Experts included professionals $(n=7)$, extensionists $(n=$ $17)$, and beef producers $(n=6)$.

Results: Through thematic analysis, 4 themes were identified: (1) quality of meat, (2) priorities in the meat industry, (3) support needed, and (4) disconnect between consumers and educators. (1) Perceptions of meat quality varied greatly among professionals and beef producers. Professionals believe marbling was a key factor for quality. Extensionists and beef producers related quality with local products, animal welfare, low-fat content, tenderness, and grass-fed. Interestingly, a slaughterhouse professional did not consider meat color as a quality factor. (2) Priorities items differed among groups starting with hobby farmers struggling to compete with dairy cattle, the largest sector, as others focused on higher-quality forages necessary to improve meat quality (external fat) to providing a fresh product and food security. (3) With experts and resources lacking, slaughterhouses and number of cattle are not comparable. On the other hand, a participant believed the support needed is that "[the industry should] modify how beef is being marketed to better benefit the producers." According to another participant, support for producers is limited and "communication is mostly supported by phone calls." (4) Several interviewees believe that the government should educate consumers, increasing interest in local Puerto Rican beef production and meat consumption. From the professionals perspective, producers are financially impacted because slaughterhouses can set their own prices without using a standard base price; therefore, beef producers struggle to grow. A professionals comment included that "everyone [professionals, extensionists, and beef producers] is too focused on their side of the business. There is a lack of communication with consumers who maintain their interest in low prices."

Conclusion: This research shows that the meat industry of Puerto Rico has significant opportunities for growth. The disconnect, and varying levels of knowledge and awareness, between groups hinders the meat industry, overall. Better communication, education, and teamwork is necessary to assist in strengthening the needs of Puerto Rico. Further studies are recommended to better understand the major voids of existing gaps. Identifying the definition of "quality" should be an initial priority in order to keep a goal (RMT1) in promoting consumer desired meat products economically benefiting Puerto Rico as whole.

Keywords: beef, consumer perception, meat industry, Puerto Rico

\section{Environment, Production Systems}

\section{4 - COMPARISON OF WINTER COW FEEDING STRATEGIES ON OFFSPRING CARCASS CHARACTERISTICS AND MEAT QUALITY}

E. Gubbels ${ }^{1 *}$, R. R. Salverson ${ }^{1}$, C. L. Wright ${ }^{1}$, K. M. Cammack $^{1}$, J. F. Legako ${ }^{2}$, Z. K. Smith ${ }^{1}$, J. K. Grubbs ${ }^{1}$, K. R. Underwood ${ }^{1}$, K. C. Olson ${ }^{1}$, and A. D. Blair ${ }^{1}$, ${ }^{1}$ Animal Science, South Dakota State University, Brookings, SD, USA, ${ }^{2}$ Animal \& Food Sciences, Texas Tech University, Lubbock, TX, USA, *erin.gubbels@sdstate.edu

Objectives: The objective of this study was to investigate the effects of maternal prepartum dietary energy source (forage vs. concentrate) during mid and late gestation on offspring carcass characteristics and meat quality.

Materials and Methods: Angus $\times$ Simmental crossbred cows from a single source were stratified by body weight and age into 2 groups, transported to a drylot, and randomly assigned to one of 2 treatments ( $n=48 /$ treatment): Concentrate (dams provided a limit-fed concentrate-based diet) or Forage (dams provided an ad libitum forage-based diet). Treatment diets were isocaloric and isonitrogenous. Offspring from both treatments were weaned and transported to the Cow Calf Education and Research Facility in Brookings, South Dakota, where they received a finishing ration common to feedlots in the Upper Midwest. Ultrasound fat thickness and intramuscular fat percentage were estimated and used to project marketing dates. Carcass measurements were recorded at the time of harvest and included hot carcass weight $(\mathrm{HCW}), 12$ th rib backfat $(\mathrm{BF})$, ribeye area (REA), USDA Yield grade and Quality grade, marbling score, and instrumental color measurements $\left(L^{*}, a^{*}\right.$, and $\left.b^{*}\right)$. A striploin was collected from each carcass and portioned into 2.54-cm steaks. Four steaks were aged 3, 7, 14, or $21 \mathrm{~d}$ for Warner-Bratzler Shear Force (WBSF) analysis. Additional steaks were designated for Fatty Acid Methyl Ether Synthesis, determination of crude fat, and trained sensory panel evaluation of 14-d aged samples. Statistical analyses were conducted using generalized linear mixed model procedures (SAS GLIMMIX, SAS Inst. Inc., Cary, NC) for effects of treatment, sex, and their interaction. WBSF were analyzed for effects of treatment, sex, aging period (AP), and their interaction, with AP considered as a repeated measure and peak cooking temperature included as a covariate. Separation of least-squares means was conducted using protected least significant difference assuming an alpha level of 0.05 .

Results: No treatment $\times$ sex interactions $(P>0.05)$ were detected for carcass or meat quality traits. Additionally, maternal treatment did not influence $(P>0.05)$ offspring $\mathrm{HCW}$, REA, Quality grade, marbling score, $L^{*}$ values, WBSF, crude fat content of steaks, or trained sensory ratings. 
Offspring from the concentrate treatment had increased $(P<$ $0.05) a^{*}(2.65 \%)$ and $b^{*}(4.02 \%)$ values and tended to have increased $(P=0.06) \mathrm{BF}(6.56 \%)$ and $(P=0.08)$ Yield grade (6.67\%), compared with offspring from the forage treatment. The concentration $(\mathrm{mg} / \mathrm{g})$ of arachidonic, nervonic, and docosapentaenoic acids were increased in samples from the concentrate treatment $(P<0.05)$; treatment did not influence $(P>0.05)$ concentration of other fatty acids. Steers had heavier $(P<0.05) \mathrm{HCW}$ and larger $(P<0.05)$ REA than heifers. Heifers had increased $(P<0.05) \mathrm{BF}$ and marbling scores, as well as increased $(P<0.05) a^{*}$ and $b^{*}$ values, percent moisture, and crude fat content than steers. Tenderness improved $(P<0.05)$ with AP; however, there was a tendency $(P=0.08)$ for a sex $\times$ AP interaction for WBSF. WBSF of heifers did not differ $(P=0.79)$ from steers at day 3 but were more tender $(P=0.08)$ at day 7,14 , and 21 .

Conclusion: Collectively, these data suggest that a forage-based diet provided to cows during mid and late gestation programmed offspring to decrease deposition of subcutaneous fat without compromising marbling score, tenderness, or other sensory attributes.

Keywords: beef, carcass characteristics, concentrate, forage, meat quality

\section{5 - FABRICATION YIELDS OF SERIALLY HARVESTED IMPLANTED OR NONIMPLANTED STEERS}

K. Wesley ${ }^{1 *}$, T. Kirkpatrick ${ }^{1}$, S. Pillmore ${ }^{1}$, K. Cooper ${ }^{1}$, T. Tennant $^{1}$, W. Nichols ${ }^{2}$, J. Hutcheson ${ }^{2}$, and T. Lawrence ${ }^{1}$, ${ }^{1}$ Beef Carcass Research Center, West Texas A\&M University, Canyon, TX, USA, ${ }^{2}$ Technical Services, Merck Animal Health, Madison, NJ, USA,*kwesley@wtamu. edu

Objectives: Cattle administered growth-promoting implants exhibit an increase in carcass weights, often composed of increased lean and bone mass, with variable results observed in fat mass. Specific carcass components that experience this increase in mass, and the rate to which this change occurs, are limited in the literature. The objective of this study was to quantify differences in fabrication yields of implanted and nonimplanted steers.

Materials and Methods: Steers (Charolais $\times$ Angus cross, $n=80$; initial body weight [BW] $271 \pm 99 \mathrm{~kg}$ ) were paired by genetic group, estimated finished BW, frame score, and days to target $\mathrm{BW}$ and randomized to harvest date (day 0, 42, 84, 126, 168, 210, 252, 294, 336, 378), and individuals within pairs were randomized to nonimplanted negative control or REV (Revalor-XS on day 0 and 190) treatments. Right sides of each animal were fabricated after a 48 -h chill into primals, denuded subprimals, lean trim (80/20), trimmed fat (>95/5), and bone; weights were recorded individually. Data were analyzed via mixed models.

Results: Administration of REV increased $(P \leq 0.03)$ absolute mass of cold side weight (CSW) $7.7 \%$, bone mass $4.9 \%$, and red meat mass $8.5 \%$ but did not alter fat mass $(P=0.78)$. Muscling (muscle:bone) and leanness (muscle:fat) were unaffected by treatment $(P \geq 0.27)$. Leanness decreased in a quadratic manner across days on feed (DOF) $(P<0.01)$, while muscling was unaffected by DOF $(P=0.90)$. Administration of REV increased $(P \leq 0.02)$ brisket and foreshank primals by $6.9 \%$ and $7.2 \%$, respectively. Chuck primals from REV steers were $8.4 \%$ heavier $(P \leq 0.02)$, with similar trends in the shoulder clod, flat iron, petite tender, chuck eye roll, and mock tender subprimals. Rib primals from REV steers were 5.2\% heavier $(P \leq 0.04)$, along with the ribeye roll and rib blade meat. Plate primals did not differ $(P=0.13)$ between treatments; however, the inside skirt, outside skirt, and outside skirt as \% CSW were heavier $(P \leq 0.04)$ from REV steers. Loin primals from REV steers were $7.0 \%$ larger $(P<0.01)$, along with the striploin, tenderloin, top sirloin butt, top sirloin butt cap, and bottom sirloin tri tip subprimals. Flank primals from REV steers were $8.6 \%$ heavier $(P \leq$ $0.04)$, bottom sirloin flap and flank steak were also heavier, and the elephant ear tended to be heavier $(P=0.08)$. Round primals from REV steers were $6.3 \%$ heavier $(P \leq 0.03)$, and the top round, eye of round, bottom round, and sirloin tip all exhibited similar upward trends. Proportion of plate $(0.009 \%)$, flank $(0.007 \%)$, rib $(0.004 \%)$, and brisket $(0.002 \%)$ to $\% \mathrm{CSW}$ all increased with increasing DOF, whereas the loin $(-0.001 \%)$, chuck $(-0.001 \%)$, foreshank $(-0.002 \%)$, and round $(-0.017 \%)$ decreased. Fat yield increased at $0.043 \%$ of CSW per day, whereas red meat yield and bone decreased at $0.023 \%$ and $0.013 \%$ of CSW per day, respectively. Length of feeding period notably affected $(P<0.01)$ all primal weights and \% CSW, with the exception of the chuck and loin as \% CSW ( $P \leq$ $0.17)$. These data indicate that carcasses from implanted steers are more likely to have heavier side weights, consisting of heavier quantities of bone and red meat with no change in fat. Although implants increased tissue mass, they did not alter the percentage of primal or subprimal components.

Conclusion: These data indicate that carcasses from implanted steers are more likely to have heavier side weights, consisting of heavier quantities of bone and red meat with no change in fat. Although implants increased tissue mass, they did not alter the percentage of primal or subprimal components.

Keywords: fabrication yields, implants, leanness, muscling, serial harvest 


\section{6 - COMPARISON OF CONVENTIONAL GRAIN-FED AND GRASS-FED FEEDING SYSTEM EFFECTS ON THE MICROBIOTA OF FECES FROM CALIFORNIA BEEF CATTLE}

F. Yang ${ }^{1}$, S. Klopatek ${ }^{1}$, J. Oltjen ${ }^{1}$, and X. Yang ${ }^{1},{ }^{1}$ Animal Science, University of California, Davis, Davis, CA, USA, *frpyang@ucdavis.edu

Objectives: Cattle feeding systems are a major factor influencing cattle gut microbiota, which can have potential impact on public health if unfavorable shifts in the microbiota are followed by fecal contamination of food production systems. The objective of this study was to compare the effects of grain-based and grass-based feeding systems on the microbial communities of feces from beef cattle.

Materials and Methods: Postweaning cattle were blocked by weight and randomly assigned to 4 treatment groups by feeding system: (1) conventional feedlot (CON, $n=22$ ) harvested in April, (2) 20-mo grass-fed (20GF, $n=$ 17) harvested in June, (3) grass-fed then fed grain $45 \mathrm{~d}$ (GR45, $n=13$ ) harvested in July, and (4) 26-mo grass-fed (26GF, $n=16$ ) harvested in October. Rectal fecal samples from the cattle were collected at 14 mo of age as baseline prior to treatment group assignment, and collected again 1 wk before each treatment group's harvest. Microbial DNA was extracted from samples, amplified targeting the V4 region of $16 \mathrm{~S}$ ribosomal RNA, and sequenced via Illumina MiSeq. Raw sequences were demultiplexed and imported into QIIME2 for processing. Analysis of differences in microbiota between treatments and sampling points was conducted in $\mathrm{R}$ version 3.6.2. Alpha diversity was assessed by Chaol and Shannon indices, beta diversity was assessed using PCoA via Bray-Curtis dissimilarity, and differences in relative abundances between treatments were assessed using Kruskal-Wallis and Mann-WhitneyWilcoxon tests.

Results: At baseline, there were no differences $(P>$ $0.05)$ in alpha diversity among treatment groups. As cattle grew older, alpha diversity of microbiome for $20 \mathrm{GF}$ and GR45 cattle increased $(P<0.05)$ at the time of harvest. At harvest, the alpha diversity of microbiome of fecal samples from 20GF and GR45 cattle was larger $(P<$ $0.05)$ than from CON cattle. Beta diversity analysis of sample OTU showed samples from GR45 cattle formed distinct clusters when mapped via PCoA, indicating a different bacterial composition from other groups. Organizing OTU by phyla showed that Bacteroidetes and Firmicutes were the most dominant phyla for all treatments and sampling points. The CON cattle had larger $(P<0.05)$ Bacteroidetes abundances compared to GR45 cattle, supporting prior studies showing Bacteroidetes members' role in the digestion of complex carbohydrates such as undigested starch, which can be found in higher proportions in the rumen of grain-fed cattle than in grass-fed cattle. No differences $(P>0.05)$ in Firmicutes abundance were detected. Proteobacteria abundances of GR45 and $25 \mathrm{GF}$ cattle increased $(P<0.05)$ from baseline to harvest and were larger $(P<0.05)$ than $\mathrm{CON}$ Proteobacteria abundances.

Conclusion: The results suggest that use of grain in cattle feeding systems had an impact on bacterial communities. Fecal bacterial communities from grain-fed cattle had lower bacterial diversity than grass-fed cattle. The results support the idea that Bacteroidetes play an important role in complex carbohydrate digestion. Differences in Proteobacteria abundance in the cattle primarily on grassbased systems may suggest an association between grass-fed systems and increased risk of presence of common foodborne Proteobacteria pathogens of concern. Increased alpha diversity from cattle harvested in summer months may warrant further study of seasonal effects on cattle fecal microbiota.

Keywords: beef, feces, grain-fed, grass-fed, microbiota

\section{7 - EMPTY BODY COMPOSITION OF STEERS IMPLANTED WITH TRENBOLONE ACETATE + ESTRADIOL-17 $\beta$ VERSUS NON- HORMONE-TREATED CATTLE ACROSS MULTIPLE MARKETING ENDPOINTS}

T. J. Kirkpatrick ${ }^{1 *}$, K. Wesley ${ }^{1}$, S. Pillmore ${ }^{1}$, K. Cooper ${ }^{1}$, F. Francis $^{1}$, T. Tennant ${ }^{1}$, W. Nichols ${ }^{2}$, J. Hutcheson ${ }^{2}$, T. Lawrence $^{1}$, and Beef Carcass Research Center, ${ }^{1}$ Agricultural Sciences, West Texas A\&M University, Canyon, TX, USA, ${ }^{2}$ Technical Services, Merck Animal Health, Madison, NJ, USA, *tkirkpatrick@wtamu.edu

Objectives: Administration of growth-promoting implants has been shown to alter chemical composition of the empty body in beef cattle. A serial harvest study evaluated days on feed (DOF) and trenbolone acetate + estradiol- $17 \beta$ administration on empty body composition across various harvest endpoints.

Materials and Methods: Charolais $\times$ Angus steers ( $n=80$; start of trial body weight [BW] $271 \pm 99 \mathrm{~kg}$ ) were randomly allocated to implant treatment and harvest date in a $2 \times 10$ factorial design. Steers were paired to minimize variation in genetic group, initial BW, frame score, and adjusted final BW. Within each pair, a steer was randomly allocated to one of 2 treatments: implanted with RevalorXS (REV) on day 0 and day 190 or nonimplanted control $(\mathrm{CON})$. Eight steers comprising 4 pairs were randomly assigned to one of 10 harvest dates at day $0,42,84,126$, $168,210,252,294,336$, and 378 DOF. Empty body composition was determined via proximate analysis of blood, hide, internal cavity components (all thoracic and abdominal components dissected and homogenized), bone, and carcass soft 
tissue (all lean and fat dissected and homogenized from the whole carcass). Proximate analysis of each tissue was multiplied by mass to assimilate empty body percentages of moisture, crude protein, ether extractable fat (EBF), and ash. Data were analyzed using mixed models with day $0 \mathrm{BW}$ as a covariate.

Results: No Treatment $\times$ DOF interaction was exhibited $(P \geq 0.17)$ for any variable. Empty body moisture decreased $(P<0.01)$ in a quadratic fashion at approximately $0.04 \% / \mathrm{d}$ beginning at $61.9 \%$ on day 0 and ending at $47.8 \%$ on day 378. Empty body protein decreased $(P<0.01)$ linearly by approximately $0.007 \% / \mathrm{d}$ beginning at $18.7 \%$ on day 0 and ending at $16.0 \%$ on day 378 . EBF increased $(P<0.01)$ in a quadratic fashion at approximately $0.05 \% / \mathrm{d}$ beginning at $14.0 \%$ on day 0 until day 294 and plateaued at approximately $32.0 \%$ through day 378 . Empty body ash remained constant $(P=0.52)$ across the feeding period $(5.5 \%-5.8 \%)$. Empty body protein $(16.7 \%$ CON vs. $17.4 \%$ REV) and empty body ash $(5.4 \% \mathrm{CON}$ vs. $5.7 \% \mathrm{REV})$ were greater $(P<0.01)$ for REV steers, and empty body moisture $(50.7 \%$ CON vs. $51.6 \% \mathrm{REV})$ tended to be greater $(P=0.07)$ for REV steers. In contrast, EBF $(25.3 \% \mathrm{REV})$ was greater $(P<0.01)$ for CON steers (27.3\%). Ratio of empty body protein to EBF was $0.61: 1$ for CON steers compared to $0.69: 1$ for REV steers $(P<0.05)$ while decreasing $(P<0.01)$ approximately $0.002 / \mathrm{d}$ beginning at 1.34 on day 0 and ending at 0.52 on day 378 .

Conclusion: Composition of gain is important to commercial industry in regard to protein and fat accretion changing over time. These data indicate that growth-promoting implants alter composition of gain during the finishing period.

Keywords: chemical composition, fat, implants, protein, serial harvest

\section{8 - COMPARISON OF METHANE GAS EMISSIONS FROM FRESH AND COOKED CHICKEN, PORK, BEEF, AND TURKEY}

T. Price ${ }^{*}$, J. Warren ${ }^{2}$, G. Mafi ${ }^{1}$, and R. Ramanathan ${ }^{1}$, ${ }^{1}$ Department of Animal and Food Sciences, Oklahoma State University, Stillwater, OK, USA, ${ }^{2}$ Department of Plant and Soil Sciences, Oklahoma State University, Stillwater, OK, USA, *mdenzer@okstate.edu

Objectives: Meat is a nutrient-dense food, and it is estimated that in the United States, Canada, Australia, and New Zealand, approximately $22 \%$ of total meat and poultry production is discarded annually. Meat waste can result from a variety of factors, including cooking or serving a larger portion than is consumed, expiration or overpurchase in the home or in food service, or a lack of marketability due to discoloration. There have been various studies to determine greenhouse gas emissions throughout the meat animal and poultry production life cycles, but currently, there is limited knowledge on the impact of meat waste on greenhouse gas production. Therefore, the objective of this study was to evaluate levels of methane production from raw and cooked meat proteins during $7-d$ in vitro storage. Individual fresh raw ground loaves of chicken, pork, beef, and turkey, all of similar composition respective to species, were purchased from a local retailer; aging time was not considered for this study to reproduce consumer purchasing habits. Methane production from both raw and cooked samples was determined on day 0 and day 7 of storage.

Materials and Methods: To determine the methane production from raw meat, $5 \mathrm{~g}$ of meat samples from day 0 were incubated at room temperature for $24 \mathrm{~h}$ in a sealed vial (to simulate if samples were discarded outside). For the preparation of cooked meat samples, poultry patties were cooked to $73^{\circ} \mathrm{C}$, and beef and pork were cooked to $71^{\circ} \mathrm{C}$; following cooking, 5 -g samples were incubated identically to raw meat. For 7-d storage, both raw and cooked meat were kept in an airtight zip-lock bag (to imitate home storage conditions) and placed in a refrigerator. Following $7 \mathrm{~d}$ of storage, all raw and cooked samples were incubated for $24 \mathrm{~h}$ at room temperature in a sealed vial to determine final greenhouse gas production. A gas chromatographer with a headspace analyzer was utilized to determine gas formation within the vial; for each storage and species combination, 8 treatment combinations in duplicates were measured, and data were analyzed using the Proc GLM procedure of SAS (SAS Institute Inc., Cary, NC).

Results: Cooked meat had greater $(P<0.05)$ methane gas production than raw meat, and the duration of storage time increased methane production. Within species, pork and turkey had the lowest $(P<0.05)$ methane production in comparison with beef. More specifically, cooked beef had significantly higher $(P<0.05)$ greenhouse production than any other cooked or raw species. The bacteria that produce methane are referred to as methanogens; their preferred environments are anaerobic, which were simulated during incubation, and can be found in landfills. They can be vital in the breakdown of organic matter, and these results indicate that cooked products could have a higher susceptibility to methanogen growth. The current research assumes significance as the Environmental Protection Agency reported that $15.1 \%$ of human methane production in 2018 originated from municipal waste.

Conclusion: Developing strategies to preserve nutrientdense animal proteins is critical in limiting meat waste and for decreasing human methane production.

Keywords: food waste, greenhouse gases, meat, methane 


\section{9 - INFLUENCE OF CATTLE BACKGROUNDING SYSTEMS ON PERFORMANCE AND CARCASS CHARACTERISTICS}

\author{
J. A. Langlie ${ }^{1 *}$, B. Omontese ${ }^{1}$, R. Cox ${ }^{1}$, and M. Webb ${ }^{1}$, \\ ${ }^{1}$ Animal Science, University of Minnesota, St. Paul, MN, \\ USA, *lang1144@umn.edu
}

Objectives: The objective of this study was to determine the influence of backgrounding systems on cattle performance, carcass characteristics, and meat quality when finished on a common feedlot diet.

Materials and Methods: Postweaning Angus and Angus $\times$ Simmental calves $(N=65)$ were assigned in a completely randomized design to one of 3 treatments: (1) dry lot (DL, $n=22)$; (2) perennial pasture (PP, $n=$ $21)$; and (3) cover crop (CC, $n=21)$ during backgrounding for $55 \mathrm{~d}$. Concluding backgrounding, the CC and PP treatments were transported to pens and acclimated to a finishing ration over a period of $14 \mathrm{~d}$ and continued 3 step-up diets over the next $15 \mathrm{~d}$. Two separate pens were utilized to house all heifers and steers during the finishing segment, and 28-d rotations eliminated pen effect. Body weight, hip height, and average daily gain were recorded every $28 \mathrm{~d}$. Five periodic carcass ultrasound measures were recorded to evaluate ultrasound ribeye area (REA), ultrasound rib fat thickness, and ultrasound percent intramuscular fat. At harvest, carcass measurements included hot carcass weight, REA, 12th rib backfat fat thickness, marbling and maturity score, and objective color $\left(L^{*}, a^{*}, b^{*}\right)$. Fortyeight-hour postmortem 2.54 -cm steaks were cut from the loin and evaluated for ether analysis, water content, cook loss, 7- and 14-d aged Warner-Bratzler shear force, and sensory analysis. Statistical analyses were conducted using mixed model procedures, and animal weaning weight was used as a covariate. Least-squares means were computed and recorded as significant at $P \leq 0.05$.

Results: No differences were observed for ultrasound intramuscular fat $(P=0.23)$, ultrasound rib fat thickness $(P=0.07)$, USDA marbling $(P=0.06)$, USDA maturity $(P=0.22)$, USDA Yield grade $(P=0.28)$, fat content $(P=0.32)$, or moisture content $(P=0.75)$. Ultrasound REA was recorded largest in CC $(P \leq 0.001)$, while PP resulted in the greatest REA $(P \leq 0.001)$. Hot carcass weight was similar between CC and PP, both heavier than DL $(P \leq$ $0.001)$. Lean tissue $L^{*}, a^{*}$, and $b^{*}$ color values were highest in DL $(P \leq 0.001 ; P=0.01 ; P \leq 0.001)$. Cook loss was lowest in PP $(P=0.02)$, but there was no difference between age periods in all treatments $(P=0.24)$. Lower Warner-Bratzler shear force values were recorded for PP loin steaks $(P=$ $0.05)$. Treatment had no effect on most steak subjective sensory attributes: overall liking $(P=0.26)$, flavor liking $(P=$ $0.36)$, toughness $(P=0.25)$, juiciness $(P=0.80)$, or offflavor $(P=0.43)$. The texture of CC steaks was least desirable $(P=0.05)$.
Conclusion: Overall, cattle backgrounded on PP and CC experienced greater compensatory growth and average daily gain during finishing compared to DL that resulted in similar USDA Yield and Quality. Cattle backgrounded on alternative diets to DL provide steaks of similar desirability and qualitative characteristics.

Keywords: backgrounding, cover crops, grading, sensory analysis, ultrasound

\section{0 - THE MICROBIOME OF A NEWLY CONSTRUCTED MEAT PROCESSING FACILITY DIFFERS BASED ON ROOM FUNCTION AND TIME}

A. D. Belk ${ }^{1 *}$, N. Frazier ${ }^{1}$, L. Fuerniss ${ }^{1}$, I. Geornaras ${ }^{1}$, B. Borlee $^{2}$, R. Delmore ${ }^{1}$, J. Martin ${ }^{1}$, and J. L. Metcalf ${ }^{1}$, ${ }^{1}$ Animal Science, Colorado State University, Fort Collins, CO, USA, ${ }^{2}$ Microbiology, Immunology, and Pathology, Colorado State University, Fort Collins, CO, USA, *aeriel. belk@colostate.edu

Objectives: Significant progress has been made towards reducing the occurrence of foodborne illness in the United States; however, foodborne pathogens still present a public health risk. These organisms, especially Listeria, find unique niches in meat processing facilities where they can then persistently contaminate food products. This phenomenon is well-described, but there is a knowledge gap in where these organisms originate and how they colonize these spaces. Recently, a new meat processing facility was constructed at Colorado State University providing a unique opportunity to study the succession of microbiota and the potential establishment of Listeria in a new built environment. The objectives of this study were to determine the patterns of microbial community composition within a new meat processing facility and how they associate to both Listeria presence as well as facility function.

Materials and Methods: To investigate this, a longitudinal experiment was designed to characterize microbial communities and detect Listeria throughout the processing facility at 10 time points, beginning immediately after postconstruction cleaning and continuing monthly after the start of production. At each time point, surface samples were collected from the internal surfaces of all drains and select door handles in all production rooms, coolers, storage rooms, and human corridors. Listeria samples were collected using a premoistened, buffered sponge and quantified using realtime polymerase chain reaction. The microbial communities were collected via sterile swabs, then DNA was extracted using a commercial kit, and $16 \mathrm{~S}$ ribosomal RNA gene amplicons were sequenced. These data were analyzed using the QIIME2 platform and R software. Statistics used an alpha level of 0.05 . 
Results: During the first year of processing at the newly constructed facility, no resident population of Listeria was observed in any production room, though transient populations were identified in several locations. Principal coordinates analysis revealed that microbial communities identified in the facility associated more closely with the function of the room (i.e., harvest, fabrication) than the sampling time point, indicating that the microbes present in a room were influenced by the room activity. This trend was confirmed by permutational analysis of variance analysis $(P<0.05)$. Within a room, there were changes in the microbial communities over time, though it did not appear that a consistent community was established within the first year of sampling. However, samples taken at later time points appear more similar to each other than samples collected in the months after opening, suggesting that a resident community will form as production continues. Moreover, an analysis of the composition of microbiomes demonstrated that there are several key taxa that are significantly different across facility spaces and time $(W=161)$. Clostridiales were more closely associated with live animal and harvest spaces, Pseudomonadales were associated with processing and fabrication spaces and became more persistent at later time points, and Bacteriodales were common in processing spaces.

Conclusion: The microbial community of the meat processing facility differs among rooms and may be associated with specific functions of the room. Furthermore, results suggest that, with daily cleaning and sanitation practices, a resident Listeria community does not form in the first year of facility production.

Keywords: 16S, facility microbiome, Listeria spp., microbiome, processing environment

\section{1 - NON-CARCASS COMPONENT YIELDS OF TRENBALONE ACETATE + ESTRADIOL- $17 \beta$ IMPLANTED STEERS VERSUS NON- HORMONE TREATED STEERS ACROSS VARIOUS HARVEST ENDPOINTS}

\section{S. L. Pillmore ${ }^{1 *}$, T. J. Kirkpatrick ${ }^{1}$, K. Wesley ${ }^{1}$, K. B. Cooper ${ }^{1}$, F. Francis ${ }^{1}$, T. Tennant ${ }^{1}$, W. Nichols ${ }^{2}$, J. Hutcheson ${ }^{2}$, and T. Lawrence ${ }^{1},{ }^{1}$ Department of Agricultural Sciences, West Texas A\&M University Meat Lab, Canyon, TX, USA, ${ }^{2}$ Technical Services, Merck Animal Health, Madison, NJ, USA, *spillmore@wtamu.edu}

Objectives: Growth-promoting implants containing trenbolone acetate (TBA) and estradiol-17 $\left(\mathrm{E}_{2}\right)$ are commonly administered to improve rate of weight gain and feed efficiency of beef cattle. Although non-carcass components are not considered lean muscle foods or contribute to traditional carcass value, many (1) are highly metabolic organs that determine efficiency of the live animal, (2) fulfill nutritive roles in global society, and (3) are important raw materials that support numerous industries. A serial-harvest study was conducted to investigate the growth of non-carcass components of implanted or nonimplanted Charolais $\times$ Angus steers.

Materials and Methods: Steers $(n=80)$ were paired by genetic similarity and projected endpoint composition and were randomly appointed to harvest date $(0,42,84,126$, $168,210,252,294,336$, or 378 days on feed [DOF]). Steers within pairs were then randomly allocated to implant treatment; REV received a Revalor-XS (200 mg TBA/40 mg $\mathrm{E}_{2}$ ) on day 0 and day 190 , whereas control $(\mathrm{CON})$ received no implant throughout the study. A $2 \times 10$ factorial treatment structure was utilized in a completely randomized experimental design. Variables were analyzed using mixed models. Four pairs ( $n=8$ steers) were harvested upon each feeding endpoint. Weights of non-carcass components were obtained upon removal, and gastrointestinal tracts were separated into individual components and weighed, flushed clean, and reweighed to obtain empty tract weight.

Results: No treatment $\times \mathrm{DOF}$ interactions $(P \geq 0.65)$ were observed for body or hot carcass weights (HCW), fill or dressed yield. Main effects of implant were observed; shrunk body weight (SBW), empty body weight (EBW), and $\mathrm{HCW}$ were $6 \%$ greater $(P<0.01)$ in REV steers than $\mathrm{CON}$, indicating a consistent increase in tissue components. No treatment effects $(P \geq 0.14)$ were observed for fill or dressed yield; however, SBW and HCW increased ( $P \leq$ $0.01)$ and percentage fill decreased as a main effect of DOF. Absolute fill weight was not affected $(P \geq 0.51)$ by DOF. A REV $\times$ DOF interaction tended to occur $(P=$ $0.07)$ for the absolute weight of the kidneys. Implanted animals had greater $(P \leq 0.05)$ absolute mass of blood, head, hide, liver, spleen, omasum, small intestine, heart, total viscera, total splanchnic tissue, and total offal and tended to have a heavier $(P \leq 0.08)$ tongue and pizzle than CON steers. Implanted steers also had diminutive $(P=0.05)$ thymus glands and less $(P=0.02)$ kidney-pelvic-heart fat than nonimplanted steers on an absolute weight basis. Absolute weight of the spinal cord and the small intestine remained constant across DOF; all other tissue weights increased $(P \leq 0.05)$ with DOF. No REV $\times$ DOF interactions $(P \geq$ $0.15)$ occurred on an EBW basis. However, the brain, limbs, abomasum, thymus, and kidney-pelvic-heart fat of REV steers weighed less $(P \leq 0.05)$ on an EBW basis, and the lungs and trachea tended $(P=0.09)$ to weigh less than those of CON steers. Conversely, the reticulum increased $(P=$ 0.03 ) approximately $7 \%$ on an EBW basis, and the pizzle tended to weigh more $(P=0.09)$ in REV than CON. Weight of oxtail on an EBW basis was similar for all DOF, while the gallbladder, esophagus, and spleen tended $(P \leq 0.10)$ to vary and all other variables differed $(P \leq$ $0.04)$ across DOF. 
Conclusion: These results suggest that $\mathrm{TBA}+\mathrm{E}_{2}$ implants increase SBW and HCW and many non-carcass components while reducing excess internal fat accumulation.

Keywords: implant, non-hormone-treated cattle, noncarcass components, serial harvest

\section{2 - MEAT ANALOGUE PRODUCTION FROM FERMENTED OKARA, TO IMPROVE SENSORY AND FUNCTIONAL PROPERTIES, AS WELL AS OXIDATIVE STRESS REDUCTION}

A. Salaseviciene ${ }^{1 *}$, S. Razavizadeh ${ }^{1}$, G. Alencikiene ${ }^{1}$, and L. Vaiciulyte-Funk ${ }^{1},{ }^{1}$ Food Institute, Kaunas University of Technology, Kaunas, Lithuania, *alvija.salaseviciene@ktu.lt

Objectives: The aim of this research was to develop meat analogues based on okara (a byproduct of soy milk production, usually used as animal feed) with sensory properties similar to those of meat products, as plant-based diets are more sustainable and have greater environmental benefits by utilizing considerably fewer natural resources than meat-based diets.

Materials and Methods: Chemical compositions of okara (raw protein content, fat content, saturated fatty acids, total fiber content, and carbohydrate content) were analyzed by standard methodologies, besides evaluating sensory and textural properties. Protein content was calculated, according to ISO 20483: 2007. Fiber content was determined, according to AOAC 985.29: 1990. Fat content and saturated fatty acid were measured, according to ISO 12966-1:2015 and ISO 12966-2:2017, respectively. Total amino acid composition was analyzed, according to ISO 17025:2005. Water as well as oil absorption capacity were measured. Protein oxidation levels of press cakes were measured by applying the methods described by Soglia et al. (2016).

Results: Chemical composition of okara clearly shows that it is a rich source of protein, fiber, essential amino acids, and energy with a low amount of saturated fatty acids. Normally, microbiological contamination of byproducts, such as okara, is high, therefore thermal treatment of okara is necessary before further usage. Due to the low sensory quality of okara, lactic acid fermentation was applied with the aim to reduce its unpleasant flavor, besides improving the protein digestibility. Okara samples were fermented by 4 specific Lactobacillus strains. After fermentation, meat analogues were produced based on fermented okara. The functional properties (water-/oil-holding capacity; effective on juiciness/oiliness of products) and protein oxidation levels of products were analyzed. Instrumental color testing showed no significant differences $(P<0.05)$ on lightness, yellowness, and redness of the meat analogue products, in comparison with the control (vegan) sample. There were no significant differences between okara-based meat analogue products, regarding water-holding capacity values or meat analogues mass spreadability or hardness, measured instrumentally. Results of sensory analysis revealed that meat analogues from okara fermented by $L$. plantarum and $L$. brevis strains had more neutral and slightly acidic smell and taste and decreased bitterness perceived in okara. However, those products incorporated with okara fermented by $L$. acidophilus strains showed more dry texture and significant increase of oil-holding capacity values than other strains, resulting in an intensive oily mouthfeel of products. Carbonyl content analysis of those products using L. acidophilus strains for fermentation of okara showed a more significant reduction in protein oxidation between products.

Conclusion: This research demonstrated the potential of fermentation of okara by applying $L$. plantarum and $L$. brevis strains (as sensory properties' improver) and L. acidophilus strains (as functional properties' enhancer and protein oxidation reducer) beside the pasteurization process to convert okara into a suitably applied ingredient in the matrices of the meat analogues. However, further research is necessary for protein quality analysis, and to get juiciness and mouthfeeling similar to meat products.

Keywords: lactic acid fermentation, meat analogue, okara, protein oxidation, water- and oil-holding capacity

\section{3 - PILOT STUDY FOR OCCUPATIONAL EXPOSURE TO POTENTIAL MICROBIAL CONTAMINATION IN DAIRY FACILITY, CATTLE FEEDLOT, AND RANGELAND}

\author{
L. Yang ${ }^{1 *}$ and X. Yang ${ }^{1},{ }^{1}$ Department of Animal Science, \\ University of California, Davis, Davis, CA, USA, *ylhyang@ \\ ucdavis.edu
}

Objectives: Workers handling livestock in beef and dairy facilities may be exposed to environmental contamination, posing potential microbial risk to workers due to pathogenic contamination through surface contact and aerosol routes. Some pathogenic bacteria such as Salmonella and Yersinia are associated with beef and dairy production and can threaten the health of farm workers. The objective was to assess the microbial contamination level of farm workers in 3 different production systems as a result of occupational exposure.

Materials and Methods: This study was conducted at 3 facilities within a 2-mo period: the University of California Davis Dairy Farm, the Beef Cattle Feedlot, and the Sierra Foothill Research \& Extension Center rangeland. Four sample matrices were collected at each facility: pen floor feces, animal hide swab, floor surface swabs, and employees' outerwear swabs (hats, clothes, pants, and boots). At each facility, 1 sponge was used to swab floor surfaces of 2 randomly selected pens, and 1 sponge was used to swab the 
outerwear of 1 employee at the end of their shift. Two sponges were utilized to swab hides from 8 animals from 2 randomly selected pens. Twenty feces pellets $(20 \mathrm{~g}$ per pellet) were collected from the floor of 2 pens. All samples were serially diluted and plated on 3M Petrifilm EB Plates and Aerobic Plate Count (APC) Petrifilms. After incubation, viable bacterial colonies for all swab samples were reported as $\log \mathrm{CFU} / \mathrm{mL}$. Pen floor fecal bacterial counts were expressed as $\log$ CFU/g. Sample collections were repeated 4 times for each production system. Data were analyzed using one-way analysis of variance via $\mathrm{R}$ version 3.6. Linear models were built with alpha level 0.05 .

Results: The EB counts from employee outerwear samples ranged from $4.32 \log \mathrm{CFU} / \mathrm{mL}$ to $5.67 \log \mathrm{CFU} / \mathrm{mL}$ for all facilities (Table 1). All other sample matrices ranged from $3.67 \log \mathrm{CFU} / \mathrm{mL}$ to $6.76 \log \mathrm{CFU} / \mathrm{g}$ (Table 1 ). Within each facility, employee outerwear EB counts were not different $(P>0.05)$ from floor, hides, and feces. The APC counts for employees' swabs across 3 locations ranged from 6.89 $\log \mathrm{CFU} / \mathrm{mL}$ to $7.72 \log \mathrm{CFU} / \mathrm{mL}$. The APC counts of all other sample matrices ranged from $5.87 \log \mathrm{CFU} / \mathrm{mL}$ to $8.48 \log$ CFU/g. No differences $(P>0.05)$ among the 4 sampling matrices were detected from feedlot samples. At the dairy facility, employees' swabs had lower $(P<0.05)$ APC counts than floor surface samples. At rangeland, APC counts from employees were higher $(P<0.05)$ than from hides. Among the 3 facilities, APC counts from employee swab samples were not significantly different $(P>0.05)$ from each other. Similarly, the same pattern was identified for EB counts. As a pilot study, this investigation may potentially have reduced statistical power due to the relatively small sample size.

Conclusion: The EB and APC counts from employee outerwear, pen floor, and fecal samples among all 3 facilities were similar. This suggests that employees working on the floor and in the places covered with animal feces were more likely to be exposed to higher levels of potential microbial contamination. Personal hygiene practices such as disinfection of the surface of employee outerwear and other practices such as manure management are needed to reduce the transmission of zoonotic bacteria from environment to farmworkers and subsequently improve occupational health.

Table 1. Least squares means (LSmeans; standard error) of the counts for Enterobacteriaceae from different samples after 24 -hour incubation at $37^{\circ} \mathrm{C}$.

\begin{tabular}{lcccc}
\hline \hline Location & \multicolumn{1}{c}{ Hide } & Feces & Floor & Employee \\
\hline Feedlot & $5.08(0.23)^{\mathrm{bcd}}$ & $6.76(0.23)^{\mathrm{a}}$ & $6.01(0.23)^{\mathrm{abc}}$ & $5.67(0.23)^{\mathrm{abc}}$ \\
Dairy & $3.85(0.27)^{\mathrm{a}}$ & $6.17(0.27)^{\mathrm{ab}}$ & $5.62(0.27)^{\mathrm{abc}}$ & $4.84(0.27)^{\mathrm{bcd}}$ \\
Rangeland & $3.67(0.28)^{\mathrm{d}}$ & $6.65(0.28)^{\mathrm{abc}}$ & $5.43(0.28)^{\mathrm{bcd}}$ & $4.32(0.28)^{\mathrm{cd}}$ \\
\hline \multicolumn{2}{c}{${ }^{\mathrm{a}-\mathrm{d}}$ Least Squares means with distinct superscript letters are significantly } \\
different $(\mathrm{P}<0.05)$.
\end{tabular}

Keywords: microbiological contamination, occupational health, production system of beef and dairy cattle

\section{Meat and Poultry Processing, Ingredient Technology and Packaging}

\section{5 - EFFECT OF PROCESSING PARAMETERS AND STORAGE TIME ON THE SPOILAGE MICROBIOME OF TURKEY PRODUCTS}

\author{
R. A. Furbeck ${ }^{1 *}$, S. C. Fernando ${ }^{1}$, and G. A. Sullivan ${ }^{1}$, \\ ${ }^{1}$ Department of Animal Science, University of Nebraska, \\ Lincoln, NE, USA, *rfurbeck@huskers.unl.edu
}

Objectives: This study aimed to identify changes in the microbial ecology of processed turkey products, as a function of storage time and degree of processing.

Materials and Methods: Three replicates of products each representing varying degrees of processing were prepared from 3 separate lots of turkey breast meat: T1: raw ground turkey; T2: raw ground turkey with salt added; T3: raw ground turkey with salt and spices added; T4: cooked link; T5: cured cooked link; T6: sliced deli; and T7: sliced cured deli. Cooked products were processed to $74^{\circ} \mathrm{C}$ and chilled to $4^{\circ} \mathrm{C}$. Aerobic plate counts (APC), anaerobic plate counts, lactic acid bacteria, Pseudomonas spp. Cephaloridine fucidin cetrimide agar and psychrotrophic plate counts (PPC) were evaluated in raw treatments every $7 \mathrm{~d}$ for $21 \mathrm{~d}$, and cooked treatments were evaluated every $28 \mathrm{~d}$ for $84 \mathrm{~d}$. Microbial communities were evaluated by sequencing the V4 region of $16 \mathrm{~S}$ ribosomal RNA gene using $250 \mathrm{bp}$ paired end sequencing on the Illumina MiSeq platform (Illumina Inc., San Diego, CA). Sequence reads generated were quality filtered and processed within $\mathrm{R}$ and Mothur. The DADA2 pipeline was used to identify amplicon sequence variants (ASV). ASV were assigned taxonomy using the Silva database 132. Alpha diversity was estimated using observed ASV and Chaol estimates; beta diversity was evaluated using weighted and unweighted UniFrac distance matrices. Raw ( 3 treatment $X$ 4 storage times) and cooked ( 4 treatment $\times 4$ storage times) samples were analyzed independently for plate counts, alpha diversity with storage time as a repeated measure with an independent covariance structure using the nlme and emmeans packages.

Results: In raw treatments, main effect of storage time on all plate count methods $(P<0.01)$ was identified, which can be observed as growth; there was a main effect of treatment on APC and PPC $(P<0.05)$. Treatments 2 and 3 had lower APC and PPC, implying that salt may be an effective microbial inhibitor. In cooked samples, storage time was 
significant on cephaloridine, fucidin, cetrimide plates $(P<$ 0.01), as Pseudomonas spp. proliferated regardless of treatment. Treatment by storage time interaction was observed in APC, anaerobic plate counts, PPC, and lactic acid bacteria $(P<0.04)$. Sliced and noncured samples had more growth, illustrating post-process contamination and nitrite inhibition. Storage time significantly influenced Chaol and observed ASV alpha diversity measures in raw samples $(P<0.01)$ and main effects of treatment $(P<0.05)$ and storage time $(P<0.03)$ on observed ASVs in cooked products. For both Chao1 and observed ASVs in raw treatments, storage times of 0 and $7 \mathrm{~d}$ had more bacterial richness than 14 and $21 \mathrm{~d}$. Observed ASVs decreased over time in cooked products. There were main effects of storage time and treatment on beta diversity in both the weighted and unweighted UniFrac distance matrix $(P<0.01)$. Raw samples clustered by "freshness," with day 0 and 7 samples clustering apart from day 14 and 21.

Conclusion: Microbial communities are modulated by degree of processing, ingoing ingredients, and storage time. Bacterial diversity decreases as products spoil and spoilage species start to predominate. This loss of richness can be explained by the overgrowth of spoilage taxa, such as Pseudomonadaceae, Enterobacteriaceae, and Lactobacillaceae. Shelf life extension may be better achieved by employing methodology that is more targeted at these core organisms.

Keywords: poultry, Pseudomonas, shelf life

\section{6 - SENSORY EFFECTS OF HICKORY, CHERRY, AND MESQUITE WOOD USED TO GENERATE HIGH AND LOW TEMPERATURE SMOKE FOR AMERICAN- STYLE BARBECUE PORK RIBS}

J. A. Ascencio ${ }^{1}$, W. Harwood ${ }^{1}$, M. A. Drake ${ }^{1}$, and D. J. Hanson ${ }^{1}$, ${ }^{1}$ Department of Food, Bioprocessing and Nutrition Sciences, North Carolina State University, Raleigh, NC,USA, *dana_hanson@ncsu.edu

Objectives: The study objective was to determine differences in flavor, color, aroma, and acceptance of smoked pork ribs using descriptive and consumer sensory analysis.

Materials and Methods: Cherry, hickory, and mesquite wood were used to create high- (HT) and low-temperature (LT) smoke treatments. Pork ribs, Institutional Meat Purchase Specifications 416A $(n=246)$, were cooked and smoked using one of 2 Enviro-Pak MP-2500 ovens (Enviro-Pak, Clackamas, OR). The HT smoke treatments denoted as cherry high, hickory high, and mesquite high were created using a log burning smoke generator. LT smoke treatments denoted as cherry low, hickory low, and mesquite low were generated using a wood chip burning smoke generator. HT smoke combustion temperature was controlled between $540^{\circ} \mathrm{C}$ and $650^{\circ} \mathrm{C}$, with LT smoke combustion temperatures being controlled between $260^{\circ} \mathrm{C}$ and $315^{\circ} \mathrm{C}$. All smoke treatments were coupled with an identical 4 -h thermal process $\left(30 \mathrm{~min}\right.$ at $63^{\circ} \mathrm{C}, 30 \mathrm{~min}$ at $71^{\circ} \mathrm{C}$ and $3 \mathrm{~h}$ at $88^{\circ} \mathrm{C}$; maintaining a $35 \%$ relative humidity for all stages). Fully cooked ribs were chilled prior to color measurements $\left(L^{*}, a^{*}, b^{*}\right)$ being recorded. Racks of the same treatments were then vacuum packaged 3 to a bag and stored for $5-7 \mathrm{~d}$ at $3{ }^{\circ} \mathrm{C}$, prior to sensory panel evaluation. All treatments were evaluated by descriptive analysis by highly trained panelists $(n=8)$, following Sensory Spectrum ${ }^{\circledR}$ methods. Consumer acceptance test utilized prescreened barbecue consumers $(n=205)$ who were divided into primed consumers $(n=103)$, for whom wood smoke treatment was revealed prior to samples being served, and unprimed consumers $(n=102)$, for whom treatment was not revealed prior to being served.

Results: Principal component analysis of trained panelists and color data coupled with external preference mapping suggested that mesquite treatments were associated with higher $L^{*}$ and $b^{*}$ values, higher smoke aroma, lower fruity/cherry flavor, lower sweet taste perception, and lower overall liking scores compared to cherry and hickory wood treatments. Analysis of variance and penalty analysis from consumer acceptance data suggested that both wood type and smoke temperature were significant $(P<0.05)$ determinants of overall liking, smoke flavor liking, appearance/color liking, and aroma liking. The highest preference was for hickory and cherry woods, as well as the HT smoke treatments for all wood types. Priming had no impact on consumer liking/acceptance scores $(P>0.05)$. When consumers were asked follow-up questions related to smoke flavor characteristics, they were generally unable to identify descriptors for specific smoke flavors of different treatments. Primed consumers more aptly assigned differentiating features to different wood type treatments. This signals that consumers may have underlying assumptions about different wood sources.

Conclusion: Trained panelists effectively distinguished flavor differences due to wood type and smoke temperature used to produce American-style barbecue pork ribs. Temperature of smoke generation appears to impact flavor liking of smoked meats, with HT smoke resulting in higher consumer acceptability scores. Unprimed consumers show low ability to differentiate flavor characteristics of specific wood used for smoking. Primed consumers are able to describe smoke flavor differences, signaling opportunities for labeling/marketing of smoked meats.

Keywords: barbeque, pork, sensory analysis, smoked ribs 


\section{7 - BREADFRUIT FLOUR ENGINEERED WITH DIFFERENT TWIN-SCREW EXTRUSION CONDITIONS AND THE EFFECTS ON PHYSICOCHEMICAL PROPERTIES OF FLOUR AND TECHNOLOGICAL PROPERTIES OF MEAT EMULSIONS}

S. Huang ${ }^{1 *}$, L. Roman ${ }^{2}$, M. M. Martinez ${ }^{2,3}$, and B. M. Bohrer ${ }^{1}$, ${ }^{1}$ Food Science, University of Guelph, Guelph, Canada, ${ }^{2}$ Engineering, University of Guelph, Guelph, Canada, ${ }^{3}$ Food Science, Aarhus University, Aarhus, Denmark,*bbohrer@uoguelph.ca

Objectives: The objective was to determine the structural and technological properties of beef emulsion modeling systems prepared with breadfruit flour engineered with different twin-screw extrusion conditions.

Materials and Methods: Native breadfruit flour was extruded with twin-screw extrusion technology (Coperion ZSK MV PLUS 27, Ramsey, NJ) under different conditions, which included feed moisture content $(17 \%$ or $30 \%)$ and last barrel temperature $\left(80^{\circ} \mathrm{C}\right.$ or $\left.120^{\circ} \mathrm{C}\right)$. Based on these different conditions, 4 extruded flours with different mechanical and thermal energy were obtained and defined as (1) Low Specific Mechanical Energy (SME; $74 \mathrm{~kJ} / \mathrm{kg}$ )-Low Temperature $\left(83^{\circ} \mathrm{C}\right)$ (LS-LT), (2) Low SME $(74 \mathrm{~kJ} / \mathrm{kg})$-High Temperature $\left(105^{\circ} \mathrm{C}\right)(\mathrm{LS}-\mathrm{HT})$, (3) High SME $(145 \mathrm{~kJ} / \mathrm{kg})$ Low Temperature $\left(100^{\circ} \mathrm{C}\right)$ (HS-LT), and (4) High SME $(145 \mathrm{~kJ} / \mathrm{kg})-$ High Temperature $\left(126^{\circ} \mathrm{C}\right)$ (HS-HT). Proximate composition, water-binding capacity, oil-binding capacity, swelling power, and pasting property were assessed in the native and extruded flours. Beef emulsion modeling systems $(n=3$ for each treatment) were used to determine the effects of the 4 extruded breadfruit flours at an inclusion level of $3 \%$ on the structural and technological properties of beef emulsion modeling systems. $\mathrm{pH}$, cooking loss, texture, instrumental color, microstructure, and dynamic rheological characteristics were evaluated. Statistical analysis was performed with PROC GLIMMIX of SAS (SAS Institute Inc., Cary, NC) with a fixed effect of treatment and a random effect of replication. Least-squares means were separated using the PDIFF option with a Tukey-Kramer adjustment.

Results: Extrusion treatment did not change $(P>0.05)$ the main compositional attributes of the flour, namely starch and protein. Lower SME $(74 \mathrm{~kJ} / \mathrm{kg})$ extrusion treatments (LS-LT and LS-HT) resulted in an increased $(P<0.05)$ capacity for water absorption compared to higher SME $(145 \mathrm{~kJ} / \mathrm{kg})$ extrusion treatments (HS-LT and HS-HT). Setback viscosity was decreased $(P<0.05)$ in all extruded flours compared with the native flour. Replacement of ground beef with native or extruded flours in meat emulsions did not change $(P>0.05)$ cooking loss, instrumental Minolta redness, and viscoelasticity. Addition of LS-HT, HS-LT, or
HS-HT extruded flours decreased $(P<0.05)$ hardness, while hardness of meat emulsions prepared with native flour or LS-LT extruded flour was not different $(P>0.05)$ compared with control (no-flour-added) samples. Replacement of ground beef in the meat emulsions with 3\% native or any of the extruded flours did not change $(P>0.05)$ springiness and adhesiveness. Moreover, microstructure and dynamic rheology showed that extruded flours behaved differently in the meat matrix compared with the native flour.

Conclusion: This research is beneficial to the meat industry since it provides a characterization of the structural network and functional changes apparent when meat emulsions were formulated with native and pre-gelatinized extruded breadfruit flours, which are novel, previously unresearched flour ingredients. Overall, it can be concluded that incorporation of extruded breadfruit flours can modify the structural and technological attributes of beef emulsions compared with the incorporation of native flour, but technological functions of beef emulsions formulated with different extruded flours were not different.

Keywords: breadfruit flour, extruded flour, meat emulsion modeling

\section{8 - NITRIC OXIDE PRODUCTION IN POST-RIGOR SEMIMEMBRANOSUS PORK MUSCLE}

K. M. Modrow*, W. Osburn, and Osburn, Wesley N. 2019. Amino acid alternative curing system. Patent Attorney Docket No. TAMU-19489 December 2019, issued 2019., ${ }^{1}$ Animal Science, Texas A\&M University, College Station, TX, USA, *kmmodrow2017@gmail.com

Objectives: This study was conducted to validate that Larginine activated nitric oxide synthase (NOS) system in living skeletal muscle can produce nitric oxide (NO) and residual nitrite in pre-rigor porcine muscle, and to investigate whether post-rigor semimembranosus pork produces NO.

Materials and Methods: Pre-rigor pork semimembranosus muscle samples $(60-75 \mathrm{~g})$ were collected from 4 prerigor pork carcasses at 3 separate times over a $3-d$ period $(N=12)$. Post-rigor samples were collected from the same carcasses after $18 \mathrm{~h}$ of refrigerated storage to ensure rigor completion $(N=12)$. Subsamples $(\sim 5 \mathrm{~g})$ were placed in separate test tubes and treated with one of 5 concentrations of 2 $\mathrm{mL}$ L-arginine solution $(2,4,8,16$, and $32 \mathrm{mM})$ with the control water $(0 \mathrm{mM})$. Additionally, a solution containing $72 \mathrm{mg} \mathrm{NaCl}, 576 \mathrm{ppm}$ sodium erythorbate, and $8 \mathrm{~mL}$ of water was added to each sample $(n=288)$. Muscle $\mathrm{pH}$ was determined before and after treatment. Samples were either heated (water bath cooking samples to $62^{\circ} \mathrm{C}$ within $60 \mathrm{~min}$ ) or left raw (uncooked). All treated samples were analyzed for residual nitrite by supernatant and pellet sampling by modified ultraviolet/visible spectrophotometric 
method. Cooked sample supernatant and pellets were analyzed for nitrosylation (determination of nitrosylhemochromagen) after a 1 -wk storage period $\left(-30^{\circ} \mathrm{C}\right.$; freezer, then thawed in $0^{\circ} \mathrm{C}$ cooler).

Results: For residual nitrite, all L-arginine treated raw pre-rigor supernatants were higher $(P<0.05$; range 11.9 $44.6 \mathrm{ppm})$ than the control $(0.26 \mathrm{ppm})$. For all raw post-rigor supernatants, L-arginine treatments had higher residual nitrite levels $(P<0.05$; range $9.5-23.5 \mathrm{ppm})$ compared to the control (2.06 ppm). The pre-rigor cooked $4 \mathrm{mM}$ supernatants had the highest level of residual nitrite $(61.9 \mathrm{ppm}$; $P<0.05)$, while $2 \mathrm{mM}(61.1 \mathrm{ppm})$ and $16 \mathrm{mM}$ (53.7) had higher levels compared to other treatments and the control. Cooked pre-rigor pellets were not different for residual nitrite with the highest level at $32 \mathrm{mM}(40.1 \mathrm{ppm})$. In cooked post-rigor pellets, $4 \mathrm{mM}(23.7 \mathrm{ppm}), 8 \mathrm{mM}(22.1 \mathrm{ppm}), 16$ $\mathrm{mM}(22.2 \mathrm{ppm})$, and $32 \mathrm{mM}$ (32.9 ppm) were different from the control $(11.02 \mathrm{ppm} ; P<0.05)$. All pre-rigor cooked supernatants and pellets were greater than the control for nitrosylhemochromagen (NO-heme), total heme pigment, and nitrosylation. The same results were observed for post-rigor cooked supernatants and pellets. Nitrosylation for post-rigor pellets were different for all concentrations to the control. Nitrosylation for pre-rigor pellets were highest at $32 \mathrm{mM}(86.1 \%)$ and different than the control (37.1\%). Nitrosylation for post-rigor supernatants were highest at $32 \mathrm{mM}$ (104.3\%). The NO-heme content was greatest at $32 \mathrm{mM}$ concentration (27.3 ppm), while $4 \mathrm{mM}$ (19.7 ppm), $8 \mathrm{mM}(18.3 \mathrm{ppm})$, and $16 \mathrm{mM}(18.4 \mathrm{ppm})$ concentrations were greater than the control $(9.16 \mathrm{ppm})$. The observed bimodal effect is similar to findings from previous research regarding NO production in endogenous skeletal muscle. As NO converts to nitrite by increasing 0.5 to $5 \mathrm{mM}$ concentrations, addition of L-arginine in pre-rigor meat $(4 \mathrm{mM})$ and at higher concentrations $(32 \mathrm{mM}$ ) resulted in more efficient $\mathrm{NO}$ generation. This likely occurred by reducing interference in NOS competition to regenerate L-arginine as a substrate for $\mathrm{NO}$, residual nitrite, and NO-hemachromagen formation.

Conclusion: Based upon the results of this study, there is evidence that the NOS system is activated by addition of L-arginine to produce $\mathrm{NO}$ and residual nitrite in post-rigor meat. These results suggest that an L-arginine alternative meat curing system may be viable.

Keywords: L-arginine, nitric oxide synthase, nitrite, postrigor meat

\section{9 - PHYSICOCHEMICAL CHANGES IN EDIBLE OILS AND FRIED DUCK MEAT DEPENDING ON FRY NUMBER}

\author{
Y. Kim ${ }^{1 *}$, S.-H. Cho ${ }^{1}$, K.-H. Seol ${ }^{1}$, S.-M. Kang ${ }^{1}$, and J.-H. \\ $\mathrm{Kim}^{1},{ }^{1}$ Animal Product Utilization Division, National
}

Institute of Animal Science, Wanju-gun, Korea, Republic of Korea, *cdangmaro@naver.com

Objectives: The objective was to determine the effects of different oils and fry numbers on the quality of edible oils and fried duck meat.

Materials and Methods: Sunflower oil, canola oil, corn oil, and soybean oil were used as edible oils in this study. Duck carcasses were purchased from a commercial abattoir at $1 \mathrm{~d}$ postmortem. Thirty duck carcasses were deep-fried for each oil. Whole duck carcasses were divided into subdivision meat: wings, breasts, legs, and body. Duck meats were cooked at $170^{\circ} \mathrm{C}$ for 10 min using an electric deep fry machine. After frying, oils were removed from the deep fry machine and then frozen at $-72^{\circ} \mathrm{C}$. According to the frying number $(0,5,10,15,20,25,30)$, the edible oils from the deep fryer were used as experimental samples. To investigate the degree of lipid oxidation during frying, acid value and color of samples were examined. Measuring of acid value was done following the protocol of the Ministry of Food and Drug Safety, Korea. Color was measured with a Minolta Chroma Meter.

Results: The acid value of sunflower oil was lowest (2.94) and of soybean oil was highest (4.49) at 30 times. The Ministry of Food and Drug Safety regulations permit edible oils to have an acid value of $<3.0 \mathrm{mg} \mathrm{KOH} / \mathrm{g}$. Each oil exceeded the regulation permit at different frying times: canola oil at 30 times, corn oil at 25 times, and soybean oil at 20 times. The lightness was highest in the sunflower oil and lowest in the soybean oil. The yellowness was highest in the soybean oil and lowest in the sunflower oil.

Conclusion: Taken together, sunflower oil showed the most suitable properties for fried duck meat. Further studies are needed to determine the effects of different edible oils on fried duck meat quality.

Keywords: acid value, deep frying, duck meat, lipid oxidation

\section{0 - YIELD ENHANCEMENT IN OVEN- ROASTED TURKEY BREAST USING A CLEAN-LABEL INGREDIENT SOLUTION}

M. Matney ${ }^{1 *}$, G. Mccoy ${ }^{1}$, D. Unruh ${ }^{1}$, S. Lasuer ${ }^{1}$, T. Rourke ${ }^{2}$, and S. Kumar ${ }^{2},{ }^{1} R D A$, Corbion Purac America, Lenexa, KS, USA, ${ }^{2}$ Business Development, Corbion Purac America, Lenexa, KS, USA, *garrett.mccoy@corbion.com

Objectives: The objective of this study was to examine the efficacy of Verdad $\AA$ Avanta $₫$ Y200 (Corbion, Lenexa, KS), a clean-label yield enhancement solution composed of vegetal protein, fiber, and natural flavor. Testing was con-

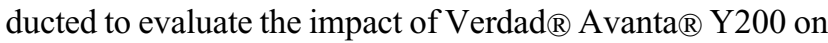
cook yield, sliceability, texture, and purge loss in ovenroasted turkey breast. 
Table 1: Cook yield, slice yield, $\mathrm{pH}$, TPA hardness, TPA chewiness, purge loss, and color measurements for different yield enhancement treatments

\begin{tabular}{lcccccc}
\hline \hline & & & & TPA & TPA & \\
Treatment & $\mathrm{pH}$ & Yield & Yield & $\begin{array}{c}\text { Hardness } \\
(\mathrm{g})\end{array}$ & $\begin{array}{c}\text { Chewiness } \\
(\mathrm{g})\end{array}$ & $\begin{array}{c}\text { Purge } \\
\text { Loss }\end{array}$ \\
\hline Control & 6.08 & $85.90 \%$ & $0.0 \%$ & 9951.16 & 6957.47 & $5.55 \%$ \\
STP & 6.29 & $90.63 \%$ & $100.0 \%$ & 15126.21 & 11544.81 & $4.48 \%$ \\
CLY & 6.29 & $90.10 \%$ & $100.0 \%$ & 16069.15 & 12649.63 & $4.94 \%$ \\
\hline
\end{tabular}

Materials and Methods: Ground turkey breast was vacuum tumbled with a salt water brine and varying levels of yield enhancement treatments. The control treatment contained no yield enhancement ingredients, whereas the treatment groups contained $0.5 \%$ sodium tripolyphosphate (STP) and $0.55 \%$ CLY. Each treatment was vacuum tumbled for 60 min, stuffed, cooked to an internal temperature of $72.8^{\circ} \mathrm{C}$, and chilled overnight to $4.4^{\circ} \mathrm{C}$. After chilling, treatments were sliced, and slice yield was measured as the percentage of intact slices. For each treatment, $\mathrm{pH}$, texture profile analysis (TPA) hardness, and TPA chewiness were measured upon slicing. Sliced samples from each treatment were vacuum packaged and aged for $14 \mathrm{~d}$ at $4.4^{\circ} \mathrm{C}$. At day 14 , purge loss was measured and reported as percentage of total sample weight.

Results: Table 1 illustrates the treatment structure along with $\mathrm{pH}$, cook yield, slice yield, TPA values for hardness and chewiness, and purge loss for the study. While both STP and CLY treatments had $\mathrm{pH}$ values greater $(P<0.05)$ than control, the CLY treatment did not result $(P>0.05)$ in finished product with $\mathrm{pH}$ values greater than the STP treatment. Additionally, STP and CLY both exhibited increased $(P>0.05)$ cook yield compared to control. Furthermore, CLY demonstrated similar $(P>0.05)$ textural properties compared to STP. Both treatments (STP and CLY) produced samples that were significantly $(P<0.05)$ harder and chewier than control in addition to increasing $(P<0.05)$ slicing yield.

Conclusion: This study authenticates the efficacy of Verdad $₫$ Avanta $₫$ Y 200 in providing cook yield enhancement, sliceability, and textural properties similar to that of sodium phosphate, thus providing the meat industry with a clean-label, fully functional alternative to sodium phosphate.

Keywords: clean label, processed meat, texture improvement, yield improvement

\section{1 - USE OF CULTURED CANE SUGAR FOR QUALITY ENHANCEMENT IN BEEF JERKY}

\author{
D. Unruh ${ }^{1}$, S. Lasuer ${ }^{1}$, G. Mccoy ${ }^{1}$, M. Matney ${ }^{1}$, N. Sevart ${ }^{2}$, \\ R. Ames $^{2}$, and S. Kumar ${ }^{2},{ }^{1} R D A$, Corbion Purac America,
}

Lenexa, KS, USA, ${ }^{2}$ Business Development, Corbion Purac America, Lenexa, KS, USA, *garrett.mccoy@corbion. com

Objectives: This study evaluates the effectiveness of Verdad ${ }^{\circ}$ Powder N20 (cultured cane sugar [CCS]) (Corbion, Lenexa, KS) at enhancing the cook yield and moisture of beef jerky.

Materials and Methods: Beef semitendinosus were sorted according fresh meat $\mathrm{pH}$ values, and a total of 25 beef semitendinosus were selected with $\mathrm{pH}$ values between 5.40 and 5.55. Selected subprimals were trimmed, sliced $0.2 \mathrm{in}$. thick, and further portioned to $1.2 \mathrm{~cm} \times 1.6 \mathrm{~cm}$ pieces. Portioned slices were randomly assigned to treatment group of control (no yield enhancement ingredient) or $1.0 \%$ CCS. Both treatment groups were vacuum tumbled with a brine containing $1.0 \%$ salt and $100 \mathrm{ppm}$ sodium nitrite, with treatment (control or $1.0 \%$ CCS) at $10 \%$ of green meat weight. Samples were dried in a smokehouse using a stage cook schedule to a target water activity of 0.88 . After reaching target water activity, samples were vacuum packaged, and cook yield, $\mathrm{pH}$, water activity, and moisture were reported. A trained sensory panel of 31 participants evaluated beef jerky samples from the $1.0 \%$ CCS treatment using a 7-point scale to report differences in color, aroma, sweetness, saltiness, off-flavors, and overall likeability compared to control samples. Cook yield was calculated as percent of original (raw) weight.

Results: Panelists did not detect any differences in color, aroma, or sweetness and did not detect any off-flavors between the 2 groups. Panelists reported that beef jerky samples containing $1.0 \% \mathrm{CCS}$ had greater salt flavor $(P<0.05)$ and subsequently reported greater overall preference for it than control $(P<0.05)$. Samples formulated with $1.0 \%$ CCS had a lower water activity than the negative control but had a higher $(P<0.05)$ moisture content and cook yield.

Conclusion: Verdad® Powder N20 (CCS) increases cook yield and desirability of beef jerky without negatively impacting $\mathrm{pH}$, water activity, or other factors impacting shelf life. Verdad $($ Powder N20 provides the meat industry with clean-label yield and flavor enhancement solution.

Keywords: cook yield, jerky, quality

Table 1: Cook yield, $\mathrm{a}_{\mathrm{w}}, \mathrm{pH}$, and moisture content for different jerky treatments.

\begin{tabular}{lcccc}
\hline \hline & Cook Yield & $\mathrm{a}_{\mathrm{w}}$ & $\mathrm{pH}$ & Moisture \\
\hline Control & $29.86 \%$ & 0.879 & 5.678 & $32.64 \%$ \\
$\mathbf{1 . 0 \%}$ Cultured Cane Sugar & $35.36 \%$ & 0.858 & 5.512 & $34.59 \%$ \\
\hline
\end{tabular}




\section{2 - EFFECTS OF TEA POLYPHENOLS AND GRAPE SEED EXTRACTS ON THE PREMATURE BROWNING OF BEEF PATTIES IN DIFFERENT PACKAGES}

H. Lei ${ }^{1}$, X. Luo ${ }^{1}$, Y. Zhang ${ }^{1}$, Y. Mao ${ }^{1}$, P. Dong ${ }^{1}$, X. Yang ${ }^{1}$, and R. Liang ${ }^{1 *},{ }^{1}$ Food Science and Engineering, Shandong Agricultural University, Taian, China, *1rr327@126. com

Objectives: Premature browning (PMB) is a cooked color problem for ground beef that can occur at internal temperatures below $71^{\circ} \mathrm{C}$. The denaturation of myoglobin results in a tan color, and it causes consumer misjudgment on the endpoint of beef cooking, which may result in food safety problems. Studies have found that some natural antioxidants can inhibit PMB. Therefore, the objective of this study was to investigate the effect of tea polyphenols (TP) and grape seed extracts (GSE) in inhibiting or minimizing PMB in beef patties combined with different packages.

Materials and Methods: The ground beef $(15 \%$ fat + 85\% lean) was divided into 4 groups: (1) Control group, (2) $0.1 \mathrm{~g} / \mathrm{kg}$ TP group, (3) $0.1 \mathrm{~g} / \mathrm{kg}$ GSE group, and (4) $0.1 \mathrm{~g} / \mathrm{kg} \mathrm{TP}+0.1 \mathrm{~g} / \mathrm{kg}$ GSE combined group. Each natural antioxidant was dissolved together with $2 \%$ salt in $10 \%$ water, added to the ground beef, mixed well, and chopped for $15 \mathrm{~s}$ before making patties. A total of 27 patties of each group were randomly divided into 3 groups and packaged in aerobic packaging (AP), high-oxygen packaging (HiOx-MAP, $80 \% \mathrm{O}_{2} / 20 \% \mathrm{CO}_{2}$ ), and vacuum skin packaging (VSP), respectively, and then stored at $4^{\circ} \mathrm{C}$ for $0 \mathrm{~d}, 3 \mathrm{~d}$, and $6 \mathrm{~d}$.
The patties were cooked to a central temperature of $66^{\circ} \mathrm{C}$, and then immediately removed and placed in ice bath for $5 \mathrm{~min}$ in a bag to prevent the temperature from rising. The center color was recorded using a X-Rite spectrometer (CR400, Konicamino, Japan). The experiments were performed in duplicate. The data were analyzed using a MIXED model (SAS version 9.0; SAS Institute Inc., Cary, NC) with natural antioxidation, package, storage time, and the interaction as fixed factors.

Results: Lower $a^{*}$ value means less redness and more browning. Sensory evaluation found that browning was observed when the $a^{*}$ value reached 9 in this study. The browning of beef patties in the HiOx-MAP was the greatest on day 6 when cooked to $66^{\circ} \mathrm{C}$ in each treatment. TP and GSE alone or in combination inhibited $\mathrm{PMB}$ in patties stored under AP, but they were ineffective under HiOx-MAP. VSP had the best effect on PMB inhibiting (Table 1).

Conclusion: HiOx-MAP can cause more PMB for beef patties, while VSP can inhibit PMB. The addition of TP or GSE can minimize PMB for beef patties in the AP but has no effect in the HiOx-MAP.

Acknowledgments: This work was supported by funds from the National Natural Science Foundation of China (31871731), Key R. and D. Plan of Shandong Province (2019GNC106050), and China Agriculture Research System-Beef (CARS-37).

Keywords: beef patty, natural antioxidant, package, premature browning

Table 1. Effects of tea polyphenol and grape seed extract, storage time, and package on cooked color of a* value of beef patties cooked to $66^{\circ} \mathrm{C}$

\begin{tabular}{|c|c|c|c|c|}
\hline \multirow[b]{2}{*}{ Natural antioxidant } & \multirow[b]{2}{*}{ Packaging } & \multicolumn{3}{|c|}{ Storage time $(\mathrm{d})$} \\
\hline & & 0 & 3 & 6 \\
\hline \multirow[t]{3}{*}{ Control } & $\mathbf{A P}$ & $10.98 \pm 0.45^{\mathrm{axm}}$ & $8.96 \pm 0.24^{\text {byn }}$ & $9.01 \pm 0.14^{\text {byn }}$ \\
\hline & HiOx-MAP & $10.98 \pm 0.45^{\mathrm{axm}}$ & $9.30 \pm 0.43^{\text {ayn }}$ & $8.37 \pm 0.12^{\mathrm{azn}}$ \\
\hline & VSP & $10.98 \pm 0.45^{\mathrm{axn}}$ & $11.80 \pm 0.70^{\mathrm{bxn}}$ & $13.80 \pm 0.49^{\mathrm{axm}}$ \\
\hline \multirow[t]{3}{*}{$\mathbf{T P}$} & $\mathbf{A P}$ & $11.12 \pm 0.37^{\mathrm{axm}}$ & $9.14 \pm 0.25^{\mathrm{abyn}}$ & $10.59 \pm 0.11^{\mathrm{aym}}$ \\
\hline & HiOx-MAP & $11.12 \pm 0.37^{\mathrm{axm}}$ & $9.79 \pm 0.05^{\text {ayn }}$ & $8.91 \pm 0.17^{\text {azn }}$ \\
\hline & VSP & $11.12 \pm 0.37^{\mathrm{axn}}$ & $13.00 \pm 0.15^{\mathrm{axm}}$ & $13.77 \pm 0.17^{\mathrm{axm}}$ \\
\hline \multirow[t]{3}{*}{ GSE } & $\mathbf{A P}$ & $11.18 \pm 0.21^{\mathrm{axm}}$ & $9.72 \pm 0.23^{\text {abyn }}$ & $10.48 \pm 0.24^{\mathrm{aymn}}$ \\
\hline & HiOx-MAP & $11.18 \pm 0.21^{\mathrm{axm}}$ & $9.76 \pm 0.18^{\mathrm{ayn}}$ & $9.03 \pm 0.27^{\mathrm{azn}}$ \\
\hline & VSP & $11.18 \pm 0.17^{\text {axo }}$ & $13.28 \pm 0.21^{\mathrm{axm}}$ & $12.87 \pm 0.52^{\mathrm{abxn}}$ \\
\hline \multirow[t]{3}{*}{ TP and GSE } & $\mathbf{A P}$ & $11.36 \pm 0.53^{\mathrm{axm}}$ & $9.99 \pm 0.16^{\text {ayn }}$ & $10.77 \pm 0.07^{\text {aymn }}$ \\
\hline & HiOx-MAP & $11.36 \pm 0.53^{\mathrm{axm}}$ & $9.84 \pm 0.26^{\mathrm{ayn}}$ & $9.09 \pm 0.10^{\mathrm{azn}}$ \\
\hline & VSP & $11.36 \pm 1.14^{\mathrm{axn}}$ & $13.04 \pm 0.53^{\mathrm{axm}}$ & $12.71 \pm 0.15^{\mathrm{bxm}}$ \\
\hline
\end{tabular}




\section{3 - EFFECT OF NATURAL ANTIOXIDANTS ATTACHED TO THE PACKAGING ON THE SHELF LIFE OF COOKED HAM}

R. Bermúdez Piedra ${ }^{1 *}$, N. Echegaray ${ }^{2}$, M. Pateiro ${ }^{1}$, P. E. Munekata $^{1}$, O. López-Fernández ${ }^{1}$, D. Franco ${ }^{1}$, and J. M. Lorenzo ${ }^{1},{ }^{1}$ Food Science and Technology, Centro Tecnológico de la Carne, Galicia (Spain), ${ }^{2}$ Centro Tecnológico de la Carne de Galicia, Centro Tecnológico de la Carne de Galicia, Ourense, Spain, *robertobermudez@ceteca.net

Objectives: Cooked ham is a meat product that is susceptible to oxidation. There is a deterioration in color, texture, and nutritional value that generates bad odors and decreases acceptability through this process. One of the main objectives of the meat industry is to prevent oxidation to increase the shelf life of the final product. In this regard, the use of films and coatings with natural extracts as packaging has increased in recent years. Therefore, the aim of this work was to evaluate the effect of packaging with natural antioxidants (Eugenol [Eu], Thymol [Th], and Carvacrol [Ca]) on physicochemical parameters during $21 \mathrm{~d}$ on cooked ham.

Materials and Methods: The hams were deboned and cleaned of connective tissue in order to facilitate the injection of $2 \%$ brine solution. Then, the pieces were injected, macerated, cooked $\left(75^{\circ} \mathrm{C} / 45 \mathrm{~min}\right)$, and refrigerated. Cooked ham pieces were cut in slices and packaged in polyethylene-based packaging, in which 3 different kinds of terpene natural antioxidants $(0.9 \% \mathrm{Eu}, 0.9 \% \mathrm{Th}, 0.9 \% \mathrm{Ca})$ have been incorporated, antioxidants previously absorbed by an inorganic compound with retention capacity. At the same time, a control sample without antioxidants was analyzed. All batches were packaged under a protective atmosphere $\left(70 \% \mathrm{~N}_{2} / 30 \%\right.$ $\mathrm{CO}_{2}$ ) supplied by PRAXAIR (Madrid, Spain). The $\mathrm{pH}$ values (digital portable $\mathrm{pH}$ meter equipped with a penetration probe), color parameters (portable colorimeter to estimate cooked ham color in the CIELAB space), chemical composition, and textural parameters were determined. An analysis of variance using the general linear model was performed for all variables considered in the study, and the least-squares means were separated using Duncan's test $(P<0.05)$ using the IBM-SPSS Statistics 23.0 program (IBM Corp., Armonk, NY).

Results: Chemical composition of samples was determined on day 0 , showing mean values of $79.55 \%$ moisture, $2.31 \%$ fat, $12.36 \%$ protein, and $3.97 \%$ ashes. The $\mathrm{pH}$ values ranged from 5.92 to 6.48 , which are normal values for cooked ham. Regarding color parameters, lightness showed a decrease $(P<0.001)$ in Eu batch, whereas Th and Ca treatments displayed an increase over time. Regarding $a^{*}$ values, Eu samples showed the highest redness $(7.21 ; P<0.05)$ at the end of the storage period. Finally, texture parameters also did not show significant differences among treatments probably due to high variability on samples of sliced cooked ham. Hardness values ranged between $28.69 \mathrm{~N}$ and $20.49 \mathrm{~N}$, for $\mathrm{C}$ and $\mathrm{Ca}$ samples, respectively, after $21 \mathrm{~d}$ of storage.
Conclusion: The use of these active packaging did not show significant differences among treatments for chemical composition, $\mathrm{pH}$ value, and hardness. However, Eu treatment presented the highest $a^{*}$ values after $21 \mathrm{~d}$ of storage. Further studies need to be done in order to test a longer period as well as other concentrations of the extracts.

Acknowledgments: The authors are grateful to RTA2017-00021-00-00 (INIA-MINECO) for the financial support. Acknowledgments to Consellería de Cultura, Educación e Ordenación Universitaria (Xunta de Galicia) for granting Noemi Echegaray with a predoctoral scholarship (Grant Number IN606A-2018/002). Paulo E. S. Munekata acknowledges postdoctoral fellowship support from the Ministry of Economy and Competitiveness (MINECO, Spain) "Juan de la Cierva" program (FJCI2016-29486). José M. Lorenzo is a member of the HealthyMeat network, funded by CYTED (reference 119RT0568).

Keywords: active packaging, color parameters, cooked ham, texture parameters

\section{4 - EFFECT OF FISH SAUCE AS A SUBSTITUTE FOR REFINED SALT ON THE PHYSICOCHEMICAL AND SENSORY QUALITY OF SMOKED AND COOKED PORK SAUSAGE}

S. M. Kang ${ }^{1 *}$, A. R. Maeng ${ }^{1}$, H. V. Ba ${ }^{1}$, Y. Kim ${ }^{1}$, K.-H. Seol $^{1}$, S. Cho ${ }^{1}$, P.-N. Seong ${ }^{2}$, and J.-H. Kim ${ }^{1},{ }^{1}$ Animal Products Utilization Division, National Institute of Animal Science, RDA, Wanju-gun, Republic of Korea, ${ }^{2}$ Planning and Coordination Bureau, RDA, Jeonju-si, Republic of Korea, *smkang1014@naver.com

Objectives: Fish sauce is one of the traditional seasonings that people have enjoyed eating in many Asian countries, including Korea, Vietnam, and Thailand. This seasoning contains a high content of salt and has a unique flavor because it is made by fermenting salted seafood. Therefore, this study estimated the effect of fish sauce as a substitute for refined salt on the physicochemical and sensory quality of smoked and cooked pork sausage.

Materials and Methods: Experimental sausages were prepared for 4 treatments ( $n=3 /$ treatment) either with $1.125 \%$ refined salt (control), $0.75 \%$ refined salt and $1.50 \%$ fish sauce (T1), $0.375 \%$ refined salt and $3.00 \%$ fish sauce (T2), or $4.50 \%$ fish sauce (T3). The salinity of fish sauce was $25 \%$, and all sausage treatments contained the same proportion $(1.125 \%)$ of salt. Pork lean $(80 \%$ hind leg meat) and fat ( $20 \%$ backfat) were ground using a meat chopper equipped with a $5-\mathrm{mm}$ diameter plate and mixed for 15 min using a vacuum bowl cutter with the following ingredients: $240 \mathrm{~g} / \mathrm{kg}$ ice, $10 \mathrm{~g} / \mathrm{kg}$ white sugar, $0.15 \mathrm{~g} / \mathrm{kg}$ 
sodium nitrite, $2 \mathrm{~g} / \mathrm{kg}$ phosphates, $0.5 \mathrm{~g} / \mathrm{kg}$ ascorbic acid, 15 $\mathrm{g} / \mathrm{kg}$ white pepper, $0.3 \mathrm{~g} / \mathrm{kg}$ coriander, $0.3 \mathrm{~g} / \mathrm{kg}$ nutmeg, $1 \mathrm{~g} /$ $\mathrm{kg}$ cinnamon, $5 \mathrm{~g} / \mathrm{kg}$ garlic, $15 \mathrm{~g} / \mathrm{kg}$ onion, $5 \mathrm{~g} / \mathrm{kg}$ monosodium glutamate, $15 \mathrm{~g} / \mathrm{kg}$ baking powder, and $30 \mathrm{~g} / \mathrm{kg}$ potato starch. The salinity of fish sauce was $25 \%$. After stuffing into 60-mm fibrous casings, sausages were dried and smoked at $55^{\circ} \mathrm{C}$, and then cooked to a core temperature of $70^{\circ} \mathrm{C}$ with steam-heating. The $\mathrm{pH}$ value was measured using a $\mathrm{pH}$ meter immediately after homogenizing sausage samples with deionized water. Lipid oxidation was expressed as the 2thiobarbituric acid reactive substances content. The internal color was determined as CIE $L^{*}, a^{*}$, and $b^{*}$ values. Textural parameters, such as hardness, cohesiveness, springiness, gumminess, and chewiness, were tested using a texture analyzer equipped with a cylindrical probe. The attributes of internal color, aroma, taste, and texture were evaluated by 10 untrained panelists using a 9-point hedonic scale $(9=$ like extremely, $1=$ dislike extremely).

Results: The $\mathrm{pH}$ value was significantly $(P<0.05)$ higher in the following order: T2 and T3 $>\mathrm{T} 1>$ control. There were no significant differences for thiobarbituric acid reactive substances content, hardness, cohesiveness, springiness, gumminess, and chewiness among all sausage treatments. The CIE $L^{*}$ value was significantly $(P<0.05)$ higher in the following order: control and T1 $>\mathrm{T} 2>\mathrm{T} 3$. Taste preference and overall liking with sensory evaluation were significantly $(P<0.05)$ higher in the $\mathrm{T} 3$ sausage than in the control sausage.

Conclusion: These findings suggest that the addition of $4.5 \%$ fish sauce as a substitute for $1.125 \%$ refined salt improves the sensory attributes of smoked and cooked pork sausage.

Keywords: fish sauce, salt, sausage, color, sensory attributes

\section{8 - BEEF ABATTOIR WATER USAGE MAY BE REDUCED THROUGH LOW-VOLUME SPRAY CHILLING WITHOUT DETRIMENTAL IMPACTS ON BEEF QUALITY OR SALEABLE YIELD}

N. C. Hardcastle ${ }^{1 *}$, J. F. Legako ${ }^{1}$, J. C. Brooks ${ }^{1}$, M. F. Miller $^{1}$, S. R. Pohlman' ${ }^{2}$, S. R. Howard ${ }^{2}$, G. Dolezal ${ }^{2}$, and D. R. Woerner ${ }^{1},{ }^{1}$ Animal and Food Science, Texas Tech University, Lubbock, TX, USA, ${ }^{2}$ Cargill Protein, Cargill, Wichita, KS, USA, *nicholas.hardcastle@ttu.edu

Objectives: Commercial beef processing plants utilize spray chilling, or the intermittent spraying of cold water, to reduce postmortem beef temperatures. Conventional spray chilling requires 40 to $150 \mathrm{~L}$ of water per side, and this study was aimed at evaluating the effect of a sustainable reduced water spray protocol. In this study, 2 spray chilling protocols were evaluated for chilling rate, saleable yield, microbial load, and carcass quality attributes.

Materials and Methods: Over a 6-wk period, 1,905 carcasses, of varying composition and breed $(97.18 \%$ Beef and $2.82 \%$ Holstein), free of dressing defects, were selected at a commercial beef abattoir. For each side $(N=1,905$ paired sides), hot side weight and removed kidney and pelvic weight were obtained on the harvest floor. Alternating sides were assigned to either conventional, high-volume water (HW; $104 \mathrm{~L}$ water/side), or reduced, low-volume water (LW; $3 \mathrm{~L}$ water/side) chilling treatments. Treatment application lasted for $28 \mathrm{~h}$ postmortem with a fixed spray pattern of chilled water $\left(3^{\circ} \mathrm{C}-4^{\circ} \mathrm{C}\right)$ and circulating air $\left(56.63 \mathrm{~m}^{3} / \mathrm{min}\right)$. Each week, 12 carcasses ( $n=72$ paired sides) were selected for continuous temperature monitoring of the deep muscle and subcutaneous fat and pre- and post-chill aerobic plant count and coliform load. After chilling, sides $(N=1,905$ paired sides) were subject to USDA grading and weighed to obtain chilled side weight. Eighty-five carcasses $(n=85$ paired sides) were selected for a cutout to determine saleable yield, trim, fat, and bone. Cutout weigh back allowed for $\pm 1 \%$ of the side weight. One steak was collected from each cut strip loin ( $n=85$ paired steaks) for a fresh 3-d WarnerBratzler shear force (WBSF) and slice shear force analysis (SSF). Data were analyzed using the GLIMMIX and Freq procedures of SAS (SAS Institute Inc., Cary, NC). For all models, side was nested in carcasses; chilling treatment was observed as the fixed effect, and replication as a random effect $(\alpha=0.05)$. Cooked peak temperature was included as a covariate for WBSF and SSF $(P<0.01)$.

Results: Spay protocols did not affect hot side weight $(P=0.53)$ or kidney and pelvic weight $(P=0.60)$. HW sides had greater $(P<0.001)$ chilled side weight yielding a $1.12 \%$ lower $(P<0.001)$ shrink than LW. No differences in preand post-chill aerobic plant count $(P \geq 0.17)$ and coliform $(P \geq 0.10)$ counts occurred between treatments. Though HW resulted in greater $(P=0.02)$ marbling scores than LW, USDA quality grade distribution did not differ $(P=$ 0.43). Sides of LW possessed a lesser fat thickness $(P<$ $0.01)$ opposite the ribeye, resulting in a lower $(P<0.01)$ numerical yield grade than $\mathrm{HW}$; however, no difference in USDA yield grade distribution occurred $(P=0.59)$. The HW chilling protocol resulted in a greater $(P=0.01)$ total cutout weight, due to increased cutout weights of trim $(P=0.03)$ and fat $(P<0.01)$, but no difference in total subprimal yield occurred $(P=0.34)$. There were no differences in WBSF $(P=0.31)$ or SSF $(P=0.20)$ between treatments.

Conclusion: Results show that the LW protocol utilized $97.52 \mathrm{~kg}$ less water $/$ side than HW and yielded a $2.08 \mathrm{~kg}$ chilling side loss. The loss of trimmings and fat, which were affected by water volumes, should be taken into consideration, as HW resulted in $0.37 \mathrm{~kg}$ more trim and $0.56 \mathrm{~kg}$ more fat per side. Ultimately, processors operating in areas where water is limited can reduce water volumes during spray 
chilling to increase sustainability without altering beef quality, safety, and saleable subprimal yield.

Keywords: beef, carcass cutout, quality, spray chill, water

\section{9 - TOWARDS THE REDUCTION OF NITRITE IN CURED MEAT: ROLE OF ASCORBATE?}

A. Bonifacie ${ }^{1 *}$, P. Gatellier ${ }^{1}$, G. Nassy ${ }^{2}$, A. Promeyrat ${ }^{2}$, V. Santé-Lhoutellier ${ }^{1}$, and L. Théron ${ }^{1}$, ${ }^{1}$ QuaPA UR370, INRAE, 63122 Saint Genès-Champanelle France, ${ }^{2} I F I P$, IFIP - Institut du Porc, 35651 Le Rheu, France, *aline. bonifacie@inrae.fr

Objectives: Nitrite and/or nitrate added in cured meat is involved in the formation of nitroso-compounds (nitrosamines, nitrosothiols, and nitrosylheme) and potentially mutagenic. Oxidation in meat affects health by formation of mutagenic aldehydes. An excessive consumption of cured meat is associated with an increased risk of colorectal cancer. Therefore, the objective of this study was to evaluate the role of ascorbate regarding the reduction of nitrites in cured meat.

Materials and Methods: Cured meat was prepared with pork shoulder muscle, with different amounts of sodium nitrite (0 to $120 \mathrm{ppm})$ with or without ascorbate (300 ppm), and cooked at $68.5^{\circ} \mathrm{C}$. A recooking at $180^{\circ} \mathrm{C} / 7 \mathrm{~min}$ was also studied. The free iron content was measured by ferrozine assay. Nitrosylated heme iron was evaluated by extraction in acetone. Lipid oxidation was evaluated by measurement of the thiobarbituric acid reactive substances. To determine nitrosothiol and nitrosamine contents, the S-NO and N-NO bonds were cleaved, and the nitrite released was measured.

Results: Addition of nitrite decreased significantly free iron level. This can be due to the oxidative cleavage of myoglobin heme by hydrogen peroxide $\left(\mathrm{H}_{2} \mathrm{O}_{2}\right)$. Nitrite prevents this cleavage by neutralizing superoxide radical at the origin of $\mathrm{H}_{2} \mathrm{O}_{2}$ formation. In meat, free iron is implicated in oxidation via the formation of hydroxyl radical. Thus, by decreasing $\mathrm{H}_{2} \mathrm{O}_{2}$ and oxygenated free radicals, nitrite acts as a powerful antioxidant. For the uncured condition, lipid oxidation was significantly higher than in cured meat products, demonstrating again the efficiency of nitrite as antioxidant. Ascorbate did not show any significant effect. Reducing nitrite from 120 to 40 ppm did not affect nitrosylheme content. So, a nitrite decrease could be considered without major impact on color. The level of nitrosothiols increased in cured meat from $40 \mathrm{ppm}$ of added nitrite. This effect was especially pronounced in the absence of ascorbate. Nitrosothiols can transfer NO group to heme iron. Nitrosamines were not detected in this model with the exception of a low level at $40 \mathrm{ppm}$ of nitrite. However, with the recooking, mimicking a second cooking process in pizza for instance, an important production of nitrosamines was observed in absence of ascorbate. This demonstrated the necessity of adding ascorbate in this process in order to protect human health.

Conclusion: The protective effect of nitrite on lipid oxidation is counterbalanced by production of nitrosothiol and nitrosylheme. Ascorbate reduces nitrosamine formation due to recooking. In the future, we will examine the protective effect of some vegetable antioxidants against nitrosation and nitrosylation.

Keywords: cured meat, lipid oxidation, nitrite, nitrosamines, nitrosothiols, nitrosylheme

\section{0 - EFFECT OF IN-THE-BAG DRY AGING ON THE FLAVOR PROFILE OF BEEF LOW- VALUE CUTS}

L. Evasiuk ${ }^{1}$, J. L. Aalhus ${ }^{1}$, O. Lopez-Campos ${ }^{1}$, I. L. Larsen ${ }^{1}$, and N. Prieto ${ }^{1 *},{ }^{1}$ Lacombe Research and Development Centre, Agriculture and Agri-Food Canada, Lacombe, Canada,*nuria.prietobenavides@canada.ca

Table. Effect of nitrite $\left(\mathrm{NO}_{2}\right)$ and ascorbate $(\mathrm{AH})$ and of recooking on iron content, oxidation, nitrosation and nitrosylation in ham model $(n=6$; superscripts differ if $P<0.01)$

\begin{tabular}{|c|c|c|c|c|c|c|c|}
\hline & $\begin{array}{c}0 \mathrm{ppm} \\
\mathrm{NO}_{2}+\mathrm{AH}\end{array}$ & $\begin{array}{c}40 \mathrm{ppm} \\
\mathrm{NO}_{2}+\mathrm{AH}\end{array}$ & $\begin{array}{c}80 \mathrm{ppm} \\
\mathrm{NO}_{2}+\mathrm{AH}\end{array}$ & $\begin{array}{c}120 \mathrm{ppm} \\
\mathrm{NO}_{2}+\mathrm{AH}\end{array}$ & $\begin{array}{c}120 \mathrm{ppm} \\
\mathrm{NO}_{2}\end{array}$ & $\begin{array}{c}120 \mathrm{ppm} \mathrm{NO} \mathrm{N}_{2}+\mathrm{AH} \\
180^{\circ} \mathrm{C} / 7 \mathrm{~min} \\
\end{array}$ & $\begin{array}{c}120 \mathrm{ppm} \mathrm{NO} \\
180^{\circ} \mathrm{C} / 7 \mathrm{~min}\end{array}$ \\
\hline $\begin{array}{l}\text { Free iron } \\
(\mu \mathrm{M})\end{array}$ & $\begin{array}{l}82.1^{\mathrm{a}} \\
+/-1.7\end{array}$ & $\begin{array}{l}28.4^{\mathrm{b}} \\
+/-1.6\end{array}$ & $\begin{array}{c}26.6^{\mathrm{b}} \\
+/-0.9\end{array}$ & $\begin{array}{l}34.9^{\mathrm{c}} \\
+/-1.2\end{array}$ & $\begin{array}{l}35.5^{\mathrm{c}} \\
+/-0.8\end{array}$ & - & - \\
\hline $\begin{array}{l}\text { TBARS } \\
(\mathrm{mg} \text { MDA } / \mathrm{kg})\end{array}$ & $\begin{array}{l}1.37^{\mathrm{a}} \\
+/-0.03\end{array}$ & $\begin{array}{l}0.69^{\mathrm{b}} \\
+/-0.06\end{array}$ & $\begin{array}{l}0.72^{\mathrm{b}} \\
+/-0.15\end{array}$ & $\begin{array}{c}0.51^{\mathrm{b}} \\
+/-0.03\end{array}$ & $\begin{array}{c}0.51^{\mathrm{b}} \\
+/-0.04\end{array}$ & - & - \\
\hline $\begin{array}{l}\text { Nitrosylated } \\
\text { heme iron }(\mu \mathrm{M})\end{array}$ & $\begin{aligned} & 1.2^{\mathrm{a}} \\
&+/-0.1\end{aligned}$ & $\begin{array}{l}83.1^{\mathrm{b}} \\
+/-7.5\end{array}$ & $\begin{array}{c}98.5^{\mathrm{b}} \\
+/-0.9\end{array}$ & $\begin{array}{l}89.1^{\mathrm{b}} \\
+/-6.1\end{array}$ & $\begin{array}{l}82.2^{\mathrm{b}} \\
+/-1.6\end{array}$ & - & - \\
\hline $\begin{array}{l}\text { Nitrosothiols } \\
(\mu \mathrm{M})\end{array}$ & $\begin{aligned} & 7.7^{\mathrm{a}} \\
+ & /-2.2\end{aligned}$ & $\begin{array}{l}30.6^{\mathrm{ab}} \\
+/-2.0\end{array}$ & $\begin{array}{l}63.0^{\mathrm{b}} \\
+/-9.1\end{array}$ & $\begin{array}{l}35.2^{\mathrm{ab}} \\
+/-14.1\end{array}$ & $\begin{array}{l}207.4^{\mathrm{c}} \\
+/-15.4\end{array}$ & - & - \\
\hline $\begin{array}{l}\text { Nitrosamines } \\
(\mu \mathrm{M})\end{array}$ & 0 & $\begin{array}{c}6.1 \\
+/-3.2\end{array}$ & 0 & 0 & 0 & $\begin{array}{l}188.2^{\mathrm{a}} \\
+/-34.4\end{array}$ & $\begin{aligned} & 439^{\mathrm{b}} \\
+ & /-41.5\end{aligned}$ \\
\hline
\end{tabular}


Objectives: This study evaluated the effects of in-the-bag dry aging and different aging periods on the flavor profile of low-value cuts from steers.

Materials and Methods: In total, 20 carcasses (AAA) from Angus cross youthful animals were used in this study. The flat iron (infraspinatus), clod heart (triceps brachii), and brisket (pectoralis profundi) muscles from both right and left carcass sides ( $\mathrm{n}=40$ from muscle type) were collected at the Lacombe Research and Development Centre (Agriculture and Agri-Food Canada, Canada). Controlling for side, muscles were randomly assigned to wet or dry aging, using conventional oxygen/moisture impermeable vacuum bags (Winpak Vak 3.0 R, Winnipeg, MB, Canada) or specifically manufactured oxygen impermeable/moisture permeable vacuum bags (Umai ${ }^{\circledR}$, Minneapolis, MN), respectively. After vacuum packing, muscles were placed in a conventional $2^{\circ} \mathrm{C}$ cooler. Half the muscles $(n=20$ from muscle type; 10 wet and 10 dry aged) were aged for $21 \mathrm{~d}$, and half ( $n=20$ from muscle type; 10 wet and 10 dry aged) for $42 \mathrm{~d}$. Following aging, muscles were removed from their respective packaging, and one $2.5-\mathrm{cm}$ steak from each muscle was cut and grilled to an internal temperature of $71^{\circ} \mathrm{C}$ for subsequent flavor profile analyses. Steaks were presented in a balanced design to a 9-member trained sensory panel to rate the intensity of aromas $(n=19)$, tastes $(n=5)$, and flavors $(n=19)$ using $15-\mathrm{cm}$ line scales. Flavor profile data were analyzed for each muscle using the MIXED model procedure of SAS version 9.4 (SAS Institute Inc., Cary, NC), with the main effects of aging treatment and period and their interactions in the model, and panel session and assessor and their interactions were included as random effects.

Results: Aging treatment $\times$ period interactions $(P<$ 0.05 ) were observed in briskets and flat irons. Livery aroma and sour taste were higher at 42- than 21-d dry-aged but similar in both 21- and 42-d wet-aged briskets, whereas bloody/ serumy aroma and corn flavor were higher at 42- than 21-d wet-aged but similar for both aging periods in dry-aged briskets. Cruciferous aroma was higher and corn aroma was lower at 42- than 21-d wet-aged but similar in 21- and 42-d dry-aged flat irons, whereas the highest bitter taste was found at 21-d dry-aged, the lowest at 21-d wet-aged, and intermediate at 42-d dry- and wet-aged flat irons. Overall, dry aging decreased $(P<0.05)$ bloody/serumy and sour dairy flavors in briskets and clod hearts, respectively, whereas it increased unidentified aroma/flavor in briskets, beef identity flavor in clod hearts, and metallic aroma and brown-roasted flavor in flat irons compared to wet aging. The 42-d aging period increased $(P<0.05)$ livery aroma in briskets, sour/dairy aroma and green/hay flavor in clod hearts, and metallic aroma in flat irons, whereas it decreased beef identity flavor in clod hearts and sweet taste and beef identity and bloody/serumy flavors in flat irons compared to $21 \mathrm{~d}$ of aging.

Conclusion: These results show in-the-bag dry aging to be an effective strategy to increase some flavors identified in the literature as desirable and decrease others that could be perceived as undesirable flavors by consumers in low-value cuts of beef, thus creating potential value-added alternatives for specialty marketers to meet the demand of the new generation of foodies. Overall, extending the aging period from 21 to $42 \mathrm{~d}$ did not result in flavor enhancement of beef lowvalue cuts.

Keywords: aging period, beef, dry aging, flavor, lowvalue cuts

\section{1 - SPOILAGE PSEUDOMONAS SURVIVE THERMAL PROCESSING AND GROW DURING VACUUM-PACKAGED STORAGE IN AN EMULSIFIED MEAT SYSTEM}

S. C. Watson ${ }^{1 *}$, R. A. Furbeck ${ }^{1}$, B. D. Chavez ${ }^{2}$, and G. A. Sullivan ${ }^{1},{ }^{1}$ Department of Animal Science, University of Nebraska, Lincoln, NE, USA, ${ }^{2}$ Department of Food Science and Technology, University of Nebraska, Lincoln, NE, USA, *swatson13@huskers.unl.edu

Objectives: Pseudomonas are considered the predominant microbial spoilers of aerobically stored raw meat products with minor roles in vacuum-packed product spoilage. However, recent findings have challenged this principle and opened new avenues for research on the role of Pseudomonas in the spoilage of thermally processed, vacuum-packaged meat. The natural food microbiome and contamination from the processing environment contribute to the Pseudomonas presence in vacuum-packed cooked product. Given the potential thermal resistance of Pseudomonas, residual post-process populations may also be responsible for product spoilage. Therefore, an experiment was conducted with the objectives of determining whether spoilage Pseudomonas can survive thermal processing and grow anaerobically through refrigerated storage in an emulsified model meat system.

Materials and Methods: Three Pseudomonas isolates from spoiled meat were grown individually in Luria-Bertani broth and combined to create an inoculation cocktail. Two kilograms of coarse ground beef were inoculated to approximately $5 \log _{10}$ of Pseudomonas and emulsified with ice, salt ( $2 \%$ meat block basis), sodium nitrite ( $156 \mathrm{ppm}$ meat block basis), sodium erythorbate, black pepper, and garlic in a food processor (Hobart FP41; Hobart Corporation; Troy, OH). Batter samples (approximately $20 \mathrm{~g},<1.5 \mathrm{~cm}$ thickness) were vacuum packaged individually and split into 3 treatments: 2 cooked treatments $\left(71^{\circ} \mathrm{C}\right.$ for $1 \mathrm{~s}$ or $54^{\circ} \mathrm{C}$ for $\left.121 \mathrm{~min}\right)$ and 1 uncooked treatment. Samples were cooked in water baths using sous vide units to target temperatures and then chilled in an ice bath for $15 \mathrm{~min}$. Pseudomonas concentrations were enumerated after inoculation, after chilling for cooked samples, and after emulsifying for uncooked samples, as well as at 14,28 , and $56 \mathrm{~d}$ of storage at $4^{\circ} \mathrm{C}$ and $10^{\circ} \mathrm{C}$. At each 
sampling time, $10 \mathrm{~g}$ of an individually packed sample were stomached with $20 \mathrm{~mL}$ of buffered peptone water. Homogenates were serially diluted and plated onto Pseudomonas Agar Base plates supplemented with Cetrimide-FucidinCephalosporin Selective Supplement. The experiment was conducted in 3 independent replications with duplicate samples. Data were reported as $\log _{10} \mathrm{CFU} / \mathrm{g}$ and analyzed using the GLIMMIX procedure with least significant difference means separation in SAS version 9.4 (SAS Institute Inc., Cary, NC).

Results: Pseudomonas concentrations in uncooked treatments decreased by $1.66 \log _{10}$ and $1.39 \log _{10}(P<$ 0.05 ) after $56 \mathrm{~d}$ of storage for $10^{\circ} \mathrm{C}$ and $4^{\circ} \mathrm{C}$ storage, respectively. In both cooked treatments, concentrations were reduced below the detection limit $\left(0.18 \log _{10}\right)$ immediately following cooking $(P<0.05)$. After $56 \mathrm{~d}$ of storage, Pseudomonas populations increased to $>0.5 \log _{10}$ in both cooked treatments stored at $10^{\circ} \mathrm{C}$ and $4{ }^{\circ} \mathrm{C}$ and were greater than concentrations from samples taken immediately after cooking $(P<0.05)$.

Conclusion: Pseudomonas was recovered from all cooking and storage treatment combinations, indicating the ability to survive thermal processing. The increasing concentration of Pseudomonas during storage in intact vacuum packages warrants further investigation into the ability of Pseudomonas to recover and grow in anaerobic environments and cause spoilage.

Keywords: meat spoilage, Pseudomonas, thermal survival

\section{2 - NITRIC OXIDE PRODUCTION IN PRE-RIGOR SEMIMEMBRANOSUS PORK MUSCLE}

W. Osburn ${ }^{1}$, K. Modrow ${ }^{1 *}$, and W. N. Osburn ${ }^{1} .2019$. Amino acid alternative curing system. Patent Attorney Docket No. TAMU-19489 December 2019, issued 2019., ${ }^{1}$ Animal Science, Texas A\&M University, College Station, TX, USA, *kmmodrow2017@gmail.com

Objectives: The objective of this study was to determine whether the L-arginine activated nitric oxide synthase (NOS) system found in living skeletal muscle is still functional in pre-rigor porcine muscle via the addition of Larginine to produce nitric oxide (NO) and residual nitrite.

Materials and Methods: Pre-rigor pork semimembranosus muscle samples $(60-75 \mathrm{~g})$ were collected from 4 prerigor pork carcasses harvested at 6 separate times over a 4 -d period $(n=24)$. Subsamples ( $\sim 5 \mathrm{~g})$ were placed in separate test tubes and treated with one of 5 concentrations of $2 \mathrm{~mL}$ L-arginine solution $(2,4,8,16$, or $32 \mathrm{mM})$ with control water $(0 \mathrm{mM})$. Additionally, a solution containing $72 \mathrm{mg}$ $\mathrm{NaCl}, 576 \mathrm{ppm}$ sodium erythorbate, and $8 \mathrm{~mL}$ of water was added to each sample $(n=288)$. Samples were either heated (water bath cooking samples to $62^{\circ} \mathrm{C}$ within $60 \mathrm{~min}$ ) or left raw (uncooked). All treated samples were analyzed for residual nitrite by analyzing supernatant and pellet samples using modified ultraviolet/visible spectrophotometric method. Cooked sample supernatant and pellets were analyzed for nitrosylation (determination of nitrosylhemochromagen) after storage $\left(-30^{\circ} \mathrm{C}\right.$; freezer for $3 \mathrm{wk}$, then thawed in $0^{\circ} \mathrm{C}$ cooler).

Results: For raw supernatants, the $32 \mathrm{mM} \mathrm{L}$-arginine treatment had higher residual nitrite levels $(14.3 \mathrm{ppm})$ compared to the control $(0.08 \mathrm{ppm} ; P<0.05)$. The $4 \mathrm{mM}(8.00$ $\mathrm{ppm}), 8 \mathrm{mM}(9.60 \mathrm{ppm})$, and $16 \mathrm{mM}(9.23 \mathrm{ppm})$ treatments were different $(P<0.05)$ than the control and $32 \mathrm{mM}$ treatment. Residual nitrite levels of $4 \mathrm{mM}$ cooked supernatants were higher $(8.79 \mathrm{ppm} ; P<0.05)$ compared to other treatments and control $(0.07 \mathrm{ppm})$. The observed bimodal effect is similar to endogenous activation of the NOS system by Larginine and NO synthesis. The NO produces stable nitrite when extracellular L-arginine concentrations increase from 0.5 to $5 \mathrm{mM}$. The L-arginine addition to pre-rigor samples activated the NOS system $(4 \mathrm{mM})$ and at higher L-arginine concentrations $(32 \mathrm{mM})$ resulted in more efficient NO generation. This occurred by reducing the interference of citrulline slowing NOS system to regenerate L-arginine as a substrate for the NOS system for residual nitrite and nitrosylhemachromagen (NO-heme) formation. For cooked muscle pellets, the $32 \mathrm{mM}$ L-arginine treatment exhibited the highest residual nitrite values $(15.7 \mathrm{ppm} ; P<0.05)$ compared to other treatments and the control $(0.04 \mathrm{ppm})$. The $2 \mathrm{mM}$ (10.5 ppm), $4 \mathrm{mM}$ (9.35 ppm), $8 \mathrm{mM}$ (11.2) and $16 \mathrm{mM}$ (14.1 ppm) L-arginine treatments were different than the control but not significantly different between each other. In cooked supernatants, no differences were observed for any L-arginine concentration for NO-heme or nitrosylation. However, all L-arginine concentrations exhibited nitrosylation values over $100 \%$. This suggests that the NOS system did generate $\mathrm{NO}$ and residual nitrite. In cooked pellets, there was a difference $(P<0.05)$ in NO-heme levels for all L-arginine treatments compared to the control. For total heme levels, all treatment concentrations were higher than the control, while no differences existed between the treatments. Percent nitrosylation was greatest for the $32 \mathrm{mM} \mathrm{L}$-arginine treatment $(156.72 \%)$ and was different $(P<0.05)$ than the other concentrations.

Conclusion: In conclusion, raw supernatant and pellet samples had higher residual nitrite levels compared to cooked supernatants and pellets. Cooked pellets' nitrosylation was significantly higher compared to the control. Based upon these study results, there was evidence that the NOS system can generate NO and residual nitrite by $\mathrm{L}$-arginine addition in pre-rigor pork muscle.

Keywords: L-arginine, nitric oxide synthase, nitrite 


\section{3 - EFFECT OF INJECTING GINGER PROTEASE ON IMPROVING TENDERNESS IN M. BICEPS FEMORIS FROM CULLED DAIRY COWS}

Z. B. Naqvi ${ }^{1,2}$, M. A. Campbell ${ }^{2}$, P. C. Thomson ${ }^{2,3}$, D. M. McGill $^{4}$, R. D. Warner ${ }^{4}$, and M. A. Friend ${ }^{1,2}$, ${ }^{1}$ School of Animal and Veterinary Sciences, Charles Sturt University, Wagga Wagga, Australia, ${ }^{2}$ Graham Centre for Agricultural Innovation, Charles Sturt University, Wagga Wagga, Australia, ${ }^{3}$ School of Life and Environmental Sciences, The University of Sydney, Sydney, Australia, ${ }^{4}$ Faculty of Veterinary and Agricultural Sciences, The University of Melbourne, Melbourne, Australia, *zanaqvi@csu.edu.au

Objectives: Muscles of culled/old animals exhibit substantial toughness due to the strength developed in the connective tissues and/or insufficient endogenous proteolytic enzyme capacity to tenderize the meat postmortem. Marination of muscles with exogenous proteases acquired from plants, bacteria, fungi, and their commercially available products have shown tenderizing effects on tough beef meat. Zingibain or ginger protease is a cysteine protease found in ginger rhizome, and its proteolytic activity appears to have manifold effects on collagen compared with actomyosin, and the combined proteolysis of these 2 muscle proteins could result in improved beef tenderness. The study was designed to investigate the tenderization of low-valued meat using commercially available ginger powder containing proteolytic enzymes including zingibain.

Materials and Methods: This study used biceps femoris $(n=30)$ acquired from Angus/Angus cross culled cows of similar age (6-7 y old). Each biceps femoris was cut into 4 portions and injected with 1 or $2 \mathrm{gL}^{-1}$ ginger powder solution, control was with water only, and another had no injection. Each portion was cut into 3 blocks, vacuum packed, and cooked for $1 \mathrm{~h}$, $8 \mathrm{~h}$, and $18 \mathrm{~h}$ in temperature-equilibrated water baths at $65^{\circ} \mathrm{C}$ and $75^{\circ} \mathrm{C}$, and internal temperature was monitored by thermocouples attached to a Grant data logger. Warner Bratzler shear force (WBSF), cooking loss, collagen content, and myofibrillar fragmentation index (MFI) were measured to assess change in meat tenderness. The data were analyzed using a linear mixed effect model fitted by REML in R version 3.5.2 (with packages "nlme" and "emmeans"; R Core Team, 2018).

Results: Results revealed significant (all $P<0.005$ ) interactions between enzyme treatment, cooking temperature, and time on WBSF. WBSF in low-valued meat reduced with injecting ginger powder solution and cooking duration (Table 1). Cooking loss increased with increasing cooking temperature and time $(P \leq 0.0001)$ (Table 2$)$. Results of collagen content and MFI are presented in Table 3. Collagen solubility increased by injecting ginger powder solution $(P=0.0045)$ and cooking time $(P=0.0001)$ but was not impacted by cooking temperature $(P=0.38)$. MFI increased with increasing concentration of enzyme $(P=0.029)$. The results have indicated weakening of myofibrillar proteins
Table 1. Effect of zingibain treatment and cooking time on the Warner-Bratzler shear force (WBSR N) of biceps femoris. Superscripts (letters a-g) refer to the significant differences $(P<0.05)$ in mean WBSF values.

\begin{tabular}{lrcccc}
\hline \hline & & \multicolumn{4}{c}{ Injection treatment } \\
\cline { 3 - 6 } & Time & No injection & Only water & $1 \mathrm{gL}^{-1} \mathrm{GP}$ & $2 \mathrm{gL}^{-1} \mathrm{GP}$ \\
\hline \multirow{2}{*}{ WBSF (N) } & $1 \mathrm{~h}$ & $48.8^{\mathrm{ab}}$ & $49.9^{\mathrm{a}}$ & $37.9^{\mathrm{cd}}$ & $27.3^{\mathrm{f}}$ \\
& $8 \mathrm{~h}$ & $42.9^{\mathrm{abc}}$ & $42.8^{\mathrm{bc}}$ & $27.9^{\mathrm{ef}}$ & $24.7^{\mathrm{f}}$ \\
& $18 \mathrm{~h}$ & $36.0^{\mathrm{d}}$ & $32.7^{\mathrm{de}}$ & $20.2^{\mathrm{g}}$ & $20.4^{\mathrm{g}}$ \\
\hline
\end{tabular}

Table 2. Effect of cooking temperature $\left(65^{\circ} \mathrm{C}, 75^{\circ} \mathrm{C}\right)$ and cooking time ( $1 \mathrm{~h}, \mathrm{~S} \mathrm{~h} ; 18 \mathrm{~h})$ on cooking loss (\%) of biceps femoris. Superscripts (letters a-e) refer to the significant differences $(P<0.05)$ in mean values.

\begin{tabular}{llllllll}
\hline \hline Temperature & \multicolumn{3}{c}{$65^{\circ} \mathrm{C}$} & & & $75^{\circ} \mathrm{C}$ \\
\cline { 2 - 4 } \cline { 6 - 8 } Time & $1 \mathrm{~h}$ & $8 \mathrm{~h}$ & $18 \mathrm{~h}$ & & $1 \mathrm{~h}$ & $8 \mathrm{~h}$ & $18 \mathrm{~h}$ \\
\hline Cooking loss & $32.3^{\mathrm{a}}$ & $39.3^{\mathrm{c}}$ & $41.3^{\mathrm{e}}$ & & $43.0^{\mathrm{b}}$ & $47.0^{\mathrm{d}}$ & $47.3^{\mathrm{d}}$ \\
\hline
\end{tabular}

Table 3. Effect of zingibain treatment on soluble collagen (mg. $\mathrm{g}^{-1}$ meat) and myofibrillar fragmentation index (MFI) of of biceps femoris. Superscripts (letters a-c) refer to the significant differences $(P<0.05)$ in mean soluble collagen and MFI values.

\begin{tabular}{lccrr}
\hline & \multicolumn{3}{c}{ Injection treatment } \\
\cline { 2 - 5 } & No injection & Only water & $1 \mathrm{gL}^{-1} \mathrm{GP}$ & $2 \mathrm{gL}^{-1} \mathrm{GP}$ \\
\hline Soluble collagen & $0.21^{\mathrm{a}}$ & $0.28^{\mathrm{ab}}$ & $0.41^{\mathrm{c}}$ & $0.39^{\mathrm{bc}}$ \\
MFI & $139.6^{\mathrm{b}}$ & $147.1^{\mathrm{ab}}$ & $150.2^{\mathrm{a}}$ & $153.7^{\mathrm{a}}$ \\
\hline
\end{tabular}

and connective tissue contribute to denaturation of proteins and solubilization of collagen.

Conclusion: Injecting zingibain improved tenderness of bicep femoris, therefore ginger protease has potential to add value to low-valued muscle from older cows with reduced cooking duration.

Keywords: ginger protease, tenderness, beef, older cows, collagen solubility

\section{4 - COMBINED EFFECT OF HIGH HYDROSTATIC PRESSURE WITH REDUCED SODIUM CHLORIDE AND SODIUM PHOSPHATE CONTENT ON THE STRUCTURE AND PALATABILITY OF BEEF GELS}

A. Maksimenko ${ }^{1 *}$, R. Kikuchi ${ }^{1}$, S. Tsutsuura ${ }^{2}$, and T. Nishiumi ${ }^{1}$, ${ }^{1}$ Graduate School of Science and Technology, 
Niigata University, Niigata, Japan, ${ }^{2}$ Institute for Research Promotion, Niigata University, Niigata, Japan, *anastasia. 2603@mail.ru

Objectives: The objective of this study was to investigate the effects of high hydrostatic pressure (HHP) treatment (100$200 \mathrm{MPa}, 10 \mathrm{~min}, 20^{\circ} \mathrm{C}$ ) in combination with sodium chloride $(0 \%-2 \%)$ and sodium phosphate $(0 \%-0.5 \%)$ addition on the microstructure, sensory characteristics, and free amino acid content of beef gels.

Materials and Methods: Meat batters were prepared from minced beef with the addition of various concentrations of sodium chloride $(\mathrm{NaCl})(0 \%-2 \%)$ and sodium pyrophosphate (SPP) (0\%-0.5\%) and subjected to HHP treatment at 0.1-200 MPa for $10 \mathrm{~min}$ using a high-pressure food processor (Dr. CHEF, Kobe Steel, LTD, Japan). After that, the heat treatment was carried out at $80^{\circ} \mathrm{C}$ for $30 \mathrm{~min}$ and, finally, cooled down with ice water to the temperature at the center $-20^{\circ} \mathrm{C}$. Sensory evaluation was conducted by students and staff of Niigata University (an untrained panel of 11-13 healthy men and women in their twenties) using the Scheffe paired comparison method. Participants were asked to assess the softness, juiciness, no residual taste, easy to swallow, cohesiveness, moistness, springiness (elasticity), pleasant odor, and pleasant taste of thermal beef gels on a 7 -point scale from $-3 \sim+3$. Friedman's test was used to compare the significant differences between the score for each evaluation item at the $5 \%$ level. The microstructure of thermal beef gels was examined using a scanning electron microscopy (JSM-6510LA, JEOL, LTD, Japan). The free amino acid content was analyzed using an amino acid analyzer (JLC-500/V, JEOL, LTD, Japan). A confidence level of $5 \%$ was used to compare significant differences among means using Student $t$ test.

Results: A large number of muscle fiber fragmentations and coarse protein aggregates were observed in the unpressurized beef gels containing $0 \%$ sodium chloride and/or $0 \%$ sodium phosphate (Figure 1a). On the other hand, the beef gels treated by high pressure at $150 \mathrm{MPa}$ contained many solubilized filaments that formed filamentous network structure (Figure 1b). Compared to the control beef gel containing $2 \% \mathrm{NaCl}+0.5 \%$ SPP (Figure 1c), the low-salt and/or lowphosphate beef gels treated at $150 \mathrm{MPa}$ contained an overlapping filamentous network that formed a denser structure with an increased number of small cavities for water retention (Figure 1d-1e). Sensory evaluation revealed that irrespective of the sodium chloride and/or sodium phosphate content, the sensory characteristics of beef gels were improved by HHP treatment at $150-200 \mathrm{MPa}$, compared to the unpressurized beef gels. Pressurized beef gels received high scores for the "Juiciness," "Easy to swallow," "Cohesiveness," "Springiness," and "Pleasant tasty" items $(P<0.05)$. HHP treatment at $150 \mathrm{MPa}$ significantly increased the cohesiveness and springiness (elasticity) while decreasing the softness in the low-salt and/or low-phosphate beef gels $(P<0.05)$. The panelists reported that the texture of pressurized beef gels was firmer, more elastic, and more pleasant to bite. The total free amino acid content (Asp, Thr, Ser, Glu, Gln, Gly, Ala, Val, Met, Ile, Leu, Trp, Phe, His, Lys, Arg) in low-salt and

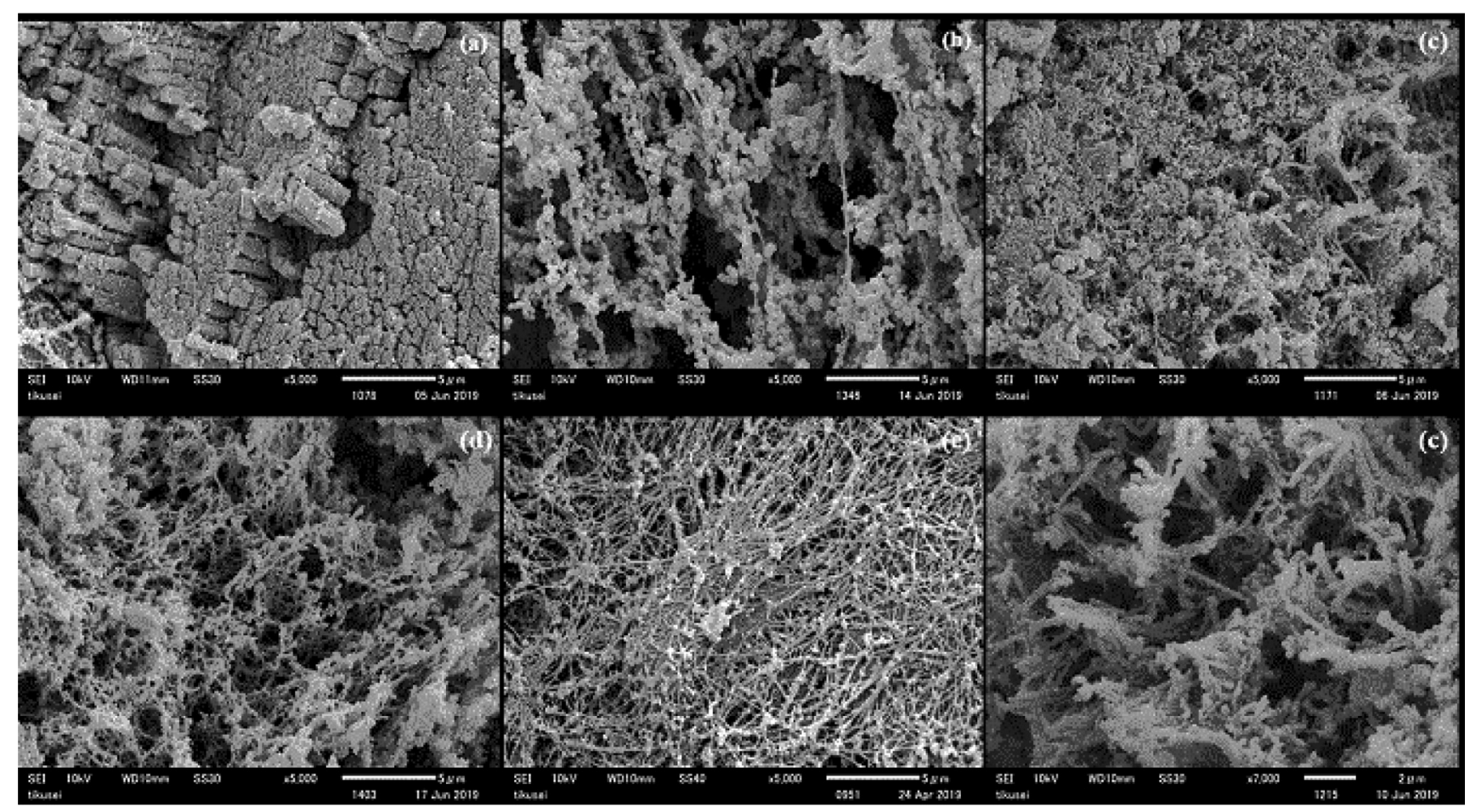

Figure 1. SEM of gel microstructure. (a) beef gel, $0 \% \mathrm{NaCl} / \mathrm{SPP}, 0.1 \mathrm{MPa}$; (b) beef gel, $0 \% \mathrm{NaCl} / \mathrm{SPP}, 150 \mathrm{MPa}$; (c) beef gel, $2 \% \mathrm{NaCl}+0.5 \% \mathrm{SPP}$, $0.1 \mathrm{MPa}$; (d) beef gel, $1 \% \mathrm{NaCl}+0.5 \% \mathrm{SPP}, 150 \mathrm{MPa}$; (e) beef gel, $1 \% \mathrm{NaCl}, 150 \mathrm{MPa}$. 
low-phosphate beef gels increased after HHP treatment at $150-200 \mathrm{MPa}$, compared to non-pressurized beef gels $(P<0.05)$.

Conclusion: These results were consistent with the results of the physicochemical parameters in our previous study and showed that HHP treatment of $150 \mathrm{MPa}$ for $10 \mathrm{~min}$ and low-salt concentration in combination allowed us to produce low-salt and/or low-phosphate meat gels with superior textural and organoleptic properties.

Keywords: high hydrostatic pressure, low-phosphate meat products, low-salt meat products, myofibrillar proteins

\section{6 - EFFECT OF NATURAL \\ ANTIMICROBIALS AGAINST CLOSTRIDIUM PERFRINGENS OUTGROWTH DURING COOLING OF TURKEY BREAST}

K. Peterson ${ }^{1 *}$, E. Boyle ${ }^{2}$, K. Myers ${ }^{1}$, T. O'Quinn ${ }^{2}$, and J. Cannon ${ }^{1},{ }^{1}$ Research and Development, Hormel Foods, Austin, TX, USA, ${ }^{2}$ Animal Sciences \& Industry, Kansas State University, Manhattan, KS, USA, *kihanson@ hormel.com

Objectives: The study objective was to investigate the efficacy of 2 natural antimicrobials in uncured deli-style turkey breast meat on outgrowth of Clostridium perfringens during chilling and consumer acceptance.

Materials and Methods: Four treatments of low-sodium, deli-style turkey breast products with standardized levels of $1.5 \%$ salt and $1 \%$ sugar were prepared. The treatments included a control, 1.0\% fruit/spice extract (FS) (Natpre T-10 Plus S, Prosur, Inc., Naperville, IL), 1.0\% dried vinegar only (DV) (Durafresh 2016, Kerry Ingredients, Beloit, WI), and $1.0 \%$ fruit/spice extract and $1.0 \%$ dried vinegar (FS \& DV). Treatments were inoculated with a 3-strain mixture of C. perfringens spores (ATCC 13124, ATCC 12915, and ATCC 12916) to target an inoculation level of 2.0-log

Table 1. Effect of treatment by hour interaction on Clostridium perfringens population means on uncured turkey breast during 15-hour cooling cycle.

\begin{tabular}{llccc}
\hline \hline \multicolumn{5}{c}{ Hours } \\
\hline Treatment $^{1}$ & 0 & 5 & 10 & 15 \\
\hline Control & $1.92^{\text {gfh }}$ & $5.39^{\mathrm{b}}$ & $6.42^{\mathrm{a}}$ & $6.37^{\mathrm{a}}$ \\
FS & $1.89^{\text {gfh }}$ & $1.56^{\text {ih }}$ & $233^{\text {ed }}$ & $1.96^{\text {gh }}$ \\
DY & $2.10^{\text {ef }}$ & $2.54^{\mathrm{d}}$ & $3.10^{\mathrm{c}}$ & $2.91^{\mathrm{c}}$ \\
FS \& DV & $2.06^{\text {ef }}$ & $1.60^{\text {gih }}$ & $1.59^{\text {gih }}$ & $1.39^{\mathrm{i}}$ \\
SEM & 0.1420 & 0.1420 & 0.1420 & 0.1420 \\
\hline
\end{tabular}

${ }^{1} \mathrm{FS}$ - fruit and spice extract. DV - dried vinegar

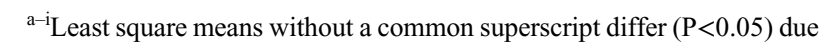
to treatment $\mathrm{x}$ hour interaction.
$\mathrm{CFU} / \mathrm{g}$. Individual 11 -g portions were vacuum packaged, cooked to $71^{\circ} \mathrm{C}$, and chilled from $54.4^{\circ} \mathrm{C}$ to $26.7^{\circ} \mathrm{C}$ in $5 \mathrm{~h}$ and from $26.7^{\circ} \mathrm{C}$ to $7.2^{\circ} \mathrm{C}$ in an additional $10 \mathrm{~h}$. Triplicate samples were analyzed for growth of $C$. perfringens after heat shock and at $0,5,10$, and $15 \mathrm{~h}$ during the cooling cycle by plating on tryptose-sulfite-cycloserine agar. The study was replicated 3 times. Consumers $(n=96)$ evaluated 4 uninoculated samples (1/treatment) in random order and recorded ratings on an electronic tablet (Model $5709 \mathrm{HP}$ Stream 7; Hewlett-Packard, Palo Alto, CA) using a digital survey (Qualtrics Software Version 2417833, Provo, UT). Panelists were asked to rate each sample for traits of appearance, texture, flavor, aftertaste, and overall liking on $100-\mathrm{mm}$ line scale. Anchors were located at $0 \mathrm{~mm}$ and $100 \mathrm{~mm}$, with $0 \mathrm{~mm}$ labeled as dislike extremely and $100 \mathrm{~mm}$ labeled as like extremely. Panelists also evaluated each trait as either acceptable or unacceptable.

Results: There was a more than 4-log outgrowth of $C$. perfringens $(P<0.05)$ in the control treatment compared to treatments containing antimicrobials after $15 \mathrm{~h}$ of cooling. FS \& DV inhibited outgrowth by nearly $0.6-\log$ than FS and $1.5-\log$ than DV $(P<0.05)$. Treatments did not affect $(P>$ 0.05 ) consumer liking for palatability ratings of appearance, texture, flavor, aftertaste, and overall liking. Consumers did indicate that FS and FS \& DV were more acceptable $(P<$ 0.05 ) for flavor and expectation acceptability over other treatments.

Conclusion: There is a synergistic effect against the outgrowth of $C$. perfringens when using FS \& DV together, and the addition of these ingredients did not diminish the acceptability of the final product for consumers.

Keywords: Clostridium perfringens, natural, turkey

\section{7 - IMPROVEMENT OF PROTEIN FUNCTIONALITY AND GEL-FORMING ABILITY IN FAT-REDUCED PORK HOT DOGS USING HOT-BONING AND COLD-BATTER MINCING TECHNOLOGY}

C. Naylor ${ }^{1 *}$, M. P. Wonderly ${ }^{1}$, H. C. Lee ${ }^{1}$, S. Pokharel ${ }^{1}$, and I. Kang ${ }^{1},{ }^{1}$ Animal Science, California Polytechnic State University San Luis Obispo, San Luis Obispo, CA, USA, *ctnaylor@calpoly.edu

Objectives: Cold-batter mixing of meat is an emerging technology that can be used to improve protein functionality and gel-forming ability for fat reduction in pork hot dogs. When mixing meat batters at near-freezing temperatures, it is postulated that internal residues of hydrophobic protein are subtly twisted outward and exposed to the surface during the cold mixing, thereby improving protein functionality and gel-forming ability. The purpose of this research was to generate low-fat hot dogs without textural quality loss using the 
cold-batter mixing technology of hot-boned (HB; or prerigor)/crust-frozen muscle.

Materials and Methods: Ham muscles of eviscerated pork carcasses were subjected to either $\mathrm{HB}$ /crust-freezeair-chilling or chill-boned (CB)/crust-freeze-air-chilling. The muscles were then minced in a bowl chopper for 9 min for control batters using $2 \%$ salt, $2 \%$ starch, $20 \%$ backfat, and $16 \%$ ice, whereas low-fat batters were similarly minced using the same ingredients except using $0 \%$ backfat and $36 \%$ ice. Protein solubility was determined using the method of centrifuge force and filtration. Sulphydryl content was measured using Ellman's reagent and molar extinction coefficient. Data in 4 replications were evaluated by oneway analysis of variance, using the PASW 18 statistics program and a completely randomized design. A post hoc analysis was performed using Duncan's multiple range test to evaluate difference among treatments at $P<0.05$.

Results: During cold-batter mincing, more protein was solubilized from HB batters than CB batters, regardless of fat content. After mincing, the values of surface reactive and total sulfhydryl contents in HB batters were lower than $\mathrm{CB}$ batters, indicating that the proteins of $\mathrm{HB}$ gels were less denatured than those of CB batters. During batter heating, higher storage modulus (G') and higher loss modulus (G') were observed in $\mathrm{HB}$ batters than $\mathrm{CB}$ batters up to $60^{\circ} \mathrm{C}$, except the $\mathrm{G}^{\prime}$ of $\mathrm{CB}$ control-fat gel after $60^{\circ} \mathrm{C}$. These results were expected due to more extracted protein, less protein denaturation, and more protein-protein interaction in $\mathrm{HB}$ batters than $\mathrm{CB}$ batters.

Conclusion: Based on these results, the combination of $\mathrm{HB} /$ crust-freeze-air-chilling and cold-batter mincing technologies can produce low-fat hot dogs without textural quality loss via the improvement of protein extraction, protein functionality, and gel-forming ability over the cold-batter mincing of $\mathrm{CB}$ muscle $(P<0.05)$.

Keywords: crust freezing, cold-batter mincing, hot-boning, low fat, protein functionality

\section{8 - YIELD ENHANCEMENT IN COOKED BEEF PATTIES USING CULTURED CANE SUGAR}

M. Matney ${ }^{1 *}$, G. Mccoy ${ }^{1}$, D. Unruh ${ }^{1}$, S. Lasuer ${ }^{1}$, T. Rourke ${ }^{2}$, and S. Kumar ${ }^{2},{ }^{1} R D A$, Corbion Purac America, Lenexa, KS, USA, ${ }^{2}$ Business Development, Corbion Piracy America, Lenexa, KS, USA, *garrett.mccoy@corbion.com

Objectives: The objective of this study was to evaluate the efficacy of Verdad $\AA$ Powder N20, a novel cultured cane sugar (CCS) product, as a yield enhancement solution in fully cooked beef patties.

Materials and Methods: Ground beef at 78\% lean was mixed with $1.0 \%$ salt and varying levels of CCS outlined in Table 1. The control treatment contained no yield
Table 1: Cook yield for varying inclusion levels of cultured cane sugar (CCS)

\begin{tabular}{lc}
\hline \hline Treatment & Cook Yield \\
\hline Control & $61.88 \%$ \\
$1.0 \%$ CCS & $65.44 \%$ \\
$2.0 \%$ CCS & $69.88 \%$ \\
\hline
\end{tabular}

enhancement ingredients, whereas the treatments included the addition of either $1.0 \%$ CCS or $2.0 \%$ CCS. Individual treatments were mixed for $10 \mathrm{~min}$ and formed into fifteen 150 -g patties. Patties were then cooked in a preheated steam-injected convection oven at $260^{\circ} \mathrm{C}$ and $20 \%$ humidity until internal temperature of the patties reached $73.8^{\circ} \mathrm{C}$. Patties were then cooled to $4.4^{\circ} \mathrm{C}$, and cook yields were measured for each treatment. All treatments were manufactured and cooked in duplicate. Cook yield was calculated as a percentage of original weight.

Results: Table 1 illustrates the treatment structure and cook yields for this study. All of the treatments with the CCS inclusion resulted in a cook yield increase $(P<0.05)$ compared to the control treatment. Increasing the inclusion rate of CCS from $1.0 \%$ to $2.0 \%$ led to further increase $(P<$ $0.05)$ in cook yield. The $2.0 \%$ CCS treatment indicated an $8.0 \%$ yield increase compared to the control.

Conclusion: This study validates the efficacy of Varda $\mathbb{R}$ Powder N20 (CCS) as a clean-label yield enhancement solution for the meat industry.

Keywords: beef, ground beef, yield enhancement

\section{9 - A VINEGAR-BASED ANTIMICROBIAL SOLUTION EXTENDS THE SHELF LIFE OF VACUUM-PACKAGED RAW CHICKEN PARTS}

S. Lasuer ${ }^{1}$, D. Unruh ${ }^{1 *}$, M. Matney ${ }^{1}$, G. Mccoy ${ }^{1}$, N. Sevart ${ }^{2}$, R. Ames ${ }^{2}$, and S. Kumar ${ }^{2},{ }^{1} R D A$, Corbion Purac America, Lenexa, KS, USA, ${ }^{2}$ Business Development, Corbion Purac America, Lenexa, KS, USA, * garrett.mccoy@corbion.com

Objectives: The study objective was to quantify the shelf life extension of freshly harvested, raw chicken parts following application of $\operatorname{Verdad} \circledast \mathrm{N} 100$ (vinegar-based solution) and subsequent vacuum packaging. Poultry shelf life is determined by the outgrowth of spoilage bacteria with or without the presence of undesirable organoleptic properties, for example, presence of off-aroma or textural changes.

Materials and Methods: Chicken parts (skin-on drumsticks and boneless skinless breasts) were obtained from a processing facility prior to any parts-level antimicrobial intervention. Approximately $24 \mathrm{~h}$ postmortem, samples were fully submerged (by part type in fresh treatment solutions 
each time) in one of 2 treatments (10\% concentration vinegar-based solution and 5\% concentration vinegar-based solution) with agitation for $20 \mathrm{~s}$, or they were not subjected to dip (negative control). Treated samples were permitted to drip dry for $5 \mathrm{~min}$. Samples were packaged into 5-mil bags and vacuum sealed ( 3 breasts per bag; 4 drums per bag) and were stored at $2.2^{\circ} \mathrm{C}$ throughout the study. Aerobic plate counts (APC) and lactic acid bacteria counts were generated on days $0,7,14,18$, and 21 . At each date, a breast and drum (composing a sample) were analyzed in triplicate. Samples were transferred to a sterile poultry rinse bag to which $100 \mathrm{~mL}$ of neutralizing buffer was added, and the bag was thoroughly shaken. Rinsate was appropriately diluted in Butterfield's buffer; APC bacteria were plated on $3 \mathrm{M}^{\mathrm{TM}} \mathrm{APC}$ Petrifilm (incubated $48 \mathrm{~h}$ at $35^{\circ} \mathrm{C}$ ), and lactic acid bacteria were plated on De Man, Rogosa, and Sharpe agar (incubated $48 \mathrm{~h}$ at $30^{\circ} \mathrm{C}$ ). Organoleptic qualities (aroma, texture, color, purge loss) were measured throughout the study by subjective panel analysis (aroma and texture) and instrumental measurement (color and purge loss). The experiment was conducted as a completely randomized design, and statistical analysis was performed using Fisher's least significant difference method in Minitab, with a significance level of $P=0.05$.

Results: Using a spoilage threshold of $6 \log$ CFU/ sample, the shelf life of breasts and drums was extended by application of vinegar solution treatment at $10 \%$ and $5 \%$ concentration. In drums, shelf life was extended by as many as $7 \mathrm{~d}$; in breasts, shelf life was extended by as many as $10 \mathrm{~d}$ (Table 1). On days 7, 14, and 18, populations were lower $(P<0.05)$ in treated samples compared to control (no dip). Therefore, when examining shelf life of raw poultry using microbial populations, the application of vinegar solution treatment extends the shelf life significantly. No difference was noted in the aroma of the samples until day 21 , texture passed panel evaluation as acceptable on all dates, and $L^{*} a^{*} b^{*}$ colorimetric values were similar among treatments throughout the shelf life. Purge loss was higher in treated and nontreated chicken breasts compared to drumsticks from day 7 onward. By day 21, purge loss ranged from a low of $2.35 \%$ in control to a high of $3.34 \%$ in $5 \%$ vinegar solution treatment for breasts; in drumsticks, purge loss

Table 1. Days to reach $6 \log$ CFU/sample by chicken part and media type (aerobic plate count, APC; lactic acid bacteria, LAB) following treatment application and vacuum packaging

\begin{tabular}{lccccc}
\hline \hline & \multicolumn{5}{c}{ Days } \\
\cline { 2 - 5 } & \multicolumn{2}{c}{ Drums } & & \multicolumn{2}{c}{ Breasts } \\
\cline { 2 - 3 } \cline { 5 - 6 } Treatment & APC & LAB & APC & LAB \\
\hline Control (no dip) & 14 & 12 & & 7 & 7 \\
Vinegar-based solution (10\% concentration) & 19 & 19 & 17 & 14 \\
Vinegar-based solution (5\% concentration) & 16 & 16 & & 15 & 14 \\
\hline
\end{tabular}

ranged from $0.84 \%$ in control and $5 \%$ vinegar solution treatment to $1.38 \%$ in $10 \%$ vinegar solution treatment.

Conclusion: The application of $\operatorname{Verdad} \AA \mathrm{N} 100$ on raw chicken parts (drums and breasts) extended product shelf life by $5-10 \mathrm{~d}(10 \%$ concentration) and $2-8 \mathrm{~d}(5 \%$ concentration). Use of this treatment solution may serve as a complementary processing step to further extend poultry shelf life.

Keywords: antimicrobial, fresh poultry, shelf life

\section{0 - INCLUSION OF COFFEE BAGASSE EXTRACT ENHANCES TOTAL ANTIOXIDANT ACTIVITY IN PORK MEAT}

M. Estrada-Alanis ${ }^{1}$, R. D. Vargas-Sánchez ${ }^{2}$, B. D. M. Torres-Martínez ${ }^{2}$, G. R. Torrescano-Urrutia ${ }^{2}$, N. HuertaLeidenz $^{3 *}$, A. Sánchez-Escalante ${ }^{2},{ }^{1}$ Facultad de Medicina Veterinaria, Universidad Autónoma de Nayarit, Tepic, Mexico, ${ }^{2}$ Coordinación de Alimentos de Origen Animal, Centro de Investigación en Alimentación y Desarrollo, A.C., Hermosillo, Mexico, ${ }^{3}$ Department of Animal \& Food Sciences, Texas Tech University, Lubbock, TX, USA, *armida-sanchez@ciad.mx

Objectives: The study objective was to evaluate the effect of adding extract of coffee bagasse (i.e., coffee spent grounds) on total antioxidant activity in pork patties.

Materials and Methods: Fresh ground pork (M. semimembranosus, $48 \mathrm{~h}$ postmortem) and pork backfat were procured from a local processor. The lean and fat ingredients were homogenized ( $20 \%$ fat in the final formulation) with water $(5 \%)$ and salt $(1.5 \%)$ for patties' elaboration (40 g each). In 2 replications, 6 patties were allocated to each of 3 treatments $(N=36)$ as follows: untreated patties (Control), patties added with coffee bagasse at $0.05 \%$ (T1), and patties added with butylated hydroxytoluene at $0.01 \%$ (T2). Samples were placed in polypropylene trays and wrapped with polyvinyl chloride film $\left(17,400 \mathrm{~cm}^{3} \mathrm{O}_{2} / \mathrm{m}^{2} /\right.$ $24 \mathrm{~h}$ at $\left.23^{\circ} \mathrm{C}\right)$ and stored $\left(4^{\circ} \mathrm{C} / 9 \mathrm{~d} /\right.$ under darkness $)$. Each sampling day, patties were subjected to an aqueous extraction by ultrasound-assisted method to yield the pork meat juice. The meat juice total antioxidant activity was determined by total phenolic content (TPC), free-radical scavenging activity (FRSA), and reducing power activity (RPA) methods. Data were subjected to analysis of variance, and means were separated by Tukey's test $(P<0.05)$.

Results: As shown in Figure 1, TPC, FRSA, and RPA were significantly affected by treatment and storage time $(P<0.05)$. TPC values decreased during storage, and at day 9 , higher TPC values $(P<0.05)$ resulted from patties treated with extract of coffee bagasse (T1) and butylated hydroxytoluene (T2). All FRSA values were reduced during storage, and at day 9, patties from T1 showed the highest antiradical activity $(P<0.05)$. Also, RPA was reduced $(P<0.05)$ during storage in T1 patties, while no significant 

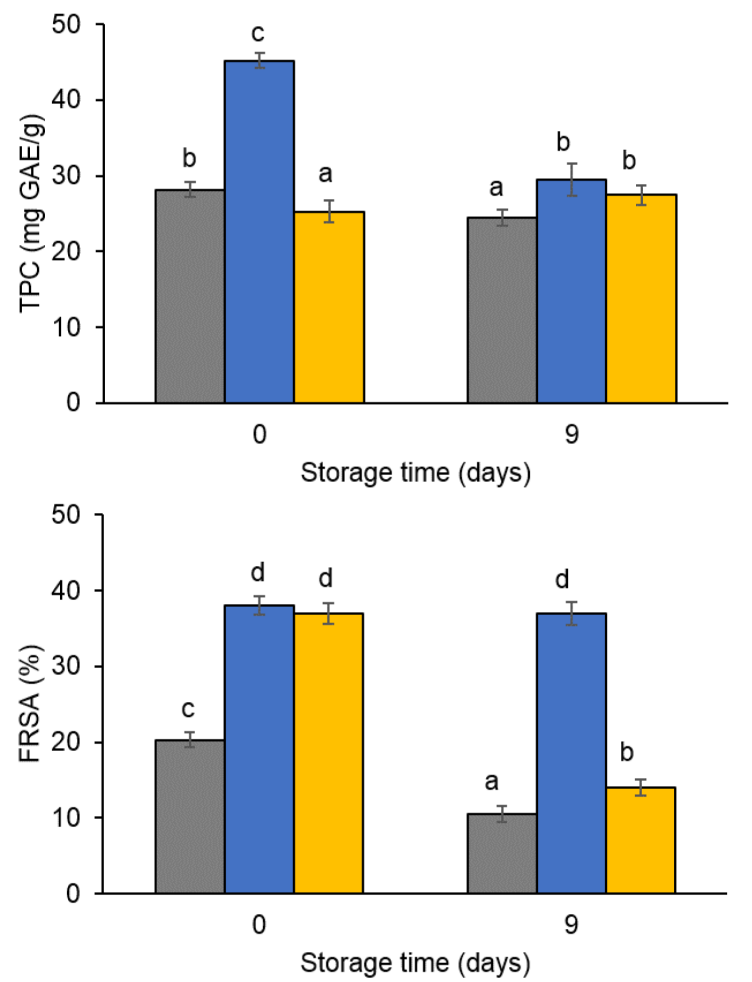

(B)

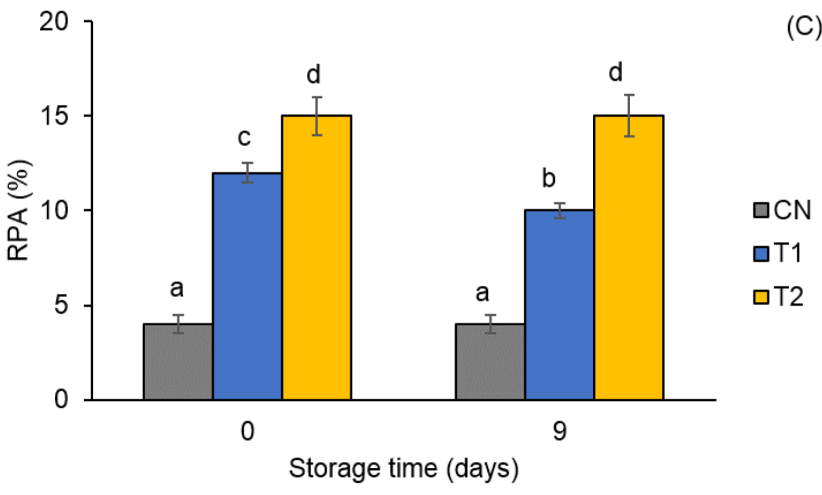

Figure 1. Image

changes were detected in patties from the Control and $\mathrm{T} 2$ groups $(P>0.05)$.

Conclusion: Coffee bagasse extract is a valuable source of antioxidants, and their addition in pork meat increases the presence of phenolic compounds, enhancing their antioxidant properties (FRSA and RPA) during storage.

Keywords: antioxidant, coffee residues, polyphenols, pork

\section{1 - REDUCING MOISTURE OF VACUUM AGED BEEF USING MEAT PADS}

D. P. Candido ${ }^{1}$, A. P. M. de Paula ${ }^{1}$, and S. B. Pflanzer ${ }^{*}$, ${ }^{1}$ Department of Food Technology, State University of Campinas, Campinas, Brazil, *spflanzer@gmail.com
Objectives: Aging is an important process to improve tenderness. Dry aging has been getting attention, mainly due to its improvement in flavor. This improvement may be associated with water loss and possible concentration of flavor compounds. However, in addition to moisture loss, there is formation of a dry surface, which must be removed, reducing the yield of this process. Vacuum aging has a higher yield; however, it does not guarantee the characteristic of flavor concentration from dry-aged meat. The aim of this work was to investigate how the use of moisture absorbers (meat pads) could act on the water loss dynamics of vacuumpacked meat.

Materials and Methods: A total of 6 pieces of strip loin (Longissimus lumborum) was used for this experiment. Each piece was divided in half, with one portion destined for the control treatment (traditional vacuum) and the other half destined for the treatment using moisture absorbers (meat pads), also vacuum-packed. For the latter, the loins were completely wrapped with absorbers (MG175S; McAirlaid's, Germany) before vacuum packaging. Before aging, a steak of each piece was used to determine meat quality characteristics. Before vacuum packaging, the loins portions were weighed to estimate purge loss. Aging was carried out at $2^{\circ} \mathrm{C} \pm 1{ }^{\circ} \mathrm{C}$ for $33 \mathrm{~d}$. After aging, the packages were opened, and the loins were weighed. Samples from the superficial region of aged meat, $\sim 3 \mathrm{~mm}$ thick, were first collected to characterize the meat surface. Then the loins were cut into 2.54-cm-thick steaks for the analysis of the central region. Analysis of $\mathrm{pH}$, water activity $\left(\mathrm{a}_{\mathrm{w}}\right)$, and moisture of the superficial and central regions was performed. A steak from the central region was also used for color stability analysis during display storage for $12 \mathrm{~d}$. The $\mathrm{pH}, \mathrm{a}_{\mathrm{w}}$, and moisture data were analyzed as a $2 \times 2$ factorial analysis of variance ( 2 treatment and 2 positions) and the color data as repeated measures analysis of variance. The averages were tested by the Tukey test at the 5\% level.

Results: The fresh, not aged, samples showed a $\mathrm{pH}$ of $5.58 \pm 0.17$, water activity of $0.9940 \pm 0.0016$, and moisture of $75.39 \% \pm 0.93 \%$. The samples without meat pads had a lower $(P<0.05)$ water loss $(2.26 \% \pm 0.76 \%)$ compared to samples with meat pads $(7.02 \% \pm 1.97 \%)$. There was no significant interaction $(P>0.05)$ between treatments and positions for the variables $\mathrm{pH}, \mathrm{a}_{\mathrm{w}}$, and moisture. The use of meat pads did not affect $(P>0.05)$ the $\mathrm{pH}(5.68 \pm 0.20)$ and the water activity $(0.9916 \pm 0.0008)$. However, samples with meat pads $(73.80 \% \pm 1.04 \%)$ had lower $(P<0.05)$ moisture content than samples without meat pads $(74.71 \% \pm 0.77 \%)$. There was no effect of position on $\mathrm{pH}$ and $\mathrm{a}_{\mathrm{w}}$ values $(P>0.05)$. A trend $(P=0.09)$ of lower moisture for the samples analyzed on the surface $(73.99 \% \pm 1.12 \%)$ was verified, in contrast with those analyzed in the central region $(74.62 \% \pm 0.87 \%)$. The color parameters $L^{*}(40.49 \pm$ $3.50), a^{*}(18.02 \pm 0.57)$, and $b^{*}(16.89 \pm 3.04)$ were not affected by the use of meat pads $(P>0.05)$. The $L^{*}$ coordinate was not affected by time $(P>0.05)$, while $a^{*}$ and $b^{*}$ 
coordinates decreased with the days of exposure $(P<0.05)$, mainly after day 8 of exposure.

Conclusion: The results indicated that the use of meat pads/moisture absorbers in vacuum-packed meats can be a strategy for aging processes where the objective is to reduce the moisture of the product, without drastically affecting the surface of the meat, reducing the process losses. Otherwise, further studies on sensory analysis and maximization strategies for water loss are suggested for better results.

Keywords: aging, moisture absorbers, water loss

\section{Meat and Poultry Quality}

\section{2 - EFFECTS OF GENETIC STRAIN OF BROILERS ON PROTEOME PROFILES OF NORMAL AND WOODY BREAST MUSCLE}

K. V. To ${ }^{1 *}$, T. Jarvis ${ }^{1}$, M. W. Schilling ${ }^{1}$, X. Zhang ${ }^{1}$, Y. L. Campbel1 $^{1}$, S. P. Suman ${ }^{2}$, J. D. Hendrix ${ }^{1}$, W. Zhai ${ }^{3}$, S. $\mathrm{Li}^{2}$, J. Chen ${ }^{4}$, and H. Zhu ${ }^{4},{ }^{1}$ Food Science, Nutrition and Health Promotion, Mississippi State University, Starkville, MS, USA, ${ }^{2}$ Animal and Food Sciences, University of Kentucky, Lexington, KY, USA, ${ }^{3}$ Poultry Science, Mississippi State University, Starkville, MS, USA, ${ }^{4}$ Proteomics Core Facility, University of Kentucky, Lexington, KY, USA, *kvt34@msstate.edu

Objectives: Wooden or woody breast (WB) is a myopathy in the Pectoralis major of fast-growing broilers and downgraded due to undesirable appearance and impaired nutritional quality. The objective of this study was to evaluate differences in growth and proteomes of normal (NB) and WB among 5 genetic broiler strains.

Materials and Methods: For each of 5 strains, 128 chicks were randomly assigned to 8 pens ( 1 pen/block) and raised at the Mississippi State University Poultry Farm according to an approved animal welfare protocol (IACUC-16-542). After $8 \mathrm{wk}, 4 \mathrm{birds} / \mathrm{pen}$ were randomly selected and evaluated for WB at $24 \mathrm{~h}$ postmortem according to Tijare et al. (2006) where breast samples were grouped into NB and WB. Completely randomized designs were used to evaluate the differences among 5 genetic strains with respect to the body, carcass, and breast weights for both $\mathrm{NB}$ and WB groups. Tukey's Honestly Significant Difference test was used to separate treatment means among strains $(P<$ $0.05)$. A two-tailed $t$ test was used to evaluate the difference between NB and WB within each strain $(P<0.05)$. A group of live male birds (1 bird/pen) were also evaluated for WB myopathy by manual palpation. Within each strain, 4 birds with NB and 4 birds with WB were selected. Next, the birds' cranial breast muscles were collected and snap-frozen in liquid nitrogen. The whole muscle proteomes (3 samples/ strain, 2 gels/sample) were analyzed using two-dimensional gel electrophoresis and liquid chromatography-tandem mass spectrometry for protein spots that exhibited a fold change of 2.0 threshold in spot intensity $(P<0.05)$.

Results: Birds from strains 1, 3, and 5 in the WB group were heavier than those in the NB group $(P<0.05)$. Although there were no differences in breast weights within WB meat $(P>0.05)$, WB meat from all strains was heavier than breast meat from NB $(P<0.05)$. Within WB, strain 5 had a greater breast percentage than strains 1,3 , and 4 $(P<0.05)$. WB from strains $2-5$ had a greater breast percentage than that of NB $(P<0.05)$. Within NB-when compared with strain 5-alpha-actin, ubiquitin carboxyl-terminal hydrolase, cofilin-2 muscle isoform, and voltage-dependent anion-selective channel protein 2 were underexpressed $(P<$ $0.05)$ in strain 2 ; nucleoside diphosphate kinase and voltagedependent anion-selective channel protein 2 were underexpressed $(P<0.05)$ in strain 3; and myozenin-1 isoform X2 was underexpressed $(P<0.05)$ in strain 4 . Within WBwhen compared with strain 5-alpha-actinin-2 and gelsolin were overexpressed $(P<0.05)$ in strain 1 ; elongation factor 2 and myozenin-3 were underexpressed $(P<0.05)$ in strain 1; adenosylhomocysteinase and LIM domain-binding protein 3 were underexpressed $(P<0.05)$ in strain 2 ; and myozenin-3 and LIM domain-binding protein 3 were underexpressed $(P<0.05)$ in strain 3 . Desmin, serum albumin precursor, annexin A5, actin-related protein 3, ubiquitin carboxyl-terminal hydrolase, and troponin $\mathrm{T}$ were underexpressed $(P<0.05)$, while mitochondrial $60 \mathrm{kDa}$ heat shock protein and myosin-binding protein $\mathrm{H}$ were overexpressed $(P<0.05)$ in strain 4 compared to strain 5 .

Conclusion: Protein profiles of broiler breast were affected by genetic strains and the presence of WB myopathy. This helps us understand how breast muscle proteins affect the growth performance of broiler strains and further elucidates the etiology of WB development in different commercially available genetic strains.

Keywords: genetics, poultry, proteomics, woody breast myopathy

\section{3 - EFFECT OF EARLY POSTMORTEM OXIDATIVE STRESS ON MITOCHONDRIAL REDOX STABILITY AND APOPTOSIS OF TWO PORCINE MUSCLES}

\author{
M. Abdelhaseib ${ }^{1 *}$, J. Zhang ${ }^{1}$, and Y. H. B. Kim ${ }^{1},{ }^{1}$ Animal \\ Science, Purdue University, West Lafayette, IN, USA, \\ *maha@purdue.edu
}

Objectives: Apoptosis has been suggested as a novel mechanism affecting meat quality attributes. Oxygendeprivation-induced apoptosis is dependent on the intrinsic mitochondrial pathway, which is closely associated with 
metabolic status and mitochondria dysfunction of the muscle cells. Generation of reactive oxygen species (ROS) under oxidative stress could lead to further mitochondrial damage. However, the exact mechanisms of ROS-mediated mitochondrial apoptosis remain largely unknown. The objective of this study was to evaluate effects of oxidative stress on early postmortem muscles and its impacts on mitochondrial functionalities and apoptosis of 2 porcine muscles.

Materials and Methods: Longissimus dorsi (LD) and Psoas major (PM) muscles were excised from 6 pork carcasses at $2 \mathrm{~h}$ postmortem. Each muscle sample was subdivided into 2 fractions and allocated to 2 treatments as follows; oxidizing condition as being immersed in $20 \mathrm{mM}$ $\mathrm{H}_{2} \mathrm{O}_{2}$ for 60 min or control as being treated with distilled water. Upon treatments, all samples were vacuum packaged and stored for $0,6,24$, and $48 \mathrm{~h}$ at $4^{\circ} \mathrm{C}$. Biochemical attributes, including mitochondrial membrane permeability, mitochondrial lipid peroxidation, mitochondrial $\mathrm{Ca}^{2+}$, and cytochrome c redox stability, were determined at each storage time. Data were analyzed by SAS (SAS Institute Inc., Cary, NC) to compare the traits across muscle types and treatments over time. Least-squares means were separated by least significant differences.

Results: Overall, the mitochondrial membrane permeability was increased at $0-48 \mathrm{~h}$ post treatment in both muscles. PM had a higher membrane permeability than $\mathrm{LD}$ at $0-24 \mathrm{~h}(P<0.05)$. The effect of oxidative stress on the mitochondrial membrane permeability was only significant in LD at 6-24 h. Oxidative stress resulted in a significant increase in mitochondrial lipid peroxidation and $\mathrm{Ca}^{2+}$ flux in both muscles. However, PM had a greater mitochondrial lipid peroxidation and $\mathrm{Ca}^{2+}$, compared to $\mathrm{LD}(P<0.05)$. While the cytochrome $\mathrm{c}$ redox stability decreased in both muscles, PM had a greater cytochrome $\mathrm{c}$ redox stability than LD $(P<0.05)$. These observations further support the hypothesis that ROS-mediated oxidative stress plays a major role in the activation of mitochondrial apoptosis by influencing important regulators of the mitochondrial apoptotic pathway. These results also imply that oxidative stress could induce the rate and extent of apoptosis process, but it could be muscle specific.

Conclusion: The ROS generated by $\mathrm{H}_{2} \mathrm{O}_{2}$ significantly increased the mitochondrial oxidative stress levels. The results from the present study found that ROS-mediated oxidative stress enhanced the mitochondrial apoptotic pathway indicated by increased mitochondrial membrane permeability, mitochondrial lipid peroxidation, and overload of the production of mitochondrial $\mathrm{Ca}^{2+}$, from early postmortem muscles. However, the rate and extent of response to the given oxidative-stress-induced apoptosis could be muscle specific, where PM showed more ROS-mediated mitochondrial apoptosis compared to LD. Further research looking into the impact of oxidative-stress-mediated apoptosis on proteolytic enzyme activity and subsequent myofibrillar protein degradation is currently underway.
Keywords: apoptosis, pork loins, reactive oxygen species

\section{4 - EFFECT OF COUNTRY OF ORIGIN AND COOKING ENDPOINT TEMPERATURE ON THE FLAVOR PROFILE OF BEEF}

\author{
N. Prieto ${ }^{*}$, J. L. Aalhus ${ }^{1}$, I. L. Larsen ${ }^{1}$, Z. Pietrasik ${ }^{2}$, and N. J. \\ Gaudette $^{2},{ }^{1}$ Lacombe Research and Development Centre, Agri- \\ culture and Agri-Food Canada, Lacombe, Canada, ${ }^{2}$ Food \\ Processing Development Centre, Ministry of Agriculture and \\ Forestry, Leduc, Canada,*nuria.prietobenavides@canada.ca
}

Objectives: The aim of this study was to evaluate the effects of country of origin and cooking endpoint temperature on the flavor profile of beef.

Materials and Methods: Full ribeye primals (Longissimus thoracis) were purchased from Australia $(n=18)$, Canada $(n=16)$, and the US $(n=20)$ to represent Meat Standards Australia Grade 4 star mixed grain, Canadian AAA barley, or USDA Choice corn-finished beef steers, respectively. Upon arrival at the Lacombe Research and Development Centre (Agriculture and Agri-Food Canada, Canada), all ribeyes were aged at $2^{\circ} \mathrm{C}$ until $45 \mathrm{~d}$ to equal the aging time incurred during shipping of the Australian product, and subsequently frozen at $-35^{\circ} \mathrm{C}$ until analyses. Two $2.5-\mathrm{cm}$ steaks from each loin were cut, thawed overnight under refrigeration, and randomly grilled to an internal endpoint temperature of either $63^{\circ} \mathrm{C}$ (rare) or $71^{\circ}$ $\mathrm{C}$ (medium-well). Steaks were presented in a balanced design to a 9-member trained sensory panel to rate the intensity of aromas $(n=19)$, tastes $(n=5)$, and flavors $(n=$ $19)$ using a $15-\mathrm{cm}$ line scale. Flavor profile data were analyzed using the MIXED model procedure of SAS version 9.4 (SAS Institute Inc., Cary, NC), with the main effects of country of origin and endpoint cooking temperature and their interaction in the model, and panel session and assessor and their interactions included as random effects.

Results: Beef identity and buttery flavors were rated higher $(P<0.05)$ in Canadian and US compared to Australian steaks, whereas bitter taste and barnyard, liver-like, and rancid flavors were higher $(P<0.05)$ in Australian than in both Canadian and US steaks. Canadian steaks presented higher $(P<0.05)$ fat-like flavor than Australian and US steaks, whereas brown roasted flavor was higher in US, lower in Australian, and intermediate in Canadian steaks $(P<0.05)$. Steaks cooked to a $63^{\circ} \mathrm{C}$ endpoint presented higher $(P<0.05)$ cruciferous aroma, sour taste, and bloody/serumy and sour/dairy flavors and tended $(P<0.1)$ to have higher metallic flavor than the $71^{\circ} \mathrm{C}$ steaks. Steaks cooked at $71^{\circ} \mathrm{C}$ had higher $(P<0.05)$ sweet taste and beef identity flavor and tended $(P<0.1)$ to have higher buttery flavor and brown roasted aroma and flavor than the $63^{\circ} \mathrm{C}$ steaks. There was only one significant interaction between endpoint cooking temperature and country of origin; the 
$71^{\circ} \mathrm{C}$ treatment significantly $(P<0.05)$ increased the beef identity flavor compared to the $63^{\circ} \mathrm{C}$ in Canadian steaks, but no cooking endpoint temperature effect was observed for this flavor attribute in either US or Australian steaks.

Conclusion: Even after $45 \mathrm{~d}$ of aging, trained panelists were able to discern variations in beef taste/flavor intensities arising from production differences in country of origin. Cooking beef steaks to a low endpoint temperature of $63^{\circ} \mathrm{C}$ resulted in a combination of aromas, tastes, and flavors that may be attributed to limited formation of Maillard reaction products. Increasing endpoint temperature to $71^{\circ} \mathrm{C}$ lengthened the cooking time and contributed to additional flavor development. Nevertheless, the endpoint temperature increasing from $63^{\circ} \mathrm{C}$ to $71{ }^{\circ} \mathrm{C}$ did not enhance umami taste regardless of country of origin, which suggests that higher temperatures/longer cooking times may be required to promote the formation of umami compounds.

Keywords: beef, cooking endpoint temperature, country of origin, flavor

\section{5 - THE RELATIONSHIP BETWEEN THE HINDGUT MICROBIOMES AND CARCASS MERIT IN ANGUS STEERS}

T. Krause ${ }^{1 *}$, J. Lourenco ${ }^{1}$, C. Welch ${ }^{1}$, T. Callaway ${ }^{1}$, and T. D. Pringle ${ }^{1},{ }^{1}$ Department of Animal and Dairy Sciences, University of Georgia, Athens, GA, USA, *trk73598@ uga.edu

Objectives: It is well known that microbial populations in the gastrointestinal tract of cattle contribute protein and energy to meet the host's nutrient requirements. These nutrients go on to influence animal performance and ultimately carcass composition. While the rumen is often accredited with the largest contribution of nutrients to requirements, recent research has suggested that the secondary fermentation occurring in the hindgut may also provide significant levels of nutrients that may explain portions of the variation seen in animal performance. Therefore, variation in carcass value, through the quality and quantity of beef produced, could be partially attributed to variation in the microbial fermentation occurring in the hindgut. The objective of this study was to determine whether dissimilarities in microbial taxa from the cecum and rectum contents of Angus steers could be correlated to differences in carcass lipid accretion in terms of intramuscular and subcutaneous fat deposition.

Materials and Methods: Twenty-four Angus steers were slaughtered to collect cecum contents, feces, longissimus muscle samples, and measurements for the calculation of carcass quality and yield grades. Cecal and fecal microbial DNA extraction and 16S ribosomal RNA gene sequencing were performed to determine microbial relative abundances. Proximate analysis was performed to determine chemical lipid content of longissimus muscle samples. A variety of correlation and regression analyses were performed to investigate the relationships between the cecal and fecal microbiomes and carcass traits.

Results: The relative abundance of the phylum Proteobacteria in the feces was negatively correlated to marbling score $(P=0.009)$ and longissimus lipid content $(P=$ 0.015 ), indicating that lower abundances of bacteria within the phylum Proteobacteria may be linked to greater intramuscular adipose accumulation. The abundance of the family Succinivibrionaceae in the feces was negatively correlated to longissimus lipid content $(P=0.017)$, and within that family, the genus Succinivibrio was negatively correlated to longissimus lipid content $(P=0.018)$. In the feces, the abundance of the genus Ruminococcus was positively correlated to longissimus lipid content $(P=0.031)$. In the cecum, the abundance of the genus Mycoplasma was positively correlated to marbling score $(P=0.029)$. In contrast, the abundance of the genus Methanosphaera in the cecum was negatively correlated to marbling score $(P=0.03)$, suggesting that lower abundances of methane-producing bacteria in the cecum may be linked to greater marbling deposition through reductions in energy wasting in the hindgut. The genus Bulleidia was positively correlated to 12 th rib adjusted backfat thickness $(P=0.002)$ and yield grade $(P=0.019)$, suggesting that greater abundances of bacteria within this genus in the cecum may be linked to increased subcutaneous fat deposition.

Conclusion: No other bacterial taxa in the hindgut were correlated with 12th rib adjusted backfat thickness or yield grade, indicating that the hindgut microbiome may not play a substantial role in subcutaneous fat deposition. The hindgut microbiome may, however, influence intramuscular fat deposition through the fermentation end products of various microbial taxa. Overall, these results suggest that the microbiomes of the cecum and rectum impact adipose accumulation in the carcass via distinct mechanisms due to the lack of overlap in significant taxa.

Keywords: hindgut microbiome, lipid, marbling, subcutaneous fat, yield grade

\section{6 - PALATABILITY ASSESSMENTS OF BEEF TOP LOIN STEAKS PORTIONED BY WEIGHT OR BY THICKNESS SOURCED FROM VARIOUS CARCASS WEIGHT/RIBEYE SIZE COMBINATIONS}

M. K. Foster ${ }^{1}$, K. R. Caldwell ${ }^{1}$, R. R. Kirkpatrick ${ }^{1 *}$, A. N. Arnold $^{1}$, D. B. Griffin ${ }^{1}$, K. B. Gehring ${ }^{1}$, J. W. Savell ${ }^{1}$, ${ }^{1}$ Animal Science, Texas A\&M University, College Station, TX,USA,*j-savell@tamu.edu

Objectives: The study objectives were (1) to determine whether carcass weights and ribeye sizes influence consumer 
acceptance of beef top loin steaks and (2) to determine whether differences exist in palatability between steak portions cut by thickness and those by weight.

Materials and Methods: Beef carcass sides $(n=90)$ were selected to be USDA Choice (Small marbling only) and to meet a $3 \times 3$ treatment scheme of ribeye area (REA) sizes ( 83.9 to $89.8 \mathrm{~cm}^{2}, 90.3$ to $96.1 \mathrm{~cm}^{2}$, and 96.7 to $102.6 \mathrm{~cm}^{2}$ ) and hot carcass weights (HCW) (340.6 to $385 \mathrm{~kg}, 386.0$ to $430.9 \mathrm{~kg}$, and 431.4 to $476.3 \mathrm{~kg}$ ). Beef strip loins were obtained, vacuum packaged, boxed, and shipped to a collaborating purveyor. Subprimals were passed through a Marel $\AA$ intelligent portion cutter (M Series 3000 , Marel $\AA$, Lenexa, KS) intelligent portion cutter to generate steaks with 2 target endpoints: (1) portion thickness of $3.18 \mathrm{~cm}$ and (2) portion weight of $340 \mathrm{~g}$. Steaks were assigned to either consumer sensory panel $(n=360)$ or Warner-Bratzler shear (WBS) force $(n=180)$ determinations, individually vacuum packaged, transported in insulated containers, and flash frozen for $24 \mathrm{~h}$ at $-40^{\circ} \mathrm{C}$ before storage at $-20^{\circ} \mathrm{C}$ until analyses were performed. Steaks were thawed $\left(2^{\circ} \mathrm{C}\right.$ to $\left.4^{\circ} \mathrm{C}\right)$ for approximately $28 \mathrm{~h}$ before being cooked on grated, nonstick electric grills preheated to a surface temperature of $177^{\circ} \mathrm{C} \pm$ $2^{\circ} \mathrm{C}$. Steaks were flipped at internal temperatures of $35^{\circ} \mathrm{C}$ and were removed from the grills at $70^{\circ} \mathrm{C}$. Cooked steaks for WBS force evaluation were placed on metal trays in a single layer, covered with plastic wrap, and stored $\left(2^{\circ} \mathrm{C}\right.$ to $4^{\circ} \mathrm{C}$ ) for approximately 12 to $16 \mathrm{~h}$ before six $1.3-\mathrm{cm}$ cores were removed for WBS force determinations. Steaks for consumer panels (IRB2019-0820M) were held in an AltoShaam oven set at $60^{\circ} \mathrm{C}$ for not more than 20 min before serving to panelists, who scored steak samples on 9-point scales $(9=$ like extremely; $1=$ dislike extremely). Analysis of variance was performed wherein the main effects of REA, HCW, and portioning method (thickness or weight) along with the REA $\times \mathrm{HCW}$ interaction were included.

Results: There were REA $\times$ HCW interactions for WBS values for both steaks portioned by thickness $(P=0.031)$ and by weight $(P=0.014)$. In both cases, the trend was for the lowest WBS values to be from the lightest HCW/ smallest REA combinations with the highest WBS values among the largest REA categories regardless of HCW category. Regardless of these trends, WBS values were all extremely low and met most thresholds for "very tender" classifications. When portioned by thickness, there were REA $\times$ HCW interactions for overall liking $(P=0.042)$ and flavor liking $(P=0.006)$. In both cases, there were significant differences among the palatability ratings for HCW within the largest REA category, whereas $\mathrm{HCW}$ did not impact these ratings within the other 2 REA categories. It is not clear why this occurred. When portioned by weight, only overall liking $(P=0.42)$ and tenderness liking $(P=$ 0.009 ) were impacted by REA with the highest ratings given to the smallest REA category. No palatability trait was influenced by HCW.

Conclusion: Carcass weight and ribeye size do impact beef steak palatability. Fortunately, all palatability ratings and shear force values were in ranges thought to be highly acceptable.

Keywords: beef, carcass weight, palatability, ribeye area, Warner-Bratzler shear force

\section{7 - CONSUMER PALATABILITY OF AUSTRALIAN GRASS- AND GRAIN-FED SMOKED BEEF RIBS}

J. C. Sarchet ${ }^{1 *}$, N. C. Hardcastle ${ }^{1}$, A. J. Garmyn ${ }^{1}$, R. J. Polkinghorne $^{2}$, and M. F. Miller ${ }^{1},{ }^{1}$ Animal and Food Science, Texas Tech University, Lubbock, TX, USA, ${ }^{2}$ Birkenwood, Birkenwood, Hawthorne, Australia, *jcsarchet@ sbcglobal.net

Objectives: The objective of this study was to identify the effects of grade, diet (grass vs. grain), muscle, and hold time on the consumer palatability of smoked beef ribs.

Materials and Methods: Beef ribs of varying quality grades $(2,3,4,5)$ were collected from 2 commercial abattoirs in Australia, equally representing grass- and grain-fed carcasses. The subprimals were then vacuum packaged, frozen at $3 \mathrm{~d}$ postmortem, and shipped frozen to Texas Tech University in Lubbock, Texas. Upon arrival, the ribs were sorted into cook batches and returned to frozen storage $\left(-20^{\circ} \mathrm{C}\right)$. Prior to cooking, ribs were thawed, excess external fat was trimmed, and ribs were lightly seasoned with salt and pepper. On their designated testing day, ribs were placed in electric pellet smokers (Green Mountain Grills set to $120^{\circ} \mathrm{C}$ ) and cooked until reaching an internal temperature of $93.3^{\circ} \mathrm{C}$. After removal from the smoker and resting for at least $30 \mathrm{~min}$, the rib meat was deboned and separated into intercostales externus et internis (INT), as well as chuck and rib portions of the serratus ventralis (SV). The samples were allocated to serve time treatment. Half of the samples were held warm $\left(\sim 60^{\circ} \mathrm{C}\right)$ and fed to consumers; the other half were vacuum packaged, refrigerated for $7 \mathrm{~d}$, reheated, and served the following week. Hot and reheated samples were fed to consumers and evaluated for tenderness, juiciness, flavor liking, overall liking, and satisfaction. Consumer data were analyzed to determine the effects of Meat Standards Australia (MSA) grade, diet, muscle, serve time (hot vs. reheat), and their interactions. Only MSA grade $\times$ diet remained in the model as a fixed effect $(P<0.05)$.

Results: There was an interaction $(P<0.01)$ between quality grade and diet for all palatability attributes and consumer satisfaction scores. Grass-fed samples from grade 2 (lowest) carcasses were scored lower $(P<0.05)$ than their grain-fed counterparts for all traits. Tenderness was greater $(P<0.05)$ for grass-fed samples from grade 3 carcasses compared to grain-fed samples, but all other palatability traits were similar for grade 3 carcasses. Flavor liking, overall liking, and satisfaction were greater $(P<0.05)$ for grade 4 grass-fed carcasses compared to grain fed, but the opposite 
trend was observed for grade 5 (highest grade) carcasses, where grain-fed samples were preferred $(P<0.05)$. Additionally, consumers were able to distinguish $(P<0.01)$ between muscle for all palatability traits and consumer satisfaction. The SV rib portion was scored greater $(P<0.05)$ for all traits compared to the SV chuck portion or INT. The SV chuck portion was scored greater $(P<0.05)$ for flavor and overall liking and satisfaction compared to INT, but those samples did not differ for tenderness or juiciness. Panelists also noted a difference in serve time for juiciness, flavor liking, overall liking, and consumer satisfaction $(P<$ 0.01 ), where hot samples were scored more favorably than reheated samples.

Conclusion: Quality grade and diet interacted to affect the eating quality of smoked beef ribs, with inconsistent trends between MSA grade depending on the diet. The palatability of the posterior end of the SV was preferred by consumers compared to the anterior portion and to the rib fingers, when prepared using a smoked cookery method. Samples served hot were scored more favorably for all palatability traits, except tenderness, compared to reheated samples.

Keywords: beef ribs, consumer, palatability, quality grade, smoking

\section{8 - EVALUATION OF DIETARY ZINC SUPPLEMENTATION ON LAMB PERFORMANCE AND CARCASS CHARACTERISTICS}

J. G. Logar ${ }^{1 *}$, C. L. Gifford ${ }^{1}$, R. M. Knuth ${ }^{1}$, H. C. Cunningham-Hollinger ${ }^{1}$, A. L. Julian ${ }^{1}$, C. M. Page ${ }^{1}$, J. R. Whaley ${ }^{1}$, B. Bisha ${ }^{1}$, and W. C. Stewart ${ }^{1},{ }^{1}$ Animal Science, University of Wyoming, Laramie, WY, USA, *jakelogar57@ gmail.com

Objectives: The objectives of this study were to assess the impacts of feeding 3 concentrations of a dietary zinc supplement pellet on lamb feedlot performance, carcass characteristics, and nutrient composition.

Materials and Methods: Commercial Rambouillet lambs $(n=33)$ were weighed $(49.0 \pm 1.0 \mathrm{~kg})$ and randomly assigned to one of 3 treatment groups consisting of a zincsulfate $(80 \%)$ and zinc amino acid $(20 \%)$ complex supplementation pellet (Zinpro Corp., Eden Prairie, MN) included in feedlot diets. Treatment groups consisted of diets with a zinc concentration of $72.7 \mathrm{mg} / \mathrm{kg}$ (T1), $95.5 \mathrm{mg} / \mathrm{kg}$ (T2), or $315 \mathrm{mg} / \mathrm{kg}$ (T3). Individual feed intake and body weights were recorded at day 10, 24, 38, 52, 66, and 73. Following the feeding phase, lambs were transported to a commercial harvest facility, and samples of the liver were collected for mineral analysis. At $24 \mathrm{~h}$ postmortem, carcasses were allowed at least $20 \mathrm{~min}$ to bloom after being ribbed between the 12th and 13th rib and carcass characteristics were measured. Objective color $\left(L^{*}, a^{*}\right.$, and $\left.b^{*}\right)$ scores were measured from the Longissimus dorsi and Rectus abdominis (flank) by obtaining 3 scans and recording a mean reading (HunterLab MiniScan EZ 45/O-L; Reston, VA). Samples of the Longissimus dorsi were removed from the 13th rib region of each carcass for proximate composition. All data were analyzed using the GLIMMIX procedure of SAS version 9.4 (SAS Institute Inc., Cary, NC).

Results: An interaction between treatment and feeding period was observed for dry matter intake $(P<0.001)$. No treatment effects were identified for growth performance, but average daily gain $(P<0.001)$ and grain/feed ratio $(P=0.04)$ was greatest during the final feeding period. Treatment impacted $a^{*}$ values $(P<0.05)$ of the flank, resulting in greater $a^{*}$ values of the flank from lambs in the T2 treatment group compared to the T3 treatment group. Treatment did not affect $L^{*}(P=0.37)$ and $b^{*}(P=0.11)$ values from either muscle. No treatment differences were identified for other carcass characteristics, proximate composition, or mineral analysis, but a trend of higher sodium $(P=0.06)$ among the $\mathrm{T} 1$ treatment group and lower calcium $(P=0.07)$ among the T3 treatment group was observed.

Conclusion: Results suggest that dietary zinc supplementation impacts dry matter intake dry matter intake and redness values in the flank of lamb carcasses. This information will be beneficial to future work assessing optimal level of zinc supplementation in feedlot lambs.

Keywords: carcass characteristics, lamb performance, zinc supplementation

\section{9 - INFLUENCE OF CARCASS CHILLING SYSTEM ON CHUCK, LOIN, AND ROUND TEMPERATURE DECLINE}

L. M. Hite ${ }^{1 *}$, C. E. Bakker ${ }^{1}$, T. C. DeHaan ${ }^{1}$, A. D. Blair ${ }^{1}$, K. R. Underwood ${ }^{1}$, and J. K. Grubbs ${ }^{1},{ }^{1}$ Animal Science, South Dakota State University, Brookings, SD, USA, *lydia.hite@sdstate.edu

Objectives: Spray chilling has been utilized to replace loss of moisture and increase yields during beef chilling. The objective of this study was to evaluate the effect of spray chilling beef carcasses on temperature decline of the chuck, loin, and round.

Materials and Methods: Ten beef animals were slaughtered at the South Dakota State University Meat Laboratory across 2 slaughter days $(n=5 / \mathrm{d})$. Hot carcass weight for each side was recorded. The left side of each carcass was chilled by air movement only (AIR), and the right side was spray chilled (SPRY). Air temperature of the cooler was $3.8^{\circ} \mathrm{C}$ (range $2.5^{\circ} \mathrm{C}-6.0^{\circ} \mathrm{C}$ ), and air movement was $3,500 \mathrm{~m}^{2} / \mathrm{min}$. The SPRY treatment was chilled under similar conditions to the AIR treatment with addition of a timed spray of chilled water at $5.5^{\circ} \mathrm{C}$ with a volume of $3.78 \mathrm{~L}$ for $80 \mathrm{~s}$ repeated every $32 \mathrm{~min}$ for $24 \mathrm{~h}$ via 10 sprinkler heads. Temperature 
data loggers were placed in the round, loin, and chuck of each side $60 \mathrm{~min}$ postmortem, and temperature was recorded at multiple positions every $30 \mathrm{~min}$. Loggers were placed in the center of the round in the semimembranosus at depths of $20.32,15.24,10.16$, and $5.08 \mathrm{~cm}$ from the surface. Loggers were placed in the chuck immediately posterior to the foreleg in the pocket between the brisket and the chuck in the serratus ventralis at depths of 20.32, 15.24, and $10.16 \mathrm{~cm}$. A data logger was placed $10.16 \mathrm{~cm}$ deep into the longissimus lumborum of the loin, at the third lumbar vertebra. Data were analyzed using the MIXED procedure of SAS (SAS Institute Inc., Cary, NC). Hot carcass weight was used as a covariate for all data. Temperature decline from the round and chuck were analyzed as repeated measures with day, time, treatment, and logger depth and their interactions as fixed variables. Temperature decline from the loin was analyzed as a repeated measure with day, time, and treatment and their interactions as fixed variables. Statistical significance was considered at an alpha of $P<0.05$.

Results: Sides exposed to SPRY treatment were cooler $(P<0.001)$ than AIR chilled sides in the round, loin, and chuck. No treatment by time interactions were observed for any primal $(P>0.05)$. A treatment by depth interaction was observed in the chuck $(P=0.001)$ with SPRY sides having lower temperatures than AIR sides at the 10.16-cm depth $\left(21.4^{\circ} \mathrm{C}\right.$ vs. $\left.22.5^{\circ} \mathrm{C} ; P<0.0001\right)$ and $20.32-\mathrm{cm}$ depth $\left(23.4^{\circ} \mathrm{C}\right.$ vs. $\left.24.0^{\circ} \mathrm{C} ; P=0.0006\right)$. A depth by time interaction was observed in the round $(P<0.0001)$. In the round at time 0 , the $5.08-\mathrm{cm}$ probe recorded lower $\left(30.0^{\circ} \mathrm{C} ; P<0.0001\right)$ temperatures compared to the $10.16-, 15.24-$, and $20.32-\mathrm{cm}$ probes, which were similar $\left(39.5^{\circ} \mathrm{C}, 40.2^{\circ} \mathrm{C}\right.$, and $39.8^{\circ} \mathrm{C}$, respectively; $P>0.05$ ). At $1,440 \mathrm{~min}$, as depth in the round increased, temperature increased and differed at each depth $\left(5.2^{\circ} \mathrm{C}\right.$ at $5.08 \mathrm{~cm}, 10.2^{\circ} \mathrm{C}$ at $10.16 \mathrm{~cm}, 12.2^{\circ} \mathrm{C}$ at 15.24 $\mathrm{cm}$, and $13.6^{\circ} \mathrm{C}$ at $20.32 \mathrm{~cm} ; P<0.0001$ ).

Conclusion: Spray chilling of beef carcasses resulted in lower internal temperatures in the round, loin, and chuck compared to the air chilled sides. Internal temperature decline differs between carcass locations and muscle depth. Tissue closer to the surface reached lower temperatures and chilled faster than deep tissue in both AIR and SPRY treatments. These data suggest that spray chilling helps chill carcasses more rapidly; however, additional research is needed to optimize spray chilling systems.

Keywords: beef, internal, spray chilling, temperature

\section{0 - SMART TUMBLING IMPROVED QUALITY AND PALATABILITY ATTRIBUTES OF FRESH BEEF M. LONGISSIMUS LUMBORUM AND M. SEMITENDINOSUS}

\author{
J. R. Tuell ${ }^{1 *}$ and Y. H. B. Kim ${ }^{1},{ }^{1}$ Animal Sciences, Purdue \\ University,West Lafayette, IN, USA, *tuell@purdue.edu
}

Objectives: Beef round muscles often exhibit unacceptable tenderness and require extensive aging to reach targeted eating quality. Our recent study indicated a feasibility in improving tenderness of beef loins immediately following the tumbling process without the use of brine enhancement, as well as promoting enzymatic degradation of muscle fiber structure with aging (termed Smart Tumbling). However, it is currently unknown whether consumers would find a tumbled fresh beef product to be acceptable, as well as what role muscle type (i.e., tender vs. tough) may have. As such, the purpose of this study was to evaluate the impact of Smart Tumbling on the quality attributes and consumer acceptability of 2 beef muscles.

Materials and Methods: Beef loin (M. Longissimus lumborum [LL]) and eye of round (M. Semitendinosus [ST]) muscles ( $n=16$; USDA Low Choice) at $5 \mathrm{~d}$ postmortem were cut into 4 sections and allocated among 4 tumbling (T) treatments (in minutes: T0 [control], T40, T80, T120). The beef sections were individually vacuum packaged, tumbled in a Lance LT-30 at $8.5 \mathrm{rpm}$, and aged either $0 \mathrm{~d}$ or $10 \mathrm{~d}$. Meat quality attributes, including $\mathrm{pH}$, water-holding capacity, Warner-Bratzler shear force, and instrumental color, were analyzed. Consumer sensory evaluations $(n=$ 120) per each muscle were conducted by assessing their liking of various attributes including tenderness, juiciness, flavor, and overall acceptability. A model per each muscle was created using the MIXED procedure of SAS (version 9.4; SAS Institute Inc., Cary, NC) with tumbling and aging durations serving as fixed effects and carcass included as a random effect.

Results: Both Smart Tumbling and aging main effects decreased Warner-Bratzler shear force in LL, but only aging improved instrumental tenderness in ST $(P<0.05)$. The consumer panel could clearly distinguish tenderness differences between Smart Tumbled and control (T0) beef samples from both LL and ST. Consumers found LL steaks tumbled for any duration to be more tender than the control $(P<$ $0.05)$, whereas juiciness, flavor, and overall liking were unaffected $(P>0.05)$. In particular, Smart Tumbled steaks from LL (T120) without further aging had equivalent tenderness liking values to control steaks with additional $10-\mathrm{d}$ aging $(P>0.05)$. Consumer panelists found improved tenderness values for Smart Tumbled (T120) steaks from ST $(P=0.050)$ after $10 \mathrm{~d}$ of aging, while no positive aging impact was found in the non-tumbled control (T0) counter$\operatorname{part}(P>0.05)$. This may be attributed to a similar interaction observed in liking of juiciness in ST $(P<0.05)$. Smart Tumbling increased cooking loss in $\operatorname{LL}(P<0.05)$ regardless of aging duration, while 10-d aging appeared to mitigate tumbling-induced detriments to cooking loss in ST $(P<$ $0.05)$. Smart Tumbling did not impact color attributes at either aging duration $(P>0.05)$.

Conclusion: Overall, the results suggest that tumbling vacuum-packaged fresh beef (Smart Tumbling) can improve tenderness and possibly other eating quality attributes of beef LL and ST muscles. Smart Tumbling may allow fresh beef 
muscles to reach targeted eating quality outcomes with shorter aging. As the effects of combined tumbling and aging treatment appear to be muscle specific, further study on other muscles (i.e., round and sirloin) would be warranted.

Keywords: beef tenderness, consumer evaluation, meat tumbling, natural processing, sensory panel

\section{1 - EFFECT OF REPLACING COTTONSEED MEAL AND SORGHUM GRAIN WITH CORN DRIED DISTILLERS GRAINS WITH SOLUBLES IN LAMB FEEDLOT DIETS ON VOLATILE AROMA COMPOUNDS OF LAMB CHOPS}

K. M. Hodges ${ }^{1}$, Z. Hicks ${ }^{1 *}$, C. R. Kerth ${ }^{1}$, T. R. Whitney ${ }^{2}$, K. R. Wall ${ }^{1}$, R. K. Miller ${ }^{1}$, W. S. Ramsey ${ }^{1}$, and D. R. Woerner ${ }^{3}$, ${ }^{1}$ Department of Animal Science, Texas A\&M University, College Station, TX, USA, ${ }^{2}$ Department of Animal Science, Texas A\&M AgriLife Research, San Angelo, TX, USA, ${ }^{3}$ Department of Animal and Food Sciences, Texas Tech University, Lubbock, TX, USA, *zmhicks@tamu.edu

Objectives: The objective of this project was the examine the presence or absence of volatile aroma compounds in lamb chops from lambs fed diets differing in percentage of dried distillers grains with solubles (DDGS).

Materials and Methods: Frozen Longissimus muscles $(n=45)$ were received from lambs fed one of 5 different treatment diets: cotton seed meal, sorghum grain, and cotton seed hulls, but no DDGS (CNTL), and 4 treatment diets that were similar to CNTL but did not contain cotton seed meal where corn DDGS replaced 0\% (0DDGS), 33\% (33DDGS), $66 \%$ (66DDGS), or $100 \%$ (100DDGS) of the sorghum grain in the treatment diets ( 9 lambs/treatment). Each frozen loin was cut into $2.54-\mathrm{cm}$ chops and stored at $-10^{\circ} \mathrm{C}$ until cooking. Chops were thawed $\left(4^{\circ} \mathrm{C}\right)$ for 12 to $24 \mathrm{~h}$, then cooked on a 2.54 -cm-thick flat-top grill set at $177^{\circ} \mathrm{C} \pm 2.8^{\circ} \mathrm{C}$. Chops were cooked until the internal temperature reached $35^{\circ} \mathrm{C}$, were turned, and continued cooking to a final internal temperature of $71^{\circ} \mathrm{C}$. Chops were cut into cubes $(1.3 \mathrm{~cm} \times 1.3$ $\mathrm{cm} \times$ chop thickness), trimmed of excess fat and connective tissue, frozen in liquid nitrogen, and stored at $-80^{\circ} \mathrm{C}$ until volatile analysis. Samples were placed in a glass jar with a Teflon lid, placed in a water bath $\left(60^{\circ} \mathrm{C}\right)$, and thawed for $60 \mathrm{~min}$. Then, a solid-phase micro-extraction portable filed sampler was placed in the headspace for $2 \mathrm{~h}$ to collect volatile compounds. The solid-phase micro-extraction was then injected into a multidimensional gas chromatographer/mass spectrometer that desorbed, separated, and identified each volatile compound. Data were analyzed using JMP version 14.0 (SAS Institute Inc., Cary, NC) using model fitment for a completely randomized design with finishing diet as fixed effects. Treatments were testing using one of the following: orthogonal contrasts of CNTL versus 0DDGS; or linear or quadratic effects of 0DDGS, 33DDGS, 66DDGS, and 100DDGS diets with the highest order relationship (linear or quadratic; $P \leq 0.05$ ) discussed.

Results: The volatile aroma compounds 2-heptenal (fried, buttery), heptanal (fatty), and 2-pentyl furan (caramel-like) linearly increased $(P<0.03)$ as DDGS increased. Conversely, 2-butanone (fruity) linearly decreased $(P=0.004)$ as DDGS increased. Furthermore, 2-heptanone (banana, fruity aroma) tended $(P=0.065)$ to increase linearly with increasing DDGS. 2-(hexyloxy)-ethanol had a quadratic decrease with up to 33DDGS in the diet and then an increase to 100DDGS in the diet $(P=0.005)$; however, 2,3-octanedoine and methyl pyrazine quadratically increased to 33DDGS then decreased to 100DDGS $(P<0.05)$. 2-ethyl-5-methyl pyrazine tended $(P=0.089)$ to increase quadratically to 33DDGS then decrease as DDGS increased above that, and 2,5-dimethyl pyrazine tended $(P=0.089)$ to decrease with increasing DDGS in the diet. Decanal (orange, citrus flavor) tended $(P=0.054)$ to be lower in the CNTL diet when contrasted with the 0DDGS diet.

Conclusion: Replacing cottonseed meal with dried distillers grains in lamb feedlot diets alters the composition of volatile compounds of lamb chops. Aromatic compounds that are perceived as fried, fatty, and caramel aromas increased as DDGS increased, suggesting that higher levels of $33 \%$ to $66 \%$ DDGS may improve flavor in lamb chops.

Keywords: gas chromatography/mass spectrometry, lamb, volatile compounds

\section{2 - INFLUENCE OF FEEDING COMPOSITION AND STORAGE TIME ON PHYSIC-CHEMICAL PARAMETERS OF CHURRA LAMB MEAT}

P. E. S. Munekata ${ }^{1 *}$, R. Bermudez ${ }^{1}$, M. Pateiro ${ }^{1}$, R. Dominguez $^{1}$, A. Maggiolino ${ }^{2}$, D. Franco ${ }^{1}$, and J. M. Lorenzo ${ }^{1},{ }^{1}$ Food Science and Technology, Centro Tecnológico de la Carne de Galicia, Ourense, Spain, ${ }^{2}$ Department of Veterinary Medicine, University of Bari Aldo Moro, Bari, Italy, *paulosichetti@ceteca.net

Objectives: The use of natural and residual sources from food processing in animal feeding is an important strategy to evolve the meat productive sector towards more sustainable practices and also reduce the environmental impact of food processing. Apple is an important source of nutrients, and heather (Calluna vulgaris) is a relevant source of bioactive compounds found in several countries of Europe. In this sense, the present study aimed to study the effect of apple, heather, and their combination and the storage time in the physic-chemical parameters of Churra lamb meat. 
Materials and Methods: The handling of animals was performed according to the Directive 2010/63/EU of the European Parliament and the Council of the European Union (2010). Sixty Churra male lambs (already weaned, 6 to 8 wk old) were used. The animals were separated in 5 groups according to diet composition: Control (14), Concentrate (12), Apple (12), Heather (11), or Heather + Apple (11). The water and feeding were offered ad libitum. The lambs were slaughtered at age $4 \mathrm{mo}$ in a commercial abattoir ( $1.5 \mathrm{~h}$ transport) where they were stunned, exsanguinated (jugular vein), eviscerated, and skinned. The carcasses were chilled for $24 \mathrm{~h}$ at $4^{\circ} \mathrm{C}$. The meat samples were collected from the longissimus thoracis muscle, vacuum packaged, and stored for 3, 9, and $15 \mathrm{~d}$ at $4^{\circ} \mathrm{C}$. During the storage period, the meat samples were evaluated regarding the $\mathrm{pH}$, color, thiobarbituric acid reactive substances, and texture. The influence of feeding composition and storage time on quality characteristics of Churra lamb meat were examined using analysis of variance, with IBM SPSS Statistics 23 software package (IBM Corp., Armonk, NY).

Results: Regarding the $\mathrm{pH}$, the lowest value $(P<0.05)$ was obtained from animals fed with Concentrate after $3 \mathrm{~d}$ of storage. This difference was not observed on other storage days wherein the $\mathrm{pH}$ values ranged from 5.61 to 5.74 . In the case of $L^{*}$, not-significant differences were obtained from storage time and feeding composition. Differently, the $a^{*}$ value increased during storage for all animals regardless of feeding composition. A similar outcome was obtained for $b^{*}$ for animals fed with Control, Concentrate, and Heather + Apple. The storage time reduced the firmness, shear force, and total work of Churra lamb meat, particularly after $9 \mathrm{~d}$ of storage. The formation of lipid oxidation increased gradually $(P<0.05)$ during storage, but it was not affected by feeding composition.

Conclusion: The inclusion of apple, heather, and their combination were associated with minimal effect on physic-chemical parameters of Churra lamb meat and could be used in its production.

Acknowledgments: This work was supported by European Research Area on Sustainable Animal Production Systems (SusAn, ERA-NETSUSAN 2016/48 [PCIN-2017053], EcoLamb project). Thanks to GAIN (Axencia Galega de Innovación) for supporting this study (Grant Number IN607A2019/01). Paulo E. S. Munekata acknowledges postdoctoral fellowship support from the Ministry of Economy and Competitiveness (MINECO, Spain) "Juan de la Cierva" program (FJCI-2016-29486). José M. Lorenzo is a member of the HealthyMeat network, funded by CYTED (reference 119RT0568).

Keywords: apple, heather, lipid oxidation, meat quality, texture parameters

\section{3 - INVESTIGATION INTO MECHANISMS UNDERPINNING DRY-AGING IMPACTS ON BEEF QUALITY ATTRIBUTES AND FLAVOR-RELATED COMPOUNDS}

D. Setyabrata ${ }^{1 *}$, S. Xue ${ }^{1,2}$, K. Vierck ${ }^{3}$, J. Legako ${ }^{3}$, B. R. Cooper $^{4}$, T. J. Sobreira ${ }^{4}$, and Y. H. B. Kim ${ }^{1},{ }^{1}$ Meat Science and Muscle Biology Lab, Department of Animal Sciences, Purdue University, West Lafayette, IN, USA, ${ }^{2}$ Key Laboratory of Meat Products Processing, Ministry of Agriculture; Jiangsu Collaborative Innovation Center of Meat Production and Processing, Quality and Safety Control; College of Food Science and Technology, Nanjing Agricultural University, Nanjing, China, ${ }^{3}$ Department of Animal and Food Science, Texas Tech University, Lubbock, TX, USA, ${ }^{4}$ Bindley Bioscience Center, Purdue University, West Lafayette, IN, USA, *dsetyabr@purdue.edu

Objectives: Postmortem aging has been well known to improve meat palatability. Dry aging, specifically, has been known to improve flavor of the meat, generating unique flavor such as beefy and umami. However, compounds that are associated with dry-aged beef flavor have not been fully identified and understood. Thus, the objective of this study was to identify flavor-related compounds that can be naturally liberated through different aging methods.

Materials and Methods: Paired beef loins (M. longissimus lumborum) from 13 cull cow carcasses (42 mo +, Holstein) were collected $5 \mathrm{~d}$ postmortem, split into 4 equal portions, and randomly assigned to 4 different aging treatments: wet aging (WA), conventional dry aging, dry aging in water-permeable bag, and conventional dry aging with ultraviolet light ( 2 treatment $/ \mathrm{d}, 5 \mathrm{~J} / \mathrm{s} /$ treatment) for $28 \mathrm{~d}$ at $2{ }^{\circ} \mathrm{C}, 65 \%$ relative humidity, and $0.8 \mathrm{~m} / \mathrm{s}$ air flow. After aging, loins were trimmed, and steaks were collected for sensory evaluation, biochemical analyses, and metabolomics analysis. Steaks were evaluated for various flavor and palatability attributes by both trained $(n=11)$ and consumer $(n=$ 130) panelists. The free amino acid concentration, fatty acid profile, and volatile content were measured for all treatments. Metabolomics were analyzed using an ultraperformance liquid chromatography electrospray ionization mass spectrometry system. All data were analyzed using PROC GLIMMIX of SAS (SAS Institute Inc., Cary, NC). Leastsquares means for all traits were separated (F-test, $P<$ $0.05)$ using the PDIFF option. Principal component analysis was performed on the metabolites using $\mathrm{R}$ software.

Results: From the analysis, 1,407 metabolites were detected, and 60 were significantly affected by the treatments $(P<0.05)$. Of these, 44 were able to be identified. Greater abundance of protein metabolites was observed in all dry aging treatments compared to WA. Similarly, free amino acids analysis also showed significant treatment effect, where dry-aged samples had greater abundance of amino acids compared to WA $(P<0.05)$, except for aspartate, 
hydroxyproline, and cysteine. No difference in the fatty acid profile was found between different aging treatments $(P>0.05)$. More lipid-related metabolites, however, were identified in the WA treatment, potentially from the limited exposure to the environment. Volatile analysis indicated that dry aging generated more volatile compounds, especially from hydrocarbon and alcohol groups, compared to WA. Additionally, more sulfur containing compounds, such as thioproline and erysothiopine, were observed in dry aging treatments compared to WA. Sulfur containing compounds have been often related to desirable flavor in beef product. The trained panel identified that conventional dry aging steaks and steaks dry-aged in water-permeable bag had significantly lower fat and sour flavor and a trend of lower oxidized flavor $(P=0.07)$ compared to steaks from WA and conventional dry aging with ultraviolet light. The consumer panel, however, found no differences in sensory attributes between treatments $(P>0.05)$.

Conclusion: The results showed that dry aging treatments resulted in greater amino acids and sulfur containing compounds, whereas more lipid-related compounds were observed in WA. This observation potentially indicates that different postharvest aging methods could affect the liberation of flavor-related compounds of beef. Further research correlating these compounds to sensory quality and conducting pathway analyses to elucidate the underlying mechanisms by which dry-aged beef flavor-related compounds can be generated is currently underway.

Keywords: cull cow, dry aging, metabolomics, volatile compounds

\section{4 - TASTE-RELATED COMPONENTS IN HANWOO STEER AND COW LONGISSIMUS DORSI MUSCLES}

S. Cho ${ }^{1}$, K.-H. Seol ${ }^{1}$, S. M. Kang ${ }^{1}$, Y. Kim ${ }^{1}$, H.-W. Seo ${ }^{1}$, J.-H. Kim ${ }^{1}$, and H. V. Ba ${ }^{1 *},{ }^{1}$ Animl Products Utilization Division, National Institute of Animal Science, RDA, Wanju-gun, Republic of Korea, *smkang1014@naver.com

Objectives: Taste (e.g., sweetness and umami, etc.) is an important factor affecting the eating quality of beef. Cow and steer are the 2 main sex classifications of Hanwoo (Korean native cattle) beef in the Korean meat market. Therefore, the main objective of this study was to determine and compare the taste-related components of beef from Hanwoo steers and cows.

Materials and Methods: Longissimus dorsi (LD) muscles ( $n=10$ per sex class) with the same quality grade $\left(1^{+}\right.$grade) collected from the left sides of Hanwoo steers (31-32 mo old) and cows (47-53 mo old) at $24 \mathrm{~h}$ postmortem were used in the present investigation. The average marbling scores and intramuscular fat content of the LD muscles used were 6.8 and 6.0 , and $17.82 \%$ and $16.69 \%$, for the steers and cows, respectively. After trimming of visual fats, the lean muscles were used for analysis of taste-related components including free amino acids (FAA), metabolites, and nucleotides. The FAA were analyzed using an ultraperformance liquid chromatography, and the results were expressed as milligram per $100 \mathrm{~g}$ meat. Nucleotides were analyzed using ultraperformance liquid chromatography, and then were identified by using external standards. For metabolite analysis, each sample $(20 \mathrm{mg})$ was extracted with acetonitrile/ water $(1: 1, \mathrm{v} / \mathrm{v})$ mixture. The extracts were freezing-dried and then dissolved in deuterated water containing $2 \mathrm{mM}$ 3-trimethylsilyl-2,2,3,3-tetradeuteropropionicacid-d4 as an internal standard. ${ }^{1} \mathrm{H}-\mathrm{NMR}$ (nuclear magnetic resonance) spectra were acquired on a $600 \mathrm{MHz}$ NMR spectrometer equipped with $600 \mathrm{MHz} 4-\mathrm{mm}$ gHX NanoProbe at a ${ }^{1} \mathrm{H}$ frequency of $599.93 \mathrm{MHz}$. Metabolites were tentatively identified using a $600 \mathrm{MHz}$ library database and NMR.

Results: Based on their similar taste qualities, researchers have classified the FAA into several classes, such as umami, saltiness, sweetness, bitterness, and sourness. The outcome of our analysis revealed that the levels of some FAA associated with umami (e.g., glutamic acid and lysine), sweetness (e.g., proline and glutamic acid), and saltiness (e.g., histidine and glutamic acid) in the LD muscles were significantly higher in the cows compared to the steers $(P<0.05)$. No differences were found in amounts of all the detected nucleotides (hypoxanthine, uridine, guanosine monophosphate, inosine $5^{\prime}$-phosphate) between the 2 types of beef $(P>0.05)$. A total of 27 metabolites were identified in the LD muscles of both cattle genders. Out of them, 8 compounds (e.g., acetate, creatine, creatinine, glucose, glycine, inosine, trimethylalanine, and tyrosine) showed their significantly higher amounts in the cow meat compared with those in steer meat $(P<0.05)$.

Conclusion: The cattle sex type partly showed its effects on the levels of taste-related components (e.g., FAA and metabolites) in the $1^{+}$grade LD muscles. However, further study is needed to determine the associations of the identified taste-related compounds with eating quality attributes of cooked beef.

Keywords: Hanwoo, taste, free amino acid, metabolite, nucleotide

\section{5 - THE INVESTIGATION OF DIFFERENT LEVELS OF VITAMIN A AND ITS EFFECTS ON CARCASS TRAITS, AND THE CONVERSION RATE OF EXTERNAL FAT COLOR IN CULL COWS}

J. T. Parkinson ${ }^{1 *}$, H. J. Cochran ${ }^{1}$, A. E. Relling ${ }^{1}$, S. L. Boyles $^{1}$, and L. G. Garcia ${ }^{1},{ }^{1}$ Animal Science, The Ohio State University, Columbus, OH, USA,*parkinson.78@ osu.edu 
Objectives: The study objective was to evaluate different feeding strategies and their effects on fat color, carcass yield, and quality traits in cull cows.

Materials and Methods: Angus crossbreed cull cows $(n=98)$ were used with a feeding time of $56 \mathrm{~d}$ under clean bunk management. Cows were blocked by location and year and then divided into 2 feed treatments ( 8 pens with 7 to 22 cows per pen). Each treatment had 4 replications, and pens were used as the experimental unit. Treatments included low vitamin A (LVA) and high vitamin A (HVA) diets. The LVA diet was formulated using corn, soybean hulls, soybean meal, and a mineral-vitamin supplement. The HVA diet was formulated using corn, fescue hay, distillers dried grains with solubles, and a mineralvitamin supplement. A subcutaneous fat biopsy was taken in the rump area on $0 \mathrm{~d}$. Fat biopsy consisted of $1 \times 1 \mathrm{~cm}$ size sample removed after area was shaved and scrubbed, followed by $5 \mathrm{~mL}$ of lidocaine used locally. Upon $3 \mathrm{~min}$, a cut with a sterile scalpel was made to remove the subcutaneous fat tissue, bagged, then placed in dry ice and transported back to Ohio State University. Visual evaluation of fat color was conducted by using a 1 to 5 number scale published by the American Meat Science Association ranging as follows: 1 , white; 2 , creamy white; 3 , slightly yellow; 4 , moderately yellow; and 5, yellow. Upon day 56, cows were harvested; carcass data were collected $48 \mathrm{~h}$ postmortem. Yield and quality grade was evaluated at the 12th/13th rib interface using USDA grading standards. Additionally, subjective visual color score of carcasses was conducted using the same American Meat Science Association 1-5 fat color scale as on $0 \mathrm{~d}$. Statistical analysis was conducted using a proc mixed procedure. The color of the fat from the 0-d biopsy was used as covariable for 56-d fat color. Differences between treatments showing significance was determined at $P \leq 0.05$.

Results: Carcass external fat, ribeye size, internal fat percent, and hot carcass weight did not differ $(P>0.10)$ among treatment groups. Similarly, quality traits including skeletal maturity, lean maturity, marbling, and overall quality grade were not significantly affected $(P>0.10)$ by treatment group. Subcutaneous adipose color scores did differ significantly $(P<0.01)$ by treatment. Cows on the LVA treatment exhibited lower color scores compared to HVA (2.19 vs. 2.59$)(P=0.0039)$.

Conclusion: Results indicate that feeding strategies with feed ingredients differing in concentration of vitamin A had no effect on carcass yield or quality traits in cull cows; however, the reduction of subjective subcutaneous fat color scores seen in the LVA treatment group lends merit to the feeding of an LVA diet to cull cows prior to slaughter in order to target a "white-cow" premium.

Keywords: beef, quality traits, subcutaneous fat color, yield

\section{6 - QUALITY DIFFERENCES IN TRADITIONAL AND CLEAN-LABEL CHICKEN PATTIES FORMULATED WITH WOODY BREAST MEAT}

T. Jarvis ${ }^{1}{ }^{*}$ X. Zhang ${ }^{1}$, C. Rowe ${ }^{2}$, B. Smith ${ }^{3}$, C. Crist ${ }^{1}$, and M. W. Schiling ${ }^{1}{ }^{1}$ Food Science, Nutrition \& Health Promotion, Mississippi State University, Starkville, MS, USA, ${ }^{2}$ Research \& Development, Perdue Foods Inc., Salisbury, MD, USA, ${ }^{3}$ Business Development, Hawkins Inc., Birmingham, AL, USA, *trj215@msstate.edu

Objectives: The objective of this research was to evaluate quality differences between chicken patties composed of different percentages of normal (NOR) and severe (SEV) woody breast (WB) meat and formulated with a control (salt), traditional (salt, sodium phosphate), or clean-label (salt, potassium carbonate) marinade.

Materials and Methods: Chicken breasts were collected from broilers with an average live weight of $4.2-4.3 \mathrm{~kg}$ at a commercial poultry plant. Breasts collected were graded NOR and SEV and stored at $2^{\circ} \mathrm{C}-3^{\circ} \mathrm{C}$ for $6 \mathrm{~d}$. On the processing day, NOR and SEV WB were ground through a $1.27 \mathrm{~cm}$ plate, then a $0.48 \mathrm{~cm}$ bone-extracting plate. Meat was combined into $18.1 \mathrm{~kg}$ batches. Each meat block was blended with control, traditional, or clean-label marinades. Each batch was vacuum blended at $25 \mathrm{mmHg}$, chilled with $\mathrm{CO}_{2}$ to $-2.7^{\circ} \mathrm{C}$, then formed into patties using a 11 $3 / 4 \times 104 / 5 \times 25 \mathrm{~cm}^{3}$ plate $(166 \mathrm{~g})$. Ten raw patties were frozen to $-62.2^{\circ} \mathrm{C}$ in a $\mathrm{CO}_{2}$ cabinet. The remaining patties were belt grilled at $257^{\circ} \mathrm{C}$ for $75 \mathrm{~s}$, cooked for $12 \mathrm{~min}$ at $163^{\circ} \mathrm{C}$, $82^{\circ} \mathrm{C}$ dew point, and $800 \mathrm{rpm}$ fan speed, then individually frozen for $25 \mathrm{~min}$ at $-62.2^{\circ} \mathrm{C}$. Samples were stored at $-23^{\circ} \mathrm{C}$ and evaluated within 3 mo of processing. A $3 \times 4$ factorial structure within a randomized complete block design with 3 replications (processing dates) was used to evaluate the impact of marinade (control, traditional, clean label) and $\%$ NOR $(0 \%$ NOR, 33\% NOR, 67\% NOR, $100 \%$ NOR) on $\mathrm{pH}$, cook yields, texture profile analysis attributes, and protein bind. A 2 (traditional vs. clean label) $\times 4$ (\% NOR) factorial structure within a randomized complete block design with 2 replications (processing dates) was used to determine the impact of $\%$ NOR meat on descriptive sensory attributes ( $n=10$ trained panelists) and consumer acceptability ( $n=105$ for traditional and 113 consumers for clean label) of chicken patties within each marinade treatments (traditional and clean label). Means were separated using Fisher's protected least significant difference test. Orthogonal contrasts were also conducted to determine whether there were linear or quadratic effects $(P<0.05)$.

Results: The $100 \%$ NOR patties had greater cook yields than $33 \%$ and $0 \%$ NOR patties $(P<0.05)$ and better protein bind than other treatments $(P<0.05)$. Traditional patties had greater cook yields and better protein bind than clean-label and control patties $(P<0.05)$. For texture profile analysis, $100 \%$ NOR patties were harder, gummier, chewier, and

American Meat Science Association. 
springier than $33 \%$ and $0 \%$ NOR patties $(P<0.05)$. For descriptive analysis, $100 \%$ NOR patties were chewier and more cohesive than $0 \%$ NOR patties $(P<0.05)$, and traditional patties were springier, gummier, chewier, juicier, more cohesive, uniform, and fracturable than clean-label patties $(P<0.05)$. Consumers rated all patties acceptable for appearance, aroma, texture, flavor, and overall acceptability $(>6)$.

Conclusion: Protein functionality was hindered when WB meat was included in chicken patties, which contributed to decreased cook yields. In addition, use of sodium phosphate in the traditional marinade maximized yields and protein bind in the $100 \%$ NOR treatment and was superior to the clean-label and control samples, but was less effective in formulations with WB meat. It appears that all WB formulations could be used without a major impact on acceptability, but yields and protein bind decreased as WB amount increased in the formulation and when potassium carbonate was used in place of sodium phosphate.

Keywords: chicken breast, marination, myopathy, waterholding capacity

\section{7 - MUSCLE WATER PROPERTIES BY TIME- DOMAIN NUCLEAR MAGNETIC RESONANCE IN RAW INTACT BROILER PECTORALIS MAJOR WITH THE WOODY BREAST CONDITION}

H. Zhuang ${ }^{1 *}$, B. Pang ${ }^{2}$, and B. C. Bowker ${ }^{1},{ }^{1}$ US National Poultry Research Center, USDA-Agricultural Research Service, Athens, GA, USA, ${ }^{2}$ Food Science and Engineering, Qingdao Agricultural University, Qingdao, China, *hong.zhuang@usda.gov

Objectives: In addition to having unique tactile properties in the raw state, woody breast (WB) fillets (pectoralis major) also exhibit altered muscle water characteristics, with increased moisture content and decreased water-holding capacity (WHC) compared to normal fillets. Time-domain nuclear magnetic resonance (TD-NMR) is a rapid analytical methodology that provides information about muscle water properties, such as mobility, compartmentalization, and relative content. Previous studies have shown a relationship between water properties measured with TD-NMR and the WB condition. However, these studies utilized only small subsamples of the pectoralis major and did not analyze the relationships between the water properties and WHC. The objective of this study was to investigate effects of WB on muscle water properties determined by TD-NMR and their relationship with WHC in raw intact broiler breast fillets.

Materials and Methods: Pectoralis major muscles ( $n=$ 144) from 8 -wk-old broilers deboned at $3 \mathrm{~h}$ postmortem were collected from a commercial plant and categorized as normal, moderate WB, or severe WB based on fillet hardness and rigidity. For WHC, fillet purge loss was determined based on weight differences before and after overnight storage at $4^{\circ} \mathrm{C}$. Muscle water properties were determined by TDNMR based on the following measurements: relaxation parameters mobility (time constant of peak, T), distribution (area of peak relative to other peaks, P), and relative abundance (peak area per $100 \mathrm{~g}$ meat, A); values were transformed using an inverse Laplace algorithm.

Results: There were 3 water components identified in raw broiler breast meat: hydration water (T-HW), intramyofibrillar water (T-INTRA), and extra-myofibrillar water (T-EXTRA). As WB scores increased (from normal to severe), time constant T-HW increased $(P<0.05)$ from 0.39 to $0.44 \mathrm{~ms}$, T-INTRA from 49 to $57 \mathrm{~ms}$, and T-EXTRA from 205 to $235 \mathrm{~ms}$. There was also an increase in the proportion of extra-myofibrillar water (P-EXTRA) from $18 \%$ to $39 \%(P<0.05)$ and increases in the relative abundance of intra-myofibrillar water (A-INTRA) from 385 to 404 unit/ $100 \mathrm{~g}$ meat and extra-myofibrillar water (A-EXTRA) from 88 to 273 unit $/ 100$ g. However, P-HW decreased from $0.79 \%$ to $0.35 \%$, P-INTRA from $81 \%$ to $60 \%$, and A-HW from 3.65 to $2.25 \mathrm{unit} / 100 \mathrm{~g}$. In addition, the WB condition resulted in increased $(P<0.05)$ purge loss from $1.14 \%$ to $1.84 \%$. Correlation analysis revealed that the strongest overall correlation $(r=0.64)$ was between A-EXTRA and purge loss. There was no correlation $(P>0.05)$ between A-INTRA and purge loss. Within normal fillets, purge loss correlated to T-HW, T-INTRA, A-INTRA, and A-EXTRA $(\mathrm{r} \geq 0.38, P<$ $0.001)$. Within the severe WB group, only the A-EXTRA correlated to purge loss $(r=0.5 ; P<0.001)$.

Conclusion: The WB condition has a significant impact on water mobility, compartmentalization, and abundance within raw breast fillets. The WB condition results in increases in water mobility, P-EXTRA, A-INTRA, and AEXTRA but decreases in P-HW and P-INTRA. In normal broiler pectoralis major, T-HW, T-EXTRA, A-INTRA, and A-EXTRA are involved in meat purge loss. However, A-EXTRA may be a key factor responsible for the poor WHC in WB meat.

Keywords: extra-myofibrillar water, intra-myofibrillar water, meat water-holding capacity, nuclear magnetic resonance, wooden breast myopathy

\section{8 - PINUS TAEDA POLYPHENOLS ADDITION IN BEEF FEEDING: EFFECTS ON VOLATILE COMPOUNDS AND ANTIOXIDANT ENZYME ACTIVITY}

A. Maggiolino $^{1 *}$, E. Casalino ${ }^{1}$, M. Faccia ${ }^{2}$, J. M. Lorenzo Rodriguez $^{3}$, and P. De Palo ${ }^{1},{ }^{1}$ Department of Veterinary Medicine, University of Bari, Valenzano, ${ }^{2}$ Department of 
Soil, Plant and Food Sciences, University of Bari, Bari, Italy, ${ }^{3}$ Centro Tecnológico de la Carne de Galicia, Parque Tecnológico de Galicia, Oruense, Spain, *aristide. maggiolino@uniba.it

Objectives: Natural antioxidants obtained from leaves, seeds, or flowers of plants, containing bioactive substances such as phenolic compounds, flavonoids, and volatile oils, have been used in animal feeding. Consumers perceive this strategy positively, as these compounds have an effectiveness also in human health, they are natural, and usually they are from agricultural byproducts, in a context of increasing circularity of the economic activities. The aim of the work was to evaluate the effects of Pinus taeda Hydrolyzed Lignin (PTHL) polyphenols' inclusion on the volatile organic compounds (VOC) profile and antioxidant enzyme activity of beef meat stored at $4^{\circ} \mathrm{C}$ for $15 \mathrm{~d}$ of aging.

Materials and Methods: Forty Limousin bulls ( $340 \pm 42$ $\mathrm{kg}$ ) were fed ad libitum on a total mixed ration (TMR) for $120 \mathrm{~d}$. The control group received exclusively TMR, while the experimental group received the same TMR as the control group but supplemented with PTHL polyphenols (Oxifenol, I-Green, Padua, Italy; $35 \mathrm{~g} / \mathrm{d}$ per head at $1-90$ $\mathrm{d}$ and $70 \mathrm{~g} / \mathrm{d}$ per head at 91-120 d). Samples of longissimus thoracis muscle (mean weight of 2,000 $\pm 100 \mathrm{~g}$ ) were removed from the right carcass side between the 11th and 13th thoracic vertebra from each animal and placed in a dry aging meat chamber $\left(2^{\circ} \mathrm{C}, 82 \%\right.$ of humidity) until analyses. Steaks of $2.5 \mathrm{~cm}$ of thickness were obtained from the sample at 2 different storage times ( 1 and $15 \mathrm{~d}$ ) and analyzed. At each aging time, samples were grill cooked at $130-150^{\circ} \mathrm{C}$ for $5 \mathrm{~min}$, and VOC profile was performed by solid-phase microextraction and gas chromatography-mass spectrometry. Moreover, superoxide dismutase, catalase, and glutathione peroxidase meat activity were detected on raw samples at both aging times and in both experimental groups. VOC profile and enzyme activity were determined by gas chromatography-mass spectrometry and the spectrophotometric method, respectively, and the results obtained were analyzed using a nested analysis of variance using the general linear models procedure by SAS software (version 9.4, SAS Institute Inc., Cary, NC), where aging time and PTHL polyphenols' inclusion were set as independent variables. Means were compared using the Tukey test for repeated measures.

Results: Ketones and aldehydes were the most common VOC from cooking meat, representing around $60 \%$ of the total VOC. The ketones showed to be produced less in meat from the polyphenols group at 15 aging days $(P<0.01)$; however, they increased in both groups during aging $(P<$ 0.01 ). They usually derived from lipid oxidation, in particular from fatty acid oxidation, and showed how these processes are probably characterized by lower intensity if animals are fed with added polyphenols. Also aldehydes, derived from lipid oxidation although through different pathways than ketones, did not show any difference between groups $(P>0.05)$. However, they increased after aging $(P<0.05)$. Superoxide dismutase and catalase activity did not change during aging, with no differences between groups $(P>0.05)$; instead, glutathione peroxidase activity showed different trends between experimental groups, increasing during aging time in PTHL group $(P<0.01)$ and decreasing in the control group $(P<0.05)$, with higher activity in meat from animals fed with polyphenol additives at 15 aging days $(P<0.01)$.

Conclusion: Some changes are present in beef meat after grilling cooking, and these changes involved primarily those indicative of lipid oxidation, with less production in animals fed with PTHL polyphenols.

Keywords: aging time, antioxidants, enzymes, volatile organic compounds

\section{9 - IMPACT OF PRODUCTION CLAIMS AND COLOR SCORING UNDER CONTROLLED LIGHTING ON PORK QUALITY TRAITS}

K. Philipps ${ }^{1 *}$, C. Carr ${ }^{1}$, D. Newman ${ }^{2}$, S. Beauchamp ${ }^{3}$, C. Burdett $^{3}$, X. Sun ${ }^{4}$, J. Apple ${ }^{5}$, J. Young ${ }^{6}$, and C. Roland ${ }^{7}$, ${ }^{1}$ Animal Sciences, University of Florida, Gainesville, FL, USA, ${ }^{2}$ Animal Sciences, Arkansas State, Jonesboro, AR, USA, ${ }^{3}$ Animal Science, Arkansas State, Jonesboro, AR, USA, ${ }^{4}$ Agricultural and Biosystems Engineering, North Dakota State University, Fargo, ND, USA, ${ }^{5}$ Animal Science, Texas A\&M University-Kingsville, Kingsville, $T X$, USA, ${ }^{6}$ Animal Science, North Dakota State University, Fargo, ND, USA, ${ }^{7}$ Animal Science, University of Florida, Gainesville, FL, USA, *kphilipps@ufl.edu

Objectives: The two objectives of this study were to (1) investigate whether center loin chops marketed with a production claim impacted retail pork quality and to (2) categorize chops by National Pork Board color scores under controlled lighting to determine impact on weight loss and slice shear force.

Materials and Methods: A total of 1,289 center loin chops from 746 retail packages were purchased from 85 retail and wholesale/club stores in 15 cities, representing 7 different regions. Auditors purchased up to 10 retail packages of center loin pork chops per retail brand, per retailer. A mobile quality laboratory was set up in each city. All chops were weighed individually to calculate retail purge as compared with retail sale weight. Chops were individually evaluated for National Pork Board (NPB) color and marbling scores under controlled lighting prior to being vacuum sealed and frozen and shipped to University of Florida. Chops were weighed frozen, thawed at $4{ }^{\circ} \mathrm{C}$, then weighed prior to and after cooking on grills to an internal temperature of $65^{\circ} \mathrm{C}$; they were then evaluated for tenderness using slice 
Impact of grouping pork loin chops by the presence of a production claim or by subjective NPB colors scores evaluated under controlled lighting on objective water-holding and slice shear force values

\begin{tabular}{llllll}
\hline \hline & $\begin{array}{l}\text { With claim } \\
(n=385)\end{array}$ & $\begin{array}{l}\text { Without claim } \\
(\mathrm{n}=904)\end{array}$ & $P$ value & $\begin{array}{l}\text { Light } \\
(n=850)\end{array}$ & $\begin{array}{l}\text { Dark } \\
(n=435)\end{array}$ \\
\hline Retail Purge, \% & $4.2 \pm 0.3$ & $5.1 \pm 0.2$ & 0.003 & $5.1 \pm 0.2$ & $4.5 \pm 0.3$ \\
Thaw Loss, \% & $3.2 \pm 0.2$ & $2.6 \pm 0.1$ & $<0.001$ & $2.8 \pm 0.1$ & $2.7 \pm 0.1$ \\
Cook Loss, \% & $18.0 \pm 0.6$ & $18.2 \pm 0.4$ & 0.73 & $18.5 \pm 0.4$ & $17.4 \pm 0.5$ \\
Total Weight Loss, \% & $26.1 \pm 0.9$ & $26.8 \pm 0.5$ & 0.44 & $27.3 \pm 0.5$ & $24.8 \pm 0.7$ \\
Slice Shear Force, Kg & $15.8 \pm 0.4$ & $16.1 \pm 0.3$ & 0.52 & $16.3 \pm 0.3$ & 0.02 \\
\hline
\end{tabular}

shear force protocol and recorded as KG of force. Total weight loss was calculated by summing retail, thaw, and cooking loss.

Results: Seventy percent of purchased chops $(n=904)$ contained no production claim on the label, which represented 427 different retail packages. The top 3 label claims were hormone or steroid free, antibiotic free, and vegetarian fed. The presence of a production claim did not affect $(P \geq$ 0.18 ) NPB color or marbling score under controlled lighting (data not shown in table). Center loin chops with production claims had less retail purge $(P=0.003)$ than chops without production claims. The opposite was found with thaw loss, where chops with production claims had a greater percentage $(P<0.001)$ than chops without production claims. Production claim did not affect cooking loss, total weight loss, or slice shear force $(P \geq 0.44)$. Chops determined to be light (NPB color score 1 or $2 ; n=145$ and 705 , respectively) under controlled lighting had more retail purge, cooking loss, and total weight loss $(\mathrm{P} \leq 0.04)$ than chops determined to be dark (NPB color score 3 or $4 ; n=408$ and 27, respectively). Additionally, dark colored chops tended $(P=0.07)$ to have lower slice shear force values than light colored chops.

Conclusion: Grouping pork loins by the presence of a production claim had less influence over total post-packaging weight loss and slice shear force than evaluating subjective NPB color scores under controlled lighting. Subjective evaluation of color under controlled lighting can distinguish differences in weight loss and potentially objective tenderness, when chops are purchased from multiple retailers.

Keywords: color scoring, pork quality, production claim, retail benchmarking

\section{1 - EFFECTS OF FEEDING BREWERS GRAINS, MAIZE STARCH, AND OMEGA 3 FATTY ACIDS ON GROWTH PERFORMANCE, MEAT QUALITY ATTRIBUTES, AND BLOOD STRESS INDICATORS OF VEAL}

F. M. Giotto ${ }^{1 *}$, E. L. Shebs ${ }^{1}$, A. M. Cavender ${ }^{1}$, D. E. Gerrard $^{2}$, M. A. Fonseca ${ }^{1}$, and A. S. De Mello ${ }^{1}$,
${ }^{1}$ Agriculture, Veterinary, and Rangeland Sciences, University of Nevada, Reno, NV, USA, ${ }^{2}$ Animal and Poultry Sciences, Virginia Polytechnic Institute and State University, Blacksburg,VA, USA, *amilton@cabnr.unr.edu

Objectives: From to 2008 to 2018, the veal production in the United Sates decreased from 143 to 76 million pounds. Therefore, research focusing on developing and promoting veal production in the US is needed. In this study, we evaluated the effects of feeding starch and omega 3 oils on growth performance, blood stress indicators, $\mathrm{pH}$, tenderness, sensory attributes, and fatty acid profile of veal as a feeding strategy to improve animal welfare and overall veal quality.

Materials and Methods: Twenty-three veal calves approximately 2 mo old and $94.67 \pm 12.07 \mathrm{~kg}$ were randomly assigned to one of 3 dietary treatments (Control $n=$ 7, Starch, $n=8$, and Omega $3 n=8$ animals per treatment). Diets included the following: milk replacer, $200 \mathrm{~g}$ of brewers grain, and mineral supplement (Control); Control + maize starch (Starch); and Control $+3 \%$ of omega 3 (Omega 3). All animals were individually fed and offered ad libitum amounts of milk replacer. Diet composition changed among treatments mostly on ethereal extract and energy content. Calves were fed for $68 \mathrm{~d}$, and blood samples were collected during exsanguination for creatinine and cortisol analysis. After slaughter, hot carcass weight was recorded as well as weight after $24 \mathrm{~h}$ (cold carcass weight). Values of $\mathrm{pH}$ were assessed using a Hanna ${ }^{\circledR} \mathrm{pH}$ meter (Hanna Instruments, RI), and instrumental color $\left(L^{*}, a^{*}, b^{*}\right)$ was recorded by using a CR-400 Konica Minolta $₫$ (Konica Minolta Sensing Inc., Osaka, Japan) color reader. Approximately $24 \mathrm{~h}$ postmortem, the M. longissimus dorsi et lumborum was excised from the loins and aged for $14 \mathrm{~d}$. Steaks $(2.5 \mathrm{~cm})$ were fabricated and evaluated for proximate composition, fatty acid profile, Warner-Bratzler shear force, and sensory attributes. Data were analyzed as a completely randomized design using the GLIMMIX procedure of SAS (SAS Institute Inc., Cary, NC). When significance was detected at $P \leq$ 0.05 , means were separated using LSMEANS.

Results: Dietary treatments did not affect hot carcass weight, cold carcass weight, $\mathrm{pH}$, moisture, color, WarnerBratzler shear force, sensory attributes, and creatinine. Veal from calves fed Control and Omega 3 diets were significantly fatter than veal from calves fed Starch. Calves fed Starch and Omega 3 had lower levels of blood cortisol compared to 
calves fed Control. Veal from calves fed Control had the highest concentrations of $\mathrm{C} 20: 2 \mathrm{n} 6, \mathrm{C} 22: 4 \mathrm{n} 6$, and $\mathrm{C} 20: 3 \mathrm{n} 3$, whereas feeding Starch increased C18:2n6 and C20:4n6 compared to Omega 3 and increased C22:5n 3 compared to control. Veal fed Starch also showed higher total omega 6 when compared to veal fed Omega 3. Feeding Omega 3 increased the deposition of eicosapentaenoic acid (C20:5n3) and docosahexaenoic acid (C22:6n3) fatty acids.

Conclusion: Supplementing veal calves with maize starch and omega 3 oils does not affect growth performance and veal quality attributes. Animals fed supplemented diets had lower blood cortisol levels. Feeding milk replacer with brewers grains with or without maize starch increased omega 6 fatty acid deposition in the lean, whereas feeding Omega 3 favored deposition of eicosapentaenoic acid and docosahexaenoic acid. Feeding management is a viable strategy to decrease blood cortisol and manipulate nutritional values of veal.

Keywords: omega 3, quality, stress, veal

\section{2 - EFFECT OF FAT LEVEL ON PREMATURE BROWNING OF MARBLING BEEF}

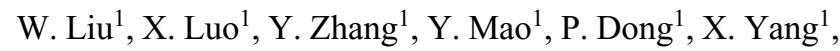
and R. Liang ${ }^{1 *}$, ${ }^{1}$ Food Science and Engineering, Shandong Agricultural University, Taian, China,*1rr327@126.com

Objectives: The problem of premature browning (PMB) leads to consumers' wrong judgment on the endpoint of cooking and can cause food safety problems. Highly marbled beef has more intramuscular fat, and the lipid oxidation is more severe during storage and cooking. Lipid oxidation will probably cause more PMB in cooked meat. However, very little of the literature can be found that focuses on the impact of lipid level on PMB in cooked beef. Therefore, the objective of this study was to investigate the effect of lipid level on PMB in marbled beef.

Materials and Methods: Longissimus lumborum muscles with 2 different Japanese marbling levels, A1 (Fat $\%=8.08 \pm 1.15)$ and A3 (Fat $\%=15.81 \pm 2.28)$, were collected from Angus $\times$ Black Waygu crossed cattle $(28 \mathrm{mo})$ in an abattoir. The cattle were fed with the same fodder using the same farming practices. Six Longissimus lumborum muscles were collected for each marbling level from 6 different cattle after grading, and 12 Longissimus lumborum were obtained in total. Then, the muscles were vacuum packaged and transported to the laboratory within $2 \mathrm{~h}$ in boxes on ice. Muscles were divided into steaks of $2.54 \mathrm{~cm}$, and all were packaged under high-oxygen packaging $\left(80 \% \mathrm{O}_{2}+20 \%\right.$ $\mathrm{CO}_{2}$ ) and stored in a dark environment of $0^{\circ} \mathrm{C}-4^{\circ} \mathrm{C}$ for $0 \mathrm{~d}$, $5 \mathrm{~d}$, and $10 \mathrm{~d}$. Four steaks from each cattle were prepared for each sampling day, and thus 72 steaks from 6 cattle were used in each treatment. On each day, the steaks were cooked to a central temperature of $55^{\circ} \mathrm{C}, 66^{\circ} \mathrm{C}$, and $72^{\circ} \mathrm{C}$,
Table 1. Effect of fat level on the cooked color ( $a^{*}$ values) of beef steaks

\begin{tabular}{|c|c|c|c|c|c|}
\hline \multirow[t]{2}{*}{ Grade } & \multirow[t]{2}{*}{$\begin{array}{c}\text { Center } \\
\text { temperature } \\
\left({ }^{\circ} \mathrm{C}\right) \\
\end{array}$} & \multicolumn{3}{|c|}{ Storage time (day) } & \multirow[t]{2}{*}{ SE } \\
\hline & & 0 & 5 & 10 & \\
\hline \multirow[t]{3}{*}{ A1 } & 55 & $14.65^{\mathrm{aAX}}$ & $14.31^{\mathrm{aAX}}$ & $11.26^{\mathrm{bAX}}$ & 0.2007 \\
\hline & 66 & $13.68^{\mathrm{aAX}}$ & $7.31^{\mathrm{bAY}}$ & $7.04^{\mathrm{bAY}}$ & \\
\hline & 72 & $10.54^{\mathrm{aAY}}$ & $6.85^{\mathrm{bAZ}}$ & $6.57^{\mathrm{bAY}}$ & \\
\hline \multirow[t]{3}{*}{ A3 } & 55 & $13.29^{\mathrm{aBX}}$ & $11.02^{\mathrm{bBX}}$ & $9.15^{\mathrm{cBX}}$ & 0.4439 \\
\hline & 66 & $10.99^{\mathrm{aBY}}$ & $6.51^{\mathrm{bAY}}$ & $6.52^{\mathrm{bBY}}$ & \\
\hline & 72 & $8.56^{\mathrm{aBZ}}$ & $6.59^{\mathrm{bAY}}$ & $6.38^{\mathrm{bAY}}$ & \\
\hline
\end{tabular}

${ }^{\mathrm{a}-\mathrm{b}}$ Within the same grade, the same temperature with different letter differ between storage times at $P<0.05$.

${ }^{\mathrm{A}-\mathrm{B}}$ Within the same storage time, the same temperature with different letter differ between fat levels at $P<0.05$.

${ }^{\mathrm{X}-\mathrm{Y}}$ Within the same storage time, the same fat level with different letter differ between different temperatures at $P<0.05$.

respectively, and the center cooked color was recorded immediately using an X-Rite spectrometer (SP62, $8 \mathrm{~mm}$ diameter aperture, Illuminant A, $10^{\circ}$ observer, Grand Rapids, MI).

Results: There was a significant interaction of fat level, storage time, and center temperature on the cooked color of $a^{*}$ values $(P<0.05$, Table 1$)$. As the central temperature increased, the central $a^{*}$ values of both fat levels decreased significantly. And with the extension of storage time, the $a^{*}$ values decreased as well. Significant differences were observed between 2 fat levels. On day 0 , the $a^{*}$ values were much lower in high fat level compared to the lower fat level. This indicated that steaks with a high fat level had more PMB problem even on day 0 of storage. When stored to day 5 and day 10, PMB was observed in steaks from both marbling levels, but it was more obvious in A3 steaks. The fat level showed no significant impact on $L^{*}$ values and $b^{*}$ values $(P<0.05$, data not shown).

Conclusion: Fat levels impacted PMB in high-oxygen packaging.

Acknowledgments: This work was supported by the fund of National Natural Science Foundation of China (31871731), China Agriculture Research System-beef (CARS-37), and Key R. and D. Plan of Shandong Province (2019GNC106050).

Keywords: $a^{*}$ value, fat level, high-oxygen packaging, marbling beef, premature browning

\section{3 - EFFECTS OF PACKAGING METHODS AND FROZEN TEMPERATURE ON THE COLOR OF FROZEN BEEF ROLLS}

F. Wang ${ }^{1}$, R. Liang ${ }^{1}$, Y. Zhang ${ }^{1}$, X. Luo ${ }^{1}$, and Y. Mao ${ }^{1 *}$, ${ }^{1}$ College of Food Science and Engineering, Shandong 
Agricultural University, Taian, China, *maoyanwei@ 163.com

Objectives: Beef rolls are popular among Chinese consumers, which makes them a widely used ingredient in hot pot meals. Beef rolls are usually stored and transported in a frozen state, during which the color deteriorates quickly due to freezing. Therefore, a number of storage methods and package methods for frozen beef rolls aiming to extend color shelf life were investigated.

Materials and Methods: Samples were obtained from the Longissimus lumborum muscle of 6 Chinese Luxi yellow cattle. The Longissimus lumborum were removed from carcasses at $48 \mathrm{~h}$ postmortem, and then frozen and cut into thin slices of $0.1 \mathrm{~cm}$ and rolled into solid beef rolls with a diameter of about $2 \mathrm{~cm}$. The slices were assigned randomly to 10 package $\times$ temperature groups $\left(50 \% \mathrm{O}_{2}: 50 \% \mathrm{O}_{2}+\right.$ $30 \% \mathrm{CO}_{2}+20 \% \mathrm{~N}_{2} ; 60 \% \mathrm{O}_{2}: 60 \% \mathrm{O}_{2}+40 \% \mathrm{~N}_{2} ; 80 \%$ $\mathrm{O}_{2}: 80 \% \mathrm{O}_{2}+20 \% \mathrm{CO}_{2}$; $\mathrm{CO}: 0.4 \% \mathrm{CO}+30 \% \mathrm{CO}_{2}+$ $69.6 \% \mathrm{~N}_{2}$ and overwrap packaging, stored at $-12^{\circ} \mathrm{C}$ or $-18^{\circ} \mathrm{C}$ ). The surface color of beef rolls was determined by using an X-Rite spectrophotometer (Model SP62, $4 \mathrm{~mm}$ diameter aperture, Illuminant $\mathrm{D}_{65}, 10^{\circ}$ observer, X-Rite, USA). The first measurement was carried out after blooming for $30 \mathrm{~min}$ at day 0 , and the other measurements were taken immediately in frozen state after opening packages at days 30, 90, 120, and 180 .

Results: Combined with the results in Table 1 and the consideration of economic factors, the following conclusions are drawn. When stored at $-12^{\circ} \mathrm{C}$ and the storage period is within $30 \mathrm{~d}$, it is recommended to use overwrap packaging, and when the storage period needs to reach $30-90 \mathrm{~d}, 60 \%$ $\mathrm{O}_{2}$ modified atmosphere packaging (MAP) is recommended, for CO MAP is forbidden in China. When stored at $-18^{\circ} \mathrm{C}$ and the storage period is within $90 \mathrm{~d}$, overwrap packaging is recommended, and when the storage period needs to reach $90-180 \mathrm{~d}, 60 \% \mathrm{O}_{2}$ MAP is recommended.
Conclusion: This study established a number of storage methods suitable for different conditions of frozen beef rolls in terms of color stability.

Keywords: color, frozen beef rolls, modified atmosphere packaging, shelf life

\section{4 - ANALYSIS OF FOAL MEAT SHELF LIFE UNDER VACUUM AND MAP PACKAGING CONDITIONS}

D. Franco ${ }^{1 *}$, R. Bermúdez ${ }^{1}$, P. E. S. Munekata ${ }^{1}$, L. CutillasBarreiro $^{1}$, L. Purriños ${ }^{1}$, and J. M. Lorenzo ${ }^{1},{ }^{1}$ Food Science and Technology, Centro Tecnológico de la Carne de Galicia, Ourense, Spain,*danielfranco@ceteca.net

Objectives: Horsemeat is characterized by low fat and cholesterol content, higher amounts of iron and vitamins of B group, as well as a favorable dietetic fatty acid profile and balanced protein profile. For this reason, horsemeat could be an alternative to conventional red meat, and it could play an important role. One of the main concerns of the meat industry is preserving food to extend its shelf life while ensuring its safety and quality. The aim of this preliminary study was to evaluate the effect of packaging under vacuum or modified atmosphere packaging (MAP) during $21 \mathrm{~d}$ at $4^{\circ} \mathrm{C}$ on main quality attributes (color, microbial spoilage, lipid oxidation, and odor) of fresh foal meat.

Materials and Methods: Five foals of Galician breed reared in an extensive system were slaughtered at 15 mo of age. Steaks of longissimus dorsi were stored in polystyrene tray and packaged with a gas mixture of $80 \% \mathrm{O}_{2} / 20 \% \mathrm{CO}_{2}$ (MAP group) and under vacuum conditions (VAC group) at $4^{\circ} \mathrm{C}$. Instrumental colorimeter parameters (luminosity $\left[L^{*}\right]$, redness index $\left[a^{*}\right]$, and percentage of metmyoglobin),

Table 1. Effects of packaging methods on $a^{*}$ values of beef rolls at different temperatures during storage

\begin{tabular}{|c|c|c|c|c|c|c|}
\hline \multirow[b]{2}{*}{ Storage temperature $\left({ }^{\circ} \mathrm{C}\right)$} & \multirow[b]{2}{*}{ Storage times $(\mathrm{d})$} & \multicolumn{5}{|c|}{ Packaging methods } \\
\hline & & $50 \% \mathrm{O}_{2}$ & $60 \% \mathrm{O}_{2}$ & $80 \% \mathrm{O}_{2}$ & $\mathrm{CO}$ & $\mathrm{OP}$ \\
\hline \multirow[t]{5}{*}{-12} & 0 & $22.27 \pm 0.38^{\mathrm{akx}}$ & $22.27 \pm 0.38^{\mathrm{akx}}$ & $22.27 \pm 0.38^{\mathrm{akx}}$ & $22.27 \pm 0.38^{\mathrm{akx}}$ & $22.27 \pm 0.38^{\mathrm{akx}}$ \\
\hline & 30 & $19.89 \pm 1.02^{\mathrm{akx}}$ & $20.05 \pm 0.74^{\mathrm{aklx}}$ & $15.67 \pm 0.98^{\text {bly }}$ & $17.81 \pm 1.53^{\mathrm{ablx}}$ & $18.73 \pm 1.38^{\mathrm{ablx}}$ \\
\hline & 90 & $15.82 \pm 1.94^{\mathrm{bclx}}$ & $17.98 \pm 1.52^{\mathrm{ablmx}}$ & $9.96 \pm 1.42^{\mathrm{dmy}}$ & $20.88 \pm 2.60^{\mathrm{aklx}}$ & $14.67 \pm 1.84^{\mathrm{cmx}}$ \\
\hline & 120 & $11.81 \pm 2.04^{\text {bcmy }}$ & $14.84 \pm 1.44^{\mathrm{bmx}}$ & $9.07 \pm 1.97^{\mathrm{cmy}}$ & $23.05 \pm 2.20^{\mathrm{akx}}$ & $11.28 \pm 1.40^{\text {cny }}$ \\
\hline & 180 & $11.14 \pm 2.07^{\mathrm{cmy}}$ & $15.05 \pm 1.86^{\mathrm{bmx}}$ & $10.87 \pm 2.16^{\mathrm{cmy}}$ & $20.39 \pm 2.39^{\mathrm{aklx}}$ & $12.49 \pm 1.76^{\mathrm{bcmnx}}$ \\
\hline \multirow[t]{5}{*}{-18} & 0 & $22.27 \pm 0.38^{\mathrm{akx}}$ & $22.27 \pm 0.38^{\mathrm{akx}}$ & $22.27 \pm 0.38^{\mathrm{akx}}$ & $22.27 \pm 0.38^{\mathrm{akx}}$ & $22.27 \pm 0.38^{\mathrm{akx}}$ \\
\hline & 30 & $20.29 \pm 0.99^{\text {aklx }}$ & $21.36 \pm 0.74^{\mathrm{akx}}$ & $21.18 \pm 0.96^{\mathrm{aklx}}$ & $16.83 \pm 1.53^{\mathrm{blx}}$ & $20.15 \pm 1.76^{\text {aklx }}$ \\
\hline & 90 & $18.33 \pm 1.10^{\text {almx }}$ & $19.39 \pm 0.86^{\mathrm{aklx}}$ & $18.11 \pm 0.84^{\text {almx }}$ & $17.64 \pm 2.29^{\text {alx }}$ & $16.70 \pm 0.90^{\mathrm{almx}}$ \\
\hline & 120 & $15.39 \pm 1.32^{\mathrm{amx}}$ & $16.90 \pm 0.96^{\text {alx }}$ & $15.55 \pm 1.76^{\mathrm{amx}}$ & $17.04 \pm 1.57^{\text {aly }}$ & $15.83 \pm 1.49^{\mathrm{amx}}$ \\
\hline & 180 & $15.75 \pm 0.43^{\mathrm{amx}}$ & $17.19 \pm 0.61^{\mathrm{alx}}$ & $16.90 \pm 0.49^{\mathrm{amx}}$ & $18.01 \pm 1.23^{\mathrm{alx}}$ & $15.50 \pm 0.87^{\mathrm{amx}}$ \\
\hline
\end{tabular}


microbial spoilage (counts of CFU mesophiles and enterobacteria), and lipid oxidation expressed as thiobarbituric acid reactive substances values were measured at $0,7,14$ and $21 \mathrm{~d}$ of storage. Additionally, an acceptance test with 12 trained panelists for discoloration and odor was carried out using a 5-point hedonic scale ( $1=$ excellent and $5=$ not acceptable). An analysis of variance was performed using the IBM SPSS Statistics 23.0 program (IBM Corp., Armonk, NY) and considering storage time and type of packaging as fixed effects. The least-squares means were separated using Duncan's post hoc test for a significance level $P<0.05$.

Results: Regarding instrumental color, there were significant differences between VAC and MAP packaging after $14 \mathrm{~d}$ of storage. Long-term storage strongly affected metmyoglobin and redness index of MAP samples which increased (159\%) and decreased (259\%) from 0 to $21 \mathrm{~d}$. Concerning lipid oxidation, foal steaks from the VAC group did not show significant $(P>0.05)$ changes during storage time, displaying values below $2 \mathrm{mg}$ malondialdehyde/ $\mathrm{kg}$ meat considered for several authors as rancidity threshold. On the contrary, the thiobarbituric acid reactive substances values of the MAP group increased significantly $(P<0.001)$ from 0.12 to $10.56 \mathrm{mg}$ malondialdehyde $/ \mathrm{kg}$ meat during storage time. With respect to microbial spoilage, there was no significant $(P>0.05)$ difference between the 2 types of packaging (VAC vs. MAP). As expected, the number of counts significantly raised as storage time increased in all cases. This increment was slower for enterobacteria than for mesophilic microorganisms, reaching average final values of 3.6 and $6.6 \log \mathrm{CFU} / \mathrm{g}$ at $21 \mathrm{~d}$ storage, respectively. Finally, sensory attributes of the acceptance test (discoloration at surface and odor) for the VAC group obtained scores within the acceptability range $(<3)$; meanwhile, MAP samples exceed this score for discoloration at surface and odor at seventh day of storage.

Conclusion: From the findings of this preliminary study, we can conclude that VAC is preferable to MAP packaging maintaining the color, avoiding the discoloration at the surface, and improving the odor acceptance and lipid rancidity.

Acknowledgments: This research received funding by INIA-Spain RTA2017-00081-C04-00. José M. Lorenzo and Daniel Franco are members of the HealthyMeat network, funded by CYTED (119RT0568). Thanks to GAIN (Axencia Galega de Innovación) for supporting this research (IN607A2019/01).

Keywords: foal, lipid oxidation, packaging, sensory analysis, spoilage

\section{5 - MATHEMATICAL MODELS WILL HELP YOU TO DETERMINE SHELF LIFE OF COOKED MEAT PRODUCTS}

\author{
L. Meinert ${ }^{1 *}$, A. G. Koch ${ }^{1}$, and T. Jacobsen ${ }^{1},{ }^{1}$ Food Safety, \\ Danish Meat Research Institute, Taastrup, Denmark, \\ *lme@dti.dk
}

Objectives: The aim was to develop mathematical models that describe the microbial development and sensory shelf life of cooked pork products. The hypothesis is that it is possible to estimate shelf life, when you know the number of bacteria in the cold cuts at the time of packaging combined with storage temperature and preservations used.

Materials and Methods: The dataset used to develop the models consists of 37 individual trial series in which approximately 2,000 packages of cold cuts were analyzed for psychrotrophic colony forming units and lactic acid bacteria. Furthermore, approximately 10,000 sensory assessments were made of the appearance, smell, and taste of the cold cuts by 5 expert assessors. The sensory assessment was based on scores from 1-4 (1-2 acceptable; 3-4 unacceptable)

The growth models: As primary model a logistic function of the form (see below in the Image section) was applied. Here, $\mathrm{I}_{0}$ is the aerobic initial counts in the used meat, and $t$ is the storage time in days. The variable $\phi$ constitutes the secondary model depending on water phase salt content, temperature, and whether nitrite and lactate were added.

The sensory models: The time dependency of all sensory profiles is all linear or slightly logistic. These profiles were modeled using a small neural network with 6 input neurons, 2 hidden neurons with logistic response functions, and a single output neuron. The 6 inputs were, nitrite $(\mathrm{Y} / \mathrm{N})$, lactate $(\mathrm{Y} / \mathrm{N})$, water phase salt, temperature, $\mathrm{I}_{0}$, and storage time. This neural network model incorporates both primary and secondary models.

Results: The results in Figure 1 show an example of a dataset used in the model. It can be seen from Figure 1 that, after $28 \mathrm{~d}$ of storage at $8^{\circ} \mathrm{C}$, some samples were assessed as unacceptable (above 2). At this time, the number of bacteria was between 7 and $8 \log \mathrm{CFU} / \mathrm{g}$. The psychrotrophic colony forming units reached a maximum after approximately $30 \mathrm{~d}$. At that time, the sensory assessments were above an average score of 2, which means that most of the assessors assessed the product as sensory unacceptable.

$$
\log (c f u(t))=8 /\left(1+\left(\frac{8}{\log \left(I_{0}\right)}-1\right) e^{-t} / \varphi\right.
$$

Conclusion: The data showed variations in the number of bacteria in the cold cuts packages, both between different products and within the same product, as expected. These variations are a part of the real life situation for meat producers when they determine end dates of their products. But even so, it is possible to predict shelf life of cooked meat products with the use of the mathematical models, which will be of great help for the quality managers in companies producing cooked meat products.

Find the shelf life model (and other predictive models) at DMRIPredict.dk. It is free of charge to use the models. 

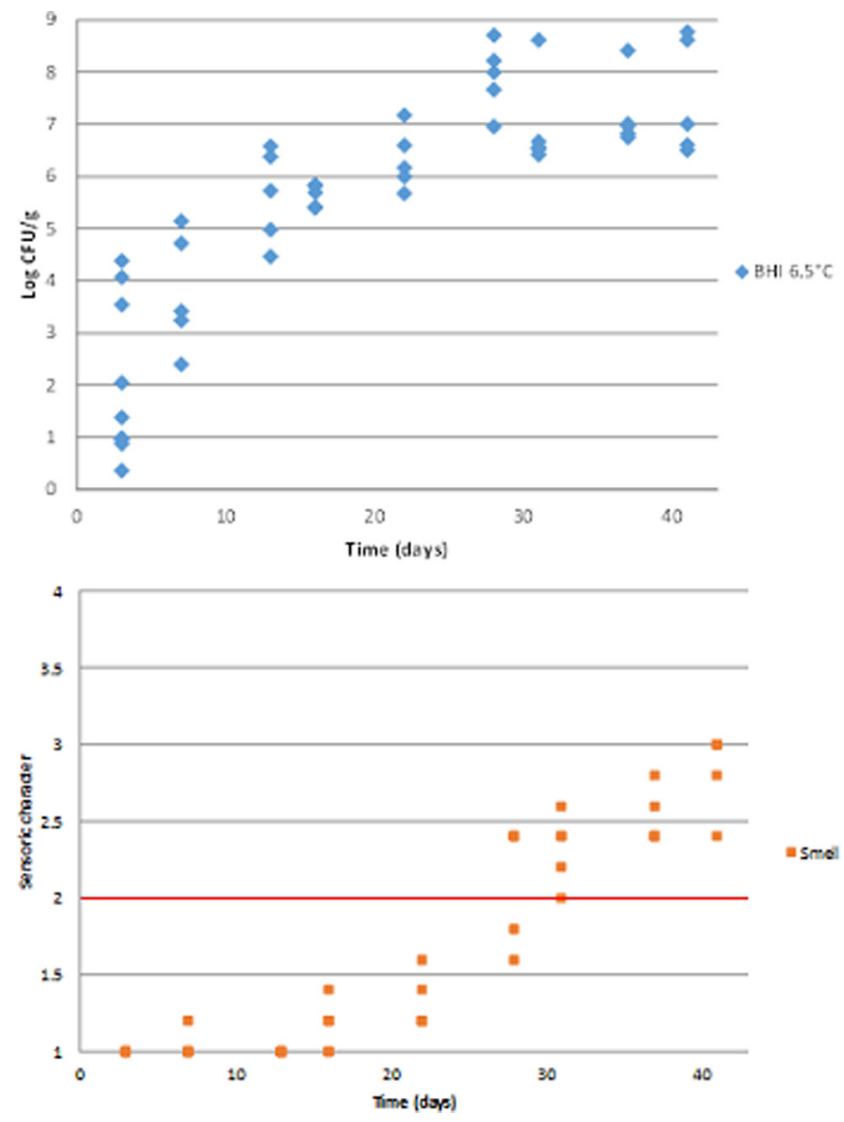

Figure 1. Microbial growth (psychrotrophic colony forming units grown in BHI-agar) and sensory development in sliced cold cuts $(2.2 \%$ salt/water, nitrite and lactate) stored at $8^{\circ} \mathrm{C}$. Initial counts: $2-24,000 \mathrm{cfu} /$ $\mathrm{g}$. The odour (smell) character is the mean value of 5 assessors' assessment of cold cuts from the same package.

\section{6 - MORPHOLOGICAL, COMPOSITIONAL AND MECHANICAL CHARACTERISTICS OF BELLIES FROM IMMUNOCASTRATED AND INTACT MALE PIGS}

\section{Font I Furnols ${ }^{*}$ Institute of Agrifood Research and Technology, Barcelona, Catalonia, Spain,*maria.font@ irta.cat}

Objectives: Firmness or softness of bellies is an important quality characteristic because it is related to fat quality, processing difficulties, and visual appearance. It depends on intrinsic and extrinsic production factors. Non-castration versus castration can affect fat content and composition and, consequently, firmness. There are few studies in which the effect of immunocastration over the quality of the bellies is studied, and it is worthwhile to study this effect when comparing to bellies from intact male pigs. The aim of the present work was to determine the effect of the noncastration versus the immunocastration of pigs on the morphological, compositional, and mechanical (firmness) characteristics of bellies.
Materials and Methods: A total of 17 bellies were used from intact male (EM) and 13 from immunocastrated (IM) pigs fed the same diet. Bellies were cut using as a reference anatomical points of the carcass and weighted. Length and width were measured in the central part. Fat content was measured with dual X-ray equipment. Firmness was determined by means of the flop distance and angle measured skin-side up and skin-side down using the bar-suspension method. The height of each belly was measured skin-side up in the center of the dorsal side. Then the skin was stretched with tweezers until the base of the belly gets up and the height was measured. The difference between these 2 heights is calculated as a measure of firmness in terms of fat separation. Subjective firmness was also measured by 2 trained technicians, applying pressure with a finger, using a 5-point scale from 1 (very firm) to 5 (very soft). Analysis of variance was performed with SAS software (version 9.4; SAS Institute Inc., Cary, NC) including the castration condition (non-castrated and immunocastrated) as fixed effect.

Results: No significant differences in bellies' weight, width, and thickness were found between IM and EM. The length was significantly $(P<0.05)$ higher in IM than EM bellies. As expected, fatness was higher in IM than EM since IM pigs behave as surgically castrated after the second dose of the immunocastration vaccine. Flop distance and angle, both skin-side up and skin-side down, were significantly higher in bellies from IM than those from EM, indicating that IM bellies were firmer. This was also confirmed with significantly lower measure of fat separation and subjective firmness value in the center of the cranial side of the bellies.

\begin{tabular}{|c|c|c|c|c|}
\hline & Intact males & Immunocastrated & RMSE & P-value \\
\hline $\mathrm{n}$ & 17 & 13 & & \\
\hline Weight (kg) & 44 & 4.7 & 0.56 & 0.204 \\
\hline Width $(\mathrm{cm})$ & 24.0 & 23.6 & 1.34 & 0494 \\
\hline Length (cm) & 42.1 & 45.9 & 2.57 & 0.001 \\
\hline Thickness $^{1}(\mathrm{~cm})$ & 3.9 & 3.7 & 0.57 & 0.322 \\
\hline Fat $(\%)$ & 29.3 & 33.9 & 4.29 & 0.008 \\
\hline \multicolumn{5}{|l|}{ Flop distance $(\mathrm{cm})$} \\
\hline Skin-side up & 13.9 & 18.8 & 4.88 & 0.010 \\
\hline Skin-side down & 16.2 & 22.5 & 6.28 & 0.011 \\
\hline \multicolumn{5}{|l|}{ Flop angle $\left(^{\circ}\right)$} \\
\hline Skin-side up & 38.5 & 48.3 & 12.32 & 0.040 \\
\hline Skin-side down & 45.6 & 59.8 & 18.25 & 0.044 \\
\hline \multicolumn{5}{|l|}{ Firmness $^{2}$} \\
\hline Fat separation $(\mathrm{cm})$ & 2.0 & 1.5 & 0.45 & 0.013 \\
\hline Pressure $^{3}$ & 4.3 & 3.6 & 0.57 & 0.005 \\
\hline
\end{tabular}

${ }^{1}$ Measured in the center of the dorsal side

${ }^{2}$ Measured in the center of the cranial side

${ }^{3} 5$-point scale from livery firm to 5 :very soft 
Conclusion: Firmness of the bellies is affected by the castration condition of the animals. In this sense, bellies from IM pigs are firmer and fattier than those from EM pigs. More work is needed to determine the effect of immunocastration on fat composition.

Keywords: fat content, firmness, flop angle, flop distance

\section{8 - POTENTIAL OF 2D QUANTITATIVE NUCLEAR MAGNETIC RESONANCE (2D qNMR) SPECTROSCOPY ANALYSIS FOR CHICKEN BREAST MEAT METABOLITES}

H. C. Kim ${ }^{1 *}$, Y.-J. $\mathrm{Ko}^{2}$, K. H. Baek ${ }^{1}$, and C. Jo ${ }^{1,3}$, ${ }^{1}$ Department of Agricultural Biotechnology, Center for Food and Bioconvergence, and Research Institute of Agriculture and Life Science, Seoul National University, Seoul, Republic of Korea, ${ }^{2}$ National Center for InterUniversity Research Facilities, Seoul National University,
Seoul, Republic of Korea, ${ }^{3}$ Institute of Green Bio Science and Technology, Seoul National University, Pyeongchang, Republic Of Korea, *eoenhc@naver.com

Objectives: Acquiring accurate metabolic information is important to understand and/or elucidate biological changes in meat samples. Among the different quantitative analyses, one-dimensional (1D) $1 \mathrm{H}$ nuclear magnetic resonance (NMR) analysis is rapid, reproducible, and simultaneous for various metabolites without derivatization. However, despite these advantages, $1 \mathrm{D}{ }^{1} \mathrm{H}$ NMR analyses still need to overcome the problem of chronic overlap, which is critical in cases of mixtures such as meat extracts. For this problem, the application of two-dimensional quantitative nuclear magnetic resonance (2D qNMR) can be helpful to reduce overlap issues via its dimensional expansion. Hence, in this study, 2D qNMR analytical methods were applied for metabolite analysis in meat, investigating its potential in comparison to high-performance liquid chromatography (HPLC) and 1D ${ }^{1} \mathrm{H}$ NMR.

Table 1. Metabolite identification and quantification of chicken breast meat from HPLC, ${ }^{\mathrm{H}} \mathrm{H}$ NMR, and 2D HSQC spectra

\begin{tabular}{|c|c|c|c|c|}
\hline \multirow{2}{*}{ Compound } & HPLC & ${ }^{1} \mathrm{H}$ NMR & 2D HSQC & \multirow{2}{*}{$R^{2,1)}$} \\
\hline & \multicolumn{3}{|c|}{$(\mathrm{mg} / \mathrm{kg})$} & \\
\hline Alanine & $304.80=8.24$ & $308.88 \pm 5.08$ & $306.58=14.48$ & 0.9964 \\
\hline Arginine & $215.83 \pm 8.03^{x}$ & $\mathrm{nd}^{2]}$ & $85.36=5.23^{b}$ & 0.9957 \\
\hline Asparagine & $77.67 \pm 5.08^{2}$ & $76.10 \pm 2.38^{a}$ & $44.82=4.22^{b}$ & 0.9954 \\
\hline Aspartic acid & $181.26 \pm 12.00$ & $186.05 \pm 2.45$ & $170.75 \pm 6.11$ & 0.9972 \\
\hline Glutamate & $324.28=9.03$ & $525.65=5.12$ & $363.23=16.43$ & 0.9955 \\
\hline Proline & $116.81=23.91^{b}$ & $(\mathrm{Glu}+\text { Pro })^{3)}$ & $158.85 \pm 3.37^{2}$ & 0.9678 \\
\hline Glutamine & $269.27 \pm 11.57^{b}$ & $269.11 \pm 1.32^{b}$ & $430.98=12.20^{x}$ & 0.9950 \\
\hline Glycine & $214.95=4.79$ & $216.57 \pm 6.42$ & $222.36=14.49$ & 0.9950 \\
\hline Histidine & $178.23 \pm 3.61^{2}$ & nd & $126.59 \pm 3.76^{b}$ & 0.9942 \\
\hline Isoleucine & $82.65 \pm 1.45^{c}$ & $100.81 \pm 2.46^{x}$ & $92.40=1.42^{b}$ & 0.9900 \\
\hline Leucine & $165.09 \pm 1.31^{2}$ & $168.49 \pm 3.02^{\mathrm{a}}$ & $153.85 \pm 6.01^{b}$ & 0.9939 \\
\hline Lysine & $87.98 \pm 6.17$ & nd & $68.74 \pm 13.14$ & 0.9957 \\
\hline Methionine & $73.00 \pm 1.01$ & $73.08 \pm 3.72$ & $75.00=0.92$ & 0.9912 \\
\hline Phenylalanine & $87.87 \pm 0.86^{b}$ & $89.43 \pm 0.28^{b}$ & $151.52=14.72^{2}$ & 0.9964 \\
\hline Serine & $197.97=5.54$ & $\mathrm{Nd}$ & $193.15=11.37$ & 0.9981 \\
\hline Tryptophan & $56.27 \pm 2.59$ & $\mathrm{Nd}$ & $58.94=9.80$ & 0.9968 \\
\hline Tyrosine & $135.63 \pm 1.25^{2}$ & $135.04 \pm 6.52^{\mathrm{s}}$ & $112.70 \pm 4.94^{b}$ & 0.9969 \\
\hline Valine & $125.70 \pm 2.37^{x}$ & $127.10 \pm 2.42^{x}$ & $83.32=3.35^{b}$ & 0.9955 \\
\hline
\end{tabular}

E- Mean values ( $\mathrm{n}=3$ ) with different letters within the same row differ significantly $(\mathrm{p}<0.05)$

1) $R^{\prime}: R$-squared was calculated based on HSQC using artificial standard mixture

${ }^{2}$ nd : not defected in the $1 \mathrm{D}^{1} \mathrm{H}$ spectrum.

3) 1D 'H NMR data of both glutamate and proline were excluded from the calculation. 
Materials and Methods: Chicken breasts meat were purchased from a local market and pooled prior to the analyses. The breast meat $(5 \mathrm{~g})$ was extracted with $0.6 \mathrm{M}$ perchloric acid, neutralized with potassium hydroxide, and lyophilized for elimination of the water. Lyophilized samples were reconstituted with $1 \mathrm{~mL}$ of $20 \mathrm{mM}$ phosphate buffer in $\mathrm{D}_{2} \mathrm{O}$ solution (1 mM 3-(trimethylsilyl)propionic acid, $\mathrm{pH}$ 7.0) and applied for $1 \mathrm{D}^{1} \mathrm{H}$ NMR, 2D qNMR (heteronuclear single nuclear quantum coherence [HSQC]), and HPLC. Prior to setting up 2D qNMR, artificial free amino acid mixture was prepared manually and acquired on HSQC for quantification. All results were triplicated, and statistical analysis was performed using the procedure of the general linear model. Significance of differences among mean values was determined by a Student-Newman-Keul test $(P<0.05)$.

Results: A total of 18 free amino acids were found in chicken breast meat extracts using 3 different analyses (Table 1). Compared to HPLC and HSQC, $1 \mathrm{D}{ }^{1} \mathrm{H}$ NMR showed good precision with lower variation. However, some metabolites (arginine, glutamate, proline, histidine, lysine, serine, and tryptophan) were quantified in $1 \mathrm{D}{ }^{1} \mathrm{H}$ NMR because of its overlap. Unlike 1D ${ }^{1} \mathrm{H}$ NMR, HSQC could qualify and quantify all metabolites without overlap. Different quantification in 2D qNMR was observed in the contents of arginine, proline, glutamine, histidine, leucine, phenylalanine, tyrosine, and valine, while other free amino acids were not different when compared to the conventional HPLC method $(P<0.05)$. This inconsistency can be improved by further optimization as it is possibly due to difference of ionic strength between breast meat extracts and artificial standard mixture, different $90^{\circ}$ pulse (p1) of metabolites, and/or NMR acquisition parameters. In addition, although quantification of was not consistent on HSQC, standard of each free amino acid showed good linearity ( $R^{2}=0.97$ in proline and $R^{2}>0.99$ in others).

Conclusion: Based on the results, 2D qNMR can be helpful in acquiring interactive and accurate information, which could be advantageous when compared to traditional chromatographic analysis. However, further optimization is needed for more accurate numerical quantification.

Keywords: one-dimensional quantitative nuclear magnetic resonance, two-dimensional quantitative nuclear magnetic resonance, chicken breast, high-performance liquid chromatography, heteronuclear single nuclear quantum coherence

\section{9 - SOW CLASSIFICATION BASED ON WEIGHT AND PARITY AFFECTS PORK QUALITY}

\author{
J. J. Nehls ${ }^{1 *}$, K. E. Hwang ${ }^{1}$, and J. R. Claus ${ }^{1},{ }^{1}$ Animal \\ Sciences, University of Wisconsin, Madison, WI, USA, \\ *jrclaus@wisc.edu
}

Objectives: The objective of this study was to determine the effects of sow classification used by the industry based on a combination of slaughter weight and parity on various meat quality attributes.

Materials and Methods: Six sows representing 2 commercial classification groups (low weight $[\mathrm{LW}]=155-165 \mathrm{~kg}$; high weight $[\mathrm{HW}]=278-308 \mathrm{~kg}$ ) were harvested. The weight classes were further controlled by age and parity (LW average age $=1 \mathrm{y}$, average parity $=1$; HW average age $=3.8 \mathrm{y}$, average parity $=8$ ). Sows were electrically stunned, bled, skinned, eviscerated, cut into anatomical lateral sides, and fabricated ( 45 min postmortem). One side was sampled for waterholding capacity (WHC) and $\mathrm{pH}$. The other side was hotboned, and the meat was ground through a $3 / 4$ " plate. The ground meat was mixed with salt $(1.5 \%$ meat weight basis) and dry ice (28\% meat weight basis) and stored ( $3 \mathrm{~d})$ before being re-ground (5/32" plate). Ground samples were homogenized and then analyzed for moisture, fat, and protein. Additional samples $(\sim 150 \mathrm{~g})$ were placed on Styrofoam trays, overwrapped with polyvinyl chloride, and continuously displayed $\left(1,615 \operatorname{lux}, 3^{\circ} \mathrm{C}\right)$. Color measurements were taken on 1,4 , and $7 \mathrm{~d}$ (CIE $L^{*} a^{*} b^{*}$; reflectance: chemical states of myoglobin). Oxygen consumption (OC) and metmyoglobin reductase activity (MRA) were calculated using a reflectance method. Ability of the meat to hold water was measured using a centrifugal method (expressible moisture [EM]\%) on ground meat and Carver Press (WHC \%) on Longissimus lumborum (LL) and Semimembranosus (SM) muscle samples. Ground meat samples premixed with $40 \%$ water added (meat weight basis) prior to being centrifuged $\left(15,000 \mathrm{G}, 5 \mathrm{~min}, 4^{\circ} \mathrm{C}\right)$ were used to determine EM\%. Patties ( $\sim 125 \mathrm{~g}$ each) were prepared and cooked in an oven until $160^{\circ} \mathrm{F}$, cooled $(30 \mathrm{~min})$, and weighed for cook loss. Animal served as the experimental unit. Data were analyzed using PROC MIXED procedures (SAS Institute Inc., Cary, NC), and significance was set at $P<0.05$.

Results: Ground meat from LW compared to HW had more moisture $(68.59 \%$ vs. $66.42 \%, P<0.05)$, less fat $(8.14 \%$ vs. $10.57 \%, P<0.05)$, and slightly lower protein $(20.83 \%$ vs. $21.19 \%, P<0.05)$. During lighted display, the HW ground meat had lower $L^{*}$ values, higher $a^{*}$ values, and greater oxymyoglobin $(P<0.05)$ than LW. Also, the $L^{*}$ and $a^{*}$ values decreased while the metmyoglobin estimate increased with increasing display time $(P<0.05)$. The calculated $\mathrm{OC}$ was higher $(P<0.05)$ in the $\mathrm{HW}$ ground meat compared to LW, but no differences were found in MRA. WHC $\%$ was greater $(P<0.05)$ in HW than LW for both the $S M$ and $L L$. In contrast EM\% was not affected $(P>0.05)$ by weight class. The $\mathrm{pH}$ of HW (5.74) was higher than LW (5.55). In addition, the $\mathrm{pH}$ of the $S M$ (5.77) was higher $(P<0.05)$ than in the $L L(5.52)$. The cook loss percentage was almost identical for the HW and LW patties, and the HW cooked patties were noticeably darker in color.

Conclusion: Sow classification, based on weight relative to age and parity, had a significant effect on moisture content, fat content, OC, WHC percentage and meat color during lighted display. However, sow classification did 
not have an influence on the protein content, MRA, EM\%, $\mathrm{pH}$, and cook loss. Time in lighted display had a significant effect on meat color. The results serve as valuable information for pork harvest facilities making decisions on which sow classification provides the most positive impact on meeting consumer quality expectations.

Keywords: meat quality, parity, pre-rigor, slaughter weight, sows

\section{0 - EFFECT OF SUPPLEMENTATION WITH MANGANESE AND CHONDROITIN SULPHATE ON CARCASS TRAITS AND BROILER MEAT QUALITY}

J. A. Muñoz ${ }^{*}$, T. S. Martins ${ }^{2}$, T. A. J. Sant'Ana², P. L. M. Garbossa $^{2}$, L. F. Mueller ${ }^{1}$, A. M. Ferrinho ${ }^{1}$, G. V. de Moura ${ }^{1}$, I. H. S. Fuzikawa ${ }^{1}$, J. L. Gemelli ${ }^{1}$, T. R. Amorim ${ }^{1}$, H. C. Rocha $^{2}$, L. B. Ferreira ${ }^{1}$, C. B. Barbalho ${ }^{1}$, M. M. da Silva ${ }^{1}$, M. D. Poleti ${ }^{1}$, and A. S. C. Pereira ${ }^{2},{ }^{1}$ Department of Animal Science, Faculty of Animal Science and Food Engineering, University of Sao Paulo, São Paulo, Brazil, ${ }^{2}$ Department of Animal Nutrition and Production, School of Veterinary Medicine and Animal Science, University of São Paulo, Pirassununa, Brazil, *julianmunoz@usp.br

Objectives: The goal of this study was to verify whether different levels of chondroitin sulphate (CS) and manganese (Mn) could influence the presence of myopathy, macroscopic measurements of breast, carcass traits, and quality of broiler breast meat.

Materials and Methods: A total of 1,152 male Cobb chicks were housed during $47 \mathrm{~d}$ and distributed in a completely randomized design, in a $4 \times 3$ factorial arrangement: 4 doses of CS $(0.00 \%, 0.06 \%, 0.12 \%$, and $0.18 \%)$ and 3 doses of $\mathrm{Mn}(0,40$, and $80 \mathrm{mg} / \mathrm{kg})$, totaling 12 treatments of 8 repetitions with 12 birds each. In the slaughter process, one breast was collected per experimental unit to verify the presence of white striping myopathy and to perform macroscopic analysis (area, width, and length). After $24 \mathrm{~h}$ of chilling, the carcasses were weighed to calculate the yield. During boning, the breasts, thighs, drumsticks, and wings were weighed to calculate the yield of the cuts. Then, 3 breasts per experimental unit were collected to determine the meat quality by analyzing $\mathrm{pH}$, color (CIELAB system: $L^{*}, a^{*}$, and $b^{*}$ ), cooking loss, and shear force. The results were submitted to analysis of variance and the means compared by the Tukey test at $5 \%$ probability.

Results: It was observed that CS and Mn supplementation levels did not influence $(P>0.05)$ carcass yields, macroscopic breast characteristics, nor the cooking loss, shear force, and luminosity $\left(L^{*}\right)$ of broiler breasts. However, there was an effect $(P<0.05)$ on breast yield, with the highest percentages presented by the birds supplemented with $0.18 \%$
CS compared to the breasts of the diet without CS inclusion. There was interaction $(P<0.05)$ for thighs-drumsticks and wings yield, in which the highest yields for thighs and drumsticks were presented by diets without the inclusion of additives (CS and $\mathrm{Mn}$ ) and it was evident that the different relationships between CS and Mn levels did not provide increases in thigh and drumstick yields. For the wings, the diet of greatest prominence was that formulated from $0.18 \%$ of CS and $80 \mathrm{mg} / \mathrm{kg}$ of $\mathrm{Mn}$, since it presented the highest yields of wings. There was an effect $(P<0.05)$ of the CS levels in $a^{*}$ values, where the highest levels of red were present in the breasts from $0.18 \%$ CS diet compared to those of the diet without added CS. The $\mathrm{pH}$ and $b^{*}$ values were influenced by Mn levels $(P<0.05)$. The breasts from $0 \mathrm{mg} / \mathrm{kg}$ Mn diet had a higher $b^{*}$ than the breasts of chickens that consumed the diet with $80 \mathrm{mg} / \mathrm{kg} \mathrm{Mn}$. The $40 \mathrm{mg} / \mathrm{kg} \mathrm{Mn}$ diet reached the highest $\mathrm{pH}$ values concerning the other $\mathrm{Mn}$ inclusion levels. Regarding the presence of myopathies, it was found that, regardless of CS or Mn levels, the breasts showed moderate white striping myopathy. However, it is noteworthy that the control diet without the inclusion of $\mathrm{CS}$ and $\mathrm{Mn}$ provided the breasts with the largest striation sizes $(0.78 \mathrm{~mm})$ when compared to the striations of the breasts of the diets of $0.18 \% \mathrm{CS}$ and $80 \mathrm{mg} / \mathrm{kg} \mathrm{Mn}(0.64 \mathrm{~mm})$ and the diet of $40 \mathrm{mg} / \mathrm{kg} \mathrm{Mn}(0.64 \mathrm{~mm})$.

Conclusion: The levels of CS and Mn were not sufficient to provide improved carcass traits and quality of breast meat. However, the levels of $0.18 \% \mathrm{CS}$ and $80 \mathrm{mg} / \mathrm{kg} \mathrm{Mn}$ may result in an interesting alternative for use in chicken feeding because it interacts with breast yields and the size of white striations, two aspects that influence the consumer in the initial selection and final satisfaction with the product.

Keywords: broiler chicken, glycosaminoglycans, mineral, myopathy

\section{1 - PACKAGING TYPE IMPACTS PROTEIN DEGRADATION AND FREE AMINO ACID PRODUCTION DURING POSTMORTEM STORAGE IN BEEF STEAKS}

K. R. Vierck ${ }^{1}$, J. F. Legako ${ }^{1}$, J. Kim²,3, B. J. Johnson ${ }^{1}$, and J. C. Brooks ${ }^{1},{ }^{1}$ Department of Animal and Food Sciences, Texas Tech University, Lubbock, TX, USA, ${ }^{2}$ Department of Animal Science, Texas Tech University, Lubbock, TX, USA, ${ }^{3}$ Department of Food Science and Human Nutrition, Michigan State University, East Lansing, MI, USA, *kelly. vierck@ttu.edu

Objectives: The objective of this study was to determine the influence of packaging type on postmortem degradation and production of free amino acids in beef steaks.

Materials and Methods: Beef strip loins and top sirloin butts were chosen from USDA Choice carcasses $(n=40,20$ / 
subprimal). Subprimals were fabricated into 2.54-cm steaks $7 \mathrm{~d}$ postmortem and randomly assigned to packaging types: carbon monoxide motherbag $(\mathrm{CO})\left(0.4 \% \mathrm{CO} / 30 \% \quad \mathrm{CO}_{2} /\right.$ $69.6 \% \mathrm{~N}_{2}$ ), high-oxygen modified atmosphere packaging ( $\mathrm{HIOX})\left(80 \% \mathrm{O}_{2} / 20 \% \mathrm{CO}_{2}\right.$ ), and rollstock (ROLL). Steaks designated for the polyvinyl chloride overwrap (OW) treatment were placed in ROLL treatment until retail display. A steak was also removed from each subprimal and immediately frozen at $-20^{\circ} \mathrm{C}$ with no packaging or aging treatment applied. Steaks were aged in the dark for $14 \mathrm{~d}$, then displayed in coffin-style cases for $48 \mathrm{~h}$ under continuous fluorescent lighting. Steaks were then vacuum packaged and frozen at $-20^{\circ} \mathrm{C}$. Prior to analysis, steaks were homogenized in liquid nitrogen. To quantify degradation of desmin and troponin-T (TnT), Western blot analysis was conducted using sodium dodecyl sulfate-polyacrylamide gel electrophoresis and immunofluorescence staining for imaging. Desmin was measured at bands located at $55 \mathrm{kDA}$, and TnT was measured at bands at $30 \mathrm{kDA}$. Free amino acid analysis was conducted using gas chromatography-mass spectrometry following derivatization and separation. Data were analyzed as a $2 \times 4$ factorial design with packaging, muscle, and their interaction serving as fixed effects, and gel was incorporated into the model as a random effect for Western blot analysis.

Results: Desmin degradation was impacted by packaging type $(P<0.001)$. Initial samples taken at $7 \mathrm{~d}$ postmortem and HIOX samples possessed $(P<0.05)$ the greatest relative intensity of desmin compared to all other packaging types, which indicates a higher concentration of desmin and less degradation during postmortem aging. No differences were observed in TnT degradation for packaging type $(P=0.442)$. Six amino acids (alanine, cystine, glycine, proline, tyrosine, and valine) were impacted $(P \leq$ $0.01)$ by both the main effects of packaging and muscle. For all 6 amino acids, longissimus lumborum steaks possessed a greater $(P<0.05)$ concentration of each respective amino acid compared to gluteus medius steaks. Tyrosine was present $(P<0.05)$ in the highest concentration in HIOX steaks compared to ROLL steaks. Additionally, $7 \mathrm{~d}$ initial samples possessed the lowest $(P<0.05)$ concentration of all free amino acids, likely the result of limited postmortem proteolysis during aging. The majority of free amino acids $(n=12)$ were impacted solely by the packaging main effect $(P<0.04)$. Initial samples exhibited the lowest concentration of amino acids compared to all other treatments, with the exception of histidine $(P<$ 0.05). ROLL and OW steaks possessed $(P<0.05)$ the greatest concentration of the remaining free amino acids, followed by CO steaks $(P<0.05)$ and then HIOX steaks $(P<0.05)$. Histidine was present $(P<0.05)$ in greater concentrations in HIOX steaks in comparison to ROLL, CO, and initial steaks.

Conclusion: These results indicate that packaging type has a strong influence over postmortem proteolysis and production of important flavor precursors, such as free amino acids. With retail transitioning to more case-ready products, processors should consider anaerobic packaging to optimize tenderness and flavor.

Keywords: aging, desmin, flavor, free amino acids, packaging

\section{2 - VASCULAR RINSING AND CHILLING EFFECTS ON MEAT QUALITY ATTRIBUTES FROM CULL DAIRY COWS WITH DIFFERENT BODY CONDITION SCORES AND WITH DIFFERENT CHILLING TEMPERATURES}

S. C. Kethavath ${ }^{1 *}$, K. E. Hwang ${ }^{1}$, M. A. Mickelson ${ }^{2}$, R. E. Campbell $^{2}$, and J. R. Claus ${ }^{1},{ }^{1}$ Animal Sciences, University of Wisconsin, Madison, WI, USA, ${ }^{2}$ Technical Services, MPSC Inc., Hudson, WI, USA, *jrclaus@wisc.edu

Objectives:Experiments were conducted to determine the meat quality effects of vascularly rinsing (Rinse \& Chill ${ }_{\circledR}$ [RC]) carcasses from commercially harvested cull dairy cows with different body condition scores (BCS) and the impact of 2 different $\mathrm{RC}$ rinse solution temperatures (ST).

Materials and Methods: Immediately after exsanguination, carcasses with 2 different BCS (Lean, Light) were conventionally chilled ( $\mathrm{CN} ; n=15$ each $\mathrm{BCS})$ or vascularly rinsed (RC; $n=18$ each $\mathrm{BCS})$. RC carcasses were vascularly rinsed with a chilled isotonic solution $\left(3^{\circ} \mathrm{C}\right.$; 98.5\% water; balance: glucose, polyphosphates, maltose). Carcass temperature (0-24 h) and $\mathrm{pH}(1,4,8,12,24 \mathrm{~h})$ were recorded. On 4 d postmortem, muscles (Longissimus lumborum [LL]; Triceps brachii [TB]) were ground (3 mm plate), packaged (polyvinyl chloride, vacuum), and displayed continuously $\left(3^{\circ} \mathrm{C}, 1,615 \mathrm{lux} ; 1,4,7 \mathrm{~d}\right)$, or stored in the dark. In the $\mathrm{ST}$ experiment, $\mathrm{RC}$ carcasses were vascularly rinsed (RCT3; solution $3{ }^{\circ} \mathrm{C} ; n=13$; RCT14; solution $\left.14^{\circ} \mathrm{C} ; n=15\right)$. Carcass temperature $(0-24 \mathrm{~h})$ and $\mathrm{pH}$ $(1,4,8,12,24 \mathrm{~h})$ were recorded. At $2 \mathrm{~d}$ postmortem, muscles were processed and packed as in the BCS experiment. Color, chemical states of myoglobin, purge, moisture, fat content, total pigments, water-holding capacity, oxygen consumption, and lipid oxidation measurements were determined. Data from each experiment were analyzed separately using PROC MIXED procedures (SAS Institute Inc., Cary, NC). Significance was set at $P<0.05$.

Results: $\mathrm{RC}$ resulted in a lower $\mathrm{pH}$ at each time postmortem than $\mathrm{CN}$ with an overall average of 5.93 and 6.30 , respectively. Generally, RC resulted in lower temperatures during chilling ( $24 \mathrm{~h})$. After $1 \mathrm{~h}$ of bloom, RC was more red (CIE $\left.a^{*}, 22.1\right)$ than $\mathrm{CN}(19.9)$. CIE $a^{*}$ values pooled over display times found that $\mathrm{RC}$ was redder (Lean, 16.9; Light, 16.0) than $\mathrm{CN}$ (Lean, 13.6; Light, 14.1). RC resulted in more oxymyoglobin than in the $\mathrm{CN}$ Lean. RC did not affect moisture fat-free (MFF) percent in 
LL. However, in the TB, MFF was greater in the RC by $1.4 \%$ (Light) and $2.0 \%$ (Lean) than CN. RC Lean LL had less purge loss compared to $\mathrm{CN}$ Lean. $\mathrm{RC}$ resulted in greater total pigments than the $\mathrm{CN}$. RC ground $\mathrm{TB}$ had greater oxygen consumption than $\mathrm{CN}$. RC had lower thiobarbituric acid reactive substances compared to $\mathrm{CN}$. In the ST experiment, $\mathrm{RCT} 3$ and RCT14 influenced $\mathrm{pH}$ decline during the first $12 \mathrm{~h}$ postmortem. In the LL, both RC treatments had a more rapid temperature decline than $\mathrm{CN}$ through $4 \mathrm{~h}$ postmortem and through $8 \mathrm{~h}$ postmortem for RCT14. RCT14 SM resulted in a more rapid temperature decline than RCT3. No differences were found in MFF, and total pigments. RC ground TB had greater expressible moisture and greater purge. RCT3 and RCT14 produced greater redness (CIE $a^{*}$ ) associated with blooming and display times. RCT3 and RCT14 ground beef resulted in greater oxymyoglobin. $\mathrm{RC}$ ground beef had less metmyoglobin during display.

Conclusion: Cull dairy cows often represent stress-susceptible animals, which negatively affects meat quality. RC could improve color, potentially decrease the incidence of dark-cutting beef, and reduce lipid oxidation. Differences in rinse ST may influence meat quality outcomes. The warmer RC ST had the ability to chill carcasses faster and may contribute to an extension in the shelf life of meat.

Keywords: carcass chilling method, cull dairy cows, meat color, meat quality

\section{3 - DETERMINING BEEF LONGISSIMUS LUMBORUM STEAK COLOR LIFE THRESHOLD AND EFFECT OF POSTMORTEM AGING TIME USING META-ANALYSIS}

F. Najar ${ }^{\text {* }}$, E. Boyle ${ }^{1}$, C. Vahl ${ }^{2}$, Q. Kang ${ }^{2}$, J. Kastner ${ }^{1}$, J. Amamcharla $^{1}$, and M. Hunt ${ }^{1},{ }^{1}$ Animal Science and Industry, Kansas State University, Manhattan, KS, USA, ${ }^{2}$ Department of Statistics, Kansas State University, Manhattan, KS, USA, *fnajar@ksu.edu

Objectives: The study objective was to determine the color life threshold for longissimus lumborum (LL) steaks during retail display and the effect of postmortem (PM) aging time on the display color life of LL steaks using meta-analysis.

Materials and Methods: This meta-analysis was divided into 2 phases: (1) threshold determination and (2) the PM impact on display color life of steaks. (1) To estimate the color threshold in phase $1, a^{*}$ redness using illuminant $\mathrm{A}$ and subjective visual score data from 14 referred journal articles, published from 2000 to 2020, with a total of 22 experiments and 153 observations, were used. Visual scores from each experiment were standardized to an 8-point line scale in which $1=$ very bright red, $2=$ bright red, $3=$ dull red, $4=$ slightly dark red, $5=$ moderately dark red, $6=$ dark red to tannish red, $7=$ dark reddish tan, and $8=\tan$ to brown - which is the recommended AMSA guideline (AMSA, 2012). Previously, others have found that a discoloration score of 4.75 was borderline for color acceptability for beef aerobically packaged LL steaks during simulated retail display. This value was used to estimate the confidence intervals for the $a^{*}$ redness threshold for display color life of LL steaks. The MIXED procedure of SAS version 9.4 (SAS Institute Inc., Cary, NC) was used to estimate the display color life threshold of steaks. Data were analyzed using the linear mixed model with visual color scores being the independent variable (fixed effect). Using a confidence interval of $95 \%, a^{*}$ redness threshold was calculated. In phase 2 , data from 23 referred journal articles were used, totaling 35 experiments with 237 observations. PM aging times and $a^{*}$ redness score values were used in this phase of the analysis. PM aging times were grouped into 7 categories: $0-7 \mathrm{~d} P M=7 \mathrm{~d} \mathrm{PM} ; 8-14 \mathrm{~d}$ $\mathrm{PM}=14 \mathrm{~d} \mathrm{PM} ; 15-21 \mathrm{~d} \mathrm{PM}=21 \mathrm{~d} \mathrm{PM} ; 22-28 \mathrm{~d} \mathrm{PM}=28 \mathrm{~d}$ PM; 29-35 d PM = 35 d PM; 36-42 d PM $=42$ d PM; and $43-62 \mathrm{~d}$ PM $=62 \mathrm{~d}$ PM. Data were analyzed using the linear mixed model with visual color scores being the independent variable (fixed effect). Intercepts and the slope of each experiment were assumed to be normally distributed with variancecovariance matrix of type "unstructured." To account for heterogeneous errors, the inverse of squared SEM was used with the WEIGHT statement for the 2 phases.

Results: For phase 1, the estimates for $a^{*}$ redness as a borderline for the display color life of LL steaks were 22.92 for the estimate, and 24.98 and 20.92 for the higher and lower bounds, respectively, using a $95 \%$ confidence interval. Using the lower bound value as the threshold for borderline acceptability, the display color life was estimated for the $7 \mathrm{~d}$ PM aging time groups for the second phase. Steaks up to $21 \mathrm{~d}$ PM had the longest display color life of $7 \mathrm{~d}$, while steaks that were $22-28 \mathrm{~d} \mathrm{PM}$ had a display color life of $4 \mathrm{~d}$. For PM d 29-35, 36-42, and 42-62, the display color life of LL steaks was 3, 2, and $2 \mathrm{~d}$, respectively.

Conclusion: Knowing the PM age of LL subprimals could serve as a tool for retailers to identify the potential display color life of LL steaks displayed under aerobic packaging conditions. Estimations from this meta-analysis demonstrate that using LL subprimals having a PM age of $21 \mathrm{~d}$ or less would maximize retail display color life of aerobically packaged steaks.

Keywords: beef, color life, meta-analysis

\section{4 - INFLUENCE OF FEEDING A COVER CROP MIXTURE INCLUDING BRASSICAS DURING BACKGROUNDING ON CARCASS CHARACTERISTICS AND BEEF TENDERNESS}

C. E. Bakker ${ }^{1 *}$, L. M. Hite ${ }^{1}$, A. D. Blair ${ }^{1}$, J. K. Grubbs ${ }^{1}$, C. L. Wright $^{1}$, D. W. Brake ${ }^{2}$, A. J. Smart ${ }^{3}$, and K. R. Underwood ${ }^{1}$, 
${ }^{1}$ Animal Science, South Dakota State University, Brookings, SD, USA, ${ }^{2}$ Animal Sciences, University of Missouri, Columbia, MO, USA, ${ }^{3}$ Natural Resource Management, South Dakota State University, Brookings, SD, USA, *christina.bakker@sdstate.edu

Objectives: Brassica cover crops are an option for producers to plant to improve soil health and utilize as a feedstuff for cattle. While brassica cover crops have been widely used for grazing cows, their use as a backgrounding feedstuff is relatively unknown. The objective of this study was to determine the impact of feeding a brassica cover crop mixture during backgrounding on carcass characteristics and beef tenderness.

Materials and Methods: Thirty Angus-based steers were assigned to one of 2 dietary treatments during backgrounding: (1) ad libitum access to freshly cut brassica cover crop forage containing radish, turnip, rapeseed, rye grass, and liquid supplement or (2) common midwestern dry lot backgrounding diet containing silage, soybean meal, grass hay, and liquid supplement (Control). Steers were assigned to electronic feed bunks (Insentec, Hokofarm Group, the Netherlands) for collection of individual feed intake. Diets were formulated to be nutritionally similar on a dry matter basis. Steers were paired by weight across treatments and pair fed. Dry matter intake was calculated daily for steers in the Cover Crop treatment, and the following day Control steers were allowed access to an equal amount of dry matter. Steers were weighed weekly and backgrounded for $44 \mathrm{~d}$ before transitioning to a common finishing diet and weighed every $28 \mathrm{~d}$. Steers were harvested at an estimated average backfat thickness of $1.02 \mathrm{~cm}$. Standard carcass data were measured, striploins were collected, and steaks were aged 3, 7, 14, or $21 \mathrm{~d}$ for analysis of Warner-Bratzler shear force (WBSF), autolysis of calpain-1, proteolysis of desmin and troponin-T, and subjective tenderness of 14-d aged steaks evaluated by a trained sensory panel. Data were analyzed using PROC Mixed of SAS (SAS Institute Inc., Cary, NC) with fixed effect of treatment, aging day, and their interaction where appropriate. Proteolysis, calpain-1 autolysis, and shear force data were deemed repeated measures. Significance was considered at $P<0.05$.

Results: Treatment did not influence live animal growth or carcass characteristics $(P>0.05)$. A treatment by day interaction was observed for WBSF values $(P=0.02)$. Control steaks were less tender than Cover Crop steaks at day 3 and 7 but did not differ on day 14 and 21. Also, WBSF values were increased for Control steaks at day 3 compared to day 7. Steaks from Cover Crop steers had increased WBSF values at day 3 compared to day 7, 14, and 21. No treatment differences were detected for calpain-1 autolysis, degradation of desmin and troponin- $\mathrm{T}$, or subjective tenderness $(P>0.05)$. An increased abundance of autolyzed calpain-1 (76 kDa subunit) and decreased abundance of the active $(78 \mathrm{kDa})$ and inactive $(80 \mathrm{kDa})$ subunits was detected with increasing aging day $(P<0.001)$. The abundance of intact desmin and troponin-T decreased with aging day $(P<0.0001)$.

Conclusion: Feeding a brassica mixture cover crop during the backgrounding phase of production did not impact live growth, carcass characteristics, subjective tenderness, autolysis of calpain-1, or proteolysis of desmin and troponin-T. However, steaks from the Cover Crop treatment reached their ultimate tenderness earlier postmortem than the Control treatment. Further investigation into the mechanisms regulating these differences in tenderness is warranted. However, producers can use cover crops to improve soil quality and be assured that feeding them in backgrounding diets of beef steers will not negatively impact tenderness.

Keywords: backgrounding, beef, brassica, cover crops, tenderness

\section{5 - IMPACTS OF IN UTERO HEAT STRESS ON CARCASS AND MEAT QUALITY TRAITS OF PORK LOINS}

M. J. Nondorf ${ }^{*}$, J. R. Tuell ${ }^{1}$, J. Maskal ${ }^{1}$, J. S. Johnson ${ }^{2}$, and Y. H. B. Kim ${ }^{1},{ }^{1}$ Animal Sciences, Purdue University, West Lafayette, IN, USA, ${ }^{2}$ Livestock Behavior Research Unit, USDA-ARS, West Lafayette, IN, USA, *mnondorf@, purdue.edu

Objectives: Heat stress (HS) has been demonstrated to negatively affect animal growth and performance, which leads to adverse impacts on the livestock industry. Swine are particularly prone to experiencing HS, due to their lack of functional sweat glands and large body size. Gestating sows are more susceptible to HS due to increased metabolic heat production as gestation progresses. Furthermore, in utero heat stress (IUHS) may cause more issues with pork production. While it is well known that IUHS has negative impacts on the offspring's growth and performance postnatally, it is largely unknown what effects IUHS has on meat quality attributes. Therefore, the objective of this study was to evaluate the impacts of IUHS on carcass and meat quality traits of market weight pigs.

Materials and Methods: A total of 24 pregnant gilts were blocked by body weight and assigned to a thermoneutral (TN; $\left.n=12 ; 17.5^{\circ} \mathrm{C} \pm 2.1^{\circ} \mathrm{C}\right)$ or $\mathrm{HS}\left(n=12\right.$; cyclic $26^{\circ} \mathrm{C}$ to $36^{\circ} \mathrm{C}$ ) chamber during the first half of gestation (day 6 to 59). All pregnant gilts were exposed to $\mathrm{TN}$ conditions $\left(20.9^{\circ} \mathrm{C} \pm 2.3^{\circ} \mathrm{C}\right)$ from day 60 of gestation to farrowing. From all of the litters, gilts were randomly selected from the TN group (in utero thermoneutral [IUTN]; $n=10$ ) and from the HS group (IUHS; $n=10$ ), raised to market weight $(117.3 \pm 1.7 \mathrm{~kg}$ body weight), and harvested under standard conditions. Carcass and organ weights were recorded, and the rate and extent of temperature and $\mathrm{pH}$ declines were measured during $24 \mathrm{~h}$ postmortem. After the carcass quality traits were measured, the right-side longissimus muscles were 
sectioned and randomly assigned to either no aging or $7 \mathrm{~d}$ of aging at $2^{\circ} \mathrm{C}$ in vacuum packaging. Chops $(2 \mathrm{~cm}$ thick $)$ were used to measure Warner-Bratzler shear force (WBSF), surface meat color, water-holding capacity (WHC), and other chemical attributes, such as fatty acid profile, lipid oxidation, and protein denaturation. The data were analyzed using the PROC MIXED procedure of SAS version 9.4 (SAS Institute Inc., Cary, NC) in a randomized complete block design with IUHS treatment as main effect and dam as a random effect. Statistical significance was separated at $P<0.05$.

Results: Carcasses from IUHS pigs had lower head and heart weights $(P<0.05)$ compared to IUTN pigs. The IUHS treatment had a decreased loin muscle area $(P<0.05)$. No differences in other carcass quality traits, such as marbling score, fat depth, and muscle/lean firmness/lean color scores, were found $(P>0.05)$. Pork chops from IUHS carcasses had significantly higher WBSF values compared to IUTN counterparts $(P<0.05)$, regardless of aging. No impacts of IUHS on WHC were found $(P>0.05)$, but postmortem aging reduced drip, freezing/thawing, and cooking losses of loin samples $(P<0.05)$. Temperature and $\mathrm{pH}$ decline, surface color attributes, fatty acid profile, lipid oxidation, and protein denaturation were all unaffected by in utero environment $(P>0.05)$.

Conclusion: These results suggest that IUHS negatively impacts some carcass and meat quality traits, evidenced by the decreased loin muscle area and increased WBSF values. However, there appeared to be limited impact on other traits, including $\mathrm{pH}$, color attributes, WHC, and antioxidative capacity. To the best of our knowledge, this is the first study reporting the impacts of IUHS on carcass and meat quality traits of market weight pigs. These results provide practical implications for the pork industry to prioritize minimizing IUHS during the first half of gestation to improve yield and quality.

Keywords: carcass composition, gestation, in utero heat stress, pork quality

\section{6 - APPLICATION OF GUAVA LEAF EXTRACT IN FRESH PORK SAUSAGE}

T. T. T. Tran ${ }^{1}$, N. M. N. Ton ${ }^{1}$, T. T. Nguyen ${ }^{1}$, V. V. M. Le ${ }^{1}$, D. Sajeev ${ }^{2}$, M. W. Schilling ${ }^{3}$, and T. T. Dinh ${ }^{2 *},{ }^{1}$ Department of Food Technology, Ho Chi Minh City University of Technology, Ho Chi Minh City, Viet Nam, ${ }^{2}$ Department of Animal and Dairy Sciences, Mississippi State University, Starkville, MS, USA, ${ }^{3}$ Department of Food Science, Nutrition, and Health Promotion, Mississippi State University, Starkville, MS, USA, *ds2823@msstate.edu

Objectives: The objective of the current study was to determine the antioxidant efficacy of guava leaf (GL) extract in comparison with butylated hydroxytoluene (BHT) in fresh pork sausage during refrigerated storage
Materials and Methods: GL were cut, extracted in 48\% ethanol, and concentrated under vacuum to a solution with an IC50 inhibitory concentration value of $0.04 \mathrm{mg}$. Treatments included a negative control (no antioxidant [CON]), $200 \mathrm{ppm}$ BHT, and GL extract at 3,000 ppm (GL3000), 4,000 ppm (GL4000), 5,000 ppm (GL5000), and 6,000 ppm (GL6000) on a fat content basis. Pork lean and fat trimmings were ground a $6-\mathrm{mm}$ plate at a 4:6 ratio. Ground meat was mixed with seasonings (wheat flour, sodium chloride, sucrose, black pepper powder, monosodium glutamate, and antioxidants), hand-mixed for $5 \mathrm{~min}$, stuffed into collagen casing to produce $35-\mathrm{g}$ sausage links, and placed in a polyamide bags (maximum moisture and oxygen transmission rates of $180 \mathrm{~g} / \mathrm{m}^{2} / \mathrm{d}$ and $15 \mathrm{~cm}^{3} / \mathrm{cm}^{2} /$ $\mathrm{d} /$ bar, respectively) at $4^{\circ} \mathrm{C}$ for $0,1,4,7,10$, and $14 \mathrm{~d}$ in the dark. The study was conducted as a randomized complete block design with 3 replications. On each storage day, 3 sausage links per replication were randomly sampled for acidic value (AV), peroxide value (PV), conjugated dienes (CD), thiobarbituric acid reactive substances (TBARS), and total antioxidant capacity (TAC). A generalized linear mixed model was used to analyze the variances with treatment, time, and treatment $\times$ time being fixed effects and replication being a random effect, using the GLIMMIX procedure of SAS version 9.4 (SAS Institute Inc., Cary, $\mathrm{NC}$ ). Actual probability was reported with the determination of statistical significance.

Results: There was a two-way treatment $\times$ time interaction for TAC and oxidation products $(P \leq 0.017)$. On day 0 , GL5000 and GL6000 had 117 and $131 \mu \mathrm{mol}$ trolox/g, greater than that in CON sausage $(101 \mu \mathrm{mol} / \mathrm{g} ; P \leq 0.014)$. However, only GL6000 had more TAC than BHT $(113 \mu \mathrm{mol} / \mathrm{g}$; $P=0.011)$. On day 7 and 10 , all treatments had similar TAC $(P \geq 0.231)$ and more than the CON $(P<0.001)$. CON had at least $0.10 \mathrm{mmol} / \mathrm{kg}(25 \%)$ more $\mathrm{CD}$ than all other treatments on day $4(P \leq 0.013)$. On day 7, both BHT and GL6000 had similar CD $(0.42$ and $0.45 \mathrm{mmol} / \mathrm{kg} ; P=0.567), 0.1 \mathrm{mmol} /$ $\mathrm{kg}$ less than CON, GL3000, GL4000, and GL5000 ( $P \leq$ 0.030 ). On day 1 , the PV of GL4000, GL5000, and GL6000 was similar to that of BHT ( 3.4 to $4.7 \mathrm{mEq} / \mathrm{kg} ; P \geq 0.068$ ) but was less than that of CON and GL3000 $(8.4$ and $8.6 \mathrm{mEq} / \mathrm{kg}$, respectively; $P \leq 0.001)$. On day 4,7 , and $10, \mathrm{CON}$ had 1.68 to $10.0 \mathrm{mEq} / \mathrm{kg}$ more $\mathrm{PV}$ than all other treatments $(P \leq$ $0.001)$. On day 1, CON sausage had similar AV to other treatments $(0.71$ to $1.55 \mathrm{~g} \mathrm{KOH} / \mathrm{kg} ; P \geq 0.057)$, with the exception of GL6000 $(0.52 \mathrm{~g} / \mathrm{kg} ; P=0.020)$. On day 4 and 7, CON had 1.33 to $2.05 \mathrm{~g} / \mathrm{kg}$ greater AV than GL4000, GL5000, GL6000, and BHT $(P \leq 0.004)$. On day 10 and 14 , CON had 2.66 to $4.42 \mathrm{~g} / \mathrm{kg}$ greater AV than all other treatments $(P \leq 0.001)$. BHT had similar AV to that of GL treatments $(P \geq 0.057)$ from day 1 to 14 . From day 1 to $14, \mathrm{CON}$ had greater TBARS value than other treatments $(P \leq 0.001)$. BHT, GL5000, and GL6000 were similar $(P \geq$ $0.147)$ and more effective at controlling TBARS than GL3000 and GL4000 $(P \leq 0.001)$ from day 1 to 14 . 
Conclusion: GL extract was as effective at slowing the process of lipid oxidation in fresh pork sausage as $200 \mathrm{ppm}$ BHT. Therefore, GL extract has potential as a natural antioxidant in clean-label meat products pending further sensory testing.

Keywords: clean label, guava leaf, lipid oxidation, natural antioxidant, pork sausage

\section{7 - GENDER STATUS AND MUSCLE TYPE MODULATES FATTY ACID COMPOSITION ASSOCIATED TO TRANSCRIPTS LEVELS OF ADIPOGENESIS-RELATED GENES IN BEEF}

L. F. Mueller ${ }^{1 *}$, P. Garbossa ${ }^{2}$, A. Bridi ${ }^{3}$, T. Amorim ${ }^{1}$, T. Martins $^{2}$, J. Gemelli ${ }^{1}$, A. Ferrinho ${ }^{1}$, J. Muñoz ${ }^{1}$, E. Fabricio $^{1}$, V. Ramos ${ }^{1}$, A. Pereira ${ }^{1}$, T. Sant Ana ${ }^{1}$, F. Baldi $^{4}$, J. Silveira ${ }^{3}$, M. Poleti ${ }^{1}$, and A. Pereira ${ }^{1},{ }^{1}$ Animal Science, ${ }^{2}$ Animal Nutrition and Production, University of Sao Paulo, Pirassununga, Brazil, ${ }^{3}$ Veterinary Medicine, University of Sao Paulo, Pirassununga, Brazil, ${ }^{4}$ Animal Science, State University of Sao Paulo, Jaboticabal, Brazil,*lelemueller@usp.br

Objectives: The goal of this study was to associate the fatty acid (FA) composition and adipogenesis related genes in Longissimus (LO) and Triceps brachii (TB) muscles of Angus $\times$ Nellore cattle from heifers, bulls, and steers.

Materials and Methods: In the feedlot, 150 animals were separated by gender, fed with same diet, and subsequently harvested. The final mean weight was $431.3 \pm 26.3 \mathrm{~kg}$, $488.9 \pm 30.7 \mathrm{~kg}$, and $452.5 \pm 24.9 \mathrm{~kg}$ for heifers, bulls, and steers, respectively. Lipids were extracted for the total lipid determination and FA composition from samples of LO and TB muscles. Total RNA from homogenous portions of samples was extracted using TRIzol $($ ). The quantitative PCR reactions were analyzed in RT-PCR QuantStudio 6 (Applied Biosystems, Thermo Fisher Scientific, Foster City, CA). To calculate relative expression, target gene cycle threshold was normalized to the $\mathrm{Ct}$ values of the GAPDH, and the relative levels were represented as $2^{-\mathrm{DCt}}$. The statistical analyses were performed by the PROC MIXED procedure of SAS (SAS Institute Inc., Cary, NC) for the meat FA analyses and JMP14 ${ }^{\circledR}$ (SAS Institute Inc.) for gene expression. Nested gender was included as covariate for the final live weight and total lipid determination. The level of statistical significance was considered at $P \leq 0.05$.

Results: There was a difference for the total saturated FA (SFA) for gender and muscle $(P<0.01)$. The meat from bulls $(43.31 \%)$ had higher levels of SFA compared to heifers $(41.09 \%)$ and had no differences in relation to meat from steers $(42.42 \%)$. Among muscle type, total SFA increased in LO muscle (43\%) compared with TB (41.6\%). The FA 14:0 and 16:0 were affected by muscle type only, and the higher levels were detected in LO muscle $(P<0.01)$. There were differences in gender for total monounsaturated FA (MUFA; $P=0.015)$. Meat from bulls $(42.65 \%)$ had lower levels of MUFA compared with other genders. The higher levels of MUFA observed in meat from heifers $(48.04 \%)$ and steers $(46.23 \%)$ is possibly due to the higher content of the major FA in this group (18:1 n-9c). The level of $18: 1 \mathrm{n}-9 \mathrm{c}$ was higher in the LO muscle $(32.35 \%)$ than the TB $(31.26 \% ; P=0.022)$. The total polyunsaturated FA (9.97\%), total $\mathrm{n} 3(2.32 \%)$ and $\mathrm{n} 6(7.25 \%)$, polyunsaturated FA/SFA $(0.24 \%)$ ratio, healthy index $(1.65 \%)$, and the FA $18: 2 \mathrm{n}-6 \mathrm{c}(6.75 \%), 20: 3-\mathrm{n} 3(1.78 \%)$, and 22:4-n6 (0.26\%) were higher $(P<0.01)$ in TB compared to LO muscle. Otherwise, the atherogenicity and thrombogenicity indexes were higher in the LO muscle $(\mathrm{P}<0.01)$. Regarding the transcript levels, there was an effect for muscle type on the expression of the $C E B P$ a and $L P L(P<0.01)$ genes, both which had higher expression in TB muscle. Gender affected the transcript level of $A C C(P=0.005)$. This gene was increased in bulls, intermediated in steers, and decreased in heifers. Additionally, gender and muscle type interaction were observed on transcript levels of $F A B P 3, T P M 2$, and TPM3 $(P<0.01)$.

Conclusion: The gender status and muscle type interact and modulate some transcripts levels related to the adipogenesis pathway by regulating the expression of enzymes and proteins involved in synthesis, transport, distribution, degradation, and oxidation of long-chain FA in adipose and muscle tissues. Therefore, these physiological and molecular changes are leading to different lipid profiles within each muscle, whereas LO muscle is more favorable to human health than $\mathrm{TB}$, suggesting changes in consumer behavior based on healthy necessities and risk to develop different diseases.

Acknowledgments: This work was supported by FAPESP Grant Number 2017/08075-0.

Keywords: gene expression, healthy index, beef cattle, crossbred, lipid accumulation

\section{8 - MYOWATER CHANGES DURING THE FIRST 24 H POSTMORTEM RELATED TO BROILER BREAST FINAL MEAT QUALITY}

\author{
G. Tasoniero ${ }^{1 *}$, H. Zhuang ${ }^{1}$, and B. Bowker ${ }^{1},{ }^{1}$ U.S. National \\ Poultry Research Center, USDA-ARS, Athens, GA, USA, \\ *brian.bowker@usda.gov
}

Objectives: The objective of this study was to provide insight on changes in myowater (muscle tissue water) mobility and distribution in broiler breast fillets during the first $24 \mathrm{~h}$ postmortem (PM) related to final meat quality.

Materials and Methods: From 12 broiler carcasses, right breast fillets were deboned at $15 \mathrm{~min}$ PM (hot deboned), and the left fillets were deboned at $2 \mathrm{~h} \mathrm{PM}$ (post-chilling). Nuclear magnetic resonance (NMR) traits $\left(T_{2 B}, T_{21}, T_{22}, P_{2 B}, P_{21}, P_{22}\right)$ of 
individual fillets $(197 \pm 35 \mathrm{~g})$ were repeatedly assessed at 0.75 , 1.25, 2, 3, 4 and $24 \mathrm{~h} \mathrm{PM}$ (right fillets) or 2, 3, 4, and $24 \mathrm{~h}$ PM (left fillets). At $24 \mathrm{~h} \mathrm{PM}$, color $\left(L^{*} a^{*} b^{*}\right)$ was measured on the middle and caudal portions of fillets (bone side), drip loss and cook loss were determined on subsamples excised from the cranial portion, and total moisture content was determined. A oneway analysis of variance mixed model was performed to evaluate PM time as a fixed effect within deboning time with carcass as a random effect. Pearson correlation analysis within deboning time was performed to assess the relationships between NMR and quality traits.

Results: In hot-deboned fillets, the mobility $(T)$ and proportion $(P)$ of the water tightly bound to macromolecules $\left(T_{2 B}\right.$ and $\left.P_{2 B}\right)$, intra-myofibrillar $\left(T_{21}\right.$ and $\left.P_{21}\right) \mathrm{H}_{2} \mathrm{O}$, and extramyofibrillar $\mathrm{H}_{2} \mathrm{O}\left(T_{22}\right.$ and $\left.P_{22}\right)$ were strongly influenced by PM time. Bound water exhibited the highest mobility $(P<$ $0.0001)$ coupled with the lowest proportion $(P=0.0036)$ between 2 and $4 \mathrm{~h}$ PM. The mobility of the intra-myofibrillar water compartment $\left(T_{21}\right)$ decreased from 0.75 to $2 \mathrm{~h} \mathrm{PM}$ and then increased from 3 to $24 \mathrm{~h} \mathrm{PM}(P=0.0002)$. The proportion of intra-myofibrillar water $\left(P_{21}\right)$ was at its minimum at $1.25 \mathrm{~h}$ and then reached its peak at $4 \mathrm{~h}$ PM $(1.25 \mathrm{~h}=$ $69.8 \%$ vs. $4 \mathrm{~h}=79.2 \% ; P<0.0001)$. The mobility of the extra-myofibrillar water compartment $\left(T_{22}\right)$ was steady between 0.75 and $4 \mathrm{~h} \mathrm{PM}$ and then decreased to reach its minimum at $24 \mathrm{~h}(P<0.0001)$, while $P_{22}$ reached its peak at $1.25 \mathrm{~h}$ and then decreased to its minimum at $4 \mathrm{~h} P M(1.25 \mathrm{~h}=29.5 \%$ vs. $4 \mathrm{~h}=20.1 \% ; P<0.0001)$. The NMR traits of fillets subjected to post-chill deboning exhibited similar trends. The correlation analysis revealed that, at $24 \mathrm{~h} \mathrm{PM}$, total moisture content was highly correlated to $T_{21}(r=0.78)$ and $P_{22}(r=$ $0.76)$, but negatively related to $P_{21}(r=-0.75)$. However, moisture content at $24 \mathrm{~h}$ PM was most highly correlated to $T_{21}$ measured at 2 and $3 \mathrm{~h} \mathrm{PM}(r=-0.85)$. For drip loss, the only significant correlation found was with $T_{22}$ at 1.25 $\mathrm{h}(r=0.71)$. Cook loss was moderately related to $T_{21}$ at all the time points $(r=0.70)$ and with $T_{2 B}$ and $T_{22}$ at 2 and $3 \mathrm{~h}$ PM. As for the color traits, lightness values exhibited a moderate correlation with $T_{21}$ at both $1.25 \mathrm{~h}(r=0.64)$ and $2 \mathrm{~h}$ PM $(r=0.52)$. At $0.75 \mathrm{~h}$, redness was correlated with $T_{2 B}(r=$ 0.72 ); at both 1.25 and $2 \mathrm{~h} \mathrm{PM}, a^{*}$ was moderately and positively correlated with bound water fractions but negatively related with $P_{22}(r=-0.60)$. Yellowness was only related to $T_{2 B}$ at $0.75 \mathrm{~h}(r=0.64)$.

Conclusion: Data from this study indicate that changes in myowater distribution and mobility occur in breast fillets within the first $24 \mathrm{~h}$ PM and suggest that myowater properties within the first $3 \mathrm{~h}$ PM may play a role in determining the final moisture content, water-holding capacity, and color traits of broiler breast meat.

Keywords: chicken breast meat, final meat quality, nuclear magnetic resonance, postmortem time

American Meat Science Association.

\section{9 - EVALUATION OF BEEF CAMERA GRADING TECHNOLOGY TO ASSESS BISON CARCASS CHARACTERISTICS}

J. K. Janssen ${ }^{*}$, K. M. Cammack ${ }^{1}$, W. J. Horne ${ }^{2}$, J. K. Grubbs $^{1}$, K. R. Underwood ${ }^{1}$, J. Hansen ${ }^{3}$, C. Kruse ${ }^{3}$, and A. D. Blair ${ }^{1},{ }^{1}$ Animal Science, South Dakota State University, Brookings, SD, USA, ${ }^{2}$ Livestock and Poultry Program, USDA-AMS, Washington, DC, USA, ${ }^{3}$ Conservation, Science, and Research, Turner Enterprises, Inc., Bozeman, MT, USA, *jessica.janssen@ sdstate.edu

Objectives: The objective of this study was to evaluate the effectiveness of beef camera grading technology on bison carcass characteristics.

Materials and Methods: Bison heifers were randomly assigned to finishing treatments: grain-finished $(n=108$; backgrounded on pasture and finished for $130 \mathrm{~d}$ with ad libitum access to grass hay, alfalfa, and a corn and dry distillers grain concentrate prior to slaughter) or grass-finished ( $n=$ 93; remained on pasture until slaughter). Heifers were transported $(\sim 720 \mathrm{~km})$ to a commercial packing facility and slaughtered at 28 mo of age over a 2 -d period. Carcass measurements and camera images were collected at $\sim 20 \mathrm{~h}$ postmortem. Carcasses were ribbed between the 12th and 13th rib and allowed to bloom for approximately $30 \mathrm{~min}$. An expert USDA grader evaluated ribeye area, backfat thickness, and marbling score of one side of each carcass. USDA personnel then captured images of the exposed ribeye from the same side evaluated by the grader using the handheld camera portion of a VBG2000 image processing system. The system automatically determined carcass parameters from the images, including preliminary yield grade, yield grade, ribeye area, and marbling. To assess the ability of the beef grading camera to evaluate bison carcass characteristics, both camera and grader measurements were analyzed using the MIXED procedures of SAS (SAS Institute Inc., Cary, NC), while yield grade data were analyzed using the GLIMMIX procedures for the main effect of finishing treatment; slaughter date was included as a random effect. Separation of least-squares main effect means was performed using least significant difference with a Tukey adjustment, assuming $\alpha=0.05$. Additionally, correlations between grader and camera measurements were analyzed using the CORR procedures of SAS.

Results: Grain-finished bison heifers had increased $(P<0.0001)$ backfat thickness and marbling scores compared to grass-finished carcasses when evaluated by both the camera and expert grader. Across both finishing treatments, means for ribeye area and marbling were increased, while mean backfat thickness was decreased when evaluated by the camera in comparison to the expert grader. Regardless of evaluation by camera or grader, yield grade was not impacted $(P>0.10)$ by finishing system, with the exception of increased $(P<0.0001)$ proportion of yield grade 1 carcasses 
in the grass-finished treatment when evaluated by the camera, and a tendency for increased $(P=0.0965)$ proportion of yield grade 2 in the grass-finished treatment when evaluated by the expert grader. Correlations were positive $(P<0.0001)$ between expert grader and camera measurements for yield grade, backfat thickness, and ribeye area. Correlations between the camera and grader were highest $(r=0.978, P<0.0001)$ for yield grade, and lowest ( $r=0.451, P<0.0001$ ) for marbling score measurements. Additional camera measurements identified as unknown pixels were found to be positively correlated $(r=0.621, P<$ $0.0001)$ with ribeye area but not correlated $(r=0.002$, $P=0.9807$ ) with marbling.

Conclusion: Collectively, these data indicate that bison ribeye images collected with a beef grading camera were correlated with expert grader evaluations. However, accuracy of measurements and validation of a suitable camera grading system for bison will require additional investigation, including calibration and adjustments for bison carcass characteristics.

Keywords: bison, camera grading technology, carcass measurements, finishing systems

\section{0 - INFLUENCE OF BISON HARVEST SYSTEMS ON ANIMAL STRESS RESPONSE, CARCASS TRAITS, AND MEAT QUALITY CHARACTERISTICS}

J. K. Janssen ${ }^{1 *}$, K. M. Cammack ${ }^{1}$, J. K. Grubbs ${ }^{1}$, K. R. Underwood $^{1}$, J. Hansen ${ }^{2}$, C. Kruse ${ }^{2}$, and A. D. Blair ${ }^{1}$, ${ }^{1}$ Animal Science, South Dakota State University, Brookings, SD, USA, ${ }^{2}$ Conservation, Science, and Research, Turner Enterprises, Inc., Bozeman, MT, USA, *jessica.janssen@ sdstate.edu

Objectives: The objectives of this study were to evaluate the influence of harvest system (on-ranch or commercial harvest system) on (1) the stress response of bison heifers, (2) carcass characteristics and meat quality of bison heifers, and consumer preference for bison steaks.

Materials and Methods: Grass-finished bison heifers were randomly assigned to harvest treatments: commercial ( $n=93$, transported $\sim 720 \mathrm{~km}$ to a commercial harvest facility) or on-ranch ( $n=40$, harvested on-ranch using a sharpshooter and mobile slaughter unit). Blood samples were collected immediately following exsanguination and analyzed for serum cortisol and haptoglobin concentrations. Approximately $20 \mathrm{~h}$ postmortem, ribeye area, backfat thickness, marbling score, and instrumental color of the exposed ribeye and subcutaneous fat opposite the ribeye were recorded. A subsample ( $n=30$ carcasses closest to the average hot carcass weight for each treatment) was selected, and striploins were removed from one side of each carcass.
Ultimate $\mathrm{pH}$ was recorded, and striploins were fabricated into 2.54-cm steaks. One steak was designated for crude fat determination. Two steaks were aged for $14 \mathrm{~d}$ and frozen for Warner-Bratzler shear force analysis, cook loss determination, and consumer sensory evaluation. Serum cortisol and haptoglobin concentrations, body weight, carcass characteristics, and meat quality data were analyzed using the MIXED procedure of SAS (SAS Institute Inc., Cary, NC) for the main effect of harvest treatment; slaughter date was included as a random effect, and peak temperature was included as covariate for Warner-Bratzler shear force and cook loss. Consumer preference data were analyzed using the MIXED procedures for the main effects of harvest treatment and serving order; serving time and panelist were included as random effects. Separation of least-squares means was performed using least significant difference with a Tukey adjustment, assuming $\alpha=0.05$.

Results: Commercially harvested bison heifers had elevated $(P<0.01)$ cortisol concentrations compared to heifers harvested on-ranch. Carcass weight, dressing percent, and ribeye area were greater $(P<0.01)$ for heifers harvested commercially. Instrumental color values $\left(L^{*}, a^{*}, b^{*}\right)$ recorded at the ribeye area and $L^{*}$ value of backfat opposite the ribeye were increased $(P<0.05)$ for heifers in the commercially harvested treatment. However, $a^{*}$ and $b^{*}$ values recorded for backfat opposite the ribeye were decreased $(P<0.01)$ in commercially harvested heifers. Heifers harvested on-ranch produced striploins with increased $(P<$ $0.01)$ ultimate $\mathrm{pH}$. Steaks from heifers harvested commercially had increased $(P<0.01)$ ether extractable fat percentage. Steaks from the on-ranch harvest system had less $(P<$ $0.01)$ cook loss than steaks from the commercial system. Harvest treatment did not influence $(P>0.05)$ haptoglobin concentration, live body weight, backfat, marbling scores, tenderness, or sensory attributes.

Conclusion: Collectively, these data indicate that harvest systems influence short-term stress response, and some carcass and meat quality characteristics of bison heifers. However, harvest systems had no impact on consumer preference for bison.

Keywords: bison, carcass characteristics, harvest systems, meat quality, stress

\section{1 - INFLUENCE OF BISON FINISHING SYSTEMS ON CARCASS CHARACTERISTICS AND MEAT QUALITY}

J. K. Janssen ${ }^{1 *}$, K. M. Cammack ${ }^{1}$, J. F. Legako ${ }^{2}$, J. K. Grubbs $^{1}$, K. R. Underwood ${ }^{1}$, J. Hansen ${ }^{3}$, C. Kruse ${ }^{3}$, and A. D. Blair ${ }^{1},{ }^{1}$ Animal Science, South Dakota State University, Brookings, SD, USA, ${ }^{2}$ Animal and Food Sciences, Texas Tech University, Lubbock, TX, USA, ${ }^{3}$ Conservation, 
Science, and Research, Turner Enterprises, Inc., Bozeman, MT, USA, *jessica.janssen@sdstate.edu

Objectives: This study evaluated the influence of finishing system (grain or grass finishing) on carcass characteristics and meat quality of bison heifers, and consumer preference for bison steaks.

Materials and Methods: Bison heifers were randomly assigned to treatments: grain-finished $(n=108$, backgrounded on pasture and finished in a drylot for $130 \mathrm{~d}$ with ad libitum access to hay and a corn and dry distillers grain diet) or grass-finished ( $n=93$, remained on pasture until slaughter). Heifers were slaughtered at $28 \mathrm{mo}$ of age. Carcass measurements were recorded, and striploins were collected from a subsample of carcasses ( $n=30$ carcasses closest to the treatment average hot carcass weight). Ultimate $\mathrm{pH}$ was recorded, striploins were fabricated, and the longissimus lumborum was isolated and cut into 2.54$\mathrm{cm}$ steaks. One steak was designated for analysis of fatty acid profile, cholesterol content, and proximate analysis. Two steaks were aged for $14 \mathrm{~d}$ for consumer sensory evaluation; 4 steaks were aged for $4,7,14$, or $21 \mathrm{~d}$ for analysis of Warner-Bratzler shear force and cook loss. All data were analyzed using the MIXED procedure of SAS (SAS Institute Inc., Cary, NC). Carcass and meat quality data were analyzed for the main effect of finishing treatment, with slaughter date as a random effect. Cook loss and WarnerBratzler shear force were analyzed as repeated measures using the ante-dependence covariance structure for effects of finishing treatment, aging, and their interaction, with peak temperature as a covariate. Consumer preference was analyzed for the main effects of finishing treatment and serving order; serving time and panelist were included as random effects. Separation of least-squares means was performed using least significant difference with a Tukey adjustment, assuming $\alpha=0.05$.

Results: Grain-finished bison heifers had greater $(P<$ $0.01)$ live and hot carcass weights, dressing percentage, ribeye area, backfat, and marbling scores compared to grass-finished heifers. Instrumental color values $\left(L^{*}, a^{*}\right.$, $\left.b^{*}\right)$ of the ribeye and $a^{*}$ value of backfat opposite the ribeye were increased $(P<0.01)$ for grain-finished heifers. However, $L^{*}$ and $b^{*}$ values of backfat opposite the ribeye were decreased $(P<0.01)$ in carcasses from the grain-finished system. Steaks from grain-finished heifers had increased $(P<0.05)$ crude protein and fat content and decreased $(P<0.01)$ moisture, while percentage of ash did not differ $(P>0.05)$ between treatments. The grain-finishing system produced steaks with increased $(P<0.01)$ cholesterol, palmitic, stearic, oleic, linoleic, arachidonic, and total fatty acids ( $\mathrm{mg} / \mathrm{g}$ of wet tissue). However, when expressed as a percentage of total lipid, grass-finished samples had increased $(P<0.05)$ proportion of polyunsaturated fatty acids and saturated fatty acids. The grain-finished system produced more tender $(P<0.05)$ steaks than grass-finished. Tenderness of all steaks improved $(P<0.01)$ with postmortem aging. Cook loss was affected $(P<0.05)$ by the interaction of treatment with aging period. Overall cook loss was reduced $(P<0.01)$ for grain-finished and increased $(P<0.05)$ in steaks aged $4 \mathrm{~d}$ compared with $7 \mathrm{~d}$ or $21 \mathrm{~d}$. Finishing system did not influence $(P>0.05)$ ultimate $\mathrm{pH}$ or consumer sensory ratings.

Conclusion: Collectively, these data indicate that finishing systems influence bison carcass characteristics and meat quality, yet these differences do not translate to changes in consumer preferences. Additionally, finishing system influenced nutrient content and fatty acid composition, which may have health implications.

Keywords: bison, carcass characteristics, consumer sensory, finishing system, meat quality

\section{2 - GROWTH PROMOTANT AND POSTMORTEM AGING EFFECTS ON COLLAGEN CHARACTERISTICS AND MEAT QUALITY OF SEMIMEMBRANOSUS MUSCLE FROM CROSSBRED ANGUS STEER CARCASSES}

P. Coleman ${ }^{1 *}$, B. C. Roy ${ }^{1}$, and H. L. Bruce ${ }^{1},{ }^{1}$ Agricultural, Food and Nutritional Sciences, University of Alberta, Edmonton, Canada, *patience@ualberta.ca

Objectives: Our objective was to relate insulin-like growth factor I-1 blood concentrations to collagen and meat quality characteristics of the semimembranosus (SM) muscle as affected by selection for low residual feed intake (RFI) and use of steroids and ractopamine hydrochloride (RH).

Materials and Methods: Forty-seven Angus steers selected for low (efficient, $n=26)(-0.33 \pm 0.08 \mathrm{~kg} / \mathrm{d})$ RFI or as controls (inefficient, $n=21)(0.63 \pm 0.09 \mathrm{~kg} / \mathrm{d})$ were randomly assigned to one of 4 treatments in a $2 \times$ $2 \times 2$ factorial design within RFI status: control (no implant; no RH) $(n=12)$; implant and RH $(n=11)$; RH only $(n=$ $12)$; and implant only $(n=12)$. Implanted steers received a first implant (200 $\mathrm{mg}$ progesterone, $20 \mathrm{mg}$ estradiol benzoate, $29 \mathrm{mg}$ tylosin tartrate) at $332.21 \pm 2.54 \mathrm{~d}$ of age and $383.19 \pm 2.88 \mathrm{~kg}$ live weight, and a terminal implant (120 mg trenbolone acetate, $24 \mathrm{mg}$ estradiol) about $100 \mathrm{~d}$ before slaughter. Blood samples were taken by jugular venipuncture $30 \mathrm{~d}$ after first implantation. RH was fed $28 \mathrm{~d}$ before slaughter at $200 \mathrm{mg} \mathrm{head}^{-1} \mathrm{day}^{-1}$. SM muscles were excised $48 \mathrm{~h}$ postmortem from the right carcass side and cut in half. Halves were randomly assigned to 3 and $12 \mathrm{~d}$ of aging at $4{ }^{\circ} \mathrm{C} \pm 2{ }^{\circ} \mathrm{C}$ and balanced within treatment for position within muscle. Warner-Bratzler shear force (WBSF), blood IGF-1 concentration, intramuscular fat (IMF) content, collagen heat solubility, and pyridinoline (PYR) concentrations were determined. Data were analyzed using RStudio as a split plot blocked by slaughter group with animal as the 
experimental unit in the whole plot and muscle halves as the experimental unit in the split-plot. Differences were considered significant at $P<0.05$. Pearson correlation coefficients were computed between meat quality and collagen solubility characteristics with significance at $P<0.05$.

Results: Mean IGF-1 levels were unrelated to meat quality and collagen characteristics and were unaffected by RFI, steroids, RH, or their interactions $(P>0.05)$. Collagen did not contribute to increased mean WBSF $(47.11 \pm 1.02 \mathrm{~N})$ of muscles from low-RFI steers relative to control steers $(41.97 \pm 1.14 \mathrm{~N})(P=0.0012)$ as there was no effect of RFI on collagen characteristics $(P>0.05)$. This difference may be due to increased IMF in muscles from control steers, as there was a negative correlation between IMF and WBSF $(r=-0.30)(P=0.05)$. Aging increased collagen heat solubility $(P<0.05)$, and collagen solubility percentage was negatively correlated with WBSF at day 12 postmortem $(P=0.0279)(r=-0.32)$ indicating that postmortem weakening of collagen occurred and became more important with postmortem aging. PYR concentration was positively correlated with WBSF at day 3 and 12 postmortem $(r=0.35$ and $r=0.32$, respectively $(P<0.05)$, indicating that this crosslink contributes to SM toughness regardless of postmortem aging. Muscles from steers supplemented with RH had a higher mean collagen content than muscles from steers not supplemented with RH $(5.38 \pm 0.20$ vs. $4.74 \pm 0.19 \mathrm{mg}$ collagen/g raw meat) $(P=0.0032)$, suggesting muscle transformation in supplemented steers.

Conclusion: Circulating IGF-1 was not affected by RFI status or steroid and RH use and was unrelated to collagen or meat quality characteristics, suggesting that localized IGF-1 is of importance. This study confirmed that PYR concentration affects WBSF in the SM and can affect final product toughness even after aging.

Keywords: beef tenderness, collagen, collagen cross-links, insulin-like growth factor I-1, implants

\section{3 - EFFECT OF DRY AGING ON QUALITY ATTRIBUTES OF PORK LOINS}

A. Wagner ${ }^{1 *}$, D. Setyabrata ${ }^{1}$, and Y. H. B. Kim ${ }^{1},{ }^{1}$ Meat Science and Muscle Biology Lab, Department of Animal Sciences, Purdue University, West Lafayette, IN, USA, *wagne259@purdue.edu

Objectives: Dry aging is a traditional method known to improve the palatability attributes of meat. Unique flavors such as buttery and nutty have often been attributed to the dry aging process. While the benefits have often been observed in beef products, the effect of dry aging on pork product is not fully known yet. Additionally, given some eating quality issues associated with pork loins, such as toughness, dryness, and bland flavor, there is a need to improve the palatability attributes of fresh pork loins. Therefore, the objective of this study was to evaluate the effect of dry aging on meat quality and palatability characteristics of pork loins.

Materials and Methods: Pork loins from one side of 10 pork carcasses were collected, split into 3 equal portions, and aged for $21 \mathrm{~d}$ at $2{ }^{\circ} \mathrm{C}, 65 \%$ relative humidity, and $0.8 \mathrm{~m} / \mathrm{s}$ air flow, using 3 different aging methods (wet aging, dry aging, and UV-light dry aging at $5 \mathrm{~J} / \mathrm{cm}^{2}$ ). After the completion of aging, sections were trimmed, and total saleable yield was determined for each aging method. Multiple chops were made from each section for several meat quality and chemical attributes. Color stability of the chops were determined using Hunter MiniScan through simulated retail display for $7 \mathrm{~d}$. The lipid oxidation of the samples was measured by conducting 2-thiobarbituric acid reactive substances assay. Water-holding capacity of the samples was determined through multiple measurements including freezing/ thawing loss, drip loss, and cooking loss. Warner-Bratzler shear force analysis and consumer sensory evaluation $(n=120)$ of pork chops from each treatment were conducted. The experiment was a complete block design with animal set as random. Data collected were analyzed using PROC GLIMMIX from SAS (SAS Institute Inc., Cary, $\mathrm{NC}$ ), and least-squares means were separated with the PDIFF options (F-test, $P<0.05$ ).

Results: Both dry aging and UV-light dry aging resulted in lower yield compared to wet aging $(P<0.05)$. However, increase in water-holding capacity was observed in dry aging treatment compared to wet aging $(P<0.05)$ shown by lower loss in both freeze/thaw and drip loss. Warner-Bratzler shear force value was found to have no difference between all treatments $(P>0.05)$. Color was not different initially, until day 5 of display $(P>0.05)$, where both dry aging and UVlight dry aging had a significant increase in hue angle and decrease in redness, yellowness, and chroma values at the end of display $(P<0.05)$. Lipid oxidation increased during display, but no difference among the treatments was found $(P>0.05)$. Consumer panelists indicated that there were no significant differences in flavor, tenderness, juiciness, and overall liking between the treatments. Overall, aging in general improved pork quality, indicated by high rating from consumers.

Conclusion: The results of the current study showed that dry aging would not negatively impact pork loin quality. Application of dry aging could potentially improve sensory acceptance due to greater water-holding capacity of the final product. Additionally, both dry aging and UV-light dry aging had a good color and oxidative stability, which could further suggest the benefit of dry aging application on pork product. Further study to identify flavor precursor changes as well as palatability profiling by trained panelists would be of interest.

Keywords: color stability, dry aging, pork, sensory panel 
104 - CARCASS MERIT OF FIVE BRAHMANINFLUENCED BREED TYPES AND EFFECTS OF ELECTRICAL STIMULATION AND WET AGING ON PALATABILITY OF GRASS-FED BULLOCK MEAT

T. Riera-Sigala ${ }^{1}$, N. Huerta-Leidenz ${ }^{2 *}$, N. Jerez-Timaure ${ }^{3}$, M. Arispe-Zubillaga ${ }^{4}$, and A. R. Rodas-Gonzalez ${ }^{5}$, ${ }^{1}$ Ingenieria de Produccion Animal, Universidad Rafael Urdaneta, Maracaibo, Venezuela, Bolivarian, ${ }^{2}$ Animal and Food Sciences, Texas Tech University, Lubbock, TX, USA, ${ }^{3}$ Instituto de Ciencia Animal, Facultad de Ciencias Veterinarias, Universidad Astral de Chile, Valdivia, Chile, ${ }^{4}$ Gerente de Produccion, Matadero Industrial Centro Occidental C.A., Barquisimeto, Venezuela, Bolivarian, ${ }^{5}$ Animal Science, University of Manitoba, Winnipeg, Canada,*argenis.rodasgonzalez@umanitoba.ca

Objectives: The study objective was to explore differences in carcass traits and meat quality among 5 types of Brahmaninfluenced bullocks, and to examine the influence of electrical stimulation and/or wet aging on shear force and sensory traits of bullock meat.

Materials and Methods: Fifty grass-fed bullocks of 5 breed types (Brahman, F1 Angus, F1 Chianina, F1 Romosinuano, and F1 Simmental) were harvested at ca. $480 \mathrm{~kg}$. Carcasses were split, and the right side of each carcass was electrically stimulated $(\mathrm{ES} ; 550 \mathrm{~V}, 60 \mathrm{~Hz}$, $2 \mathrm{amp}$ ). At $2 \mathrm{~d}$ postmortem, chilled carcasses were evaluated, a 20-cm-long striploin roast (longissimus lumborum) from each side was excised and subsampled to yield eight 2.5 -cm-thick steaks in 4 segments of 2 steaks each. The 8 steaks from each roast were allocated to each of the two (2-d or 10-d) aging treatments (a pair for descriptive sensory analysis and another pair for Warner Bratzler shear force [WBSF]) vacuum packaged and accordingly identified. Anatomical position bias was avoided by alternating analysis designations (WBSF and descriptive sensory analysis) and by rotating ES or aging treatments. Steaks representing control samples (2-d aging time) were immediately blast-frozen and stored at $-30^{\circ} \mathrm{C}$ for subsequent analyses. The other steaks representing the 10-d aging (AG) treatment were stored at $4^{\circ} \mathrm{C}$ and, at the end of that period, were frozen $\left(-30^{\circ} \mathrm{C}\right)$ and stored for further analysis. Data were analyzed with a split-plot arrangement.

Results: Except for the fat cover finish score, carcass traits were not affected by breed type $(P>0.05$; ca. A maturity, 1.44-mm backfat thickness, "traces" of marbling). Carcasses of Brahman exhibited the most abundant fat cover of all breed types $(P<0.01)$. No difference in WBSF was detected between breed types $(P>0.05)$. Steaks from F1 Angus scored highest $(P<0.05)$ for muscle fiber tenderness (MFT; "slightly tender"), overall tenderness (OT; "slightly tough"), and amount of connective tissue (ACT; "moderate"), whereas F1 Simmental scored lowest in MFT ("slightly tough"), OT ("moderately tough"), and ACT ("slightly abundant"). Brahman, F1 Chianina, and F1 Romosinuano did not differ $(P>0.05)$ in sensory attributes and exhibited intermediate values. Bullock steaks were more responsive to $\mathrm{ES}+\mathrm{AG}$ in WBSF reduction and increased scores for MFT ("slightly tender"), OT ("slightly tough"), and ACT ("moderate") compared with other postmortem treatments $(P<0.05)$. ES + AG steaks reached the largest proportion $(72 \%)$ of the "tender steaks" class $(<4.09 \mathrm{~kg}$ WBSF) compared to those from the AG (48\%), ES (36\%), and control $(24 \%)$ groups $(P<0.01)$.

Conclusion: Because of their superiority in tenderness, steaks from F1 Angus are expected to be more acceptable than those from F1 Simmental. The combined treatment of $\mathrm{ES}+\mathrm{AG}$ resulted in the most effective postmortem treatment for tenderizing bullock meat of Bos indicus influence.

Keywords: beef palatability, bullock, crossbreeding, electrical stimulation, vacuum aging

\section{5 - CALPAIN AUTOLYSIS AND PROTEOLYSIS OF BOS TAURUS AND BOS INDICUS LONGISSIMUS LUMBORUM DURING 14-DAY AGING}

M. McKinney ${ }^{1 *}$, B. Hawryluk ${ }^{1}$, C. Carr ${ }^{1}$, P. Ramos ${ }^{1,2}$, and T. Scheffler ${ }^{1},{ }^{1}$ Department of Animal Sciences, University of Florida, Gainesville, United States, ${ }^{2}$ Department of Animal Sciences, University of Sao Paulo, Piracicaba, Brazil,*tscheffler@ufl.edu

Objectives: The objective of this study was to determine calpain autolysis and protein degradation in aged Bos taurus (Angus) and Bos indicus (Brahman) steaks and their relationship with tenderness.

Materials and Methods: Angus and Brahman steers ( $n=14$ per breed) were reared in the same conditions. Samples from the longissimus lumborum (LL) were taken at $1 \mathrm{~h}$ and $24 \mathrm{~h}$ postmortem, immediately frozen in liquid nitrogen, and stored at $-80^{\circ} \mathrm{C}$ until analysis. At $48 \mathrm{~h}$, an approximately $8-\mathrm{cm}$ section was removed posterior to the 12 th and 13th rib, which was subsequently cut into three 2.54-cm-thick steaks. Steaks were vacuum packaged and aged at $3^{\circ} \mathrm{C}$ for $14 \mathrm{~d}$ postmortem. After aging, 2 steaks were frozen at $-40^{\circ} \mathrm{C}$ until further analysis of tenderness, and the remaining steak was used for protein biochemistry. For protein extraction, pulverized LL was diluted in extraction buffer, homogenized, and centrifuged. Protein concentration of supernatants was determined using a bicinchoninic acid protein assay, and samples were diluted to obtain equal protein concentrations. Proteins were separated using sodium dodecyl sulfate-polyacrylamide gel electrophoresis. Proteins were then transferred to nitrocellulose membranes. Membranes were stained for total protein, which was used as 
an index to normalize protein content. Membranes were blocked and incubated with primary antibodies for desmin, troponin- $T$, and $\mu$-calpain, followed by secondary antibody conjugated with fluorescent dye. Bands were detected using the LI-COR Odyssey and quantified using ImageStudio software. For tenderness evaluation, steaks were thawed at $4^{\circ} \mathrm{C}$ and cooked to an internal temperature of $71{ }^{\circ} \mathrm{C}$. Tenderness was determined objectively by Warner-Bratzler shear force (WBSF) and a trained sensory panel $(1=$ extremely tough; $8=$ extremely tender). Data were analyzed using SAS mixed procedure (SAS Institute Inc., Cary, NC) with fixed effects of time, breed, and the interaction; time was considered a repeated measure for proteolysis. Tenderness and WBSF were analyzed using the fixed effect of breed.

Results: Breed affected $\mu$-calpain autolysis $(P=$ 0.0085). Brahman showed less $\mu$-calpain autolysis at $24 \mathrm{~h}$ $(P=0.0040)$ than Angus. Degradation of troponin-T $(P=$ $0.0028)$ and desmin $(P=0.0210)$ were lower for Brahman compared to Angus. Troponin-T degradation was lower at $24 \mathrm{~h}(P=0.0288)$ and $14 \mathrm{~d}(P=0.0106)$ in Brahman compared to Angus. Desmin degradation was also lower at $24 \mathrm{~h}$ $(P=0.0421)$ in Brahman. Brahman were less tender than Angus, evidenced by higher WBSF $(P=0.0029)$ and lower sensory panel scores $(P=0.0007)$. Brahman also showed greater variability in tenderness than Angus (sensory: $4.51 \pm 0.91$ vs. $5.60 \pm 0.55$, respectively). Within Brahman samples, protein degradation (14 d) of desmin $(P=0.0161)$ and troponin-T $(P=0.0090)$, and $\mu$-calpain autolysis at $24 \mathrm{~h}$ $(P=0.0211)$, were associated with tenderness.

Conclusion: As expected, there are differences between proteolysis of steaks from Angus and Brahman breeds. There is a large amount of tenderness variability within Brahman. Tender Brahman do exist, and greater $\mu$-calpain autolysis is positively associated with proteolysis and tenderness. In order to enhance value of Brahman and reduce variability, it is important to identify ante- and postmortem predictors of tenderness specifically aimed at Brahman.

Keywords: Brahman, calpain, desmin, tenderness, troponin- $\mathrm{T}$

\section{6 - IMPACT OF DIET AND QUALITY GRADE ON PROTEOMIC ANALYSIS OF OXIDIZED PROTEINS IN BEEF}

\author{
N. A. Bland ${ }^{1 *}$, N. J. Herrera ${ }^{1}$, F. A. Ribeiro ${ }^{1}$, M. L. Henriott ${ }^{1}$, \\ K. B. Hart ${ }^{1}$, and C. R. Calkins ${ }^{1},{ }^{1}$ Department of Animal \\ Science, University of Nebraska, Lincoln, NE, USA, *nicolas. \\ bland@huskers.unl.edu
}

Objectives: The explanation of the relationship between marbling and beef tenderness is still unclear, highlighting the need to evaluate the relationship between these valuable aspects of a positive consumer eating experience. This study was conducted to evaluate the effects of feeding diets with different types of processed corn and with or without distillers grains on byproducts of oxidative stress and how that relates to tenderness and oxidative damage of proteins in beef steaks of differing quality grade.

Materials and Methods: Steers $(n=240)$ were finished on diets containing dry rolled corn (DRC), DRC $+30 \%$ dried distillers grains, steam flaked corn (SFC), or SFC + $30 \%$ dried distillers grains. Cattle were fed 10 per pen. Only pens with both upper 2/3 Choice and Select-grade carcasses were sampled, with a goal of 2 (and a minimum of 1 ) of each grade per pen. Three pens per treatment met the selection criterion, and 36 carcasses were chosen (21 upper $2 / 3$ Choice and 15 Select). Strip loins aged for $2 \mathrm{~d}$ postmortem were fabricated in steaks and evaluated for WarnerBratzler shear force and proteomic analysis evaluating oxidative damage in proteins.

Results: Upper 2/3 Choice grade steaks were found to be more tender than Select grade steaks $(P<0.05)$. Across comparisons, proteomic analysis revealed increased oxidative damage of proteins like heat shock, glycolytic, apoptotic, and structural proteins. Upper 2/3 Choice steaks were found to generally have increased oxidative damage in glycolytic, structural, and heat shock proteins, compared to Select quality grade, similar to those identified in previous research evaluating tender meat $(P<0.05)$. Samples from cattle fed DRC had increased oxidative damage from feeding distillers grains, while the SFC-related treatments had the inverse.

Conclusion: Overall results support the relationship between marbling and tenderness and suggest that oxidative stress may be a factor involved in the phenomenon.

Keywords: distillers grains, marbling, oxidative stress, proteomics, tenderness

\section{7 - MEAT CONSUMPTION-WHAT DO FRENCH CONSUMERS FEEL ABOUT THE QUALITY OF BEEF?}

M.-P. Ellies-Oury ${ }^{1}$ and J.-F. Hocquette ${ }^{1 *},{ }^{1} U M R H 1213$, INRA, Saint Genès Champanelle, France, *marie-pierre. ellies@agro-bordeaux.fr

Objectives: The French beef sector developed a new trading system for beef in self-service aisles published in 2014 in a ministerial decree. It is based on labels indicating a simplified name of cut, the potential quality of each cut expressed in a number of stars, and the preferred cooking method. After a few years of implementation, the aim of the current survey was to study beef consumption habits and to get feedback from consumers about this new meat labelling system.

Materials and Methods: To investigate consumers' purchasing behaviors, attitudes, and knowledge about the new labelling system, an ad hoc questionnaire was designed. A total of 625 individuals participated in a survey with 
questions related to sociodemographic characteristics, focused on meat purchasing behaviors and consumption, or related to consumer perceptions of the new beef labelling system.

Results: Respondents say they eat beef because they like its taste $(87 \%)$ but also because meat is a source of various important nutrients such as protein and iron $(50 \%)$. Over the last few years, the respondents' consumption has remained stable or has diminished. The reasons for the decline in meat consumption are numerous: too-high price, the possible health risks, the lack of sensory consistency, animal welfare concerns, and the impact of health scandals. While the respondents indicated that they paid close attention to the labels (81\%), a large proportion of them (72\%) had not noticed the presence of stars on labels of beef in supermarket self-service aisles. About $75 \%$ of the respondents, who do use this potential quality ranking, consider that eating quality should be reflected by the number of stars indicated on the label. When both old and new labels were shown to the respondents, $57 \%$ said they prefer the new labelling system, $75 \%$ of them found the new system clearer due to the presence of stars, and $10 \%$ like the cooking method tips. The respondents who prefer the old labelling system (13\%) do not like not knowing the name of the cut (55\%) and criticize the "marketing" aspect, which does not "educate" consumers $(22 \%)$. A proposal would be to place the name of the cut in italics under the generic name in order to satisfy both educated consumers and those who do not know the names of cuts. Of the respondents, $88 \%$ would be interested in a system that would ensure a guaranteed level of meat tenderness/taste at the time of purchase. Consumers that often eat meat appear to be more interested in such a system. This type of system could encourage consumers to purchase and eat more beef undoubtedly or probably for all consumers or for the restricted population who had decreased their meat consumption.

Conclusion: The beef industry should, in addition to maximize meat yields and profit, guarantee good sensory and nutritional quality in order to better meet consumers' expectations (2). Consumers generally highlight the importance of the cut at the time of purchase, which is highly relevant since cuts have different tenderness potential. This explains why some quality prediction systems (e.g., the Meat Standards Australia [MSA] grading scheme) are based on the cut and not the carcass as in Europe (3). In this respect, the star system is a real step forward in satisfying consumer expectations. However, it is still incomplete as, unlike MSA, it does not take into account other factors that contribute to the variability of beef eating quality. Further developing the star system to something comparable to MSA would be judicious, as it will be appreciated by European consumers (4).

Keywords: consumer behavior, French labelling system, meat consumption, survey

American Meat Science Association.

108 - EFFECTS OF BONE AND SUBCUTANEOUS FAT ON THE YIELD AND PHYSICAL-CHEMICAL TRAITS OF DRYAGED BEEF

A. P. S. Bernardo ${ }^{1}$, F. A. Ribeiro ${ }^{2}$, C. R. Calkins ${ }^{2}$, and S. B. Pflanzer ${ }^{1 *}$, ${ }^{1}$ Department of Food Technology, State University of Campinas, Campinas, Brazil, ${ }^{2}$ Animal Science, University of Nebraska, Lincoln, NE, USA, *spflanzer@gmail.com

Objectives: Compared to wet aging, dry aging is considered a costly process, and requires strict control of cooling conditions and larger spaces in chambers. Bone and subcutaneous fat could reduce weight loss during dry aging, preventing liquid evaporation from the lean. So, the present study aimed to evaluate the individual and combined effects of bone and subcutaneous fat on total yield and physical-chemical traits of dry-aged beef from grass-fed zebu cattle.

Materials and Methods: Eight paired, bone-in strip loins from grass-fed zebu steers ( $\sim 30$ mo old; $\sim 290 \mathrm{~kg}$ of carcass weight; $\sim 6.0 \mathrm{~mm}$ of subcutaneous fat thickness) were collected. Each loin was cut in the middle, and each half-loin was assigned to one of the 4 treatments: bone-in with subcutaneous fat, bone-in without subcutaneous fat, boneless with subcutaneous fat, and boneless without subcutaneous fat. The loin sections were aged for $21 \mathrm{~d}\left(2^{\circ} \mathrm{C}\right.$ and $70 \%$ relative humidity). After aging, the evaporation and trimming losses and total yield were determined. After trimming, the loins were cut into steaks and analyzed for surface water activity (crust), internal $\mathrm{pH}$ and moisture content, shear force, and instrumental color stability during retail display up to $9 \mathrm{~d}$ (with no lights). The experimental design was a $2 \times 2$ factorial (bone-in and boneless; with and without fat). The data obtained were statistically analyzed using Statistica Version 10.0 by two-way analysis of variance. Color results were analyzed by SAS version 9.2 (SAS Institute Inc., Cary, NC), using the PROC GLIMMIX procedure with bone, fat, and time as a fixed factor and carcasses as a random factor. When significance $(P<0.05)$ was indicated, LSMEANS and DIFF functions were used to separate the means.

Results: No interactions between bone and subcutaneous fat were found for evaporation, trimming, and yield traits, and for the $\mathrm{pH}$, surface water activity, moisture content, shear force, and color attributes $(P>0.05)$. Boneless samples showed higher evaporation $(22.76 \% \pm 0.96 \%)$ and trimming losses $(18.18 \% \pm 0.65 \%)$ and lower yield $(40.79 \% \pm 1.42 \%)$ compared to bone-in samples $(15.63 \%$ $\pm 0.76 \%, 8.16 \% \pm 0.55 \%$, and $49.77 \% \pm 1.15 \%$, respectively). Similarly, samples without subcutaneous fat had higher evaporation $(21.81 \% \pm 1.23 \%)$ and trimming losses $(14.64 \% \pm 1.53 \%)$ and lower yield $(41.64 \% \pm 1.46 \%)$ compared to samples with subcutaneous fat $(16.58 \% \pm 0.88 \%$, $11.70 \% \pm 1.21 \%$, and $48.92 \% \pm 1.46 \%$, respectively). Shear force $(3.25 \pm 0.25 \mathrm{kgf})$ and $\mathrm{pH}(5.44 \pm 0.01)$ were not affected by the presence or absence of bone or fat $(P>0.05)$. 
Boneless samples had lower values of moisture $(72.97 \% \pm$ $0.21 \%)$ and surface water activity $(0.9324 \pm 0.0021)$ than bone-in samples $(74.06 \pm 0.23$ and $0.9409 \pm 0.0020$, respectively). Samples without subcutaneous fat showed lower surface water activity values $(0.9336 \pm 0.0024)$ than samples with subcutaneous fat $(0.9396 \pm 0.0020)$. For color, there was a bone and display time interaction $(P<0.05)$ for $\mathrm{CIE} a^{*}$ and $b^{*}$ parameters. After $6 \mathrm{~d}$ of display, bone-in samples had a greater decrease of $a^{*}$ values, and the $b^{*}$ values were lower after $8 \mathrm{~d}$, compared to boneless samples.

Conclusion: Results indicated that bone and subcutaneous fat had a protective effect on the lean beef, reducing evaporation and trimmings, resulting in a more economically feasible process. However, the presence of bone could lead to a decrease in color stability during retail display.

Keywords: aging, dry-aged, yield

\section{9 - IMPACT OF BEEF CARCASS SIZE ON CHILLING RATE, PH, DISPLAY COLOR, AND TENDERNESS OF TOP ROUND SUBPRIMALS}

J. M. Lancaster ${ }^{*}$, B. J. Buseman ${ }^{1}$, T. M. Weber ${ }^{1}$, J. A. Nasados $^{1}$, G. K. Murdoch ${ }^{1}$, W. J. Price ${ }^{2}$, M. J. Colle ${ }^{1}$, and P. D. Bass ${ }^{1},{ }^{1}$ Animal and Veterinary Science, University of Idaho, Moscow, ID, USA, ${ }^{2}$ Statistical Programs, University of Idaho, Moscow, ID, USA, *lanc6314@vandals.uidaho. edu

Objectives: The objective of the study was to investigate the impact of beef carcass size on chilling rate, $\mathrm{pH}$ decline, retail display color, and tenderness of the beef top round (North American Meat Institute \#169A).

Materials and Methods: Eight beef carcasses identified to be near the US industry average hot carcass (AW; 341$397 \mathrm{~kg}$ ) and 8 beef carcasses identified as "oversized" (OW; 433-500 kg) were evaluated for the study. Temperature loggers were placed at superficial $(2.54 \mathrm{~cm}$ from surface) and deep (approximate sagittal center, touching femur bone of respective carcass) locations of the top round, and temperatures were logged continuously for $48 \mathrm{~h}$ post USDA inspection. During the initial $12 \mathrm{~h}$, and every $5 \mathrm{~h}$ subsequently, $\mathrm{pH}$ measurements were observed on all carcasses at a consistent superficial and deep anatomical location of the respective top rounds. Carcasses were fabricated into subprimals at $48 \mathrm{~h}$, and top rounds were aged at $2^{\circ} \mathrm{C}$ for an additional $12 \mathrm{~d}$. Six steaks were cut from each top round proximally to distally. Steak assignment was systematically rotated to account for steak location. Steaks were assigned to one of the following analyses: color analysis, lipid oxidation using the thiobarbituric acid reactive substances (TBARS) method, and Warner-Bratzler Shear Force (WBSF) tenderness analysis. Steaks for color analysis and TBARS were placed on Styrofoam trays, overwrapped with an oxygenpermeable film, and displayed in a glass-front retail display case at $3{ }^{\circ} \mathrm{C}$ for a simulated retail display of $4 \mathrm{~d}$. Steaks for color analysis and TBARS were evaluated daily. The steaks allocated to WBSF were tempered for $24 \mathrm{~h}$ at $4{ }^{\circ} \mathrm{C}$ and were subsequently cooked on a two-sided electric grill to an internal temperature of $71^{\circ} \mathrm{C}$. Six cores per deep and superficial portion of each steak were removed and sheared perpendicular to the muscle fibers. Calpain samples from the deep and superficial portion were snap frozen on day 2 and 14 and stored at $-80^{\circ} \mathrm{C}$. Subsequently calpain samples were extracted and evaluated using casein zymography. Data were analyzed using SAS (SAS Institute Inc., Cary, NC), and significance was determined at $P<0.05$.

Results: The superficial anatomical location on the top round of both AW and OW carcasses cooled at a faster rate $(P<0.01)$ than the deep locations. The deep location of OW carcasses had a lower $\mathrm{pH}$ and a more rapid $(P<0.01)$ initial $\mathrm{pH}$ decline. Quantitative color of steaks from $\mathrm{OW}$ carcasses had higher mean $L^{*}$ (lightness; $P<0.01$ ) and $b^{*}$ (yellowness; $P<0.001$ ), and lower $a^{*}$ (redness; $P<0.001$ ), values. There was an interaction of day and carcass weight for TBARS $(P=0.01)$ with AW steaks having greater TBARS values as the retail display progressed. Mean WBSF values were not different $(P=0.24)$. The superficial portion had a greater percentage of native calpain-2 activity compared to the deep portion $(P=0.01)$, and the AW carcasses had a lower percentage of autolyzed calpain- 2 activity compared to OW carcasses.

Conclusion: The delayed temperature decline in combination with accelerated $\mathrm{pH}$ decline of the deep portion of the top round of OW carcasses occurs at different rates than AW carcasses. This rate change leads to irreversible impacts on steak appearance characteristics and potential eating quality of top round steaks fabricated from OW beef carcasses when compared to AW carcasses.

Keywords: beef, carcass size, $\mathrm{pH}$, temperature, top round

\section{0 - FREE CALCIUM CONCENTRATION, CALPAIN-2 ACTIVITY, AND FINAL PRODUCT TENDERNESS OF ELECTRICALLY STIMULATED BEEF}

B. Buseman ${ }^{1 *}$, T. Weber ${ }^{1}$, J. Nasados ${ }^{1}$, P. Bass ${ }^{1}$, J. Van Buren $^{1}$, J. Lancaster ${ }^{1}$, J. Smart ${ }^{1}$, M. Doumit ${ }^{2}$, G. Murdoch ${ }^{1}$, W. Price ${ }^{2}, \mathrm{~K}$. Insausti ${ }^{3}$, and M. Colle ${ }^{1},{ }^{1}$ Animal and Veterinary Sciences, University of Idaho, Moscow, ID, USA, ${ }^{2}$ College of Agriculture and Life Sciences, University of Idaho, Moscow, ID, USA, ${ }^{3}$ Agricultural Engineering School-IS FOOD, Public University of Navarra, Pamplona, Spain,*buse2360@vandals.uidaho.edu 
Objectives: The objective of this study was to evaluate timing of electrical stimulation on free calcium concentration, calpain-2 activity, Warner-Bratzler shear force (WBSF), and consumer sensory analysis.

Materials and Methods: Twenty-three crossbred beef steers were harvested and stimulated using extra-low voltage 21) $\mathrm{V}$ for $20 \mathrm{~s}$ ) or not stimulated at exsanguination and at $1 \mathrm{~h}$ postmortem, resulting in 4 stimulation treatments: Not Stimulated-Not Stimulated, Not Stimulated-Stimulated, Stimulated-Not Stimulated, or Stimulated-Stimulated. Samples were cut from the longissimus lumborum (LL) and semimembranosus (SM) for free calcium and calpain2 analysis on days 1,4 , and 14 postmortem. On day 4, steaks were cut from the LL and SM for WBSF and consumer sensory analysis and assigned to an aging period of 4 or $14 \mathrm{~d}$. Free calcium concentration was analyzed by mixing samples with calcium ion strength adjuster (Hanna Instruments, Woonsocket, RI) and measuring ionic strength of the solution using a calcium selective electrode. Ionic strength of each sample was compared to a calibration curve to determine final concentration. Calpain analysis was conducted utilizing casein zymography. The volume of each band was recorded as a percentage of the day 0 control band ran on each gel. For WBSF analysis, six $1.27-\mathrm{cm}$ cores were removed from each steak parallel to the muscle fibers, with care to avoid connective tissue and fat. Cores were sheared perpendicular to the muscle fibers, and peak shear force was recorded. One consumer sensory panel for each muscle group was held, with panelists receiving 5 samples. Samples were assigned to the panelists using an incomplete block design. Data were analyzed using the Mixed Model procedure of SAS (SAS Institute Inc., Cary, NC), with significance determined at $P<0.05$.

Results: There was a tendency for an aging period by stimulation treatment interaction for LL free calcium concentration $(P=0.0503)$. While all treatments had similar initial and final values, the Not Stimulated-Not Stimulated treatment showed an increase in free calcium concentration relative to the other treatments at $4 \mathrm{~d}$ of aging. No difference was observed for free calcium concentration in the SM between stimulation treatments $(P=0.44)$; aging, however, significantly increased SM free calcium concentration $(P<0.01)$. Stimulation did not impact native calpain-2 activity in the LL $(P=0.71)$ or SM $(P=0.89)$. Aging period did not significantly influence native clapain-2 activity in the LL $(P=0.11)$; however, it did show higher activity in the SM on day $14(P=0.08)$. Furthermore, stimulation treatment did not improve WBSF in the LL $(P=0.69)$ or SM $(P=0.61)$. Aging period was significant in the LL, with steaks aged $14 \mathrm{~d}$ being more tender than those aged only $4 \mathrm{~d}(P<0.01)$. Conversely, aging period did not significantly influence WBSF values in the SM $(P=0.61)$. Stimulation treatment did not influence consumer sensory scores for tenderness in the LL $(P=$ $0.56)$ or SM $(P=0.36)$. In the $\mathrm{LL}$, aging period did not show a significant influence on tenderness $(P=0.71)$. In the SM, consumers preferred samples aged $14 \mathrm{~d}$ over those aged $4 \mathrm{~d}(P<0.01)$.

Conclusion: In conclusion, the timing of electrical stimulation utilized in the current study did not influence free calcium concentration, calpain-2 activity, or beef tenderness; however, aging did improve tenderness.

Keywords: beef, calcium, calpain, electrical stimulation, tenderness

\section{1 - ENDPOINT TEMPERATURE AND VARIABILITY IN PORK AND BEEF COOKED TO VARYING DEGREES OF DONENESS USING TWO DIFFERENT COOKING METHODS}

\author{
D. Shirey ${ }^{1 *}$, E. Bryan ${ }^{1}$, J. Redifer $^{1}$, B. Harsh ${ }^{1}$, and A. Dilger ${ }^{1}$, \\ ${ }^{1}$ Animal Sciences, University of Illinois, Urbana- \\ Champaign, IL, USA, *dshirey2@illinois.edu
}

Objectives: The objective was to determine endpoint temperature (EPT) and Warner-Bratzler shear force variability differences in pork and beef cooked to varying degrees of doneness using grilling and sous vide.

Materials and Methods: Ten beef semitendinosus muscles were cut into four $2.54-\mathrm{cm}$ steaks $(n=40)$ and aged for $21 \mathrm{~d}$. Fifty-one pork loins were cut into four $2.54-\mathrm{cm}$ chops starting at the 10th rib each loin $(n=204)$ and aged for $7 \mathrm{~d}$. Steaks and chops were randomly allotted within whole muscle to 4 treatments: grilled or sous vide to $63^{\circ} \mathrm{C}$, or grilled or sous vide to $71^{\circ} \mathrm{C}$. An analysis of variance (ANOVA) precision immersion cooker (ANOVA Applied Electronic, Inc., San Francisco, CA) in $18 \mathrm{~L}$ of water, and a Farberware Open Hearth grill (model 455N; Walter Kidde, Bronx, NY) were used. Sous vide chops and steaks remained in the water bath for $90 \mathrm{~min}$ to complete cooking. A thermocouple was inserted into the geometric center of each grilled chop or steak. When thermocouple readings reached the allotted EPT, chops or steaks were removed from the grill. Regardless of cooking method, 2 handheld thermometers were inserted into each end of the chop or steak after cooking to determine actual EPT. Chops and steaks were cooled to room temperature before 6 cores were excised parallel to the muscle fibers and analyzed for Warner-Bratzler shear force. Data were analyzed using two-way ANOVA with originating semitendinosus or loin muscle and cook day as blocking factors. Least-squares means were separated using the PDIFF option in the MIXED procedure of SAS (SAS Institute Inc., Cary, NC).

Results: Among pork chops cooked to $63^{\circ} \mathrm{C}$, the 2 recorded EPT within grilled chops differed by 4.05 degrees but differed by only 0.23 degrees in sous vide chops $(P<$ 0.01). When cooked to $71^{\circ} \mathrm{C}$, the 2 recorded EPT within 
grilled chops differed by 4.16 degrees but differed by only 0.21 degrees in sous vide chops $(P<0.01)$. Among chops cooked to $63^{\circ} \mathrm{C}$, average recorded EPT of grilled chops deviated 9.46 degrees from target temperature, whereas recorded EPT of sous vide chops deviated 1.57 degrees $(P<0.01)$. When cooked to $71^{\circ} \mathrm{C}$, average recorded EPT of grilled chops deviated 8.90 degrees from target temperature, whereas recorded EPT of sous vide chops deviated $1.69 \mathrm{deg}-$ rees $(P<0.01)$. Among beef steaks cooked to $63^{\circ} \mathrm{C}$, the 2 recorded EPT within grilled steaks differed by 3.08 degrees but differed by only 0.26 degrees in sous vide chops $(P<$ $0.01)$. When cooked to $71^{\circ} \mathrm{C}$, the 2 recorded EPT within grilled steaks differed by 2.97 degrees but differed by only 0.48 degrees in sous vide chops $(P<0.01)$. Among beef steaks cooked to $63^{\circ} \mathrm{C}$, average recorded EPT of grilled steaks deviated 4.04 from the target temperature, whereas recorded EPT of sous vide steaks deviated 0.34 degrees $(P<0.01)$. When cooked to $71^{\circ} \mathrm{C}$, average recorded EPT of grilled steaks deviated 5.64 degrees from target temperature, whereas recorded EPT of sous vide steaks deviated 0.64 degrees $(P<0.01)$. Sous vide pork chops were more tender at $63^{\circ} \mathrm{C}$ than grilled chops but less tender at $71^{\circ} \mathrm{C}$
$(P \geq 0.06)$. There were no differences between cooking methods in tenderness of beef steaks at either temperature $(P \geq 0.49)$.

Conclusion: Overall, these data indicate that using sous vide results in less EPT variability in both pork and beef cooked to varying degrees of doneness.

Keywords: endpoint temperature, grilling, sous vide, variability

\section{2 - STUDY OF THE FATTY ACID PROFILE FROM FILLETS OF BROILERS AFFECTED BY DEEP PECTORAL MYOPATHY}

A. Giampietro Ganeco ${ }^{1 *}$, H. Borba ${ }^{2}$, M. R. Pereira ${ }^{2}$, E. A. Villegas-Cayllahua $^{2}$, É. N. F. Cavalcanti ${ }^{2}$, F. B. Ferrari ${ }^{2}$, R. F. Oliveira ${ }^{2}$, and M. A. Trindade ${ }^{1},{ }^{1}$ Department of Food Engineering, University of São Paulo-USP, Pirassununga, Brazil, 2Department of Technology, São Paulo State University-UNESP, Jaboticabal, Brazil, *giampietroganeco@gmail.com

Table 1. Mean values and standard deviation of fatty acid profile of Pectoralis major muscle samples affected by deep pectoral myopathy (DPM).

\begin{tabular}{|c|c|c|c|c|}
\hline Fatty acid & Unaffected & DPM score 2 & DPM score 3 & $P$-value \\
\hline $\mathrm{Cl} 10: 0$ & 0,000 & 0,000 & 0,000 & 0,0500 \\
\hline $\mathrm{Cl} 2: 0$ & $0,000^{\mathrm{B}}$ & $0,013^{\wedge}$ & $0,003^{\mathrm{AB}}$ & 0,0202 \\
\hline $\mathrm{Cl} 4: 0$ & $0,283^{\text {в }}$ & $0,380^{A}$ & $0,383^{A}$ & 0,0003 \\
\hline $\mathrm{Cl} 15: 0$ & 0,083 & 0,076 & 0,078 & 0,5801 \\
\hline $\mathrm{Cl} 6: 0$ & $22,001^{\text {В }}$ & $23,060^{A}$ & $23,210^{\wedge}$ & 0,0083 \\
\hline $\mathrm{Cl} 7: 0$ & 0,130 & 0,135 & 0,125 & 0,5229 \\
\hline $\mathrm{Cl} 8: 0$ & $11,561^{A}$ & $8,043^{\text {в }}$ & $9,595^{\mathrm{B}}$ & 0,0069 \\
\hline $\mathrm{C} 20: 0$ & 0,076 & 0,085 & 0,081 & 0,1144 \\
\hline Total SFA ${ }^{3}$ & $56,501^{\wedge}$ & $31,792^{\mathrm{B}}$ & $33,475^{B}$ & 0,0005 \\
\hline $\mathrm{Cl} 4: 1$ & $0,030^{\mathrm{B}}$ & $0,058^{\wedge}$ & $0,055^{A}$ & 0,0050 \\
\hline $\mathrm{Cl} 6: 1$ & $1,258^{\mathrm{B}}$ & $2,121^{A}$ & $2,198^{A}$ & 0,0094 \\
\hline C $17: 1$ & $0,125^{\wedge}$ & $0,050^{\mathrm{B}}$ & $0,038^{\mathrm{B}}$ & 0,0006 \\
\hline $\mathrm{C} 18 \mathrm{l} \ln 9 \mathrm{c}$ & $20,913^{\text {в }}$ & $28,798^{\wedge}$ & $25,592^{\wedge}$ & 0,0005 \\
\hline $\mathrm{C} 18: \ln 7$ & $2,758^{\wedge}$ & $1,798^{\mathrm{B}}$ & $2,758^{\wedge}$ & $<0,001$ \\
\hline $\mathrm{C} 20: \ln 9$ & $0,285^{\wedge}$ & $0,260^{A B}$ & $0,238^{\mathrm{B}}$ & 0,0455 \\
\hline Total MUFA ${ }^{3}$ & $25,369^{\mathrm{B}}$ & $33,085^{\wedge}$ & $30,879^{\wedge}$ & 0,0006 \\
\hline $\mathrm{Cl} 8: 2 \mathrm{n} 6 \mathrm{c}$ & $19,535^{\mathrm{B}}$ & $27,758^{\wedge}$ & $25,422^{A}$ & 0,0001 \\
\hline $\mathrm{Cl} 8: 2 \mathrm{c} \underline{\underline{\mathrm{t}} 11}$ & 0,015 & 0,011 & 0,013 & 0,8752 \\
\hline $\mathrm{C} 20: 2$ & $1,245^{\wedge}$ & $0,540^{\mathrm{B}}$ & $0,668^{\mathrm{B}}$ & 0,0005 \\
\hline $\mathrm{Cl} 8: 3 \mathrm{n} 6$ & $0,118^{\mathrm{B}}$ & $0,176^{A}$ & $0,195^{A}$ & 0,0026 \\
\hline $\mathrm{Cl} 8: 3 \mathrm{n} 3$ & $0,5517^{\mathrm{c}}$ & $1,926^{A}$ & $1,506^{\mathrm{B}}$ & $<0,001$ \\
\hline$C 20: 3 n 6$ & $1,341^{\wedge}$ & $0,691^{\text {в }}$ & $1,030^{\mathrm{AB}}$ & 0,0062 \\
\hline $\mathrm{C} 20: 3 \mathrm{n} 3$ & $0,158^{A}$ & $0,065^{\mathrm{B}}$ & $0,086^{\mathrm{B}}$ & 0,0013 \\
\hline $\mathrm{C} 20: 4 \mathrm{n} 6$ & $9,328^{\wedge}$ & $2,960^{\mathrm{B}}$ & $4,545^{\mathrm{B}}$ & $<0,001$ \\
\hline $\mathrm{C} 22: 4 \mathrm{n} 6$ & $3,048^{\wedge}$ & $0,991^{\text {в }}$ & $1,386^{\mathrm{B}}$ & $<0,001$ \\
\hline $\mathrm{C} 20: 5 \mathrm{n} 3$ & $0,430^{\wedge}$ & $0,125^{\mathrm{B}}$ & $0,201^{\mathrm{B}}$ & $<0,001$ \\
\hline $\mathrm{C} 22: 5 \mathrm{n} 3$ & $1,905^{\wedge}$ & $0,556^{\mathrm{B}}$ & $0,823^{\mathrm{B}}$ & 0,0002 \\
\hline $\mathrm{C} 22: 6 \mathrm{n} 3$ & $1,016^{\wedge}$ & $0,316^{\mathrm{B}}$ & $0,481^{\mathrm{B}}$ & $<0,001$ \\
\hline Total PUFA $^{3}$ & $38,70^{\wedge}$ & $36,115^{\mathrm{B}}$ & $36,356^{\mathrm{B}}$ & $\mathbf{0 , 0 3 9 9}$ \\
\hline
\end{tabular}


Objectives: Deep pectoral myopathy (DPM) is characterized by necrosis and atrophy of the supracoracoideus muscle, which exhibits variations in color ranging from a pinkish hemorrhage-like appearance to a grayish-green discoloration. This study aimed to evaluate the fatty acid profile in Pectoralis major (fillet) muscle from broiler affected by DPM.

Materials and Methods: Breast samples were collected from broilers affected by DPM, Cobb 500 strain and slaughtered with $45 \mathrm{~d}$ of age. The classification was according to the methodology used by Bilgilie and Hess (2008): DPM score 2-muscles with coagulative necrosis, fibrous tissue texture, and pink to plumb; DPM score 3-muscles with green necrotic area. The Pectoralis major muscle (fillet) was removed from the poultry carcasses, and the fatty acid profile was isolated by the method described by Bligh and Dyer (1959), which extracts the lipid phase from the sample; the esterification of fatty acids was performed according to the method proposed by Maia and Rodriguez-Amaya (1993) using a Shimadzu 14B gas chromatograph (Shimadzu Corporation, Kyoto, Japan) equipped with a flame ionization detector and a fused silica capillary column (Omegawax 250) with $\mathrm{H} 2$ used as the carrier gas. The identification of peaks was made by comparison with retention times of standards with known composition. This study used a randomized design (Unaffected, DPM score 2, and DPM score 3) with 50 repetitions each; results were submitted to analysis of variance using the GLM procedure of SAS operating system (SAS Institute Inc., Cary, NC), and means were compared by a Tukey test at $5 \%$ significance.

Results: There was no difference $(P<0.05)$ in fatty acid profile only for $\mathrm{C} 10: 0, \mathrm{C} 15: 0, \mathrm{C} 17: 0, \mathrm{C} 20: 0$, and $\mathrm{C} 18: 2 \mathrm{c} 9$, $\mathrm{t} 11$. The fatty acid profile of $\mathrm{C} 20: 5 \mathrm{n} 3$ and $\mathrm{C} 22: 6 \mathrm{n} 3$ are considered to be important for human health, and there was a difference $(P>0.05)$ between meats unaffected by myopathy (normal) and the affected group (DPM score 2 and 3 ). Polyunsaturated fatty acids are considered to be important nutrients and structural components in cell membranes to regulate human health. Diet as a way of controlling the risk of disease in humans is of considerable interest, and higher values were found in myopathy-unaffected meats too. However, C18:1n9c and C18:2n6c are considered to be important for human health too and are higher in meat from broilers with myopathy. For C14:0 and C16:0, there was a difference $(P>0.05)$, and normal meat had lower values. Meat from broiler fillets affected by DPM showed higher values for monounsaturated fatty acids in poultry unaffected meat by myopathy.

Conclusion: DPM that occurs in the supracoracoideus muscle influences the composition of fatty acid profile of the Pectoralis major (fillet) muscle of broilers. This is a disadvantage for the consumer market in the search for healthier meat, and there is clear evidence of the nutritional benefits of consumption.
Acknowledgments: To the Foundation for Research Support of the State of São Paulo (FAPESP) for the support provided (case number 2019/09707-6).

Keywords: breast, Pectoralis major muscle, supracoracoideus muscle

\section{3 - THE RELATIONSHIP BETWEEN THE RUMEN MICROBIOME AND CARCASS MERIT IN ANGUS STEERS}

T. Krause ${ }^{1 *}$, J. Lourenco ${ }^{1}$, C. Welch ${ }^{1}$, M. Rothrock ${ }^{2}$, T. Callaway ${ }^{1}$, and T. D. Pringle ${ }^{1},{ }^{1}$ Department of Animal and Dairy Sciences, University of Georgia, Athens, GA, USA, ${ }^{2}$ Egg Safety and Quality Research Unit, Richard B. Russell Research Center, Agricultural Research Service, USDA, Athens, GA, USA, *trk73598@uga.edu

Objectives: Profitability in the beef industry is heavily influenced by the quality and quantity of end products. Therefore, opportunities to improve both the quality and yield grades of beef carcasses at the producer level should be fully explored. The ruminal microbial ecosystem has a significant impact on overall host energetic status and nutrient availability, and thus alterations in the microbial populations in the rumen could impact energy partitioning and subsequent carcass composition. The objective of this study was to explore the relationships between ruminal microbial populations and adipose accumulation in Angus steers that were divergent in carcass merit.

Materials and Methods: Twenty-four Angus steers were slaughtered, and ruminal contents and carcass data were collected. Microbial DNA extraction and 16S ribosomal RNA gene sequencing were performed on the ruminal contents to determine microbial relative abundances, estimate microbial diversity, and to predict microbial metabolic pathways. Longissimus muscle samples were collected $48 \mathrm{~h}$ postmortem for proximate analysis to determine chemical lipid content. A variety of correlation analyses and one-way analysis of variance were performed to investigate the relationships between the rumen microbiome and carcass traits. For the analysis of variance, steers were grouped into high- and low-marbling classes ( $n=10$ steers/class) with average marbling scores of 760 (low Prime) and 579 (average Choice), respectively.

Results: Marbling score and longissimus lipid content were positively correlated to both Chaol richness index $(P=0.001$ and $P=0.009)$ and the number of operational taxonomic units $(P=0.017$ and $P=0.043)$, respectively, suggesting that increased intramuscular fat was associated with increased numbers of ruminal microbial species. The phyla Tenericutes and TM7 were negatively correlated $(P \leq 0.05)$ to marbling score and longissimus lipid content, indicating that lower abundances of these phyla in the rumen were linked to improvements in intramuscular fat content. Greater abundances of the bacterial family S24-7 in the rumen 
were positively correlated $(P=0.002)$ to marbling score. Analysis by marbling classification revealed differences in microbial richness $(P \leq 0.063)$, diversity $(P=0.044)$, and S24-7 abundances $(P<0.001)$, such that the high-marbling steers had greater microbial richness, diversity, and abundance of bacteria within the family S24-7 in their rumen compared to the low-marbling steers. However, computational prediction of the microbial metabolic pathways revealed no differences $(P \geq 0.05)$ in metabolic function between steers in the high- and low-marbling classes. No estimates of microbial richness, evenness, or diversity were correlated $(P \geq 0.05)$ to 12 th rib adjusted backfat thickness or yield grade. Several phyla, families, and genera, however, were correlated $(P \leq$ 0.05 ) to both 12th rib adjusted backfat thickness and yield grade, each in a positive nature, suggesting that increased abundances of bacteria within these taxa were associated with increased subcutaneous fat deposition.

Conclusion: Collectively, our results imply that differences in ruminal microbial abundances and diversity are linked to differing performance in carcass adipose traits. Overall, most of the bacterial taxa correlated to the intramuscular and subcutaneous fat depots did not overlap, suggesting that the microbial population impacted adipose accumulation largely via separate biological mechanisms within the host animal.

Keywords: lipid, marbling, rumen microbiome, subcutaneous fat, yield grade

\section{4 - IMPACTS OF SMART TUMBLING ON MUSCLE ULTRASTRUCTURE, PROTEOLYSIS, AND QUALITY ATTRIBUTES OF FRESH BEEF LOINS (M. LONGISSIMUS LUMBORUM)}

J. R. Tuell ${ }^{1 *}$, Q. Yu ${ }^{1}$, and Y. H. B. Kim ${ }^{1},{ }^{1}$ Animal Sciences, Purdue University, West Lafayette, IN, USA, *tuell@ purdue.edu

Objectives: Developing natural postharvest processing systems to improve meat tenderness is crucial for the beef industry. Tumbling is one of the most widely applied methods in processed meat applications. However, no studies have evaluated the impacts of tumbling fresh beef products without the use of brine enhancement on meat quality attributes. As tumbling disrupts the muscle structure and disintegrates muscle fibers, it is reasonable to postulate that tumbling will increase activity of endogenous proteolytic enzymes and thus tenderness of fresh beef products without using brine solution. The objective of this study was to determine the impacts of tumbling of vacuum-packaged beef (termed Smart Tumbling) on muscle ultrastructure, proteolysis, and quality attributes of fresh beef loins.

Materials and Methods: Beef loin (M. Longissimus lumborum) muscles ( $n=9$; USDA Select grade) at $7 \mathrm{~d}$ postmortem were sectioned into 3 equal length sections and allocated among 3 tumbling $(\mathrm{T})$ treatments (in minutes, $\mathrm{T} 0$ [control], T60, and T90). Beef sections were individually vacuum sealed and tumbled for the respective duration in a meat tumbler (Lance LT-30) at $8.5 \mathrm{rpm}$. Following tumbling, each section was further divided into 3 equal-length sections and randomly allocated among 3 additional aging durations ( $0 \mathrm{~d}, 7 \mathrm{~d}$, and $14 \mathrm{~d})$. Warner-Bratzler shear force (WBSF), water-holding capacity, myofibril fragmentation index (MFI), degradation of desmin and troponin- $\mathrm{T}$, and transmission electron microscopy were performed. Quantitative data were analyzed using the PROC MIXED procedure of SAS (version 9.4; SAS Institute Inc., Cary, $\mathrm{NC}$ ), and least-squares means were separated at $P<0.05$.

Results: Smart Tumbling resulted in an immediate positive impact on tenderness of beef loin sections shown by WBSF, where T90 samples exhibited a significantly lower WBSF value compared to T0 and T60 samples $(P<0.05)$. It is of interest to note that beef loins assigned Smart Tumbling without any further aging $(0 \mathrm{~d})$ had numerically lower shear force values $(2.5 \mathrm{~kg}$ and $2.2 \mathrm{~kg}$ for T60 and $\mathrm{T} 90$, respectively) compared to $\mathrm{T} 0$ (control) beef loins with additional $14 \mathrm{~d}$ of aging $(2.6 \mathrm{~kg})$. A considerable increase in MFI was observed with both Smart Tumbling and aging $(P$ $<0.001)$. Western blot results showed the appearance of structural protein degradation products with tumbling at all postmortem aging durations, in line with the MFI result. The transmission electron microscopy images demonstrated distinct appearance of initial muscle fracture along with substantial degradation at the Z-line of beef loin muscles subjected to tumbling with further aging. Tumbling treatment did not affect purge or display weight losses $(P>0.05)$ but increased cooking loss $(P<0.05)$.

Conclusion: The results of this study indicate the feasibility of tumbling vacuum-packaged fresh beef products to improve product tenderness. Smart Tumbling would result in a marked increase in tenderness through the result of physical disruptions generated by mechanical forces, whereas tumbling with further aging appears to increase proteolytic potential. This will in turn accelerate the tenderization process and subsequently shorten aging times for targeted eating quality outcomes.

Keywords: instrumental tenderness, meat tumbling, muscle ultrastructure, postmortem proteolysis, transmission electron microscopy

\section{5 - PROCESSING CHARACTERISTICS BETWEEN COMMERCIAL DUROC-SIRED AND HERITAGE BREED LARGE BLACK PIGS}

\author{
Y. Guo $^{1 *}$, K. Sharp ${ }^{1}$, S. Thayer ${ }^{1}$, B. Richert ${ }^{1}$, and S. Zuelly ${ }^{1}$, \\ ${ }^{1}$ Animal Sciences, Purdue University, West Lafayette, IN, \\ USA,*guo335@purdue.edu
}


Objectives: Heritage-bred pork is praised as premium pork for its unique quality characteristics; however, there are very little data evaluating Large Black pork quality or processing characteristics and commercial breeds. Therefore, the objectives of this study were to examine differences in pork processing characteristics between commercial Duroc-sired (DS) genetics and Large Black (LB) genetic lines fed high forage or commercial diets.

Materials and Methods: Fifty pigs were utilized in the study: DS ( $n=25$ pigs) and LB sired ( $n=25$ pigs). All the pigs were weighed and allocated to 2 dietary treatments: Fiber (Fib) or Control (Con), (LB Fib, $n=14$; LB Con, $n=$ 11; DS Fib, $n=14$; DS Con $n=11$ ). Dietary treatments were fed throughout the grow-finish period (101 or $140 \mathrm{~d}$ ) in 6 phases. Con diet was corn-soybean meal distillers dried grains with solubles based, and Fib diet used increasing amounts of wheat middlings $(1 \%-10 \%)$ and dehydrated alfalfa meal (7.5\%-20\%) replacing corn and soybean meal in the Con diet, from phase 1 to 6 . Pigs were harvested at a common age, but body weight varied between genetics (DS $125 \pm 2.23 \mathrm{~kg}, \mathrm{LB} 99 \pm 2.28 \mathrm{~kg} ; P<0.001$ ). Bellies (Institutional Meat Purchase Specifications 408) were measured for thickness, length, and firmness. Lean and fat trim was obtained from the shoulder of each carcass, formulated to $80 \%$ lean: $20 \%$ fat, ground, seasoned, and mixed to create individual sausage batches. Sausage patties (136 g each) from each batch were placed in polyvinyl chloride packaging under retail display lighting for 0,3 , and $7 \mathrm{~d}$. Fat smear was determined on day 0 by a trained evaluator using a scale of 1 (excessive fat smearing) to 8 (little/no fat smearing). Retail display effect on color (Minolta colorimeter) and lipid oxidation (thiobarbituric acid reactive substances) were examined each display day. Data were analyzed with breed and diet as fixed effects using $\mathrm{R}$ software (version 1.2.1335) with least-squares means separated at $P<0.05$.

Results: Results showed differences in $L^{*}(P=0.0051)$, $a^{*}(P<0.001)$, and $b^{*}(P<0.001)$ among display days, consistent with color deterioration over time. DS patties were lighter $\left(L^{*}, P<0.001\right)$ and less red $\left(a^{*}, P<0.001\right)$ than LB patties with no breed differences in patty $b^{*}(P=$ $0.71)$. No diet differences were found for patty $L^{*}(P=$ $0.47), a^{*}(P=0.13)$ or $b^{*}(P=0.77)$. Breed $\times$ diet showed no differences for patty $L^{*}(P=0.53), a^{*}(P=0.50)$, or $b^{*}(P=0.74)$. For patty fat smear, Fib had better particle definition than Con $(P=0.0064)$, but no differences were observed in breed $(P=0.36)$, or breed $\times \operatorname{diet}(P=0.27)$. DS bellies were longer $(P<0.001)$ but thinner $(P=$ $0.0035)$ than LB. Fib bellies were longer $(P<0.001)$ but thinner $(P<0.001$; $)$ than Con. No breed $\times$ diet interactions were found in belly length $(P=0.63)$ or thickness $(P=$ $0.40)$. Partial thiobarbituric acid reactive substances analysis showed days under retail display $(P<0.001)$ and breed $x$ diet interaction $(P=0.0043)$ to be significant. LB Con had the most lipid oxidation, and DS Con had the least amount of lipid oxidation, particularly at day 3 .
Conclusion: This experiment found variations in processing characteristics between DS and LB genetic lines and their diets. DS and Fib bellies were longer but thinner when compared to LB and Con bellies, respectfully. Fib patties had more fat smearing than Con patties. This provides novel insight into the comparison between these breeds and diets. Further studies focusing on fatty acid analysis in LB to improve their lean quality could lead to enhanced and diverse marketing products.

Keywords: heritage breed, pork quality, processing characteristics

\section{6 - COMPARISON OF THE PHYSICAL ATTRIBUTES OF PLANT-BASED GROUND BEEF ALTERNATIVES TO GROUND BEEF}

K. Harr*, S. G. Davis ${ }^{1}$, S. B. Bigger ${ }^{2}$, D. U. Thomson ${ }^{3}$, M. D. $\mathrm{Chao}^{1}$, J. L. Vipham ${ }^{1}$, M. D. Apley ${ }^{2}$, D. A. Blasi ${ }^{1}$, S. M. Ensley $^{2}$, M. D. Haub ${ }^{4}$, M. D. Miesner ${ }^{2}$, A. J. Tarpoff ${ }^{1}$, K. C. Olson ${ }^{1}$, and T. G. O'Quinn ${ }^{1},{ }^{1}$ Animal Science and Industry, Kansas State University, Manhattan, KS, USA, ${ }^{2}$ College of Veterinary Medicine, Kansas State University, Manhattan, KS, USA ${ }^{3}$ Animal Science, Iowa State University, Ames, IA, ${ }^{4}$ Nutrition, Dietetics, and Health, Kansas State University, Manhattan, KS, USA, *keaylah@ksu.edu

Objectives: The objective of this study was to evaluate the physical attributes of 3 different plant-based ground beef alternatives (GBA) in comparison to ground beef (GB) of 3 different fat percentages.

Materials and Methods: GB of 3 different fat percentages $(10 \%, 20 \%$, and $30 \%)$, a retail pea-protein-based GBA (RGBA), and a traditional soy-flour-based GBA (TGBA) were obtained from retail stores in the Manhattan, Kansas, area over several weeks in order to obtain different production lots for each product ( $n=15$ lots/treatment). Additional samples from 15 production lots of a foodservice soy-protein-based GBA (FGBA) were obtained from a commercial foodservice chain. GB, RGBA, and FGBA were fabricated into $151 \mathrm{~g}$ (approximately 13-cm diameter; $1-\mathrm{cm}$ thick) patties using a manual patty former and randomly assigned to one of 4 assays: color analysis, texture profile analysis (TPA), shear force (SF), and pressed juice percentage (PJP). Patties used for TPA and SF were cooked to $71^{\circ} \mathrm{C}$ on a clamshell-style grill with three $2.54-\mathrm{cm}$ cores taken from each patty for TPA and two 2.54 -cm-wide strips taken from each patty for SF. Patties were evaluated for $L^{*}, a^{*}$, and $b^{*}$ both in the raw, precooked state as well as after cooking for both external and internal color. PJP measured the percentage of weight lost from $1 \mathrm{~cm}^{3}$ cooked samples that were compressed for $30 \mathrm{~s}$ at $8 \mathrm{~kg}$ of force. During cooking for TPA, SF, and PJP, patty weights, diameters, and thicknesses were measured for determination of size change through 
cooking. All data were analyzed as a completely randomized design.

Results: When evaluating raw color, TGBA had the highest $(P<0.05) a^{*}$ value and were redder when compared to all other treatments, with RGBA having the lowest $(P<$ $0.05) a^{*}$ value. TGBA and RGBA had the highest $(P<0.05)$ $a^{*}$ value, whereas FGBA and $30 \%$ and $10 \%$ fat GB had the lowest $(P<0.05) a^{*}$ value for cooked surface color. Additionally, $30 \%$ and $20 \%$ fat GB had higher $(P<0.05)$ $L^{*}$ values for internal cooked color than all other treatments, with all GBA patties having the lowest $(P<0.05) L^{*}$ values. For texture attributes, RGBA and FGBA had lower $(P<$ $0.05)$ values for cohesiveness, gumminess, hardness, and chewiness, as well as higher values for springiness, than all other treatments evaluated. Few differences were found between TGBA and $20 \%$ and $30 \%$ fat GB for texture, with TGBA only found softer and less chewy $(P<0.05)$ than both GB treatments. For SF, the 3 GBA were more tender $(P<$ $0.05)$ than all 3 GB treatments, with FGBA and RGBA being more tender $(P<0.05)$ than all treatments. The 3 GB treatments had greater $(P<0.05)$ PJP values than all GBA, indicating that the GB was juicier than any of the GBA evaluated. Finally, during cooking, the $3 \mathrm{~GB}$ treatments had a greater $(P<0.05)$ cook loss percentage and decrease in patty diameter and thickness than the $3 \mathrm{GBA}$, with FGBA and RGBA increasing in thickness during cooking.

Conclusion: For physical attributes, the GBA evaluated differed significantly from GB. RGBA and FGBA had the greatest differences, with the TGBA being the most similar to $20 \%$ and $30 \%$ fat GB for some traits. This provides evidence that that, though these products attempt to mimic GB, they provide very different color, texture, tenderness, and cooking characteristics than traditional GB.

Keywords: alternative proteins, color, ground beef, ground beef alternatives, texture

\section{7 - POSTMORTEM PROTEOMIC CHANGES IN NORMAL AND WOODY BROILER BREAST MUSCLE}

X. Zhang ${ }^{1 *}$, M. W. Schilling ${ }^{1}$, W. Zhai ${ }^{2}$, S. P. Suman ${ }^{3}$, S. Li ${ }^{3}$, J. Chen ${ }^{4}$, H. Zhu ${ }^{4}$, and D. S. Antonelo ${ }^{5},{ }^{1}$ Food Science, Nutrition, and Health Promotion, Mississippi State University, Mississippi State, MS, USA, ${ }^{2}$ Department of Poultry Science, Mississippi State University, Mississippi State, MS, USA, ${ }^{3}$ Department of Animal and Food Sciences, University of Kentucky, Lexington, KY, USA, ${ }^{4}$ Proteomics Core Facility, University of Kentucky, Lexington, KY, USA, ${ }^{5}$ Department of Animal Nutrition and Production, University of São Paulo, Pirassununga, Brazil,*xz206@msstate.edu

Objectives: Woody breast (WB) is a stress-related pectoral myopathy in fast-growing broilers. WB muscle exhibits changes in energy homeostasis, osmotic balance, protease/ lipase system, and proteins compared to normal breast (NB). These antemortem differences can influence the biochemical changes postmortem. In the current study, the proteomic profiles in NB and WB were studied early postmortem (0-24 h after death) in order to characterize proteome changes in live muscle, pre-rigor muscle, and postrigor muscle to improve our knowledge regarding the postmortem biochemical changes exerted by the WB condition.

Materials and Methods: A total of 128 one-day-old mixed-sex chicks were selected and randomly assigned to 8 pens that were evenly distributed in a chicken house at the Poultry Research Farm at Mississippi State University (IACUC-16-542). At $8 \mathrm{wk}$ of age, live male broilers were evaluated by palpation for WB myopathy. Four birds with NB were sampled from 4 pens (1 bird per pen), and 4 birds with WB were sampled from the rest of 4 pens ( 1 bird per pen). Birds were euthanized using $\mathrm{CO}_{2}$ gas. The muscle from the cranial portion of the right breast was collected and snapfrozen in liquid nitrogen at death $(0 \mathrm{~min}), 15 \mathrm{~min}, 4 \mathrm{~h}$, and $24 \mathrm{~h}$ postmortem. To confirm the manual palpation technique, the WB defects were evaluated at each time point, where $0=$ normal, $1=$ slight, $2=$ moderate, and $3=$ severe. Three NB samples $(n=3)$ that were scored 0 and 1 , and three WB samples $(n=3)$ that were scored 2 and 3 throughout $24 \mathrm{~h}$ were selected for proteomic analysis. Whole muscle proteins were separated using two-dimensional gel electrophoresis (6 gels per treatment) and identified using liquid chromatography-tandem mass spectrometry. A 1.5-fold change threshold in abundance between samples collected at 4 time points was considered significant $(P<0.05)$ using the Student's $t$ test.

Results: Four proteins, including EH domain-containing protein 2, elongation factor 2, phosphoglycerate mutase 1 , and T-complex protein 1 subunit gamma, were changed in both NB and WB muscles during postmortem storage. Twenty proteins were uniquely changed in either NB (6 proteins) or WB (14 proteins) postmortem muscles, indicating the differences in their postmortem metabolism. In postmortem WB meat, the changes in protein degradation products were observed as indicated by the presence of desmin fragment, ovotransferrin chain A, and troponin I chain I. In addition, a few glycolytic proteins in WB consisted of post-translational modified postmortem, including enolase, phosphoglucomutase-1, phosphoglycerate mutase 1, and pyruvate kinase.

Conclusion: WB meat exhibited a greater number of changes in structural proteins, metabolic proteins, stressrelated proteins, and transport proteins. In addition, WB had a greater rate of postmortem metabolism and a greater number of post-translational modifications postmortem due to the differences in antemortem conditions that exist in NB and WB. These differences indicate a faster and more oxidative muscle to meat conversion process for WB. These protein biomarkers and their post-translational modification products improve our knowledge of the biochemical processes in postmortem WB meat and provide novel insights 
into the development of WB and the potential strategies to reduce WB incidence, such as alleviating oxidative stress and regulating protein degradation and oxidation.

Keywords: postmortem change, protein degradation, proteomics, woody breast

\section{8 - EFFECT OF TERMINAL SIRE LINE AND TIMING OF IMMUNOCASTRATION ON PERFORMANCE, CARCASS, AND MEAT QUALITY}

E. Kowalski ${ }^{1,2 *}$, E. Vossen ${ }^{2}, \mathrm{~S}$. Millet $^{1}$, M. Aluwé ${ }^{1}$, and S. De Smet ${ }^{2},{ }^{1}$ Animal Sciences, ILVO, Ghent University Melle, Belgium, ${ }^{2}$ Animal Sciences and Aquatic ecology, Ghent University, Ghent, Belgium, *eline.kowalski@ilvo. vlaanderen.be

Objectives: In past decades, pig breeding companies in Europe have focused strongly on producing the highest quantity of lean meat at the lowest cost. A side effect of this intense selection towards leanness is a lowered eating quality of the pork. The intention to stop surgical castration in the European Union makes the meat quality issues even more prominent. In this respect, immunocastration could offer an alternative for entire male production to guarantee acceptable meat quality. However, more insight is needed into the effect of timing of the second vaccination and the influence of the genetics used to further optimize this alternative for meat quality as well as carcass quality.

Materials and Methods: This study aimed to evaluate the effect of timing of the second vaccination of Improvac $\mathbb{R}$ (Zoetis, Belgium) (V2) in crossbred fattening pigs of 3 different terminal sire lines on lean meat content and meat quality traits $\left(\mathrm{pH}_{35 \mathrm{~min}} \mathrm{pm}\right.$, drip loss, intramuscular fat [IMF]) of the loin. A homozygous stress positive $(R Y R 1)(\mathrm{BP}+)$ and homozygous stress negative Piétrain sire line (BP-) of Belgian origin and a Duroc sire line of Canadian origin (CD) were included for comparison. Within $\mathrm{BP}+$ and $\mathrm{BP}-$, there were 4 treatment groups: gilts $(\mathrm{G})$, and immunocastrates (IC) with a V2 at average pen weight of $55 \mathrm{~kg}$ $\left(\mathrm{IC}_{\text {early }}\right), 70 \mathrm{~kg}\left(\mathrm{IC}_{\text {middle }}\right)$, and $90 \mathrm{~kg}\left(\mathrm{IC}_{\text {late }}\right)$. Within $\mathrm{CD}$, there were 3 treatment groups: G, and IC with V2 at $65 \mathrm{~kg}$ $\left(\mathrm{IC}_{\text {middle }}\right)$ and $85 \mathrm{~kg}\left(\mathrm{IC}_{\text {late }}\right)$. Across 5 rounds, 440 animals were selected and allocated to these 11 treatment groups. Twenty animals per treatment were selected to assess meat quality. For all analyses, pen was used as the experimental unit. A linear mixed model was used with sire line and treatment group and their interaction as fixed factors and cold carcass weight as covariable. Slaughter date and pen number was included as random effect to account for repeated measurements within pens. A Tukey post hoc partial F-test split per sire line and treatment group was used to compare means in order to deal with the unbalanced experimental setup.
Results: Lean meat content was significantly higher for $\mathrm{BP}+$ and $\mathrm{BP}-$ compared to $\mathrm{CD}$ within the treatment groups $(P<0.05)$. For meat quality, $\mathrm{pH}_{35 \text { minpm }}$ was significantly lower for the $\mathrm{BP}+$ compared to $\mathrm{BP}-$ and $\mathrm{CD}$ within all treatment groups $(P<0.05)$, except within $\mathrm{IC}_{\text {late }}(P=0.103)$. Drip loss differed between sire lines: in the case of $\mathrm{IC}_{\text {late }}$ : $\mathrm{CD}<\mathrm{BP}+$ and $\mathrm{BP}-(P<0.001) ; \mathrm{IC}_{\text {middle }}$ and $\mathrm{G}: \mathrm{CD} \leq$ $\mathrm{BP}+\leq \mathrm{BP}-(P<0.05)$; and $\mathrm{IC}_{\text {early }}: \mathrm{BP}-<\mathrm{BP}+(P=$ 0.052). IMF was higher for $\mathrm{CD}$ compared to $\mathrm{BP}+$ within $\mathrm{IC}_{\text {middle }}$ and compared to $\mathrm{BP}+$ and $\mathrm{BP}-$ within $\mathrm{G}(P<$ $0.05)$, whereas there was no effect of sire line within $\mathrm{IC}_{\text {early }}(P=0.669)$ or $\mathrm{IC}_{\text {late }}(P=0.102)$. Lean meat content differed between treatment groups: for $\mathrm{BP}+$ : $\mathrm{IC}_{\text {early }} \leq$ $\mathrm{IC}_{\text {middle }} \leq \mathrm{IC}_{\text {late }} \leq \mathrm{G}(P=0.003) ; \mathrm{BP}-$ : $\mathrm{IC}_{\text {early }} \leq \mathrm{IC}_{\text {middle }}$ and $\mathrm{IC}_{\text {late }} \leq \mathrm{G}(P=0.006)$; and $\mathrm{CD}$ : $\mathrm{IC}_{\text {early }} \leq \mathrm{IC}_{\text {late }} \leq \mathrm{G}$ $(P=0.017)$. There was no effect of treatment group on the meat quality traits $\left(\mathrm{pH}_{35 \mathrm{~min}} \mathrm{pm}\right.$, drip loss, and IMF) (all $P>0.05)$.

Conclusion: In general, meat quality was best in offspring of $\mathrm{CD}$, followed by $\mathrm{BP}-$, and was the lowest for $\mathrm{BP}+$; however, $\mathrm{BP}+$ and $\mathrm{BP}-$ had the highest lean meat content. Meat quality did not differ significantly between G and IC, nor between IC treatments. Lean meat content was higher for G compared to IC, and a trend to a lower lean meat content with a longer interval between V2 and slaughter was observed.

Keywords: gilts, immunocastrates, pork quality, terminal sire line, timing immunocastration

\section{9 - EFFECT OF BOER GOAT AGE ON MEAT QUALITY}

A. Abhijith ${ }^{1 *}$, R. D. Warner ${ }^{2}$, F. R. Dunshea ${ }^{2}$, M. $\mathrm{Ha}^{2}$, B. J. Leury $^{2}$, M. H. Zhang ${ }^{2}$, A. Joy ${ }^{2}$, R. Osei-Amponsah ${ }^{2}$, and S. S. Chauhan ${ }^{2},{ }^{1}$ Veterinary and Agricultural Science, The University of Melbourne, North Melbourne, Australia, ${ }^{2}$ Faculty of Veterinary and Agricultural Sciences, The University of Melbourne, Melbourne, Australia, *archana. abhijith@student.unimelb.edu.au

Objectives: With the growing recognition of goat meat in western countries, research needs to be prioritized on factors affecting the goat meat quality from processing conditions needs to be prioritized. The main objective of this study was to document the $\mathrm{pH}$-temperature decline in postmortem $M$. longissimus of young and adult Boers slaughtered and processed under commercial processing conditions in Australia. Effects of slaughter age and aging period on tenderness and cooking loss in Boer goat meat was assessed.

Materials and Methods: Twenty-four farmed Boer goats (12 young goats of 6-9 mo,12 adult goats of 2 y), sourced from Myrrhee Farm, Victoria, Australia, were transported to Cedar Meats, Brooklyn, Australia, for $2.5 \mathrm{~h}$, kept in lairage overnight, and slaughtered the next morning following 
the standard slaughter protocol (Halal) of the commercial abattoir in Victoria, Australia, without stimulation. Both postmortem $\mathrm{pH}$ and temperature of $M$. longissimus muscles were recorded repeatedly from $30 \mathrm{~min}$ post-slaughter every $8 \mathrm{~h}$ and then at $24 \mathrm{~h}$. Grams of fat was measured (total tissue depth over the 12th rib, $110 \mathrm{~mm}$ from the midline) using GR knife. The M. longissimus thoracis et lumborum samples designated into 2 aging periods ( $1 \mathrm{~d}$ and $14 \mathrm{~d}$ ), cooked to internal temperature of $70^{\circ} \mathrm{C}$, was used for WarnerBratzler shear force (WBSF) and hardness. A total of 5 measurements were taken for each sample and presented as means for WBSF and hardness. Glycogen was estimated in the samples collected $5 \mathrm{~min}$ after slaughter using assay kit (MAC016A, Sigma-Aldrich, St. Louis, MO). Carcass and meat quality traits were analyzed using restricted maximum likelihood with GenStat using linear mixed model (16th Edition, VSN International Ltd., Hemel Hempstead, UK).

Results: The GR fat depth was significantly higher $(P<$ 0.01 ) in old goats (Table 1$)$. The $\mathrm{pH}$ declined rapidly in both goat groups, with a faster decline in young goats (Figure 1). The ultimate $\mathrm{pH}\left(\mathrm{pH}_{24}\right)$ of young goat meat was significantly $(P<0.01)$ higher compared to adult goats. The predicted $\mathrm{pH}$ ( Temp18 was above 6 for both groups, with significantly $(P<0.01)$ higher value in young goats (Table 1$)$. An optimal $\mathrm{pH}$-temperature window for eating quality has been defined as $18^{\circ} \mathrm{C}-35^{\circ} \mathrm{C}$. Postmortem aging of meat for $14 \mathrm{~d}$ significantly $(P<0.01)$ reduced WBSF in both age groups. Muscle glycogen concentration at slaughter was lower than optimum concentration in both age groups, with a significantly $(P<$ 0.01 ) lower level in young goat meat compared to that of the adults.

Conclusion: Results show toughness in goat meat irrespective of age. However, results highlight the potential of aging to enhance goat meat tenderness in both age groups.

\begin{tabular}{|c|c|c|c|c|c|c|}
\hline Traits & \multicolumn{2}{|c|}{ Adult Goats } & \multicolumn{2}{|c|}{ Young Goats } & \multicolumn{2}{|c|}{ Age } \\
\hline Live weight (kg) & \multicolumn{2}{|c|}{$45.8 \pm 1.19$} & \multicolumn{2}{|c|}{$29.0 \pm 1.19$} & & $* *$ \\
\hline HCW (kg) & \multicolumn{2}{|c|}{$18.9 \pm 0.52$} & \multicolumn{2}{|c|}{$11.3 \pm 0.52$} & & $* *$ \\
\hline $\begin{array}{l}\text { GR fat depth } \\
(\mathrm{mm})\end{array}$ & \multicolumn{2}{|c|}{$5.5 \pm 0.40$} & \multicolumn{2}{|c|}{$3.7 \pm 0.40$} & & $* *$ \\
\hline pH@Temp18 & \multicolumn{2}{|c|}{$6.7 \pm 0.04$} & \multicolumn{2}{|c|}{$7.1 \pm 0.04$} & & $* *$ \\
\hline $\mathrm{pH}_{24}$ & \multicolumn{2}{|c|}{$5.8 \pm 0.06$} & \multicolumn{2}{|c|}{$6.13 \pm 0.06$} & & $* *$ \\
\hline \multirow[t]{2}{*}{ Glycogen $(\mu \mathrm{mol} / \mathrm{g})$} & \multicolumn{2}{|c|}{$27.4 \pm 2.58$} & \multicolumn{2}{|c|}{$11.6 \pm 2.58$} & & $* *$ \\
\hline & $1 \mathrm{~d}$ & $14 \mathrm{~d}$ & $1 \mathrm{~d}$ & $14 \mathrm{~d}$ & Age & Aging \\
\hline WBSF (kg) & $\begin{array}{l}7.1 \pm \\
0.29\end{array}$ & $\begin{array}{l}4.1 \pm \\
0.29\end{array}$ & $\begin{array}{c}6.21 \pm \\
0.29\end{array}$ & $\begin{array}{l}4.1 \pm \\
0.29\end{array}$ & NS & $* *$ \\
\hline Hardness (N) & $\begin{array}{c}47.8 \pm \\
1.87\end{array}$ & $\begin{array}{l}39.2 \pm \\
1.87\end{array}$ & $\begin{array}{c}39.2 \pm \\
1.87\end{array}$ & $\begin{array}{l}37.8 \pm \\
1.87\end{array}$ & $*$ & NS \\
\hline
\end{tabular}

$* * \mathrm{P}<0.01$

$* \mathrm{P}<0.05$.

1 d, 1-d aged; 14 d, 14-d aged; HCW, hot carcass weight; NS nonsignificant.

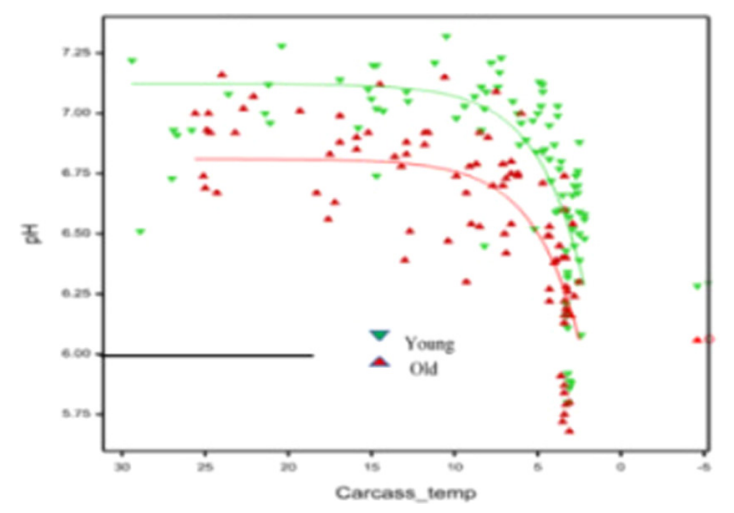

Figure 1. Lines of best fit based on $\mathrm{pH}$ and temperature for young (green) versus old (red) goats with the 'ideal' $\mathrm{pH} /$ temperature window shown as the solid black line $(\mathrm{pH} /$ temperature window defined as temperature at $\mathrm{pH} 6$ in the M. longissimus $<35^{\circ} \mathrm{C}$ and $>12^{\circ} \mathrm{C}$.

This reinforces the need for further research to better understand the goat meat supply chain in Australia to develop meat quality standards for goat meat similar to Meat Standards Australia.

Keywords: glycogen, goat, $\mathrm{pH}$ decline, toughness

\section{0 - EXTENDING THE SHELF LIFE OF BEEF CHUCK ROLL STEAKS USING ACEROLA CHERRY POWDER AND ROSEMARY EXTRACT}

J. B. Van Buren ${ }^{1 *}$, B. J. Buseman ${ }^{1}$, T. M. Weber ${ }^{1}$, J. A. Nasados $^{1}$, J. M. Lancaster ${ }^{1}$, J. H. Smart ${ }^{1}$, P. D. Bass ${ }^{1}$, and M. J. Colle ${ }^{1},{ }^{1}$ Animal \& Veterinary Science, University of Idaho, Moscow, ID, USA, *vanb2144@vandals.uidaho.edu

Objectives: Exported beef is aged for an extended period and therefore has a shorter shelf life. Improving the shelf life of steaks from beef chuck rolls, which are commonly exported, will increase demand and producer profits. The objective of Experiment 1 (Exp 1) was to determine the effect of the topical application of acerola cherry powder and rosemary extract from various suppliers-as efficacy can differ-on beef chuck roll shelf life, including color stability and lipid oxidation. The objective of Experiment 2 (Exp 2) was to determine the effect of applying a combination of the topperforming acerola cherry powder and rosemary extract from Exp 1 on beef chuck roll shelf life.

Materials and Methods: In both Exp 1 and Exp 2, beef chuck rolls (Institutional Meat Purchase Specifications 116A) ( $n=8$, Exp $1 ; n=9$, Exp 2) from USDA Choice carcasses were purchased from Washington Beef in Toppenish, Washington, and aged $\left(0^{\circ} \mathrm{C}\right)$ for $28 \mathrm{~d}$ post-fabrication at Vandal Brand Meats, Moscow, Idaho. Following aging, steaks-1.02 cm thick-were cut and systematically assigned to their respective treatment based on location. 
Exp 1 treatments included the following: untreated control, topically sprayed $(\sim 2 \mathrm{~mL})$ with an acerola cherry powder solution $(0.05 \%)$ from one of 3 suppliers $(\mathrm{C} 1, \mathrm{C} 2, \mathrm{C} 3)$, or topically sprayed $(\sim 2 \mathrm{~mL})$ with a rosemary extract solution $(0.10 \%)$ from one of 3 suppliers (R1, R2, R3). Exp 2 treatments included the following: untreated control or topically sprayed $(\sim 2 \mathrm{~mL})$ with a treatment of an acerola cherry powder solution $(0.05 \% \mathrm{C} 1)$, rosemary extract solution $(0.10 \% \mathrm{R} 3)$, or a mixture of the acerola cherry powder and rosemary extract $(\mathrm{M} 1=0.05 \% \mathrm{C} 1+0.1 \% \mathrm{R} 3 ; \mathrm{M} 2=$ $0.1 \% \mathrm{C} 1+0.1 \% \mathrm{R} 3 ; \mathrm{M} 3=0.05 \% \mathrm{C} 1+0.2 \% \mathrm{R} 3$; and $\mathrm{M} 4=0.1 \% \mathrm{C} 1+0.2 \% \mathrm{R} 3)$. In both Exp 1 and Exp 2, steaks were assigned to either day 0 thiobarbituric acid reactive substances (TBARS) to measure lipid oxidation or to $4 \mathrm{~d}$ of retail display followed by day-4 TBARS analysis. Throughout retail display, color was measured twice daily. Objective color measurements $\left(L^{*}, a^{*}, b^{*}\right)$ were taken using a Nix Pro 2 Color Sensor. Subjective color-including lean color, browning, discoloration, surface discoloration, and uniformity-was evaluated by a team following the American Meat Science Association guidelines. Data were analyzed using the Mixed Model procedure of SAS (SAS Institute Inc., Cary, NC). Significance was determined at $P<0.05$ and tendencies at $P<0.10$.

Results: In Exp 1, antioxidants differed in $a^{*}(P<0.03)$, but not $L^{*}(P=0.31)$ or $b^{*}(P=0.19)$. R3 had the highest $a^{*}$ value. Additionally, antioxidants influenced lean color $(P<0.01)$, surface discoloration $(P<0.01)$, and uniformity $(P<0.02) . \mathrm{C} 1$ and R3 had less surface discoloration than the control, which led to the use of $\mathrm{C} 1$ and R3 in Exp 2. In Exp 2, antioxidants did not impact TBARS values on day $0(P=$ $0.16)$ but did on day $4(P<0.01)$. After $4 \mathrm{~d}$ of retail display, steaks treated with M3 and M4 had less lipid oxidation than the control. Additionally, antioxidants affected $L^{*}(P<0.02)$ and $a^{*}(P<0.01)$, but not $b^{*}(P=0.15) . \mathrm{C} 1, \mathrm{M} 2$, and M3 were redder than the control. Antioxidant treatments differed in lean color $(P<0.03)$, browning $(P<0.02)$, discoloration $(P<0.02)$, and uniformity $(P<0.02)$ but not in surface discoloration $(P=0.43)$. Interestingly, M4 had a brighter colored lean than that of the control.

Conclusion: Applying topical antioxidants individually or in combination can improve shelf life stability of beef chuck rolls aged for an extended period.

Keywords: antioxidants, extended aging, shelf life

\section{1 - EFFECTS OF ROSEMARY AND GREEN TEA ANTIOXIDANTS ON GROUND BEEF PATTIES IN TRADITIONAL AND MODIFIED ATMOSPHERE PACKAGING}

L. Yoder ${ }^{*}$, D. VanOverbeke ${ }^{1}$, R. Ramanathan ${ }^{1}$, and G. Mafi ${ }^{1}$, ${ }^{1}$ Department of Animal and Food Sciences,
Oklahoma State University, Stillwater, OK, USA, *lyoder@okstate.edu

Objectives: Consumers purchase ground beef more than any other beef product in the US, with $88 \%$ of consumers preferring traditional foam trays with overwrap (polyvinyl chloride [PVC]); however, PVC has a very limited shelf life. Utilizing gases like carbon monoxide (CO) and antioxidants like rosemary and green tea have been shown to extend shelf life. The objective of this study was to understand the effects of rosemary and green tea in ground beef in PVC and modified atmosphere packaging (MAP).

Materials and Methods: Four treatments were used in this study: control, 2,500 ppm rosemary, $300 \mathrm{ppm}$ green tea, and 2,500 rosemary $+300 \mathrm{ppm}$ green tea. Patties from each treatment $(n=42)$ were randomly packaged into one of 3 types: PVC, MAP, or master packages (MP), MAP and MP flushed with $0.4 \% \mathrm{CO}, 69.6 \% \mathrm{~N}_{2}$, and $30 \% \mathrm{CO}_{2}$. Patties in $\mathrm{PVC}$ and MAP were put directly into simulated retail display for $7 \mathrm{~d}$; MP were put in dark storage for $7 \mathrm{~d}$, then removed and put in display cases for $7 \mathrm{~d}$. Subjective and objective color were measured utilizing a spectrophotometer, lipid oxidation and trained sensory panel measurements were measured. A $3 \times 4$ factorial design was used to evaluate 3 packaging types and 4 treatment types. The MIXED procedure of SAS version 9.4 (SAS Institute Inc., Cary, NC) was utilized, and when there was a significant F-test $(P<$ $0.05)$, least-squares means were separated using a pairwise $t$ test with the PDIFF option.

Results: As expected, patties in MAP had significantly $(P<0.05)$ lower lipid oxidation values and were significantly higher $(P<0.05)$ in all color values than patties in PVC after display. In MAP, green tea significantly $(P<$ $0.05)$ improved $a^{*}$ and chroma values on day 6 of retail display and had lower surface discoloration scores from day 3 to 6 compared to the rosemary + green tea treatment. In MP patties, green tea statistically $(P<0.05)$ improved $L^{*}$ values, display color, and surface discoloration scores compared to the combination treatment. However, green tea did not significantly $(P>0.05)$ improve values when compared to rosemary for these parameters. Trained taste panelists could not detect a difference between control and green tea patties $(P>0.05)$ for the green-hay attribute; however, rosemary was highly detectable in the green-hay attribute.

Conclusion: In conclusion, utilizing MAP and MP can improve shelf life duration of ground beef due to the formation of carboxymyoglobin. Green tea improved subjective color measurements and $L^{*}$ values; however, other objective measurements were not improved with either green tea or rosemary. Sensory panelists were unable to detect green tea, which can be utilized in the future for label-friendly antioxidant replacements.

Keywords: antioxidants, beef, meat color, packaging 


\section{2 - RETAIL LIGHTING AND POSTMORTEM AGING IMPACT THE PALATABILITY OF VACUUM-PACKAGED BEEF STEAKS}

S. N. Barker ${ }^{1 *}$, J. F. Legako ${ }^{1}$, J. C. Brooks ${ }^{1}$, and D. R. Woerner ${ }^{1},{ }^{1}$ Department of Animal and Food Science, Texas Tech University, Lubbock, TX, USA, *sam.barker@, ttu.edu

Objectives: The objective of this study was to evaluate the eating quality of individually vacuum-packaged beef steaks displayed under 2 lighting sources: fluorescent (FLUR) and light-emitting diode (LED).

Materials and Methods: USDA Low Choice paired beef top sirloin butts, striploins, and tenderloins $(n=32)$ were collected at a commercial beef processing facility. Subprimals were aged for $7 \mathrm{~d}$ postmortem in the absence of light. Subprimals were fabricated into 2.54-cm-thick steaks representing the Gluteus medius (GM), Longissimus lumborum (LL), and Psoas major (PM). Steaks were packaged in rollstock vacuum packaging and aged for an additional $7 \mathrm{~d}$ before being randomly assigned to a lighting display of either FLUR or LED for 0,2 , 6, or $10 \mathrm{~d}$. Following the assigned aging periods, steaks were frozen at $-20^{\circ} \mathrm{C}$ until further analysis. Trained sensory panelists evaluated samples for beef flavor identity, brown/roasted, bloody/serumy, fatlike, liver-like, oxidized, fishy, buttery, umami, bitter, sour, overall juiciness, and overall tenderness. Data were analyzed as a split-split plot with subprimal serving as the whole plot, lighting as the sub plot, and days of age as the sub-sub plot. Peak cooked temperatures and cook loss percentages were used as covariates.

Results: Lighting type and days of display interacted to impact tenderness and umami flavor scores. Umami flavor scores for both LED and FLUR had similar $(P>0.05)$ initial flavor intensity at $0 \mathrm{~d}$ and showed a decrease $(P<0.05)$ in umami flavor over time. However, FLUR umami intensity scores at $10 \mathrm{~d}$ were lower $(P<0.05)$ than those in LED lighting at $10 \mathrm{~d}$. Tenderness scores were similar $(P>0.05)$ for steaks in both the LED and FLUR lighting types at the initial $0 \mathrm{~d}$ of display and the final $10 \mathrm{~d}$ of display, with an increase $(P<0.05)$ in tenderness over time. Nonetheless, tenderness scores under FLUR were similar $(P>0.05)$ from 0 to $6 \mathrm{~d}$; however, day-10 FLUR tenderness scores were similar $(P>0.05)$ to day-10 LED scores. Tenderness was also impacted by the interaction between lighting type and muscle cuts. For the LL and PM, panelists scored steaks more tender $(P<0.05)$ under LED lighting compared to those in FLUR displays. The GM was unaffected $(P>$ $0.05)$ by lighting type. A third interaction between muscle cut and days of age was also determined, showing an increase $(P<0.05)$ in sour intensity for the LL and PM, with no change $(P>0.05)$ in values for the GM. Tenderness increased $(P<0.05)$ for the GM and LL from 0 to $10 \mathrm{~d}$, but values did not change $(P>0.05)$ for the $\mathrm{PM}$ during display. Lighting type influenced $(P<0.05)$ overall juiciness values, with panelists scoring steaks displayed under LED more desirable $(P<0.05)$ than those displayed under FLUR lighting.

Conclusion: These results suggest that LED lighting will not be detrimental to the eating quality of individually vacuum-packaged beef steak during retail displays. Moreover, the data that suggest beef flavor attributes of the muscles evaluated were more desirable under LED lighting than steaks displayed under FLUR lighting. However, more research is needed to fully understand the impact of lighting displays on flavor.

Keywords: aging, lighting, retail display, trained descriptive panel, vacuum packaged

\section{3 - EFFECT OF TENDERNESS CLASSIFICATION AND AGING TIME ON ABUNDANCE OF PEROXIREDOXIN-6 IN PORCINE LONGISSIMUS}

\section{Y. Zhang ${ }^{1 *}$, L. Johnson ${ }^{2}$, E. Steadham ${ }^{2}$, E. Huff-Lonergan ${ }^{2}$, and S. Lonergan ${ }^{2},{ }^{1}$ College of Food Science and Engineering, Gansu Agricultural University, Lanzhou, China, ${ }^{2}$ Animal Science, Iowa State University, Ames, IA, USA,*slonerga@iastate.edu}

Objectives: Meat tenderness significantly influences meat palatability, consumer satisfaction, and industry profitability. Protein oxidation does influence the development of meat tenderness. Peroxiredoxin-6 (PRDX6), an antioxidant protein, contributes to hydrogen peroxide degradation. Previous studies have suggested that PRDX6 might be a protein marker for tenderness in bovine biopsies and samples collected shortly after slaughter. However, the relationship between PRDX6 and ultimate meat tenderness in porcine skeletal muscle is not defined. This study aimed to examine PRDX6 during postmortem aging to further understand the relationship between PRDX6 and meat tenderness.

Materials and Methods: Fresh pork loins were collected at $1 \mathrm{~d}$ postmortem. Pork chops $(2.5 \mathrm{~cm})$ containing the longissimus dorsi muscle were fabricated and aged for $1,8,14$, or $21 \mathrm{~d}$ postmortem. After aging, chops were cooked to $68^{\circ} \mathrm{C}$, and instrumental tenderness was measured using of the star probe attachment on an Instron (Instron, Norwood, MA). Star probe values were used to classify chops into high (star probe $>7.0 \mathrm{~kg}, n=6$ ) and low (star probe $<5.8 \mathrm{~kg}, n=6$ ) star probe groups ( $21 \mathrm{~d}$ values). Sarcoplasmic proteins from longissimus dorsi at each aging time were solubilized in icecold, low-ionic strength buffer $(50 \mathrm{mM}$ Tris- $\mathrm{HCl}$ and $1 \mathrm{mM}$ EDTA; $p H$ 8.5), and samples with a reducing agent were prepared. A pooled reference representing every sample in the experiment was prepared. Western blot analysis was employed to identify PRDX6. Each sample was fractionated 
on a $15 \%$ acrylamide separating gel and transferred to PVDF membrane. After 1-h blocking within 5\% nonfat dried milk, membranes were incubated overnight with primary antibody (1:10,000 anti-PRDX6; AB1333, AbCam) at $4^{\circ} \mathrm{C}$. Membranes were incubated with secondary antibody (1:20,000 Goat anti-rabbit IgG-HRP) for $1 \mathrm{~h}$ at room temperature. The results were visualized using the chemiluminescent detection kit (ECL Prime; GE Healthcare, Piscataway, NJ), and images were obtained and analyzed using a ChemiImager 5500 (Alpha Innotech Corp., San Leandro, CA) and Alpha Ease FC software (version 3.03; Alpha Innotech Corp.). Samples were run in duplicate and reported as a ratio to the reference that was run on each gel. PRDX6 abundance (as a ratio to a within gel reference) was analyzed using PROC MIXED of SAS version 9.4 (SAS Institute Inc., Cary, NC) with fixed effects of days aging and star probe category and a random effect of gel. Significance was denoted with $P<0.05$.

Results: Star probe classification (at $21 \mathrm{~d}$ postmortem) did not affect PRDX6 abundance (high star probe, 1.16; low star probe, 1.11). PRDX6 abundance was not affected by aging time (Day 1, 1.11; Day 8, 1.19; Day 14, 1.04; Day 21, 1.20).

Conclusion: These results indicate that PRDX6 abundance is not changed during postmortem aging. Star probe classification did not result in a difference in PRDX6 abundance. PRDX6 may not be a substrate for degradation by proteinases in postmortem muscle. Alternatively, changes in PRDX6 that do occur in postmortem muscle may be complete by $1 \mathrm{~d}$ postmortem. PRDX6 undergoes various posttranslational modifications, which, when analyzed under nonreducing conditions, may provide further insight into the relationship between PRDX6 and meat tenderness.

Keywords: peroxiredoxin-6, pork, postmortem aging, tenderness

\section{4 - ASSESSING BIOCHEMICAL CHANGES IN NORMAL AND HIGH-PH BEEF LONGISSIMUS LUMBORUM IN RELATION TO BIOELECTRICAL IMPEDANCE ANALYSIS}

F. Najar ${ }^{1 *}$, E. Boyle ${ }^{1}$, T. Houser ${ }^{1}$, C. Vahl ${ }^{2}$, J. Wolf ${ }^{1}$, J. Gonzalez $^{3}$, J. Kastner ${ }^{1}$, T. O'Quinnn ${ }^{1}$, M. Chao ${ }^{1}$, K. Cox ${ }^{4}$, S. Stroda ${ }^{1}$, and M. Hunt ${ }^{1},{ }^{1}$ Animal Science and Industry, Kansas State University, Manhattan, KS, USA, ${ }^{2}$ Department of Statistics, Kansas State University, Manhattan, KS, USA, ${ }^{3}$ Department of Animal and Dairy Science, University of Georgia, Athens, GA, USA, ${ }^{4}$ Quality Assurance, Seafood Analytics, Juneau, AK, USA, *fnajar@ksu.edu

Objectives: The study objective was to use external bioelectrical impedance analysis (BIA) to assess postmortem chemical changes in normal- and high-pH beef longissimus lumborum steaks during simulated retail display.

Materials and Methods: The experiment was designed as a split-plot with loin as the whole plot. Display day and meat $\mathrm{pH}$ were treated as the sub-plot treatments. Data were analyzed using SAS version 9.4 (SAS Institute Inc., Cary, NC). Treatment effects were treated as fixed effects and evaluated using the PROC MIXED procedure in SAS. Differences were considered significant at $P<0.05$. Beef strip loins $(n=20$; postmortem age $=14 \mathrm{~d})$ obtained from a commercial processor were sorted into 2 treatments: normal pH $(5.61-5.64 ; n=11)$ and high $\mathrm{pH}(6.2-7.0 ; n=9)$. Loins were fabricated into five $2.54-\mathrm{cm}$-thick steaks $(n=$ $100)$ and randomly assigned to one of 5 display days: 1 , $3,5,7$, and 9 . Steaks were aerobically packaged and displayed in coffin-style retail cases under fluorescent lights at $0^{\circ} \mathrm{C}-4.4^{\circ} \mathrm{C}$. External BIA, oxygen consumption (OC), metmyoglobin reducing activity (MRA), protein degradation, water-holding capacity (WHC), and $\mathrm{pH}$ were assessed on each display day.

Results: There was no meat- $\mathrm{pH} \times$ display-day interaction $(P>0.05)$ for external BIA values; however, an effect on meat $\mathrm{pH}$ and display day was found $(P<0.05)$. External BIA was $20 \%$ higher $(P<0.05)$ for high-pH meat than normal-pH meat. Steaks on day 1 had lower external BIA values $(P<0.05)$ compared to day 5 and 7 , but similar $(P>0.05)$ to day 3 and 9 . There was a meat-pH $\times$ display-day interaction for OC, MRA, WHC, and $\mathrm{pH}(P<0.05)$. During retail display, normal-pH steaks had lower $(P<0.05)$ OC values than high-pH steaks. Additionally, MRA values were similar $(P>0.05)$ from day 1 to day 7; however, MRA values decreased $9 \%(P<0.05)$ by day 9 for normal-pH meat, while high-pH meat remained constant $(P>0.05)$ over the $9 \mathrm{~d}$ of retail display. There was no meat-pH $\times$ display-day interaction $(P>0.05)$ or display-day effect for intact or degraded desmin; however, a meat-pH effect $(P<0.05)$ was found. Normal-pH meat had $33 \%$ and $43 \%$ higher $(P<0.05)$ amount of intact and degraded desmin, respectively, than high-pH meat. There tended to be a meat-pH $\times$ displayday interaction for troponin- $\mathrm{T} 40$ and $30 \mathrm{KDa}(P=$ $0.0601)$. In addition, no meat- $\mathrm{pH} \times$ display-day interaction $(P>0.05)$ was found for troponin-T 36, 34, and $30 \mathrm{KDa}$. High-pH steaks had a greater $(P<0.05) \mathrm{WHC}$ and $\mathrm{pH}$ than normal-pH beef over the display time. In high-pH beef, external BIA values were moderately correlated with OC, MRA, and WHC $(r=0.35 ; P<0.05)$. Additionally, a negative correlation occurred between external BIA and $\mathrm{pH}$ $(r=-0.48 ; P<0.05)$. External BIA was low to moderately correlated with degraded troponin-T $(30 \mathrm{KDa})$, degraded portion, and WHC $(r=0.28 ; P<0.05)$ for normal-pH beef. External BIA values were negatively correlated with $\mathrm{pH}$, intact and degraded desmin, and intact and degraded troponin-T (36 KDa) $(r=-0.24 ; P<0.05)$.

Conclusion: External BIA is a method that could be used to separate normal- and high-pH strip loins with potential for rapid, in-plant use to identify dark-cutting beef; 
however, BIA is not as strongly correlated to changes steaks undergo during retail display and after $14 \mathrm{~d}$ of aging.

Keywords: beef, impedance, $\mathrm{pH}$

\section{5 - EFFECT OF SIRE BREED AND AGE AT HARVEST ON SENSORY AND VOLATILE ATTRIBUTES OF LAMB LEG ROASTS}

Z. M. Hicks ${ }^{1 *}$, K. R. Wall ${ }^{1}$, C. R. Kerth ${ }^{1}$, T. W. Murphy ${ }^{2}$, W. Stewart $^{3}$, R. K. Miller ${ }^{1}$, and J. A. Boles ${ }^{4},{ }^{1}$ Department of Animal Science, Texas A\&M University, College Station, TX, USA, ${ }^{2}$ USDA ARS, Roman L. Hruska Meat Animal Research Center, Clay Center, NE, USA, ${ }^{3}$ Department of Animal Science, University of Wyoming, Laramie, WY, USA, ${ }^{4}$ Department of Animal and Range Science, Montana State University, Bozeman, MT, USA, *zmhicks@tamu.edu

Objectives: The objective of this study was to identify differences in tenderness and flavor of lamb semimembranosus roasts from wethers differing in sire breed and age at harvest.

Materials and Methods: Frozen lamb semimembranosus muscles $(N=60 ; n=30 /$ age; $n=20 /$ sire breed $)$ were received from wethers sired by one of 3 different breeds (Rambouillet, South African Meat Merino, or Suffolk) and harvested at either 11 or 18 mo of age. Packages were opened, and slices were removed from the frozen roasts for chemical analyses, including total and soluble collagen content. Roasts were then assigned a 3-digit code, repackaged, and kept in frozen storage at $-10^{\circ} \mathrm{C}$ until sensory analyses. Roasts were thawed at $4^{\circ} \mathrm{C}$ for 12 to $24 \mathrm{~h}$ prior to cooking, placed on a baking rack in a baking pan that contained $237 \mathrm{~mL}$ of water in the bottom, and roasted in an oven set at $177^{\circ} \mathrm{C}$ to an internal temperature of $71^{\circ} \mathrm{C}$. Roasts were cut into 2.54-cm slices; trimmed of external fat and connective tissue; and cut into cubes $(1.3 \mathrm{~cm} \times 1.3 \mathrm{~cm} \times$ slice thickness). Cubes were served to a trained descriptive sensory panel and evaluated for 17 flavors and 3 texture attributes. Extra cubes were frozen in liquid nitrogen and stored at $-80^{\circ} \mathrm{C}$ until volatile analyses. Five grams of meat was placed in a glass collection vile with a Teflon lid and heated on a heating block at $65^{\circ} \mathrm{C}$. Volatile compounds were collected using a solid phase microextraction portable field sampler placed into the headspace for $20 \mathrm{~min}$. The solid phase microextraction was then injected into a multidimensional gas chromatographer/mass spectrometer that desorbed, separated, and identified each volatile compound. Data were analyzed using JMP version 14.0 (SAS Institute Inc., Cary, NC) for a 3 (breed) by 2 (aging time) factorial arrangement of a completely randomized design.

Results: Total collagen $(\mathrm{mg} / \mathrm{g})$ and the percentage of soluble collagen determined from chemical analyses slices did not differ among sire breed $(P>0.05)$. Although total collagen did not differ between age groups $(P>0.05)$, lambs harvested at 11 mo of age had a higher percentage of soluble collagen $(P<0.05)$. Sensory attributes did not differ among any of the age or sire breed treatments $(P>0.05)$. Hentriacontane volatile was greatest in roasts from Suffolksired wethers $(P<0.05)$. Wethers slaughtered at $18 \mathrm{mo}$ had greater nonadecane $(P<0.05)$, whereas wethers slaughtered at $11 \mathrm{mo}$ had greater eicosane, 2-pentanone (a sweet, fruity aroma), pentanal (fermented, bready), and thiobis-methane (sulfurous, tomato, or creamy depending on the concentration; $P<0.05)$. There was a moderate positive correlation $(r=0.510, P<0.05)$ between tenderness and juiciness and a weak positive correlation $(r=0.301, P<0.05)$ between juiciness and connective tissue. Tenderness and connective tissue had a strong, positive correlation $(r=0.729$, $P<0.05)$.

Conclusion: Lambs harvested at 11 mo of age had greater soluble collagen; however, sensory panelists did not detect a difference in tenderness. Sire breed did not largely affect volatile compounds present. Although no flavor differences were found, the concentration of certain volatile compounds differed based on age at harvest.

Keywords: gas chromatography-mass spectrometry, lamb, sensory, volatile compounds

\section{6 - EVALUATING SHELF LIFE OF BEEF STEAKS OF CATTLE RAISED FROM DIFFERENT GRASS AND GRAIN FEEDING SYSTEMS}

\author{
B. Bolkenov ${ }^{1 *}$, S. Klopatek ${ }^{1}$, J. Oltjen ${ }^{1}$, X. Yang ${ }^{1},{ }^{1}$ Animal \\ Science, University of California, Davis, Davis, United \\ States, bbakytzhan@ucdavis.edu
}

Objectives: Current consumer perceptions commonly view grass-fed beef to be healthier and more environmentally sustainable compared to conventional grain-fed beef. Consumer and retailer trends indicate that shelf life is an important factor in making purchasing decisions. The objective of this study was to compare the shelf life of beef steaks from different grass and grain feeding systems. The 4 treatments include (1) conventional grain-fed beef (CON, $n=12)$, (2) 20-mo grass-fed beef (20GF, $n=12$ ), (3) 25 -mo grass-fed beef (25GF, $n=12$ ), and (4) 20-mo grass-fed +45 -d grain-fed beef ( $45 \mathrm{CON}, n=9)$.

Materials and Methods: After the slaughter, the strip loins were aged for $14 \mathrm{~d}$ in vacuum packages at $5.5^{\circ} \mathrm{C}$, then cut into steaks. Immediately, the steaks were wrapped into the oxygen-permeable film and placed under a retail display for $6 \mathrm{~d}$ at $4^{\circ} \mathrm{C}-6^{\circ} \mathrm{C}$. During the retail display, color of lean muscle and external fat surfaces of each steak $\left(L^{*}, a^{*}\right.$, and $\left.b^{*}\right)$ were measured at 3 positions every $12 \mathrm{~h}$ daily for $6 \mathrm{~d}$. Bacterial counts for total aerobic mesophilic bacteria, aerobic psychrotrophic bacteria (APB), and lactic acid bacteria 
for steaks from each strip loin were assessed on days 0,3 , and 6 of retail display. The $\mathrm{pH}$ of the same steak samples for microbial analysis was also measured. Data were analyzed using $\mathrm{R}$ version 3.6.1 for Windows. Packages analysis of variance, emmeans, and Cld were used. Two-way analysis of variance was conducted for each variable to investigate treatment effects, display time effect, and corresponding interactive effect.

Results: Steaks from 20GF and 25GF treatment groups had lower $(P<0.05) L^{*}$ values than steaks from the CON treatment group, while $L^{*}$ values of steaks from CON and $45 \mathrm{CON}$ were not significantly different. The $a^{*}$ values of $20 \mathrm{GF}$ and $45 \mathrm{CON}$ treatment group steaks were lower $(P<$ $0.05)$ than those of $\mathrm{CON}$ and $25 \mathrm{GF}$ treatment group steaks. However, external fat $L^{*}$ values of steaks from CON and 45CON treatment groups were significantly lower $(P<$ 0.05 ) than those of the $20 \mathrm{GF}$ and $25 \mathrm{GF}$ treatment groups. The $b^{*}$ values of external fat were significantly higher $(P<0.05)$ in the steaks from CON and 20GF treatment groups than in the steaks from $25 \mathrm{GF}$ and $45 \mathrm{CON}$. The aerobic mesophilic bacteria counts were significantly higher $(P<0.05)$ in steaks from $20 \mathrm{GF}$ and $45 \mathrm{CON}$ treatment groups than in beef muscle from CON and 25GF treatment groups, while APB and lactic acid bacteria were significantly higher $(P<0.05)$ in steaks from grass-fed treatment groups than in steaks from grain-fed and $45 \mathrm{CON}$ treatment groups. Initial counts for APB were between 4.80 and 5.85 on day 0 in the steaks from all the treatments. However, on day 3, APB counts for the steaks from gras-fed steers approached the indicative spoilage level ( $7 \log \mathrm{CFU} / \mathrm{g})$. On day 6, APB counts for all the treatments exceeded spoilage level. The thiobarbituric acid reactive substances values were significantly higher $(P<0.05)$ in the steaks from 20GF and CON treatment groups than $25 \mathrm{GF}$ and $45 \mathrm{CON}$ treatment groups. On day 6 , thiobarbituric acid reactive substances values were $4.71 \mathrm{mg} / \mathrm{kg}$ for $\mathrm{CON}$, $3.47 \mathrm{mg} / \mathrm{kg}$ for $20 \mathrm{GF}$, and $2.84 \mathrm{mg} / \mathrm{kg}$ for $45 \mathrm{CON}$ and $25 \mathrm{GF}$ treatment groups, respectively, indicating the spoilage of the steaks due to lipid oxidation. There was no significant difference in muscle $\mathrm{pH}$ among treatments.

Conclusion: The results indicated that the shelf life of the steaks from the grass feeding system might be shorter due to the appearance and faster microbial spoilage rate than the steaks from cattle raised on grain-incorporated feeding systems.

Keywords: shelf life, beef steaks, feeding systems, grass-fed beef, grain-fed beef

\section{7 - INCREASED INTERNAL TEMPERATURE IN HEAVY BEEF CARCASSES INFLUENCES PRODUCT QUALITY}

\author{
S. Egolf ${ }^{*}$, A. Egolf ${ }^{1}$, H. Rode ${ }^{2}$, L. Hite $^{1}$, C. Bakker ${ }^{1}$, A.
} Blair $^{1}$, K. Underwood ${ }^{1}$, and J. Grubbs ${ }^{1}$, ${ }^{1}$ Animal Science
Department, South Dakota State University, Brookings, SD, USA, ${ }^{2}$ Tyson Fresh Meat, Tyson Fresh Meat, Dakota Dunes, SD, USA, *samantha.egolf@jacks.sdstate.edu

Objectives: Increases in beef hot carcass weights (HCW) the last $30 \mathrm{y}$ in the United States may negatively impact beef quality by slowing carcass chilling. The objectives of this study were to assess the impact of HCW on (1) internal carcass temperature of the chuck, loin, and round in USDA Select (Se) and Low Choice (LC) carcasses and (2) ultimate $\mathrm{pH}\left(\mathrm{pH}_{\mathrm{u}}\right)$, objective color $\left(\mathrm{CIE} L^{*}, a^{*}, b^{*}\right)$, sarcomere length, Warner-Bratzler shear force, cook loss, and protein degradation of the serratus ventralis (SV), longissimus lumborum (LL), and semitendinosus (ST) from Se and LC carcasses.

Materials and Methods: Carcasses $(n=116)$ were selected by weight (light $[\mathrm{LW}]=295.5-341 \mathrm{~kg}$; middle $[\mathrm{MW}]=386.4-431.8 \mathrm{~kg}$; heavy $[\mathrm{HW}]=465.9-522.7 \mathrm{~kg}$ ), and data loggers were placed in the muscle portion of the deep chuck $(20.32 \mathrm{~cm})$, loin $(10.16 \mathrm{~cm})$, and round $(20.32$ $\mathrm{cm})$ to record internal temperature for $26 \mathrm{~h}$. Carcass and temperature data were collected following $26 \mathrm{~h}$ of chilling, and USDA Se and LC carcasses were selected for fabrication following chilling. Samples from each subprimal were aged for 5, 10, and $14 \mathrm{~d}$ for Warner-Bratzler shear force, cook loss, and protein degradation, and $5 \mathrm{~d}$ for sarcomere length. Data were analyzed using PROC MIXED in SAS (version 9.4; SAS Institute Inc., Cary, NC) with HCW and Quality Grade as main effects, 12 th rib backfat as a covariate, and cooler location and collection day as random effects. A significance level of $\alpha=0.05$ was used.

Results: A QG $\times \mathrm{HCW}$ interaction was observed for loin and round temperatures $(P<0.04)$. During chilling, Se LW had the lowest temperatures and Se and LC HW had the highest temperatures in both subprimals. At $26 \mathrm{~h}$, Se and LC HW carcasses were highest and Se LW were lowest in the round. No differences were detected in the loin between weight groups at $26 \mathrm{~h}$. Temperature in the chuck was higher for HW throughout chilling and at $26 \mathrm{~h}$. No differences were observed between weight groups for $\mathrm{pH}_{\mathrm{u}}$ $(P>0.05)$; however, LC SV had an increased $\mathrm{pH}_{\mathrm{u}}(P=$ $0.01)$ compared to Se SV. Increased $L^{*}$ and $a^{*}$ values were observed for $\mathrm{HW}$ carcasses compared to LW in all muscles $(P<0.001)$. A QG $\times$ HCW interaction was observed for sarcomere length in the LL $(P=0.03)$, with LC LW having shorter sarcomeres than Se LW and LC MW. The sarcomere length of the ST was influenced by $\mathrm{HCW}(P<0.01)$, with HW having the shortest sarcomeres. Sarcomere length in the SV was not influenced by HCW. Tenderness of steaks from the SV and ST were not impacted by QG or HCW. Tenderness tended to increase in MW and HW in the LL $(P=0.08)$. Increased tenderness was observed in HW LL at $5 \mathrm{~d}$ of aging $(P<0.04)$, but no differences were observed at 10 and $14 \mathrm{~d}(P>0.05)$. Cook loss was influenced by aging day $(P<0.03)$, but not HCW or QG $(P>0.05)$. A QG $\times$ $\mathrm{HCW}$ interaction was observed for the $55 \mathrm{kDa}$ desmin 
product in the LL $(P=0.04)$ and $30 \mathrm{kDa}$ troponin-T product in the SV $(P<0.01)$. Decreased desmin degradation was observed in Se LW LL at $5 \mathrm{~d}(P<0.02)$, and increased troponin-T degradation was observed in LC HW at $5 \mathrm{~d}$ in the SV $(P<0.02)$. No differences in proteolysis were observed at 10 and $14 \mathrm{~d}$ of aging across the muscles.

Conclusion: Heavier carcasses had increased temperatures at the end of the 26-h chilling period in the chuck and round, which may influence product quality. The HW carcasses had brighter, redder muscles, and tenderness was improved at earlier aging times; however, the underlying mechanisms explaining the relationship of $\mathrm{HCW}$ with $\mathrm{Se}$ and LC beef quality grades require further exploration.

Keywords: beef, beef quality, hot carcass weight, temperature decline

\section{8 - HEAVIER BEEF HOT CARCASS WEIGHTS IMPACT SENSORY TRAITS}

S. Egolf ${ }^{*}$, J. Janssen ${ }^{1}$, R. $\operatorname{Cox}^{2}$, A. Egolf ${ }^{1}, H . R^{2}{ }^{3}$, L. Hite $^{1}$, C. Bakker ${ }^{1}$, A. Blair ${ }^{1}$, J. Grubbs ${ }^{1}$, and K. Underwood ${ }^{1},{ }^{1}$ Animal Science Department, South Dakota State University, Brookings, SD, USA, ${ }^{2}$ Department of Animal Science, University of Minnesota, St. Paul, MN, USA, ${ }^{3}$ Tyson Fresh Meat, Tyson Fresh Meat, Dakota Dunes, SD, USA, *samantha.egolf@jacks.sdstate.edu

Objectives: Tenderness and flavor are two of the most important beef sensory attributes driving consumer satisfaction. Heavier carcasses may influence product quality and consistency. The objective of this study was to determine the effect of increased hot carcass weight (HCW) on the sensory attributes of the Denver cut (DEN), strip loin (SL), and eye of round (EOR) from USDA Select (Se) and Low Choice (LC) carcasses.

Materials and Methods: Carcasses $(n=116)$ were selected at a commercial packing facility by weight [light $[\mathrm{LW}]=295.5-341 \mathrm{~kg}$; middle $[\mathrm{MW}]=386.4-431.8 \mathrm{~kg}$; and heavy $(\mathrm{HW})=465.9-522.7 \mathrm{~kg})$. After $26 \mathrm{~h}$ of chilling, carcass data were collected. Carcasses meeting weight and grade criteria were fabricated (20 per group; 16 for Se HW), and samples were transported to the South Dakota State University meat lab. Steaks (DEN, SL, and EOR) were collected on day 5 , day 10 , and day 14 and assessed by consumer sensory panels for tenderness, juiciness, off-flavors, flavor liking, texture liking, and overall liking. Data were analyzed using PROC MIXED in SAS (version 9.4, SAS Institute Inc., Cary, NC) for the main effects of $\mathrm{HCW}$ and aging day. Correlations were determined using PROC CORR. An $\alpha=0.05$ was used to determine significance.

Results: In Se DEN steaks, LW had decreased tenderness and overall liking on day 5 compared to MW and HW day-5 steaks $(P<0.03)$, but by day 10 , no differences were observed. No differences were observed between weight groups for flavor in Se DEN steaks. For LC DEN steaks, a HCW $\times$ aging day interaction was observed for tenderness $(P<0.0001)$. On day 5, LW were the least tender, and on day 14, HW were the most tender. There were no differences between HCW for flavor or overall liking for the LC DEN $(P>0.05)$. In Se SL steaks, tenderness was increased in MW and HW compared to LW $(P<0.02)$. Flavor was more intense on day 10 and day 14 compared to day 5 in LW Se SL steaks $(P<0.04)$. Overall liking of Se SL was influenced by aging day, with higher ratings given to day-10 and day-14 steaks $(P<0.01)$. A HCW $\times$ aging day interaction was observed for tenderness and overall liking $(P$ $<0.04)$ for LC SL steaks. Tenderness was decreased in day$5 \mathrm{LW}$ and day-10 HW. Overall liking was higher for day-5 MW and decreased for day-10 HW. By day 14, all LC SL steaks had similar ratings for tenderness and overall liking. Flavor in LC SL steaks was more intense for day-5 MW and day-14 MW compared to day- $5 \mathrm{LW}$ and day-14 LW, respectively $(P<0.05)$. A $\mathrm{HCW} \times$ aging day interaction was observed for tenderness $(P=0.01)$ in Se EOR steaks. Tenderness was increased in day-10 MW compared to day-14 MW and decreased in day-5 HW compared to day-10 and day-14 HW. Aging day influenced flavor and overall liking in Se EOR. The day-5 and day-14 MW steaks were rated higher than day-10 for both sensory attributes $(P<0.04)$. For LC EOR steaks, a HCW $\times$ aging day interaction was observed for tenderness $(P<0.05)$. Tenderness was decreased in LW, but similar between MW and HW. Flavor was more intense in day-5 MW compared to day-5 LW $(P<$ $0.01)$. Overall liking was higher in MW compared to $L W$ and HW $(P<0.01)$. A moderate negative relationship was found between overall liking and tenderness $(r=-0.41 ; P<$ 0.0001 ), but a stronger positive correlation was observed between flavor and overall liking $(r=0.85 ; P<0.0001)$.

Conclusion: In this study, consumers were more apt to rate MW steaks higher for sensory attributes, but a definitive pattern was not observed between HCW or aging day. The relationship between $\mathrm{HCW}$ and aging time is unclear and warrants further investigation to determine whether quality grade impacts these ratings.

Keywords: beef, hot carcass weight, sensory

\section{9 - PLECTIN POSTMORTEM DEGRADATION AND ITS RELATION TO WATER DISTRIBUTION IN FRESH PORK}

\author{
W. Zhang ${ }^{1 *}$ and X. Tian ${ }^{1},{ }^{1}$ College of Food Science and \\ Technology, Nanjing Agricultural University, Nanjing, \\ China, *wangang.zhang@njau.edu.cn
}

Objectives: Plectin is an intermediate filament (IF)-based versatile cytolinker protein of the plakin family. Plectin links the desmin IF and anchors them to specific cytoskeletal structures and cytoplasmic organelles in skeletal muscle, 
forming a complex IF network that has a great influence in sustaining and strengthening the integrity of cytoarchitecture. A previous study has shown that plectin was a potential substrate of calpain-1 during the conversion of muscle to meat. Therefore, the current study aimed to evaluate the contribution of plectin postmortem degradation to the changes in water distribution in fresh pork.

Materials and Methods: Longissimus thoracis muscles (between the 10th and 14th thoracic vertebra with the size of $15 \mathrm{~cm} \times 8 \mathrm{~cm} \times 4 \mathrm{~cm}$ ) were collected from both sides of 44 carcasses within $1 \mathrm{~h}$ postmortem and aged at $4{ }^{\circ} \mathrm{C}$ for $0 \mathrm{~h}, 6 \mathrm{~h}, 12 \mathrm{~h}, 1 \mathrm{~d}, 3 \mathrm{~d}, 7 \mathrm{~d}$, and $13 \mathrm{~d}$ under vacuum package. Longissimus thoracis samples from one side were used to prepare the whole protein samples of $5 \mathrm{mg} / \mathrm{mL}$ for the Western blot analysis of plectin degradation. Samples from the other side were used for low field-nuclear magnetic resonance analysis (LF-NMR) based on the spin-spin transverse relaxation time $\left(T_{2}\right)$ analysis. Statistical significance of the differences among individual means was assessed by Duncan's multiple-range tests. Pearson correlation coefficients were obtained to evaluate the correlation between different protein degradation and LF-NMR parameters.

Results: Figure 1 shows that plectin was found to be significantly degraded during postmortem aging. The amount of intact plectin was rapidly reduced at the early postmortem aging $(P<0.05)$ and almost disappeared at day 3. Meanwhile, the degraded plectin under the intact band emerged at $0 \mathrm{~h}$ and accumulated fast during the first $3 \mathrm{~d}$ aging $(P<$ $0.05)$. However, the amount of the degraded product reduced between day 3 and day $13(P<0.05)$, indicating a further degradation of plectin during later stage of postmortem aging. Table 1 shows the correlations between LF-NMR $\mathrm{T}_{2}$ parameters (water population [P] and transverse relaxation time [T]) and plectin postmortem degradation. The amount of intact plectin during 1-d aging was significantly correlated to $\mathrm{P}_{21}$ at day 1 and $3(P<0.01)$. There were significantly positive correlations between plectin intensity within $1 \mathrm{~d}$ and $\mathrm{P}_{22}$ at $1 \mathrm{~d}$ and $3 \mathrm{~d}(P<0.01)$. Moreover, plectin content at $0 \mathrm{~h}$ was significantly correlated to $\mathrm{T}_{21}$ and $\mathrm{T}_{22}$ at $1 \mathrm{~d}(P<0.01)$. As $\mathrm{P}_{21}$ and $\mathrm{P}_{22}$ represent the intra- and extra-myofibrillar water, respectively, these results indicate that plectin might contribute to the water distribution during condition including the decrease of intra-myofibrillar water and the increase of extra-myofibrillar water early postmortem. As $T_{21}$ and $T_{22}$ reflect the changes in intra- and extra-myofibrillar space, the results above imply the contribution of plectin to the structural changes including the decrease of intra-myofibrillar space and the space between myofibrils and sarcolemma early postmortem.

Conclusion: Plectin degradation was found to be negatively correlated to $\mathrm{P}_{21}, \mathrm{~T}_{21}$, and $\mathrm{T}_{22}$ and positively correlated to $\mathrm{P}_{22}$. The correlations were established between plectin content at the earlier aging time and the population of $T_{2}$ water at the later days, indicating the potential contribution of plectin degradation to the changes of water distribution during postmortem aging.

Keywords: plectin, pork, postmortem aging, water-holding capacity
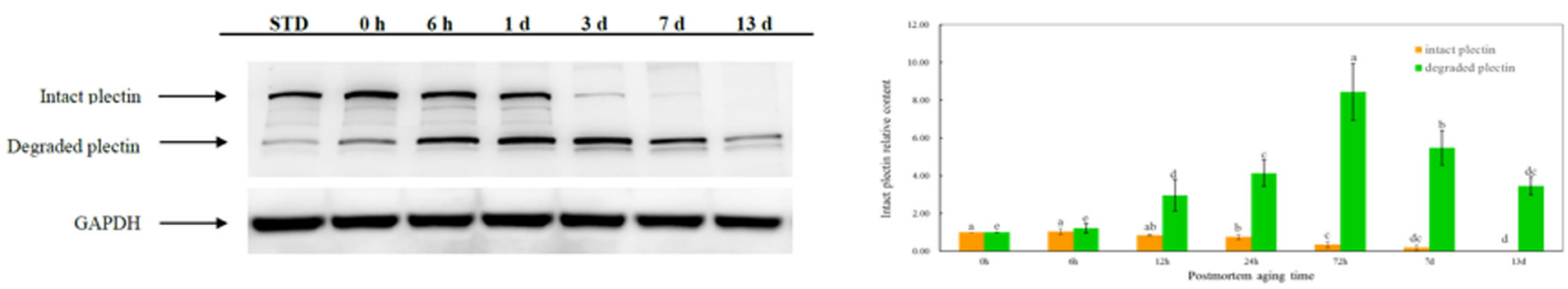

Figure 1. Western blot analysis of plectin expression in postmortem pork LT muscle.

Note: $50 \mu \mathrm{g}$ of total protein samples was loaded per lane. The standard was a sample with a stable band in the trial test and was loaded onto each gel

Table 1 Pearson correlation coefficients and levels of significance for the correlations between plectin degradation and LF-NMR T21, T22, P21, P22.

\begin{tabular}{|c|c|c|c|c|c|c|c|c|c|c|c|c|c|c|c|c|}
\hline \multirow[b]{2}{*}{ Intact plectin } & \multicolumn{4}{|c|}{$\mathrm{P}_{21}$} & \multicolumn{4}{|c|}{$\mathrm{P}_{22}$} & \multicolumn{4}{|c|}{$\mathrm{T}_{21}$} & \multicolumn{4}{|c|}{$\mathrm{T}_{22}$} \\
\hline & $1 \mathrm{~d}$ & $3 \mathrm{~d}$ & $7 \mathrm{~d}$ & $13 \mathrm{~d}$ & $1 \mathrm{~d}$ & $3 d$ & $7 \mathrm{~d}$ & $13 \mathrm{~d}$ & $1 \mathrm{~d}$ & $3 d$ & $7 \mathrm{~d}$ & $13 \mathrm{~d}$ & $1 \mathrm{~d}$ & $3 \mathrm{~d}$ & $7 \mathrm{~d}$ & $13 \mathrm{~d}$ \\
\hline Oh & $-0.549 * *$ & $-0.500^{* *}$ & $-0.421 * *$ & -0.002 & $0.576^{* *}$ & $0.544 * *$ & $0.551^{* *}$ & $0.386^{* * *}$ & $-0.445^{* *}$ & -0.119 & -0.031 & -0.264 & $-0.415^{* *}$ & 0.026 & -0.253 & $-0.338^{*}$ \\
\hline $6 \mathrm{~h}$ & $-0.404 * *$ & $-0.394 * *$ & -0.051 & -0.117 & $0.428 * *$ & $0.406^{* *}$ & 0.269 & 0.275 & -0.172 & 0.133 & 0.022 & -0.239 & $-0.322 *$ & -0.029 & -0.195 & -0.187 \\
\hline $1 \mathrm{~d}$ & $-0.486^{* *}$ & $-0.380 *$ & -0.246 & -0.070 & $0.480 * *$ & $0.414 * *$ & $0.409^{* *}$ & $0.310^{*}$ & -0.230 & -0.142 & -0.058 & -0.238 & $-0.350^{*}$ & 0.052 & -0.221 & -0.198 \\
\hline $3 d$ & $-0.549 * *$ & $-0.338^{*}$ & $-0.354 *$ & 0.043 & $0.566^{* *}$ & $0.425^{* *}$ & $0.421^{* *}$ & 0.118 & $-0.431 * *$ & -0.182 & -0.034 & -0.145 & $-0.466^{* *}$ & 0.116 & -0.140 & -0.054 \\
\hline
\end{tabular}

Note: **: Correlation is significant at the 0.01 level. *: Correlation is significant at the 0.05 level. $(\mathrm{n}=44)$. 


\section{1 - EFFECTS OF DRY AND WET AGING ON VOLATILE AND AMINO ACID PROFILE OF USDA CHOICE AND PRIME STRIP LOINS}

A. M. Cavender ${ }^{1 *}$, D. Miner ${ }^{2}$, F. M. Giotto ${ }^{1}$, M. R. Ugur ${ }^{3}$, T. Dinh $^{3}$, and A. S. De Mello ${ }^{1},{ }^{1}$ Agriculture, Veterinary, and Rangeland Sciences, University of Nevada, Reno, NV, USA, ${ }^{2}$ Chemistry, University of Nevada, Reno, NV, USA, ${ }^{3}$ Animal and Dairy Sciences, Mississippi State University, Starkville, MS, USA,*amilton@cabnr.unr.edu

Objectives: Dry aging beef is suggested to enhance the flavor profile compared with conventional wet aging. Proteolysis and changes in chemical profile are thought to enhance the flavor profile of beef, improving the overall eating experience. To better understand this hypothesis, this study aimed to investigate the volatile compounds and amino acid profile of dry-aged beef.

Materials and Methods: A total of 48 short loins (Institutional Meat Purchase Specifications 174; 24 Prime and 24 Choice) were commercially acquired and assigned to a $2 \times 2 \times 2$ factorial design. Fixed effects were USDA quality grade (Choice and Prime), aging method (dry and wet), and aging length ( $21 \mathrm{~d}$ and $42 \mathrm{~d}$ ). Dry-aged samples were held at $2^{\circ} \mathrm{C} \pm 2^{\circ} \mathrm{C}$. Cooler humidity was maintained at $80 \%-85 \%$ and air speed at $2 \mathrm{~m} / \mathrm{s}$. Wet-aged samples were stored under the same temperature in their original vacuumsealed bag. Strip loins were fabricated, and two $2.54-\mathrm{cm}$ steaks were obtained from each short loin, cooked, and evaluated for volatile compound profile. Volatiles were collected by Solid-phase microextraction fibers and analyzed using gas chromatography-mass spectrometry. For amino acids, fresh steaks were pulverized with liquid nitrogen and analyzed using EZfaast Amino Acid kit. Data were analyzed using PROC GLIMMIX of SAS (SAS Institute Inc., Cary, NC). When significance was detected at $P \leq 0.05$, means were separated using LSMEANS.

Results: Overall, the majority of the amino acids were affected only by aging length. As aging length increased, the concentration of alanine, asparagine, aspartic acid, glutamic acid, glycine, isoleucine, leucine, lysine, methionine, ornithine, phenylalanine, proline, serine, threonine, tyrosine, and valine increased. Dry-aged beef had higher tyrosine and lower sarcosine concentrations when compared to wet-aged. Interactions between aging length and aging method were observed for 3-hydroxyproline and 4-hydroxyproline. Dry aging increased concentrations of both amino acids when compared to wet aging. For the volatile profile, a significant three-way interaction was observed for 2,3-butanedione, and for the sum of ketone compounds. An interaction between aging method and aging length showed that 21-d dry-aged loins had higher concentrations of 2-ethyl-3,5/6-dimethyl pyrazine compared to 21-d wet-aged loins. Interactions between aging method and grade were observed for pentanal, 2-butanone, and methyl pyrazine. Choice wet-aged loins had higher concentrations of pentanal compared to Choice dry-aged and Prime wet-aged. Prime dry-aged loins had higher concentrations of 2-butanone compared to Choice. Prime dry-aged loins also showed higher methyl pyrazine concentrations compared to wet-aged Prime and dry-aged Choice. Overall, dry aging increased concentrations of octane compared to wet aging.

Conclusion: The amino acid profile of beef was primarily affected by aging length. The individual effect of aging method (dry or wet aging) played a minimal role in determining the volatile profile of beef. However, when combined with USDA grade, dry aging may affect the volatile profile. Quality grade seems to be the major driving effect for changes in volatiles of beef.

Keywords: amino acids, beef, dry aging, volatiles

\section{2 - OXIDATIVE STRESS AS A MEASURE OF POSTMORTEM MEAT QUALITY IN CROSSBRED LAMBS}

N. J. Herrera ${ }^{1 *}$, N. A. Bland ${ }^{1}$, F. A. Ribeiro ${ }^{1}$, M. L. Henriott ${ }^{1}$, J. L. Petersen ${ }^{1}$, and C. R. Calkins ${ }^{1},{ }^{1}$ Animal Science, University of Nebraska-Lincoln, Lincoln, NE, USA, *nherrera18@huskers.unl.edu

Objectives: The objective of this study was to evaluate the effects of different levels of lipopolysaccharide (LPS)-mediated oxidative stress on fresh meat quality in Longissimus lumborum of crossbred lambs.

Materials and Methods: Crossbred lambs $(n=29)$ were blocked by weight and fed a standard finishing ration for the duration of the study. Lambs were individually housed, and treatment groups were administered one of 3 injections every $72 \mathrm{~h}$ across a 3-injection (9-d) cycle: a saline control (Control), $50 \mathrm{ng} \mathrm{LPS} / \mathrm{kg}$ body weight (LPS50), or $100 \mathrm{ng}$ LPS $/ \mathrm{kg}$ body weight (LPS100). Rectal temperatures were measured to indicate inflammatory response. Lambs were harvested at the Loeffel Meat Laboratory, and $80 \mathrm{mg}$ of pre-rigor Longissimus lumborum were collected in Control and LPS100 treatments within 30 min postmortem for RNA analysis. Loins were split and randomly assigned for 1 or $14 \mathrm{~d}$ of aging. Chops were fabricated after aging and placed under retail display (RD) conditions for 0 or $7 \mathrm{~d}$. Using SAS (version 9.4, SAS Institute Inc., Cary, NC), objective and subjective color data were analyzed as a split-plot repeated measures design with treatment as the whole-plot, aging period as the split-plot, and days of RD for repeated measures. Tenderness, troponin-T, desmin, calcium, and $\mathrm{pH}$ were analyzed as a split-plot design with treatment as the whole-plot and aging period for split-plot. Lipid oxidation was a split-split-plot design with treatment as the whole-plot, aging period as the split-plot, and RD time as the split-split-plot. Transcriptomics, sarcomere length, fatty acids, and isoprostanes were analyzed as a completely 
randomized design. Data were analyzed using the PROC GLIMMIX procedure of SAS using animal as the experimental unit. All means were separated with the LSMEANS statement with an $\alpha$ level of 0.05 for significance. Tendencies were considered at an $\alpha$ level of 0.10 .

Results: LPS-treated lambs had increased $(P<0.05)$ rectal temperatures at $1,2,4$, and $24 \mathrm{~h}$ post-injection. Transcriptomics exhibited significant $\left(P_{\text {raw }}<0.05\right)$ upregulation in RNA pathways related to generation of oxidative stress in LPS100 compared to Control. A trend was found for tenderness (Warner-Bratzler shear) $(P=0.10)$, with chops from LPS50 having a lower shear force compared with Control at $1 \mathrm{~d}$ postmortem. Additionally, the LPS50 treatment exhibited greater troponin-T degradation $(P=0.02)$ compared to all treatments at $1 \mathrm{~d}$. No statistical differences were found at $14 \mathrm{~d}$ postmortem for shear force or troponin- $\mathrm{T}$ $(P>0.05)$. Aging decreased WBSF $(P<0.0001)$ and increased free calcium concentration $(P<0.0001), \mathrm{pH}$ $(P<0.0001)$, and proteolytic degradation $(P<0.0001)$ across all treatments. After $7 \mathrm{~d}$ of $\mathrm{RD}$, following aging periods, chops increased discoloration as RD increased $(P<$ $0.0001)$, with Control chops aged for $14 \mathrm{~d}$ being the most discolored. Chops from lambs given LPS had higher $(P<$ 0.05) $a^{*}$ values compared to Control at $14 \mathrm{~d}$ of aging. The $L^{*}$ values were greater $(P<0.05)$ in LPS100 compared to both LPS50 and Control. Aging tended $(P=0.0608)$ to increased lipid oxidation (thiobarbituric acid reactive substances) during RD across either aging period. There were no significant differences $(P>0.05)$ in sarcomere length, proximate composition, fatty acid composition, or isoprostane content.

Conclusion: These results suggest that defined upregulation of oxidative stress has no detriment on fresh meat color but may alter biological pathways responsible for muscle composition and enzymatic processes, resulting in changes in tenderness early postmortem.

Keywords: apoptosis, color stability, lamb, meat quality, oxidative stress

\section{3 - EFFECT OF DIETARY SUPPLEMENTATION WITH SILKWORM (BOMBYX MORI L.) OIL ON FATTY ACID PROFILE OF RABBIT MEAT}

A. Dalle Zotte ${ }^{1^{*}}$, Y. Singh ${ }^{1}$, M. Cullere ${ }^{2}, Z$. Gerencsér ${ }^{3}, Z$. Matics $^{3}$, S. Tenti ${ }^{2}$, and S. Cappellozza ${ }^{4},{ }^{1}$ Animal Medicine, Production and Health, Padova University, Legnaro, Italy, ${ }^{2}$ Animal Medicine, Production and Health, Padova University, Legnaro, Italy, ${ }^{3}$ Faculty of Agricultural and Environmental Sciences, Kaposvàr University, Kaposvàr, Hungary, ${ }^{4}$ Research Centre for Agriculture and Environment-Sericulture, Council for Agricultural
Research and Economics, Padova, Italy, *antonella. dallezotte@unipd.it

Objectives: The experiment was conducted to investigate the effect of the dietary incorporation of oil extracted from silkworm (Bombyx mori L.) pupae in rabbit diets, as a complete replacement of sunflower oil, on the fatty acid (FA) composition of rabbit hind leg meat.

Materials and Methods: For this purpose, sixty-four 7-wk-old Pannon White rabbits were assigned to 2 feeding groups ( $n=32$ rabbits/group): the first group received a standard commercial pelleted diet containing $1.30 \%$ sunflower oil (Control), and the second group received a diet in which sunflower oil was completely replaced with $1.30 \%$ silkworm oil (SWO). Rabbits were pair-housed in wire-mesh cages $(n=16 /$ treatment $)$ and fed with the experimental diets until slaughter (10 wk of age). After slaughter, one rabbit/cage was randomly selected ( $n=16$ rabbits/group), and the hind legs were excised and used for FA profile determination. Data were analyzed by a one-way analysis of variance with diet as the fixed effect.

Results: The dietary inclusion of SWO affected the FA profile of the hind leg; both $n-6$ and $n-3$ fractions were affected; meat from the SWO rabbits displayed a lower $n-6$ and a higher $n-3$ percentage, respectively $(P<$ $0.0001)$, compared to the Control group. This led to a lower, and therefore better, $n-6 / n-3$ ratio in rabbits fed with the SWO diet. The main single FA responsible of this change were linoleic $(P<0.0001)$ and linolenic $(P<0.0001)$ acid among the $n-6$ and the $n-3$ FA, respectively. Despite this, the total saturated fatty acid, monounsaturated fatty acid, and polyunsaturated fatty acid proportions remained unaffected by the dietary treatment, but thrombogenic index of meat was improved $(P<0.0001)$ with the dietary incorporation of SWO.

Conclusion: SWO showed to be a promising feed ingredient for growing rabbits as it improved the healthiness of rabbit meat lipids.

Keywords: silkworm oil, rabbit nutrition, rabbit meat, fatty acids

\section{4 - BACKFAT THICKNESS AS A PRIMARY INDEX REFLECTING THE PORK MEAT YIELD AND TECHNOLOGICAL QUALITY TRAITS}

H. Van-Ba ${ }^{1}$ on behalf of Hyun-Woo Seo ${ }^{1}$, Pil-Nam Seong ${ }^{1}$, Soo-Hyun $\mathrm{Cho}^{1}$, Sun-Moon Kang ${ }^{1}$, Yun-Seok Kim ${ }^{1}$, Jin-Hyoung Kim ${ }^{1}$, S.-S. Moon ${ }^{2}$, Y.-M. Choi ${ }^{3}$, and K.-H. Seol $^{1 *}$, ${ }^{1}$ Animal Product Utilization Division, National Institute of Animal Science, RDA, Wanju-gun, Republic Of Korea, ${ }^{2}$ Meat Research Center, Sunjin, Ansung, Republic Of Korea, ${ }^{3}$ National Institute of Agricultural Science, 
RDA, Wanju-gun, Republic Of Korea,*skh0205@naver. com

Objectives: Predicting aspects of lean meat yield and eating quality of pork based on particular carcass traits has become increasingly important from an economic profitability point of view. Therefore, the main objective of this study was to determine the effects of backfat (BF) thickness on the lean meat yield and meat quality traits of growing-finishing pigs.

Materials and Methods: A total of 240 ([Landrace $\times$ Yorkshire] gilts $\times$ Duroc boars) crossbred pigs reared under identical conditions and harvested at around $180 \mathrm{~d}$ old were used. Twenty-four hours postmortem, the left side of each carcass was dissected for screening BF thickness. The BF thickness was measured at (i) between the 11th and 12th rib and (ii) between the last rib and first lumber vertebra using a Vernier caliper. The final BF thickness (millimeters) was determined by averaging the BF thickness at the 2 above measured areas. Four BF groups-12-15, 16-20, 21-25, and $26-30 \mathrm{~mm}$-were classified. To determine the total lean meat yield, each carcass was separated into primal and subprimal cuts. After deboning, skinning, and trimming of all visual fats, their bones, skins, fat, and lean meat weights were separately recorded. The yields (percent) for the total lean meat and fat per carcass were calculated by their corresponding weights divided by the carcass weight multiplied by 100 . Finally, longissimus dorsi muscles from BF groups were collected and used for meat quality analysis. Protein and intramuscular fat (IMF) were determined using a Food Scan ${ }^{\mathrm{TM}}$ Lab 78810. Meat $\mathrm{pH}$ was determined using a $\mathrm{pH}^{*} \mathrm{~K} 21$ meter. Instrumental color was determined using a Minolta Chroma Meter. Water-holding capacity (WHC) was determined using a centrifugation-based method. Cooking loss and shear force both were measured on a same block $(3.0 \mathrm{~cm}$ in thickness) of each sample. The sample blocks were cooked to an internal temperature of $70^{\circ} \mathrm{C}$. The shear force was determined by cutting cores ( 5 cores per sample) with a V-shaped blade of a Warner-Bratzler shear device. All data were analyzed by using the General Linear Model procedure of SAS (SAS Institute Inc., Cary, NC), and means were compared using Duncan's Multiple Range Test. Significance was set at $P<0.05$. Pearson correlation coefficients between BF thickness and carcass and meat quality traits were also determined.

Results: BF thickness was positively correlated with live weight and total fat yield $(r=0.686, P<0.001)$, whereas it was negatively correlated with loin area and total meat yield $(r=-0.532, P<0.001)$. Increasing the BF thickness significantly decreased the total lean meat yield and loin lean area but increased the total fat yield $(P<0.05)$. Regarding the meat quality, $\mathrm{BF}$ thickness was positively correlated with IMF $(r=0.237, P<0.05), L^{*}(r=0.420, P<$ $0.05)$, and WHC $(r=0.235, P<0.05)$, whereas it was negatively correlated with shear force $(r=-0.249, P<0.05)$. The thickest BF group (26-30 mm) was higher in IMF content, $\mathrm{pH}$, and $\mathrm{WHC}$, whereas it was lower in shear force values compared to the thinnest $\mathrm{BF}$ group $(12-15 \mathrm{~mm})$ $(P<0.05)$. No differences occurred in the IMF, $\mathrm{pH}$, and WHC between the 21-25 and 26-30 BF groups $(P>0.05)$.

Conclusion: When the preharvest factors are kept the same, BF thickness could be considered as a primary index for predicting lean meat yield, and the minimal BF thickness of $21-30 \mathrm{~mm}$ is required to improve pork meat quality.

Keywords: backfat thickness, meat yield, pork, quality

\section{5 - IMPACT OF NITRITE-EMBEDDED PACKAGING AND ENHANCEMENT ON DARK-CUTTING RAW AND COOKED BEEF COLOR}

M. Denzer ${ }^{1 *}$, D. VanOverbeke ${ }^{1}$, G. Mafi ${ }^{1}$, and R. Ramanathan ${ }^{1},{ }^{1}$ Department of Animal and Food Sciences, Oklahoma State University, Stillwater, OK, USA, *mdenzer@ okstate.edu

Objectives: A greater than normal $\mathrm{pH}(>\mathrm{pH} 6)$ provides a darker appearance upon blooming and a pink cooked internal color. Therefore, dark-cutting (DC) beef can be negatively perceived by consumers, resulting in a discount in carcass value. Novel nitrite-embedded packaging (NEP) has been shown to improve the redness of DC beef. However, the understanding of retail color stability, cooked color, and palatability of enhanced DC steaks stored in NEP and the effects repackaging in polyvinyl chloride (PVC) overwrap on retail color are limited. Therefore, the objectives of this study were to (1) determine the effects of NEP and enhancement on DC beef color, (2) investigate the impact of repackaging in PVC on the redness of DC beef, and (3) evaluate the effect of enhancement and packaging on cooked color and palatability.

Materials and Methods: DC beef strip loins $(n=8 ; \mathrm{pH}$ $6.39)$ and USDA Choice beef strip loins ( $n=6$; pH 5.56) were selected at a commercial packing plant. DC loins were divided into 2 sections and randomly selected as nonenhanced dark-cutting (DCNE) and enhanced dark-cutting (DCE) treatments with $110 \%$ pump of the green weight. From the enhancement, a final concentration of $0.5 \%$ glucono delta-lactone and $0.1 \%$ rosemary was in the loins. Steaks $(1.91 \mathrm{~cm})$ were removed from nonenhanced normal-pH, DCNE, and DCE loins with steaks randomly assigned to 3, 6, or $9 \mathrm{~d}$ in dark storage. Normal-pH and DCNE steaks were vacuum packaged, while DCE steaks were packed in NEP. All steaks were stored in dark storage until 3, 6, or $9 \mathrm{~d}$ and then repackaged in PVC and displayed for $6 \mathrm{~d}$. During dark storage, instrumental color was evaluated every $24 \mathrm{~h}$ using a HunterLab MiniScan Spectrophotometer twice on the surface of the steak, and upon repackaging, instrumental and visual color $(n=6)$ was evaluated every $12 \mathrm{~h}$. For objective 3 , steaks for cooked color 
were placed in dark storage for $72 \mathrm{~h}$ after packaging the DCE steak in NEP and the DCNE and normal-pH steaks in vacuum packaging. A trained sensory panel $(n=6)$ evaluated sensory steaks for beef palatability. The data were analyzed using the Mixed Procedure of SAS (SAS Institute Inc., Cary, NC).

Results: The DCE steaks had an increase $(P<0.05)$ in $a^{*}$ value in $24 \mathrm{~h}$ of dark storage, aligning with an increase $(P<0.05)$ in nitric oxide myoglobin. Upon repackaging DCE steaks, nitric oxide myoglobin decreased $(P<0.05)$ during the first $12 \mathrm{~h}$ of display. The loss of nitric oxide myoglobin corresponded with a darker red appearance, increased surface discoloration, and decreased $a^{*}$ values. The cooked DCE steaks had lower $(P>0.05)$ internal $a^{*}$ values than cooked DCNE steaks, and panelists reported a decrease in internal redness for DCE steaks. However, the external cooked color of DCE had a pinker $(P<0.05)$ appearance than normal-pH and DCNE steaks. The sensory panel determined no difference in tenderness for all treatments; however, DCE steaks had more detectable $(P<0.05)$ sour flavor than DCNE steaks.

Conclusion: In conclusion, DCE steaks packaged in NEP improved surface redness during retail display. However, repackaging DCE steaks in PVC decreased color stability and redness of steaks within $12 \mathrm{~h}$ of the display. The enhancement decreased internal redness upon cooking, and palatability was impacted by the enhancement. The current research suggests that adopting appropriate packing and enhancement technology has the potential to increase the value of DC beef.

Keywords: dark-cutting beef, Freshcase $₫$, meat color, myoglobin, nitrite packaging

\section{6 - FROZEN-THAWED BISON MEAT DISPLAY AND SHELF LIFE}

S. Wirt ${ }^{*}$, T. Bergholz ${ }^{2}$, J. Young ${ }^{1}$, W. Keller ${ }^{1}$, and R. Maddock ${ }^{1},{ }^{1}$ Animal Sciences, North Dakota State University, Fargo, ND, USA, ${ }^{2}$ Microbiological Sciences, North Dakota State University, Fargo, ND, USA, *robert. maddock@ndsu.edu

Objectives: The study objective was to determine the display life of previously frozen and then thawed bison retail ribeye steaks, sirloin steaks, stew meat, and ground bison.

Materials and Methods: All Bison (Bison bison) retail products were received vacuum sealed, frozen, and in boxes from the same source. All whole muscle cuts were packaged and frozen on the same day, and all ground bison was packaged and frozen on the same day. Product was thawed at approximately $4^{\circ} \mathrm{C}$ and then placed on racks in a $4^{\circ} \mathrm{C}$ cooler still in its vacuumed package to simulate retail display under continuous fluorescent lighting. Product was moved on the shelf on average every $6 \mathrm{~d}$. Three packages of each product type were evaluated on days $0,7,14,21,25,32,35,39,42$, and 46 for aerobic plate counts (APC), objective color $\left(L^{*}\right.$, $\left.a^{*}, b^{*}\right)$, and oxidation by thiobarbituric acid reactive substances (TBARS). A $5-\mathrm{cm} \times 5-\mathrm{cm}$ area on each whole muscle cut was swabbed with an EZ-Reach Polyurethane Sponge Sampler saturated in a $10-\mathrm{mL}$ Letheen broth and sent to a commercial lab for APC. A 25-g sample of ground bison was removed from its package, placed in a Whirlpak bag, and sent to the same lab for APC. After a 45-min bloom time, color scores were measured with a Minolta colorimeter using a $\mathrm{D}_{65}$ illuminant in the center of steaks, in the center of the formed patty of ground, and in the center of stew meat formed into one solid sample. Samples for TBARS were ground, and 2-g subsamples were vacuum packaged and frozen at $-80^{\circ} \mathrm{C}$ until analysis by the OxiSelect TBARS Assay Kit (Cell Biolabs STA-330). Data were analyzed using the Mixed Procedure of SAS (SAS Institute Inc., Cary, NC) within each cut with day as the fixed effect and replication as the random effect.

Results: APC for ribeye, sirloin, and stew meat were greater $(P<0.05)$ at day 32 compared with those at earlier time points, with ground bison showing no increases over display days. Ground bison and sirloin had decreased $(P<0.05) L^{*}$ between day 21 and day 32 . The $a^{*}$ value was decreased between day 21 and day 32 for ground bison $(P<0.1)$, sirloin $(P<0.05)$, and stew meat $(P<0.05)$. Ground bison $(P<0.1)$, sirloin $(P<0.1)$, and stew meat $(P<0.15)$ tended to have decreased $b^{*}$ between days 21 and 32. TBARS ( $\mu$ mol malondialdehyde/g protein) trended upwards from day 0 to day 46 in both ribeye and sirloin.

Conclusion: Based on changes in objective color score, microbial growth measured by APC, and oxidation by TBARS, frozen-thawed bison ribeye steaks, sirloin steaks, and stew meat had a display life range of 21 to $32 \mathrm{~d}$, whereas frozen-thawed ground bison had a display life range up to $32 \mathrm{~d}$.

Keywords: bison, lipid oxidation, meat color, microbial growth, shelf life

\section{7 - EFFECTS OF ANGUS SIRE BREEDING METHOD TO PREDOMINATELY ANGUS COWS ON STEER OFFSPRING GROWTH PERFORMANCE AND CARCASS TRAITS}

F. L. Yang ${ }^{1 *}$, K. S. Anschutz ${ }^{1}$, J. G. Powell ${ }^{1}$, J. T. Richeson ${ }^{2}$, and F. W. Pohlman ${ }^{1},{ }^{1}$ Department of Animal Science, University of Arkansas, Fayetteville, AR, USA, ${ }^{2}$ Department of Agricultural Sciences, West Texas A\&M University, Canyon, TX, USA, *flyang@uark.edu

Objectives: The study objective was to determine the effect of breeding method (bull or artificial insemination) on steer offspring performance and carcass traits. 
Materials and Methods: Over 6 y, 244 fall-calving, mixed-aged Angus and Angus-crossbred cows were bred by either bull or artificial insemination with Angus sires. Cattle were housed with access to pasture at the University of Arkansas' beef unit. At birth, cattle were processed and weaned early to middle of May. Steers grazed at the University farm for 2 mo before being transported to West Texas A\&M research feedlot, located in Canyon, Texas, and remained there until harvest. Steers were harvested when backfat thickness reached a minimum of 1.0 $\mathrm{cm}$. Steers were transported to a meat processing plant in Friona, Texas, for harvest. Carcass data were collected at the processing plant for analysis. For analysis and results, steers bred from bulls were referred to as B steers, and steers bred from artificial insemination were referred to as $\mathrm{AI}$ steers.

Results: B steers had greater birth weight $(P<0.05)$ and adjusted weaning weight $(P<0.05)$ than AI steers. The hot carcass weight of AI steers was greater $(P<0.05)$ than $\mathrm{B}$ steers. The ribeye area, yield grade, backfat thickness, and marbling number were not affected $(P<0.05)$ by breeding methods. B steers had a shorter $(P<0.05)$ time to harvest than AI steers.

Conclusion: Breeding method affected growth and carcass performance but not carcass quality.

Keywords: breeding, carcass characteristics, growth performance

\section{Meat and Poultry Quality and Composition - Measurement and Prediction}

\section{9 - SUBSTITUTING CORN FOR BARLEY IN BEEF FINISHER DIETS HAS ONLY MINOR EFFECTS ON BACKFAT FATTY ACID COMPOSITION}

P. Vahmani ${ }^{*}$, J. A. Johnson ${ }^{2}$, B. D. Sutherland ${ }^{2}$, G. B. Penner $^{2}$, N. Prieto ${ }^{3}$, J. L. Aalhus ${ }^{3}$, M. Juárez ${ }^{3}$, O. LópezCampos $^{3}$, and M. E. Dugan ${ }^{4},{ }^{1}$ Department of Animal Science, University of California, Davis, CA, USA, ${ }^{2}$ Department of Animal and Poultry Science, University of Saskatchewan, Saskatoon, Canada, ${ }^{3}$ Agriculture and AgriFood Canada, Lacombe Research and Development Centre, Lacombe, Canada, ${ }^{4}$ Agriculture and Agri-Food Canada, Lacombe Research and Development Centre, Lacombe, Canada,*pvahmani@ucdavis.edu

Objectives: Surveys of beef fatty acid composition in Canada where corn instead of barley are fed have shown instances of higher levels of trans $(t)$ fatty acid isomers associated with unhealthy lipoprotein profiles. The objective of the present research was to examine direct effects of feeding corn, barley, or an equal mixture of corn and barley grain together with either corn or barley silage on the comprehensive fatty acid composition of backfat.

Materials and Methods: Steers weighing $465 \pm 28.0 \mathrm{~kg}$ were assigned to one of 24 pens (12 steers/pen) in a completely randomized design for an 89-d finishing study. Diets were arranged in a $2 \times 3$ factorial with either corn silage or barley silage included at $8 \%$ dry matter (DM). Within silage source, diets contained dry-rolled barley grain ( $86 \%$ of DM), dry-rolled corn grain $(85 \%$ of DM), or an equal blend of barley and corn grain ( $85 \%$ of DM). Cattle were fed to a final body weight of $659 \pm 4.9 \mathrm{~kg}$ and transported to a federally inspected slaughter plant. Bone-in ribeyes were collected from 4 steers per pen, and subcutaneous fat was sampled and subjected to base catalyzed methylation and fatty acid methyl ester analyzed by GC. Fatty acid proportions were subjected to two-way analysis of variance using the MIXED procedure in SAS version 9.2 (SAS Institute Inc., Cary, NC). Pen was the experimental unit, and the statistical model included forage and grain source as main effects, the forage by grain interaction, and pen as a random effect.

Results: Silage source had minimal effects on the fatty acid composition of backfat. Feeding corn grain increased dietary fat and 18:2n-6 contents leading to small increases in 18:2n-6, total n-6 fatty acids, and n-6/n-3 ratios in backfat $(P<0.05)$. There were no changes in proportions of major trans-18:1 isomers (t10-18:1 or t11-18:1), the main natural isomer of conjugated linoleic acid (cis9,t11-18:2), or n-3 fatty acids. Substitution of corn grain for barley grain in finisher diets can therefore be done without substantially altering the fatty acid composition of beef fat, including polyunsaturated fatty acid (PUFA) biohydrogenation products.

Conclusion: The slight increase in the $n-6 / n-3$ PUFA ratio in beef backfat when feeding corn grain will likely have little influence on its nutritional/health value. Substitution of corn grain for barley grain did not lead to increases in total trans 18:1 in backfat and can be included in beef finisher diets without fear of promoting greater risk of cardiovascular disease. Likewise, the type of silage included in diets had very little effect on the fatty acid composition of beef fat. In all likelihood, during surveys when higher levels of unhealthy trans fatty acids have been found, diets were supplemented with ingredients enriched with trans 18:1 precursors (i.e., PUFA), and investigations on limiting their inclusion levels are warranted.

Keywords: beef, backfat, trans fatty acid, vaccenic acid, rumenic acid 


\section{0 - AN INVESTIGATION INTO THE DEVELOPMENT OF ELECTROCHEMICAL SCREEN-PRINTED BIOSENSORS FOR FATTY ACID ANALYSIS IN MEAT}

\author{
A. Smart ${ }^{1 *}$, J. P. Hart ${ }^{1}$, A. Crew ${ }^{1}$, and O. Doran ${ }^{1},{ }^{1}$ Health \\ and Applied Sciences, University of the West of England, \\ Bristol, United Kingdom, *amy.smart@uwe.ac.uk
}

Objectives: The composition of fatty acids (FA) in meat is an important indicator of quality, particularly relative amounts of saturated and unsaturated FAs. Current chromatographic methods of measuring FA on-line are impractical. The novel screen-printed biosensor approach is rapid and simple to use, and producible at low cost on a large scale. Developing biosensor technology for measurement of FA in meat could streamline abattoir processing. The objective of this project is to develop novel screen-printed biosensors for the direct measurement of FA in meat.

Materials and Methods: Mediated screen-printed carbon electrodes were drop-coated with a selected enzyme to measure polyunsaturated FA. Enzyme was immobilized on the electrode surface using a cross-linking agent. Applied voltage was optimized using hydrodynamic voltammetry, and amperometry in stirred solution was used to optimize measurement conditions and perform calibration and precision studies for $\alpha$-linolenic and linoleic acid.

Results: The applied voltage for the operation of the biosensor was optimized. From the position of the plateaus, $+0.5 \mathrm{~V}$ versus $\mathrm{Ag} / \mathrm{AgCl}$ was selected for both $\mathrm{FA}$, which provides maximum sensitivity without excessive voltage (low voltages minimizes potential interferences, improving selectivity). Enzyme loading and concentration of the cross-linking agent was optimized with reference to potential commercial manufacture. The reproducibility of the optimized biosensor was assessed by examining responses of 3 individual biosensors to additions of $\alpha$-linolenic acid. The coefficient of variation was $2.77 \%$, which demonstrates good precision. $\mathrm{pH}$ and temperature were optimized; neutral $\mathrm{pH} 8$ was optimal for both $\mathrm{FA}$, and a linear response was seen at $37^{\circ} \mathrm{C}$ (suitable for abattoir carcasses). Calibration studies at the optimized conditions show a steady state current proportional to concentration of FA over an extended linear concentration range $\left(R^{2}\right.$ values of 0.98 were achieved). This indicates that the biosensor holds promise for the measurement of the selected FA in meat.

Conclusion: This investigation demonstrates that novel amperometric screen-printed biosensors can successfully measure $\alpha$-linolenic and linoleic acid. This technology holds promise for the measurement of polyunsaturated FA present in meat. Future work will evaluate the application of the biosensors to meat samples.

Keywords: biosensor, fat composition, fatty acid, meat quality

\section{1 - IMPLEMENTATION OF THE CANADIAN RETAIL CUT BEEF YIELD GRADES}

J. Segura ${ }^{1}$, J. L. Aalhus ${ }^{1}$, I. L. Larsen ${ }^{1}$, N. Prieto ${ }^{1}$, M. E. Dugan ${ }^{1}$, and O. Lopez-Campos ${ }^{1 *},{ }^{1}$ Lacombe Research and Development Centre, Agriculture and Agri-Food Canada, Lacombe, Canada, *oscar.lopezcampos@canada.ca

Objectives: As a means to facilitate North American crossborder beef trade, Canada is adapting its grading system from a 3-class total lean yield (TLY) to a 5-class retail cut yield (RCY). The relationships and implications of this transition from total lean meat yield to a harmonized RCY provide important historical grading benchmarks. The aim of this study was to interpret the relationships and implications of the transition from TLY to RCY estimations and to adapt the traditional Canadian grading system of 3 TLY classes to a new system of 5 RCY classes.

Materials and Methods: A total of 720 beef carcasses were used first to determine the relationship between the United States Department of Agriculture (USDA) and Canada TLY systems and then develop a modified grade ruler, harmonizing the Canadian grades into 5 classes similar to the USDA RCY grades. Additionally, the developed RCY matrix was validated with a population of 750 carcasses from 3 Canadian beef plants with capabilities for Camera Vision Systems RCY evaluations. The predictive ability (R2, root mean squared error) was evaluated using SAS version 9.4 (SAS Institute Inc., Cary, NC).

Results: Carcass weight $(192.2-536.4 \mathrm{~kg})$, grade fat $(2.0-32.0 \mathrm{~mm})$, ribeye area $\left(52-124 \mathrm{~cm}^{2}\right)$, estimated lean yield (42.5-65.6\%), USDA RCY (45.1\%-55.3\%), and marbling score (220-710) values of the carcass population used in the present study were within the actual range of the Canadian beef carcass market. The prior 3-class TLY grade system in Canada was based on the percentage estimation of the total carcass lean. Harmonization with the USDA RCY resulted in the classification of carcasses into 5 retail yield classes defined by the percentage of closely trimmed (0.5inch fat, or less) and boneless retail cuts from the 4 major primal cuts (chuck, rib, loin, and round) derived from a carcass. The relationship between the Canada TLY and the USDA RCY fit a linear regression model with a considerably high regression coefficient $(\mathrm{R} 2=0.80$; root mean squared error $=0.8472 ; P<0.0001)$. By considering both the regression model and the breakpoints for the 5 classes of the USDA RCY, a matrix of the estimated RCY percentage was developed and implemented into the Canadian grading ruler, characterized by 4 muscle scores and 15 fat classes (Jones et al., 1991) (Figure 1). The validation of the adapted Canadian Grade Ruler, including the RCY grade matrix against the Camera Vision Systems RCY estimations, showed $R^{2}$ values between 0.60 and 0.75 .

Conclusion: Yield grade evaluations using the Grade Ruler are still required in beef plants where camera 

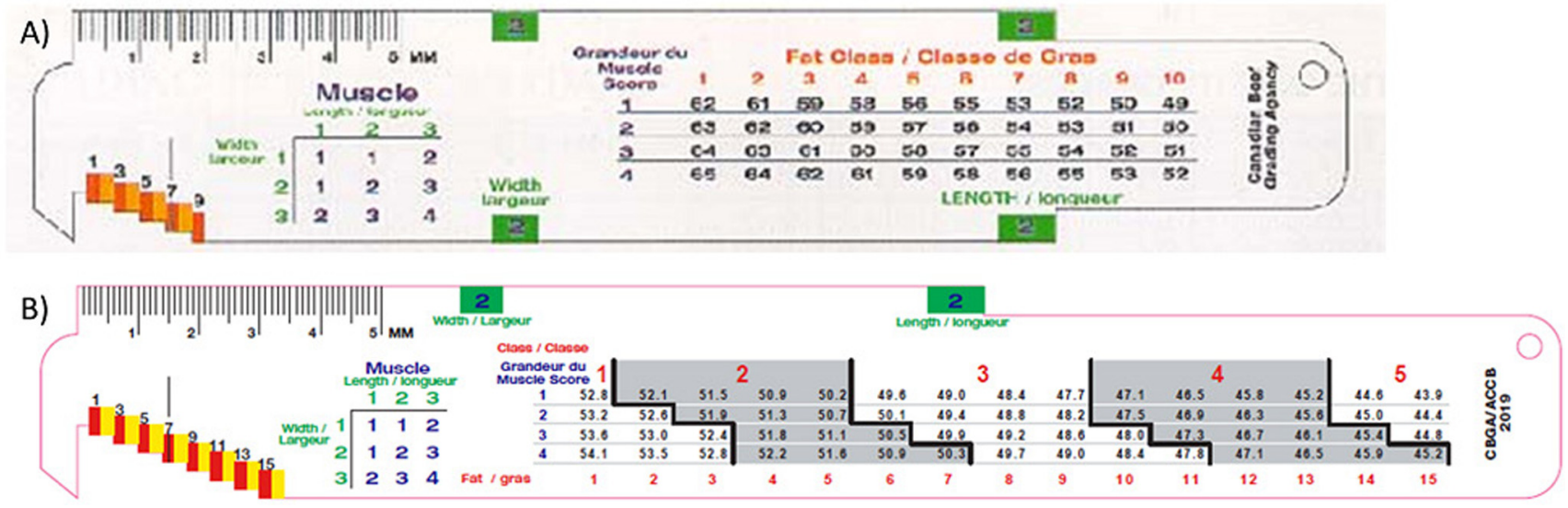

Figure 1. (A) Traditional Canadian grade ruler defined by 3 total lean yield classes and 4 muscle scores and 10 fat classes. (B) Updated Canadian grade ruler defined by 5 retail cut yield classes and 4 muscle scores and 15 fat classes.

technology has not been implemented, or only partially introduced. Regression analyses of the Canada TLY and USDA RCY showed a strong relationship $\left(R^{2}=0.80\right)$ in a research population. A successful adaptation of the Canadian grade ruler to a Canada RCY percentage matrix with 5 yield class breakpoints (Canada 1-Canada 5) was carried out. The adapted Canadian grade ruler is an accurate and reliable grading tool and can be implemented where camera technology is absent to provide harmonization between Canadian and USDA yield estimations, facilitating crossborder beef trading.

Keywords: grade ruler, grading, saleable yield, total lean

\section{2 - EFFECT OF POSTMORTEM AGING AND GROWTH PROMOTANT USE ON M. GLUTEUS MEDIUS COLLAGEN CHARACTERISTICS AND MEAT QUALITY IN CROSSBRED ANGUS STEERS}

\section{P. Coleman ${ }^{1 *}$, B. C. Roy ${ }^{1}$, and H. L. Bruce ${ }^{1},{ }^{1}$ Agricultural, Food and Nutritional Sciences, University of Alberta, Edmonton,Canada,*patience@ualberta.ca}

Objectives: The beef industry aims to enhance production efficiency without compromising beef tenderness. Tenderness is influenced by myofibrillar proteins and surrounding connective tissues, mainly composed of collagen. The influence of collagen has been considered immutable and deemed responsible for the "background toughness" of beef. Our objective was to determine whether its contribution to beef toughness is indeed immutable through investigation of the effects of residual feed intake (RFI), steroids, ractopamine hydrochloride (RH), and postmortem aging on collagen solubility and meat quality of the $m$. gluteus medius (GM).
Materials and Methods: Forty-seven crossbred Angus steers from low (efficient, $n=26)(-0.33 \pm 0.08 \mathrm{~kg} / \mathrm{d})$ and control (inefficient, $n=21)(0.63 \pm 0.09 \mathrm{~kg} / \mathrm{d})$ RFI breeding lines were randomly assigned to one of 3 treatments in a $2 \times$ $2 \times 2$ factorial design within RFI status: control (no implant, no RH) $(n=12)$; implant and RH $(n=11)$; RH only $(n=12)$ and implant only $(n=12)$. Steers' actual RFI performance was confirmed during finishing using GrowSafe ${ }^{\mathrm{TM}}$ technology. Implanted steers received a first implant (200 mg progesterone, $20 \mathrm{mg}$ estradiol benzoate, and $29 \mathrm{mg}$ tylosin tartrate) at $332.21 \pm 2.54 \mathrm{~d}$ of age and $383.19 \pm 2.88 \mathrm{~kg}$ live weight, and a terminal implant $(120 \mathrm{mg}$ trenbolone acetate and $24 \mathrm{mg}$ estradiol) at about $100 \mathrm{~d}$ before slaughter. RH was fed $28 \mathrm{~d}$ before slaughter at $200 \mathrm{mg}$ head ${ }^{-1}$ day $^{-1}$. GM muscles were excised $48 \mathrm{~h}$ postmortem from the right carcass side. Muscles were cut in half and halves randomly assigned to 3 and $12 \mathrm{~d}$ of aging at $4{ }^{\circ} \mathrm{C} \pm 2^{\circ} \mathrm{C}$ balanced within treatment for position within muscle. Warner-Bratzler shear force (WBSF), muscle proximate content, and collagen heat solubility were measured. Data were analyzed using RStudio as a split plot blocked by slaughter group with experimental units of animal in the main plot and muscle half in the split plot. RFI, steroids, RH, aging, and their interactions were fixed variables. Models were considered significant at $P<0.05$. Pearson correlations were performed between meat quality and collagen solubility measurements with significance at $P<0.05$.

Results: Steaks from implanted muscles had a higher mean peak shear force $(37.70 \pm 1.30 \mathrm{~N})$ than nonimplanted steers $(33.38 \pm 1.29 \mathrm{~N})(P=0.0217)$. WBSF was correlated with protein content $(P=0.0131)(r=0.38)$, suggesting that muscles undergoing active protein deposition were tougher. Total collagen increased from day 3 to 12 due to biological variation in the steaks (Table 1), and at day 12 was positively correlated with WBSF $(P=0.0278)(r=0.34)$, indicating that collagen contributed to GM toughness more so after aging. There was an interaction between steroid, $\mathrm{RH}$, and aging on collagen solubility, where steaks from cattle with no growth promotion had the highest increase in solubility 
Table 1. Effect of ageing on collagen characteristics and WBSF

\begin{tabular}{|c|c|c|c|}
\hline \multirow[b]{2}{*}{ Measurement $(\mathrm{n}=46)$} & \multicolumn{2}{|c|}{ Day } & \multirow[b]{2}{*}{$\operatorname{Pr}>F^{3}$} \\
\hline & Day $03^{1}$ & Day $12^{2}$ & \\
\hline $\begin{array}{l}\text { Soluble collagen (mg } \\
\text { collagen/g raw meat) }\end{array}$ & $0.60 \pm 0.17^{\mathrm{a}}$ & $1.09 \pm 0.17^{\mathrm{b}}$ & 0.0208 \\
\hline $\begin{array}{l}\text { Total collagen (mg } \\
\text { coliagen/g raw meat) }\end{array}$ & $2.53 \pm 0.31^{\mathrm{a}}$ & $3.49 \pm 0.31^{\mathrm{b}}$ & 0.0067 \\
\hline $\begin{array}{l}\text { Warner-Bratzler } \\
\text { Shear Force }(N)\end{array}$ & $39.32 \pm 131^{\mathrm{b}}$ & $31.76 \pm 1.31^{\mathrm{a}}$ & 0.0001 \\
\hline
\end{tabular}

$a, b$ Least square means within a row lacking a common letter differ at $\mathrm{P}<0.05$. ${ }^{1}$ Muscles aged for 3 days. ${ }^{2}$ Muscles aged for 12 days. ${ }^{3}$ Probability of the $\mathrm{F}$ test, with significance at $\mathrm{P}<0.05$.

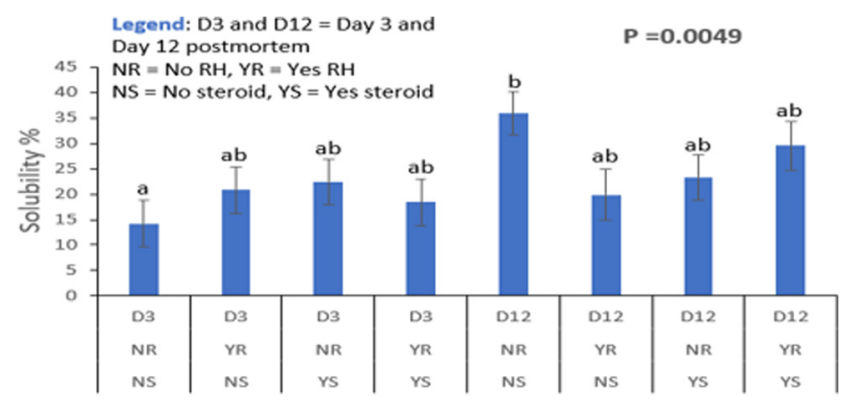

Figure 1. Interaction effect between ageing, RH and steroid on collagen solubility

percentage with postmortem aging $(P=0.0049)$ (Figure 1). RFI status did not affect collagen characteristics $(P>0.05)$.

Conclusion: This study confirmed that steroid use in beef steers increased cooked GM toughness, but that this increase can be resolved with postmortem aging. This study also showed that the effect of collagen on beef toughness is not immutable as collagen solubility can increase with postmortem aging, and that the contribution of collagen to WBSF can become more prominent after aging.

Keywords: beef tenderness, collagen, ractopamine, residual feed intake, steroids

\section{3 - DIMENSIONAL MEASUREMENTS AND RETAIL YIELDS OF INDIVIDUAL MUSCLES AND SUBPRIMALS SOURCED FROM VARIOUS BEEF CARCASS WEIGHT/ RIBEYE SIZE COMBINATIONS}

K. R. Caldwell ${ }^{1}$, M. K. Foster ${ }^{1}$, J. L. Hunt ${ }^{1 *}$, A. N. Arnold ${ }^{1}$, D. B. Griffin ${ }^{1}$, K. B. Gehring ${ }^{1}$, and J. W. Savell ${ }^{1},{ }^{1}$ Animal Science, Texas A\&M University, College Station, TX, USA, *j-savell@tamu.edu

Objectives: The objectives were (1) to determine how various combinations of carcass weights and ribeye sizes impact weight, size, and shape of individual muscles and subprimals, and (2) to calculate primary and total yields of muscles and subprimals when manufactured into case-ready products.

Materials and Methods: Beef carcasses $(n=36)$ were selected from a beef processing plant in Nebraska to be upper 2/3's USDA Choice (Modest and Moderate marbling only) and to meet a $3 \times 3$ treatment scheme of ribeye area (REA) sizes (REA: 83.9 to $89.8 \mathrm{~cm}^{2}, 90.3$ to $96.1 \mathrm{~cm}^{2}$, and 96.7 to $102.6 \mathrm{~cm}^{2}$ ) and hot carcass weights (HCW: 340.6 to $385 \mathrm{~kg}, 386.0$ to $430.9 \mathrm{~kg}$, and 431.4 to $476.3 \mathrm{~kg}$ ). One hindquarter per carcass was identified for individual muscle dissection-M. gluteobiceps, M. gluteus medius, M. longissimus lumborum, M. semitendinosus, and M. semimembranosus - whereas the other was designated for conventional fabrication-strip loin, boneless; top sirloin butt, centercut, cap off, boneless; top sirloin butt, cap (coulotte); top (inside) round, cap off; outside round (flat); and eye of round. Muscles and subprimals were vacuum packaged, boxed, and transported to a case-ready manufacturing facility. At the facility, products were removed from their packaging and weighed; length, width, depth, and circumference measurements were taken. From each, primary cuts (steaks and roasts), secondary items (stew meat and lean trimmings), and fat trimmings and refuse were generated. Weights were taken to calculate primary steak/roast yield and total saleable yield. Analysis of variance was performed to investigate the main effects of REA and HCW along with their interaction.

Results: For dissected muscles, there were increases $(P<0.05)$ in weight with increasing HCW category, but only the M. longissimus lumborum and M. semimembranosus were impacted by REA. Only four dimensional measurements differed significantly across REA categories, whereas there were 15 measurements that differed $(P<0.05)$ across the $\mathrm{HCW}$ categories indicating how HCW influenced the size and shape of these muscles more than REA. For the subprimals, weight increases were similar to those seen for dissected muscles with all becoming heavier $(P<$ $0.05)$ as HCW increased, but only the strip loin and inside round became heavier with increasing REA. Significant dimensional differences occurred 3 times more often ( $31 \mathrm{vs.}$ 10) with $\mathrm{HCW}$ categories compared to REA categories although there were $5 \mathrm{REA} \times \mathrm{HCW}$ interactions. With the exception of one interaction for the $M$. gluteobiceps, neither REA nor HCW impacted primary or total yield for dissected muscles and subprimals. If steak/roast weights were impacted, significant differences were most likely due to $\mathrm{HCW}$ rather than the REA category. Weights for dissected muscles generally increased with increasing REA and $\mathrm{HCW}$; however, when muscle-to-muscle variation was calculated, these ratios were quite similar, indicating that these muscles remained proportional regardless of the REA/ HCW category.

Conclusion: Increasing carcass weights played a greater role than increasing REA in the size, shape, and weights of 
muscles or subprimals. Primary and total saleable yields were unaffected by REA or HCW. Even in carcasses that varied in weight and REA, muscle-to-muscle relationships appear to be similar.

Keywords: beef, carcass weight, composition, retail cutting, ribeye area

\section{4 - RELATIONSHIP OF SUBCUTANEOUS FAT COVERAGE TO INTRAMUSCULAR FAT EXPRESSION DETERMINED USING DIGITAL IMAGE ANALYSIS}

B. B. Grimes ${ }^{1 *}$, T. Kirkpatrick ${ }^{1}$, K. Wesley ${ }^{1}$, S. Pillmore ${ }^{1}$, K. B. Cooper ${ }^{1}$, F. Francis ${ }^{1}$, T. Tennant ${ }^{1}$, W. Nichols ${ }^{2}$, J. Hutcheson $^{2}$, and T. Lawrence ${ }^{1},{ }^{1}$ Agricultural Sciences, West Texas A\&M University, Canyon, TX, USA, ${ }^{2}$ Technical Services, Merck Animal Health, Madison, NJ, USA, *bbgrimes1@buffs.wtamu.edu

Objectives: Evaluation of subcutaneous fat coverage of beef carcasses has been used extensively in the European Union for subjectively categorizing carcasses into marketing groups rather than via evaluation of 12th rib intramuscular fat used in North America. Research that evaluated a 378 day of feed (DOF) duration and trenbolone acetate + estradiol-17 $\beta$ administration provided opportunity to investigate biometric measurements of extremely lean and overfinished beef steers.

Materials and Methods: Charolais $\times$ Angus steers $(n=$ 80; start of trial body weight [BW] [271 $\pm 99 \mathrm{~kg}]$ ) were randomly allocated to implant treatment and harvest date in a $2 \times 10$ factorial design. Steers were paired to minimize variation in genetic group, initial BW, frame score, and adjusted final BW. Within each pair, a steer was randomly allocated to one of 2 treatments; implanted with Revalor-XS on day 0 and day 190 or nonimplanted control (CON). Eight steers comprising 4 pairs were randomly assigned to one of 10 harvest dates at day $0,42,84,126,168,210,252,294,336$, or 378 DOF. Digital images of the lateral aspect of the right side of each carcass was captured $48 \mathrm{~h}$ postmortem, immediately prior to grading. Carcass grading data, evaluated by a human grader, included 12th rib fat thickness, marbling score (MARB), weighed percentage of kidney-pelvic-heart fat (KPH), and calculated yield grade (YG). Pixel analysis software objectively distinguished between fat (white) and lean (red) pixels of the entire carcass side via color thresholding. Percentage carcass surface fat was analyzed using mixed models to test $(\alpha=$ 0.05 ) the fixed effects of DOF and treatment.

Results: No treatment $\times$ DOF effect was exhibited $(P=$ $0.94)$ for percentage carcass surface fat. Additionally, no treatment effect was exhibited $(P=0.87)$ for percentage carcass surface fat. A DOF effect was exhibited $(P<0.01)$ in which percentage carcass surface fat increased by approximately
$0.0895 \% / \mathrm{d}$ in a quadratic manner from $47 \%$ at day 0 to $83 \%$ at day 378 . Pearson correlation coefficients revealed relationships $(P<0.01)$ between percentage of carcass subcutaneous fat coverage $(\% \mathrm{FAT})$ and carcass characteristics used to determine value. Strong positive relationships were observed between \%FAT and MARB $(r=0.74), \%$ FAT and 12 th rib fat thickness $(r=0.76), \%$ FAT and KPH $(r=$ $0.64)$, and \%FAT and YG $(r=0.75)$.

Conclusion: These data suggest that utilizing a color thresholding method to evaluate subcutaneous fat coverage based on the area of lean and fat pixels could be an effective tool to estimate carcass outcomes. These data also suggest that the outer deposition of subcutaneous fat coverage is strongly correlated to deposition of MARB, KPH, and YG of the carcass being evaluated.

Keywords: fat, image analysis, lean, marbling, steers

\section{5 - THE RELATIONSHIP OF CARCASS GRADING FACTORS AND YIELD TRAITS OF HANWOO COW BEEF BY DIFFERENT SLAUGHTER AGES}

H.-W. Seo ${ }^{1^{*}}$ on behalf of Hoa Van $\mathrm{Ba}^{1}$, Pil-Nam Seong ${ }^{1}$, Sun-Moon Kang ${ }^{1}$, Yun-Seok Kim ${ }^{1}$, Jin-Hyoung Kim ${ }^{1}$, K.-H. Seol ${ }^{1}$, S.-S. Moon ${ }^{2}$, and S.-H. Cho ${ }^{1},{ }^{1}$ Animal Product Utilization Division, National Institute of Animal Science, RDA, Wanju-gun, Republic Of Korea, ${ }^{2}$ Meat Research Center, Sunjin, Ansung, Republic Of Korea, *skh0205@naver.com

Objectives: The current study was conducted to determine the relationship between carcass grading traits and effect of slaughter age on carcass of Hanwoo cow beef.

Materials and Methods: A total of 106 Hanwoo cows were slaughtered at the National Institute of Animal Science (Wanju, Korea). The day following slaughter, cow carcasses were randomly selected by official meat graders within 3 levels of maturity (Group $1=$ less than 35 mo of age, $n=20$; Group $2=36-60$ mo of age, $n=36$; Group $3=61-80$ mo of age, $n=29$; Group $4=$ above 81 mo of age, $n=21$ ), and their carcass traits were collected according to the Animal Products Grading Service manual. Carcasses were classified into one of 5 quality grades $\left(1^{++}, 1^{+}, 1,2\right.$, or 3 ) and one of 3 yield grades (A, B, or C). Quality grade was primarily determined by marbling score and additionally adjusted by other carcass traits such as meat color, fat color, texture, and maturity when there was a particular defect in these traits. Yield grade was determined on the basis of estimated retail cut percentage, which was a function of backfat thickness, ribeye area, and cold carcass weight. Data were analyzed using Proc REG and CORR of SAS (SAS Institute Inc., Cary, NC). Simple correlation coefficients of carcass grading traits with the various slaughter ages were carried out using Pearson's correlations. 
Results: Average carcass weights were $364.50 \mathrm{~kg}$ for Group 1, $373.80 \mathrm{~kg}$ for Group 2, $390.80 \mathrm{~kg}$ for Group 3, and $387.10 \mathrm{~kg}$ for Group 4. Increased-maturity carcasses had the darker meat color, lower quality grade, and a higher yield grade $(P<0.05)$. The longissimus area was strongly associated with live weight, carcass weight, yield of retail cut, fat, and bone $(P<0.001)$. Retail cut yield index, yield grade, and meat color score were negatively correlated with live weight, carcass weight, meat, and fat weight. Maturity score was positively correlated with slaughter age $(P<$ $0.001)$. Increased maturity score was linked to older and more live weight and meat weight $(P<0.01)$. Slaughter age showed a negative relationship $(r=-0.318, P<$ $0.001)$ with quality grade. In this study, however, the carcasses with a high marbling score had the greatest fat thickness and a better quality grade.

Conclusion: In the Korean grading system, the quality grade depends on the marbling level of longissimus muscle. However, this study identified that the maturity level affected carcass grading traits of cow carcasses as much as the retail cut yield index, quality grade, yield grade, and meat color score. The older-maturity group showed obvious differences in meat quality compared to other groups.

Keywords: carcass grading, correlation, Hanwoo cow, retail cut

\section{6 - BIOCHEMICAL CHARACTERIZATION OF BEEF TONGUE AS VALUE-ADDED MEAT PRODUCT}

\author{
A. Mohan ${ }^{1 *}$, B. Bowker ${ }^{2}$, S. E. Warren ${ }^{3}$, and R. $\operatorname{Singh}^{3}$, \\ ${ }^{1}$ Food Science and Technology, University of Georgia, \\ Athens, GA, USA, ${ }^{2}$ U.S. National Poultry Research \\ Center, USDA-ARS, Athens, GA, USA, ${ }^{3}$ Food Science and \\ Technology, University of Georgia, Athens, GA, USA, \\ *anandmohan@uga.edu
}

Objectives: This research study was undertaken to evaluate and characterize the physicochemical properties of beef tongue as a meat product. Specifically, objectives were to (1) determine the proximate composition, proteomic profile, and lipid profile of beef tongue; and (2) evaluate the fiber type, connective tissue, and histological composition (muscle structure, fiber size, and fiber type) of beef tongue.

Materials and Methods: Proximate composition including sarcoplasmic and myofibrillar protein fractions $(n=6)$ were determined from the anterior, anteromedial, posteromedial, and posterior regions of a thawed, peeled beef tongue. Protein profiling $(n=3)$ was performed using liquid chromatography-mass spectrometry (MS) analysis on the isolated sarcoplasmic fractions of beef tongue and $L$. dorsi. An LTQ Orbitrap Elite Mass Spectrometer coupled with a Proxeon Easy NanoLC system was used for MS analysis of the samples. MS data were acquired by the data-dependent acquisition method. The MS and MS/MS scans were acquired at resolutions of 120,000 and 30,000, respectively. Fatty acid composition $(n=6)$ was analyzed by derivatizing fatty acid to methyl esters before being analyzed by gas chromatography-flame ionization detection. Adenosine triphosphatase staining was performed to determine the percentage of type I (slow-twitch) and type II (fast-twitch) muscle fibers in beef tongue.

Results: Proximate composition analysis of beef tongue revealed differences in moisture, protein, fat, and ash content along the length of the tongue. The anterior and anteromedial regions exhibited lower $(P<0.05)$ percent fat values than the posteromedial and posterior regions. The protein band patterns resulting from the separations reveal that beef tongue has a protein profile distinct from both $L$. dorsi and heart muscles. The band pattern seen in the myofibrillar fraction of beef tongue more closely resembles that of $L$. dorsi than that of heart. However, beef tongue's sarcoplasmic fraction band pattern is distinct from that of $L$. dorsi. It more closely resembles that of heart than that of $L$. dorsi. Polyunsaturated fatty acids constituted a smaller percentage of fatty acids in all regions of the tongue compared with monounsaturated fatty acids and saturated fatty acids. The sarcoplasmic protein fraction of beef tongue has an abundance of mitochondrial proteins and enzymes involved in oxidative metabolic pathways compared with other skeletal muscles. The majority of beef tongue muscle fibers are type I ( $75 \%$; slow-twitch) oxidative fibers. The surface of the anterior region of beef tongue is the only location where type II (60\%; fast-twitch) glycolytic fibers predominate.

Conclusion: Our findings indicate that the anterior region of beef tongue has higher protein and lower fat content than the posterior. The findings also show that the fat content of the anterior region is richer in polyunsaturated fatty acids and lower in saturated fatty acids than the posterior, and that the dominant fatty acid in beef tongue is the monounsaturated fatty acid oleic acid (C18:1 n-9). Primarily oxidative muscles are abundant in mitochondria and are known for higher tenderness. The findings of this study therefore indicate that beef tongue is also likely to produce a tender meat product.

Keywords: beef tongue, meat quality, fiber type, sarcomere, fatty acids, protein

\section{8 - RELATIONSHIP OF VOLATILE COMPOUNDS AND CONSUMER SENSORY TRAITS FROM NEW ZEALAND GRASS-FED AND AMERICAN GRAIN-FED BEEF STRIP LOINS VARYING IN MARBLING LEVEL AND WET AGING TIME}

T. J. Tilton ${ }^{1 *}$, L. W. Lucherk ${ }^{1}$, T. G. O'Quinn' ${ }^{2}$ J. F. Legako ${ }^{3}$, J. C. Brooks ${ }^{3}$, and M. F. Miller ${ }^{3},{ }^{1}$ Agricultural Sciences, West Texas A\&M University, Canyon, TX, USA, ${ }^{2}$ Animal 
Sciences and Industry, Kansas State University, Manhattan, KS, USA, ${ }^{3}$ Animal and Food Sciences, Texas Tech University,Lubbock, TX, USA, *1lucherk@wtamu.edu

Objectives: Cattle diet, marbling, and wet aging can drastically affect eating quality and consumer preference of beef. Volatile compound analysis has been reported to be related to beef flavor. The objective of this study was to assess the relationship of cooked volatile compounds and consumer sensory traits of New Zealand grass-fed strip loin steaks in comparison to United States grain-fed strip loin steaks from 5 different USDA quality grades, wet-aged for 7, 21, and $42 \mathrm{~d}$.

Materials and Methods: Beef strip loins $(n=200 ; 20$ per USDA quality grade/fed cattle type) representing 5 USDA quality grades (USDA Prime, Top Choice, Low Choice, Select, and Standard) and 2 fed cattle types (New Zealand grass-finished and US grain-finished) were used in the study. Steaks were cooked to a target internal temperature of $71^{\circ} \mathrm{C}$ using a clamshell grill (Cuisinart Griddler Deluxe, East Windsor, $\mathrm{NJ})$. Consumer panelists $(n=600 ; 120$ per 5 different cities in the US) evaluated 8 samples for juiciness, tenderness, flavor liking, and overall liking. Immediately following cooking, the steak for volatile analysis $(n=600)$ was trimmed to remove external fat and connective tissue, cubed, submerged in liquid nitrogen, and homogenized. Five grams of the homogenate and ten microliters of internal standard (1,2-dicholorobenzene; $0.801 \mathrm{mg} / \mathrm{mL}$ ) was used for extraction of volatile compounds. Quantitation was performed by an internal standard calibration with authentic standards. Statistical analyses were conducted using the procedures of SAS (version 9.3; SAS Institute Inc., Cary, NC). PROC CORR was used for calculating and determining significance $(P<0.05)$ of all Pearson correlation coefficients. Principal component analysis (PCA) was conducted on volatile compounds and consumer sensory results using the FACTOR procedure of SAS.

Results: No lipid-derived volatile compounds were significantly correlated to flavor liking $(P>0.05)$; however, there was a positive correlation between dodecanal and overall liking $(r=0.093 ; P<0.05)$. Of the Maillard-derived volatile compounds, the only volatile compounds positively correlated to flavor liking were phenylacetaldehyde $(r=$ 0.119), methyl-pyrazine $(r=0.094), 2,5$-dimethylpyrazine $(r=0.110)$, trimethylpyrazine $(r=0.0 .91)$, and 2-ethyl-3, 5/6-dimethylpyrazine $(r=0.087) \quad(P<0.05)$. Methional was negatively correlated with flavor liking $(r=-0.139$; $P<0.05)$. A PCA showed relationships of volatile compounds, treatments, and consumer ratings. PC1 explained $17.17 \%$ of the variation and PC2 explained $10.96 \%$ of the variation. Consumer flavor liking was most closely associated with ethanol and treatments grass and grain Prime aged $42 \mathrm{~d}$. The majority of the alcohols and n-aldehydes were associated with the 7-d grain treatments including grain Top Choice, Low Choice, and Select. The Maillard-derived compounds were grouped together and most associated with grass Top Choice $42 \mathrm{~d}$ and the consumer attributes. Methional was closest to many grass treatments, including grass Top Choice $7 \mathrm{~d}$, Low Choice $7 \mathrm{~d}$, Standard $7 \mathrm{~d}$, and Prime $7 \mathrm{~d}$ in addition to Standard $21 \mathrm{~d}$.

Conclusion: Although associations were evident between volatile compounds and consumer sensory attributes in the PCA, they were not strongly related in this study.

Keywords: consumer, flavor, grain-fed, grass-fed, volatile compounds

\section{9 - TWO HANDHELD SPECTRAL DEVICES CAN DIFFERENTIATE GRASS- AND GRAIN-FED BEEF}

\author{
R. R. Liddle ${ }^{1}$, C. E. O. Coombs ${ }^{1 *}$, and L. A. González ${ }^{1}$, \\ ${ }^{1}$ Sydney Institute of Agriculture, School of Life and \\ Environmental Sciences, University Of Sydney, Camden, \\ Australia,*cassius.coombs@sydney.edu.au
}

Objectives: Near-infrared reflectance (NIRS) and Raman spectroscopies are frequently used in the determination of meat quality as they are nondestructive and can be used handheld in real time. Both NIRS and Raman have been used previously to differentiate grass-fed meat from grain-fed meat; however, no study has compared both NIRS and Raman scanning both lean and fat tissue of meat. It is hypothesized that NIRS and Raman can accurately classify commercial beef cuts as grass-fed or grain-fed, and in the future these handheld scanners could be used to prevent food fraud and mislabeling, as they provide certification at a quantifiable level as opposed to trusting in the labelling between producers and processors.

Materials and Methods: A total of 108 beef steaks were purchased, comprising store-labelled grass-fed $(n=54)$ and grain-fed $(n=54)$, with grain-fed beef labelled as 100,150 , and $300 \mathrm{~d}$ on feed. Grass-fed samples were sourced from a highly trustworthy supplier with a minimal and highly monitored supply chain. Scans were made using a smartphoneconnectable NIRS sensor (900-1,600 nm; NIRvascan, Allied Scientific Pro, Gatineau, Canada) and a portable

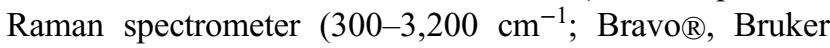
Optics, Billerica, MA) with the objective of obtaining a complete spectral profile of the lean and fat surfaces of each steak. A mean of 18 scans per sample were made for NIRS on lean and 10 on fat, while a mean of 10 scans were made for Raman on lean and 5 on fat. The NIR spectra were inverse $\log$ transformed $(\log (1 / \mathrm{R}))$. Partial least squares discriminant analysis and linear discriminant analysis models were obtained to classify beef samples as grass- or grainfed using RStudio, with $75 \%$ of the samples assigned to the training data and $25 \%$ to the test data. Model fitness was assessed using accuracy, precision, sensitivity, and specificity as metrics, and results are shown for the test data only. 
Table 1. Comparison of NIR and Raman spectroscopy abilities to correctly classify grass- and grain-fed beef using partial least square discriminant analysis (PLSDA) and linear discriminant analysis (LDA). ${ }^{1}$

\begin{tabular}{lllll}
\hline \hline Model & Sensitivity & Specificity & Precision & Accuracy \\
\hline NIR Lean PLSDA & 0.538 & 0.077 & 0.363 & 0.308 \\
NIR Lean PLSDA Max & 0.846 & 0.923 & 0.917 & 0.885 \\
NIR Lean LDA & 0.692 & 0.231 & 0.474 & 0.462 \\
NIR Fat PLSDA & 1.000 & 0.636 & 0.765 & 0.833 \\
NIR Fat PLSDA Max & 1.000 & 0.818 & 0.867 & 0.917 \\
NIR Fat LDA & 0.846 & 0.455 & 0.647 & 0.667 \\
Raman Lean PLSDA & 0.615 & 0.154 & 0.421 & 0.385 \\
Raman Lean PLSDA Max & 0.538 & 0.923 & 0.875 & 0.731 \\
Raman Lean LDA & 0.615 & 0.231 & 0.444 & 0.423 \\
Raman Fat PLSDA & 1.000 & 0.000 & 0.542 & 0.542 \\
Raman Fat PLSDA Max & 0.923 & 1.000 & 1.000 & 0.539 \\
Raman Fat LDA & 0.923 & 0.000 & 0.522 & 0.500 \\
\hline
\end{tabular}

${ }^{1}$ Results shown for the testing or evaluation dataset only.

Results: When determining correct classification of grass- or grain-fed beef, NIRS was shown to be more accurate and precise than Raman (Table 1). The best model for both lean and fat was a partial least squares discriminant analysis from NIRS $(88.5 \%$ and $91.7 \%$ accuracy, respectively), while the linear discriminant analysis model showed negligible difference ( $42.3 \%-46.2 \%$ accuracy).

Conclusion: In conclusion, the small, handheld smartphone NIRS sensor showed promise to discriminate between grass- and grain-fed beef compared to Raman, and its portability and ease of use makes it an attractive technology for industry and commercial use.

Keywords: beef meat, near-infrared spectroscopy, prediction models, Raman

\section{0 - INFLUENCE OF PRODUCTION FACTORS ON BEEF PRIMAL TISSUE COMPOSITION}

V. Sood ${ }^{1 *}$, A. Rodas-González ${ }^{2}$, S. Lam³ ${ }^{3}$, Ó. López Campos $^{3}$, J. Segura ${ }^{3}$, M. E. Dugan ${ }^{3}$, J. Basarab ${ }^{4}$, J. Aalhus ${ }^{3}$, and M. Juárez ${ }^{3},{ }^{1}$ Food and Human Nutritional Sciences, Faculty of Agricultural and Food Sciences, University of Manitoba, Winnipeg, MB, Canada, ${ }^{2}$ Animal Science, University of Manitoba, Winnipeg, MB, Canada, ${ }^{3}$ Lacombe Research and Development Centre, Agriculture and Agri-Food Canada, Lacombe, AB, Canada, ${ }^{4}$ Alberta Agriculture and Forestry, Lacombe Research and Development Centre, Lacombe, AB, Canada,*soodv@ myumanitoba.ca

Objectives: Primal composition, specifically lean meat yield, is an economically important trait that drives the final cattle payment in the beef industry. Production factors may have beneficial or costly influences on the primal composition of the carcass. Understanding the impact of certain production factors on primal composition is important to beef cattle producers in order to optimize proportions of lean, fat, and bone for improved profitability. As the study of primal cut weights has been well studied, improved understanding of factors influencing primal tissue composition is needed. Therefore, the objective of this study was to understand the influence of and relationship between production factors (production system, use of implants, and breed composition) on individual beef primal tissue composition (i.e., lean, fat, and bone).

Materials and Methods: This study used 1,083 crossbred steers, selected from previous projects conducted at the Agriculture and Agri-Food Lacombe Research and Development Centre (LRDC). Data included pedigree, environmental, performance, grading, and phenotypic information related to primal cut composition. All animals were slaughtered and processed at the federally inspected abattoir of the LRDC, and full blue tag Canadian grade data were assessed by a Canadian Beef Grading Agency certified grader, including fat thickness, grade fat, ribeye areas, estimated total lean yield, and marbling. The primal cut data were evaluated using manual dissection of individual primals (i.e., chuck, rib, brisket, flank, fore-shank, loin, round, and plate) from the left carcass sides to measure total lean, fat, and bone content. Data were analyzed using the SAS mixed model procedure (SAS Institute Inc., Cary, NC) using production system (calf-fed vs. yearling-fed), implants (implanted vs. nonimplanted), breed composition (high Angus [>70\%] vs. low Angus $[<70 \%]$ ), and their interactions as the fixed effects, research project as a random effect, and slaughter weight (kilograms) nested within production system as a covariate. Least-squares means were computed and separated using least significant differences when treatment effects were significant at $\alpha \leq 0.05$. The adjusted multiple $R^{2}$ (representing the overall fit of the proposed model or the variability of trait explained for the analyzed factors) was calculated.

Results: High Angus ( $>70 \%$ ) breed resulted in animals with increased marbling and fat content $(P \leq 0.05)$ and decreased yield and lean content $(P \leq 0.05)$, while use of implants increased lean $(P \leq 0.05)$ and decreased marbling and total fat $(P \leq 0.05)$. Additionally, the individual primal as well as total lean and fat content differed significantly for calf-fed versus yearling-fed animals $(P \leq 0.05) . R^{2}$ values for each primal's fat and lean content ranged from 0.26 to 0.42 and from 0.19 to 0.42 , respectively, while $R^{2}$ values for total fat, total lean, marbling, and muscle score were all between 0.32 and 0.38 . For most traits evaluated, the model explained $<50 \%$ of the total variation, indicating that other factors are significantly influencing primal composition.

Conclusion: Production system, use of implants, and breed significantly impacted the lean and fat composition of the animals based on both grading and cutout parameters. 
Calf-fed animals, animals with implants, and with low Angus $(<70 \%)$ breed composition were leaner than their counterparts. Future research will include additional factors, such as genetics to explore phenotypic and genetic correlations and estimate molecular breeding values for individual beef primal tissue composition traits.

Keywords: Angus, calf-fed, implant, primal composition, yearling-fed

\section{1 - EVALUATION OF THE COMPUTER VISION SYSTEM (CVS) TO PREDICT PRIMAL COMPOSITION OF MATURE COWS}

J. Segura ${ }^{1}$, J. L. Aalhus ${ }^{1}$, I. L. Larsen ${ }^{1}$, N. Prieto ${ }^{1}$, M. Juarez ${ }^{1}$, and O. Lopez-Campos ${ }^{1 *},{ }^{1}$ Lacombe Research and Development Centre, Agriculture and Agri-Food Canada, Lacombe, Canada, *oscar.lopezcampos@canada.ca

Objectives: Yield prediction using ribeye or cold carcass cameras (CCC) is not achievable on mature animals. This study was undertaken to determine the potential of the Computer Vision Systems (CVS) for the whole-side or hot carcass camera (HCC) to predict primal composition (fat, lean, and bone) of mature cows.

Materials and Methods: A total of 111 mature cows were slaughtered at the Applied Aerodynamics and Flow Control Lacombe Research and Development Centre federally inspected abattoir. Immediately after slaughter, pictures of each carcass side were taken using an HCC (VBS 2000, $\mathrm{e}+\mathrm{v}{ }^{\circledR}$ Technology GmbH, Germany). Following $72 \mathrm{~h}$ of chilling at $2^{\circ} \mathrm{C}$, carcass sides were weighed and knife-ribbed between 12th and 13th ribs. After 20 min of atmospheric exposure, CCC (VGB $2000 \mathrm{e}+\mathrm{V} \circledast$ ) Technology GmbH, Germany) pictures at the grade site from left and right ribeyes were taken. All images were analyzed using camera software to yield 166 and 99 variables from the HCC and CCC, respectively. Left carcass sides were fabricated into primal cuts, with carcass breakpoints identified following the Institutional Meat Purchase Specifications for Fresh Beef Products: Chuck (\#113), rib (\#103), loin (\#172A), and round (\#158A). Then, all primals were fully dissected into fat, lean, and bone and weighed by qualified personnel. Partial least squares regression procedure was used to predict primal composition using the CVS data as independent variables. The accuracies were assessed by the coefficient of determination $\left(R^{2}\right)$ and root mean square error of crossvalidation.

Results: Overall, proportion of variation in primal composition accounted for by HCC prediction equations was very high across the primal cuts studied (Table 1); most of the lean and fat predictions for the primal cuts showed high coefficients of determination, being $R^{2}$ values for fat $\left(R^{2} \sim 0.85\right.$ on
Table 1. Relationship $\left(R^{2}\right)^{\mathrm{a}}$ and root mean square error of cross-validation between $\mathrm{CVS}^{\mathrm{b}}$ values and the primal composition for lean, fat, and bone of mature cow carcasses.

\begin{tabular}{|c|c|c|c|c|c|c|c|}
\hline \multirow[t]{2}{*}{ Tissue } & \multirow[t]{2}{*}{ Primal } & \multicolumn{2}{|c|}{$\mathrm{HCC}^{\mathrm{c}}$} & \multicolumn{2}{|c|}{$\mathrm{CCC}^{\mathrm{d}}$} & \multicolumn{2}{|c|}{$\mathrm{HCC}+\mathrm{CCC}$} \\
\hline & & $\mathrm{R}^{2}$ & RMSE & $\mathrm{R}^{2}$ & RMSE & $\mathrm{R}^{2}$ & RMSE \\
\hline \multirow{5}{*}{ Fat } & Chuck & 0.88 & 2.3678 & 0.91 & 2.1435 & 0.87 & 2.8515 \\
\hline & Rib & 0.87 & 1.1126 & 0.78 & 2.0276 & 0.86 & 1.3099 \\
\hline & Loin & 0.81 & 2,5542 & 0.91 & 1.3322 & 0.85 & 2.1950 \\
\hline & Round & 0.85 & 0.6854 & 0.72 & 1.3811 & 0.88 & 0.6259 \\
\hline & Overall & 0.92 & 29.407 & 0.93 & 30.104 & 0.91 & 35.532 \\
\hline \multirow{5}{*}{ Lean } & Chuck & 0.85 & 4.6386 & 0.52 & 14.710 & 0.88 & 3.9094 \\
\hline & Rib & 0.66 & 1.2365 & 0.69 & 1.1224 & 0.79 & 0.7751 \\
\hline & Loin & 0.82 & 1.5263 & 0.58 & 3.5920 & 0.82 & 1.5196 \\
\hline & Round & 0.90 & 2.0669 & 0.65 & 7.2982 & 0.86 & 2.9706 \\
\hline & Overall & 0.89 & 36.092 & 0.67 & 104.53 & 0.93 & 23.044 \\
\hline \multirow{5}{*}{ Bone } & Chuck & 0.68 & 0.4167 & 0.38 & 0.8187 & 0.71 & 0.3886 \\
\hline & Rib & 0.36 & 0.1358 & 0.11 & 0.1896 & 0.36 & 0.1369 \\
\hline & Loin & 0.64 & 0.1272 & 0,09 & 0,3185 & 0.76 & 0.0848 \\
\hline & Round & 0.79 & 0.2256 & 0.36 & 0.6723 & 0.75 & 0.2622 \\
\hline & Overall & 0.82 & 2.4731 & 0.31 & 9.2266 & 0.84 & 2.1539 \\
\hline
\end{tabular}

${ }^{\mathrm{a}} \mathrm{R}^{2}$ : Coefficient of determination. ${ }^{\mathrm{b}} \mathrm{CVS}$ : Camera Vision System

${ }^{\mathrm{c}} \mathrm{HCC}$ : Hot/whole-side carcass camera. ${ }^{\mathrm{d}} \mathrm{CCC}$ : Cold/rib-eye carcass camera.

average) slightly higher than those for lean $\left(R^{2} \sim 0.75\right.$ on average). Specifically, HCC predictions showed higher $R^{2}$ values for the fat composition of rib (0.87) and round (0.85) than those observed for $\mathrm{CCC}$ with limited improvement by using data from both grading cameras $(\mathrm{HCC}+\mathrm{CCC})$. In the case of lean, HCC improved the $R^{2}$ values for chuck $\left(R^{2}=0.85\right)$, loin $\left(R^{2}=\right.$ $0.82)$, and round $\left(R^{2}=0.90\right)$ compared to the CCC, but superior prediction was obtained for the rib using the $\mathrm{CCC}\left(R^{2}=\right.$ $0.69)$ and the combined CCC + HCC $\left(R^{2}=0.79\right)$. Overall, observed $R^{2}$ values for predicting bone in the primals were lower $\left(R^{2}=0.36-0.79\right)$ using the HCC than those for lean and fat. CCC alone had limited ability to predict bone $\left(R^{2}=0.09-0.38\right)$, but when combined with the HCC, predictions for bone in the chuck and the loin were slightly improved $\left(R^{2}=0.71\right.$ and 0.76 , respectively). On average, the explained variability with $\mathrm{HCC}$ was $3.32 \%, 24.4 \%$, or $61.8 \%$ higher than that observed with $\mathrm{CCC}$ for fat, lean, or bone, respectively.

Conclusion: Early literature has reported the suitability of CCC to predict total lean or saleable carcass yields. However, the current preliminary results suggest that individual primal cut composition of mature cows can be accurately predicted by CVS using the HCC alone. This is an important finding for slaughter systems, such as those used for mature cattle in Canada, that do not routinely knife rib carcasses which negates the use of CCC.

Keywords: beef primals, cold carcass camera, cull cows, ribeye camera, whole-side camera 


\section{2 - THE RELATIONSHIP OF PORK CARCASS WEIGHT, LEANNESS PARAMETERS, AND IODINE VALUE IN THE ONTARIO COMMERCIAL PORK INDUSTRY}

\author{
Z. Zhou ${ }^{1 *}$ and B. M. Bohrer ${ }^{1},{ }^{1}$ Food Science, University of \\ Guelph, Guelph, Canada,*bbohrer@uoguelph.ca
}

Objectives: This study examined the relationships of pork carcass parameters with carcass weight and iodine value. It was hypothesized (1) that increased carcass weight would cause pork carcasses to become fatter and heavier muscled and to have lower iodine value and (2) that iodine value would decrease as pork carcasses become fatter.

Materials and Methods: Data used in this study were obtained from 37,488 pork carcasses processed in a commercial facility in southwestern Ontario in November 2019. Fat depth, muscle depth, predicted lean yield, carcass weight, and iodine value (level of unsaturation for fat tissue) were evaluated. The dataset was analyzed using 2 unique approaches. Approach \#1 analyzed the data for correlation with individual carcasses as the statistical units. Approach \#2 separated the data into categories based on carcass weight or iodine value before the averages were analyzed for correlation. The categories for carcass weight were determined based on $10-\mathrm{kg}$ increments ranging from very light (70.0 $79.9 \mathrm{~kg})$ to very heavy $(130.0-139.9 \mathrm{~kg})$. The categories for iodine value were defined as low $(<70)$, medium low (70-75), medium high (75-80), and high ( $>80)$. Pearson correlation coefficients generated from SAS (SAS Institute Inc., Cary, NC) with PROC CORR were used for both statistical approaches.

Results: Statistical analysis approach \#1: A significant weak positive correlation was detected between fat depth and hot carcass weight $(r=0.32 ; P<0.001)$, muscle depth and hot carcass weight $(r=0.09 ; P<0.001)$, iodine value and hot carcass weight $(r=0.03 ; P<0.001)$, muscle depth and iodine value $(r=0.07 ; P<0.001)$, and predicted lean yield and iodine value $(r=0.26 ; P<0.001)$. A significant weak negative correlation was detected between predicted lean and hot carcass weight $(r=-0.29 ; P<0.001)$ and fat depth and iodine value $(r=-0.26 ; P<0.001)$.

Statistical analysis approach \#2: Highly predictive regression equations were generated, which was summarized by strong correlations between fat depth and hot carcass weight $(r=0.99 ; P<0.05)$, muscle depth and hot carcass weight $(r=0.81 ; P<0.05)$, and predicted lean and hot carcass weight $(r=-0.99 ; P<0.05)$. The correlation between iodine value and hot carcass weight was moderate but not considered significant $(r=-0.52 ; P>0.05)$. Upon further investigation, iodine value reached a plateau of approximately 71 between the weight range of 80.0-129.9 $\mathrm{kg}$. When carcasses were categorized by iodine value, significant correlation was detected between hot carcass weight and iodine value $(r=0.97 ; P<0.05)$, fat depth and iodine value $(r=-0.99 ; P<0.05)$, muscle depth and iodine value $(r=0.99 ; P<0.05)$, and predicted lean yield and iodine value $(r=0.99 ; P<0.05)$.

Conclusion: It was possible to conclude that categorical analysis provided stronger correlations when compared to uncategorized correlation analysis that used individual carcass as the statistical unit. This highlights the large variation attributed to individual carcass, or pig, in this commercial dataset. It was concluded that when carcasses were categorized based on weights into $10-\mathrm{kg}$ increments, heavier carcasses were fatter and heavier muscled and had a lower predicted percentage lean. Additionally, when carcasses were categorized based on iodine value, carcasses with greater iodine value were heavier weight, less fat, and heavier muscled and had a greater predicted lean yield.

Keywords: carcass composition, iodine value, meat quality, pork

\section{3 - INVESTIGATION OF RAPID EVAPORATIVE IONIZATION MASS SPECTROMETRY (REIMS) TO CHARACTERIZE BEEF BRISKETS}

W. T. Fletcher ${ }^{1}$, B. Mills ${ }^{1}$, A. J. Garmyn ${ }^{1}$, J. F. Legako ${ }^{1}$, D. R. Woerner ${ }^{1}$, J. C. Brooks ${ }^{1}$, and M. F. Miller ${ }^{1},{ }^{1}$ Animal and Food Science, Texas Tech University, Lubbock, TX, USA, *ben.mills@ttu.edu

Objectives: The objective of this study was to evaluate the ability of rapid evaporative ionization mass spectrometry (REIMS) to predict beef brisket composition and eating quality characteristics.

Materials and Methods: Beef briskets from the USDA Prime, Average Choice, and Select quality grades $(n=54$; 18 per treatment) were collected at a commercial abattoir in Omaha, Nebraska. After selection, briskets were vacuum packaged and frozen until use. Briskets were thawed, and raw meat samples were taken from the brisket flat (pectoralis profundi) and point (pectoralis superficialis) for REIMS and compositional analysis. These samples were vacuum packaged, labeled, and frozen. The remaining brisket portions were smoked and served for sensory evaluation. Panelists $(n=360)$ scored each sample for tenderness, juiciness, flavor, and overall liking. Each consumer was served 6 samples representing all quality grade $\times$ muscle combinations. Consumers scored point portions similarly $(P>0.05)$ for all palatability traits $(P>0.05)$ regardless of quality grade. Choice and Select flat portions $(P>0.05)$ were scored similar in tenderness, flavor, and overall liking. Samples were thawed for proximate analysis and ground in a $4.5-\mathrm{mm}$ grinding plate before being analyzed in a FOSS Foodscan (FOSS North America, Eden Prairie, MN). Lipid content was highest in Prime point samples $(P<0.05)$, whereas Prime Flat, Choice point, and Select point samples had 
similar fat percentages $(P<0.05)$. Prime point samples had the lowest moisture percentage $(P<0.05)$ and the highest collagen content $(P<0.05)$. REIMS samples were thawed, and 5 "burns" were collected with an iKnife (Waters Corporation, Milford, MA) sampling tool to create a molecular fingerprint of each sample. Variables with correlation coefficients of greater than 0.75 were identified, and highly colinear variables were removed. Models were built in $\mathrm{R}$ to classify samples by brisket type and sensory attributes. Sensory models were used to assign samples into High, Medium, and Low categories based on all sensory attributes (i.e., tenderness, juiciness, flavor, and overall liking).

Results: When Prime, Choice, and Select, flat and point sections were combined, balanced prediction accuracy reached $98.2 \%$ on REIMS. Tenderness class was predicted with $90.7 \%$ balanced prediction accuracy, and Juiciness class was predicted with $88.9 \%$ balanced prediction accuracy. Additionally, Flavor class was predicted with $85.2 \%$ balanced prediction accuracy, and Overall Liking class was predicted with $91.7 \%$ balanced prediction accuracy.

Conclusion: These data suggest that with fine-tuning REIMS generates a metabolic fingerprint that can meaningfully predict beef brisket palatability with diverse composition.

Keywords: brisket, quality grade, rapid evaporative ionization mass spectrometry

\section{4 - EVALUATION OF FRESH PORK COLOR USING A MINOLTA SPECTROPHOTOMETER, NIX SENSORS, AND SUBJECTIVE SCORING}

X. Wei ${ }^{1 *}$, B. Bohrer ${ }^{2}$, B. Uttaro ${ }^{1}$, O. López-Campos ${ }^{1}, \mathrm{~N}$. Prieto $^{1}$, and M. Juarez ${ }^{1},{ }^{1}$ Lacombe Research and Development Centre, Agriculture and Agri-Food Canada, Lacombe, Canada, ${ }^{2}$ Food Science, University of Guelph, Guelph, Canada, *xwei02@uoguelph.ca

Objectives: The objective of the study was to explore the feasibility of using more affordable and portable Nix sensors (Nix Pro II, Nix QC; Nix Sensor Ltd., Ontario, Canada) to measure pork color in comparison with using the Minolta spectrophotometer (Chroma Meter CM-700D Minolta Canada Inc., Ontario, Canada) with common and different combinations of illuminant and observer angle.

Materials and Methods: Color coordinates from 140 center-loin chops of 5-cm thickness and ham sections were collected in 4 muscles (longissimus, biceps femoris, psoas major, and rectus femoris) using 2 sensors of each Nix model $\left(D_{65}\right.$ illuminant; $10^{\circ}$ observer angle; 14 and $15 \mathrm{~mm}$ aperture for Nix Pro II and Nix QC, respectively) and 2 Minolta spectrophotometers (C illuminant; $2^{\circ}$ observer angle; $8 \mathrm{~mm}$ aperture). Another set of 240 center-loin chops of 5 -cm thickness (120 commercially classified as poor color and 120 as premium color) were used to compare the Nix sensors $\left(\mathrm{D}_{65} ; 10^{\circ}\right)$ and Minolta spectrophotometers $\left(\mathrm{D}_{65} ; 10^{\circ}\right)$. Eight measurements were collected in each muscle. The CIE color space was used with $L^{*}$ (lightness), $a^{*}$ (redness), and $b^{*}$ (yellowness) values. Color of 140 loin and ham samples was also scored using the Japanese subjective color standard. Data were analyzed with PROC CORR and PROC REG of SAS version 9.4 (SAS Institute Inc., Cary, NC) to generate Pearson correlation coefficients and coefficients of determination.

Results: Typical settings for Minolta spectrophotometers in meat quality studies are $\mathrm{C}$ or $\mathrm{D}_{65}$ illuminants and $2^{\circ}$ or $10^{\circ}$ observer angle. Nix sensors do not offer the illuminant $\mathrm{C}$ option, so the $\mathrm{D}_{65}$ illuminant and $10^{\circ}$ observer angle would be the typical setting in meat quality studies. When the $\mathrm{C}$ illuminant and $2^{\circ}$ observer angle setting was used for the Minolta and the $\mathrm{D}_{65}$ illuminant and $10^{\circ}$ observer angle was used for the Nix sensors, a correlation coefficient of 0.7 was observed for $L^{*}$ and $b^{*}$ values between the Nix Pro II and QC with the Minolta. For the average $a^{*}$ value, both Nix sensors presented a strong correlation $(|r|>0.8)$ with the Minolta. When using Minolta $L^{*}$ and Minolta $a^{*}$ as dependent variables, regression analysis showed that the Nix Pro II and QC presented a coefficient of determination $\left(R^{2}\right)$ equal to 0.7 . When instrumental measurements were compared to subjective scoring, both $\mathrm{Nix}$ sensors had slightly stronger correlation $(|r|=0.8)$ for $L^{*}$ compared with the correlation $(|r|=0.7)$ between Minolta $L^{*}$ and the subjective standards. Minolta and Nix QC had the same correlation $(|r|=0.7)$ between $a^{*}$ value and the subjective standard, which was lower than the correlation $(|r|=0.8)$ between Nix Pro II $a^{*}$ and the subjective standard. For $b^{*}$ value, Nix Pro II showed the highest correlation $(|r|=0.7)$ with the subjective standard. When both the Minolta and the Nix sensors were used with the $\mathrm{D}_{65}$ illuminant and $10^{\circ}$ observer angle setting, the results showed that Nix sensors had a strong correlation $(|r|>0.8)$ with the Minolta for all coordinates. When using Minolta $a^{*}$ as the dependent variable, regression analysis showed that the Nix Pro II and the Nix QC presented a coefficient of determination $\left(R^{2}\right)$ equal to 0.8 .

Conclusion: The present study showed the potential of using Nix series sensors (Pro II and QC) to assess fresh pork color. Further data analysis should include evaluations of precision and repeatability. The small size and low price of the Nix devices can lead to the development of automated systems for online pork evaluation.

Keywords: Nix, Minolta, pork color, and CIE coordinates

\section{5 - ASSESSING OUTCOMES OF GENETIC SELECTION PANELS TO PREDICT MARBLING IN CROSSBRED BEEF CATTLE}

T. M. Weber ${ }^{1 *}$, B. J. Buseman ${ }^{1}$, J. A. Nasados ${ }^{1}$, J. M. Lancaster $^{1}$, J. B. Van Buren ${ }^{1}$, J. H. Smart ${ }^{1}$, G. K. 
Murdoch $^{1}$, K. Insausti ${ }^{2}$, P. D. Bass ${ }^{1}$, and M. J. Colle ${ }^{1}$, ${ }^{1}$ Animal and Veterinary Science, University of Idaho, Moscow, ID, USA, ${ }^{2}$ Agricultural Engineering School-IS FOOD, Public University of Navarra, Pamplona, Spain, *tmweber@uidaho.edu

Objectives: The objective of this study was to evaluate the effectiveness of genetic panel marbling indexes (Igenity ${ }^{\circledR}$ $[\mathrm{IT}]$ and PredicGEN $\left.{ }^{\mathrm{TM}}[\mathrm{PG}]\right)$ to predict marbling and tenderness of crossbred beef cattle.

Materials and Methods: Steers $(n=23)$ were harvested at the University of Idaho Meat Science Laboratory, and blood samples $(1 \mathrm{~mL})$ were submitted to Neogen ${ }$ (IT) and Zoetis $^{\mathrm{TM}}$ (PG) for genetic panel analysis. Marbling score (MS) and yield grade were determined by University of Idaho research team members using USDA quality and yield grading standards at $24 \mathrm{~h}$ postharvest and $1 \mathrm{~h}$ after carcasses were ribbed between the 12th and 13th ribs. Forty-eight hours postharvest, one boneless strip loin was collected from each carcass. On day 4 postharvest, six $2.54-\mathrm{cm}$-thick steaks were cut from the anterior end of each strip loin and assigned to an aging period of 14 or $21 \mathrm{~d}$ and evaluated using consumer sensory evaluation or Warner-Bratzler shear force (WBSF) analysis. Carcasses were grouped by their genetic panel marbling index scores into Low IT (IT indexes 3-6; $n=16$ ), High IT (IT indexes 7-10; $n=7$ ), Low PG (PG index $<50 ; n=9$ ), or High PG (PG index $\geq 50 ; n=14$ ). Steaks for all analyses were cooked to a target peak internal temperature of $71^{\circ} \mathrm{C}$. For WBSF analysis, six 1.27 -cm cores were removed from each steak parallel to the muscle fiber orientation, taking care to avoid connective tissue and fat. Cores were sheared perpendicular to the muscle fiber orientation, and peak shear force was recorded. One consumer sensory panel was conducted with 92 consumer sensory panelists. Each panelist evaluated 5 different steak samples for their perception of overall acceptability, tenderness, juiciness, and flavor. Samples were assigned to panelists using an incomplete block design. Results were analyzed using the Mixed Model procedure of SAS (SAS Institute Inc., Cary, NC). Significance was determined at $P<0.05$, and trends were determined at $P<0.10$.

Results: Mean MS was observed to be greater in High IT steaks than in Low IT steaks $(P<0.01 ; 496$ and 410, respectively). Additionally, mean MS was observed to be greater in High PG steaks than in Low PG steaks $(P=0.01 ; 458$ and 398 , respectively). A trend was observed for WBSF to be greater in High IT steaks than in Low IT steaks $(P=$ $0.06)$, but no difference in WBSF was observed between PG marbling groups $(P=0.83)$. Consumers did not report differences between IT marbling groups in terms of acceptability $(P=0.99)$ or tenderness $(P=0.24)$. Additionally, consumers could not detect differences between PG marbling groups in terms of acceptability $(P=0.75)$ or tenderness $(P=0.40)$. Regardless of IT or PG index, consumers consistently preferred Choice steaks over Select steaks in terms of acceptability $(P=0.02)$ and tenderness $(P=0.02)$.
Conclusion: In conclusion, based on these results, commercially available genetic tests could be a valuable tool for producers to be able to predict marbling by retaining ownership of feedlot steers with high genetic panel indexes. At times when the Choice-Select spread is high (\$20), genetic panels could be cost-effective for commercial producers to use at the feedlot level to make decisions about retaining ownership or for feedlot managers to make feeding, implant, and marketing decisions.

Keywords: beef quality, genetic panels, Igenity $®$, marbling, PredicGEN $^{\mathrm{TM}}$

\section{6 - POTENTIAL OF VISIBLE AND NEAR INFRARED SPECTROSCOPY TO AUTHENTICATE BARLEY-FINISHED BEEF}

W. Barragan ${ }^{1,2,3}$, J. L. Aalhus ${ }^{1}$, G. B. Penner ${ }^{4}$, M. E. Dugan ${ }^{1}$, M. Juarez ${ }^{1}$, O. Lopez-Campos ${ }^{1}$, P. Vahmani ${ }^{5}$, J. Segura ${ }^{1}$, J. Angulo $^{3}$, and N. Prieto ${ }^{1 *},{ }^{1}$ Lacombe Research and Development Centre, Agriculture and Agri-Food Canada, Lacombe, Canada, ${ }^{2}$ Faculty of Agricultural Sciences, University of Antioquia, Medellin, Colombia, ${ }^{3}$ Turipaná Research Centre, AGROSAVIA, Cereté, Colombia, ${ }^{4}$ College of Agriculture and Bioresources, University of Saskatchewan, Saskatoon, Canada, ${ }^{5}$ Department of Animal Science, University of California, Davis, CA, USA, *nuria.prietobenavides@canada.ca

Objectives: Barley production is well suited to western Canada and provides feedstuffs (i.e., grain and silage) found in most beef finisher diets. At the same time, development of low heat unit hybrid corn has provided a practical alternative to barley. The ability to discriminate between beef fed different diets creates opportunities for targeted marketing. The objective of this study was to test whether visible and near infrared spectroscopy (vis-NIRS), combined with different discrimination approaches, could be used to rapidly classify beef based on grain source in finishing diets.

Materials and Methods: A total of 85 in-bone ribeyes from barley $(n=29)$, corn $(n=27)$ and blended (50:50 barley and corn; $n=29$ ) grain-fed steers [1] were collected and shipped refrigerated to the Lacombe Research and Development Centre (Agriculture and Agri-Food Canada, Canada). After $15 \mathrm{~d}$ of aging, two $2.5-\mathrm{cm}$ steaks were fabricated from each ribeye for subsequent analyses. Sensory descriptive evaluation (9-member trained sensory panel) [2], fatty acids (FA) [3], and proximate analyses [2] were performed on the longissimus thoracis (LT), whereas color (Minolta $L^{*}, a^{*}$, $b^{*}$ ) was measured on both LT and subcutaneous fat. The vis-NIR spectra were collected on subcutaneous fat and

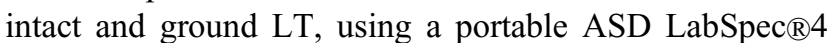
Standard-Res spectrometer from 350 to $2,500 \mathrm{~nm}$. Meat quality, FA, and sensory data were analyzed using the MIXED model procedure of SAS version 9.4 (SAS 
Institute Inc., Cary, NC). Partial least squares discriminant analysis (PLS-DA) and support vector machine in linear (L-SMV) and radial (R-SVM) kernel were applied to the spectra for source of grain-fed discrimination, using the software R-Project (R Development Core Team 2009).

Results: Dietary grain source did not affect $(P>0.05)$ meat quality but influenced $(P<0.05)$ FA profiles and sensory attributes. The LT from barley-fed steers had greater n-3 FA contents and were more tender and juicy compared to LT from corn and blended grain-fed steers. Using vis-NIRS coupled with PLS-DA and L/R-SVM kernel resulted in $<70 \%$ overall accuracy when barley, corn, and blended grain were included in the same analysis for subcutaneous fat, and intact and ground LT. When only barley and corn samples were considered in the analyses, fat samples were correctly classified with $>94 \%$ overall accuracy using PLS-DA and L/ R-SVM. Additionally, for intact LT, PLS-DA and L-SVM successfully ( $\sim 9 \%$ of accuracy) discriminated barley from corn. Spectral differences observed at 420, 540, 570, 1,200, and 1,720-1,760 nm, related to the absorption of FA and meat pigments and carotenoids stored in fat, could have contributed to the successful classification of fat and intact LT samples based on cattle feeding regime. Ground LT samples were classified with $<70 \%$ overall accuracy, probably due to the destruction of muscle integrity during grinding, which entails a loss of relevant information of muscle such as texture and juiciness.

Conclusion: Vis-NIRS measurements on fat and intact loin can be used to accurately discriminate between cornand barley-fed beef, but the ability to discriminate between diets was reduced when a blended grain diet was also considered.

References: [1] Johnson et al. (2019). Translational Animal Science 4(1):129-140. [2] Prieto et al. (2017). Meat and Muscle Biology 1:157-168. [3] Vahmani et al. (2017). Meat Sci. 131:9-17.

Keywords: barley, beef, near infrared spectroscopy, partial least squares discriminant analysis, support vector machine

\section{7 - COLOR MUSE COLORIMETER AS AN ALTERNATIVE METHOD FOR MEASURING COLOR IN MEAT}

D. S. Dang ${ }^{1 *}$, J. F. Buhler ${ }^{1}$, C. D. Stafford ${ }^{1}$, N. E. Keele ${ }^{1}$, A. N. Esco ${ }^{1}$, J. Yang ${ }^{1}$, and S. Matarneh ${ }^{1}$, ${ }^{1}$ Nutrition, Dietetics, and Food Science, Utah State University, Logan, UT, USA, *ds.dang24@gmail.com

Objectives: Among all the visual qualities of meat, color is often described as the most important factor influencing meat purchasing decisions. Color is used by consumers as the first indicator of product freshness and wholesomeness. Thus, it is important to have the ability to objectively measure color in order to understand a consumer's threshold for acceptance of meat products. However, purchasing a reliable colorimeter may be a challenge for some researchers and processors due to the high cost. Therefore, an alternative product that is cost-effective, accurate, and precise would benefit both researchers and industry workers who are interested in measuring meat color. The Color Muse (Variable, Inc., Chattanooga, TN; illuminant $\mathrm{D}_{65}$, aperture size $4 \mathrm{~mm}$, observer angle $0^{\circ}$ ) colorimeter is a small, cost-effective, handheld device that is coupled with a smartphone and can measure a variety of color space models such as CIE $L^{*} a^{*} b^{*}$, sRGB, and XYZ. However, the use of Color Muse in the food industry has not yet been explored, as it was originally designed for matching color in paint, textiles, flooring, and home décor products. Therefore, the aim of this study was to understand the efficacy of Color Muse in color measurements on various meat products. We hypothesized that Color Muse measurements of CIE $L^{*}, a^{*}, b^{*}$ values would be reproducible and similar to other colorimeters specialized for meat color evaluation.

Materials and Methods: In this study, we compared color measurements between Color Muse and a Minolta Chroma Meter CR-400 (Minolta, Inc., Ramsey, NJ; illuminant $\mathrm{D}_{65}$, aperture size $8 \mathrm{~mm}$, observer angle $2^{\circ}$ ). CIE $L^{*}, a^{*}$, and $b^{*}$ measurements were collected on a variety of retail purchased meats, including chicken breasts, beef sirloin, top round steaks, pork loin chops, and salmon $(n=4$ per sample). In order to evaluate the reproducibility of these measurements, coefficients of variation for each measurement were also compared between instruments. Collected data were analyzed using a Student $t$ test and considered significant at $P \leq 0.05$.

Results: Our results showed no significant differences in $L^{*}$ values for salmon and chicken breasts, while measured $L^{*}$ values for beef sirloin steaks and beef top round steaks were $8.3 \%$ and $14.3 \%$ higher $(P<0.05)$, respectively, when using the CR-400 compared to Color Muse. On the other hand, pork loin chop $L^{*}$ values measured with Color Muse were $7.2 \%$ higher $(P<0.05)$ than CR-400 measurement. $a^{*}$ values measured using Color Muse were 28.5\%, 139\%, 9\%, $25.1 \%$, and $60 \%$ higher $(P<0.05)$ in salmon, chicken breast, beef sirloin, round steaks, and pork loin chops, respectively. Similarly, $b^{*}$ values measured by Color Muse were $14.8 \%$, $104 \%, 20.6 \%$, and $78.1 \%$ higher $(P<0.05)$ in salmon, chicken breasts, beef round steaks, and pork loin chops, respectively, with no difference in sirloin steaks. For both colorimeters, coefficients of variation were $<5 \%$ across all measurements.

Conclusion: Although the Color Muse colorimeter generally measured higher $a^{*}$ and $b^{*}$ values — which may be due to differences in illuminant, aperture size, and observation angle between both instruments - the results indicate high reproducibility. Thus, it suggests that Color Muse could be an alternative colorimeter for researchers and industry workers, especially when comparing meat samples from the same species. 
Keywords: color, color muse, colorimeter

\section{8 - COMPARISON OF WARNER- BRATZLER SHEAR FORCE AND SLICE SHEAR FORCE IN SERIALLY SLAUGHTERED STEER CARCASSES}

K. B. Cooper ${ }^{1}$ on behalf of West Texas A\&M University, T. J. Kirkpatrick on behalf of West Texas A\&M University, K. R. Wesley ${ }^{1}$ on behalf of West Texas A\&M University, S. L. Pillmore ${ }^{1}$ on behalf of West Texas A\&M University, F. L. Francis ${ }^{1}$ on behalf of West Texas A\&M University, T. C. Tennant ${ }^{1}$, W. T. Nichols ${ }^{2}$, J. P. Hutcheson ${ }^{2}$, and T. E. Lawrence ${ }^{1 *},{ }^{1}$ Beef Carcass Research Center, West Texas A\&M University, Canyon, TX, USA,${ }^{2}$ Merck, Merck Animal Health, Madison, NJ, USA, *tlawrence@wtamu.edu

Objectives: Growth promotants are commonly administered to increase rate of weight gain and improve feed efficiency but can increase the incidence of tough beef. The objective of this study was to investigate objective mechanical tenderness of serially harvested steers.

Materials and Methods: Charolais $\times$ Angus steers $(n=$ 80 ) approximately $219 \pm 19 \mathrm{~d}$ of age were randomized to implant treatments (Revalor-XS on day 0 and day 190 or Control [no implant]) and harvest date in a $2 \times 10$ factorial design. Four pairs of steers were randomly allocated to one of 10 harvest dates and harvested in 42-d intervals $(0$, 42, 84, 126, 168, 210, 252, 294, 336, and 378). Samples of the M. longissimus dorsi from the 13th rib section were obtained from the left side of each carcass, aged for $14 \mathrm{~d}$, and frozen at $-29^{\circ} \mathrm{C}$. Frozen samples were cut into 2.54$\mathrm{cm}$-thick steaks for Warner-Bratzler shear force (WBSF) and slice shear force (SSF) measurements, and vacuum packaged. Samples were thawed for $24 \mathrm{~h}$ at $2^{\circ} \mathrm{C}$, initial weight was obtained prior to cooking, and steaks were cooked to an internal temperature of $71^{\circ} \mathrm{C}$. After a 5-min cooling period, cooked weights were recorded; WBSF samples were cooled $24 \mathrm{~h}$ at $2{ }^{\circ} \mathrm{C}$ prior to coring and shearing. SSF samples were taken immediately following attainment of cooked weight. All samples were sheared according to American Meat Science Association protocol with a texture analyzer, and peak shear forces were analyzed. Data were analyzed via Pearson correlation and mixed models.

Results: There were no treatment $x$ days on feed interactions $(P \geq 0.51)$ observed for objective tenderness determined via WBSF or SSF. Peak force values did not differ between Revalor-X (3.65 kg and $15.12 \mathrm{~kg})$ and Control $(3.36 \mathrm{~kg}$ and $14.86 \mathrm{~kg})$ for WBSF $(P=0.10)$ or SSF $(P=$ $0.83)$, respectively. Peak force via either method was not effected by $(P \geq 0.17)$. Moderate correlation was observed between WBSF and SSF $(r=0.41)$. Results from this study indicate that growth promotion had little effect on tenderness.
Conclusion: Results from this study indicate that growth promotion had little effect on tenderness.

Keywords: shear force, Warner-Bratzler shear force

\section{9 - EFFECT OF DIFFERENT AGING METHODS ON THE FORMATION OF AROMA VOLATILES IN BEEF STRIP LOINS}

\begin{abstract}
D. Lee ${ }^{1 *}$, H. J. Lee ${ }^{1}$, M. Kim ${ }^{1}$, J. W. Yoon ${ }^{1}$, and C. Jo ${ }^{1,2}$, ${ }^{1}$ Department of Agricultural Biotechnology, Center for Food and Bioconvergence, and Research Institute of Agriculture and Life Science, Seoul National University, Seoul, Republic Of Korea, ${ }^{2}$ Institute of Green Bio Science and Technology, Seoul National University, Republic of Korea, Pyeongchang, Republic Of Korea,*sptails@snu. ac.kr
\end{abstract}

Objectives: Dry-aged beef is well known for its unique flavor compared to unaged or wet-aged beef. Flavor can be perceived by 5 basic tastes combined with many different aromas. Aroma, especially, is regarded as a critical factor for meat flavor. Therefore, in order to understand the characteristic of dry-aged flavor, the analysis of aroma volatile compounds in dry-aged beef is necessary. In this study, we investigated the changes in aroma compounds of dry-aged beef in comparison to wet-aged beef during $28 \mathrm{~d}$ of aging.

Materials and Methods: Thirty beef strip loins were allocated to dry or wet aging randomly $(n=15$ for each aging method). For wet aging, the samples were vacuum packaged and stored at $4{ }^{\circ} \mathrm{C}$, while dry aging was processed at $4{ }^{\circ} \mathrm{C}$, relative humidity of $75 \%$, and air flow velocity of $2.5 \mathrm{~m} / \mathrm{s}$ without any packaging. Both aging processes continued for $28 \mathrm{~d}$, and the samples were collected on day $0,7,14,21$, and 28 ( $n=3$ for each aging period). Before sampling, the crust of dry-aged beef was trimmed off, and all samples from dryand wet-aged beef with different aging periods were ground. Then, the ground beef was weighed $5 \mathrm{~g}$ in a vial and heated for $10 \mathrm{~min}$ at $80^{\circ} \mathrm{C}$. Volatile compound analysis was conducted using electronic nose (Heracles II, Alpha MOS, Toulouse, France). After the analysis, each peak area was integrated (Alphasoft System, Alpha MOS), and statistical analysis was performed using general linear model (SAS version 9.4; SAS Institute Inc., Cary, NC). Significant differences were determined by Student-Newman-Keuls comparison test at a level of $P<0.05$.

Results: As a result, 38 volatile compounds in dry- and wet-aged beef were identified during $28 \mathrm{~d}$ of aging and were assigned to the following chemical groups: acid, alcohol, aldehyde, ester, furan, hydrocarbon, ketone, N-containing compound, S-containing compound, and others. As shown in Figure 1, it was detected that the overall levels of volatile compounds in dry-aged beef increased, which were much higher than those in wet-aged beef $(P<0.05)$. Diverse volatile changes were observed in beef during dry aging. From 
(a) Dry
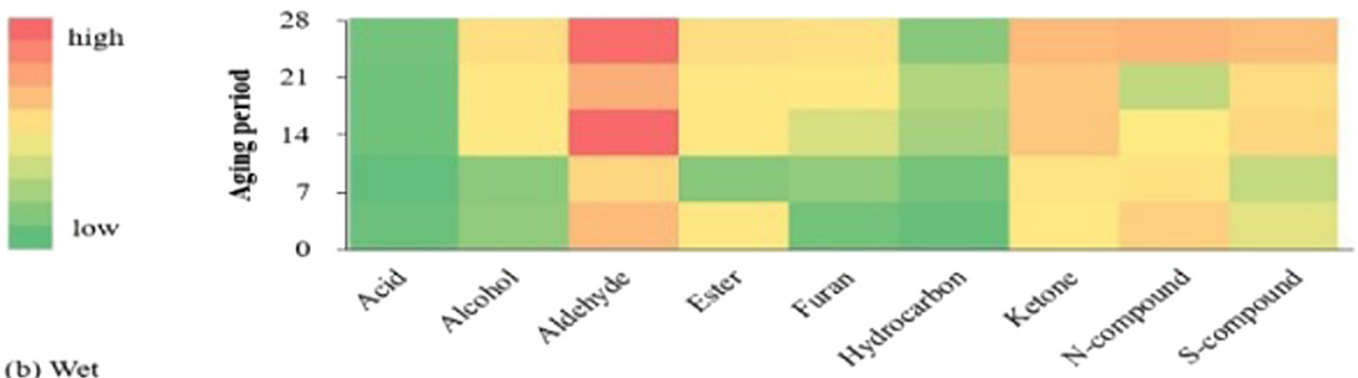

(b) Wet

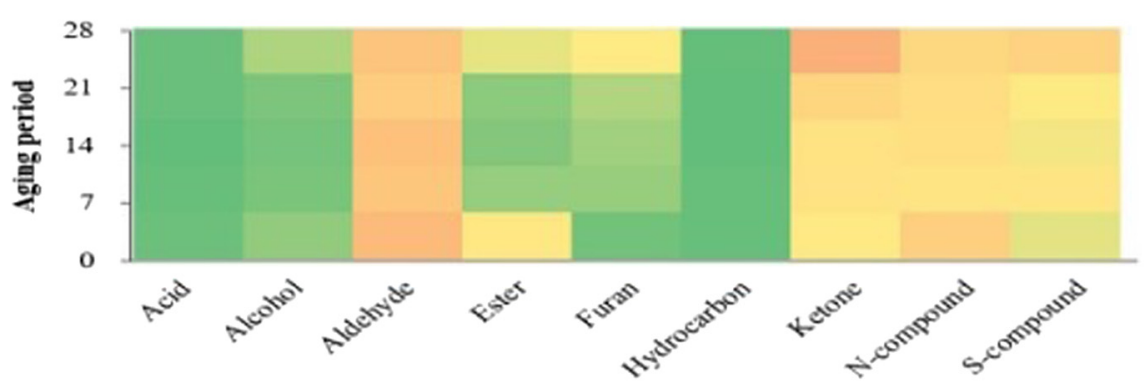

Figure 1. Heat map on the changes of aroma volatiles in (a) dry-aged beef and (b) wet-aged beef during 28 days of aging period.

day 14 , the aroma of dry-aged beef could be differentiated from those of wet-aged or unaged beef. Most changes in the amounts of volatiles of dry-aged beef were associated with aldehydes, $\mathrm{N}$-containing compounds, and S-containing compounds, which are known to be produced by lipid oxidation and/or microbial activity. We previously found an increase in lipid oxidation during dry aging, as well as increases of free fatty acids and free amino acids in dry-aged beef by microbial activity (proteolysis and lipolysis). These findings were consistent with the results from other studies on dry- and wet-aged beef.

Conclusion: Based on the results from previous and our present studies, the formation of volatile compounds in dryaged beef could be attributed to lipid oxidation and microbial activity during aging. Consequently, dry-aged beef had significantly higher amounts of volatile compounds with more distinctive changes than those in wet-aged beef, possibly resulting in the characteristic of dry-aged beef flavor.

Keywords: aging periods, aroma volatiles, dry aging, wet aging

\section{0 - IDENTIFICATION OF DIFFERENTIAL S-NITROSYLATED PROTEINS AND SITES IN DARK-CUTTING BEEF AND NORMAL BEEF USING ISOBARIC IODOTMT SWITCH ASSAY}

\author{
W. Zhang ${ }^{1 *}$, Q. Zhu ${ }^{1}$, and Q. Hou ${ }^{1},{ }^{1}$ College of Food Science \\ and Technology, Nanjing Agricultural University, Nanjing, \\ China, *wangang.zhang@njau.edu.cn
}

Objectives: Dark-cutting beef is a typical representative of abnormal meat that has dark, firm, and dry characteristics. This study aimed to compare the difference of S-nitrosylated proteins between dark-cutting beef and normal beef in the early postmortem stage.

Materials and Methods: Animal and meat samples Simmental-cross cattle with average carcass weight of 341 $\pm 20 \mathrm{~kg}$ and age of $18 \mathrm{mo}$ under the same raising and slaughter conditions were selected from a commercial abattoir (Hengdu Food Co., Ltd., Bi'yang, China). Longissimus thoracis (LT, 12th to 13th rib) were taken from the left side of the carcasses at $24 \mathrm{~h}$ postmortem. Based on the ultimate $\mathrm{pH}$, samples were divided into normal beef group $\left(5.40<\mathrm{pH}_{24} \leq 5.80, n=6\right)$ and dark- cutting beef group $\left(\mathrm{pH}_{24} \geq 6.09, n=6\right)$. Each group was added with 4 vol of lysis buffer ( $1 \%$ sodium dodecyl sulfate, $1 \%$ protease inhibitor cocktail, $50 \mathrm{mM}$ iodoacetamide). The protein solution mixed with 6 time volume of acetone at $-20^{\circ} \mathrm{C}$. The acetone-precipitated protein was redissolved in a solution (50 mM N-2-hydroxyethylpiperazine-N-ethanesulphonicacid, $1 \mathrm{mM}$ EDTA, $0.1 \%$ sodium dodecyl sulfate). The samples were labeled with iodo-tandem mass tags (iodoTMT126-129). An immobilized anti-TMT resin was used to enrich S-nitrosylated proteins.

Statistical analysis: Relative quantification was performed based on the abundance of the reported ion of the tag, and it determined the proteins that were specific through UniProt Bovine (http://www.uniprot.org/). The different sites were screened with 1.2 times as the fold change, and the coefficient of variation was less than 0.1 .

Results: A total of 856 S-nitrosylated sites from 257 proteins were identified in the current study. Among them, 803 sites on 240 proteins contained quantitative information as shown in Table 1 . The number of available effective 
Table 1. Basic statistical values of S-nitrosylated proteins from LC-MS/MS

\begin{tabular}{|c|c|c|c|c|c|c|c|}
\hline $\begin{array}{l}\text { Total spectrogram } \\
\text { number }\end{array}$ & $\begin{array}{l}\text { Matching spectrogram } \\
\text { number }\end{array}$ & Peptides & $\begin{array}{c}\text { Modified } \\
\text { peptides }\end{array}$ & $\begin{array}{l}\text { Identification } \\
\text { proteins }\end{array}$ & $\begin{array}{l}\text { Quantification } \\
\text { proteins }\end{array}$ & $\begin{array}{l}\text { Identification } \\
\text { sites }\end{array}$ & $\begin{array}{c}\text { Quantitative } \\
\text { sites }\end{array}$ \\
\hline 21991 & $4509(20.5 \%)$ & 2118 & 670 & 257 & 240 & 856 & 803 \\
\hline
\end{tabular}

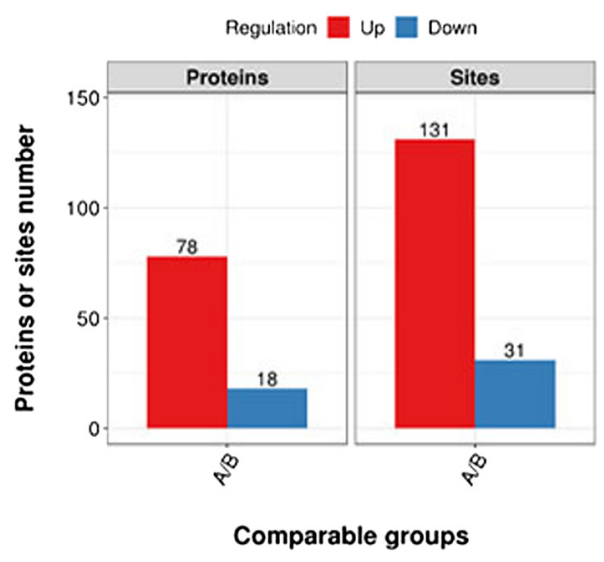

Figure 1. Histogram of the number distribution of differential Snitrosylated expressed proteins and modification sites in different comparison groups. (A represented dark cutting beef samples. B represented normal beef samples.)

spectrograms was 4,509 , and the utilization rate was $20.5 \%$ after the protein theory data database was searched. A total of 2,118 peptides and 670 S-nitrosylated peptides were identified by spectrogram analysis (Table 1). These results imply that a large number of proteins were endogenously S-nitrosylated in beef muscle. In dark-cutting beef samples, there were 131 modification sites upregulated, whereas 31 modification sites were downregulated based on the standard of fold change $>1.2$ and coefficient of variation $<0.1$ (Figure 1). Dark-cutting beef samples showed a higher number of S-nitrosylated proteins compared to normal beef samples, indicating that more cysteines and proteins were available for S-nitrosylation.

Conclusion: This study identified and compared the differential S-nitrosylated proteins between dark-cutting beef and normal beef at $24 \mathrm{~h}$ postmortem. The Longissimus thoracis muscles were labeled with iodoTMT126-129 for the liquid chromatography-tandem mass spectrometry analysis. A total of 856 S-nitrosylated sites from 257 proteins were identified. The S-nitrosylated protein intensity in dark-cutting beef samples was higher compared to that of normal beef samples along with a large number of cysteine sites. Further studies should focus on the effects of protein S-nitrosylation on the variation of beef quality during postmortem aging.

Keywords: dark-cutting beef, iodo-tandem mass tags switch assay, S-nitrosylation

161 - ANTIOXIDANT ACTIVITIES OF THREE DIFFERENT GRAPE POMACES AND SEEDS AND SENSORY ANALYSIS OF GRAPE POMACE PATTY

A. C. Pereira ${ }^{1 *}$, C. J. Wolberg ${ }^{2}$, C. Immoos ${ }^{2}$, H. C. Lee $^{3}$, and I. Kang ${ }^{3},{ }^{1}$ Food Science \& Nutrition, California Polytechnic San Luis Obispo, San Luis Obispo, CA, USA ${ }^{2}$ Chemistry \& Biochemistry, California Polytechnic San Luis Obispo, San Luis Obispo, CA, USA, ${ }^{3}$ Animal Science, California Polytechnic San Luis Obispo, San Luis Obispo, CA, USA, *acpereir@calpoly.edu

Objectives: Conversion of food wastes into value-added products can create a win-win-win situation for food waste reduction, value-added products, and additional income to product growers. The purpose of this research was to evaluate antioxidant activities of seed and seedless pomaces, generation of hamburger patties with grape pomaces, and sensory attributes of the grape patties.

Materials and Methods: Three types of grape pomaces (Syrah, Merlot, and Cabernet Sauvignon) were obtained after red-wine production in the Center for Wine and Viticulture at California Polytechnic State University. The pomaces were divided into 2 portions (grape seed and seedless pomace) by manually separating seeds from the grape pomaces. Grape seeds were dried and pulverized, and seedless pomaces were freeze-dried to measure the relative ability of antioxidants using the ABTS (2,2'-azino-bis (3-ethylbenzothiazoline-6-sulfonic acid) assay. Based on the results of antioxidation, 3 different hamburger patties were prepared using $0 \%$ (control), $2 \%$, and $4 \%$ Merlot grape pomace. The patties were then evaluated for color $\left(L^{*}, a^{*}\right.$, $\left.b^{*}\right)$ using a chromameter, cooking yield using the weight difference before/after cooking, and sensory attributes (flavor, texture, juiciness, color, taste, and overall) using a 50 sensory panel in the 9-point hedonic scale. Data in 3 replications were evaluated by one-way analysis of variance, using the PASW (Predictive Analytics SoftWare) (a pack of statistical analysis tools, formerly known as SPSS Statistics [SPSS Inc., Chicago, IL) 18 statistics program) and a completely randomized design. A post hoc analysis was performed using Duncan's multiple range test to evaluate difference among treatments at $P<0.05$.

Results: Results of the ABTS assay indicated that Merlot seed possessed the highest radical scavenging activity among the 3 types. A similar trend was observed in the seedless pomace although the activity was relatively lower than that of the seed. In color evaluation, pomace patties 
showed lower $L^{*}, a^{*}$, and $b^{*}$ values than the control, regardless of addition amount. In sensory evaluation, lower sensory scores were generally observed in the patties having fermented grape pomaces compared with the control patties, regardless of pomace amount, except the juiciness and color in $2 \%$ pomace and the color in $4 \%$ pomace.

Conclusion: Based on these results, addition of fermented-grape pomace may improve antioxidant activity and nutrition values but resulted in the loss of sensory attributes.

Keywords: grape pomace, antioxidant, phenol, hamburger patty

\section{2 - DEVELOPING AN IN SITU MODEL OF DFD BY INJECTING IODOACETIC ACID INTO PRE-RIGOR BOVINE MUSCLE}

J. F. Buhler ${ }^{1 *}$, D. S. Dang ${ }^{2}$, K. J. Thornton ${ }^{3}$, D. P. Cornforth ${ }^{2}$, and S. K. Matarneh ${ }^{1},{ }^{1}$ Nutrition, Dietetics and Food Sciences, Utah State University, Logan, UT, USA, ${ }^{2}$ Nutrition, Dietetics and Food Sciences, Utah State University, Logan, UT, USA, ${ }^{3}$ Animal, Dairy and Veterinary Sciences, Utah State University, Logan, UT, USA, *jaredbuhlertorreon (a)gmail.com

Objectives: Of all factors impacting meat quality, the rate and extent of postmortem $\mathrm{pH}$ decline are often described as the most important. Dark, firm, and dry (DFD) is a meat defect mainly observed in beef cattle and results from abnormally high ultimate $\mathrm{pH}(\mathrm{pH}>6.0)$. This condition is usually attributed to depletion of muscle glycogen resulting from long-term antemortem stress. DFD meat is characterized as having an abnormally dark color, firm texture, and dry sticky feel, which makes it a major concern for the meat industry worldwide. However, DFD has a low incidence rate in the US, making it difficult for US-based researchers to obtain DFD meat samples. Therefore, the objective of this study was to develop an in situ model for DFD meat to facilitate research in this area. We hypothesized that injection of iodoacetic acid into pre-rigor muscle samples inhibits glycolysis and $\mathrm{pH}$ decline, resulting in meat with similar characteristics to those of DFD.

Materials and Methods: The longissimus thoracis et lumborum muscle $(n=8)$ was excised within $0.5 \mathrm{~h}$ postmortem and fabricated into eight $2.5-\mathrm{cm}$ steaks. Four steaks were injected with Na-iodoacetic acid at a $5 \mu \mathrm{mol} / \mathrm{g}$ of tissue, while the other 4 steaks were injected with water (control). One steak from each treatment was either immediately used ( $0 \mathrm{~h}$ storage) or vacuum packaged and stored at $4{ }^{\circ} \mathrm{C}$ for $24 \mathrm{~h}$, $168 \mathrm{~h}(7 \mathrm{~d})$, and $336 \mathrm{~h}(14 \mathrm{~d})$ postmortem. At the end of each storage period, a portion of each steak was snap-frozen in liquid nitrogen for $\mathrm{pH}$ and metabolite analysis, and the remainder of the steak was analyzed for color, drip loss percentage, cook loss percentage, and texture profile analysis.
Data were analyzed as a $2 \times 4$ factorial arrangement using the mixed model of SAS-JMP (SAS Institute Inc., Cary, NC). Post hoc analysis was performed using a TukeyKramer multiple comparison, and differences were considered significant at $P \leq 0.05$.

Results: At $24 \mathrm{~h}$ postmortem, treated steaks had greater $\mathrm{pH}(P<0.0001)$ at 6.3 compared to 5.6 in control steaks. Treated steaks had lower lightness $(P<0.0001)$ values of 31.7 compared to 39.0 in control steaks. The $\mathrm{pH}$ and color values were similar to those of naturally occurring DFD meat. The elevated ultimate $\mathrm{pH}$ in the treated steaks was combined with lower glycogen degradation $(P=0.005)$ and lactate accumulation $(P<0.0001)$. These differences were maintained over the 336-h storage period. Further, drip loss percentage at $24 \mathrm{~h}$ was lower $(P<0.0001)$ at $1.5 \%$ in treated steaks and $4.5 \%$ in control steaks, and cook loss percentage at 168 and $336 \mathrm{~h}$ postmortem were $22.7 \%$ lower in the treated steaks $(P \leq 0.002)$ compared to controls. Hardness values measured on raw steaks were greater $(P<0.0001)$ in the treated steaks at $80.1 \mathrm{~N}$ compared to $48.1 \mathrm{~N}$ in control streaks.

Conclusion: Injecting pre-rigor muscle samples with $\mathrm{Na}$-iodoacetic acid inhibits $\mathrm{pH}$ decline and results in meat that mimics characteristics of DFD meat. Because of these DFD-like characteristics, this model may allow for DFDrelated research.

Keywords: color; dark, firm, and dry; iodoacetic acid; $\mathrm{pH}$

\section{3 - OPTIMIZATION OF A HIGH- CAPACITY SORPTIVE EXTRACTION (HI-SORB) METHOD COUPLED TO GAS CHROMATROGRAPHY-MASS SPECTROMETRY FOR THE DETERMINATION OF LIPID OXIDATION VOLATILE COMPOUNDS IN BEEF MEAT}

E. Garicano Vilar ${ }^{1 *}$, M. G. O'Sullivan ${ }^{2}$, J. P. Kerry ${ }^{3}$, and K. N. Kilcawley ${ }^{1},{ }^{1}$ Food Quality \& Sensory Science Department, Teagasc Food Research Centre, Moorepark, Fermoy, Ireland, ${ }^{2}$ Sensory Group, University College Cork, Cork, Ireland, ${ }^{3}$ Food Packaging Group, University College Cork, Cork, Ireland, *elena.garicano@gmail.com

Objectives: The main cause of quality deterioration in muscle foods is the oxidation of lipids, a decisive factor in determining the shelf life of food products. Storage changes the composition of volatiles, and the increased or decreased production of some of those volatiles could be used as indicators for lipid oxidation. This work aims to assess the changes in volatile compounds of raw beef patties over time, which could aid shelf life prediction.

Materials and Methods: A method using high-capacity sorptive extraction thermal desorption gas chromatography- 
mass spectrometry was developed and applied to the determination of volatile compounds generated in 21 samples, analyzed in triplicate after $1,3,5,8,10,12$, and $15 \mathrm{~d}$ of storage at $4^{\circ} \mathrm{C}$. Extraction time $(60,120,180 \mathrm{~min})$ and sample amount $(1 \mathrm{~g}, 3 \mathrm{~g})$ were optimized for the analysis of volatiles.

Results: The analysis indicated that time of storage caused some important changes in the volatile profile of the patties. The analysis of $3 \mathrm{~g}$ of sample at $40^{\circ} \mathrm{C}$ for 180 min produced the largest volatile signal $(P<0.001)$. The acids (hexanoic $[P=0.001]$, octanoic $[P<0.001]$ and nonanoic $[P<0.001]$ acids), alcohols (1-hexanol $[P=0.001], 3-$ methyl-1-butanol $[P<0.001]$, and 1-propanol $[P<0.001])$, aldehydes (nonanal, decanal, and furfural (all $P<0.001]$ ), furans (3-methylfuran and 2-pentylfuran [both $P<$ $0.001]$ ), and ketones (2-pentanone $[P<0.001]$ and 2-butanone $[P=0.005])$ were the most representative generated and changeable compounds during storage. The most sensitive predictors were hexanoic acid, 3-methyl-1-butanol, nonanal, 2-furamethanol, and acetol. Meat could be stored at $4^{\circ} \mathrm{C}$ for $10 \mathrm{~d}$ without severe alteration.

Conclusion: This study shows the ability of high-capacity sorptive extraction using polydimethylsiloxane probes, combined with thermal desorption-gas chromatographymass spectrometry analysis, to identify changes in the volatile organic compounds of refrigerated beef meat over time. Further work is required to elucidate and validate volatile compounds as indicators/predictors of lipid oxidation which can be significantly more reliable than thiobarbituric acid reactive substances values or other lipid qualitative oxidation parameters.

Keywords: gas chromatography-mass spectrometry, lipid oxidation, meat, storage, volatile compounds

\section{4 - INFLUENCE OF MINCING SPEED ON THE STRUCTURE OF MINCED MEAT}

\author{
F. Witte ${ }^{1}$, V. Heinz ${ }^{1}$, J. Weiss ${ }^{2}$, N. Terjung ${ }^{1 *}$, ${ }^{1}$ Product \\ Innovation, DIL e. V., Quakenbrück, Germany, ${ }^{2}$ Institute of \\ Food Science and Biotechnology, University of Hohenheim, \\ Hohenheim, Germany, *n.terjung@dil-ev.de
}

Objectives: During minced meat production, muscle cells are destroyed due to a combination of unit operations (comminution, mixing, forming). Therefore, to maintain a highvalue product, the amount of destroyed muscle cells should be as low as possible. In order to prove alternative analyses to time- and resource-consuming histology, minced meat was produced at varying mincer speed and analyzed for, e.g., water-holding capacity (WHC), heat capacity with differential scanning calorimetry (DSC), and overall structure scanning electron microscopy (SEM). The working hypothesis to prove is that a higher energy input leads to more destroyed muscle cells.

Materials and Methods: Pork shoulder was diced $\left(3 \mathrm{~cm}^{3}\right)$, minced to first $12 \mathrm{~mm}$ and then at a knife rotational speed of 100 or $400 \mathrm{rpm}$ to $2.8 \mathrm{~mm}$. Volume flow rate was constant at $25 \mathrm{~L} / \mathrm{min}$. For WHC, 10 or $5 \mathrm{~g}$ was placed on filter paper for $2 \mathrm{~h}$ or $2 \mathrm{~min}$ with $2 \mathrm{~kg}$ for extra- or extra- and intercellular water, respectively $(n=3)$. Cooking loss was calculated after separation of solid and liquid phase after steam heating of $200 \mathrm{~g}$ at $145^{\circ} \mathrm{C}$ for $30 \mathrm{~min}$ in a plastic beaker $(n=3)$. Compression and adhesiveness were measured with texture analyzer with a stamp $(\mathrm{r}=1 \mathrm{~mm})$, driven $1 \mathrm{~mm} / \mathrm{s}$ in and out in samples beaker $(n=6)$. Elasticity was analyzed frequency-dependent with oscillation rheometer at $20^{\circ} \mathrm{C}$ $(n=2)$. Heat flow was measured with DSC from $-40^{\circ} \mathrm{C}$

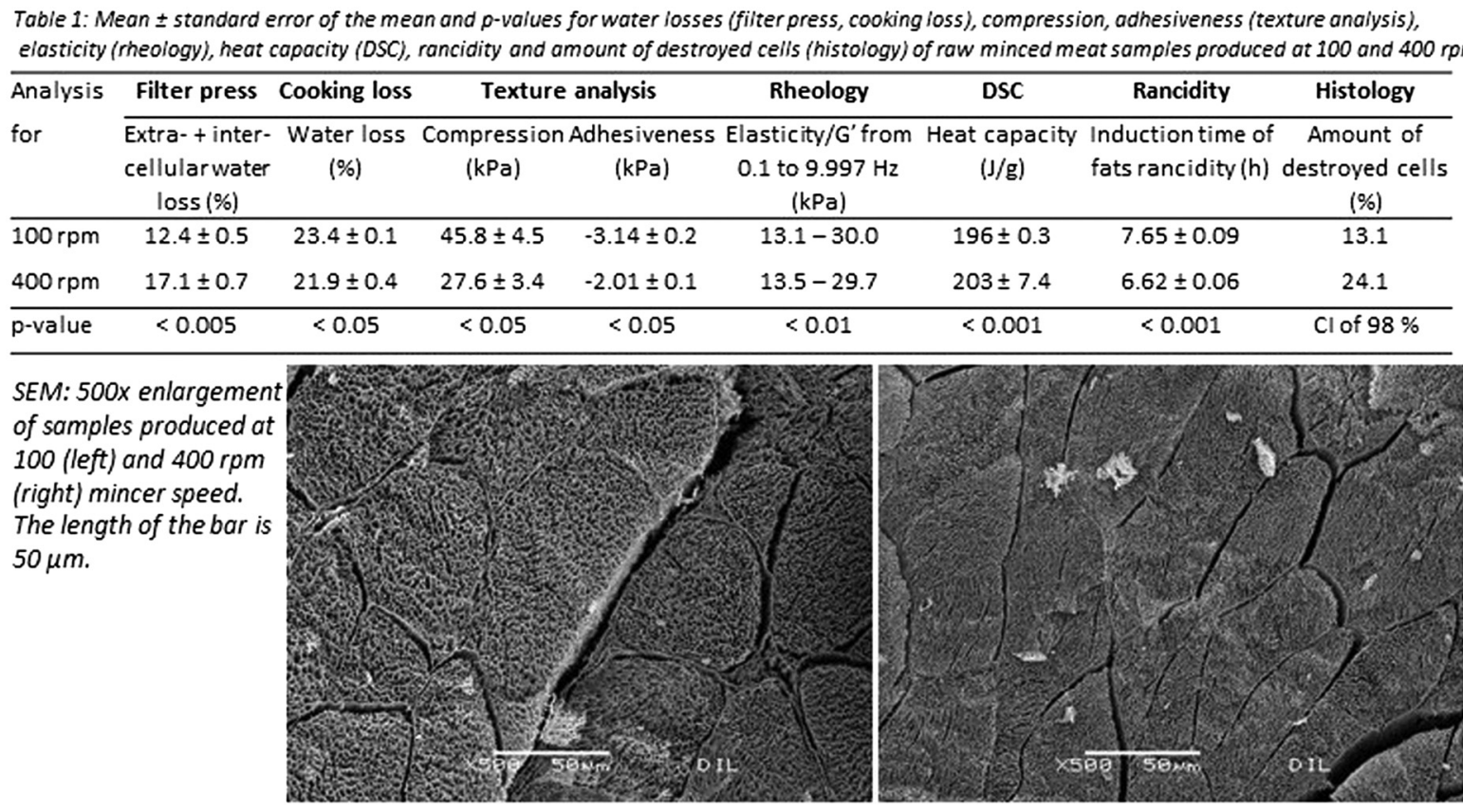


to $+40^{\circ} \mathrm{C}(n=2)$. Rancidity was analyzed from extracted fat (fat extraction with petroleum ether at $\left.40^{\circ} \mathrm{C}\right)(n=3)$. SEM images were produced to illustrate structural differences $(n$ $=1$ ). Histological analysis was conducted based on $\$ 64$ LFGB (Lebensmittel und Futtermittelgesetzbuch) L06.0013 with a calculation of threshold at confidence intervals of $98 \%(n=8)$. Significant differences were determined with paired $t$ test using Sigma Plot 14.0 (Systat Software Inc., San Jose, CA).

Results: Results and $P$ values of analyses (Table 1) show that the higher the energy input, respectively, knife rotational speed, the higher the water loss, the earlier the occurrence of rancidity, the more destroyed the structure, and the more cells are destroyed. SEM images support structural results through a finer network. The WHC of minced meat depends on the state of myofibrillar proteins and their coagulation ability, and moreover, the structural strength depends on the amount of proteins that pass from cellular to continuous phase. Since in our approach, differences can only be caused by the knife rotational speed, the higher water loss, lower cooking loss, compression, adhesiveness, and elasticity, as well as the higher heat capacity in 400-rpm sample, might be due to more coagulated proteins.

Conclusion: Hypotheses have been proven successfully as analyses show high significant differences between samples, as does histology, and a higher energy input destroys more cells. Since especially DSC and rancidity measurement show high significant differences between the samples, these analyses might correlate to histology. Nevertheless, correlations need to be proven by further experiments on a larger scale.

Keywords: histology, differential scanning calorimetry, minced meat, rancidity, scanning electron microscopy

\section{Meat and Poultry Safety}

\section{5 - ANTIMICROBIAL EFFICACY OF CHEMICAL TREATMENTS APPLIED BY IMMERSION OR SPRAYING AGAINST CAMPYLOBACTER JEJUNI INOCULATED ON CHICKEN WINGS}

S. V. Gonzalez Sanchez ${ }^{1 *}$, I. Geornaras ${ }^{1}$, J. O. Reagan ${ }^{2}$, and K. E. Belk ${ }^{1},{ }^{1}$ Animal Science, Colorado State University, Fort Collins, CO, USA, ${ }^{2}$ Strategic Beef Technical Services, Zoetis Inc., Parsippany, NJ, USA, *sara.gonzalez@ colostate.edu

Objectives: Campylobacter is the leading bacterial cause of diarrheal illness in the United States, with an estimated 1.3 million infections each year. Food-related cases of campylobacteriosis are mainly associated with consumption of unintentionally undercooked contaminated poultry products. Therefore, continued research efforts are needed on different strategies to reduce this pathogen on poultry products. The objective of this study was to evaluate the decontamination efficacy of various antimicrobial treatments, when applied by immersion or spray application, to chicken wings inoculated with $C$. jejuni.

Materials and Methods: Skin-on chicken wings were surface-inoculated (3 to $4 \log \mathrm{CFU} / \mathrm{mL}$ of wing rinsate) with a 6-strain mixture of $C$. jejuni of poultry origin. Following inoculation, samples remained untreated (control) or were treated by immersion $(500 \mathrm{~mL}$ solution per wing; $5 \mathrm{~s})$ or spray application (10 to $12 \mathrm{psi} ; 4 \mathrm{~s}$ ) with water, a proprietary blend of sulfuric acid and sodium sulfate (SSS; $\mathrm{pH} 1.2$ ), formic acid (FA; 1.5\%), peroxyacetic acid (PAA; $550 \mathrm{ppm}$ ), PAA (550 ppm) acidified with SSS (pH 1.2), or PAA (550 ppm) acidified with FA (1.5\%). Samples were analyzed for $C$. jejuni counts immediately after treatment application $(0 \mathrm{~h})$ and following $24 \mathrm{~h}$ of refrigerated $\left(4^{\circ} \mathrm{C}\right)$ storage. The study was designed as a 7 (treatments) $\times 2$ (sampling times) factorial for each application method (immersion, spraying), blocked by trial day. The experiment was repeated on 2 separate days, and 3 samples were analyzed per treatment and sampling time in each trial $(n=6$ samples per treatment and sampling time). Data were analyzed using the emmeans package in $\mathrm{R}$ (version 3.5.1). Least-squares means were separated using a significance level of $\alpha=0.05$.

Results: All chemical treatments evaluated in this study were effective $(P<0.05)$ at reducing the initial inoculated (3.9 $\log \mathrm{CFU} / \mathrm{mL}$ ) C. jejuni populations on chicken wings, regardless of treatment application method. Spray application of the chemical treatments resulted in immediate $(0 \mathrm{~h})$ pathogen reductions ranging from 0.5 (SSS) to 1.2 (PAA acidified with FA) $\log \mathrm{CFU} / \mathrm{mL}$, whereas their application by immersion resulted in reductions ranging from 1.7 (SSS) to 2.2 (PAA, and PAA acidified with SSS) $\log$ $\mathrm{CFU} / \mathrm{mL}$. The PAA and acidified PAA treatments were equally $(P \geq 0.05)$ effective at reducing initial $C$. jejuni populations, regardless of treatment application method. However, following refrigerated storage, samples that had been spray- or immersion-treated with SSS- or FA-acidified PAA had lower $(P<0.05)$ pathogen counts than those that had been treated with the nonacidified PAA treatment. Additionally, $C$. jejuni counts of wings that had been immersion-treated with SSS, FA, or one of the acidified PAA treatments, and stored for $24 \mathrm{~h}$, were lower $(P<0.05)$ than those recovered from the corresponding 0 -h samples.

Conclusion: Application of the chemical interventions by immersion resulted in greater immediate reductions of C. jejuni than when applied by spraying. Overall, regardless of treatment application method, wings treated with the acidified solutions of PAA and stored at $4^{\circ} \mathrm{C}$ for $24 \mathrm{~h}$ had the lowest pathogen counts. Findings of this study should be useful to the poultry industry as they consider new interventions against $C$. jejuni on chicken parts. 
Keywords: antimicrobials, Campylobacter jejuni, chicken wings

\section{6 - CONTROL OF E. COLI O157:H7 AND SALMONELLA DURING PRODUCTION OF ETHIOPIAN QWANTA}

G. Allen ${ }^{1 *}$, J. Brown ${ }^{1}$, A. Cavallo ${ }^{1}$, A. Diaz ${ }^{1}$, S. Calderon ${ }^{1}$, B. Woldeyohannes ${ }^{2}$, and J. M. Scheffler ${ }^{1},{ }^{1}$ Animal Sciences, University of Florida, Gainesville, FL, USA, ${ }^{2}$ School of Animal and Range Sciences, Hawassa University, Awassa, Ethiopia, *jmscheff@ufl.edu

Objectives: Animal-sourced foods (ASF), such as meat, can provide nutrients that are beneficial for physical and cognitive development. When access to ASF is limited, and general nutrition is poor, the safety of food is particularly important. Ethiopia has a $37 \%$ incidence rate of stunting in children, and diarrhea is the leading cause of childhood mortality. Improving meat preservation techniques has the potential to improve both availability and safety of ASF. Ethiopian qwanta is a whole muscle dried beef product made utilizing an intricate cutting technique to create elongated, thin strips of meat that are then seasoned and air dried. The objective of this study was to investigate the potential food safety risks associated with consumption of traditional Ethiopian qwanta.

Materials and Methods: Twenty-gram strips were cut from top round ( $<2 \%$ fat), seasoned with a mixture of salt and berbere, an Ethiopian spice from one of 2 sources. Strips were then inoculated with 5 serotypes of Salmonella enterica (Anatum, Dublin, Newport, Saintpaul, Typhimirium) and 3 strains of Escherichia coli O157:H7. Chambers were created to mimic the ambient air-drying process; 2 ventilation rates-2,600 exchanges $/ \mathrm{h}$ and 3,700 exchanges/h-were evaluated to simulate 2 different-sized drying rooms. Chambers were at an ambient temperature of $22.2^{\circ} \mathrm{C} \pm 1.2^{\circ} \mathrm{C}$ with a relative humidity of $60.8 \%-66.9 \%$. Qwanta strips remained suspended in these chambers for 1 to $7 \mathrm{~d}$; samples were plated for enumeration on days $0,1,4$, and 7 post-inoculation and were weighed at the start and finish of the drying period. Microbial results are based on the wet weight of samples.

Results: Aside from water activity (day $\times$ ventilation rate; $P=0.0030$ ), ventilation rate and berbere source showed no detectable interaction $(P>0.32)$ on any parameter. Qwanta lost $(P<0.001) 53.4 \%, 65.4 \%$, and $65.9 \%$ of weight on day 1,4 , and 7 , respectively, compared to day 0 . Water activity decreased faster $(P<0.001)$ at higher ventilation rate on day $1(0.76$ vs. $0.88 \pm 0.015)$ but resolved at a similar $(P=0.69)$ water activity by day $7(0.53$ vs. $0.54 \pm 0.018)$. The drying process resulted in $3.5 \pm 0.11 \mathrm{log} \mathrm{CFU} / \mathrm{g}$ reduction in E. coli $\mathrm{O} 157: \mathrm{H} 7$ and a $1.7 \pm 0.23 \log \mathrm{CFU} / \mathrm{g}$ reduction in $S$. enterica by day $7(<0.0001)$.
Conclusion: Air drying without heat treatment does significantly reduce pathogen load in qwanta, although substantial improvement is still needed. Additional processing interventions should be utilized to further mitigate risks.

Keywords: dehydration, dried beef, international development, risk mitigation, Salmonella reduction

\section{7 - AN EVALUATION OF SODIUM FERRATE AS A GREEN PROCESSING CHEMISTRY}

M. Barnas ${ }^{1 *}$, J. McNaughton ${ }^{1}$, A. J. Punchihewage Don $^{2}$, M. Roberts ${ }^{1}$, and S. Auman ${ }^{1},{ }^{1}$ Food and Agriculture Research, AHPharma, Inc., Hebron, MD, USA, ${ }^{2}$ Food and Agriculture Sciences, University of Maryland Eastern Shore, Princess Anne, MD, USA, *barnas@ahpharma.com

Objectives: Ferrate (VI) is an oxidized state of iron with oxidation-reduction capacity superior to all other commercial chemical oxidizers and disinfectants used in wastewater treatment. Ferrate can be produced from relatively inexpensive commercial chemicals: trivalent ferric chloride $\left(\mathrm{FeCl}_{3}\right)$, sodium hypochlorite $(\mathrm{NaOCl})$, and sodium hydroxide $(\mathrm{NaOH})$; sodium ferrate $\left(\mathrm{Na}_{2} \mathrm{FeO}_{4}\right)$, sodium chloride salt $(\mathrm{NaCl})$, ferric hydrate $\left(\mathrm{Fe}(\mathrm{OH})_{3}\right)$, and water are the odorless reaction products. The objective of this research was to determine the efficacy of sodium ferrate (SF) tested using a commercial electrostatic misting system and boneless, skinless chicken thighs.

Materials and Methods: An inoculum was made to approximately $5 \log$ CFU/mL of Salmonella Typhimurium (resistant to rifampicin) in phosphate buffered saline. Thigh meat was aseptically cut into $100 \mathrm{~g}$ samples then spread on each side with $10^{5} \mathrm{CFU}$ per sample using a sterile cell spreader. Samples were aseptically stored for $45 \mathrm{~min}$ for Salmonella attachment. After $45 \mathrm{~min}$, all samples $(n=32)$ were randomized and assigned to 4 treatments. Samples from 3 treatments passed through an electrostatic misting system treated with: deionized water (DI) positive control, DI + $0.15 \% \mathrm{SF}$, or DI $+0.30 \% \mathrm{SF}$ for $15 \mathrm{~s}$. The fourth treatment received no electrostatic misting. After treatment, samples were stored in sterile bags at $4^{\circ} \mathrm{C}$ for $2 \mathrm{~h}$ then diluted in $100 \mathrm{~mL}$ of buffered peptone water and stomached for $1 \mathrm{~min}$. Next, a $20 \mathrm{~mL}$ aliquot was removed and diluted in $80 \mathrm{~mL}$ of buffered peptone water. Duplicate $10^{-1}$ serial dilutions were made in phosphate buffered saline, then $50 \mu \mathrm{L}$ amounts were automatically spiral plated onto xylose lysine Tergitol 4 agar containing $20 \mu \mathrm{g} / \mathrm{mL}$ rifampicin. Plates were incubated at $37^{\circ} \mathrm{C}$ for $24 \mathrm{~h}$, and then colonies were counted and $\mathrm{CFU} / \mathrm{g}$ calculated.

Results: Both $0.15 \%$ and $0.30 \%$ SF significantly $(P<$ 0.0001 ) reduced Salmonella recovery by 0.65 and 0.89 $\log \mathrm{CFU} / \mathrm{g}$, respectively, as compared to NC. However, 
levels of Salmonella recovered between $0.15 \%$ and $0.30 \%$ treatments were not significantly different $(P=0.4267)$.

Conclusion: Further investigation will elucidate the antimicrobial properties of SF using various application systems in poultry processing, and measure meat quality attributes after treatment with ferrate.

Keywords: antimicrobial, ferrate, green chemistry

\section{8 - CLEAN LABEL SALMONELLA CONTROL IN RAW MINCED MEAT}

\section{E. Heintz ${ }^{1 *}$ and M. Eliasen ${ }^{1},{ }^{1}$ Niacet Corporation, Niagara Falls, NY, USA, *eelco.heintz@niacet.nl}

Objectives: This research demonstrated the possibility to increase safety, by controlling the growth of Salmonella of fresh minced beef using preservatives that meet current food trends, like sodium reduction and natural origin.

Materials and Methods: Ground beef was mixed with $0.25 \%$ and $0.75 \%(\mathrm{w} / \mathrm{w})$ dry vinegar (Provian Neutralized
Dry Vinegar). Thirty-gram portions of ground raw beef (80\% lean, $20 \%$ fat) were inoculated separately with $5-\log _{10} \mathrm{CFU} / \mathrm{g}$ Salmonella (including strains $S$. Enteritidis 6424, S. Enteritidis E40, S. Heidelberg S13, S. Typhimurium S9, and $S$. Typhimurium M-09-0001-A1), by applying a $1 \%$ inoculum into ground product. Inoculated products were sprayed with a test solution (treatments diluted in sterile deionized water) and mixed for $3 \mathrm{~min}$. The product was divided into polyethylene bags $(25 \mathrm{~g} / \mathrm{bag})$ and stored at $10^{\circ} \mathrm{C}$ and $15^{\circ} \mathrm{C}$. Triplicate samples were assayed at $0,1,7,10$, and $14 \mathrm{~d}$ for both temperatures and Salmonella populations enumerated by surface plating on selective agar (Xylose Lysine Deoxycholate agar). The results were statistically analyzed using one-way analysis of variance.

Results: Populations of Salmonella increased $\sim 1.5-\log$ in Control samples stored at $15^{\circ} \mathrm{C}$ for $1 \mathrm{~d}$, and $\sim 1-\log$ after $10 \mathrm{~d}$ of storage at $10^{\circ} \mathrm{C}$. In contrast, no growth $(<0.3 \log$ increase) was detected in any acetate-based treatments stored at $10^{\circ} \mathrm{C}$ or $15^{\circ} \mathrm{C}$ for $14 \mathrm{~d}$. The data from this study revealed that vinegar derivatives prevented Salmonella growth significantly $(P<0.05)$ at abuse temperatures $\left(15^{\circ} \mathrm{C}\right.$ and $\left.10^{\circ} \mathrm{C}\right)$ during prolonged storage $(14 \mathrm{~d})$ compared to the control without antimicrobials.
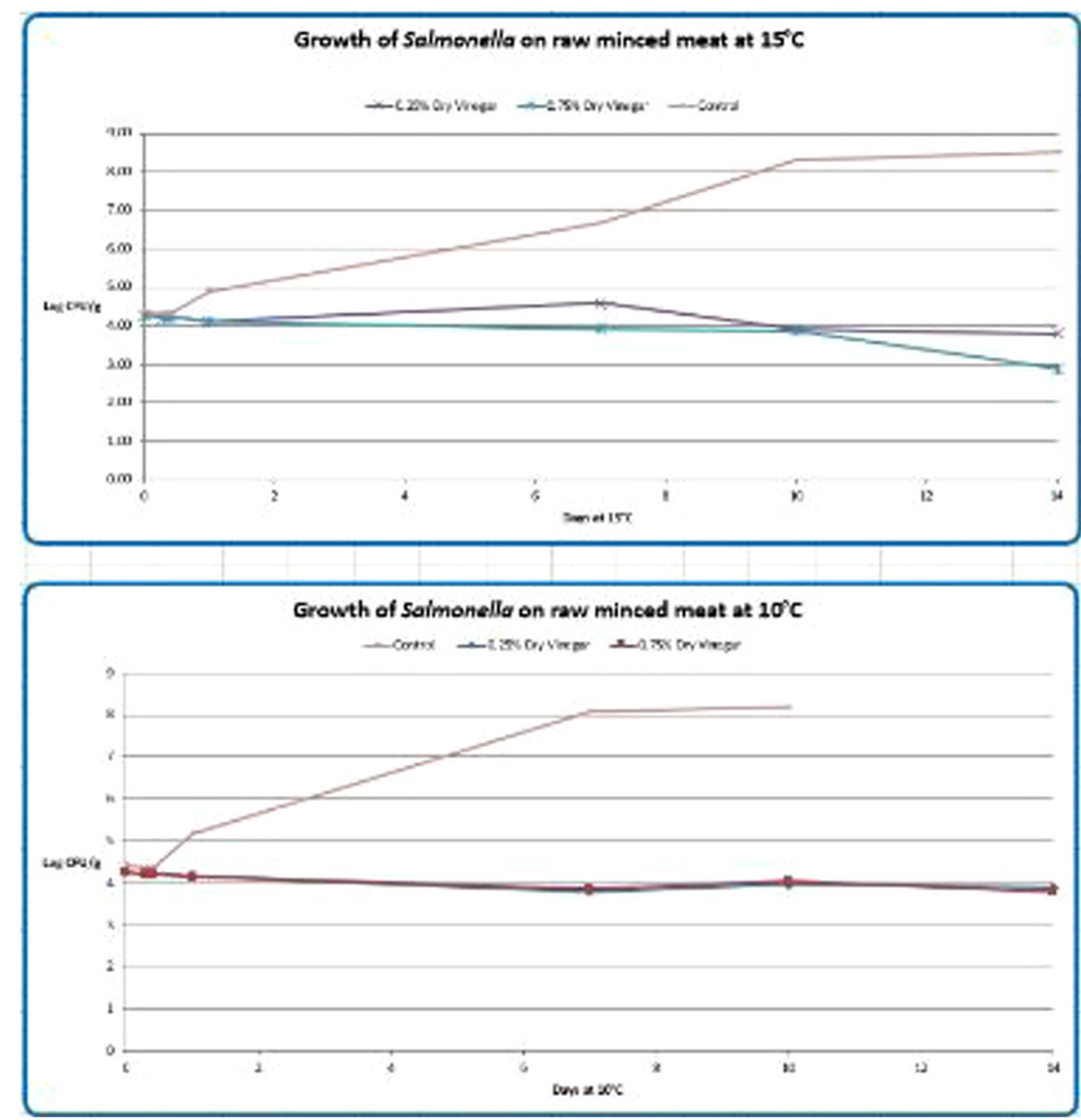
Conclusion: This research demonstrated the possibility to increase safety of fresh minced beef, by controlling the growth of Salmonella, using vinegar-derived preservatives.

Keywords: clean label, food protection, food safety, Provian, Salmonella control

\section{9 - EFFECTS OF SLAUGHTERING METHODS (SKINNING, SCALDING, AND SINGEING) ON GOAT MEAT QUALITY}

\author{
P. O. Fakolade* Osun State Ibadan, OSUN 234101 Nigeria, \\ *patience.fakolade@uniosun.edu.ng
}

Objectives: The type of slaughtering processing methods usually practiced in developing countries, especially Nigeria, could affect meat quality and expose consumers to health hazard, if care is not taken. In Nigeria, the best type of slaughtering is the singeing method, and it is not hygienically practiced.

Materials and Methods: Thirty-six West Africa Dwarf 1-year-old male goats were used, purchased from the teaching and research farm of Osun State University, Nigeria. They were reared for $2 \mathrm{wk}$, fed growers mash, and allowed to graze in the paddock with enough water. Nine animals each were exposed to 4 different slaughtering processing methods: skinning (T1), scalding (T2), singeing with kerosene (T3), and singeing with gasoline (T4). Thigh muscles from each treatment were replicated 5 times to determine their proximate and mineral compositions, palatability scores, polycyclic aromatic hydrocarbons $(\mathrm{PAH})$, and phenol status in a factorial design.

Results: T1 and T2 methods of slaughtering gave the highest significant $(P<0.05)$ water-holding capacity and lowest cooking loss, with $73.95 \%$ and $72.23 \%$ of $\mathrm{T} 1$ and

Table 1. Proximate, mineral, PAH, and phenol composition of goat meat slaughtered using different methods

\begin{tabular}{|c|c|c|c|c|c|}
\hline Parameters & $\mathrm{T} 1$ & $\mathrm{~T} 2$ & T3 & $\mathrm{T} 4$ & SEM \\
\hline Protein \% & $21.16^{\mathrm{a}}$ & $22.41^{\mathrm{a}}$ & $17.78^{\mathrm{b}}$ & $20.42^{\mathrm{ab}}$ & 0.98 \\
\hline Ash \% & $0.98^{\mathrm{b}}$ & $0.99^{\mathrm{b}}$ & $1.06^{\mathrm{a}}$ & $1.04^{\mathrm{a}}$ & 0.23 \\
\hline Either Extract \% & $10.83^{\mathrm{a}}$ & $10.27^{\mathrm{a}}$ & $8.70^{\mathrm{b}}$ & $8.38^{\mathrm{b}}$ & 0.40 \\
\hline Moisture \% & $69.37^{\mathrm{b}}$ & $68.39^{\mathrm{b}}$ & $70.07^{\mathrm{ab}}$ & $73.88^{\mathrm{a}}$ & 1.05 \\
\hline $\mathrm{Fe} \%$ & $0.89^{\mathrm{c}}$ & $0.99^{\mathrm{c}}$ & $1.74^{\mathrm{a}}$ & $1.26^{\mathrm{a}}$ & 0.79 \\
\hline Mg \% & $112.56^{\mathrm{c}}$ & $112.94^{\mathrm{c}}$ & $140.56^{\mathrm{a}}$ & $122.06^{\mathrm{b}}$ & 2.50 \\
\hline P \% & $340.33^{c}$ & $342.56^{\mathrm{c}}$ & $491.89^{\mathrm{a}}$ & $384.55^{\mathrm{b}}$ & 18.52 \\
\hline PAH $(\mu \mathrm{g} / \mathrm{g})$ & $0.0003^{\mathrm{c}}$ & $0.0003^{\mathrm{c}}$ & $0.007^{\mathrm{a}}$ & $0.006^{\mathrm{a}}$ & 0.008 \\
\hline Phenols $(\mu \mathrm{g} / \mathrm{g})$ & $0.012^{\mathrm{b}}$ & $0.013^{\mathrm{b}}$ & $0.139^{\mathrm{a}}$ & $0.129^{\mathrm{ab}}$ & 0.010 \\
\hline
\end{tabular}

${ }^{\mathrm{a}-\mathrm{d}}$ Means of different superscripts along the column are significantly different $(P<0.05)$.

$\mathrm{T} 1=$ Skinning; $\mathrm{T} 2=$ Scalding; $\mathrm{T} 3=$ Singeing with Kerosine; $\mathrm{T} 4=$ Singeing with gasoline.
T2 compared with $59.96 \%$ and $64.58 \%$ of $\mathrm{T} 3$ and T4, respectively. T1 and T2 had the highest significant $(P<0.05)$ value of protein and either extract and mineral evaluation and the lowest values for PAH and phenols. T2 processing methods had the highest palatability score, followed by T1 slaughtering methods.

Conclusion: T2 had reduced PAH, higher protein, higher water-holding capacity, lower cooking loss, and higher acceptability score. Scalding methods (T2) should be encouraged in Nigeria's abattoirs to improve meat quality and consumers' health status, though this method take time to process, except with a scalding machine.

Keywords: skinning, scalding, singeing, polycyclic aromatic hydrocarbons, phenols

\section{0 - MICROBIAL GROWTH AND SHELF LIFE STUDY ON PORK LOINS BY THE APPLICATION OF DIFFERENT ANTIMICROBIALS}

D. A. Vargas ${ }^{1 *}$, M. F. Miller ${ }^{1}$, D. R. Woerner ${ }^{1}$, and A. Echeverry ${ }^{1}$, ${ }^{1}$ Animal Science, Texas Tech University, Lubbock, TX, USA, *david.vargas95@hotmail.com

Objectives: The objective of the study was to determine the impact of antimicrobials on the microbial growth of indicator bacteria on pork loins under dark storage conditions and determine the shelf life of pork chops under display cooler conditions.

Materials and Methods: Fresh boneless loins $(n=36)$, with no more than $48 \mathrm{~h}$ of aging, were split in 5 sections, and each section was treated with any of the antimicrobials (Water, Bovibrom 225 ppm [1,3-Dibromo-5,5-dimethyl hydantoin] [BB225], Bovibrom 500 ppm [BB500], Fit Fresh 3 ppm [Chlorine dioxide] [FF3], or Washing Solution $750 \mathrm{ppm}$ [Rhamnolipid] [WS750]). Sections were packed in boxes and then distributed to any of the 4 dark storage times $(1 \mathrm{~d}, 14 \mathrm{~d}, 28 \mathrm{~d}$, or $42 \mathrm{~d})$ at refrigerated conditions $\left(2^{\circ} \mathrm{C}-4^{\circ} \mathrm{C}\right)$. Then, sections were cut into chops and displayed in a retail case for $96 \mathrm{~h}$ at refrigerated conditions $\left(2^{\circ} \mathrm{C}-4{ }^{\circ} \mathrm{C}\right)$. Mesophilic and psychrotrophic aerobic plate counts (APC$\mathrm{M}$, APC-P), mesophilic lactic acid bacteria, and coliforms counts were performed before and after treatment application, at the end of each dark storage time, and at $0 \mathrm{~h}$, $48 \mathrm{~h}$, and $96 \mathrm{~h}$ of retail case display. Instrumental and visual color analyses were performed every $12 \mathrm{~h}$ during the retail case display time. All bacterial enumeration data were converted into $\log _{10}$ for statistical analysis, and the PROC GLIMMIX procedure of SAS was used to determine differences between least-squares means. All color data were analyzed using the PROC MIXED procedure of SAS to determine differences between least-squares means (version 9.4; SAS Institute Inc., Cary, NC). 
Results: A treatment by sampling point interaction was found for coliforms, APC-M, and APC-P $(P<0.01)$ before and after treatment application. Initial counts (before treatment intervention) did not differ between treatments, whereas after treatment interventions, treatment WS750 did not effectively reduce counts for APC-M, APC-P, and coliforms $(P<0.01)$. BB500, FF3, and WS750 performed better inhibiting the growth of indicator bacteria under 6 logs until $42 \mathrm{~d}$ of dark storage, both for sections and chops. For $a^{*}, b^{*}$, hue angle, and chroma, a treatment by dark storage time interaction was found $(P<0.01)$. Dark storage time progression did not influence any of the analyzed variables for most treatments, except for treatment WS750 in which the storage time progression decreased redness $\left(a^{*}\right)$, increased yellowness $\left(b^{*}\right)$, and promoted discoloration (hue angles) and loss of vividness (chroma). For attribute lean color, fat color, and percentage discoloration, a treatment by dark storage time interaction was found $(P<0.01)$. BB225, BB500 due to its high oxidative capacity, fat color percentage, and the percentage discoloration were the worst at $28 \mathrm{~d}$ of storage when compared with other antimicrobial treatments. FF3 presented the best stability for color during storage. Principal component analysis clustered initial dark storage days with $a^{*}$ and chroma, while percentage discoloration, hue, $b^{*}$, and microorganisms were clustered with longer dark storage times.

Conclusion: In general, treatment FF3 presented the best performance, both in inhibiting microbial growth and maintaining the stability of color, thus increasing the shelf life of pork loins.

Keywords: Bovibrom, indicator bacteria, instrumental color, interventions, shelf life

\section{1 - PREVALENCE OF MICROBIOME IN SOIL AND WATER SAMPLES AROUND ANIMAL UNITS USING $16 S$ rRNA SEQUENCING}

\begin{abstract}
N. Walker ${ }^{*}$, M. A.-A. Sadek ${ }^{1}$, K. Humagain ${ }^{2}$, N. Banjara ${ }^{3}$, and S. Pokharel ${ }^{1},{ }^{1}$ Animal Science, California Polytechnic State University, San Luis Obispo, CA, USA, ${ }^{2}$ Department of Geology, The State University of New York, Potsdam, NY, USA, ${ }^{3}$ Biological and Physical Sciences, University of Holy Cross, New Orleans, LA, USA,*sirojpokharel@ yahoo.com
\end{abstract}

Objectives: Food safety is one of the defining food issues for industry, consumers, and policymakers alike. Animal habitat, water source, and soil harbor microorganisms of food safety importance, including foodborne pathogens: Escherichia coli, Salmonella, Clostridium, Listeria, and Campylobacter. These microbes can cross-contaminate our food system. Therefore, the goal of this project is to use the 16S ribosomal RNA sequencing to examine the prevalence of microbiome in soil and water samples around animal units during the Fall season. The animal units comprise a pig unit, a sheep and goat unit, a dairy farm, a poultry unit, an equine unit, and a cattle ranch around California Polytechnic State University in San Luis Obispo, California.

Materials and Methods: For this study, soil and water samples ( $n=16 /$ Fall - first phase) were aseptically collected around animal units where the collection site (latitude, longitude) was determined using a handheld Global Positioning System. In order to monitor the proper variability of soil and water samples, 3 subsamples were collected from each site (10 to $15 \mathrm{~m}$ apart) and later combined into one. Sterilized plastic spoons and tubes were used to collect the soil and water samples, respectively, approximately $10 \mathrm{~cm}$ below the surface. Samples were immediately placed in a cooler $\left(\leq 4^{\circ} \mathrm{C}\right)$ and transferred to a laboratory for further processing. DNA was extracted from samples using QIAamp PowerFecal $\AA$ DNA kit. For the $16 \mathrm{~S}$ service, $100 \mathrm{ng}$ to $1 \mu \mathrm{g}$ of genomic DNA per sample was preserved in elution buffer at a minimum concentration of $20 \mathrm{ng} / \mu \mathrm{L}$. Metagenomic analysis of the microbial population was carried out using the 16S ribosomal RNA gene. Universal polymerase chain reaction primer was designed to target the conserved regions of 16S. Next-generation sequencing ( $250 \mathrm{bp}$ Paired End, onaverage min. of $\sim 10,000-30,000$ reads per sample) was used to cluster the sequences into operational taxonomic units, alpha and beta diversity, species classification, and abundance analysis.

Results: First-phase results from the Fall season indicated that soil and water samples harbor different microbiome profiles where the phylogenetic relationship of operational taxonomic units was separated by Bray-Curtis distance of more than 0.6. Similarly, the principal component analysis $(P=0.002)$ demonstrates the clustering of the soil and water fecal groups. More than 30 bacterial phyla were detected in soil and water fecal samples. Among them, relative abundance of Proteobacteria, Bacteroidetes, and Firmicutes group was higher in water samples as compared with soil samples. However, Actinobacteria was the most abundant phylum in soil samples. Similarly, relative abundance of Chloroflexi, Planctomycetes, Acidobacteria, and Verrucomicrobia group was higher in soil samples as compared with water samples.

Conclusion: The prevalence of Proteobacteria and Firmicutes in water samples around animal units was high in the Fall season. We are still waiting for the microbial abundance data from other seasons of the year. However, the presence of indicator microorganisms such as coliform, Salmonella, and others will be analyzed on above-mentioned samples using similar sequencing technology. Further analysis will demonstrate whether the prevalence of Proteobacteria and Firmicutes around animal units is a good indicator of foodborne microorganisms. 
Keywords: 16S ribosomal RNA, microbiome, food safety, Gammaproteobacteria, Firmicutes

\section{2 - EFFECT OF UV-C IRRADIATION AND LACTIC ACID APPLICATION ON THE INACTIVATION OF INOCULATED LISTERIA MONOCYTOGENES IN VACUUM- PACKAGED BEEF MEAT}

C. Rufo ${ }^{1}$, G. Brugnini ${ }^{1 *}$, S. Rodríguez-Cortés ${ }^{1}$, and Inocuidad, Alimentos y Nutrición, ${ }^{1}$ Instituto Polo Tecnológico, Facultad de Química, Universidad de la Republica, Montevideo, Uruguay, *caterinarufod@gmail.com

Objectives: The combined effect of lactic acid application and short-wave ultraviolet radiation (UV-C) radiation to reduce Listeria monocytogenes on beef meat cuts was evaluated, using a L. monocytogenes strain isolated from a local abattoir.

Materials and Methods: The effect of different doses of UV-C and lactic acid application on L. monocytogenes survival was analyzed using a two-factor central composite design with 5 central points using Design-Expert ${ }^{\circledR}$ (StatEase Inc., Minneapolis, MN). The independent variables were lactic acid concentration (from $0 \%$ to $5 \% \mathrm{~m} / \mathrm{v}$ ) and UV-C dose (from 0 to $802 \mathrm{mWs} / \mathrm{cm}^{2}$ ), and the dependent variables were $L$. monocytogenes counts and meat color. For this purpose, 21 pieces of $10 \mathrm{~g}$ each were cut from a freshly produced vacuum-packed eye round and inoculated with $5.8 \log$ CFU of the L. monocytogenes strain LM100A1. After $10 \mathrm{~min}$, inoculated meat pieces were sprayed with 1.5 $\mathrm{mL}$ of lactic acid according to the experimental design and vacuum packaged using Cryovac T7335B bags. Then, variable doses of UV-C were applied to the vacuum-packaged meat according to the design. A second set of samples was prepared for color measurements. After treatments, samples were homogenized in sterile bags with $90 \mathrm{~mL}$ of Butterfield buffer, and appropriate dilutions were plated by duplicate on agar Palcam and incubated at $37^{\circ} \mathrm{C}$ for 48 h. L. monocytogenes colonies were counted and expressed as CFU/g. Results were $\log$ transformed for analysis and expressed as $\log$ reduction of LM100A1 per gram of meat compared to the sample with no treatments. Twenty-four hours post treatments, color measurements were performed $30 \mathrm{~min}$ after opening the packages, with a colorimeter (Chromium Minolta C10 meter from Konica Minolta) in the color space $L^{*}, a^{*}, b^{*}$. Color measurements were expressed as Chroma value, calculated as $C *=\sqrt{ }\left(a^{2}+b^{2}\right)$.

Results: Analysis of variance indicated that a quadratic model was the best-fitted model $(P<0.0001)$ that explains the reduction of LM100A1 in meat with a $95 \%$ confidence due to lactic acid and UV-C treatments. Furthermore, the similarity between $R^{2}$ and the adjusted $R^{2}$ value showed the adequacy of the model to predict the corresponding response $\left(R^{2}=0.9038\right.$, adjusted $\left.R^{2}=0.8718\right)$. Both application of lactic acid and UV-C in the ranges studied have a positive effect on reduction of LM100A1, while lactic acid application is the only significant variable $(P<0.05)$ that affected meat color. According to the model obtained, the maximum reduction on LM100A1 without significant $(P<0.05)$ color changes compared to control was $1.55 \pm$ $0.41 \log$ CFU/g with the application of $2.6 \% \mathrm{~m} / \mathrm{v}$ of lactic acid and a UV-C dose of $802 \mathrm{mWs} / \mathrm{cm}^{2}$. The optimal conditions were experimentally verified, and a reduction of 1.24 $\log \mathrm{CFU} / \mathrm{g}$ was obtained. This value is within the $95 \%$ confidence interval of the predicted outcome by the model.

Conclusion: The combined application of lactic acid and UV-C radiation under the tested conditions proved to be a useful strategy to reduce contamination against $L$. monocytogenes in meat without significantly affecting meat color. Studies are underway to evaluate the efficacy of combined application of lactic acid and UV-C against other meat pathogens.

Keywords: beef meat, lactic acid, Listeria monocytogenes, predictive model, UV-C irradiation

\section{3 - EFFECTS OF BACTERIOPHAGES AND PEROXYACETIC ACID APPLICATIONS ON BEEF CONTAMINATED WITH SALMONELLA DURING DIFFERENT GRINDING STAGES}

E. L. Shebs ${ }^{1}$, F. M. Giotto ${ }^{1}$, S. T. Laidler ${ }^{1}$, and A. S. De

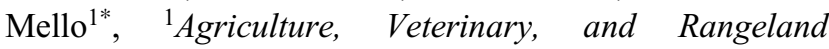
Sciences, University of Nevada, Reno, NV, USA, *amilton@ cabnr.unr.edu

Objectives: Previous research has suggested that lymph nodes in beef trim may be a source of Salmonella and therefore a potential contamination source that is incorporated into ground beef. Before grinding, beef trim is usually treated with antimicrobials to reduce risks of pathogen contamination. In this study, we tested the efficacy of bacteriophage (PhageGuard S [PGS]) and peroxyacetic acid (PAA) applications on contaminated trim, coarse ground, and fine ground beef (final ground product) to directly evaluate potential Salmonella reduction at different grinding steps.

Materials and Methods: Rose meat (m. cutaneous trunci) was fabricated into trim-like pieces and assigned to 120 -g samples $(n=36)$, coarse ground $(4 \mathrm{~mm}, n=36)$, or coarse ground followed by fine ground $(2 \mathrm{~mm}, n=36)$. Samples ( $n=108$ total) were randomly assigned to a $3 \times 4$ factorial design including fixed effects of grinding stage (trim, coarse, fine) and treatment (Control, 400 ppm PAA, $50 \%$ PGS, 5\% PGS) with 9 observations each. Nested models for each grinding stage were also tested. Samples were inoculated with a 4-Salmonella-strain cocktail to achieve $2 \times 104$ colony-forming units (CFU)/g. After $30 \mathrm{~min}$ of bacterial attachment at $5^{\circ} \mathrm{C}$, samples were treated with $1,200 \mu \mathrm{L}$ 
of either sterile buffered peptone water (Control), $400 \mathrm{ppm}$ PAA, $50 \%$ PGS $(1 \times 109$ plaque forming units $/ \mathrm{g})$, or $5 \%$ PGS $\left(1 \times 10^{8}\right.$ plaque forming units/g). After treatment, samples were hand massaged through sterile foil and allowed to dwell for $6 \mathrm{~h}$ at $5^{\circ} \mathrm{C}$ prior to grinding (for trim and coarse samples). After grinding, samples dwelled for $22 \pm 2 \mathrm{~h}$ at $5^{\circ} \mathrm{C}$. Subsequently, a $10 \mathrm{~g}$ aliquot was stomached for $2 \mathrm{~min}$ at $230 \mathrm{rpm}$ in Buffered Peptone Water. The homogenate was serially diluted before plating on Xylose Lysine Deoxycholate agar and Aerobic Plate Count (APC) Petrifilm plates. Plates were incubated at $35^{\circ} \mathrm{C}$ for $24 \mathrm{~h}$, and typical colonies were counted. APC plates were incubated for $48 \mathrm{~h}$ at $35^{\circ} \mathrm{C}$. Data were analyzed using SAS as a completely randomized design (SAS Institute Inc., Cary, NC).

Results: No interaction between both fixed effects was observed $(P=0.37)$. The same was true for grinding stage effect $(P=0.29)$. Treatment effect was significant at $P<$ 0.0001 . Overall, a significant decrease of approximately $1.5 \mathrm{log}$ was observed when comparing Control samples (3.6 log CFU/g) to 50\% PGS (2.10 log CFU/g) treatments and a significant $0.8 \mathrm{log}$ reduction for 5\% PGS (2.79 log $\mathrm{CFU} / \mathrm{g}$ ). When analyzing nested models for trim, coarse, and fine ground, the greatest reductions were seen with trim application by $5 \%$ and $50 \%$ PGS resulting in 1.13 and $1.29 \log \mathrm{CFU} / \mathrm{g}$, respectively. For coarse, only applications of 50\% PGS significantly reduced Salmonella $(1.59 \mathrm{log})$, whereas for fine, $50 \%$ PGS led to the optimal reduction (1.67 log). Overall, similar Salmonella loads were observed for Control and PAA-treated samples (3.60 and $3.53 \mathrm{log}$, respectively. Grinding stage and treatment effects individually affected mesophilic counts. Ground products had higher APC counts compared to trim, whereas bacteriophage applications led to lower APC counts compared to PAA.

Conclusion: Application of bacteriophages at any grinding stage reduce Salmonella when compared to PAA. When applied onto trim, simulating surface contamination, the lower concentration of phages promoted similar reduction compared to the higher concentration. In comminuted stages simulating possible lymph node contamination, higher concentrations of bacteriophage solutions provided the best results.

Keywords: bacteriophage, ground beef, Salmonella

\section{4 - PREVALENCE OF MCR-HARBORING SALMONELLA SPP. AND ENTEROBACTERIACEAE ISOLATED FROM MEAT PRODUCTS, FOOD-PRODUCING ANIMALS, AND THEIR ENVIRONMENTS FROM DIFFERENT LOCATIONS IN THE DOMINICAN REPUBLIC}

A. M. Perdomo ${ }^{1 *}$, M. Bugarel ${ }^{1}$, G. Loneragan ${ }^{1}$, and A. Calle ${ }^{1},{ }^{1}$ Animal and Food Science, Texas Tech University, Lubbock, TX, USA, *angela.perdomo@ttu.edu
Objectives: Estimate the prevalence of the $\mathrm{mcr}$ genes in gram-negative bacteria recovered from retailed meat, food-producing animals, and their production environment in the Dominican Republic.

Materials and Methods: A total of 80 raw beef, 94 chicken, and 88 pork samples were obtained from various types of meat markets from touristic and production areas in the Dominican Republic. In addition, 330 samples from cattle, swine, and poultry farming operations, animal feces, feed, and water were collected. Each sample was enriched overnight at $37^{\circ} \mathrm{C}$ in $90 \mathrm{~mL}$ of Buffered Peptone Water, using 3 different colistin conditions $(0 \mathrm{mg} / \mathrm{L}, 1 \mathrm{mg} / \mathrm{L}$ or $2 \mathrm{mg} / \mathrm{L}$ ). Real-time polymerase chain reaction was performed to detect $m c r$ genes in DNA extractions from the 0 $\mathrm{mg} / \mathrm{L}$-colistin overnight enrichment. All $\mathrm{mcr}$-positive samples were subjected to Enterobacteriaceae and Salmonella spp. isolation procedures. For Enterobacteria, overnight enrichments were streaked on Violet Red Bile Glucose Agar with concentrations of $0 \mathrm{mg} / \mathrm{L}, 1 \mathrm{mg} / \mathrm{L}$, and $2 \mathrm{mg} / \mathrm{L}$. For Salmonella spp., one aliquot from the overnight enrichment of Buffered Peptone Water $0 \mathrm{mg} / \mathrm{L}$ colistin was transferred to Brilliant Green Sulfa and Xylose Lysin Tergitol 4 without colistin. Isolated colonies were screened by realtime polymerase chain reaction to detect genes $\mathrm{mcr}-1$ through 8 . Positive $m c r$ isolates were further characterized using whole genome sequencing.

Results: Of the samples, $60.3 \%$ (357/592) were found to carry one or more $\mathrm{mcr}$ genes, 142 from swine, 128 from poultry, and 87 from beef cattle. From the $m c r$-positive samples, a total of 1,940 isolates were tested for $\mathrm{mcr}$ gene, from which $45.9 \%$ (34/74) were presumptive Salmonella spp. and $54.1 \%(40 / 74)$ were presumptive Enterobacteriaceae. Overall, multiplex analysis showed that $3.8 \%(74 / 1,940)$ were positive for at least 1 of $8 \mathrm{mcr}$ genes. By grouping the $m c r$-positive isolates per animal of origin, it was observed that $53(71.6 \%)$ came from swine, $10(13.5 \%)$ from poultry, and $11(14.9 \%)$ from beef cattle. A total of $27 \mathrm{mcr}$ positive isolates were analyzed by whole genome sequencing. Results showed that only $5(18.5 \%)$ of the 27 isolates were colistin resistant, from which 4 isolates were Escherichia coli carrying mcr-1 and 1 Enterobacter cloacae carrying mcr-9.

Conclusion: Results showed a high $m c r$ sample prevalence $(60.3 \%)$ compared to a very low $m c r$ isolate $(3.8 \%)$. This is perhaps due the elevated bacterial background on the samples and isolation protocols only focused on Salmonella and Enterobacteriaceae. Media used for the latter may not be fully selective for specific members of this family. It is also possible that naked DNA or injured cells carrying the genes were present in the samples.

Keywords: antimicrobial resistance, colistin, Enterobacteriaceae, $m c r$ genes 


\section{5 - EFFECTS OF PEROXYACETIC ACID AND CITRIC LACTIC ACID BLEND SPRAY ON THE SHELF LIFE OF VACUUM- PACKAGED BEEF}

H. Wang ${ }^{1 *}$ and X. Yang ${ }^{1},{ }^{1}$ Lacombe Research and Development Centre, Agriculture and Agri-Food Canada, Lacombe, Canada, *hui.wang@canada.ca

Objectives: Antimicrobial interventions are widely used for reducing enteric pathogens such as Escherichia coli O157: H7 on meat. However, information on the efficacy of acid sprays, e.g., peroxyacetic acid (PAA) and citric lactic acid blend (CLA), for spoilage bacteria and their impact on the microbiota during chiller storage is largely lacking. The aim of this work was to examine the effects of PAA and CLA spray on the shelf life of vacuum-packaged beef using a customized spray system and investigate the microbial community dynamics on acid-sprayed beef during chiller storage.

Materials and Methods: Both sides of beef short plates ( $400 \mathrm{~cm}^{2} /$ side) were sprayed with water, $400 \mathrm{ppm}$ PAA or $2.5 \% \mathrm{CLA}$ at $30 \mathrm{PSI}$ for $3 \mathrm{~min}$ at $1{ }^{\circ} \mathrm{C} \pm 1^{\circ} \mathrm{C}$ for a coverage of $>0.5 \mathrm{~mL} / \mathrm{cm}^{2}$. Each plate was then equally divided into 4 pieces, which were subsequently vacuum packaged individually. In total, 84 pieces of meat with 28 for each treatment group were stored at $1{ }^{\circ} \mathrm{C} \pm 1{ }^{\circ} \mathrm{C}$ and sampled on days $1,15,28,43,57,70$ and 86 of storage. An additional 4 pieces of meat not sprayed with any solutions were sampled on day 1 as control. At each sampling time, total aerobes, lactic acid bacteria (LAB), Enterobacteriaceae and $E$. coli were enumerated, and color, odor, and $\mathrm{pH}$ of meat were assessed. Tukey test was used to separate least-squares means. The microbiota was profiled using $16 \mathrm{~S}$ ribosomal RNA gene amplicon sequencing.

Results: Mean surface $\mathrm{pH}$ values for CLA-sprayed meat were lower $(P<0.05)$ on days 1,15 , and 43 , but no differences were noted between PAA and water groups. Off-odor intensity and discoloration increased over time. PAA delayed moderate off-odor by $14 \mathrm{~d}$ compared with water, and discoloration ( $>50 \%$ ) by 27 days compared with CLA. E. coli were only found on 2 of the water-sprayed and control meat samples on day 1 . No difference in numbers of aerobes and LAB was noted between water, PAA, and CLA at equivalent times during storage, although PAA and CLA reduced aerobes and LAB by 0.5 and 1.1 , and 0.7 and $0.9 \log$ units, compared with water spray on day $1(P<0.05)$. PAA reduced $1.1 \mathrm{log}$ units more of Enterobacteriaceae than water on day $1(P<0.05)$, but this difference was not maintained during storage. On the other hand, no difference in numbers of Enterobacteriaceae was noted between CLA- and watersprayed groups for up to $28 \mathrm{~d}$, but the numbers for CLAsprayed group were 1.4, 1.6, and $2.1 \log$ units lower $(P<$ 0.05 ) on days 43,70 , and 86 , respectively. From $16 \mathrm{~S}$ ribosomal RNA gene amplicon sequencing, LAB dominated the microbiota on the meat from each treatment during storage.
Lactobacillus, Carnobacterium, and Yersinia were the most frequently identified genera across the samples. Spray treatment and storage time affected the microbiota beta diversity $(P<0.001)$.

Conclusion: Spraying beef with 400 ppm PAA and $2.5 \%$ CLA did not affect the numbers of total aerobes, $\mathrm{LAB}$, and Enterobacteriaceae on meat with extended storage. However, PAA-sprayed meat may have an edge over water- or CLA-sprayed meat with extended storage in their organoleptic properties, which could be caused by difference in composition, rather in numbers of bacteria growing on meat from different treatment groups.

Keywords: acid spray, antimicrobial intervention, shelf life, spoilage bacteria, vacuum packaged

\section{6 - THE CHANGE OF ACID TOLERANCE RESPONSE OF SALMONELLA DURING A SIMULATED CHILLED BEEF STORAGE}

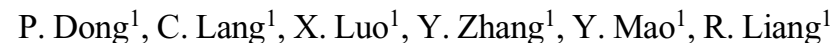
and L. Zhu ${ }^{1 *}$, College of Food Science and Engineering, Shandong Agricultural University, Taian, China, *pcdong@ sdau.edu.cn

Objectives: Salmonella is a major foodborne pathogen worldwide. It can be transferred from feces and hide to the carcasses' surface during processing and frequently causes human gastroenteritis and bacteremia. Methods such as spraying of organic acids on animal carcasses have been widely employed as decontamination treatments. However, its consequences should be considered. Besides reducing the antibacterial effect of acids, the acid tolerance response (ATR) can also trigger a cross-protective effect in bacteria, which may promote an enhanced virulence, endangering the safety of meat and meat products. This study aims to address the change of ATR of adapted and nonadapted Salmonella strains exposed to a simulated chilled beef storage environment.

Materials and Methods: The S. enterica serovar Typhimurium ATCC 14028 strain were transferred to $50 \mathrm{~mL}$ of non-glucose Tryptic Soy Broth and Tryptic Soy Broth medium containing $10 \%(\mathrm{w} / \mathrm{v})$ glucose (TSBG) and cultured at $37^{\circ} \mathrm{C}$ overnight to produce nonadapted and adapted strains, respectively. Meat extract (ME) medium was used to simulate the beef chilled storage environment of Salmonella strains. Cells were centrifuged and transferred into $100 \mathrm{~mL}$ of ME. The initial cell concentration was adjusted at $8.3 \log \mathrm{CFU} / \mathrm{mL}$, and cells were incubated at $4^{\circ} \mathrm{C}$ for $25 \mathrm{~d}$. Gradient dilutions, followed by culture on Brain Heart Infused Agar (BHIA) plates, were carried out $1 \mathrm{~d}$ before the acid tolerance assay $(0,6,12,18$, and $24 \mathrm{~d})$ to obtain the exact concentration of cells. On the day of the assay, different volumes of culture media were concentrated and transferred to $10 \mathrm{~mL}$ of BHI medium ( $\mathrm{pH} \mathrm{3}$, 

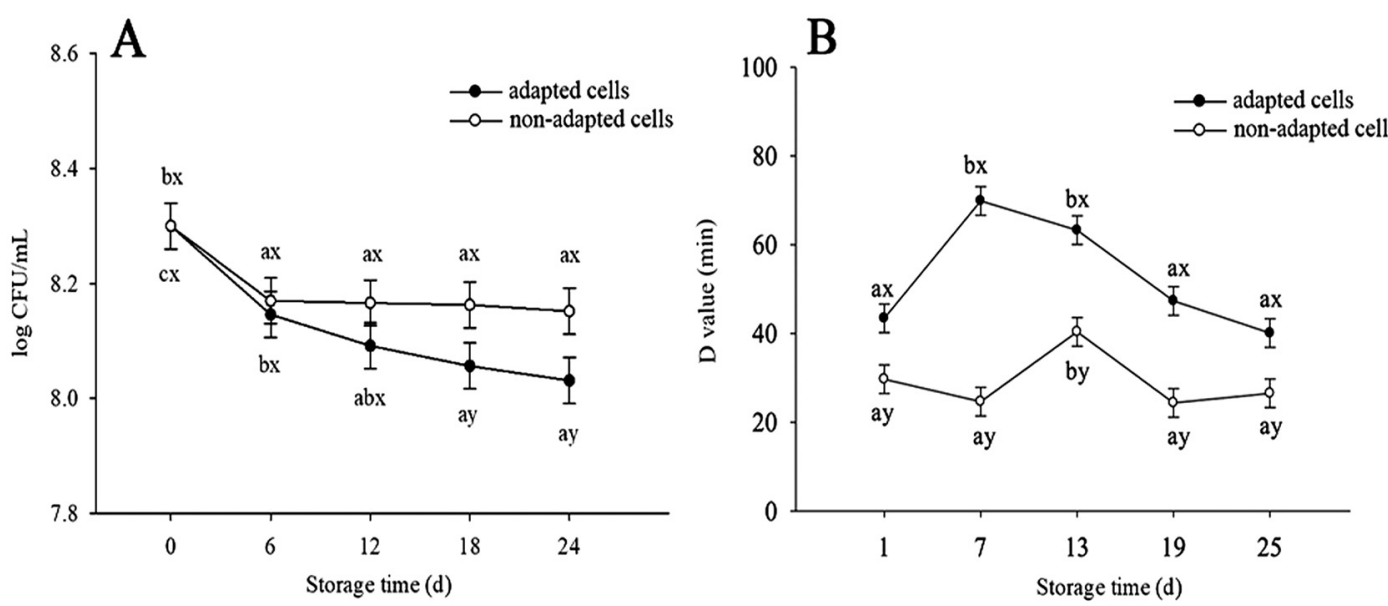

Figure 1. Changes of the colony counting of adapted and non-adapted S. typhimulium (ATCC 14028) strains in Meat Extract during the storage at $4{ }^{\circ} \mathrm{C}$ (A) and $\mathrm{D}$-values $(\mathrm{min})$ for acid treated $(\mathrm{pH}=3, \mathrm{BM})$ cells of adapted and non-adapted strains storage in Meat Extract at $4^{\circ} \mathrm{C}$ at different time intervals $(\mathrm{B})$.

adjusted with $\mathrm{HCl}$ ) to ensure the initial concentration. After incubation in acid challenge medium for $0,30,60$ and 120 min at $37^{\circ} \mathrm{C}$, cells were collected, gradient diluted, and plated on BHIA plates. The line that best fits survivor plots was determined by linear regression, and the negative reciprocal of the slope was used for the D-value.

Results: During the 25-d storage period in ME media, the cell number that was found in acid-adapted Salmonella strains was significantly lower than that of the non-acid-adapted strain. A significant decrease in the cell number was observed in both adapted and nonadapted strains $(P<0.05)$ as the storage time extended. Acid adaption significantly improved the acid tolerance of $S$. Typhimurium during storage in ME media. With regard to the acid-adapted strain, the D-value increased significantly on day 7 and then decreased to its basal level in the following days. For the nonadapted strain, the D-value was low in ME media, except for a slight increase in ME medium on day 13. Although the mild acid environment was considered the main reason for the development of ATR, and the current $\mathrm{pH}$ in ME media was very suitable for the nonadapted strain to develop an enhanced acid tolerance during storage, the D-value of nonadapted strain was not found raised over the adapted ones. Low temperature may inhibit the development of ATR, emphasizing the importance of keeping a low temperature during processing to prevent the development or survival of Salmonella spp. in meat.

Conclusion: The ATR in Salmonella was sustained for $25 \mathrm{~d}$ in the ME medium, indicating that the ATR of this bacteria occurs during the aging and distribution of beef, which is a potential threat to public health. Combined with a mild acid ME environment, low temperature had a positive effect on the acid tolerance of Salmonella.

Keywords: acid tolerance response, chilled storage, Salmonella

\section{7 - EFFECTS OF 3\% ACETIC ACID ON SALMONELLA REDUCTION IN PORK TRIMMING}

D. Sajeev ${ }^{1 *}$, P. Anderson ${ }^{1}$, C. Dahlgren ${ }^{1}$, H. Thames ${ }^{2}$, C. Fancher $^{2}$, A. Sukumaran ${ }^{2}$, S. White ${ }^{3}$, J. Feugang ${ }^{1}$, O. Rivers ${ }^{4}$, W. Schilling ${ }^{3}$, and T. Dinh ${ }^{1},{ }^{1}$ Department of Animal and Dairy Sciences, Mississippi State University, Mississippi State, MS, ${ }^{2}$ Department of Poultry Science, Mississippi State University, Mississippi State, MS, ${ }^{3}$ Department of Food Science, Nutrition, and Health Promotion, Mississippi State University, Mississippi State, MS, Institute for Imaging and Analytical Technologies, Mississippi State University, Mississippi State, MS, USA, *ds2823@msstate.edu

Objectives: The objective of the current study was to determine the efficacy of dipping pork trimmings in 3\% acetic acid on Salmonella reduction.

Materials and Methods: Pork loins and trimmings were purchased from a commercial purveyor. Pork loins were trimmed, leaving only the longissimus muscle, which was further cut into $2.5 \mathrm{~cm}$ (length) $\times 2.5 \mathrm{~cm}$ (width) $\times 1.3 \mathrm{~cm}$ (height) cubes. Pork trimmings were further ground through a kidney plate to approximately 2 -in cubes and aliquoted into $5-\mathrm{lb}$ chubs. To study the effects of temperature and time on efficacy of 3\% acetic acid in reducing Salmonella, pork cubes were inoculated at $5 \log$ of nalidixic acid-resistant Salmonella serovar Typhimurium and randomly assigned to the negative control (no inoculation, no dipping), a positive control (inoculation, no dipping), acetic acid dipping at $21^{\circ} \mathrm{C}$ (ACC), and acetic acid dipping at $50^{\circ} \mathrm{C}(\mathrm{ACH})$ for 15,45 , or $75 \mathrm{~s}$. To study the efficacy of $3 \%$ acetic acid at $50^{\circ} \mathrm{C}$ in reducing Salmonella inoculated at the geometrical center of pork trimming bulks, a pork cube inoculated at 5-log cocktail of nalidixic acid-resistant Salmonella serovar Typhimurium and Salmonella serovar Enteritidis was placed at the geometrical center of a $2.5-\mathrm{kg}$ pile of other 
uninoculated cubes in perforated canisters to create a simulation of a trimming bucket in the industry. Treatments were randomly assigned to a negative control (no inoculation; no dipping), a positive control (inoculation; no dipping), $3 \%$ acetic acid dipping at $50^{\circ} \mathrm{C}$ without $(\mathrm{ACH})$, or with shaking (ACHS) for $75 \mathrm{~s}$. After both experiments, pork cubes were retrieved and placed in Buffered Peptone Water for Salmonella extraction and plated in Xylose Lysine Deoxycholate agar for enumeration, reported as log CFU. A 9$\log$ culture of nalidixic acid-resistant Salmonella enterica serovar Typhimurium was also treated with 3\% acetic acid at $50^{\circ} \mathrm{C}$ for 45 or $75 \mathrm{~s}$ and observed by scanning electron microscopy and transmission electron microscopy for structural damages. A completely randomized design was used. Analysis of variance was performed by the GLIMMIX procedure of SAS version 9.4 (SAS Institute Inc., Cary, NC).

Results: For 15-s dipping, ACH reduced Salmonella by $0.7 \log (P<0.001), 0.5 \log$ greater than ACC $(P<0.001)$. For 45-s dipping, ACH reduced Salmonella by $1.0 \mathrm{log}$ $(P<0.001), 0.7 \log$ greater than ACC $(P<0.001)$. For 75-s dipping, the Salmonella reduction by ACH treatment was $1.4 \log (P<0.001)$, whereas the reduction was less for ACC treatment $(P=0.004)$, at only $0.5 \log (P<0.001)$. $\mathrm{ACH}$ treatment reduced Salmonella in the geometrically centered cubes by $0.2 \log (P=0.040)$. The ACHS treatment similarly $(P=0.198)$ reduced Salmonella by $0.3 \log (P=$ $0.010)$. The scanning electron microscopy images showed a less rigid surface of treated cells than the positive-control cells, especially for 75-s treatment. Moreover, transmission electron microscopy images revealed structural damages inside the treated Salmonella cells and the disappearance of the cell membrane of the treated cells, especially for 75-s dipping.

Conclusion: The present study suggests that acetic acid at $3 \%$ and $50^{\circ} \mathrm{C}$ for $75 \mathrm{~s}$ reduced Salmonella by 1.4-log by disrupting the bacterial cell membrane. Large bulks of pork trimming did not allow for adequate penetration by acetic acid solution. It is recommended to spread trimming on conveyor or loosen trimming bulks to allow for adequate penetration by acetic acid.

Keywords: acetic acid, pork trimmings, Salmonella reduction, scanning electron microscopy, transmission electron microscopy

\section{8 - EFFECT OF L28 SUPPLEMENTATION AS DIRECT FED MICROBIAL ON BEEF CATTLE PATHOGEN SHEDDING AND INDICATOR MICROORGANISM PRESENCE}

\author{
D. E. Casas $^{1 *}$, S. J. Forgey ${ }^{1}$, A. Echeverry ${ }^{1}$, M. X. Sanchez- \\ Plata $^{1}$, J. O. Sarturi ${ }^{1}$, and M. F. Miller ${ }^{1},{ }^{1}$ Animal and Food \\ Sciences, Texas Tech University, Lubbock, TX, USA, \\ *diego.casas@ttu.edu
}

Objectives: The objective of the study was to determine the effect of dietary L28 and tylosin on Salmonella, Enterococci, generic Escherichia coli, and E. coli $\mathrm{O} 157$ presence in fecal grabs, perineal and hide swabs, and subiliac lymph node samples.

Materials and Methods: Five supplementation treatments were used as follows: negative control with no additives, monensin and tylosin (MONTY), monensin and L28 (MONPRO), L28 by itself, and tylosin by itself. These 5 treatments had 12 pens assigned for each for a total of 60 pens. Fecal grab samplings were done at day 0, 100, and 200 of feed, totaling 180 samples. A completely randomized block design was used with pens as the experimental units and blocks of body weight. The individual and combined effects of tylosin and L28 were studied. Hides and perineal swabs were taken at final day of feed prior to shipping to harvesting plant. Subiliac lymph nodes were excised after harvest to analyze Salmonella presence. Generic E. coli, Enterococci, Salmonella, and E. coli O157 presence were evaluated. A chi-squared analysis was used to compare microbial presence difference between treatments using an alpha level of 0.05 .

Results: Generic E. coli and Enterococci presence was high throughout the study, $98.3 \%$ and $90.5 \%$, respectively, as expected. Salmonella presence was substantially high $(62.7 \%)$ within pens and similar among treatments. No effects $(P>0.152)$ among treatment on microbial presence was observed in any of the 4 microorganisms studied within the pen, and perineal samples taken. However, Salmonella presence within lymph nodes was affected by the treatments $(P<0.001)$. The MONPRO treatment $(34.8 \%, 26 / 46)$ had greater presence of Salmonella than the MONTY $(8.7 \%$, $4 / 46)$ and the L28-alone treatment $(0.0 \%, 0 / 42)$. The presence of Salmonella on hide samples was affected $(P=$ 0.047), where MONPRO treatment $(23.9 \%, 11 / 46)$ had the greatest presence compared to control $(4.4 \%, 2 / 45)$ and tylosin-alone treatment $(6.7 \%, 3 / 45)$.

Conclusion: Incidence of Salmonella, Enterococci, generic E. coli, and $\mathrm{O} 157$ did not increase with supplementation of L28 compared to the control. Greater presence of Salmonella in MONPRO treatment on lymph nodes and hides suggests that monensin may have an antagonistic effect with the probiotic when supplemented at the same time through the diet, and it needs to be further studied. Absence of Salmonella in L28 treatment lymph nodes suggests that supplementation of L28 may contribute to mitigating Salmonella's capacity to invade the lymphatic system. This poses a significant contribution to the beef industry as lymph nodes can be a substantial source of Salmonella in ground beef.

Keywords: direct fed microbial, Lactobacillus salivarus L28, pathogen shedding 


\section{9 - REDUCTION OF SALMONELLA SPP. BY UV-C LED IRRADIATION}

M. Fernandez ${ }^{1 *}$, A. Calle ${ }^{1}$, I. Arvelo ${ }^{1}$, B. Montoya ${ }^{2}$, and J. Thompson ${ }^{3},{ }^{1}$ Animal and Food Sciences, Texas Tech University, Lubbock, TX, USA, ${ }^{2}$ Universidad Autonoma de Honduras, Universidad Autonoma de Honduras, Tegucigalpa, Honduras, ${ }^{3}$ Chemistry, Texas Tech University, Lubbock, TX, USA, *mariana.fernandez@ttu.edu

Objectives: The objective of this study was to evaluate the effectiveness of UV-C light-emitting diode (LED) light at different exposure time points and intensities for the reduction of Salmonella spp. applied to the surface of chicken breast, stainless steel, and high-density polyethylene.

Materials and Methods: Portions of $2 \times 2 \mathrm{~cm}$ of 3 different surfaces were obtained. The upper surface of the portions was inoculated with a 5-strain Salmonella cocktail at a target concentration of $6.0 \log \mathrm{CFU} / \mathrm{cm}^{2}$. The 3 different surfaces were treated with UV-C LED irradiation: (1) boneless skinless chicken breast, (2) stainless steel, and (3) high-density polyethylene. Each treatment was based on different exposure times using 2 different irradiance intensities of $2 \mathrm{~mW} /$ $\mathrm{cm}^{2}$ (50\% intensity) and $4 \mathrm{~mW} / \mathrm{cm}^{2}$ (100\% intensity). Different time points were tested for each surface. For the chicken breast, time points of $0,1,3,5,10$, and $15 \mathrm{~min}$ of exposure to both $50 \%$ and $100 \%$ intensity were observed in triplicate. For stainless steel, time points of $0,0.25,0.5$, 0.70 , and $1 \mathrm{~min}$ of exposure to both $50 \%$ and $100 \%$ intensity were observed in triplicate. For high-density polyethylene, time points of $0,0.5,1,1.5,2,2.5,3,5,10$, and $15 \mathrm{~min}$ of exposure to both $50 \%$ and $100 \%$ intensity were observed in triplicate.

Results: The population of Salmonella on the boneless skinless chicken breast had a reduction of $1.71 \log _{10} \mathrm{CFU} /$ $\mathrm{cm}^{2}$ and $2.05 \log _{10} \mathrm{CFU} / \mathrm{cm}^{2}$ for the $50 \%$ and $100 \%$ intensity treatments, respectively. Furthermore, the stainless steel coupons showed a reduction of $1.85 \log _{10} \mathrm{CFU} / \mathrm{cm}^{2}$ for the coupons treated at $50 \%$ intensity and a $4.57 \log _{10}$ $\mathrm{CFU} / \mathrm{cm}^{2}$ for the ones at $100 \%$ intensity. Finally, for the high-density polyethylene coupons, there was a reduction of $4.48 \log _{10} \mathrm{CFU} / \mathrm{cm}^{2}$ in the $50 \%$ intensity treatment, and a complete reduction to $0 \log _{10} \mathrm{CFU} / \mathrm{cm}^{2}$ after $5 \mathrm{~min}$ of being treated at $100 \%$ intensity.

Conclusion: Many efforts to control Salmonella in poultry products and food contact surfaces involve the application of chemical treatments at different steps of process. UV LED are increasingly being used to treat food and food contact surfaces. Further testing is needed to understand the efficiency of UV-C LED treatment on food surfaces and food contact surfaces to be used while on production line.

Keywords: Salmonella reduction, ultra violet irradiation

American Meat Science Association.

180 - COMMUNITY-LEVEL PHYSIOLOGICAL PROFILING OF REDUCED OR REPLACED SALT BOEREWORS BATTERS INOCULATED WITH ESCHERICHIA COLI

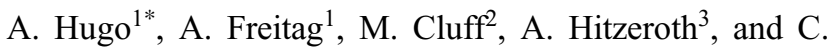
Hugo $^{1},{ }^{1}$ Food Science Division, University of the Free State, Bloemfontein, South Africa, ${ }^{2}$ Research and Development, B.T. Enterprises, Johannesburg, South Africa, ${ }^{3}$ Department of Biotechnology, University of the Western Cape, Bellville, South Africa, *hugoa@ufs.ac.za

Objectives: Since high salt intake is regarded as unsafe for human health, the South African Department of Health (2013) released regulations for a stepwise lowering of salt content in certain food products, including fresh sausages. The reduction or partial replacement of salt, with salt replacers, can affect the product safety and shelf life of fresh meat products. Boerewors is a traditional fresh and uncured South African sausage manufactured from beef and pork. No studies have yet been performed on the effect of salt reduction/replacement with potassium chloride or potassium lactate on the microbial communities in Boerewors models when inoculated with Escherichia coli. The objectives of this study were to evaluate the bacterial communities in Boerewors batters with reduced and/or partial replacement by $\mathrm{KCl}$ or K-lactate, after it was inoculated with E. coli, by determining the community-level physiological profiles of each treatment by assessing the metabolic diversity in the meat samples.

Materials and Methods: Five treatments of Boerewors batters were formulated: $\mathrm{NC}=$ negative control with no added $\mathrm{NaCl}$ or replacer; $\mathrm{K} 600=$ treatment with potassium chloride as replacer $(1.25 \%$ added $\mathrm{NaCl}+0.551 \% \mathrm{KCl})$; $\mathrm{L} 600=$ treatment with potassium lactate as replacer $(1.25 \%$ added $\mathrm{NaCl}+0.551 \% \mathrm{~K}$-lactate); N600 = treatment containing only $\mathrm{NaCl}$ and no replacer $(1.25 \%$ added $\mathrm{NaCl})$; $\mathrm{PC}=$ positive control containing the $800 \mathrm{mg} / 100 \mathrm{~g} \mathrm{Na}$ level recommended for Boerewors from 30 June $2016(1.80 \%$ added NaCl; DoH of South Africa, 2013). K600, L600, and $\mathrm{N} 600$ contained the $600 \mathrm{mg} / 100 \mathrm{~g} \mathrm{Na}$ level recommended for Boerewors from 30 June 2019. The Boerewors models were spiked with $2.61 \log \mathrm{CFU} / \mathrm{g} E$. coli and stored at $4^{\circ} \mathrm{C}$, for $3 \mathrm{~d}$. This study used community-level physiological profiling, employing the Biolog ${ }^{\mathrm{TM}}$ Ecoplates $^{\mathrm{TM}}$. The reactions of the microbial communities to the 31 carbon compounds were measured and statistically evaluated using analysis of variance, Gompertz equations (XLSTAT, 2018).

Results: When spiked with $2.61 \log \mathrm{CFU} / \mathrm{g}$ E. coli and stored at $4{ }^{\circ} \mathrm{C}$ for $3 \mathrm{~d}$, the treatment with no added salt (NC), showed the highest growth rate and maximum population size when compared to the treatments containing salt and replacers. The average well color development and 
Table 1. Growth kinetics of microbial communities from Boerewors samples with different treatments, inoculated with $E$. coli.

\begin{tabular}{lllllll}
\hline Treatment & C (hours) & a (OD values) & AWCD & $\boldsymbol{H}$ & $\boldsymbol{E}$ & $\boldsymbol{S}$ \\
\hline NC & $0.0365^{\mathrm{a}}$ & 64.915 & 39.328 & 1.383 & 0.051 & $20.454^{\mathrm{a}}$ \\
$\mathbf{K} 600$ & $0.0451^{\mathrm{b}}$ & 58.786 & 39.139 & 1.441 & 0.049 & $22.086^{\circ}$ \\
$\mathbf{L 6 0 0}$ & $0.0416^{\mathrm{ab}}$ & 59.920 & 38.600 & 1.400 & 0.048 & $21.515^{\mathrm{ab}}$ \\
$\mathbf{N 6 0 0}$ & $0.0422^{\mathrm{ab}}$ & 60.780 & 38.667 & 1.422 & 0.052 & $21.406^{\mathrm{ab}}$ \\
PC & $\mathbf{0 . 0 4 0 4 ^ { \mathrm { ab } }}$ & 60.468 & 36.333 & 1.415 & 0.053 & $20.928^{\mathrm{ab}}$ \\
\hline Sign. level & $\mathrm{p}=0.020$ & $\mathrm{p}=0.763$ & $\mathrm{p}=0.886$ & $\mathrm{p}=0.986$ & $\mathrm{p}=0.374$ & $\mathrm{p}=0.023$ \\
\hline
\end{tabular}

Means with different superscripts in the same column differed significantly. Mean \pm standard deviation.

The growth rate (c) and maximum population size (a) were calculated with the Gompertz equation (Yeh et al., 2019).

AWCD = Average well colour development; $H=$ Shannon diversity, $E=$ Shannon evenness; $S=$ Shannon richness

were calculated according to Grzadziel et al. (2018)

Shannon richness indicated that the potassium chloride treatment was significantly $(\mathrm{P}<0.05)$ more efficient in controlling the growth of the microbial communities compared to the NC (Table 1). With the single carbon oxidization, the K600 and N600 were comparable to each other in controlling the growth of the bacterial communities. It was indicated that there was a reduction in the functional diversity of microbial communities and E. coli in Boerewors with higher salt content.

Conclusion: The community-level physiological profiling in this study indicated that the sodium level mandatory in South Africa from 30 June 2019, as well as $\mathrm{KCl}$ as a salt replacer with lowered levels of $\mathrm{NaCl}$ inclusion, were effective in controlling $E$. coli in Boerewors when stored at $4{ }^{\circ} \mathrm{C}$.

Keywords: Boerewors, community-level physiological profiling, EcoPlates, Escherichia coli, salt reduction

\section{1 - EVALUATION OF UV-C IRRADIATION TO REDUCE LISTERIA MONOCYTOGENES CONTAMINATION IN MEAT PROCESSING PLANTS}

C. Rufo ${ }^{1 *}$, G. Brugnini ${ }^{1}$, S. Rodríguez-Cortés ${ }^{1}$, and Inocuidad, Alimentos y Nutrición, ${ }^{1}$ Instituto Polo Tecnológico, Facultad de Química, Universidad de la Republica, Montevideo, Uruguay,*caterinarufod@gmail.com

Objectives: The effect of UV-C (254 nm) on Listeria monocytogenes contamination on the conveyor belts surface of a high-throughput meat processing plant was evaluated.

Materials and Methods: First, the effect of different doses of UV-C on L. monocytogenes survival was analyzed by inoculating Triptone Soy Agar plates and polyethylene coupons of $150 \mathrm{~cm}^{2}$ (clean and with meat remains) with the L. monocytogenes strain ATCC19111. Agar plates were inoculated with $10^{2}$ and $10^{3} \mathrm{CFU}$ and coupons with $10^{5}$ CFU. The UV-C dose applied on inoculated Trypticas Soy Agar plates varied from 38 to $229 \mathrm{mWs} / \mathrm{cm}^{2}$ and from
76 to $405 \mathrm{mWs} / \mathrm{cm}^{2}$ on the polyethylene coupons. After exposure to UV-C, agar plates were incubated at $37^{\circ} \mathrm{C}$ for $48 \mathrm{~h}$, and then survivor colonies were manually counted. Coupons were sampled with a sterile sponge, the sponge was placed in a sterile bag with $30 \mathrm{~mL}$ of Butterfield buffer, and appropriate dilutions were plated by duplicate on agar Palcam and incubated at $37^{\circ} \mathrm{C}$ for $48 \mathrm{~h}$. In the abattoir, a UV-C germicidal emitting lamp $(53 \times 20 \mathrm{~cm})$ was placed underneath the meat conveyor belt at $7 \mathrm{~cm}$ from the surface. Each belt section received a dose of $593 \mathrm{~mW} / \mathrm{cm}^{2}$ every 18 min during the deboning process. UV-C effect was evaluated on 3 different days without interfering with the regular plant operation. Swab-surface samples were collected, every $20 \mathrm{~min}$ over a period of $7 \mathrm{~h}$, from UV-C-exposed and nonexposed areas of the conveyor belt surface. Sampling areas surface was of $900 \mathrm{~cm}^{2}$. Listeria spp., and indicator microorganisms such as total mesophilic aerobic (aerobic plate count [APC]), Escherichia coli, and total coliforms from areas exposed and not exposed to UV-C were enumerated on $3 \mathrm{M} \otimes$ Petrifilm according to the manufacturer's recommendations. Plate counts were expressed as $\mathrm{CFU} / \mathrm{cm}^{2}$ for $\mathrm{APC}$ and as $\mathrm{CFU} / 100 \mathrm{~cm}^{2}$ for coliforms, E. coli, and Listeria spp. Results were $\log$ transformed for analysis, and the mean $\operatorname{logs}$ for the treatments were compared by a paired $t$ test with a significance level of 0.05 , using SPSS IBM software (IBM Corp., Armonk, NY).

Results: L. monocytogenes reduction of $3 \log$ CFU/plate was obtained in Trypticas Soy Agar plates treated with a dose of $153 \mathrm{mWs} / \mathrm{cm}^{2}$ of UV-C radiation. The same UV$\mathrm{C}$ dose in the coupon model caused reductions of 2.1 and $1.1 \log$ for clean and dirty coupons, respectively. In the processing plant, it was observed that the application of $\mathrm{UV}-\mathrm{C}$ at $163 \mathrm{mWs} / \mathrm{cm}^{2}$ on the conveyor belt of the deboning room significantly $(P<0.05)$ reduced Listeria spp. counts by $1.2 \log \mathrm{CFU} / 100 \mathrm{~cm}^{2}$. UV-C application also produced a significant decrease $(P<0.05)$ of $1.7 \log C F U / 100 \mathrm{~cm}^{2}$ for APC, $1.8 \log \mathrm{CFU} / 100 \mathrm{~cm}^{2}$ for E. coli, and $2.0 \log$ $\mathrm{CFU} / 100 \mathrm{~cm}^{2}$ for coliforms.

Conclusion: The application of UV-C on the conveyor belts surface reduced Listeria and the overall 
microbiological contamination of the surfaces that come in contact with meat, contributing to the reduction of the microbiological contamination on the final product. The reduction in the bacterial population on the conveyor belts surface was observed over the 3 sampling days, suggesting that the UV-C effect is independent of the meat plant operating conditions. UV-C treatment of belts surface during production may add an extra hurdle to bacterial contamination.

Keywords: conveyor belt, Listeria monocytogenes, UV-C irradiation

\section{2 - EXTENDING THE SHELF LIFE OF READY-TO-EAT FOODS BY INHIBITION OF THE GROWTH AND STAPHYLOCOCCAL ENTEROTOXIN A (SEA) PRODUCTION OF STAPHYLOCOCCUS AUREUS}

\author{
S. Tsutsuura ${ }^{1 *}$, T. Nishiumi ${ }^{2}$, and M. Murata ${ }^{3},{ }^{1}$ Institute for \\ Research Promotion, Niigata, Japan, ${ }^{2}$ Faculty of Agricul- \\ ture, Niigata University, Niigata, Japan, ${ }^{3}$ Department of \\ Nutrition and Food Science, Ochanomizu University, Tokyo, \\ Japan,*tsu2ura@agr.niigata-u.ac.jp
}

Objectives: Staphylococcus aureus has been recognized as one of the food poisoning bacteria related to poor food handling. Staphylococcal food poisoning (SFP) occurs in various foods such as meat products, dairy products, and other processed foods in the world. In Japan, SFP occurs in ready-to-eat meals such as rice balls and lunch packs. To prevent SFP, it is important to inhibit the growth and staphylococcal enterotoxin A (SEA) production by S. aureus in ready-to-eat meals during retail storage. The purpose of this study was to clarify the effects of storage factors on growth and SEA production in cooked rice to extend the shelf life of ready-to-eat meals.

Materials and Methods: To examine the effects of inoculum size and incubation temperature, we used the SEAproducing strains of $S$. aureus, respectively inoculated at the rate of about $10^{2}$ or $10^{6} \mathrm{CFU} / \mathrm{g}$ in cooked rice, and incubated at $15^{\circ} \mathrm{C}-37^{\circ} \mathrm{C}$. To examine the effects of $\mathrm{pH}$, glycine, and sodium chloride $(\mathrm{NaCl})$, we used cooked rice, which was adjusted to a final $\mathrm{pH}$ of $4.0-6.3$ with acetic acid. Glycine $(0.5 \%-5 \%, \mathrm{w} / \mathrm{w})$ or $\mathrm{NaCl}(1 \%-7 \%, \mathrm{w} / \mathrm{w})$ was then added into cooked rice before inoculation with $S$. aureus at the rate of $10^{6} \mathrm{CFU} / \mathrm{g}$ and incubated at $37^{\circ} \mathrm{C}$. To screen for seasonings that are effective in inhibiting SEA production, 80 types of commercial seasonings were added to $1.5 \% \mathrm{NaCl}$-added rice, inoculated with $S$. aureus and incubated at $37^{\circ} \mathrm{C}$. The concentrations of SEA were estimated by a Western blot analysis using the enhanced chemiluminescence method. The detection limit in cooked rice was ca. $400 \mathrm{pg} / \mathrm{g}$.

Results: As incubation temperature decreased from $37^{\circ} \mathrm{C}$ to $15^{\circ} \mathrm{C}$, the growth and SEA production of $S$. aureus strains were decreased in cooked rice, while the time needed to produce SEA was prolonged. At the early stage of preservation, SEA detection required a longer time with inoculum size of $10^{2} \mathrm{CFU} / \mathrm{g}$ than $10^{6} \mathrm{CFU} / \mathrm{g}$ in cooked rice at $37^{\circ} \mathrm{C}$. In cooked rice to which $2 \%-5 \%$ glycine and $3 \%-7 \% \mathrm{NaCl}$ were respectively added, growth and SEA production of $S$. aureus strains were decreased, and the time needed to produce SEA was prolonged during the early stage of preservation. SEA production was inhibited at the early stage of preservation in cooked rice at $\mathrm{pH} 4.0$ and 4.5. In particular, SEA production was inhibited at $\mathrm{pH} 4.0$ even after $24 \mathrm{~h}$ of incubation at $37^{\circ} \mathrm{C}$. Among the 80 seasonings added to $1.5 \% \mathrm{NaCl}$-added cooked rice, vinegars, lemon extract, Worcestershire sauce, mustard paste, rose hip tea, and pickled plum (umeboshi) paste were effective in inhibiting SEA production, even after $48 \mathrm{~h}$ of incubation at $37^{\circ} \mathrm{C}$. SEA production seemed to be affected not only by $\mathrm{NaCl}$ but also by food ingredients and $\mathrm{pH}$ of the commercial seasonings because the $\mathrm{pH}$ of these cooked rice was as low as 3.5-4.5.

Conclusion: Scientific regulation and control by reducing food contamination (inoculum size), adding glycine, $\mathrm{NaCl}$, and commercial seasonings as well as $\mathrm{pH}$ adjustment were effective for extending the shelf life of cooked rice. It is expected that the methods of scientific regulation obtained in this study will be applicable to meat products as well.

Keywords: food poisoning, processed food, shelf life, staphylococcal enterotoxin A

\section{3 - PULSED ULTRAVIOLET LIGHT DECONTAMINATION OF MEAT CONVEYOR CONTACT SURFACES}

J. R. Cassar ${ }^{*}$, E. W. Mills ${ }^{1}$, and A. Demirci ${ }^{2},{ }^{1}$ Animal Science, The Pennsylvania State University, University Park, PA, USA, ${ }^{2}$ Agricultural and Biological Engineering, The Pennsylvania State University, University Park, PA, USA,*jrc5659@psu.edu

Objectives: Movement of meat on a continuous belt conveyor during processing results in opportunities for pathogenic and spoilage microorganisms to contaminate the product. Pulsed ultraviolet (PUV) light is an effective antimicrobial treatment with limited application in the food industry. PUV light is the emittance of broad-spectrum electromagnetic radiation, 100 $1,100 \mathrm{~nm}$, from a xenon flashlamp pulsed 3 times per second with $>50 \%$ of the energy deriving from the UV region. The objective of this project was to investigate the germicidal response on the surface of food-grade conveyor materials after treatment by PUV light.

Materials and Methods: Four conveyor belt types, including stainless-steel chain-link, heat-resistant fabric belt, solid pliable polymer belt, and rigid linked polymer belt, were cut into $100-\mathrm{cm}^{2}$ sample units. Materials were inoculated with an antibiotic resistant Escherichia coli K12 strain or a lactic acid bacteria (LAB) cocktail, yielding a surface 
inoculation of $10^{5}$ and $10^{4} \log _{10} \mathrm{CFU} / \mathrm{cm}^{2}$, respectively. Prior to bacterial inoculation, samples were classified as soiled or unsoiled, based on the presence or absence of pork intramuscular fluid on the surface of the sample. Using a conveyor equipped with a xenon flashlamp positioned $10 \mathrm{~cm}$ above the surface, each belt sample was exposed to PUV light at 3 fixed conveyor speeds: 3.05, 15.24, and 30.48 $\mathrm{cm} / \mathrm{s}$, resulting in a total energy exposure of $3.31,0.66$, and $0.33 \mathrm{~J} / \mathrm{cm}^{2}$, respectively. Three samples of each material were evaluated under all treatment combinations including surface condition, microorganism type, and conveyor speed. After treatment, a $10-\mathrm{cm}^{2}$ area was swabbed to retrieve surviving bacteria from each sample. Swab samples were serially diluted and plated on selective agars. Comparison of treated to control samples allowed for quantification of microbial reduction due to PUV light treatment. The main effects, treatment and surface condition and their interaction, were evaluated in a two-way analysis of variance with Tukey multiple comparison test used to detect significant differences $(P<0.05)$ among treatment means.

Results: For samples inoculated with E. coli K12 NSR, the surface condition by treatment interaction was significant for microbial inactivation on the surface of the rigid polymer linked belt $(P<0.05)$, but not for the heat-resistant fabric, the solid pliable polymer belt, and the stainless-steel chain-link $(P>0.05)$. For samples inoculated with the LAB cocktail, the surface condition by treatment interaction was significant for microbial inactivation on the surface of the heat-resistant fabric and the solid pliable polymer belt $(P<0.05)$, but not for the rigid polymer linked belt and the stainless-steel chain-link $(P>0.05)$. When $3.31 \mathrm{~J} / \mathrm{cm}^{2}$ was delivered to the samples, microbial reduction of $E$. coli $\mathrm{K} 12$ ranged from 3.34 to 5.47 and 2.07 to $4.62 \log _{10} \mathrm{CFU} / \mathrm{cm}^{2}$ for on unsoiled and soiled surfaces, respectively. Those same conditions lead to 2.47 to 5.00 and 2.44 to $5.11 \log _{10} \mathrm{CFU} / \mathrm{cm}^{2}$ microbial reductions for LAB on unsoiled and soiled surfaces, respectively. Faster conveyor speeds resulted in lower PUV energy fluence and lower microbial destruction.

Conclusion: The results of this work demonstrate that PUV light has potential to quickly reduce microbial counts on the surface of various types of conveyor belts, during operation, with reduced effectiveness as meat residue builds up on the surface.

Keywords: contact surfaces, conveyor, E. coli, lactic acid bacteria, pulsed ultraviolet light

\section{4 - FATE OF ESCHERICHIA COLI ATCC 25922 IN BEEF STEAKS DURING SOUS VIDE COOKING AT DIFFERENT HOLDING TIME AND TEMPERATURE COMBINATIONS}

H. B. Hunt ${ }^{1 *}$, S. C. Watson ${ }^{1}$, B. D. Chaves ${ }^{2}$, and G. A. Sullivan ${ }^{1},{ }^{1}$ Department of Animal Science, University of
Nebraska, Lincoln, NE, USA, ${ }^{2}$ Department of Food Science and Technology, University of Nebraska, Lincoln, NE, USA, *heather.hunt@huskers.unl.edu

Objectives: Sous vide cooking, a method of preparing food in hot water baths, can be used to achieve a precise degree of doneness throughout a steak. Due to ease of use, there is increased popularity of sous vide cooking in domestic and food service food preparation. For cooking safety, United States Department of Agriculture Food Safety and Inspection Service Appendix A guidance for the control of Salmonella is commonly referenced for the prevention of pathogenic Escherichia coli adulteration of cooked beef products. The lowest temperature included in Appendix A is $54^{\circ} \mathrm{C}$. Some sous vide manufacturers' cooking recipes suggest cooking nonintact beef products to an internal temperature as low as $46^{\circ} \mathrm{CC}$. Thus, this experiment was designed to validate a $5 \log _{10}$ thermal reduction of generic E. coli in sous vide cooked beef steaks.

Materials and Methods: The experiment was conducted in 3 independent replications. Beef semitendinosus muscles were cut into $2.54-\mathrm{cm}$ slices, vacuum packaged, and frozen until use. For each replication, steaks were thawed ( $48 \mathrm{~h}$, $4^{\circ} \mathrm{C}$ ) and exposed to ultraviolet light for $15 \mathrm{~min}$ on each side. Steaks were submerged in liquid inoculum $(2 \mathrm{~L}$ of $E$. coli ATCC 25922 overnight culture) and internally inoculated with a pin pad inserted 5 times into each side of each steak. After inoculation, steaks were air-dried $\left(30 \mathrm{~min}, 23^{\circ} \mathrm{C}\right)$, individually vacuumed sealed, and cooked in sous vide water baths. Duplicate steak samples were taken from raw, inoculated steaks and at the following hold time, temperature combinations: $150 \mathrm{~min} / 46^{\circ} \mathrm{C}, 420 \mathrm{~min} / 46^{\circ} \mathrm{C}, 150 \mathrm{~min} / 51^{\circ} \mathrm{C}$, $193.5 \mathrm{~min} / 51^{\circ} \mathrm{C}, 258 \mathrm{~min} / 51^{\circ} \mathrm{C}, 322.5 \mathrm{~min} / 51^{\circ} \mathrm{C}, 64.5 \mathrm{~min} /$ $54^{\circ} \mathrm{C}, 86 \mathrm{~min} / 54^{\circ} \mathrm{C}, 107.5 \mathrm{~min} / 54^{\circ} \mathrm{C}, 2.25 \mathrm{~min} / 62^{\circ} \mathrm{C}, 3 \mathrm{~min} /$ $62^{\circ} \mathrm{C}$, and $3.75 \mathrm{~min} / 62^{\circ} \mathrm{C}$. The median sampling times for $54^{\circ} \mathrm{C}$ and $62^{\circ} \mathrm{C}$ were taken directly from the Appendix A $5 \log _{10}$ reduction table, and the other times were $\pm 25 \%$ of the median time. The median sampling time for $51^{\circ} \mathrm{C}$ was extrapolated from the table. The $46^{\circ} \mathrm{C}$ sampling times represented potential worst-case scenarios. Core samples $(25 \mathrm{~g})$ were homogenized, serially diluted, and plated onto Charm EC Peal plates. E. coli colonies were counted after incubation $\left(24 \mathrm{~h}, 35^{\circ} \mathrm{C}\right)$ according to manufacturer guidelines and reported as $\log _{10} \mathrm{CFU} / \mathrm{g}$. Reductions were determined by subtracting concentrations at given sampling times from the raw sample. Data were analyzed using PROC GLM contrasts in SAS 9.4 (SAS Institute Inc., Cary, NC).

Results: The minimum time measured for a $5 \log _{10}$ reduction for $51^{\circ} \mathrm{C}, 54^{\circ} \mathrm{C}$, and $62^{\circ} \mathrm{C}$ was $258 \mathrm{~min}, 64.5$ $\mathrm{min}$, and $2.25 \mathrm{~min}(P<0.01)$, respectively. At $46^{\circ} \mathrm{C}$, cooking achieved a final reduction of $1.07 \log _{10}(P<0.01)$ after $420 \mathrm{~min}$.

Conclusion: The worst-case scenario conditions of this study confirm the utility of Appendix A time, temperature tables for a $5 \log _{10}$ reduction of generic E. coli at $62^{\circ} \mathrm{C}$ and $54^{\circ} \mathrm{C}$, and suggest the possibility for safely sous vide 
cooking steaks at $51^{\circ} \mathrm{C}$. Further experimentation is needed to determine the fate of pathogenic $E$. coli during sous vide cooking of steaks using time and temperature combinations at and below recommended USDA-FSIS values.

Keywords: beef, cooking temperature, sous vide

\section{5 - SURVIVAL OF LISTERIA MONOCYTOGENES ON COOKED HIGH PRESSURE TREATED BACON}

\author{
H. Cetin-Karaca ${ }^{1 *}$, S. Cruzen ${ }^{1}$, and D. Ebbing ${ }^{1},{ }^{1}$ Food Safety \\ and Quality Assurance, Smithfield Foods, Cincinnati, OH, \\ USA, *hcetinkaraca@smithfield.com
}

Objectives: Industrially produced cooked bacon product is exposed to the environment post-lethality and handled prior to final packaging after exiting continuous cooking ovens. There is a lack of information in the literature on the efficacy of high pressure processing (HPP) as a control intervention for recontamination and subsequent growth of Listeria monocytogenes on cooked bacon. The purpose of this study was to determine the survival of $L$. monocytogenes on cooked high pressure processed bacon strips water activity $\left(\mathrm{a}_{\mathrm{w}}\right)(0.805 \pm 0.072$ and $0.824 \pm 0.042)$ and bits $\left(\mathrm{a}_{\mathrm{w}} 0.807\right.$ \pm 0.052 and $0.840 \pm 0.019)$ during a 150 -d storage period at $4^{\circ} \mathrm{C}$.

Materials and Methods: Bacon strips and bits with $2 \mathrm{a}_{\mathrm{w}}$ levels $(99 \mathrm{~g})$ were inoculated with a 5 -strain L. monocytogenes cocktail at ca. $2.0-\log 10 \mathrm{CFU} / \mathrm{g}$ and then packaged under $100 \%$ nitrogen gas and high pressure processed at 86,000 psi for $5 \mathrm{~min}$. Inoculated samples were held at $4^{\circ} \mathrm{C}$, and duplicate samples were analyzed at $1,15,30$, $45,60,90,120$, and $150 \mathrm{~d}$ by direct spiral plating on modified Oxford medium. Un-inoculated samples $(n=8)$ were analyzed for lactic acid bacteria and aerobic total plate counts. Data were analyzed using SPSS version 27 (IBM Corp., Armonk, NY) and one-way analysis of variance. Triangle tests were performed using untrained panelists to determine whether flavor differences existed between uninoculated HPP and non-HPP samples and analyzed using the chi-squared distribution.

Results: There were no significant differences in $L$. monocytogenes populations between bacon strips and bits or between samples with different $\mathrm{a}_{\mathrm{w}}(P>0.05)$. HPP resulted in an initial decrease (measured at $24 \mathrm{~h}$ ) of ca. $1.0-\log 10 \mathrm{CFU} / \mathrm{g}$ compared to non-HPP samples $(P<$ 0.05). Detection limit was $20 \mathrm{CFU} / \mathrm{g}$. The $L$. monocytogenes population declined over the course of $150 \mathrm{~d}$ in non-HPP samples by $1.0-\log 10 \mathrm{CFU} / \mathrm{g}(P<0.05)$ but did not significantly decline over time in the HPP bacon. HPP and nonHPP bits and low $a_{w}$ strips were not statistically different in flavor, as determined by triangle testing. The higher $\mathrm{a}_{\mathrm{w}}$ strips, however, were statistically different $(P<0.05)$, with up to $31 \%$ of the population able to discern between HPP and
non-HPP product. Additionally, the HPP bits tended to be compressed and clumped together in the package.

Conclusion: These data suggest that HPP may be effective in reducing $L$. monocytogenes populations in cooked bacon strips and bits. However, the reduction was small, and sensory differences in bacon strips and severe clumping in bits may preclude its use from a practical standpoint.

Keywords: cooked bacon, high pressure processing, Listeria monocytogenes, sensory analysis

\section{Muscle and Lipid Biology and Biochemistry}

\section{6 - PROTEOME AND METABOLOME OF EARLY POSTMORTEM LONGISSIMUS DORSI TO EXPLORE ULTIMATE PH AND PORK QUALITY}

E. Zuber ${ }^{1 *}$, A. Outhouse ${ }^{1}$, E. Huff-Lonergan ${ }^{1}$, E. Steadham ${ }^{1}$, and S. Lonergan ${ }^{1},{ }^{1}$ Animal Science, Iowa State University, Ames, IA, USA, *bzuber@iastate.edu

Objectives: Extent of pH decline relies on postmortem (PM) muscle metabolism. Glycolytic mechanisms maintain metabolism and may influence pork quality. The objective was to identify metabolomic and proteomic features that are associated with variation in ultimate $\mathrm{pH}$ pork loin. The hypothesis was that a combination of elements in early PM Longissimus dorsi (LD) proteome and metabolome can be used to understand variation in $\mathrm{pH}$ decline.

Materials and Methods: Pigs $(N=47)$ were harvested using standard industry procedures. Samples were removed from the LD at 45 min PM, snap-frozen in liquid nitrogen, powdered, and stored at $-80^{\circ} \mathrm{C}$. A portion of the $\mathrm{LD}$ was used for $\mathrm{pH}$ and quality measurements (lightness, purge loss, star probe, and intact desmin) after $14 \mathrm{~d}$ of aging. Densitometry analysis of immunoblots was used to quantify intact desmin, resolved in the whole muscle extracts from day-14 samples. Groups were classified as normal $\mathrm{pH}$ $(\mathrm{NpH})(\mu=5.59,5.53-5.67 ; n=10)$ and low $(\mathrm{LpH})(\mu=$ $5.42,5.38-5.45 ; n=10)$ at $14 \mathrm{~d} \mathrm{PM}$ for metabolomic and proteomic analyses. Nontargeted, polar metabolites from 45 min PM were identified using gas chromatography-mass spectrometry. Proteins were solubilized in sarcoplasmic extraction buffer from 45 min PM samples. Two-dimensional difference in gel electrophoreses experiments were conducted in duplicate. Differentially abundant spots were identified with MALDI-MS. Statistical analysis for $\mathrm{pH}$ and quality was done with GLM procedures of SAS version 9.4 (SAS Institute Inc., Cary, NC) and fixed effect of $14 \mathrm{~d} \mathrm{pH}$ classification. Statistical differences between $\mathrm{NpH}$ and $\mathrm{LpH}$ 
Table 1. Spots denoted as fold change $(\mathrm{NpH} / \mathrm{LpH} ; P<0.1)$.

\begin{tabular}{|c|c|c|}
\hline ID & $\mathrm{FC}$ & $P$ value \\
\hline Fructose Bisphosphate Aldolase (ALDO) (Spot $152^{1}, 156^{1}, 155^{1}, 151^{1}$ ) & $1.25,1.29,1.30,1.27$ & $0.04,0.07,0.07,0.09$ \\
\hline Pyruvate Kinase (PK) $\left(\right.$ Spot $\left.124^{1}, 121^{1}, 63^{2}\right)$ & $1.15,1.25,1.52$ & $0.10,0.05,0.08$ \\
\hline$\alpha$ 2-Heat Shock Glycoprotein (HSG) (Spot $128^{1}$ ) & 1.33 & 0.06 \\
\hline Glyceraldehyde 3-Phosphate Dehydrogenase (GAPDH) (Spot 57²) & 1.49 & 0.02 \\
\hline L-Lactate Dehydrogenase (LLDH) (Spot $\left.49^{1}\right)$ & 1.56 & 0.03 \\
\hline Heat Shock Protein 70 (HSP70) $\left(\right.$ Spot $\left.79^{1}\right)$ & 1.44 & 0.13 \\
\hline$\beta$-enolase (Spot 39²) & 1.50 & 0.08 \\
\hline$\alpha$-Actin $\left(\operatorname{Spot} 86^{1}, 41^{2}, 40^{2}, 42^{2}\right)$ & $1.93,2.07,2.32,1.76$ & $0.01,0.04,0.04,0.06$ \\
\hline
\end{tabular}

were reported as significant $(P<0.05)$. Metabolomics and proteomics were analyzed through MetaboAnalyst (Xia Lab, McGill, CA) and Melanie 9 (Cytiva, Marlborough, MA), respectively, using a Student $t$ test. Differential abundance between $\mathrm{NpH}$ and $\mathrm{LpH}$ were reported as significantly different fold changes $(\mathrm{FC}=\mathrm{NpH} / \mathrm{LpH} ; P<0.1)$.

Results: Ultimate $\mathrm{pH}$ classification did not affect $45 \mathrm{~min}$ PM pH $(P=0.64) ; 14 \mathrm{~d} \mathrm{pH}$ was different between groups $(P<0.01)$. NpH LD were darker $(\mathrm{L}: \mathrm{NpH}, 47.63 ; \mathrm{LpH}$, $50.58 ; P=0.03)$ and had less purge loss $(\mathrm{NpH}, 2.40 ; \mathrm{LpH}$, $3.84 ; P<0.01)$, lower star probe $(\mathrm{NpH}, 5.36 ; \mathrm{LpH}, 6.31$; $P<0.01)$ and less intact desmin $(\mathrm{NpH}, 1.43$; $\mathrm{LpH}, 2.36$; $P=0.06)$. Fructose 6-phosphate $(\mathrm{FC}=0.76, P=0.06)$ and lactate $(\mathrm{FC}=0.59, P=0.09)$ were greater in $\mathrm{LpH}$; glycerate 3 -phosphate $(\mathrm{FC}=1.39, P=0.11)$, pyruvate $(\mathrm{FC}=1.98$, $P=0.01)$, and malate $(\mathrm{FC}=1.59, P<0.01)$ were greater in $\mathrm{NpH}$. Proteins involved in contraction, metabolism, and heat stress response were greater in $\mathrm{NpH}$ (Table 1).

Conclusion: Subtle changes in ultimate $\mathrm{pH}$ can influence quality even when early $\mathrm{PM} \mathrm{pH}$ is not different. However, differences in the proteome and metabolome at this early time PM are associated with differences in the extent of $\mathrm{pH}$ decline, and therefore these features deserve evaluation.

Keywords: early postmortem, metabolome, pork quality, proteome

\section{7 - POTENTIAL FACTORS RELATED TO MUSCLE DEGENERATION IN WHITE STRIPING CHICKEN BREAST}

H. Jeneske ${ }^{*}$, C. $\mathrm{Li}^{1}, \mathrm{~K} . \mathrm{McMillin}{ }^{2}$, and X. Fu ${ }^{2},{ }^{1}$ School of Animal Sciences, Louisiana State University, Baton Rouge, LA, USA, ${ }^{2}$ School of Animal Sciences, Louisiana State University, Baton Rouge, LA, USA, *hjenes1@1su.edu

Objectives: White striping (WS) in chicken breast is characterized as white lines, mostly fat and collagen deposition parallel to the muscle fiber direction, that impact palatability.
Histologically, chicken breast with WS has muscle lesions, mainly due to myodegeneration as well as increase in the fibrosis and chronic lipidosis. Research has shown a correlation between a heavier, faster-growing bird and WS myopathy. Therefore, it is postulated that the myofiber regeneration ability of fast-growing birds would be diminished and damaged muscle fibers would instead be replaced with collagen and fat deposition. The fibrosis and fat deposition are fibroblasts and adipocytes that share common fibro/adipogenic progenitor (FAP) cells. FAPs are located at the stromal vascular fraction of skeletal muscle, indicating that WS formation might be correlated with the expression of this cell lineage. In this study, the alteration of expression of FAP and satellite cells and changes in muscle fiber characteristics were examined in growing chickens.

Materials and Methods: Ross 708 broiler chicks $(n=288)$ were divided into 12 pens with 24 birds in each pen. Two broilers from each pen were harvested at 2 and 4 wk of age, and M. pectorales major and M. tibialis were removed and weighed. Two subsamples were collected from each muscle. One was fixed in $4 \%$ paraformaldehyde for $4 \mathrm{~h}$ and then left in $30 \%$ sucrose overnight. The other was frozen in isopentane chilled by liquid nitrogen. All samples were stored in $-80^{\circ} \mathrm{C}$. Tissue sections were 5 micrometers thick for immunohistochemical analysis. Tissue sections were rinsed with deionized water, followed by 3 washes of TBS and $0.3 \%$ Triton (TBST). Then, sections were blocked with blocking buffer ( $5 \%$ donkey serum, $1 \%$ BSA, and TBST) for $2 \mathrm{~h}$ at room temperature and then incubated with primary antibody overnight at $4^{\circ} \mathrm{C}$. The sections were washed 3 times with TBST and incubated with secondary antibodies for $1 \mathrm{~h}$ at room temperature. The slides were rinsed in TBS for 20 min before mounting in mounting medium with DAPI. Pictures were acquired by ECHO fluorescence microscope and processed by ImageJ software.

Results: The 4-wk-old chicken muscle had a greater amount of PDGFR $\alpha$ compared to the 2-wk-old chicken muscle. In both the thigh and breast muscles, the muscle fiber diameters in the 4-wk-old chickens were much larger than in the 2-wk-old chickens. However, there appeared to be less Pax7 in the muscles of 4-wk-old chickens than in 
muscles of the 2-wk-old chickens. The PDGFR $\alpha$ and Pax7 amounts were less pronounced in M. tibialis than in M. pectorales major. The 4-wk-old chicken breast that visibly displayed WS had more amounts of PDGFR $\alpha$ with greater reduction in Pax7 than 4-wk-old chicken breast without visible WS.

Conclusion: The increased muscle cross-section would be expected to cause greater pressure on the peripheral fibers, resulting in the myopathy. The decrease in the satellite cells could decrease the capability of repairing of damaged muscle fiber. The greater expression of PDGFR $\alpha$ suggested that the damaged muscle fiber was more apparent and would be infiltrated with the collagen or fat tissue as an indication of impaired regenerative capacity.

Keywords: chicken, fibro/adipogenic progenitors, myopathy, satellite cells, white striping

\section{8 - MITOCHONDRIAL FUNCTION AND INTEGRITY IN POSTMORTEM MUSCLE OF BRAHMAN AND ANGUS STEERS}

P. Ramos ${ }^{1,2}$, B. Hawryluk ${ }^{1}$, L. Bell ${ }^{1}$, M. McKinney ${ }^{1}$, S. Wohlgemuth $^{3}$, and T. Scheffler ${ }^{1 *},{ }^{1}$ Department of Animal Sciences, University of Florida, Gainesville, FL, USA, ${ }^{2}$ Department of Animal Sciences, University of Sao Paulo, Piracicaba, Brazil, ${ }^{3}$ Department of Aging and Geriatric Research, University of Florida, Gainesville, FL, USA, *tscheffler@ufl.edu

Objectives: Mitochondria contribute to energy and heat production in muscle. Heat-tolerant Brahman may exhibit distinct mitochondria functional characteristics that subsequently impact postmortem energy metabolism and rate of beef tenderization. Our objective was to assess mitochondrial function in Angus and Brahman cattle during $24 \mathrm{~h}$ postmortem.

Materials and Methods: Steers $(n=26)$ of primarily ( $80 \%$ to $100 \%$ ) Angus or Brahman genetics were harvested at approximately $19 \mathrm{mo}$ and $570 \mathrm{~kg}$ live weight. Samples from the longissimus lumborum were collected at 1, 3, 6, and $24 \mathrm{~h}$ postmortem. Fresh-preserved muscle samples were permeabilized using saponin, and muscle bundles $(2-4 \mathrm{mg})$ were transferred to a high-resolution oxygraphy for oxygen consumption rate ( $\rho \mathrm{mol} / \mathrm{s} / \mathrm{mg}$ tissue) measurement. A stepwise protocol was initiated by addition of pyruvate, malate, and glutamate to support the TCA cycle and assess leak respiration. The second step was injection of ADP to support electron flow through complex I (PCI), followed by succinate to stimulate complex II (PCI + II). Integrity of outer mitochondrial membrane was tested by cytochrome $c$ (cyt_c) addition, using $15 \%$ as threshold for integrity. Next, titration with uncoupler (FCCP) was performed to force electron transport system to maximum capacity (ECI + II), followed by rotenone to inhibit complex I (ECII). Antimycin A was used to terminate experiment. Data were analyzed in a randomized block design, with slaughter date as block and fixed effects of breed, time, and the interaction. Random effect considered animal within slaughter date and time used as repeated measure, with covariance structure considering heterogeneous compound symmetry. Least-squares means were separated by Bonferroni test with alpha at $10 \%$.

Results: Breed and time differentially affected leak respiration (breed*time: $P=0.020$ ) with 24-h Brahman showing a greater rate than Angus. Breed and time influenced PCI (breed: $P=0.002$; time: $P<0.001$ ), with higher rates related to Brahman longissimus lumborum. Similarly, PCI + II differed between breeds (breed: $P=0.003$ ) and time (time: $P<$ $0.001)$. For electron transport system capacity, ECI + II was affected by breed (breed: $P=0.042$ ) and time (time: $P<$ 0.001 ), and pattern for ECII was similar (breed: $P=0.052$ and time: $P=0.058$ ). Based on cyt_c, mitochondria from both breeds were intact at $1 \mathrm{~h}$ (breed: $P=0.595$ and breed*time: $P=0.368$ ). However, at $3 \mathrm{~h}$ postmortem, $46 \%$ of Brahman samples showed cyt_c response lower than $15 \%$, whereas for Angus it occurred in 23\% of samples.

Conclusion: Mitochondria were intact and functioning for both breeds at $1 \mathrm{~h}$ postmortem. Greater respiration rates related to the tropically adapted Brahman during earlier times postmortem revealed greater capacity to maintain aerobic energy production. Time postmortem consistently reduced mitochondria respiration.

Keywords: beef, energy, metabolism, respirometry

\section{9 - REDUCED AND NONREDUCED PEROXIREDOXIN-2 PROFILE OF AGED PORK LOINS CLASSIFIED BY INSTRUMENTAL STAR PROBE}

L. G. Johnson ${ }^{1 *}$, M. D. Schulte ${ }^{1}$, E. M. Steadham ${ }^{1}$, K. J. Stalder $^{1}$, E. Huff-Lonergan ${ }^{1}$, and S. M. Lonergan ${ }^{1}$, ${ }^{1}$ Animal Science, Iowa State University, Ames, IA, USA, *logan01@iastate.edu

Objectives: Meat tenderness improves through degradation of myofibrillar proteins in postmortem muscle. However, one source of variation in proteolysis can be the rate and extent of tissue and protein oxidation. Peroxiredoxin is an antioxidant protein ubiquitously expressed in cells which reduces reactive oxygen species. Peroxiredoxin-2 (PRDX-2) has been identified to be more abundant in aged pork that was less tender. The objective was to compare PRDX-2 in reduced and nonreduced forms during aging to understand further the relationship of PRDX-2 to meat tenderness. It was hypothesized that changes in oxidation state and abundance of PRDX-2 postmortem aging reflect variation in proteolysis and tenderness. 
Materials and Methods: Fresh pork loins were collected at $1 \mathrm{~d}$ postmortem. Pork chops $(2.54 \mathrm{~cm})$ were fabricated and aged for $1,8,14$, or $21 \mathrm{~d}$ postmortem. After aging, chops were cooked to $68^{\circ} \mathrm{C}$, and instrumental tenderness was measured using the star probe attachment on an Instron. Star probe values were used to classify chops into high- (HSP; star probe $>7.0 \mathrm{~kg}, n=6$ ) and low- (LSP; star probe $<5.8$ $\mathrm{kg}, n=6)$ star probe groups based on 21-d analysis. Sarcoplasmic proteins from the longissimus dorsi from each aging time were solubilized in ice-cold, low-ionic strength buffer (50 mM Tris- $\mathrm{HCl}[\mathrm{pH} 8.5]$ and $1 \mathrm{mM}$ EDTA), and samples with and without a reducing agent were prepared for immunoblot analysis. Reduced and nonreduced PRDX2 and reduced intact desmin were determined using monoclonal rabbit anti-PRDX-2 antibody (ab109367; ABCam, Cambridge, UK) and polyclonal rabbit anti-desmin antibody (ISU), respectively, and normalized by a reference sample on each gel. Intact desmin and PRDX-2 were analyzed using PROC MIXED of SAS 9.4 (SAS Institute Inc., Cary, NC) with fixed effects of days aging and classification for reduced PRDX-2 and desmin. Fixed effects of days aging, classification, and migrating band were used in nonreduced PRDX-2 analysis. Significance was denoted with $P<0.05$.

Results: There was less $(P<0.05)$ intact desmin at 14 and $21 \mathrm{~d}$ in LSP compared to HSP. In LSP chops, reduced PRDX-2 decreased between 1 and $8 \mathrm{~d}(P<0.05)$ but did not change after $8 \mathrm{~d}$ of aging. In the HSP chops, reduced PRDX2 decreased between 1 and $14 \mathrm{~d}(P<0.05)$. The LSP group had less $(P<0.05)$ PRDX-2 at 8 and $21 \mathrm{~d}$ compared with HSP. In nonreduced gels, PRDX-2 was identified by 3 distinct bands. The 2 slower migrating bands changed similarly during aging regardless of classification, where bands 1 and 2 decreased between 1 and $8 \mathrm{~d}$ and increased between 14 and $21 \mathrm{~d}$ of aging. Bands 1 and 2 were not different between 1 and $21 \mathrm{~d}$. Conversely, band 3 increased $(P<0.05)$ between 1 and $8 \mathrm{~d}$ and 8 and $14 \mathrm{~d}$ of aging but decreased from 14 to $21 \mathrm{~d}$ of aging. At $21 \mathrm{~d}$, the third band in the LSP had less $(P<0.05)$ PRDX-2 compared to HSP.

Conclusion: Reduced PRDX-2 decreases at a variable rate with postmortem aging based on differences in tenderness classification. In a nonreduced state, bands 1 and 2 changed inversely compared to band 3 , suggesting a transition of oxidation state during postmortem aging. The identification of the modifications between the 3 bands is essential to further interpret the relationship between PRDX-2 and meat tenderness. The change in PRDX-2 is dynamic and appears to be altered during postmortem aging and related to the proteolysis of desmin and the development of tenderness.

Keywords: nonreducing, peroxiredoxin-2, pork tenderness, reducing

\section{0 - NONDESTRUCTIVE SEGREGATION OF COLOR-LABILE AND COLOR-STABLE BISON MUSCLES USING NEAR-INFRARED SPECTROSCOPY}

M. M. Hasan ${ }^{1 *}$, M. M. A. Chaudhry ${ }^{2}$, C. Erkinbaev ${ }^{2}$, J. Paliwal $^{2}$, S. Suman ${ }^{3}$, and A. Rodas-Gonzalez ${ }^{4},{ }^{1}$ Food and Human Nutritional Sciences, University of Manitoba, Winnipeg, Canada, ${ }^{2}$ Biosystems Engineering, University of Manitoba, Winnipeg, Canada, ${ }^{3}$ Animal and Food Sciences, University of Kentucky, Lexington, KY, USA, ${ }^{4}$ Animal Science, University of Manitoba, Winnipeg, Canada,*hasanmm@myumanitoba.ca

Objectives: The objective of the study was to investigate the potential of visible near-infrared (Vis-NIR) and short wave near-infrared (SWIR) spectroscopy for the segregation of bison muscles longissimus lumborum (color-stable) and psoas major (color-labile) based on muscle type and storage period.

Materials and Methods: Ten longissimus lumborum (striploins) and 10 psoas major (tenderloins) muscles from A1 grade bison carcasses were collected within $48 \mathrm{~h}$ postmortem. Each muscle portion was subsampled for the determination of $\mathrm{pH}$, malondialdehyde, 4-hydroxy-2-nonenal, and protein carbonyl contents, prior to which hyperspectral images (HSI) in the Vis-NIR and SWIR regions were acquired for $2 \times 2 \mathrm{~cm}$ steaks. The remaining muscle samples were divided into 2 parts and stored at $2^{\circ} \mathrm{C}$ for an aging period of 7 and $14 \mathrm{~d}$. At the end of each aging period, 2.5 -cm-thick steaks were obtained, and HSI were acquired followed by the determination of instrumental $\left(L^{*}, a^{*}\right.$, and $\left.b^{*}\right)$ color, sensory color (color and discoloration score), purge loss, $\mathrm{pH}$, malondialdehyde, 4-hydroxy-2-nonenal, and protein carbonyl contents. Steaks were further used for HSI acquisition after the retail display period of $5 \mathrm{~d}$, followed by determination of the aforementioned parameters. Principal component analysis was used as an unsupervised classification approach for the segregation of bison muscles based on muscle type and storage period.

Results: Prior to the application of the principal component analysis, the spectral data in the Vis-NIR and SWIR ranges were mean-centered. In case of the Vis-NIR range, principal component 1 (PC1) explained $64.98 \%$ variance in the data and depicted groupings based on the muscle type. The loading plots portrayed a major peak starting from $580 \mathrm{~nm}$ to $650 \mathrm{~nm}$, which represents the red region of the Vis-NIR range. Hence, this wavelength region was significant for the discrimination of muscles based on $a^{*}$ values. On the other hand, PC2 (27.50\%) provided a clear discrimination between fresh and aged samples (considered as one class) against samples belonging to retail day 5 with the loading plots portraying major peaks around $650 \mathrm{~nm}$ to $700 \mathrm{~nm}$. The segregation based on the muscle type in the SWIR region was better portrayed by PC3, which explained $7.46 \%$ of variance in the data with significant loading peaks at $1,100 \mathrm{~nm}$ to $1,300 \mathrm{~nm}$. This region is related to the water and fat absorption bands, therefore the muscle type 
discrimination can be attributed to these factors. Moreover, PC1 (68.88\%) effectively discriminated the fresh and aged (considered as one class) against the retail display samples with major loading peaks at $1,100 \mathrm{~nm}, 1,200 \mathrm{~nm}$, and $1,400 \mathrm{~nm}$, which is also a water absorption band. Therefore, the moderate discrimination based on the muscle type and storage period was acquired with effective contribution from the red region in the Vis-NIR range. In case of the SWIR range, water and fat absorption bands were the major contributors towards achieving good discrimination based on the aforementioned categories.

Conclusion: The Vis-NIR range discriminated the bison muscles based on storage time effectively compared to the SWIR range. On the other hand, the SWIR range served better for the segregation of the 2 muscle types. These results obtained from unsupervised classification are encouraging for further processing of the spectral data using supervised classification algorithms.

Keywords: bison, color stability, principal component analysis, short wave near-infrared spectroscopy, visible nearinfrared spectroscopy

\section{1 - PRINCIPAL COMPONENT ANALYSIS OF THE MULTIVARIATE RELATIONSHIP BETWEEN OXIDATION PRODUCTS AND COLOR ATTRIBUTES IN BISON LONGISSIMUS LUMBORUM AND PSOAS MAJOR MUSCLES}

M. M. Hasan ${ }^{1 *}$, V. Sood ${ }^{1}$, C. Erkinbaev ${ }^{2}$, J. Paliwal ${ }^{2}$, S. Suman $^{3}$, N. Prieto ${ }^{4}$, and A. R. Rodas-Gonzalez ${ }^{1},{ }^{1}$ Food and Human Nutritional Sciences, University of Manitoba, Winnipeg, Canada, ${ }^{2}$ Biosystems Engineering, University of Manitoba, Winnipeg, Canada, ${ }^{3}$ Animal and Food Sciences, University of Kentucky, Lexington, KY, USA, ${ }^{4}$ Lacombe Research and Development Centre, Agriculture and Agri-Food Canada, Lacombe, Canada, *argenis. rodasgonzalez@umanitoba.ca

Objectives: Fresh bison meat color deteriorates promptly under retail aerobic packaging conditions and oxidation products are originated; however, the role of different oxidation products in bison meat color stability has not yet been evaluated. A principal component analysis was used to examine the multivariate relationship between lipid (malondialdehyde [MDA], 4-hydroxy-2-nonenal [HNE]), and protein (carbonyl content [CAR]) oxidation products and color attributes (objective and subjective) in bison longissimus lumborum (LL) and psoas major (PM) muscles.

Materials and Methods: A total of $10 \mathrm{LL}$ and $10 \mathrm{PM}$ from 5 A1-grade bison carcasses were obtained from a commercial plant within $48 \mathrm{~h}$ postmortem. The muscles were cut into 2 equal portions, vacuum-packaged, and randomly assigned to an aging period of 7 and $14 \mathrm{~d}$ at $2^{\circ} \mathrm{C}$. At the end of each aging period, muscle portions were removed from the packages, $\mathrm{pH}$ was measured, and $2.5-\mathrm{cm}$-thick steaks were obtained for sensory (muscle color and discoloration scores), instrumental $\left(L^{*}, a^{*}\right.$, and $\left.b^{*}\right)$ color measurements, and protein and lipid oxidation analyses. After $5 \mathrm{~d}$ in retail display, color and $\mathrm{pH}$ were measured, and the steaks were removed for subsequent protein and lipid oxidation determination. Principal component analysis was performed on oxidation compounds and color traits to identify the influence of the measured attributes.

Results: Correlation coefficients revealed strong relationship of MDA with $a^{*}$, color and discoloration scores (r or rs $>70 ; P<0.01$ ), followed by a moderate correlation between HNE and CAR ( $\mathrm{r}$ or rs $<0.70 ; P<0.01$ ). The factor analysis showed that the first 2 principal components (PC) with eigenvalues greater than 1 explained $73.09 \%$ of the standardized variance ( $\mathrm{PC} 1$ explained $58.48 \%$, and $\mathrm{PC} 2$ $14.61 \%)$. While the set of variables for the first PC included mainly MDA, HNE, CAR, $a^{*}, b^{*}$, and color and discoloration scores (based on the largest loading values), the PC2 was related to $\mathrm{pH}, L^{*}$, and $b^{*}$. Results showed clear segregation between steaks displayed day 0 and day 4 (regardless of the muscle and aging time), where PM and LL steaks aged for 7 and $14 \mathrm{~d}$ at day 0 of retail display were closely associated with redness and yellowness traits, and located far away of oxidation compounds and scores, indicating more red color stability and less oxidation. In contrast, steaks at day 4 of the retail display were closely associated with oxidation compounds and scores. Noticeably, 3 distinct groups within steaks displayed at day 4 were identified according to the muscle and aging time. A first group was represented by PM steaks aged for 7 and $14 \mathrm{~d}$ with more oxidation compounds and high scores (representing high oxidation level and less red color). The second (close to the central axis) and third group (negative side of PC2) of steaks displayed at day 4 were represented by LL steaks aged for 7 and $14 \mathrm{~d}$, respectively, showing different levels of color deterioration on LL muscle based on aging period.

Conclusion: These results indicate that color attributes are moderate to strongly influenced by the lipid and protein oxidation compounds in bison meat. Content of individual lipids and protein oxidation compounds were positively associated with color and discoloration scores but negatively associated with $a^{*}$ and $b^{*}$.

Keywords: bison, carbonyl content, color stability, discoloration mechanism, malondialdehyde

\section{2 - PEPTIDOMIC ANALYSIS OF PORCINE LIVER HYDROLYSATES USING SWATH-MS TO SEARCH BIOPEPTIDES}

M. Lopez-Pedrouso ${ }^{1}$, P. Borrajo ${ }^{2}$, D. Franco ${ }^{2}$, and J. M. Lorenzo ${ }^{3 *},{ }^{1}$ Department of Zoology, Genetics and 
Physical Anthropology, University of Santiago de Compostela, Santiago de Compostela, Galicia, Spain, ${ }^{2}$ Food Science and Technology, Centro Tecnológico de la Carne de Galicia, Galicia, Spain, ${ }^{3}$ Food Science and Technology, Centro Tecnologico de la Carne, Ourense, Spain,*jmlorenzo@ceteca.net

Objectives: Bioactive peptides are of great interest from a biotechnological point of view, and there is a major concern about their identification and isolation. Peptidomic analysis using SWATH-MS offers a great opportunity to identify and quantify the biopeptides in a complex mixture. On the other hand, protein-rich meat byproducts and enzymatic hydrolysis are being tested to obtain biopeptides with antioxidant, antidiabetic, or antimicrobial activity. Specifically, the porcine liver hydrolysates have proved to have antioxidant and antimicrobial activity, resulting in an effective method to produce added-value products and functional ingredients. Additionally, the use of pork liver and other offal contributes to the sustainability of meat industry.

Materials and Methods: Livers were cleaned of fat and connective tissues and cut into small pieces. The homogenization was carried out with ice in a cutter machine. Afterwards, enzymatic hydrolysis was performed using 4 different enzymes: papain, bromelain, Alcalase, and Flavourzyme. The conditions of enzymatic reaction were $37^{\circ} \mathrm{C}$ and $\mathrm{pH} 6$ for papain, $40^{\circ} \mathrm{C}$ and $\mathrm{pH} 6$ for bromelain, $50^{\circ} \mathrm{C}$ and $\mathrm{pH} 8$ for Alcalase, and $50^{\circ} \mathrm{C}$ and $\mathrm{pH} 5.5$ for Flavourzyme. To analyze the peptides by mass spectrometry, digestion of peptide mixtures with trypsin was performed. Twelve replicates were realized for each enzymatic treatment. A shotgun data-dependent acquisition approach using micro-liquid chromatography-mass spectrometry was obtained prior to peptide quantification. Finally, a data-independent acquisition from SWATH-MS analysis was used for peptide quantification for peptide mixtures.

Results: A total of 2,022 peptides showed quantitative significant differences between control and hydrolysates from enzymatic digestions $(P<0.05)$. This showed that liver proteins were largely digested by papain, bromelain, Alcalase, and Flavourzyme, indicating that these hydrolysates are an adequate choice to search biopeptides. To develop this approach, we profiled peptide mixtures from enzyme treatments, and the profiles were compared by principal component analysis. This analysis allowed us to reduce a large number of peptide quantifications of the dataset into a smaller number of dimensions without losing significant information. As expected, the principal component analysis reflected that the control samples have a peptide profile quite different from the rest. This suggests that the number of peptides in enzyme samples was far higher due to enzymatic degradation. On the other hand, the porcine liver hydrolysates from Alcalase were particularly different with respect to the other enzymes, thereby generating distinguishing peptides by Alcalase. Among enzymatic treatments, 2 different groups of peptides were found to be differentiated.

Conclusion: Therefore, the Alcalase hydrolyzation or the others may be used to search biopeptides with the required activity and subsequent isolation.

Acknowledgments: This research received external funding by Grant RTA RTA2017-00081-C04-00 from INIA (Spain). José M. Lorenzo and Daniel Franco are members of the HealthyMeat network, funded by CYTED (reference 119RT0568). Thanks to GAIN (Axencia Galega de Innovación) for supporting this research (Grant Number IN607A2019/01).

Keywords: Alcalase, antioxidant and antimicrobial activity, bioactive peptides, meat byproducts, SWATH-MS analysis

\section{3 - POSTMORTEM ENERGY METABOLISM IN LONGISSIMUS LUMBORUM OF BRAHMAN AND ANGUS STEERS}

\section{Gingerich ${ }^{1 *}$, D. Jean ${ }^{1}$, E. Hicks ${ }^{1}$, B. Hawryluk ${ }^{1}$, L. Bell ${ }^{1}$, M. McKinney ${ }^{1}$, P. Ramos ${ }^{1,2}$, and T. Scheffler ${ }^{1},{ }^{1}$ Department of Animal Sciences, University of Florida, Gainesville, FL, USA, ${ }^{2}$ Department of Animal Sciences, University of Sao Paulo, Piracicaba, Brazil, *tscheffler@ufl.edu}

Objectives: During the first several hours postmortem, the biochemical and energetic status of muscle shifts considerably. These changes influence the development of meat quality attributes and may contribute to variation in tenderization rate between Brahman and Angus. The objective of this study was to determine muscle $\mathrm{pH}$ and temperature decline, glycolysis, and energy status in these breeds.

Materials and Methods: Steers of primarily Angus or Brahman genetics $(80 \%-100 \% ; n=14$ per breed) were reared together and harvested at the University of Florida Meat Lab. Temperature and $\mathrm{pH}$ decline of the longissimus lumborum (LL) were evaluated at 1, 3, 6, 9, and 24 h postmortem, and samples of the LL were collected at 1, 3,6, and $24 \mathrm{~h}$ postmortem. Muscle samples were immediately frozen in liquid nitrogen and stored at $-80^{\circ} \mathrm{C}$ until further analysis. For metabolite analysis, LL was powdered in liquid nitrogen and diluted in perchloric acid (phosphocreatine; ATP; glucose; glucose 6-phosphate; lactate) or hydrochloric acid (glycogen). Then, samples were homogenized with ceramic beads; those diluted in perchloric acid were centrifuged, and supernatant was collected for analysis. Samples for the glycogen assay were heated at $100^{\circ} \mathrm{C}$ for $2 \mathrm{~h}$ to hydrolyze glycogen to glucose and centrifuged, and the supernatant was collected. Glycogen, glucose, glucose 6-phosphate, and lactate were quantified at all time points, whereas ATP was determined at 1,3 , and $6 \mathrm{~h}$ and phosphocreatine at $1 \mathrm{~h}$. Metabolites were quantified using enzymatic methods. 
Data were analyzed using SAS (SAS Institute Inc., Cary, NC), and the model included the fixed effects of breed, time, and their interaction. Time was considered a repeated measure.

Results: The decline in muscle $\mathrm{pH}$ postmortem tended to differ between breeds (breed $\times$ time, $P=0.07$ ). Brahman LL exhibited higher $\mathrm{pH}$ at 6 and $9 \mathrm{~h}(P=0.02)$. Temperature decline postmortem also exhibited distinct patterns between breeds (breed $\times$ time, $P=0.0008$ ), with Brahman LL being lower at 1, 3, 6, and $9 \mathrm{~h}$ postmortem. As expected, time influenced glycolytic metabolites (glycogen, glucose, glucose 6-phosphate, and lactate; $P<0.0001$ ), but pattern between breeds was similar. Glycogen declined from 1 to $24 \mathrm{~h}$ postmortem, whereas glucose and lactate increased. Breed tended to influence phosphocreatine content at $1 \mathrm{~h}(P=0.097)$, with higher values observed in Brahman LL. Both breed $(P=0.02)$ and time $(P<0.0001)$ impacted ATP. Breeds exhibited similar rates of ATP decline; however, ATP in Brahman LL was generally higher, particularly at $1 \mathrm{~h}(P<0.01)$.

Conclusion: Breeds exhibited similar changes in glycolytic metabolites postmortem. However, Brahman LL maintained improved energy status early postmortem, which may be due to increased capacity to generate ATP using pathways other than anaerobic glycolysis, or alternatively, decreased ATP utilization. This may contribute to protracted $\mathrm{pH}$ decline in Brahman LL. These results will be used in conjunction with proteolysis markers and mitochondrial function analyses to better understand meat quality development in Brahman and Angus.

Keywords: Brahman, glycolysis, $\mathrm{pH}$, postmortem metabolism, temperature

\section{4 - METHODS FOR THE PARTIAL PURIFICATION OF PEROXIREDOXIN-2 IN PORCINE SKELETAL MUSCLE}

L. G. Johnson ${ }^{1 *}$, E. M. Steadham ${ }^{1}$, E. Huff-Lonergan ${ }^{1}$, and S. M. Lonergan ${ }^{1},{ }^{1}$ Animal Science, Iowa State University, Ames, IA, USA, *logan01@iastate.edu

Objectives: Peroxiredoxins are a family of antioxidant proteins ubiquitously expressed in cells and reduce reactive oxygen species, primarily that are $\mathrm{H}_{2} \mathrm{O}_{2}$. Peroxiredoxins exist as a homodimer with 2 identical subunits involved in the active site of the protein. The catalytic cycle of peroxiredoxins involves a peroxidatic cysteine residue which is oxidized to a sulfenic acid. The peroxidatic cysteine can be further oxidized to a sulfinic acid or inactivated to a sulfonic acid. In postmortem skeletal muscle, peroxiredoxin, specifically peroxiredoxin-2 (PRDX-2), is hypothesized to play a critical role in protecting against oxidative damage by reactive oxygen species. In order to test this hypothesis, it is necessary to use purified PRDX-2 in controlled benchtop experiments. Thus, the objective was to define a method to partially purify PRDX-2 from skeletal muscle.

Materials and Methods: Porcine psoas major, diaphragm, and longissimus dorsi were collected at about $45 \mathrm{~min}$ postmortem. Muscles were immediately trimmed of visible fat and connective tissue, minced, and stored in $-80^{\circ} \mathrm{C}$. Each muscle $(20 \mathrm{~g})$ was homogenized in 3 volumes of ice-cold, low-ionic-strength buffer $(50 \mathrm{mM}$ Tris-HCl $[\mathrm{pH}$ $8.5]$ and $1 \mathrm{mM}$ EDTA) and centrifuged at $10,000 \times g$ for $30 \mathrm{~min}$ at $4^{\circ} \mathrm{C}$. The supernatant was filtered through cheesecloth and dialyzed against 40 volumes of $40 \mathrm{mM}$ Tris- $\mathrm{HCl}$ (pH 7.4), $1 \mathrm{mM}$ EDTA, and $0.1 \%$ 2-mercaptoethanol (TEM pH 7.4). Dialyzed extracts were clarified by centrifugation and filtration. Clarified dialysates were loaded onto separate $20 \mathrm{~mL}$ Q-Sepharose Fast Flow anion exchange columns, equilibrated with TEM $\mathrm{pH}$ 7.4. Columns were washed, and proteins were eluted with a linear gradient of 75 to $500 \mathrm{mM} \mathrm{NaCl}$ in TEM pH 7.4. PRDX-2 eluted in all muscles from approximately 75 to $160 \mathrm{mM} \mathrm{NaCl}$ as confirmed by immunoblot analysis. Monoclonal rabbit antiPRDX-2 antibody (ab109367; ABCam, Cambridge, UK) was used to detect PRDX-2 abundance. Immunoreactive fractions were pooled, dialyzed against $40 \mathrm{vol}$ of TEM pH 8.0 , and clarified by centrifugation and filtration. Clarified dialysates were loaded separately on a DEAE-TSK equilibrated with TEM pH 8.0. Columns were washed and eluted with a linear gradient of 0 to $500 \mathrm{mM} \mathrm{NaCl}$ in TEM pH 8.0. PRDX-2 eluted from approximately 35 to $120 \mathrm{mM} \mathrm{NaCl}$ as confirmed by immunoblot analysis. Immunoreactive fractions were pooled, dialyzed against 40 vol of TEM $\mathrm{pH}$ 8.0, and clarified by centrifugation and filtration. Protein concentration of the resulting dialysates was determined by Bradford analysis. The purity of the dialysates was analyzed by resolving with a $15 \%$ SDS-PAGE gel and visualized by Silver staining. Images were captured using a ChemiImager 5500, and whole lane analysis was conducted with Alpha Ease FC software. The percent purity of the extract was measured by comparing the densitometry of the $22 \mathrm{kDa}$ PRDX- 2 band to the sum of the total area of individual bands within the lane.

Results: The purity of the muscle extracts was determined to be approximately $45 \%-50 \%$ purity in the longissimus dorsi and psoas major samples and approximately $30 \%$ purity in the diaphragm sample.

Conclusion: To our knowledge, this is the first report of a method to partially purify PRDX-2 from skeletal muscle. The methods here describe provide a viable method for the partial purification of PRDX-2 in postmortem skeletal muscle to utilize in controlled in vitro experiments.

Keywords: column chromatography, peroxiredoxin-2, protein purification 


\section{5 - AN INVESTIGATION OF COLLAGEN CHARACTERISTICS AND COLLAGENASE ACTIVITY IN WOODY BREAST MEAT}

\author{
R. Maurer ${ }^{1 *}$, A. Welter ${ }^{1}$, W. J. Wu ${ }^{1}$, M. Chao ${ }^{1}$, B. Bowker ${ }^{2}$, \\ and H. Zhuang ${ }^{2}$, ${ }^{1}$ Animal Sciences and Industry, Kansas \\ State University, Manhattan, KS, USA, ${ }^{2}$ U.S. National \\ Poultry Research Center, USDA, Athens, GA, USA, \\ *mdchao@ksu.edu
}

Objectives: Woody breast is a relatively new quality issue in the broiler industry. Breast meat with this myopathy exhibits tough and rubbery texture. Greater collagen content is observed in woody breast meat, but the relationship between the collagen characteristics and the abnormal texture of woody breast meat has not been delineated. Therefore, the objective of this study was (1) to characterize collagen properties and (2) to assess native collagenase activity of normal and woody breast meat.

Materials and Methods: A total of fourteen Ross line chicken breast fillet samples ( 7 severe woody breast and 7 normal) were collected, packaged, and frozen at approximately $8 \mathrm{~h}$ postmortem. The cranial region of each breast was pulverized in liquid nitrogen and used to measure collagen content (hydroxyproline content), mature collagen crosslink density (pyridinoline and deoxypyridinoline concentration using liquid chromatography with a fluorescence detector), collagenase activity (gelatin-zymography measuring MMP2 activity), and pH. Warner-Bratzler shear force (WBSF) of cooked breast meat and perimysial thermal stability (differential scanning calorimeter) were conducted using the middle and caudal region of the breast meat.

Results: Woody breast meat had greater collagen content (3.89 vs. $2.08 \mathrm{mg}$ collagen/g muscle; $P<0.01)$, pyridinoline density $(0.23$ vs. $0.14 \mathrm{~mol} / \mathrm{mol}$ collagen; $P<0.05)$, deoxypyridinoline density ( 0.07 vs. $0.04 \mathrm{~mol} / \mathrm{mol}$ collagen; $P<0.01$ ), and perimysial thermal stability (peak denaturation temperature: $65.47^{\circ} \mathrm{C}$ vs. $\left.63.72^{\circ} \mathrm{C} ; P<0.05\right)$ than normal breast meat. In addition, woody breast meat had more collagenase activity (13.24\% vs. $7.84 \%$ active MMP-2; $P<0.05)$ and a greater $\mathrm{pH}(6.17$ vs. $5.83 ; P<0.01)$ than normal breast meat. Although significant differences in collagen characteristics were detected between woody breast and normal meat, WBSF did not differ between woody breast and normal meat (4.33 vs. $5.97 \mathrm{kgf}$, respectively; $P>0.10$ ). It should be noted that other studies reported similar findings with varying WBSF values, which indicates that WBSF may not be a suitable assessment method of woody breast meat texture.

Conclusion: These results indicated that woody breast meat had greater collagen concentration and mature collagen crosslink densities compared to normal breast meat, which may have resulted in the observed increase in perimysial thermal stability and the abnormal palpable hardness of the fillets. On the other hand, a greater collagenase activity was found in woody breast meat compared to normal breast meat, which was likely enhanced by the greater free calcium content and the more neutral $\mathrm{pH}$ found in woody breast meat. Additional research linking collagen characteristics and collagenase activity with the observed dissipation of woody breast meat over storage (Byron et al., 2019) is needed to develop better postmortem handling strategies to minimize the impact of this quality defect.

Keywords: collagen, collagen crosslinks, collagenase activity, differential scanning calorimetry, woody breast

\section{6 - AN INVESTIGATION OF BIOCHEMICAL FACTORS AFFECTING ASIAN CONSUMERS PREFERENCE FOR STEWED GOAT MEAT}

E. LaRoche ${ }^{1 *}$, W. J. Wu ${ }^{1}$, C. Chun ${ }^{1}$, A. Welter ${ }^{1}$, C. Jones ${ }^{1}$, A. Crane ${ }^{1}$, T. Houser ${ }^{2}$, T. O'Quinn ${ }^{1}$, and M. Chao ${ }^{1},{ }^{1}$ Animal Sciences and Industry, Kansas State University, Manhattan, KS, USA, ${ }^{2}$ Animal Sciences and Industry, Iowa State University, Ames, IA, USA, *mdchao@ksu.edu

Objectives: Many Asian cultures enjoy stewed goat meat cubes (skin-on or -off) because of its unique flavor and texture. With the growing Asian population in the US, there is potential to grow the goat meat market to meet the new demand. Therefore, the objective of this study was to investigate biochemical factors that influence Asian consumers' palatability preferences for stewed goat meat.

Materials and Methods: A total of 14 Boer goats averaging $35.6 \pm 2.96 \mathrm{~kg}$ were harvested either skin-on $(n=7)$ or -off $(n=7)$. The carcasses were fabricated into 4 primalsloin, shoulder, leg, and breast—and each primal, regardless of harvest technique, was cut into $5 \mathrm{~cm} \times 5 \mathrm{~cm}$ cubes. Fiftythree Asian consumers were recruited from Manhattan, Kanas, and evaluated tenderness, juiciness, flavor, connective tissue amount, fat amount, and overall liking of the stewed goat meat from the 8 treatments. Collagen, moisture, and lipid content; cook loss; and $\mathrm{pH}$ were also measured in this study. Finally, correlation analysis was conducted to determine the driving factors that contributed to Asian consumers' preference for goat meat.

Results: Panelists found that meat cubes from breast tended to have more connective tissue than meat cubes from other primals $(P=0.08)$. They also determined that meat cubes from shoulder and breast were more tender and juicier than meat cubes from loin and leg $(P<0.01)$. Panelists indicated that meat cubes from breast had the most fat followed by shoulder, loin, and leg $(P<0.01)$. Panelists rated meat cubes from the shoulder with the highest overall liking score compared to those from the other primals $(P<0.01)$. Finally, meat cubes from breast had less cook loss percentage compared to those from the other primals $(P<0.01)$. It was interesting to note that Asian consumers determined that meat 
cubes with skin-on were more tender, juicier, and had more fat than those without skin $(P<0.05)$. However, treatments of skin-on versus skin-off did not differ in consumer overall liking $(P>0.10)$. Meat cubes from shoulder and breast had higher $\mathrm{pH}$ than meat cubes from leg and loin. There was an inverse relationship between moisture and lipid content. Meat cubes from leg had the greatest amount of moisture, followed by loin and shoulder, with breast with the least $(P<0.01)$, whereas lipid content followed the exact opposite trend $(P<0.01)$. Meat cubes from breast and loin had more collagen than meat cubes from leg $(P<0.01)$, while meat cubes from shoulder was not different from any of the primals. In addition, meat cubes with skin-on had more collagen content than those with skin-off $(P<0.01)$. Lipid percentage had a positive correlation with overall tenderness, juiciness, flavor, amount of fat, and most importantly, overall liking $(P<0.05)$. As expected, $\mathrm{pH}$ also had a positive correlation with overall tenderness and juiciness $(P<0.01)$. To our surprise, collagen content did not exhibit a relationship with any of parameters measured in the consumer panel.

Conclusion: This study provided a missing knowledge gap that shed light on Asian consumers' eating preferences of goat meat. Asian consumers preferred goat shoulder primal with skin-on over the other treatments. Meat cuts with greater lipid percentage and $\mathrm{pH}$ were found to be the more important palatability traits to the Asian consumers, while collagen content did not seem to play a large role in Asian consumers' overall liking.

Keywords: Asian consumers, collagen, goat, pH, skin-on processing

\section{7 - COMPARATIVE TRANSCRIPTOMIC PROFILE FOR MEAT TENDERNESS IN A MULTIBREED ANGUS-BRAHMAN POPULATION}

J. C. C. Balieiro ${ }^{1 *}$, J. L. Gutierrez ${ }^{1}$, V. R. Paschoal $^{1}$, C. Carr ${ }^{2}$, M. A. Elzo ${ }^{1}$, and R. G. Mateescu ${ }^{1},{ }^{1}$ Animal Sciences Department, University of Florida, Gainesville, FL, USA, ${ }^{2}$ Animal Sciences Department, University of Sao Paulo, Gainesville, United States, *balieiro@usp.br

Objectives: The aim of this study was to evaluate the transcriptome profile of Longissimus dorsi muscle from multibreed Angus-Brahman cattle with divergent phenotypes for meat tenderness.

Materials and Methods: Animals from 4 breed groups (BG) from the University of Florida multibreed AngusBrahman herd were used: BG1 $=80 \%>100 \%$ Angus; $\mathrm{BG} 2=65 \%>79.0 \%$ Angus; $\mathrm{BG} 3=40 \%>59 \%$ Angus; and BG4 $=0.0 \%>19 \%$ Angus. Two extreme groups were identified within each BG based on the Warner-Bratzler shear force (WBSF) values (LOW and HIGH). A total of 47 animals slaughtered in 2016 and 2017 with Longissimus dorsi muscle RNA-Seq data were used to identify differentially expressed genes (DEG). A Generalized Mixed Linear model was used to identify DEG, assuming link function with negative binomial distribution. The model included the fixed effects of the BG, WBSF class, BG $\times$ WBSF class interaction, and the random effects of birth year. The DEG annotation was performed using Ensembl Release 99 file, and the Database for Annotation, Visualization and Integrated Discovery 6.8 was used to identify Gene Ontology terms and pathways with significant enrichment scores. A false discovery rate $<10 \%$ was used for DEG and Gene Ontology analyses.

Results: The WBSF LSMeans and standard deviation for the LOW groups within each $\mathrm{BG}$ were as follows: $2.77 \pm 0.24 \mathrm{~kg}(\mathrm{BG} 1, n=8), 3.11 \pm 0.28 \mathrm{~kg}(\mathrm{BG} 2, n=5)$, $3.12 \pm 0.47 \mathrm{~kg}(\mathrm{BG} 3, n=7)$, and $3.22 \pm 0.55 \mathrm{~kg}$ (BG4, $n=$ 5). The WBSF LSMeans and standard deviation for the HIGH groups within each BG were as follows: $5.00 \pm$ $0.62 \mathrm{~kg}(\mathrm{BG} 1, n=4), 5.04 \pm 0.78 \mathrm{~kg}(\mathrm{BG} 2, n=5), 5.41 \pm$ $0.36 \mathrm{~kg}(\mathrm{BG} 3, n=7)$, and $6.00 \pm 0.50 \mathrm{~kg}(\mathrm{BG} 4, n=6)$. There were no significant $\mathrm{BG} \times \mathrm{WBSF}$ class interaction effects. There were 266 significant DEG for the main effect of BG. Regression analysis of the transcripts counts on Angus percentage showed that 129 of the 266 DEG had a linear reduction in expression as the percentage of Angus increased from $0 \%$ to $100 \%$, and 135 DEG had a linear increase in expression as the percentage of Angus increased. For the main effect of $\mathrm{BG}$, the most significant pathways were related with general metabolism $\left(P=7.81 \times 10^{-8}\right)$, longevity regulating $\left(P=1.26 \times 10^{-4}\right)$, and proton pump inhibitor $\left(P=1.48 \times 10^{-4}\right)$. There were 2,038 significant DEG for the WBSF class main effect. A correlations analysis between WBSF values and transcript counts identified 353 and 47 DEG with correlation higher than -0.50 and 0.5 , respectively. The most significant pathways for the WBSF class main effect DEG were related with transport to the Golgi and subsequent modifications $\left(P=1.91 \times 10^{-12}\right)$, vesiclemediated Transport $\left(P=4.14 \times 10^{-10}\right)$, and Metabolism of Proteins $\left(P=1.34 \times 10^{-9}\right)$.

Conclusion: Our results suggest that (i) effect of WBSF class acts independently of breed composition, (ii) there are significant differences in transcript count between BG, and (iii) there are significant differences in transcript count between the LOW and HIGH WBSF groups.

Keywords: beef quality, RNA profile, RNA-Seq, WarnerBratzler shear force

\section{8 - DIFFERENTIAL BEEF PROTEOMIC ANALYSIS BETWEEN STEERS AND BULLS}

L. A. de Oliveira Stella ${ }^{1}$, M. D. Poleti ${ }^{2}$, H. B. dos Reis ${ }^{2}$, A. G. Vignato do Barco ${ }^{2}$, J. Pereira Eler ${ }^{2}$, and J. C. de Carvalho Balieiro $^{1 *}$, Department of Animal Nutrition and 
Production, School of Veterinary Medicine and Animal Science - University of São Paulo, Brazil, ${ }^{2}$ Department of Veterinary Medicine, Faculty of Animal Science and Food Engineering - University of São Paulo, Pirassununga, Brazil, *mirelep@usp.br

Objectives: The study aims to identify differentially abundant proteins between 2 sexual conditions (steers and bulls) in Nelore cattle and to associate them with beef quality traits and metabolites.

Materials and Methods: The analysis of beef quality (color, $\mathrm{pH}$, cooking losses, Warner-Bratzler shear force at 1,7 , and $14 \mathrm{~d}$ postmortem) and muscle metabolites (lactate and glycogen at 1 and $24 \mathrm{~h}$ postmortem) were carried out in Longissimus thoracis samples from a population of 200 Nelore cattle, 100 steers (castrated), and 100 bulls (noncastrated). The animals were finished in the feedlot and slaughtered at 24 mo old and live weight $505 \mathrm{~kg}$. The carcasses were chilled, and Longissimus thoracis muscle samples were excised at $24 \mathrm{~h}$ after slaughter. We used samples from 6 animals of each sexual condition $(N=12)$ chosen randomly to carried out the proteomic analysis. Total proteins were extracted by homogenizing these samples in lysis buffer (8 M urea, $2 \mathrm{M}$ thiourea, $1 \%$ DTT, $2 \%$ CHAPS, and $1 \%$ protease inhibitor cocktail), vortexing, and centrifuging. The protein concentrations of the supernatant were determined. The proteins were digested by trypsin, and the mass spectra of the peptide fragments were acquired by the bidimensional nanoUPLC tandem nanoESI-HDMS ${ }^{\mathrm{E}}$ technology system. Mass spectrometry data were processed using ProteinLynx Global Server version 2.5.1 (PLGS, Waters Corporation) software platform. False positive discovery rate of the identification algorithm was set to $4 \%$. For statistical analysis, only proteins present in at least 3 of the 6 biological repetitions were considered $(N=198)$ to detect differentially abundant proteins. Variance analyses (analysis of variance) were performed using a mixed linear model, including the fixed effect of sexual condition and origin and slaughter data as random effects. Correlations were estimated by Pearson's correlation coefficient $(r)$ and considered significant at $P<0.05$.

Results: A total of 605 proteins were identified. Of these, 330 were common between groups, 139 were found only in steers, and 136 were found only in bulls. However, we considered potential proteins exclusive for each sexual condition when they were found in 5 of the 6 biological replicates. Therefore, 5 proteins (CX6A2, ANXA2, F1MS25, F1N206, PROF1) were considered exclusive for the steers, and 2 proteins (MYH3 and ACBP) were deemed to be unique to the bulls. Nine proteins were differentially abundant $(P<0.05)$ between sexual conditions. Three proteins play a structural role (MYBPC1, MYH4, MYL6B), 5 are metabolic pathway proteins (AGL, PYGM, PRDX6, $\mathrm{ACO} 2, \mathrm{HADHA}$ ), and 1 is involved in the epigenetic regulation of gene expression (APOBEC2). APOBEC2, AGL, PRDX6, and ACO2 showed positive correlations $(r>0.65)$ with color values $\left(L^{*}, a^{*}, b^{*}\right)$, and the last 2 proteins had a negative correlation with Warner-Bratzler shear force at $14 \mathrm{~d}$ of aging ( $r=-0.64$ and $r=-0.70$, respectively). Lactate measured $1 \mathrm{~h}$ postmortem was negatively correlated with MYH4 $(r=-0.68)$, and lactate measured $24 \mathrm{~h}$ postmortem was positively correlated with HADHA abundance $(r=0.68)$.

Conclusion: Overall, our findings demonstrated that (a) sexual condition modified the beef proteome and (b) meat quality and muscle metabolite traits were differentially associated with specific proteins for steers and bulls.

Acknowledgments: We are grateful to CAPES for the scholarship (Grant Number 001). We also thank CNPq for financial support (Process \#409186 / 2018-0, \#425000/ 2018-4, and \#303461/2019-5).

Keywords: castration, meat quality, Nelore, proteome, zebu cattle

\section{9 - NOVEL PROBE-TYPE OXYGEN SENSOR TO MEASURE OXYGEN CONSUMPTION IN BEEF STEAKS}

M. Lawson ${ }^{1 *}$, M. Denzer ${ }^{1}$, G. Mafi ${ }^{1}$, and R. Ramanathan ${ }^{1}$, ${ }^{1}$ Department of Animal and Food Sciences, Oklahoma State University, Stillwater, OK, USA, *mdenzer@, okstate.edu

Objectives: Oxygen consumption (OC) is an inherent muscle property that influences beef color. More specifically, greater $\mathrm{OC}$ results in less bloom and darker meat color, whereas lower OC can promote a bright-red color. Mitochondrial function, microorganisms, and oxidative processes can influence oxygen utilization. Hence, the quantification of $\mathrm{OC}$ is important to characterize beef color changes. The OC measured using reflectance approach indirectly measure oxygen utilization based on changes in myoglobin redox forms. Here, we discuss a novel fiber optics oxygen probe to measure changes in percentage partial pressure oxygen on the surface of beef longissimus lumborum steaks. Therefore, the objective of the current study was to compare OC using near infrared (NIR)-based oxygen sensor, fiber optics oxygen probe, and reflectance approach.

Materials and Methods: Steaks from 4 beef longissimus lumborum Choice loins were used in the study ( $n=4$; postmortem age $>7 \mathrm{~d}$ ). The $\mathrm{OC}$ quantification using the reflectance method was based on the American Meat Science Association Color Guide. In brief, steaks were bloomed for $1 \mathrm{~h}$ and then vacuum packaged and incubated at $30^{\circ} \mathrm{C}$ for $30 \mathrm{~min}$ inducing conversion of oxymyoglobin to deoxymyoglobin. The color changes were measured using a HunterLab MiniScan spectrophotometer, and oxymyoglobin level was calculated as $[1-(\mathrm{K} / \mathrm{S} 610 \div \mathrm{K} / \mathrm{S} 525)]$. K/S represents the absorption and scattering coefficient determined at 610 and $525 \mathrm{~nm}$. For NIR-based approach, the 
methodology used in reflectance OC was followed, and a Peripedal MOXY-3 Muscle Oxygen Monitor was used to measure the amount of oxygen on bloomed and deoxygenated muscle. Thirdly, an Ocean Optics Neo Fox Oxygen Sensing System probe was utilized to measure percent surface oxygen of steaks. Steaks were bloomed for $1 \mathrm{~h}$ and then packaged in plastic bags flushed and filled with nitrogen gas, then incubated at $30^{\circ} \mathrm{C}$ for $30 \mathrm{~min}$ allowing for conversion to deoxymyoglobin. Percent oxygen levels were measured using the probe after a 1-h bloom period and after 30-min incubation period. Both NIR- and fiber optics oxygen probe gave oxymyoglobin/oxygen partial pressure in percentage. However, the reflectance approach determined oxymyoglobin levels in $\mathrm{K} / \mathrm{S}$ ratios. Therefore, the percentage of $\mathrm{OC}$ was measured by the differences between pre- and post-percent muscle oxygen divided by pre-incubation value $\times 100$. The assays were replicated 4 times, and data were analyzed using the Univariate Proc of SAS (SAS Institute Inc., Cary, NC).

Results: The results suggest that the fiber optics oxygen probe was very sensitive to OC $(85.7 \%$, standard deviation $=$ 6.3 , and variance $=2.5$ ) and detected a higher percentage of oxygen with the lowest variance level compared with NIR and reflectance method. NIR-based MOXY detected greater levels of OC $(12.9 \%$, standard deviation $=6.2$, and variance $=$ 40.1) with more variance than with using the HunterLab spectrophotometer $(7.3 \%$, standard deviation $=2.9$, and variance $=8.7$ ). The Neo Fox Oxygen probe directly measures the oxygen partial pressure on the surface, hence a larger difference in oxygen level was noticed before and after incubation than indirect measurements using handheld spectrophotometers.

Conclusion: The results suggest that the fiber optics oxygen probe-type approach is a viable method to determine the OC of steaks.

Keywords: beef, meat color, myoglobin, oxygen consumption, probe-type sensor

\section{0 - EFFECT OF TEMPERATURE AND PH ON THE PROTEIN DENATURATION IN MUSCLES OF DIFFERENT FIBER TYPES ANALYSED BY FOURIER TRANSFORM INFRARED (FTIR) MICRO SPECTROSCOPY}

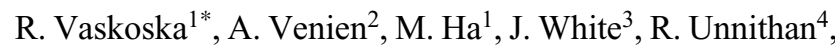
T. Astruc ${ }^{2}$, and R. Warner ${ }^{1},{ }^{1}$ Faculty of Agriculture and Food Systems, University of Melbourne, Melbourne, Australia, ${ }^{2}$ QuaPA, INRAE, Saint Genès-Champanelle, France, ${ }^{3}$ Research Office, Charles Sturt University, Wagga Wagga, Australia, ${ }^{4}$ Electrical and Electronic Engineering, University of Melbourne, Melbourne, Australia,*rspirovska@student.unimelb.edu.au
Objectives: The study objective was to determine the effect of cooking temperature and $\mathrm{pH}$ on the protein denaturation in muscle fibers and connective tissue from muscles of different fiber types.

Materials and Methods: Masseter (type I fiber) and cutaneous trunci (type II fiber) muscles were obtained from 1 bovine Angus cattle, $1 \mathrm{~d}$ postmortem. Muscles from 1 carcass were used in order to focus on the effect of the treatments and avoid the effect of animal variability, as commonly done in Fourier Transform Infrared studies in meat. Cryo-sections with $6-\mu \mathrm{m}$ thickness were incubated at 4 temperature levels $\left(25^{\circ} \mathrm{C}, 55^{\circ} \mathrm{C}, 60^{\circ} \mathrm{C}\right.$, and $\left.65^{\circ} \mathrm{C}\right)$ and $2 \mathrm{pH}$ levels (5.6 and 6.5). The temperatures were selected based on the sensitivity of myosin and collagen denaturation to differences in $\mathrm{pH}$ and temperature, and the selected $\mathrm{pH}$ levels corresponded to the measured ultimate $\mathrm{pH}$ of the muscles. Spectra $\left(4,000-650 \mathrm{~cm}^{-1}\right)$ were collected with a Nicolet $^{\mathrm{TM}}$ Continu $^{\mathrm{TM}}{ }^{\mathrm{TM}}$ Infrared Microscope (Thermo Fischer Scientific, Waltham, MA) from muscle fibers $(N=5$ for each treatment) and connective tissue areas $(N=5$ for each treatment). The data were analyzed with Unscrambler X 10.3 (Camo Analytics, Oslo, Norway) using Extended Multiplicative Scatter Correction and SavitzkyGolay smoothing. The absorbance for relevant wavenumbers in the Amide I region was analyzed using unbalanced analysis of variance in Genstat (VSN International, Hemel Hempstead, UK).

Results: The increase in temperature resulted in a decrease in $\alpha$-helices $\left(1,655 \mathrm{~cm}^{-1}\right.$ in fibers and $1,658 \mathrm{~cm}$ ${ }^{-1}$ in connective tissue) and an increase in aggregated $\beta$-sheet structures $\left(1,624 \mathrm{~cm}^{-1}\right.$ in fibers and $1,628 \mathrm{~cm}^{-1}$ in connective tissue). The difference in the thermal denaturation of the proteins in the muscle fibers, between the 2 muscle types at $55^{\circ} \mathrm{C}$, was greater than the difference between $\mathrm{pH}$, with

a)

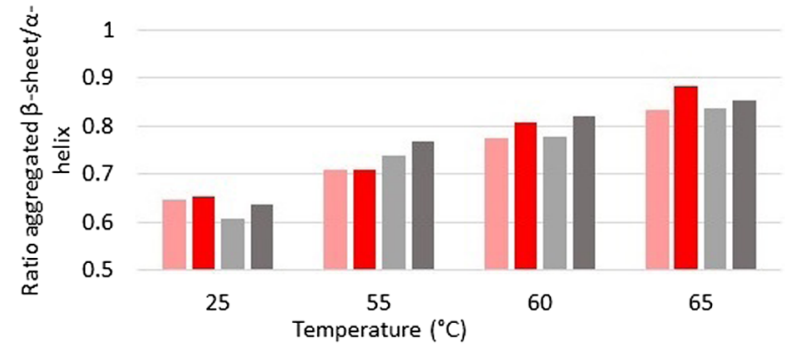

b)

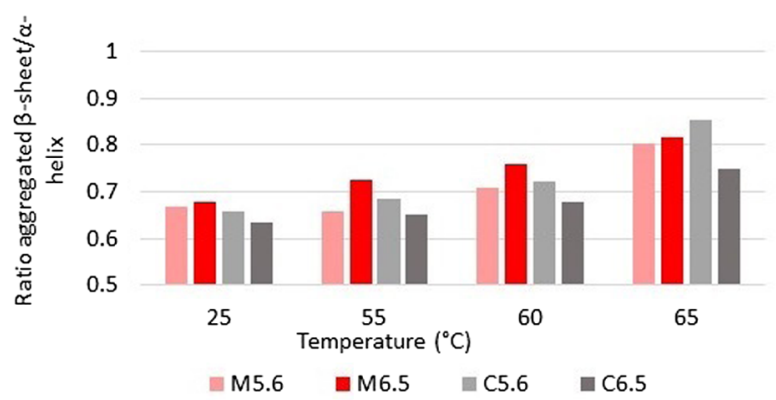

Figure 1. Protein denaturation in a) muscle fibers; b) connective tissue. M- masseter, C-cutaneous trunci. $\mathrm{N}=5$ for each data point. 
cutaneous trunci showing a greater shift of $\alpha$-helix to aggregated $\beta$-sheet than masseter (relative to $25^{\circ} \mathrm{C}$ ) (Figure 1a). This is hypothesized to be a consequence of the difference in thermostability of their myosin isoforms. However, at $60^{\circ} \mathrm{C}$ and $65^{\circ} \mathrm{C}$ in the muscle fibers, $\mathrm{pH}$ rather than temperature distinguishes the samples (Figure 1a-1b). At $60^{\circ} \mathrm{C}$ and $65^{\circ} \mathrm{C}$, proteins of the muscle fibers of masseter and cutaneous trunci undergo greater conversion of $\alpha$-helix into aggregated $\beta$-sheet at pH 6.5 compared to $\mathrm{pH} 5.6$ (Figure 1a). Distinctively, proteins in the connective tissue from masseter and cutaneous trunci seem to be more sensitive to protein denaturation at their ultimate $\mathrm{pH}, 6.5$ and 5.6, respectively, at all cooking temperatures (Figure 1b).

Conclusion: There are indications that muscle fiber proteins in cutaneous trunci show a greater thermal denaturation at $55^{\circ} \mathrm{C}$ than in masseter, which is most likely related to the different myosin isoforms and their thermal sensitivity. At all other conditions, $\mathrm{pH}$ rather than muscle type explains the differences in thermal protein denaturation of masseter and cutaneous trunci. These results might reflect differences in the water holding, texture, and digestibility of these muscles.

Keywords: beef, cooking, fiber type, $\mathrm{pH}$, protein denaturation

\section{1 - SEEDS OIL OLEOGELS BASED ON NATURAL EDIBLE WAXES: OXIDATIVE STABILITY, SENSORY PROPERTIES, AND APPLICATION IN MEAT BURGERS PORK FAT SUBSTITUTES}

A. Salaseviciene ${ }^{1 *}$, I. Hamidioglu ${ }^{1}$, G. Alencikiene ${ }^{1}$, and M. Dzedulionyte ${ }^{1},{ }^{1}$ Food Institute, Kaunas University of Technology, Kaunas, Lithuania, *alvija.salaseviciene@ktu.lt

Objectives: The objective of this study was to investigate the possibility of replacing pork fat in meat burgers by oleogels produced from hemp seed oil containing $3 \%$ and $7 \%$ waxes.

Materials and Methods: Oleogels were produced from hemp seed oil by mixing with rice bran wax or Candelila wax. Meat burgers were formulated from beef with 5 different lipid sources: (1) 20\% pork fat (control), (2) 20\% Oleogel with $3 \%$ rice bran wax, (3) $20 \%$ Oleogel with $7 \%$ rice bran wax, (4) $20 \%$ Oleogel with $3 \%$ Candelila wax, and (5) $20 \%$ Oleogel with $7 \%$ Candelila wax. Burgers were analyzed raw and cooked. Instrumental texture parameters (the firmness, stickiness, and spreadability for oleogels, texture profile analysis) and tenderness for burgers were measured with a Texture Analyzer (INSTRON 3343). Color parameters (CIE $L^{*} a^{*} b^{*}$ ) and lipid oxidation parameters were measured for oleogels and burgers. A sensory panel for the descriptive analysis consisted of 8 assessors (selected and trained according to the ISO 8586) experienced in sensory evaluation of meat and other products. The sensory attributes of the cooked samples were analyzed by using a standardized sensory descriptive method (ISO 13299). A structured numerical scale was used for the evaluation of the intensity of each attribute. The left side of a scale corresponding to the lowest intensity of an attribute was given the value of 1 , and the right side corresponding to the highest intensity was given the value of 15 . All sessions were conducted in a climate-controlled sensory analysis laboratory equipped with individual booths. A data collection system for automatic acquisition of the assessors scores and data analysis was used (FIZZ, Biosystems, France). All experiments were carried out with 3 replications. Data were analyzed using IBM SPSS version 25 (IBM Corp., Armonk, NY), and one-way analysis of variance was performed. Least significant difference was used to determine significant differences at $P<0.05$.

Results: The application of Candelila wax (3\% and 7\%) to produce the oleogels had a positive effect on hemp oil peroxide value in comparison to that of fresh hemp seed oil. Mechanical analysis of texture revealed that oleogels were able to match pork fat in some texture properties, such as spreadability and adhesiveness. Cooking losses were lower for control samples, while samples with oleogels' cooking losses were up to $20 \%$ higher $(P<0.01)$. Sensory profiles of cooked burgers revealed that off-flavor was not detected in all burgers, so it was possible to replace pork fat by oleogels without significant negative effect on the flavor properties of the burgers. However, control burger samples (formulated only with pork fat) had higher juiciness values $(P<0.001)$ and were more soft $(P<0.05)$ than samples with oleogels. During chewing process of burgers with oleogels, greasiness was perceived in mouth, which was not expressed in control burgers. This could be explained by the lower water/oil binding capacity of the burgers made with oleogels as determined by cooking losses. The results showed that wax-based oleogels from Candelila and rice bran have potential as pork fat substitutes in burgers' matrixes. However, ways to increase the oil binding capacity to keep the juiciness typical for burgers should be developed.

Conclusion: The results demonstrated a potential application of oleogels as a substitute for animal fat in meat burgers. Future studies should be focused on how to mimic pork fat sensory properties.

Keywords: natural waxes, oleogel, oxidative stability, pork fat substitutes, sensory

\section{2 - INVESTIGATING THE CONTRIBUTION OF MATURE COLLAGEN CROSSLINKS TO COOKED MEAT TOUGHNESS USING A STEWED BEEF SHANK MODEL}

W. J. Wu ${ }^{1}$, A. Welter ${ }^{1}$, C. Chun ${ }^{1}$, T. O’Quinn ${ }^{1}$, G. Magnin ${ }^{2}$, and M. Chao ${ }^{1},{ }^{1}$ Animal Sciences and Industry, Kansas State 
University, Manhattan, KS, USA, ${ }^{2}$ Anatomy \& Physiology, College of Veterinary Medicine, Kansas State University, Manhattan, KS, USA, *mdchao@ksu.edu

Objectives: Connective tissue provides the "background toughness" in meat, and past research has demonstrated that this background toughness is the result of heat insoluble collagen content in meat after cooking. However, the characteristics of heat insoluble collagen are not well studied. Therefore, the objective of this study was to investigate mature collagen crosslink densities and their relationship to cooked beef tenderness and connective tissue texture using a stewed beef shank model.

Materials and Methods: Beef shank cuts from both sides of the carcass, 3 from the foreshank (biceps brachii, deep digital flexor from foreshank [DDF-F], and extensor carpi radialis [ECR]) and 3 from the hindshank (flexor digitorum superficialis [FDS], deep digital flexor from hindshank [DDF-H], and a combination of long digital extensor, medial digital extensor, and peroneus tertius) were collected from 8 USDA Low Choice beef carcasses $(n=48)$. Each muscle was used as the experimental unit. Shanks from the left side of the carcasses were designated for the cooked treatment (stewed in water for $90 \mathrm{~min}$ at $93^{\circ} \mathrm{C}$ ), and shanks from the right side were designated as the raw treatment. Asian consumers $(n=61)$ evaluated the connective tissue texture from cooked shanks, and Warner-Bratzler shear force (WBSF) value was also obtained. Mature collagen crosslinks densities (pyridinoline [PYD] and deoxypyridinoline [DPD]) and collagen content were measured for both raw and cooked shanks. The collagen contents were adjusted to dry matter basis to account for moisture loss during the cooking process. Finally, relative percentages of soluble and insoluble collagen content were calculated.

Results: DDF-F had the toughest connective tissue texture, greatest shear force value, most cooked collagen content, and greatest insoluble collagen percentage as well as greatest raw and cooked PYD densities among all the beef shank cuts $(P<0.05)$. It was interesting to note that DDFF, FDS, and the combination of long digital extensor, medial digital extensor, and peroneus tertius all started with similar raw collagen content $(P>0.10)$, but DDF-F ended with greater cooked collagen content than the others. Cooking only decreased PYD density for DDF-F $(P<0.05)$, and PYD density for the rest of the beef shank cuts was not affected by cooking $(P>0.10)$. For DPD density, ECR had the greatest DPD density among all cuts for both raw and cooked samples $(P<0.01)$. Again, there was a cooking effect in which cooking decreased DPD density for DDF-F, ECR, and FDS $(P<0.01)$, but not for the other cuts $(P>$ $0.10)$. As expected, cooked collagen content and insoluble collagen percentage as well as raw PYD densities had positive correlations with connective tissue texture $(r=0.550$, 0.498 , and 0.560 respectively; $P<0.01)$ and WBSF $(r=$ $0.615,0.392$, and 0.730 , respectively; $P<0.05$ ). There was a noted positive correlation between cooked PYD density with connective tissue texture $(r=0.375 ; P<$ $0.05)$ and WBSF $(r=0.324 ; P<0.10)$, but the relationship was not as strong as for raw PYD density.

Conclusion: Based on the results from this study, we confirmed that PYD is a heat-stable collagen crosslink that requires extensive heat treatment to degrade and allow for the solubilization of collagen. As a result, raw PYD density is a good indicator for cooked collagen content, cooked beef connective tissue texture, and ultimately, tenderness in beef cuts with high concentration of connective tissue prepared with moist heat cookery.

Keywords: beef shanks, collagen, collagen crosslinks, connective tissue texture, tenderness

\section{3 - AN INVESTIGATION OF THE RELATIONSHIP BETWEEN MUSCLE FIBER CROSS-SECTIONAL AREA AND MEAT TENDERNESS}

P. Hammond ${ }^{1 *}$, A. Welter ${ }^{1}$, C. Chun ${ }^{1}$, W. J. Wu ${ }^{1}$, T. O'Quinn ${ }^{1}$, and M. Chao ${ }^{1},{ }^{1}$ Animal Sciences and Industry, Kansas State University, Manhattan, KS, USA,*mdchao@, ksu.edu

Objectives: Cross-sectional area (CSA) of muscle fibers represent the amount of myofibrillar substances the consumer's teeth must bite through during chewing. Therefore, it is logical to expect that CSA is positively correlated to meat tenderness. However, past studies speculated that this relationship between CSA and meat tenderness may be muscle specific, and the exact relationship is unclear. Therefore, the objective of this study was to investigate the relationship between Warner-Bratzler shear force (WBSF) and muscle fiber CSA of 10 different beef muscles with 2 aging periods.

Materials and Methods: Two separate studies were conducted. In the first study, triceps brachii (TB), supraspinatus (SS), longissimus thoracis (LT), rectus abdominus (RA), rectus femoris (RF), semitendinosus (ST), and gluteus medius (GM) were collected from 10 USDA Choice beef carcasses. Each sample was fabricated into steaks and assigned to a 2- or 21-d aging period $(n=140)$. The second study examined longissimus lumborum (LL), gastrocnemius (GC), and tensor fascia latae (TL) from 10 USDA Low Choice beef carcasses. Each sample was fabricated into steaks and assigned to a 5- or 21-d aging period $(n=60)$. For each sample, two $10-\mu \mathrm{m}$ cryosections were collected, and dystrophin was detected using the immunofluorescence method. An average of 400 muscle fibers per sample were analyzed for CSA and diameter. In addition, WBSF was measured for each sample. Correlation analysis was conducted to elucidate the relationship between CSA and WBSF. 
Results: No interaction was found between muscles and aging time for both studies. For the first study, SS and ST had the highest WBSF values followed by RA, GM, TB, and RF, with LT presenting the lowest WBSF values $(P<0.01)$. On the other hand, RA and SS had the greatest CSA and diameter, followed by LT, TB, GM, and ST, with RF possessing the lowest CSA and diameter values $(P<0.01)$. The SS and TB samples demonstrated a positive correlation $(r=0.59$, $P<0.01$ and $r=0.50, P<0.05$, respectively) between CSA and WBSF. In contrast, ST had a negative correlation $(r=-0.64 ; P<0.01)$ between CSA and WBSF. There was a minor but significant negative correlation $(r=-0.22 ; P<$ 0.01 ) between CSA and WBSF for all cuts in the first study. In the second study, GC had the highest WBSF values, followed by TL, whereas LL had the lowest WBSF values $(P<$ 0.01). The LL had the greatest CSA and diameter, and CSA and diameter for GC and TL were lower $(P<0.01)$. The LL had a positive correlation $(r=0.61 ; P<0.01)$ between CSA and WBSF. There was also a negative correlation for CSA and WBSF $(r=-0.55 ; P<0.01)$ for all cuts in the second study. No relationship was documented between CSA and WBSF for GM, LT, RA, RF, GC, and TL in the 2 studies $(P>0.10)$.

Conclusion: The results confirmed that the relationship between CSA and meat tenderness is muscle specific. However, it was interesting to note that both studies exhibited an overall negative correlation between CSA and WBSF. Although no aging effect was found for CSA, the steady decrease of WBSF values from early postmortem to $21 \mathrm{~d}$ postmortem may lead to this negative correlation with CSA. However, one cannot overlook the potential effect of muscle fiber fracture and tearing as enzymatic degradation takes places, leading to the detection of slightly larger CSA.

Keywords: beef, cross-sectional area, diameter, muscle fiber, tenderness

\section{4 - THE INFLUENCE OF BEEF CARCASS WEIGHT ON TROPONIN-T DEGRADATION AND HEAT SHOCK PROTEIN 70 IN TWO DIFFERENT MUSCLES}

S. D. Nath ${ }^{1 *}$, R. Maddock ${ }^{1}$, M. Fevold ${ }^{1}$, W. Keller ${ }^{1}$, and K. Carlin ${ }^{1}$, 1 Animal Sciences, North Dakota State University, Fargo, ND, USA, *kasey.maddockcarlin@ndsu.edu

Objectives: This study was to evaluate troponin-T (TnT) degradation and heat shock protein 70 (HSP70) in 2 muscles (semimembranosus [SM] and longissimus thoracis et lumborum [LD]) from different weight beef carcasses and how they correlate to carcass measurements during slaughter, processing, and aging.

Materials and Methods: Heavy (>430.9 kg; $n=22)$, average $(351.5$ to $385.6 \mathrm{~kg} ; n=23)$, and light $(<317.5$ $\mathrm{kg} ; n=20$ ) beef carcasses were randomly selected over 5 nonsequential days at a commercial abattoir. Carcasses were monitored for temperature and $\mathrm{pH}$ at $45 \mathrm{~min}, 4 \mathrm{~h}$, and $24 \mathrm{~h}$ after exsanguination in the SM and LD between the 12th and 13 th rib. After a 24-h chill, ribeye area, 12th rib fat, and yield grade were evaluated by trained personnel. Upon fabrication, subprimals were transported to the North Dakota State University meat laboratory and aged. After $14 \mathrm{~d}$, $2.5-\mathrm{cm}$ steaks were collected from the cranial end of the ribeye roll and the proximal end of inside round for shear force. Meat samples from the LD and SM were collected at day 3 and day 14. Western blots for TnT and HSP70 were performed on day-3 and day-14 LD and day-3 SM. Reactive bands for TnT were observed at 40, 38, 36, 34, and $30 \mathrm{kDa}$. LSMEANS were measured using PROC MIXED procedure of SAS (SAS Institute Inc., Cary, NC), and Tukey test was performed for mean comparison. Pearson correlation coefficients were determined using PROC CORR of SAS.

Results: Carcass weight did not influence $(P>0.05)$ HSP70 abundance in LD or SM. In day-3 SM, heavy carcasses had less $40-\mathrm{kDa}(P=0.02)$ and greater $34-\mathrm{kDa}(P=0.03)$ and $30-\mathrm{kDa}(P=0.03)$ TnT than light carcasses. Alternatively, in day-14 LD, light carcasses had greater $30 \mathrm{kDa}(P=0.007)$ TnT than heavy carcasses. Carcass weight was negatively correlated with $30-\mathrm{kDa}$ TnT in day-14 LD $(r=-0.34, P=0.03)$ and $40-\mathrm{kDa}$ TnT in day-3 SM $(r=-0.44, P=0.03)$ and positively correlated with $30-\mathrm{kDa}$ TnT in day-3 SM $(r=0.43, P=$ $0.04)$. There were negative correlations between $24-\mathrm{h} \mathrm{pH}$ and $30-\mathrm{kDa}$ TnT in the day-3 and day-14 LD $(r=-0.33, P=0.03$; $r=-0.319, P=0.04$, respectively) and between $4-\mathrm{h} \mathrm{pH}$ and $30-\mathrm{kDa}$ TnT $(r=-0.43, P=0.04$ ) in day-3 SM. There was a tendency of positive correlations between 4-h $\mathrm{pH}$ and 24-h pH with HSP70 abundance in day-3 SM $(r=0.30$, $P=0.06 ; r=0.27, P=0.08$, respectively). In addition, 24-h temperature and ribeye area were negatively correlated with $30-\mathrm{kDa}$ TnT in the day-3 LD $(r=-0.33, P=0.05 ; r=$ $-0.32, P=0.02$, respectively). Moreover, drip loss percentage and shear force were also negatively correlated with the 30$\mathrm{kDa}$ TnT band in day-14 LD $(r=-0.27, P=0.04 ; r=-0.31$, $P=0.03$, respectively).

Conclusion: HSP70 protein was not influenced by carcass weight. TnT degradation was greater in heavy carcasses in the day-3 SM and in light carcasses in the day-14 LD. In the LD, correlations between the $30-\mathrm{kDa}$ TnT band and carcass weight as well as ribeye area were negative, indicating that the LD may have less protein degradation as the carcass got heavier. In the SM, there was an opposite observation where the larger carcasses had more $30-\mathrm{kDa}$ TnT degradation product. Based on the correlation data, these differences do not seem to be temperature or $\mathrm{pH}$ dependent. Interestingly, the LD and SM respond differently to increases in carcass weight with regard to protein degradation during aging.

Keywords: beef, carcass size, heat shock protein, troponin-T 


\section{5 - INFLUENCE OF ZINC AND RACTOPAMINE HYDROCHLORIDE SUPPLEMENTATION ON THE PROTEOME OF EARLY POSTMORTEM BEEF LONGISSIMUS THORACIS MUSCLE}

M. D. Schulte ${ }^{1 *}$, K. G. Hochmuth ${ }^{1}$, E. M. Steadham ${ }^{1}$, S. M. Lonergan $^{1}$, S. L. Hansen ${ }^{1}$, and E. Huff-Lonergan ${ }^{1}$, ${ }^{1}$ Department of Animal Science, Iowa State University, Ames, IA, USA, *schulte1@iastate.edu

Objectives: Production practices to improve growth and efficiency can impact early postmortem metabolism and tenderness. The objective of this experiment was to investigate the impact of growth improvement strategies on the muscle proteome of longissimus thoracis (LT) muscle of beef finishing steers.

Materials and Methods: Yearling steers were assigned dry rolled corn diets based on growth potential and initial body weight: non-Zn supplemented control (CON-NO; 36 $\mathrm{mg} Z \mathrm{Zn} / \mathrm{kg}$ dry matter; $n=5$ ), supranutritional Zn supplementation (SUPZN-NO; $\mathrm{CON}$ diet $+60 \mathrm{ppm} \mathrm{Zn}$ from $\mathrm{ZnSO}_{4}+$ $60 \mathrm{ppm} Z n$ from $\mathrm{Zn}$ amino acid complex; $n=5), \mathrm{CON}+$ ractopamine hydrochloride (RAC) supplementation (CONRAC; 300 mg RAC-steer ${ }^{-1} \mathrm{~d}^{-1} ; n=5$ ), and supranutritional $\mathrm{Zn}$ supplementation + RAC supplementation (SUPZN$\mathrm{RAC} ; n=5$ ). $\mathrm{Zn}$ treatments were fed for the entire 89-d trial and RAC supplementation for the final $28 \mathrm{~d}$ for RAC treatments. At finishing weights, 1 steer per treatment was harvested at the ISU Meat Lab on 5 separate dates. LT muscle $\mathrm{pH}$ measurements were taken at $1,3,6$, and $24 \mathrm{~h}$ postmortem. LT muscle samples were taken at $1 \mathrm{~h}$ and $1 \mathrm{~d}$ postmortem and frozen until protein analysis. Steaks were fabricated and aged for $1,3,7$, or $14 \mathrm{~d}$ postmortem and Warner-Bratzler shear (WBS) force values were determined. Sarcoplasmic proteins were extracted and used for mass spectrometry (liquid chromatography-tandem mass spectrometry) analysis using tandem mass tagging. WBS and $\mathrm{pH}$ data were analyzed as a $2 \times 2$ factorial using the mixed procedure of SAS version 9.4 (SAS Institute Inc., Cary, NC) with fixed effects of Zn, RAC, and the interaction, with harvest date as a fixed block. Significance was denoted by $P<$ 0.05 and trends by $P<0.10$. Proteomic data were analyzed using $t$ tests. Significance was set at $P<0.10$.

Results: $\mathrm{Zn}$ supplementation trended for a lesser $(P=$ $0.06) \mathrm{pH}$ value at $6 \mathrm{~h}$ postmortem, and RAC supplementation resulted in a greater $(P=0.04) \mathrm{pH}$ value at $6 \mathrm{~h}$ postmortem. These differences are driven by the individual treatments, not the interaction. At $1 \mathrm{~d}$ postmortem, $\mathrm{Zn}$ supplementation trended for lesser $(P=0.06)$ WBS values, and RAC supplementation resulted in greater $(P<0.01)$ WBS values. At $1 \mathrm{~h}$ postmortem, a greater abundance of soluble actin and lesser abundance of vinculin was observed in SUPZN-RAC compared with SUPZN-NO or CON-RAC. A lesser abundance of soluble myosin 7 and troponin-T was observed in CON-NO compared with CON-RAC or SUPZN-RAC. A lesser abundance of myosin regulatory light chain 2 was detected in SUPZN-NO compared with CON-NO, CON-RAC, or SUPZN-RAC. At $1 \mathrm{~d}$ postmortem, lesser abundance of AMP deaminase and greater abundance of malate dehydrogenase was detected in SUPZN-NO compared with CON-NO or SUPZN-RAC. A greater abundance of pyruvate dehydrogenase subunit beta was detected in SUPZN-NO compared with CON-NO or CON-RAC. A greater abundance of fructose bisphosphate aldolase and glyceraldehyde phosphate dehydrogenase was observed in CON-RAC compared with CON-NO or SUPZN-RAC.

Conclusion: At $1 \mathrm{~h}$ postmortem, several structural proteins differed in abundance between rapid growth treatments. These structural proteins are not typically soluble in muscle potentially indicating differences in cellular remodeling of the muscle structure. At $1 \mathrm{~d}$ postmortem, differences in metabolic proteins in the proteome were identified which may play a key role in the observed differences in early postmortem metabolism of rapid growing animals. The results represent a complex response to management practices that can influence meat quality.

Keywords: beef tenderness, $\mathrm{pH}$ decline, proteomics, postmortem metabolism

\section{6 - POTENTIAL RELATIONSHIP OF THE PORCINE MUSCLE THANATOTRANSCRIPTOME TO PORK QUALITY}

A. King ${ }^{1 *}$, A. Dickey ${ }^{1}$, S. D. Shackelford ${ }^{1}$, T. L. Wheeler ${ }^{1}$, G. Rohrer ${ }^{1}$, and D. Nonneman ${ }^{1},{ }^{1}$ USDA-ARS, US Meat Animal Research Center, Clay Center, NE, USDA, *andy.king@ars.usda.gov

Objectives: Genome-wide association studies for pork quality traits that are defined antemortem (i.e., fatty acid profile) tend to yield clear QTL associations, while studies for traits affected by postmortem metabolism (i.e., color) are less clear. Most gene expression studies for identification of meat quality candidate genes involve tissues collected at death, though studies have reported many genes to be upregulated postmortem (thanatotranscriptome). Anaerobic glycolysis is considered the primary postmortem metabolic pathway in the conversion of muscle to meat; however, oxygen remains in postmortem muscle for hours to support mitochondrial function. The objectives of this study were to determine changes in gene expression within the postmortem interval and relate these affected genes and pathways to QTL for pork quality.

Materials and Methods: Gilts $(n=5 ; 262$ to $325 \mathrm{~d}$ of age) were harvested using electrical stunning and conventional chilling. Longissimus lumborum muscle samples were 
removed at 0, 24, and $48 \mathrm{~h}$ postmortem. Samples for the 24 and 48 -h time periods were chilled as part of the carcass. From each sample, RNA-seq libraries were prepared. An average of 58.5 million paired-end reads were collected from each library, mapped to Sscrofa 11.1 assembly, and differential gene expression was determined using DESeq2. Genes were considered differentially expressed with a false discovery rate-corrected $P \leq 0.05$. Differentially expressed genes were compared to existing QTL for pork quality traits.

Results: Compared to 0 -h samples, 4 and 1,943 genes were more highly expressed and 132 and 2,280 were more lowly expressed at 24 and $48 \mathrm{~h}$, respectively $(P<0.05)$, with $\log 2$ fold changes ranging from -7.15 to 2.55 compared to $0 \mathrm{~h}$. The most overrepresented pathways included ribosomal protein, protein translation, oxidative phosphorylation, and cytochrome-C oxidase activity $(P<0.05)$. Genes coding for mitochondrial complexes I (34 of 44), II (3 of 4), III ( 2 of 10), IV (19 of 9), and V (11 of 20) were more highly expressed $(P<0.05)$ at $48 \mathrm{~h}$ postmortem than at death. These differentially expressed genes are primarily involved in electron transport, the TCA cycle, and ATP synthesis. Differentially expressed genes $(P<0.05)$ also included ribosomal proteins (85 of 109) as well as mitochondrial ribosomal proteins (40 of 77), suggesting an increase of translational capacity. Enzyme-linked immunosorbent assay for 3 proteins (HSPA6, CCL21, and EPB42) with gene expression fold changes of 5.1, 2.94, and 4.4 at $48 \mathrm{~h}$ showed protein content changes $(P<0.05)$ of $88.01 \%, 42.19 \%$, and $-37.17 \%$ at $48 \mathrm{~h}$, respectively. One hundred and twenty more highly expressed genes $(P<0.05)$ at $48 \mathrm{~h}$ postmortem were located within QTL associations for color, $\mathrm{pH}$, water-holding capacity, and tenderness.

Conclusion: These results imply that gene expression and protein translation continue to occur in postmortem muscle. Increased gene expression resulted in protein synthesis. This contrasts with the current dogma that gene expression ends at death. The overlap of genes expressed postmortem with genes previously associated with pork quality traits indicate that postmortem gene expression could impact meat quality.

Keywords: gene expression, pork, postmortem change, quality, thanatotranscriptome

\section{7 - CITRATE SYNTHASE ACTIVITY AS A MARKER FOR MITOCHONDRIAL CONTENT IN BOVINE LONGISSIMUS LUMBORUM POSTMORTEM}

L. Bell ${ }^{1}$, B. Hawryluk ${ }^{1}$, M. McKinney ${ }^{1}$, P. Ramos ${ }^{1,2}$, and T. Scheffler ${ }^{1},{ }^{1}$ Department of Animal Sciences, University of Florida, Gainesville, FL, USA, ${ }^{2}$ Department of Animal Sciences, University of Sao Paulo, Piracicaba, Brazil, *tscheffler@ufl.edu
Objectives: Citrate synthase (CS) is a key enzyme in the Krebs cycle, and CS activity is a commonly used marker for mitochondrial content. However, it is unclear whether the decrease in $\mathrm{pH}$ of postmortem muscle affects $\mathrm{CS}$ activity. Our first objective was to determine whether CS from bovine longissimus lumborum (LL) retains activity at $\mathrm{pH}$ values observed in postmortem muscle and verify $\mathrm{pH}$ for maximal CS activity. Our second objective was to determine CS activity of bovine LL collected during the first $24 \mathrm{~h}$ postmortem.

Materials and Methods: For the first study, LL from Angus (Bos taurus) $(n=4)$ and Brahman (Bos indicus) $(n=$ 2) steer carcasses were collected at $1 \mathrm{~h}$ postmortem. Samples were immediately frozen using liquid nitrogen and stored at $-80^{\circ} \mathrm{C}$ until further analysis. Pulverized muscle was diluted in homogenization buffer, sonicated, and centrifuged. Supernatants were pipetted into 96-well plates and reaction media adjusted to $\mathrm{pH}$ 5.5, 5.9, 6.3, 6.7 (MES as buffer); 7.4 and 8.3 (Tris as buffer) was added. Background readings were taken every $20 \mathrm{~s}$ for $2 \mathrm{~min}$. Reaction was initiated by adding acetyl coenzyme $\mathrm{A}$, and the rate was monitored by following reduction of 5,5'-dithiobis-(2-nitrobenzoic acid) at $412 \mathrm{~nm}$. CS activity was calculated as $\mathrm{nmol} / \mathrm{min} / \mathrm{mg}$ tissue. Data were analyzed using SAS JMP-Pro 11 (SAS Institute Inc., Cary, NC) with the fixed effect of $\mathrm{pH}$. For the second study, LL was collected from Angus and Brahman steer carcasses $(n=14$ per breed) at $1,3,6$, and $24 \mathrm{~h}$ postmortem. Muscle processing and CS activity assay procedures were conducted as in the first study, except CS activity of muscle supernatants was determined using reaction media at $\mathrm{pH}$ 7.4. Data were analyzed using SAS mixed procedure with fixed effects of breed, time, and the interaction, with time as a repeated measure.

Results: Assay $\mathrm{pH}$ influenced CS activity $(P<0.0001)$, with the highest amount of CS activity at $\mathrm{pH} 7.4$. Activity of $\mathrm{CS}$ declined rapidly as $\mathrm{pH}$ decreased from 7.4; at $\mathrm{pH}$ 6.3, activity was $<15 \%$ of activity at $\mathrm{pH} 7.4$. For the second study, breed did not influence CS activity $(P=0.15)$; however, time postmortem affected CS activity $(P=0.012)$. CS activity was lower in 1-h LL compared to subsequent time points $(P<0.05)$, with maximal change of $10 \%$; this was primarily associated with greater CS activity in Brahman LL at 3,6 , and $24 \mathrm{~h}$ postmortem.

Conclusion: Reaction media of $\mathrm{pH} 7.4$ produced maximal CS activity, but CS activity is expected to rapidly decrease in muscle postmortem, in accordance with $\mathrm{pH}$ decline. Somewhat unexpectedly, in postmortem samples assayed at $\mathrm{pH} 7.4, \mathrm{LL}$ collected at later times $(3,6$, and $24 \mathrm{~h}$ ) exhibited greater CS activity than 1-h LL. Therefore, the $\mathrm{pH}$ decline in postmortem muscle does not irreversibly impair CS activity.

Keywords: beef, citrate synthase, mitochondria, $\mathrm{pH}$ 


\section{8 - HYDROXYL RADICAL FOOTPRINTING OF BOVINE MYOGLOBIN USING PLASMA INDUCED MODIFICATION OF BIOMOLECULES (PLIMB)}

J. Whalin 1 , D. Benjamin ${ }^{2}$, F. A. Choudhury ${ }^{3}, \mathrm{Y} . \mathrm{Wu}^{2}$, Y. Wang $^{4}$, S. P. Suman ${ }^{4}$, J. L. Shohet ${ }^{2}$, and M. P. Richards ${ }^{1 *}$, ${ }^{1}$ Department of Animal Sciences, University of WisconsinMadison, Madison, WI, USA, ${ }^{2}$ Department of Electrical and Computer Engineering, University of WisconsinMadison, Madison, WI, USA, ${ }^{3}$ Department of Biochemistry, University of Wisconsin-Madison, Madison, WI, USA, ${ }^{4}$ Department of Animal and Food Sciences, University of Kentucky, Lexington, KY, USA, *whalin@wisc.edu

Objectives: The study objective was to utilize Plasma Induced Modification of Biomolecules (PLIMB) to examine solvent access to the distal and proximal cavities of bovine oxymyoglobin. Tentative mechanisms of myoglobin oxidation and heme dissociation are predicated on solvent access to the heme pocket which can potentially be examined using PLIMB.

Materials and Methods: PLIMB: Fifty hertz and 10 volts was generated and amped to $10 \mathrm{kHz}$ and $10 \mathrm{kV}$, which was then discharged from a steel needle above protein in buffer. Fifty microliters of protein $(30 \mu \mathrm{M})$ in sodium phosphate buffer $(50 \mathrm{mM}, \mathrm{pH} 6.5)$ was used per exposure and excess hydroxyl radicals quenched with $25 \mathrm{mM}$ methionine solution.

Orbitrap tandem mass spectrometry (MS/MS): After a trypsin/LysC digest, samples were cleaned with a $\mathrm{C} 18$ OMIX tip. Samples were injected into a Pepmap C18, $3 \mu \mathrm{M}, 100 \mathrm{~A}, 25 \mu \mathrm{M}$ ID, $15 \mathrm{~cm}$ reversed phase column and ionized with an EASY-Spray Ion Source. Samples were analyzed with an Orbitrap Elite in data-dependent MS/MS mode and with Protein Metrics Byos software. Plasmainduced modifications of side chains included (I) monooxidation as $+15.99 \mathrm{Da}$, (II) di-oxidation as $+31.99 \mathrm{Da}$, (III) His to Asp as $-22.032 \mathrm{Da}$, (IV) His to Asn as $-23.016 \mathrm{Da},(\mathrm{V})$ Lys to Arg as +28.006 Da, (VI) Lys to Asn as -14.05 , (VII) Thr to Asp as +13.979 Da, (VIII) Carbamidomethyl as $+57.021 \mathrm{Da}$, (IX) Gln to pyro-Gln as $-17.026 \mathrm{Da}$, (X) Glu to pyro-Glu as $-18.010 \mathrm{Da}$, and (XI) Ser to Asp as +27.99 Da.

Results: There are 17 amino acids within 4 angstroms of the heme protoporphyrin nine ring that collectively form the distal and proximal pockets. Notably, the distal residues at E7 and CD3 (His64 and K44) and the proximal residue at C7 (K42) were modified by PLIMB after $1 \mathrm{~s}$ of exposure. Histidine modifications resulted in mass shifts of -22.03 $\mathrm{Da},-23.016 \mathrm{Da}$, and +15.99 Da. Lysine modifications resulted in a mass shift of $+28.006 \mathrm{Da}$. One experiment conducted in duplicate was done, which limited power of the statistical evaluations. There was no significant difference between the summed modifications of the proximal residues compared to the distal residues, even though the absolute value was higher for the distal residues compared to the proximal residues $(0.37 \%$ vs. $0.04 \%)$. There was no significant increase in modification in response to time, though the absolute value was higher at $1 \mathrm{~s}$ compared to $0 \mathrm{~s}(0.29 \% \mathrm{vs}$. $0.12 \%$ ). It should be noted that the -22.03 and $-23.016 \mathrm{Da}$ modifications were more numerous than the +16 Da even though additive reactions generally occur faster with hydroxyl radical footprinting. Indeed, there was no validated +15.99 Da modification of lysine, nor of the other 15 amino acids examined as part of the experiment.

Conclusion: The study demonstrates potential for the use of PLIMB in tandem with MS/MS to study solvent access to myoglobin. More distal amino acids were modified compared to proximal, consistent with solvent access to the distal pocket facilitating $\mathrm{Mb}$ oxidation. Further experiments will be required to better assess the utility of PLIMB to measure solvent access and differential modification of the distal and proximal amino acids.

Keywords: heme, myoglobin, oxidation, protein footprinting

\section{9 - DEGREE OF DONENESS OF COOKED BEEF LONGISSIMUS MUSCLE ALTERS LIPID BIOACCESSIBILITY}

E. A. L. West ${ }^{1 *}$, A. X. Xu ${ }^{1}$, B. M. Bohrer ${ }^{1}$, M. Corradini ${ }^{1}$, I. Joye $^{1}$, and M. A. Rogers ${ }^{1},{ }^{1}$ Food Science, University of Guelph, Guelph, Canada,*weste@uoguelph.ca

Objectives: The physical properties of the foods' network influences how it disintegrates along the gastrointestinal tract, modulating both fatty acid release and bioavailability during digestion. The objective of this study was to investigate whether physical state differs significantly enough by cooking to alter the metabolic response of lipids in food.

Materials and Methods: Canadian AAA boneless beef strip loins (Institutional Meat Purchase Specifications 180) containing the longissimus muscle $(n=3)$ were procured from a commercial processing facility and aged $6 \mathrm{~d}$ postmortem. Loins were cut into 2.5 -cm-thick steaks with the external fat removed and randomly assigned to different degrees of doneness $\left(\mathrm{raw} / 20^{\circ} \mathrm{C}, 50^{\circ} \mathrm{C}, 60^{\circ} \mathrm{C}, 70^{\circ} \mathrm{C}\right.$, and $80^{\circ} \mathrm{C}$ ). The steaks were individually vacuumed sealed and frozen at $-30^{\circ} \mathrm{C}$. Steaks were thawed at $4^{\circ} \mathrm{C}$ for 12 to $24 \mathrm{~h}$ prior to sous vide cooking. Proximate analysis was performed on the cooked steaks, and the amount of steak digested was standardized to $2 \%$ fat in a $300 \mathrm{~g}$ meal. The meal was prepared by blending the steak with salivary fluids to simulate mastication. Lipid bioaccessibility was assessed utilizing the advanced TIM-1 simulated gastrointestinal tract (TNO, Zeist, The Netherlands), which is a dynamic in vitro 
system that mimics human digestion. Digestion kinetics consisting of lipid digestion rate constants and bioaccessibility were measured over a $6 \mathrm{~h}$ simulated digestion using the TIM1 robotic gastrointestinal track. Ileal and jejunal filtrates and ileal efflux were collected at 30, 60, 90, 120, 180, 240, 300, and $360 \mathrm{~min}$, and the concentration of free fatty acids was measured using a non-esterified fatty acid test kit. The changes in network structure of the different degrees of doneness were analyzed using rheology and microscopy. A repeated measures two-way analysis of variance with the Geisser-Greenhouse correction and Tukey's Multiple Comparison Test was used to compare the effects of digestion time and degree of doneness on percentage free fatty acid bioaccessibility. GraphPad Prism 8 (GraphPad Software, San Diego, CA) was used, and statistical significance was defined as $P<0.05$. Digestion rate was determined from linear regression, and a statistical significance was defined as no overlap of the $95 \%$ confidence intervals.

Results: The total cumulative free fatty acid bioaccessibility (percentage) showed significant differences $(P<0.05)$ depending on the degree of doneness, with the beef cooked to $60^{\circ} \mathrm{C}$ giving the maximum bioaccessibility. Free fatty acid digestion rate was significantly different $(P<0.05)$ between all degrees of doneness except between $70^{\circ} \mathrm{C}$ and $80^{\circ} \mathrm{C}$, which showed no statistical difference $(P>0.05)$. Rheology analysis showed significant $(P<0.05)$ increases in storage and loss modulus with increasing degree of doneness; furthermore, the storage modulus dominates the loss modulus for all samples. Observations of particle size from microscopy images showed a continuous decrease in particle area in the stomach after $60 \mathrm{~min}$ for both $20^{\circ} \mathrm{C}$ and $80^{\circ} \mathrm{C}$ samples.

Conclusion: This research shows that the protein structural changes that occur during heating may impact the release of lipids during digestion. A better understanding of the mechanisms of digestion with relation to changes in food structure and matrices due to processing/preparation can serve in the development of foods that tailor the amount of free fatty acid release.

Keywords: beef, degree of doneness, lipid digestibility, sous vide

\section{0 - PROTEIN BIOMARKERS IDENTIFIED IN SUBCUTANEOUS FAT FROM ZEBU CATTLE CAN BE ASSOCIATED WITH ENDOCRINE HORMONES}

T. S. Martins ${ }^{1 *}$, M. D. Poleti ${ }^{2}$, P. L. M. Garbossa ${ }^{1}$, H. C. Rocha $^{1}$, A. G. V. do Barco ${ }^{3}$, A. M. Ferrinho ${ }^{4}$, J. Silva ${ }^{4}$, T. R. Amorim ${ }^{4}$, L. F. Mueller ${ }^{4}$, J. A. Muñoz ${ }^{4}$, I. H. S. Fuzikawa $^{4}$, G. V. de Moura ${ }^{4}$, J. L. Gemelli ${ }^{4}$, J. F. M. Gómez $^{4}$, R. Jordão ${ }^{4}$ J. C. C. Balieiro ${ }^{1}$, J. A. Negrão ${ }^{5}$, F. Baldi $^{6}$, S. L. e Silva ${ }^{4}$, and A. S. C. Pereira ${ }^{1},{ }^{1}$ Department of Animal Nutrition and Production, School of Veterinary Medicine and Animal Science, University of Sao Paulo (FMVZ/USP), Brazil, ${ }^{2}$ Department of Veterinary Medicine, Faculty of Animal Science and Food Engineering, University of São Paulo (FZEA/USP), Pirassununga, Brazil, ${ }^{3}$ Department of Biomedicine, Foundation Herminio Ometto, Uniararas, Araras, Brazil, ${ }^{4}$ Department of Animal Science, University of São Paulo (FZEA/USP), Pirassununga, Brazil, ${ }^{5}$ Department of Basic Sciences, Faculty of Animal Science and Food Engineering, University of São Paulo (FZEA/USP), Pirassununga, Brazil, ${ }^{6}$ Department of Animal Science, State University of São Paulo (UNESP), Jaboticabal, Brazil, *taianemartins@ usp.br

Objectives: This work aimed to correlate the significant electrophoretic bands of subcutaneous fat proteins with productive, biologics, and qualitative phenotypes, such as weight gain, hormones, marbling, and subcutaneous fat deposition measured by carcass ultrasound. It also aimed to identify the proteins involved in these biological processes.

Materials and Methods: One hundred and five Nelore bulls (initial weight of $350 \mathrm{~kg} \pm 15 \mathrm{~kg}$ ) 20 mo of age were confined for $100 \mathrm{~d}$ and fed with the same diet (27:73 forage/concentrate). The animals were slaughtered, and subcutaneous fat samples were collected and immediately frozen in liquid nitrogen. The animals were ranked based on their parent's Expected Progeny Difference for precocity and growth, and 14 animals were selected with either Highest Expected Progeny Difference $(N=7)$ or Lowest Expected Progeny Difference $(N=7)$ deposition of subcutaneous fat to form the groups that were tested for differential proteomic analysis. The present study analyzed the protein profile of subcutaneous fat by dodecyl sulfate-polyacrylamide gel electrophoresis and detected 3 electrophoretic bands differentially abundant (bands 24, 30, and 32) between the groups evaluated. The bands were excised from the gel and submitted to protein identification by liquid chromatography-tandem mass spectrometry. Correlations were estimated by Pearson's correlation coefficient $(r)$ within and between groups and considered significant at $P<0.05$.

Results: Independent of groups, the serum insulin content was positively correlated with band $30(r=0.63)$, while beef marbling was positively correlated with band 24 $(r=0.76)$ and band $30(r=0.65)$. The results showed a great positive correlation between the serum insulin content and band $30(r=0.93)$ for group Lowest Expected Progeny Difference. Correlations within the group Highest Expected Progeny Difference revealed a great negative correlation between band 32 and average daily weight gain $(r=$ $-0.85)$ and total weight gain $(r=-0.84)$. The list of proteins identified in each band was submitted to over-representation analysis using WebGestalt web tool. The top enriched pathways (false discovery rate $<0.05$ ) found are shown in Table 1. 
Table 1. Top over-representation KEGG pathways (FDR <0.05) for the list of proteins identified for each band differentially abundant between high and low group for deposition of subcutaneous fat in Nelore cattle

\begin{tabular}{lccc}
\hline \hline Band & KEGG & Description & Protein \\
\hline $\mathbf{2 4}$ & bta01210 & 2-oxocarboxylic acid metabolism & ACY1, CS, IDH1 \\
& bta00061 & Fatty acid biosynthesis & ACSL1, FASN \\
& bta00020 & Citrate cycle & CS, IDH1, SUCLG2 \\
$\mathbf{3 0}$ & bta00620 & Pyruvate metabolism & LDHA, LDHB, MDH1, MDH2, PDHB \\
& bta00270 & Cysteine and methionine metabolism & LDHA, LDHB, MDH1, MDH2, MPST \\
& bta00020 & Citrate cycle & MDH1, MDH2, PDHB \\
\hline 3 & bta03050 & Proteasome & PSMA1, PSME1, PSME2 \\
\hline
\end{tabular}

Conclusion: This study revealed that there is a strong relationship between the subcutaneous fat protein profile and the insulin serum hormonal profile, independent and within the groups evaluated. The proteins involved in the citrate cycle pathway may influence subcutaneous fat deposition. Besides that, the proteins involved in the proteasome pathway can be related to animal growth. Thus, the exploration of proteins present in subcutaneous fat will allow a better understanding of the metabolic pathways involved in fat deposition, animal production, carcass, and beef quality.

Acknowledgments: We are grateful to FAPESP for the scholarship (Grant Number 2017/02349-1) and financial support (process number 2016/17433-5). We also thank CNPq for financial support (process number 409186/ 2018-0).

Keywords: backfat thickness, insulin, metabolic pathways, Nelore, proteomics

\section{1 - LIPID METABOLOMIC CHANGES IN TWO MUSCLES FROM BEEF AT 2 HOURS AND 24 HOURS POSTMORTEM}

\author{
K. Carlin ${ }^{1 *}$, W. Keller ${ }^{1}$, and K. Swanson ${ }^{1},{ }^{1}$ Animal Sciences, \\ North Dakota State University, Fargo, ND, USA, *kasey. \\ maddockcarlin@ndsu.edu
}

Objectives: The longissimus and the semitendinosus muscles are economically important muscles that vary in meat quality attributes. Study of postmortem metabolism is often focused on glucose metabolism and biochemical changes associated with exhaustion of adenosine triphosphate. However, little is known about postmortem changes in lipid metabolites. The use of metabolomic analysis can provide insight into metabolic processes that have not been evaluated during the early postmortem period. Therefore, the study objective was to evaluate changes in lipid metabolites that occur in 2 muscles from beef between $2 \mathrm{~h}$ and $24 \mathrm{~h}$ postmortem.

Materials and Methods: Steers (body weight $=365 \pm$ $3.9 \mathrm{~kg}$ ) of predominately Angus and Simmental breeding were subjected to a $126-\mathrm{d}(n=46)$ or $154-\mathrm{d}(n=23)$ feeding period as part of a study evaluating supplemental ractopamine hydrochloride. The total mixed rations were delivered once daily at 0800 hours. Steers had ad libitum access to feed and water. Twelve steers ( 6 from each feeding period) were selected at random to be slaughtered at the North Dakota State University Meat Science Laboratory for tissue collection. Muscle samples ( $\sim 50 \mathrm{~g})$ were collected from the longissimus (11th rib) and semitendinosus (midpoint between origin and insertion) at $2 \mathrm{~h}$ and $24 \mathrm{~h}$ after stunning. Samples were frozen at $-80^{\circ} \mathrm{C}$ and stored until further analysis. Samples were submitted to a commercial laboratory for metabolic profile analysis. Samples were prepared and extracted for analysis by ultrahigh performance liquid chromatography-tandem mass spectrometry. Identified compounds were subjected to analysis by analysis of variance. A summary of the differences in metabolites between $2 \mathrm{~h}$ and $24 \mathrm{~h}$ that achieved statistical significance $(P \leq 0.05)$ was provided by the analytical company.

Results: In the longissimus and semitendinosus, there were 56 and 58 compounds related to lipid metabolism that changed $(P<0.05)$ between $2 \mathrm{~h}$ and $24 \mathrm{~h}$, respectively. Oleoyl CoA decreased in both muscle types from $2 \mathrm{~h}$ to $24 \mathrm{~h}$. Malonate and palmitoyl CoA decreased over time in the longissimus but not in the semitendinosus. Long chain polyunsaturated fatty acids (docosapenteaenoate [22:5n3], mead acid [20:3n9], and arachidonate [20:4n6]) increased over time in both muscle tissues. Multiple acyl carnitine species were also elevated in both muscle types; a few decreased over time in the longissimus, which was not observed in the semitendinosus. Many lysophospholipids were increased over time in the semitendinosus $(P<0.05)$ that were not changed in the longissimus.

Conclusion: Overall, these results indicate that lipolysis and release of free fatty acids increased between $2 \mathrm{~h}$ and $24 \mathrm{~h}$ in both muscle types. However, there were marked differences between the muscle types in metabolites from fatty acid and lysophospholipid metabolism. The observed differences in these metabolites could have impacts on mitochondrial membrane integrity, calcium regulation, and apoptosis resulting in differences in aging and meat quality outcomes.

Keywords: beef, lipid, metabolomics, postmortem time 


\section{2 - AN INTEGRATIVE OMICS APPROACH TO UNDERSTAND SARCOPLASMIC RETICULUM'S ROLE IN ELEVATED LEVELS OF FREE CALCIUM IN BROILER WOODY BREAST}

A. Welter ${ }^{1 *}$, W. J. Wu ${ }^{1}$, C. Chun ${ }^{1}$, P. Hammond ${ }^{1}$, T. O'Quinn ${ }^{1}$, M. Chao ${ }^{1}$, E. Geisbrecht ${ }^{2}$, D. Brooks ${ }^{2}$, S. Hartson $^{3}$, B. Bowker ${ }^{4}$, and H. Zhuang ${ }^{4},{ }^{1}$ Animal Sciences and Industry, Kansas State University, Manhattan, KS, USA, ${ }^{2}$ Biochemistry \& Molecular Biophysics, Kansas State University, Manhattan, KS, USA, ${ }^{3}$ Biochemistry \& Molecular Biophysics, Oklahoma State University, Stillwater, OK, USA, ${ }^{4}$ U.S. National Poultry Research Center, USDA, Athens, GA, USA, mdchao@ksu.edu

Objectives: Woody breast (WB) is a myopathy observed in broiler breast meat (Pectoralis major) that results in rubbery texture. A previous study from our lab noted elevated levels of free calcium $\left(\mathrm{Ca}^{2+}\right)$ and sarcomere shortening in WB meat compared to normal. We hypothesize that a sarcoplasmic reticulum (SR) dysfunctionality associated with WB may result in the additional leakage of intracellular $\mathrm{Ca}^{2+}$ into the sarcoplasm. The objective of this study was to utilize an integrative omics (lipidomics/proteomics) approach to investigate the functionality/integrity of WB SR.

Materials and Methods: Fourteen Ross line broiler breast fillets ( 7 severe WB and 7 normal) were collected, packaged, and frozen at $8 \mathrm{~h}$ postmortem from a commercial processing plant. The SR fractions of the samples were extracted via ultracentrifugation through discontinuous sucrose gradients. The lipid was extracted from the SR fractions and utilized for comprehensive phospholipid profile analysis (lipidomics) through electrospray ionization tandem mass spectrometry. The protein was extracted from the SR fractions in urea, and protein concentration was determined. Proteins were separated using high-performance liquid chromatography and identified using an Orbitrap-based mass spectrometry system (proteomics). Level of significance for statistical analysis was set at $P<0.05$ for both omics data.

Results: Sixty-six phospholipid species and 677 proteins (false discovery rate $=0.05$ ) showed differential abundance. Lipidomics data revealed that WB SR had less relative percentage of phosphatidylcholine and more phosphatidylethanolamine (PE), phosphatidylserine (PS), and lysophosphatidylcholine compared to normal SR $(P<$ 0.05). Proteomics data revealed an upregulation of $\mathrm{Ca}^{2+}$ transport proteins (e.g., sarcoplasmic/endoplasmic reticulum calcium adenosine triphosphatase) and a downregulation of proteins responsible for $\mathrm{Ca}^{2+}$ release and signaling (e.g., ryanodine receptors; $P<0.05)$ in WB SR. There was no difference in protein abundance for $\mathrm{Ca}^{2+}$ storage proteins (e.g., calsequestrin). Interestingly, carboxylic ester hydrolase (representing acetylcholinesterase $[\mathrm{AChE}]$ activity) exhibited a 7.62-fold increase in WB SR $(P<0.01)$, and there was also an upregulation of phospholipase A2 in WB SR $(P<0.01)$.

Conclusion: Changes in phospholipid composition and increases in phospholipid catabolism in SR may be partly responsible for the increased free $\mathrm{Ca}^{2+}$ levels in WB meat. WB SR membrane integrity may be compromised due to an upregulation of phospholipase A2, resulting in the hydrolysis of phosphatidylcholine, the main building block of lipid bilayers, into lysophosphatidylcholine. The PE and PS play important roles in the functionality of $\mathrm{Ca}^{2+}$ transport proteins, and the upregulation of $\mathrm{PE}, \mathrm{PS}$, and the $\mathrm{Ca}^{2+}$ transport proteins revealed there was an increase activity in $\mathrm{Ca}^{2+}$ re-sequestration of WB SR. Meanwhile, $\mathrm{Ca}^{2+}$ release and signaling proteins were downregulated, indicating attempts to control $\mathrm{Ca}^{2+}$ outflow from WB SR. So, what is the source of this elevated free $\mathrm{Ca}^{2+}$ in WB meat? The upregulation of AChE activity is a typical indicator of elevated AChE inhibitors as found in Alzheimer and Parkinson disease patients. Perhaps the inhibition of AChE extended the length of action potential and $\mathrm{Ca}^{2+}$ release from the SR. The upregulation of $\mathrm{Ca}^{2+}$ transport proteins and downregulation of $\mathrm{Ca}^{2+}$ release proteins in WB SR may be its attempt to regulate this proposed excessive signaling of $\mathrm{Ca}^{2+}$ release.

Keywords: calcium, lipidomics, proteomics, sarcoplasmic reticulum, woody breast

\section{3 - GLOBAL CHANGES IN RIBOSOMAL PROTEIN EXPRESSION REGULATES MITOCHONDRIAL PROTEIN MASS AND FUNCTION IN DARK-CUTTING BEEF}

F. Kiyimba ${ }^{1 *}$, S. Hartson ${ }^{2}$, J. Rogers ${ }^{2}$, D. VanOverbeke ${ }^{1}$, G. Mafi ${ }^{1}$, and R. Ramanathan ${ }^{1},{ }^{1}$ Department of Animal and Food Sciences, Oklahoma State University, Stillwater, OK, USA, ${ }^{2}$ Department of Biochemistry and Molecular Biology, Oklahoma State University, Stillwater, OK, USA, mdenzer@okstate.edu

Objectives: Mitochondria remain active in postmortem muscles and can influence meat color via oxygen consumption. Previous studies have shown that dark-cutting beef has a high mitochondrial protein mass compared to normal-pH beef. However, the mechanisms that regulate mitochondrial proliferation in dark-cutting beef is still unknown.

Materials and Methods: To evaluate how changes in mitochondrial protein expression may contribute to darkcutting properties, mitochondria were isolated from 11 normal-pH and 11 dark-cutting loins. Oxygen consumption properties of isolated mitochondria from normal-pH and dark-cutting were determined using a Clark-oxygen electrode. Complex specific substrates were added to assess functions of complex I, II, and IV, membrane integrity (by 
addition of cytochrome c), and uncoupled oxidative phosphorylation using a Clark-oxygen electrode. Six $(n=6)$ out of the 11 samples from dark-cutting and normal-pH beef were subjected to liquid chromatography-tandem mass spectrometry-based proteomics analysis. A completely randomized block design was utilized to determine differences in mitochondrial functionality at $P<0.05$, and the experiment was replicated 11 times $(n=11)$. The proteomics experiment was replicated 6 times $(n=6)$. The changes in mitochondrial protein expression were analyzed using multiple bioinformatics approaches and were considered significant at a false discovery rate $<0.05$.

Results: Liquid chromatography-tandem mass spectrometry-based proteomics analysis identified 1,863 proteins in the mitochondrial proteomes of dark-cutting and normal$\mathrm{pH}$ beef. Of these, 162 proteins had significant changes in expression between dark-cutting versus normal-pH beef at false discovery rate $<0.05$. In dark-cutting beef, 55 proteins were upregulated, while 37 were downregulated with a fold change $>1.5$. Functional annotation showed that these changes in protein expression comprised proteins involved in translation biological processes, structural constituent of ribosome, and large ribosomal subunit ribosomal RNA binding molecular functions. Pathway analysis showed that the majority of the upregulated DEP were involved in the ribosomal and mitochondrial electron transport pathways. The impact of these changes in mitochondrial protein expressions was further validated by assessing mitochondrial function in dark-cutting versus normal-pH beef. Dark-cutting beef had greater $(P<0.05)$ mitochondrial complex II respiration and uncoupled oxidative phosphorylation compared to normal-pH beef. However, evaluation of mitochondrial membrane integrity and respiration at complexes I, and IV, did not show significant differences $(P>0.05)$ between dark-cutting versus normal-pH beef.

Conclusion: These results showed that changes in ribosomal proteins might regulate mitochondrial protein content and function in dark-cutting beef. Thus, a greater mitochondrial function could promote greater oxygen consumption, limiting myoglobin oxygenation in dark-cutting beef.

Keywords: dark-cutting beef, mitochondria, oxygen consumption, proteomics, ribosome

\section{4 - EFFECTS OF LIGHT ON NONENZYMATIC METMYOGLOBIN REDUCTION IN VITRO}

M. Scott ${ }^{1 *}$, M. Denzer ${ }^{1}$, G. Mafi ${ }^{1}$, and R. Ramanathan ${ }^{1}$, ${ }^{1}$ Department of Animal and Food Sciences, Oklahoma State University, Stillwater, OK, USA, *mdenzer@ okstate.edu

Objectives: Predominant oxymyoglobin imparts consumerpreferred bright cherry-red meat color, but discoloration on meat negatively impacts purchasing decisions, which can lead to food waste. Discoloration occurs by the oxidation of oxymyoglobin, forming metmyoglobin, a brown pigment. Oxidation can occur more readily in common retail settings such as exposure to light and oxygen $\left(\mathrm{O}_{2}\right)$; however, meat has an inherent ability to reduce the brown pigment and form the cherry-red color through metmyoglobin reducing systems. Research has indicated that inherently present electron donors and carriers could contribute to nonenzymatic metmyoglobin reduction. However, limited knowledge is available on the impact of light on nonenzymatic reduction. The objective of this study was to evaluate the effect of light and dark storage conditions on nonenzymatic metmyoglobin reduction in vitro.

Materials and Methods: Solutions of ascorbate and NADH were used as the electron donors and cytochrome $c$ (cyt-c) and methylene blue (MB) as the electron carriers. Equine metmyoglobin solution at $\mathrm{pH} 5.6$ was combined with different electron donors and carriers in a 96-well plate. There were 6 treatments used throughout the entirety of the experiment: (1) NADH, (2) NADH + MB, (3) $\mathrm{NADH}+\mathrm{MB}+$ ethylenediaminetetraacetic acid (EDTA), (4) $\mathrm{NADH}+$ cyt-c + EDTA, (5) asborbate + MB + EDTA, and (6) ascorbate + cyt-c + EDTA. To evaluate lighting effects, the well-plate was kept under LED lighting, and readings were taken every $5 \mathrm{~min}$ for $25 \mathrm{~min}$ using a spectrophotometer set to $582 \mathrm{~nm}$. The preliminary research indicated that $25-$ min incubation provided consistent results with less variation. The same combinations were evaluated in dark storage with the previously mentioned method. The experiment was replicated 3 times with 6 wells per treatment for each replicate. The method was never repeated on the solutions in a single 96-well plate, as each plate was only evaluated once per 25 -min reading, including separate 96-well plates for each light and dark storage reading. Therefore, each treatment was evaluated 18 different times for each respective light condition and 36 times in total. The data were analyzed using the Mixed Procedure of SAS (SAS Institute Inc., Cary, NC) with a completely randomized design.

Results: There was a significant treatment by lighting effect on the metmyoglobin reduction. The combination of $\mathrm{NADH}$ and MB had an increase $(P<0.0001)$ in metmyoglobin reduction in light conditions compared to dark storage. Furthermore, in the presence of light, $\mathrm{NADH}+\mathrm{MB}+$ EDTA had an increase $(P<0.0001)$ in metmyoglobin reduction. The metmyoglobin reduction was limited in the presence of $\mathrm{NADH}+$ cyt-c in both lighting conditions. Metmyoglobin reduction was significantly higher in the presence of cyt-c and ascorbate compared with cyt-c and NADH for light and dark conditions. In addition, metmyoglobin reduction increased with the presence of light and the combination of ascorbate and MB $(P<0.001)$. The reduction of metmyoglobin in the presence of NADH alone significantly increased in light conditions.

Conclusion: In conclusion, the current in vitro research demonstrates that inherently present electron donors and 
carriers can contribute to nonenzymatic metmyoglobin reduction in retail light settings and meat $\mathrm{pH}$. Additionally, the study indicated that the characteristics of the individual cofactors impacted the reduction under various lighting conditions.

Keywords: meat color, metmyoglobin reducing ability, myoglobin 